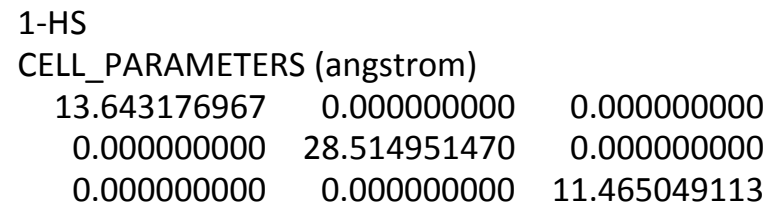

\begin{tabular}{ll}
0.5514061623 & 0.5136638727 \\
0.6989571150 & 0.9680447013 \\
0.4485938377 & 0.5136638727 \\
0.3010429140 & 0.9680447013 \\
0.0514061513 & 0.4863361273 \\
0.1989570860 & 0.0319553177 \\
0.9485938377 & 0.4863361273 \\
0.8010428850 & 0.0319553177 \\
0.5425423529 & 0.6448243638 \\
0.6217871647 & 0.5568688071 \\
0.6289805145 & 0.9263578248 \\
0.7076262964 & 0.8483593649 \\
0.4574576171 & 0.6448243638 \\
0.3782128653 & 0.5568688071 \\
0.3710194855 & 0.9263578248 \\
0.2923737036 & 0.8483593649 \\
0.0425423719 & 0.3551756062 \\
0.1217871347 & 0.4431311929 \\
0.1289805145 & 0.0736421672 \\
0.2076263104 & 0.1516406501 \\
0.9574576471 & 0.3551756062 \\
0.8782128353 & 0.4431311929 \\
0.8710194855 & 0.0736421672 \\
0.7923737036 & 0.1516406501 \\
0.5591487438 & 0.3554763732 \\
0.4779862054 & 0.5010121512 \\
0.5554859427 & 0.6120782835 \\
0.5505064846 & 0.3709526643 \\
0.7716181047 & 0.9878194081 \\
0.6909321737 & 0.1294630785 \\
0.6957482688 & 0.8587672626 \\
0.6956460650 & 0.0992643237 \\
0.4408512272 & 0.3554763732 \\
0.5220138246 & 0.5010121512 \\
0.4445140873 & 0.6120782835 \\
0.4494935444 & 0.3709526643 \\
0.2283818663 & 0.9878194081 \\
0.3090678263 & 0.1294630785 \\
0.3042517012 & 0.8587672626 \\
0.3043539350 & 0.0992643237 \\
0.0591487698 & 0.6445236568 \\
0.9779861754 & 0.4989878488 \\
0.0554859247 & 0.3879216865 \\
0.0505064626 & 0.6290473067 \\
0.2716181337 & 0.0121806029 \\
0.1909321587 & 0.8705369215 \\
0.1957482838 & 0.1412327224 \\
0.1956460650 & 0.9007356763 \\
0.9408512562 & 0.6445236568 \\
0.0220138246 & 0.4989878488 \\
0.9445140573 & 0.3879216865 \\
\hline
\end{tabular}




\begin{tabular}{|c|c|c|c|}
\hline $\mathrm{N}$ & 0.6939889296 & 0.9494935154 & 0.6290473067 \\
\hline $\mathrm{N}$ & 0.1472624848 & 0.7283818953 & 0.0121806029 \\
\hline $\mathrm{N}$ & 0.0408395219 & 0.8090678263 & 0.8705369215 \\
\hline $\mathrm{N}$ & 0.2480278585 & 0.8042517312 & 0.1412327224 \\
\hline $\mathrm{N}$ & 0.2418048445 & 0.8043539350 & 0.9007356763 \\
\hline C & 0.4375357387 & 0.5804375563 & 0.6970190459 \\
\hline C & 0.4936415466 & 0.5619772150 & 0.1428426618 \\
\hline C & 0.2190520009 & 0.4640856514 & 0.4191880113 \\
\hline C & 0.1678822858 & 0.5470811961 & 0.7275273934 \\
\hline C & 0.3999838550 & 0.6258936440 & 0.6448104401 \\
\hline C & 0.9944794730 & 0.5607123917 & 0.7224542827 \\
\hline C & 0.5452466007 & 0.5575001565 & 0.2473493465 \\
\hline C & 0.0786161092 & 0.5494907554 & 0.7859230060 \\
\hline C & 0.4275865082 & 0.6693069121 & 0.6933158073 \\
\hline C & 0.3230093454 & 0.4459490414 & 0.5742166634 \\
\hline C & 0.5281116835 & 0.6245554719 & 0.8410219998 \\
\hline C & 0.4997842350 & 0.5811596313 & 0.7931795877 \\
\hline C & 0.1956468361 & 0.4167518347 & 0.4042604506 \\
\hline C & 0.2364912986 & 0.3836764005 & 0.4793909789 \\
\hline C & 0.3005138355 & 0.3985407088 & 0.5669265015 \\
\hline C & 0.1706827281 & 0.5008023033 & 0.3430765277 \\
\hline C & 0.2361669632 & 0.5746053656 & 0.2678893213 \\
\hline C & 0.3916641979 & 0.5654946020 & 0.1468672245 \\
\hline C & 0.0026871455 & 0.5682532955 & 0.6027425943 \\
\hline C & 0.1101266624 & 0.5764314930 & 0.4221043715 \\
\hline C & 0.3445393896 & 0.5650230747 & 0.2549240217 \\
\hline C & 0.4929779518 & 0.5555333195 & 0.3512714254 \\
\hline C & 0.4871923125 & 0.6698841468 & 0.7917822063 \\
\hline C & 0.0945670118 & 0.5653841984 & 0.5498768635 \\
\hline C & 0.6010958259 & 0.8054914102 & 0.9265266622 \\
\hline C & 0.7173537659 & 0.7833788711 & 0.0653556811 \\
\hline C & 0.5955793565 & 0.6822983443 & 0.2252060943 \\
\hline C & 0.5530585382 & 0.6792415140 & 0.3357392839 \\
\hline C & 0.4428508386 & 0.6945337742 & 0.1401566852 \\
\hline C & 0.3952321490 & 0.6898173505 & 0.2466039718 \\
\hline C & 0.7732154548 & 0.7444686340 & 0.1268886401 \\
\hline C & 0.7515487938 & 0.7071955601 & 0.7446544637 \\
\hline C & 0.8368345985 & 0.7027147430 & 0.6791201964 \\
\hline C & 0.8192282084 & 0.6676015825 & 0.0400552495 \\
\hline C & 0.8299178876 & 0.6805463980 & 0.9128647205 \\
\hline C & 0.6211978913 & 0.8528148288 & 0.9426414353 \\
\hline C & 0.9210600245 & 0.6854783671 & 0.7335004574 \\
\hline C & 0.4515256397 & 0.6827146125 & 0.3467885179 \\
\hline C & 0.7402806673 & 0.8304248476 & 0.0870190664 \\
\hline C & 0.7025488592 & 0.6714184439 & 0.2037071118 \\
\hline C & 0.6915228474 & 0.8655421999 & 0.0254021921 \\
\hline C & 0.9177665002 & 0.6747424089 & 0.8519632095 \\
\hline C & 0.5624642613 & 0.4195624727 & 0.6970190459 \\
\hline C & 0.5063584534 & 0.4380227550 & 0.1428426618 \\
\hline C & 0.7809479841 & 0.5359143486 & 0.4191880113 \\
\hline C & 0.8321177442 & 0.4529187739 & 0.7275273934 \\
\hline C & 0.6000161450 & 0.3741063560 & 0.6448104401 \\
\hline C & 0.0055195740 & 0.4392876383 & 0.7224542827 \\
\hline C & 0.4547533693 & 0.4424998135 & 0.2473493465 \\
\hline C & 0.9213838758 & 0.4505092746 & 0.7859230060 \\
\hline C & 0.5724134918 & 0.3306931179 & 0.6933158073 \\
\hline C & 0.6769906546 & 0.5540509586 & 0.5742166634 \\
\hline C & 0.4718883165 & 0.3754444981 & 0.8410219998 \\
\hline
\end{tabular}




\begin{tabular}{|c|c|c|c|}
\hline C & 0.5002157650 & 0.4188403987 & 0.7931795877 \\
\hline C & 0.8043531489 & 0.5832481353 & 0.4042604506 \\
\hline C & 0.7635087164 & 0.6163235995 & 0.4793909789 \\
\hline C & 0.6994861945 & 0.6014592912 & 0.5669265015 \\
\hline C & 0.8293172569 & 0.4991977267 & 0.3430765277 \\
\hline C & 0.7638330518 & 0.4253946044 & 0.2678893213 \\
\hline C & 0.6083357721 & 0.4345053680 & 0.1468672245 \\
\hline C & 0.9973128685 & 0.4317467045 & 0.6027425943 \\
\hline C & 0.8898733306 & 0.4235684770 & 0.4221043715 \\
\hline C & 0.6554606104 & 0.4349769253 & 0.2549240217 \\
\hline C & 0.5070220782 & 0.4444666805 & 0.3512714254 \\
\hline C & 0.5128077175 & 0.3301158532 & 0.7917822063 \\
\hline C & 0.9054329882 & 0.4346158316 & 0.5498768635 \\
\hline C & 0.3989041741 & 0.1945085758 & 0.9265266622 \\
\hline C & 0.2826462041 & 0.2166210999 & 0.0653556811 \\
\hline C & 0.4044206435 & 0.3177016857 & 0.2252060943 \\
\hline C & 0.4469424458 & 0.3207584860 & 0.3357392839 \\
\hline C & 0.5571491614 & 0.3054662548 & 0.1401566852 \\
\hline C & 0.6047678510 & 0.3101826195 & 0.2466039718 \\
\hline C & 0.2267845302 & 0.2555313660 & 0.1268886401 \\
\hline C & 0.2484512212 & 0.2928044699 & 0.7446544637 \\
\hline C & 0.1631654315 & 0.2972852570 & 0.6791201964 \\
\hline C & 0.1807718066 & 0.3323984475 & 0.0400552495 \\
\hline C & 0.1700821274 & 0.3194536020 & 0.9128647205 \\
\hline C & 0.3788020787 & 0.1471851862 & 0.9426414353 \\
\hline C & 0.0789399905 & 0.3145216629 & 0.7335004574 \\
\hline C & 0.5484743603 & 0.3172853875 & 0.3467885179 \\
\hline C & 0.2597193627 & 0.1695751674 & 0.0870190664 \\
\hline C & 0.2974511118 & 0.3285815561 & 0.2037071118 \\
\hline C & 0.3084771816 & 0.1344578291 & 0.0254021921 \\
\hline C & 0.0822335068 & 0.3252575611 & 0.8519632095 \\
\hline C & 0.0624642653 & 0.0804375273 & 0.3029809541 \\
\hline C & 0.0063584434 & 0.0619772450 & 0.8571573082 \\
\hline C & 0.2809479841 & 0.9640856514 & 0.5808120187 \\
\hline C & 0.3321177142 & 0.0470812191 & 0.2724726066 \\
\hline C & 0.1000161600 & 0.1258936440 & 0.3551895599 \\
\hline C & 0.5055195140 & 0.0607123657 & 0.2775456883 \\
\hline C & 0.9547533993 & 0.0575001715 & 0.7526506535 \\
\hline C & 0.4213838758 & 0.0494907334 & 0.2140770090 \\
\hline C & 0.0724134838 & 0.1693068971 & 0.3066841627 \\
\hline C & 0.1769906546 & 0.9459490414 & 0.4257833366 \\
\hline C & 0.9718883165 & 0.1245554869 & 0.1589780302 \\
\hline C & 1.0002157650 & 0.0811596083 & 0.2068203973 \\
\hline C & 0.3043531789 & 0.9167518647 & 0.5957395494 \\
\hline C & 0.2635086864 & 0.8836764005 & 0.5206100051 \\
\hline C & 0.1994861795 & 0.8985407088 & 0.4330734985 \\
\hline C & 0.3293172869 & 0.0008022823 & 0.6569235013 \\
\hline C & 0.2638330518 & 0.0746053886 & 0.7321106487 \\
\hline C & 0.1083358021 & 0.0654946240 & 0.8531327755 \\
\hline C & 0.4973128685 & 0.0682532955 & 0.3972573757 \\
\hline C & 0.3898733306 & 0.0764315150 & 0.5778956285 \\
\hline C & 0.1554606104 & 0.0650230747 & 0.7450759783 \\
\hline C & 0.0070220582 & 0.0555333085 & 0.6487285446 \\
\hline C & 0.0128076985 & 0.1698841318 & 0.2082177937 \\
\hline C & 0.4054329882 & 0.0653841724 & 0.4501231665 \\
\hline C & 0.8989041741 & 0.3054914392 & 0.0734733158 \\
\hline C & 0.7826462341 & 0.2833789001 & 0.9346443189 \\
\hline C & 0.9044206435 & 0.1822983293 & 0.7747939057 \\
\hline
\end{tabular}




\begin{tabular}{|c|c|c|c|}
\hline C & 0.9469424758 & 0.1792415290 & 0.6642607161 \\
\hline C & 0.0571491574 & 0.1945337592 & 0.8598433148 \\
\hline C & 0.1047678650 & 0.1898173805 & 0.7533960282 \\
\hline C & 0.7267845452 & 0.2444686340 & 0.8731114189 \\
\hline C & 0.7484512062 & 0.2071955301 & 0.2553455363 \\
\hline C & 0.6631654015 & 0.2027147430 & 0.3208797736 \\
\hline C & 0.6807717916 & 0.1676015525 & 0.9599447505 \\
\hline C & 0.6700821124 & 0.1805463980 & 0.0871352945 \\
\hline C & 0.8788020487 & 0.3528147988 & 0.0573585907 \\
\hline C & 0.5789399755 & 0.1854783371 & 0.2664995726 \\
\hline C & 0.0484743563 & 0.1827146125 & 0.6532114821 \\
\hline C & 0.7597193327 & 0.3304248176 & 0.9129809336 \\
\hline C & 0.7974510818 & 0.1714184589 & 0.7962928882 \\
\hline C & 0.8084771526 & 0.3655421709 & 0.9745977479 \\
\hline C & 0.5822334998 & 0.1747424389 & 0.1480367755 \\
\hline C & 0.9375357387 & 0.9195624437 & 0.3029809541 \\
\hline C & 0.9936415466 & 0.9380227850 & 0.8571573082 \\
\hline C & 0.7190520159 & 0.0359143486 & 0.5808120187 \\
\hline C & 0.6678822558 & 0.9529188039 & 0.2724726066 \\
\hline C & 0.8999838550 & 0.8741063560 & 0.3551895599 \\
\hline C & 0.4944804860 & 0.9392876083 & 0.2775456883 \\
\hline C & 0.0452466007 & 0.9424998435 & 0.7526506535 \\
\hline C & 0.5786161242 & 0.9505092446 & 0.2140770090 \\
\hline C & 0.9275865082 & 0.8306930879 & 0.3066841627 \\
\hline C & 0.8230093454 & 0.0540508996 & 0.4257833366 \\
\hline C & 0.0281116835 & 0.8754445281 & 0.1589780302 \\
\hline C & -0.0002157650 & 0.9188403687 & 0.2068203973 \\
\hline C & 0.6956468511 & 0.0832481943 & 0.5957395494 \\
\hline C & 0.7364912836 & 0.1163235995 & 0.5206100051 \\
\hline C & 0.8005138055 & 0.1014593512 & 0.4330734985 \\
\hline C & 0.6706827431 & 0.9991976967 & 0.6569235013 \\
\hline C & 0.7361669482 & 0.9253946344 & 0.7321106487 \\
\hline C & 0.8916642279 & 0.9345053980 & 0.8531327755 \\
\hline C & 0.5026871315 & 0.9317467045 & 0.3972573757 \\
\hline C & 0.6101266694 & 0.9235685070 & 0.5778956285 \\
\hline C & 0.8445393896 & 0.9349769253 & 0.7450759783 \\
\hline C & 0.9929779218 & 0.9444666805 & 0.6487285446 \\
\hline C & 0.9871922825 & 0.8301158532 & 0.2082177937 \\
\hline C & 0.5945670118 & 0.9346158016 & 0.4501231665 \\
\hline C & 0.1010958259 & 0.6945085898 & 0.0734733158 \\
\hline C & 0.2173537659 & 0.7166211289 & 0.9346443189 \\
\hline C & 0.0955794165 & 0.8177016557 & 0.7747939057 \\
\hline C & 0.0530575242 & 0.8207584860 & 0.6642607161 \\
\hline C & 0.9428508386 & 0.8054662258 & 0.8598433148 \\
\hline C & 0.8952321490 & 0.8101826495 & 0.7533960282 \\
\hline C & 0.2732154548 & 0.7555313660 & 0.8731114189 \\
\hline C & 0.2515487938 & 0.7928044399 & 0.2553455363 \\
\hline C & 0.3368345985 & 0.7972852570 & 0.3208797736 \\
\hline C & 0.3192281484 & 0.8323984175 & 0.9599447505 \\
\hline C & 0.3299178876 & 0.8194536020 & 0.0871352945 \\
\hline C & 0.1211979513 & 0.6471851712 & 0.0573585907 \\
\hline C & 0.4210600245 & 0.8145216329 & 0.2664995726 \\
\hline C & 0.9515256397 & 0.8172853875 & 0.6532114821 \\
\hline C & 0.2402806673 & 0.6695751524 & 0.9129809336 \\
\hline C & 0.2025489182 & 0.8285815561 & 0.7962928882 \\
\hline C & 0.1915228474 & 0.6344578001 & 0.9745977479 \\
\hline C & 0.4177664412 & 0.8252575911 & 0.1480367755 \\
\hline $\mathrm{H}$ & 0.5330321989 & 0.5626645053 & 0.0600480570 \\
\hline
\end{tabular}




\begin{tabular}{|c|c|c|c|}
\hline $\mathrm{H}$ & 0.2355131383 & 0.5376045341 & 0.7729576692 \\
\hline $\mathrm{H}$ & 0.9236002816 & 0.5627113897 & 0.7662904470 \\
\hline $\mathrm{H}$ & 0.6247916690 & 0.5544176887 & 0.2468048137 \\
\hline $\mathrm{H}$ & 0.0755505784 & 0.5416132948 & 0.8789061775 \\
\hline $\mathrm{H}$ & 0.4007283007 & 0.7021462266 & 0.6562948556 \\
\hline $\mathrm{H}$ & 0.3734582978 & 0.4597993484 & 0.6395313811 \\
\hline $\mathrm{H}$ & 0.5292607204 & 0.5486912735 & 0.8294124776 \\
\hline $\mathrm{H}$ & 0.1444079589 & 0.4057971816 & 0.3363596605 \\
\hline $\mathrm{H}$ & 0.2159341320 & 0.3469095249 & 0.4706323311 \\
\hline $\mathrm{H}$ & 0.3326746893 & 0.3740856256 & 0.6291605078 \\
\hline $\mathrm{H}$ & 0.0905827789 & 0.4956071694 & 0.3480651544 \\
\hline $\mathrm{H}$ & 0.1906270457 & 0.4933997874 & 0.2517669319 \\
\hline $\mathrm{H}$ & 0.2270680981 & 0.6126353548 & 0.2812109237 \\
\hline $\mathrm{H}$ & 0.1973895862 & 0.5650691357 & 0.1871022510 \\
\hline $\mathrm{H}$ & 0.3487091197 & 0.5679164762 & 0.0669649804 \\
\hline $\mathrm{H}$ & 0.9389047812 & 0.5772795543 & 0.5504378039 \\
\hline $\mathrm{H}$ & 0.0418778927 & 0.5707719432 & 0.3722778711 \\
\hline $\mathrm{H}$ & 0.1260348426 & 0.6141980425 & 0.4156879753 \\
\hline $\mathrm{H}$ & 0.5293980639 & 0.5507603106 & 0.4350850758 \\
\hline $\mathrm{H}$ & 0.5476926895 & 0.7934526268 & 0.8624321968 \\
\hline $\mathrm{H}$ & 0.5990579899 & 0.6731985935 & 0.4120390364 \\
\hline $\mathrm{H}$ & 0.4025972759 & 0.7009622779 & 0.0594618715 \\
\hline $\mathrm{H}$ & 0.3156080764 & 0.6924701301 & 0.2508869409 \\
\hline $\mathrm{H}$ & 0.8519285426 & 0.7485333595 & 0.1070325677 \\
\hline $\mathrm{H}$ & 0.7684433582 & 0.7500601878 & 0.2217583873 \\
\hline $\mathrm{H}$ & 0.6834385656 & 0.7207256672 & 0.7070435678 \\
\hline $\mathrm{H}$ & 0.8382947056 & 0.7127798845 & 0.5873899174 \\
\hline $\mathrm{H}$ & 0.7970397112 & 0.6304374944 & 0.0436136916 \\
\hline $\mathrm{H}$ & 0.8897966539 & 0.6703892186 & 0.0862325151 \\
\hline $\mathrm{H}$ & 0.5811058628 & 0.8787982096 & 0.8918397674 \\
\hline $\mathrm{H}$ & 0.9880170467 & 0.6808048840 & 0.6829038638 \\
\hline $\mathrm{H}$ & 0.4164398348 & 0.6791667134 & 0.4317981500 \\
\hline $\mathrm{H}$ & 0.7959907945 & 0.8390748711 & 0.1518650874 \\
\hline $\mathrm{H}$ & 0.7473020241 & 0.6791344298 & 0.2812730277 \\
\hline $\mathrm{H}$ & 0.7077268924 & 0.6333787742 & 0.1874877965 \\
\hline $\mathrm{H}$ & 0.7079520229 & 0.9024519890 & 0.0411601533 \\
\hline $\mathrm{H}$ & 0.9822878063 & 0.6615360041 & 0.8973180478 \\
\hline $\mathrm{H}$ & 0.4669677711 & 0.4373355247 & 0.0600480570 \\
\hline $\mathrm{H}$ & 0.7644868767 & 0.4623954659 & 0.7729576692 \\
\hline $\mathrm{H}$ & 0.0763997184 & 0.4372886103 & 0.7662904470 \\
\hline $\mathrm{H}$ & 0.3752083020 & 0.4455823113 & 0.2468048137 \\
\hline $\mathrm{H}$ & 0.9244494516 & 0.4583867052 & 0.8789061775 \\
\hline $\mathrm{H}$ & 0.5992716993 & 0.2978537434 & 0.6562948556 \\
\hline $\mathrm{H}$ & 0.6265417022 & 0.5402006516 & 0.6395313811 \\
\hline $\mathrm{H}$ & 0.4707392796 & 0.4513087265 & 0.8294124776 \\
\hline $\mathrm{H}$ & 0.8555920411 & 0.5942028184 & 0.3363596605 \\
\hline $\mathrm{H}$ & 0.7840658830 & 0.6530905051 & 0.4706323311 \\
\hline $\mathrm{H}$ & 0.6673253107 & 0.6259143744 & 0.6291605078 \\
\hline $\mathrm{H}$ & 0.9094172061 & 0.5043928006 & 0.3480651544 \\
\hline $\mathrm{H}$ & 0.8093729243 & 0.5066002126 & 0.2517669319 \\
\hline $\mathrm{H}$ & 0.7729319169 & 0.3873646152 & 0.2812109237 \\
\hline $\mathrm{H}$ & 0.8026104138 & 0.4349308643 & 0.1871022510 \\
\hline $\mathrm{H}$ & 0.6512908803 & 0.4320835238 & 0.0669649804 \\
\hline $\mathrm{H}$ & 0.0610952188 & 0.4227204457 & 0.5504378039 \\
\hline $\mathrm{H}$ & 0.9581221153 & 0.4292280268 & 0.3722778711 \\
\hline $\mathrm{H}$ & 0.8739651354 & 0.3858019275 & 0.4156879753 \\
\hline $\mathrm{H}$ & 0.4706019361 & 0.4492397194 & 0.4350850758 \\
\hline $\mathrm{H}$ & 0.4523073105 & 0.2065474022 & 0.8624321968 \\
\hline
\end{tabular}




\begin{tabular}{|c|c|c|c|}
\hline $\mathrm{H}$ & 0.4009419801 & 0.3268014065 & 0.4120390364 \\
\hline $\mathrm{H}$ & 0.5974027531 & 0.2990377221 & 0.0594618715 \\
\hline $\mathrm{H}$ & 0.6843918936 & 0.3075298699 & 0.2508869409 \\
\hline $\mathrm{H}$ & 0.1480714424 & 0.2514666405 & 0.1070325677 \\
\hline $\mathrm{H}$ & 0.2315566568 & 0.2499398122 & 0.2217583873 \\
\hline $\mathrm{H}$ & 0.3165614644 & 0.2792743328 & 0.7070435678 \\
\hline $\mathrm{H}$ & 0.1617052944 & 0.2872201155 & 0.5873899174 \\
\hline $\mathrm{H}$ & 0.2029602738 & 0.3695624756 & 0.0436136916 \\
\hline $\mathrm{H}$ & 0.1102033531 & 0.3296107814 & 0.0862325151 \\
\hline $\mathrm{H}$ & 0.4188941072 & 0.1212017604 & 0.8918397674 \\
\hline $\mathrm{H}$ & 0.0119829463 & 0.3191951450 & 0.6829038638 \\
\hline $\mathrm{H}$ & 0.5835601362 & 0.3208332566 & 0.4317981500 \\
\hline $\mathrm{H}$ & 0.2040092055 & 0.1609251289 & 0.1518650874 \\
\hline $\mathrm{H}$ & 0.2526979759 & 0.3208655992 & 0.2812730277 \\
\hline $\mathrm{H}$ & 0.2922731076 & 0.3666212258 & 0.1874877965 \\
\hline $\mathrm{H}$ & 0.2920479771 & 0.0975479890 & 0.0411601533 \\
\hline $\mathrm{H}$ & 0.0177122237 & 0.3384639959 & 0.8973180478 \\
\hline $\mathrm{H}$ & 0.9669678011 & 0.0626644753 & 0.9399519280 \\
\hline $\mathrm{H}$ & 0.2644868767 & 0.0376045231 & 0.2270423308 \\
\hline $\mathrm{H}$ & 0.5763997184 & 0.0627113967 & 0.2337095380 \\
\hline $\mathrm{H}$ & 0.8752082720 & 0.0544176847 & 0.7531952163 \\
\hline $\mathrm{H}$ & 0.4244494216 & 0.0416132798 & 0.1210937995 \\
\hline $\mathrm{H}$ & 0.0992717073 & 0.2021462566 & 0.3437051444 \\
\hline $\mathrm{H}$ & 0.1265417172 & 0.9597993484 & 0.3604686189 \\
\hline $\mathrm{H}$ & 0.9707392796 & 0.0486912735 & 0.1705875524 \\
\hline $\mathrm{H}$ & 0.3555920411 & 0.9057971816 & 0.6636403395 \\
\hline $\mathrm{H}$ & 0.2840658830 & 0.8469094949 & 0.5293676979 \\
\hline $\mathrm{H}$ & 0.1673253107 & 0.8740856256 & 0.3708394922 \\
\hline $\mathrm{H}$ & 0.4094172361 & 0.9956071994 & 0.6519348456 \\
\hline $\mathrm{H}$ & 0.3093729543 & 0.9933997874 & 0.7482330981 \\
\hline $\mathrm{H}$ & 0.2729319169 & 0.1126353698 & 0.7187880923 \\
\hline $\mathrm{H}$ & 0.3026104138 & 0.0650691207 & 0.8128977790 \\
\hline $\mathrm{H}$ & 0.1512908803 & 0.0679164762 & 0.9330350416 \\
\hline $\mathrm{H}$ & 0.5610952188 & 0.0772795393 & 0.4495621661 \\
\hline $\mathrm{H}$ & 0.4581221153 & 0.0707719652 & 0.6277221589 \\
\hline $\mathrm{H}$ & 0.3739651654 & 0.1141980645 & 0.5843130087 \\
\hline $\mathrm{H}$ & 0.9706019361 & 0.0507602916 & 0.5649148942 \\
\hline $\mathrm{H}$ & 0.9523073105 & 0.2934525978 & 0.1375678032 \\
\hline $\mathrm{H}$ & 0.9009420101 & 0.1731985785 & 0.5879609636 \\
\hline $\mathrm{H}$ & 0.0974027311 & 0.2009622779 & 0.9405381285 \\
\hline $\mathrm{H}$ & 0.1843919086 & 0.1924701301 & 0.7491130591 \\
\hline $\mathrm{H}$ & 0.6480714574 & 0.2485333445 & 0.8929674923 \\
\hline $\mathrm{H}$ & 0.7315567018 & 0.2500601878 & 0.7782416127 \\
\hline $\mathrm{H}$ & 0.8165624474 & 0.2207256532 & 0.2929564022 \\
\hline $\mathrm{H}$ & 0.6617052944 & 0.2127798845 & 0.4126100826 \\
\hline $\mathrm{H}$ & 0.7029602888 & 0.1304375094 & 0.9563863084 \\
\hline $\mathrm{H}$ & 0.6102033461 & 0.1703892336 & 0.9137674259 \\
\hline $\mathrm{H}$ & 0.9188940772 & 0.3787982396 & 0.1081602256 \\
\hline $\mathrm{H}$ & 0.5119829533 & 0.1808048550 & 0.3170961662 \\
\hline $\mathrm{H}$ & 0.0835601652 & 0.1791667434 & 0.5682018500 \\
\hline $\mathrm{H}$ & 0.7040092055 & 0.3390748711 & 0.8481348526 \\
\hline $\mathrm{H}$ & 0.7526979759 & 0.1791344008 & 0.7187259583 \\
\hline $\mathrm{H}$ & 0.7922731076 & 0.1333787742 & 0.8125122035 \\
\hline $\mathrm{H}$ & 0.7920479771 & 0.4024520180 & 0.9588397867 \\
\hline $\mathrm{H}$ & 0.5177121937 & 0.1615360191 & 0.1026819672 \\
\hline $\mathrm{H}$ & 0.0330321989 & 0.9373354947 & 0.9399519280 \\
\hline $\mathrm{H}$ & 0.7355131233 & 0.9623954659 & 0.2270423308 \\
\hline $\mathrm{H}$ & 0.4236002816 & 0.9372886103 & 0.2337095380 \\
\hline
\end{tabular}




\begin{tabular}{|c|c|c|c|}
\hline $\mathrm{H}$ & 0.1247917280 & 0.9455823113 & 0.7531952163 \\
\hline $\mathrm{H}$ & 0.5755505484 & 0.9583867052 & 0.1210937995 \\
\hline $\mathrm{H}$ & 0.9007283007 & 0.7978537734 & 0.3437051444 \\
\hline $\mathrm{H}$ & 0.8734582978 & 0.0402006516 & 0.3604686189 \\
\hline $\mathrm{H}$ & 0.0292607204 & 0.9513087265 & 0.1705875524 \\
\hline $\mathrm{H}$ & 0.6444079589 & 0.0942028184 & 0.6636403395 \\
\hline $\mathrm{H}$ & 0.7159341170 & 0.1530905051 & 0.5293676979 \\
\hline $\mathrm{H}$ & 0.8326746893 & 0.1259144344 & 0.3708394922 \\
\hline $\mathrm{H}$ & 0.5905827939 & 0.0043928006 & 0.6519348456 \\
\hline $\mathrm{H}$ & 0.6906270757 & 0.0066001536 & 0.7482330981 \\
\hline $\mathrm{H}$ & 0.7270680831 & 0.8873646452 & 0.7187880923 \\
\hline $\mathrm{H}$ & 0.6973895862 & 0.9349308643 & 0.8128977790 \\
\hline $\mathrm{H}$ & 0.8487091197 & 0.9320835238 & 0.9330350416 \\
\hline $\mathrm{H}$ & 0.4389047512 & 0.9227204457 & 0.4495621661 \\
\hline $\mathrm{H}$ & 0.5418778847 & 0.9292280568 & 0.6277221589 \\
\hline $\mathrm{H}$ & 0.6260348646 & 0.8858019575 & 0.5843130087 \\
\hline $\mathrm{H}$ & 0.0293981229 & 0.9492396894 & 0.5649148942 \\
\hline $\mathrm{H}$ & 0.0476927495 & 0.7065473732 & 0.1375678032 \\
\hline $\mathrm{H}$ & 0.0990579899 & 0.8268014065 & 0.5879609636 \\
\hline $\mathrm{H}$ & 0.9025972469 & 0.7990377221 & 0.9405381285 \\
\hline $\mathrm{H}$ & 0.8156081064 & 0.8075298699 & 0.7491130591 \\
\hline $\mathrm{H}$ & 0.3519285426 & 0.7514666405 & 0.8929674923 \\
\hline $\mathrm{H}$ & 0.2684432982 & 0.7499398122 & 0.7782416127 \\
\hline $\mathrm{H}$ & 0.1834385656 & 0.7792743328 & 0.2929564022 \\
\hline $\mathrm{H}$ & 0.3382947056 & 0.7872201155 & 0.4126100826 \\
\hline $\mathrm{H}$ & 0.2970397112 & 0.8695625056 & 0.9563863084 \\
\hline $\mathrm{H}$ & 0.3897965949 & 0.8296107814 & 0.9137674259 \\
\hline $\mathrm{H}$ & 0.0811059228 & 0.6212017904 & 0.1081602256 \\
\hline $\mathrm{H}$ & 0.4880171057 & 0.8191951160 & 0.3170961662 \\
\hline $\mathrm{H}$ & 0.9164398638 & 0.8208332866 & 0.5682018500 \\
\hline $\mathrm{H}$ & 0.2959907345 & 0.6609251289 & 0.8481348526 \\
\hline $\mathrm{H}$ & 0.2473020841 & 0.8208655702 & 0.7187259583 \\
\hline $\mathrm{H}$ & 0.2077268324 & 0.8666212258 & 0.8125122035 \\
\hline $\mathrm{H}$ & 0.2079520229 & 0.5975480110 & 0.9588397867 \\
\hline $\mathrm{H}$ & 0.4822878063 & 0.8384639959 & 0.1026819672 \\
\hline $\mathrm{F}$ & 0.8286204962 & 0.4860859706 & 0.0373316068 \\
\hline $\mathrm{F}$ & 0.7485942213 & 0.5525052300 & 0.1070817078 \\
\hline $\mathrm{F}$ & 0.8312163158 & 0.5556515233 & 0.9301660000 \\
\hline $\mathrm{F}$ & 0.6872239293 & 0.5120099079 & 0.9453355995 \\
\hline $\mathrm{F}$ & 0.1713795038 & 0.5139140594 & 0.0373316068 \\
\hline $\mathrm{F}$ & 0.2514058087 & 0.4474947700 & 0.1070817078 \\
\hline $\mathrm{F}$ & 0.1687836762 & 0.4443484477 & 0.9301660000 \\
\hline $\mathrm{F}$ & 0.3127760407 & 0.4879900621 & 0.9453355995 \\
\hline $\mathrm{F}$ & 0.6713795038 & 0.9860859406 & 0.9626683673 \\
\hline $\mathrm{F}$ & 0.7514057787 & 0.0525052180 & 0.8929183072 \\
\hline $\mathrm{F}$ & 0.6687836842 & 0.0556515453 & 0.0698340000 \\
\hline $\mathrm{F}$ & 0.8127760707 & 0.0120099299 & 0.0546643415 \\
\hline$F$ & 0.3286204962 & 0.0139139994 & 0.9626683673 \\
\hline $\mathrm{F}$ & 0.2485942063 & 0.9474947700 & 0.8929183072 \\
\hline $\mathrm{F}$ & 0.3312163158 & 0.9443484767 & 0.0698340000 \\
\hline $\mathrm{F}$ & 0.1872239443 & 0.9879900921 & 0.0546643415 \\
\hline $\mathrm{F}$ & 0.4875622414 & 0.8357433382 & 0.5223451526 \\
\hline $\mathrm{F}$ & 0.4598049726 & 0.8414641277 & 0.7217618781 \\
\hline $\mathrm{F}$ & 0.5530664192 & 0.7798957357 & 0.6472070878 \\
\hline $\mathrm{F}$ & 0.3837735223 & 0.7827345241 & 0.6139749662 \\
\hline $\mathrm{F}$ & 0.5124377586 & 0.1642566618 & 0.5223451526 \\
\hline $\mathrm{F}$ & 0.5401950274 & 0.1585358873 & 0.7217618781 \\
\hline $\mathrm{F}$ & 0.4469335808 & 0.2201042943 & 0.6472070878 \\
\hline
\end{tabular}




$\begin{array}{llll}\text { F } & 0.6162264777 & 0.2172655059 & 0.6139749662 \\ \text { F } & 0.0124377666 & 0.3357433382 & 0.4776548764 \\ \text { F } & 0.0401950314 & 0.3414641277 & 0.2782380919 \\ \text { F } & 0.9469335808 & 0.2798957057 & 0.3527929122 \\ \text { F } & 0.1162264927 & 0.2827344941 & 0.3860250338 \\ \text { F } & 0.9875622414 & 0.6642566618 & 0.4776548764 \\ \text { F } & 0.9598049726 & 0.6585358723 & 0.2782380919 \\ \text { F } & 0.0530664192 & 0.7201042643 & 0.3527929122 \\ \text { F } & 0.8837735223 & 0.7172654759 & 0.3860250338 \\ \text { B } & 0.7732986342 & 0.5267956848 & 0.0033805125 \\ \text { B } & 0.2267013508 & 0.4732043152 & 0.0033805125 \\ \text { B } & 0.7267013658 & 0.0267956718 & 0.9966194645 \\ \text { B } & 0.2732986342 & 0.9732043152 & 0.9966194645 \\ \text { B } & 0.4722052979 & 0.8095455994 & 0.6260489489 \\ \text { B } & 0.5277937181 & 0.1904544306 & 0.6260489489 \\ \text { B } & 0.0277947051 & 0.3095455694 & 0.3739510511 \\ \text { B } & 0.9722062819 & 0.6904544006 & 0.3739510511\end{array}$

1-iS

CELL_PARAMETERS (angstrom)
13.619242860
0.000000000
0.000000000
$0.000000000 \quad 28.874655560$
0.000000000
0.000000000
0.000000000
11.256034307

ATOMIC_POSITIONS (crystal)

$\begin{array}{lll}\text { Fe1 } & 0.3106108308 & 0.5543566668 \\ \mathrm{Fe} 2 & 0.6178539278 & 0.6994849573 \\ \mathrm{Fe} 1 & 0.6893891692 & 0.4456433332 \\ \mathrm{Fe} 2 & 0.3821460422 & 0.3005150717 \\ \mathrm{Fe} 1 & 0.1893891692 & 0.0543566558 \\ \mathrm{Fe} 2 & 0.8821460722 & 0.1994849283 \\ \mathrm{Fe} 1 & 0.8106108308 & 0.9456433332 \\ \mathrm{Fe} 2 & 0.1178539868 & 0.8005150427 \\ \mathrm{O} & 0.4115900521 & 0.5460637142 \\ \mathrm{O} & 0.3439768023 & 0.6236494121 \\ \mathrm{O} & 0.5922865107 & 0.6333671083 \\ \mathrm{O} & 0.5151701761 & 0.7087553775 \\ \mathrm{O} & 0.5884099479 & 0.4539362558 \\ \mathrm{O} & 0.6560231977 & 0.3763506179 \\ \mathrm{O} & 0.4077135193 & 0.3666328917 \\ \mathrm{O} & 0.4848298239 & 0.2912446225 \\ \mathrm{O} & 0.0884099479 & 0.0460637332 \\ \mathrm{O} & 0.1560232127 & 0.1236493821 \\ \mathrm{O} & 0.9077135483 & 0.1333671083 \\ \mathrm{O} & 0.9848298239 & 0.2087553915 \\ \mathrm{O} & 0.9115900521 & 0.9539362858 \\ \mathrm{O} & 0.8439768023 & 0.8763505879 \\ \mathrm{O} & 0.0922864517 & 0.8666328917 \\ \mathrm{O} & 0.0151701761 & 0.7912446225 \\ \mathrm{~N} & 0.3952002363 & 0.5602663841 \\ \mathrm{~N} & 0.2839511184 & 0.4818736723 \\ \mathrm{~N} & 0.1744569582 & 0.5585914071 \\ \mathrm{~N} & 0.1948651923 & 0.5536542978 \\ \mathrm{~N} & 0.6384848326 & 0.7646408343 \\ \mathrm{~N} & 0.5322412994 & 0.6934972876 \\ \mathrm{~N} & 0.7240195936 & 0.6982385958 \\ \mathrm{~N} & 0.7265317799 & 0.6902805923 \\ \mathrm{~N} & 0.6047997937 & 0.4397335869 \\ \mathrm{~N} & 0.7160488816 & 0.5181263577 \\ & & \end{array}$

0.5194138085

0.9716473504

0.5194138085

0.9716473504

0.4805861915

0.0283526686

0.4805861915

0.0283526686

0.6529391774

0.5608162560

0.9356502574

0.8550871599

0.6529391774

0.5608162560

0.9356502573

0.8550871599

0.3470607926

0.4391837440

0.0643497347

0.1449128551

0.3470607926

0.4391837440

0.0643497347

0.1449128551

0.3574672191

0.5064805302

0.6173857777

0.3723236137

0.9961301082

0.1100980524

0.8544144393

0.0891256253

0.3574672191

0.5064805302 


\begin{tabular}{|c|c|c|c|}
\hline $\mathrm{N}$ & 0.8255430718 & 0.4414086229 & 0.6173857777 \\
\hline $\mathrm{N}$ & 0.8051347927 & 0.4463457312 & 0.3723236137 \\
\hline $\mathrm{N}$ & 0.3615151674 & 0.2353591367 & 0.9961301082 \\
\hline$N$ & 0.4677587296 & 0.3065027124 & 0.1100980524 \\
\hline $\mathrm{N}$ & 0.2759804064 & 0.3017613742 & 0.8544144393 \\
\hline $\mathrm{N}$ & 0.2734682201 & 0.3097194077 & 0.0891256253 \\
\hline $\mathrm{N}$ & 0.1047997717 & 0.0602664101 & 0.6425328109 \\
\hline $\mathrm{N}$ & 0.2160488816 & 0.9818736423 & 0.4935194698 \\
\hline $\mathrm{N}$ & 0.3255430418 & 0.0585913891 & 0.3826141923 \\
\hline $\mathrm{N}$ & 0.3051347927 & 0.0536542758 & 0.6276763573 \\
\hline $\mathrm{N}$ & 0.8615151674 & 0.2646408633 & 0.0038699028 \\
\hline $\mathrm{N}$ & 0.9677587006 & 0.1934972726 & 0.8899019476 \\
\hline $\mathrm{N}$ & 0.7759804064 & 0.1982386108 & 0.1455855457 \\
\hline $\mathrm{N}$ & 0.7734682201 & 0.1902805923 & 0.9108743747 \\
\hline $\mathrm{N}$ & 0.8952002063 & 0.9397336159 & 0.6425328109 \\
\hline $\mathrm{N}$ & 0.7839511184 & 0.0181263577 & 0.4935194698 \\
\hline $\mathrm{N}$ & 0.6744569282 & 0.9414085929 & 0.3826141923 \\
\hline $\mathrm{N}$ & 0.6948652073 & 0.9463457022 & 0.6276763573 \\
\hline $\mathrm{N}$ & 0.1384847736 & 0.7353591657 & 0.0038699028 \\
\hline $\mathrm{N}$ & 0.0322412404 & 0.8065027124 & 0.8899019476 \\
\hline $\mathrm{N}$ & 0.2240195936 & 0.8017614042 & 0.1455855457 \\
\hline $\mathrm{N}$ & 0.2265318399 & 0.8097194077 & 0.9108743747 \\
\hline C & 0.4406192555 & 0.5837513906 & 0.7039173470 \\
\hline C & 0.4945843883 & 0.5609662202 & 0.1410246314 \\
\hline C & 0.2217900793 & 0.4681544299 & 0.4201900109 \\
\hline C & 0.1650641567 & 0.5495955065 & 0.7344417593 \\
\hline C & 0.4019076966 & 0.6283111570 & 0.6500215157 \\
\hline C & 0.9906303082 & 0.5604698966 & 0.7256403458 \\
\hline C & 0.5456639303 & 0.5551679836 & 0.2474406333 \\
\hline C & 0.0745955897 & 0.5503628579 & 0.7918656183 \\
\hline C & 0.4294456243 & 0.6711628659 & 0.6984346108 \\
\hline C & 0.3234779334 & 0.4500616328 & 0.5804343565 \\
\hline C & 0.5327436143 & 0.6278442333 & 0.8477706687 \\
\hline C & 0.5049025690 & 0.5847598234 & 0.8007252700 \\
\hline C & 0.2016206827 & 0.4212718387 & 0.4012655575 \\
\hline C & 0.2429050595 & 0.3884004645 & 0.4770000195 \\
\hline C & 0.3039337287 & 0.4030313128 & 0.5693224005 \\
\hline C & 0.1722239117 & 0.5044989525 & 0.3436846626 \\
\hline C & 0.2377158342 & 0.5777614991 & 0.2682202300 \\
\hline C & 0.3929717769 & 0.5668526043 & 0.1449135192 \\
\hline C & 0.0002654217 & 0.5687146104 & 0.6042167939 \\
\hline C & 0.1100712308 & 0.5789898364 & 0.4230501968 \\
\hline C & 0.3456950709 & 0.5670746259 & 0.2549267983 \\
\hline C & 0.4932360108 & 0.5545500743 & 0.3532755293 \\
\hline C & 0.4909542220 & 0.6716648581 & 0.7976416261 \\
\hline C & 0.0932711380 & 0.5675482800 & 0.5525455987 \\
\hline C & 0.5895969383 & 0.7985512706 & 0.9377224460 \\
\hline C & 0.7102902478 & 0.7763639770 & 0.0738869551 \\
\hline C & 0.5786810951 & 0.6778611258 & 0.2091771374 \\
\hline C & 0.5287330298 & 0.6718266231 & 0.3160784251 \\
\hline C & 0.4346443662 & 0.7010593807 & 0.1145583836 \\
\hline C & 0.3797704930 & 0.6935136652 & 0.2168575320 \\
\hline C & 0.7643386060 & 0.7365574685 & 0.1303049465 \\
\hline C & 0.7207094342 & 0.7126150276 & 0.7401428718 \\
\hline C & 0.8010619500 & 0.7080530692 & 0.6646356936 \\
\hline C & 0.8027624868 & 0.6631025421 & 0.0238793448 \\
\hline C & 0.8084535606 & 0.6796283471 & 0.8975931967 \\
\hline C & 0.6101162439 & 0.8451671274 & 0.9556160569 \\
\hline
\end{tabular}




\begin{tabular}{|c|c|c|c|}
\hline C & 0.8875617092 & 0.6883372768 & 0.7079458099 \\
\hline C & 0.4275815347 & 0.6794593480 & 0.3204202499 \\
\hline C & 0.7334615075 & 0.8225973780 & 0.0968770378 \\
\hline C & 0.6836365361 & 0.6636528766 & 0.1899194435 \\
\hline C & 0.6830793248 & 0.8575478886 & 0.0368699382 \\
\hline C & 0.8913828994 & 0.6743848835 & 0.8264194726 \\
\hline C & 0.5593807445 & 0.4162486384 & 0.7039173470 \\
\hline C & 0.5054156117 & 0.4390337498 & 0.1410246314 \\
\hline C & 0.7782099057 & 0.5318455701 & 0.4201900109 \\
\hline C & 0.8349358733 & 0.4504044635 & 0.7344417593 \\
\hline C & 0.5980923034 & 0.3716888430 & 0.6500215157 \\
\hline C & 0.0093687388 & 0.4395301334 & 0.7256403458 \\
\hline C & 0.4543360397 & 0.4448319864 & 0.2474406333 \\
\hline C & 0.9254043953 & 0.4496371721 & 0.7918656183 \\
\hline C & 0.5705543757 & 0.3288371641 & 0.6984346108 \\
\hline C & 0.6765220666 & 0.5499383672 & 0.5804343565 \\
\hline C & 0.4672563857 & 0.3721557367 & 0.8477706687 \\
\hline C & 0.4950974310 & 0.4152402066 & 0.8007252700 \\
\hline C & 0.7983793023 & 0.5787281313 & 0.4012655575 \\
\hline C & 0.7570949555 & 0.6115995355 & 0.4770000195 \\
\hline C & 0.6960663013 & 0.5969686872 & 0.5693224005 \\
\hline C & 0.8277760733 & 0.4955010775 & 0.3436846626 \\
\hline C & 0.7622841808 & 0.4222384709 & 0.2682202300 \\
\hline C & 0.6070281931 & 0.4331473657 & 0.1449135192 \\
\hline C & 0.9997345923 & 0.4312853896 & 0.6042167939 \\
\hline C & 0.8899287622 & 0.4210101336 & 0.4230501968 \\
\hline C & 0.6543049291 & 0.4329253741 & 0.2549267983 \\
\hline C & 0.5067640192 & 0.4454499257 & 0.3532755293 \\
\hline C & 0.5090458080 & 0.3283351419 & 0.7976416261 \\
\hline C & 0.9067288620 & 0.4324517500 & 0.5525455987 \\
\hline C & 0.4104030617 & 0.2014487154 & 0.9377224460 \\
\hline C & 0.2897097222 & 0.2236359940 & 0.0738869551 \\
\hline C & 0.4213189049 & 0.3221389042 & 0.2091771374 \\
\hline C & 0.4712679542 & 0.3281733769 & 0.3160784251 \\
\hline C & 0.5653556338 & 0.2989406483 & 0.1145583836 \\
\hline C & 0.6202295070 & 0.3064863048 & 0.2168575320 \\
\hline C & 0.2356613790 & 0.2634425315 & 0.1303049465 \\
\hline C & 0.2792905808 & 0.2873850024 & 0.7401428718 \\
\hline C & 0.1989380800 & 0.2919469308 & 0.6646356936 \\
\hline C & 0.1972375282 & 0.3368974879 & 0.0238793448 \\
\hline C & 0.1915464544 & 0.3203716529 & 0.8975931967 \\
\hline C & 0.3898837261 & 0.1548328876 & 0.9556160569 \\
\hline C & 0.1124383058 & 0.3116627532 & 0.7079458099 \\
\hline C & 0.5724184653 & 0.3205406520 & 0.3204202499 \\
\hline C & 0.2665385225 & 0.1774026370 & 0.0968770378 \\
\hline C & 0.3163634349 & 0.3363471234 & 0.1899194435 \\
\hline C & 0.3169207042 & 0.1424521404 & 0.0368699382 \\
\hline C & 0.1086171076 & 0.3256150865 & 0.8264194726 \\
\hline C & 0.0593807485 & 0.0837513616 & 0.2960826530 \\
\hline C & 0.0054156017 & 0.0609662502 & 0.8589753386 \\
\hline C & 0.2782099057 & 0.9681544299 & 0.5798100191 \\
\hline C & 0.3349358433 & 0.0495955295 & 0.2655582407 \\
\hline C & 0.0980923184 & 0.1283111570 & 0.3499784843 \\
\hline C & 0.5093686788 & 0.0604698706 & 0.2743596252 \\
\hline C & 0.9543360697 & 0.0551679986 & 0.7525593667 \\
\hline C & 0.4254043953 & 0.0503628359 & 0.2081343967 \\
\hline C & 0.0705543677 & 0.1711628509 & 0.3015653592 \\
\hline C & 0.1765220666 & 0.9500616328 & 0.4195656435 \\
\hline
\end{tabular}




\begin{tabular}{|c|c|c|c|}
\hline C & 0.9672563857 & 0.1278442483 & 0.1522293613 \\
\hline C & 0.9950974310 & 0.0847598004 & 0.1992747150 \\
\hline C & 0.2983793323 & 0.9212718687 & 0.5987344425 \\
\hline C & 0.2570949255 & 0.8884004645 & 0.5230009645 \\
\hline C & 0.1960662863 & 0.9030313128 & 0.4306775995 \\
\hline C & 0.3277761033 & 0.0044989315 & 0.6563153664 \\
\hline C & 0.2622841808 & 0.0777615221 & 0.7317797400 \\
\hline C & 0.1070282231 & 0.0668526263 & 0.8550864808 \\
\hline C & 0.4997345923 & 0.0687146104 & 0.3957831761 \\
\hline C & 0.3899287622 & 0.0789898584 & 0.5769498032 \\
\hline C & 0.1543049291 & 0.0670746259 & 0.7450732017 \\
\hline C & 0.0067639992 & 0.0545500633 & 0.6467244407 \\
\hline C & 0.0090457890 & 0.1716648431 & 0.2023583739 \\
\hline C & 0.4067288620 & 0.0675482540 & 0.4474544313 \\
\hline C & 0.9104030617 & 0.2985512996 & 0.0622775320 \\
\hline C & 0.7897097522 & 0.2763640060 & 0.9261130449 \\
\hline C & 0.9213189049 & 0.1778611108 & 0.7908228626 \\
\hline C & 0.9712679842 & 0.1718266381 & 0.6839215749 \\
\hline C & 0.0653556298 & 0.2010593657 & 0.8854416164 \\
\hline C & 0.1202295210 & 0.1935136952 & 0.7831424680 \\
\hline C & 0.7356613940 & 0.2365574685 & 0.8696951125 \\
\hline C & 0.7792905658 & 0.2126149976 & 0.2598571282 \\
\hline C & 0.6989380500 & 0.2080530692 & 0.3353642764 \\
\hline C & 0.6972375132 & 0.1631025121 & 0.9761206552 \\
\hline C & 0.6915464394 & 0.1796283471 & 0.1024068183 \\
\hline C & 0.8898836961 & 0.3451670974 & 0.0443839691 \\
\hline C & 0.6124382908 & 0.1883372468 & 0.2920542201 \\
\hline C & 0.0724184613 & 0.1794593480 & 0.6795797501 \\
\hline C & 0.7665384925 & 0.3225973480 & 0.9031229622 \\
\hline C & 0.8163634049 & 0.1636528916 & 0.8100805565 \\
\hline C & 0.8169206752 & 0.3575478596 & 0.9631300018 \\
\hline C & 0.6086171006 & 0.1743849135 & 0.1735805124 \\
\hline C & 0.9406192555 & 0.9162486094 & 0.2960826530 \\
\hline C & 0.9945843883 & 0.9390337798 & 0.8589753386 \\
\hline C & 0.7217900943 & 0.0318455701 & 0.5798100191 \\
\hline C & 0.6650641267 & 0.9504044935 & 0.2655582407 \\
\hline C & 0.9019076966 & 0.8716888430 & 0.3499784843 \\
\hline C & 0.4906313212 & 0.9395301034 & 0.2743596252 \\
\hline C & 0.0456639303 & 0.9448320164 & 0.7525593667 \\
\hline C & 0.5745956047 & 0.9496371421 & 0.2081343967 \\
\hline C & 0.9294456243 & 0.8288371341 & 0.3015653592 \\
\hline C & 0.8234779334 & 0.0499383082 & 0.4195656435 \\
\hline C & 0.0327436143 & 0.8721557667 & 0.1522293613 \\
\hline C & 0.0049025690 & 0.9152401766 & 0.1992747150 \\
\hline C & 0.7016206977 & 0.0787281903 & 0.5987344425 \\
\hline C & 0.7429050445 & 0.1115995355 & 0.5230009645 \\
\hline C & 0.8039336987 & 0.0969687472 & 0.4306775995 \\
\hline C & 0.6722239267 & 0.9955010475 & 0.6563153664 \\
\hline C & 0.7377158192 & 0.9222385009 & 0.7317797400 \\
\hline C & 0.8929718068 & 0.9331473957 & 0.8550864808 \\
\hline C & 0.5002654077 & 0.9312853896 & 0.3957831761 \\
\hline C & 0.6100712378 & 0.9210101636 & 0.5769498032 \\
\hline C & 0.8456950709 & 0.9329253741 & 0.7450732017 \\
\hline C & 0.9932359808 & 0.9454499257 & 0.6467244407 \\
\hline C & 0.9909541920 & 0.8283351419 & 0.2023583739 \\
\hline C & 0.5932711380 & 0.9324517200 & 0.4474544313 \\
\hline C & 0.0895969383 & 0.7014487294 & 0.0622775320 \\
\hline C & 0.2102902478 & 0.7236360230 & 0.9261130449 \\
\hline
\end{tabular}




\begin{tabular}{|c|c|c|c|}
\hline C & 0.0786811551 & 0.8221388742 & 0.7908228626 \\
\hline C & 0.0287320158 & 0.8281733769 & 0.6839215749 \\
\hline C & 0.9346443662 & 0.7989406193 & 0.8854416164 \\
\hline C & 0.8797704930 & 0.8064863348 & 0.7831424680 \\
\hline C & 0.2643386060 & 0.7634425315 & 0.8696951125 \\
\hline C & 0.2207094342 & 0.7873849724 & 0.2598571282 \\
\hline C & 0.3010619500 & 0.7919469308 & 0.3353642764 \\
\hline C & 0.3027624268 & 0.8368974579 & 0.9761206552 \\
\hline C & 0.3084535606 & 0.8203716529 & 0.1024068183 \\
\hline C & 0.1101163039 & 0.6548328726 & 0.0443839691 \\
\hline C & 0.3875617092 & 0.8116627232 & 0.2920542201 \\
\hline C & 0.9275815347 & 0.8205406520 & 0.6795797501 \\
\hline C & 0.2334615075 & 0.6774026220 & 0.9031229622 \\
\hline C & 0.1836365951 & 0.8363471234 & 0.8100805565 \\
\hline C & 0.1830793248 & 0.6424521114 & 0.9631300018 \\
\hline C & 0.3913828404 & 0.8256151165 & 0.1735805124 \\
\hline $\mathrm{H}$ & 0.5341006593 & 0.5605982560 & 0.0568116132 \\
\hline $\mathrm{H}$ & 0.2326580662 & 0.5409688583 & 0.7819589589 \\
\hline $\mathrm{H}$ & 0.9188224136 & 0.5610333250 & 0.7685523950 \\
\hline $\mathrm{H}$ & 0.6248496810 & 0.5501390333 & 0.2469258441 \\
\hline $\mathrm{H}$ & 0.0707916091 & 0.5421376230 & 0.8862473932 \\
\hline $\mathrm{H}$ & 0.4023708098 & 0.7035893303 & 0.6608524545 \\
\hline $\mathrm{H}$ & 0.3719454992 & 0.4637357942 & 0.6492034842 \\
\hline $\mathrm{H}$ & 0.5347817639 & 0.5527444693 & 0.8374922144 \\
\hline $\mathrm{H}$ & 0.1528338002 & 0.4105256599 & 0.3294066453 \\
\hline $\mathrm{H}$ & 0.2249396464 & 0.3518897878 & 0.4654484348 \\
\hline $\mathrm{H}$ & 0.3361359830 & 0.3786388818 & 0.6321216327 \\
\hline $\mathrm{H}$ & 0.0921296459 & 0.4990751916 & 0.3501336186 \\
\hline $\mathrm{H}$ & 0.1912826032 & 0.4974112711 & 0.2503148822 \\
\hline $\mathrm{H}$ & 0.2303211210 & 0.6152976249 & 0.2836203884 \\
\hline $\mathrm{H}$ & 0.1983517504 & 0.5693660684 & 0.1854858345 \\
\hline $\mathrm{H}$ & 0.3502411776 & 0.5704147656 & 0.0635483417 \\
\hline $\mathrm{H}$ & 0.9367122926 & 0.5769137350 & 0.5496024424 \\
\hline $\mathrm{H}$ & 0.0423357397 & 0.5733855229 & 0.3710916170 \\
\hline $\mathrm{H}$ & 0.1258671646 & 0.6163192464 & 0.4173235653 \\
\hline $\mathrm{H}$ & 0.5291738784 & 0.5491061460 & 0.4387042378 \\
\hline $\mathrm{H}$ & 0.5349035651 & 0.7869729373 & 0.8738144025 \\
\hline $\mathrm{H}$ & 0.5685501001 & 0.6600168114 & 0.3944963710 \\
\hline $\mathrm{H}$ & 0.4014338068 & 0.7128743355 & 0.0320100539 \\
\hline $\mathrm{H}$ & 0.3007313375 & 0.6993288509 & 0.2149640885 \\
\hline $\mathrm{H}$ & 0.8434493188 & 0.7391372708 & 0.1109147745 \\
\hline $\mathrm{H}$ & 0.7596277458 & 0.7386147130 & 0.2276921142 \\
\hline $\mathrm{H}$ & 0.6517445453 & 0.7283081850 & 0.7113908006 \\
\hline $\mathrm{H}$ & 0.7967277001 & 0.7201908392 & 0.5730360696 \\
\hline $\mathrm{H}$ & 0.7791716085 & 0.6266309980 & 0.0226606687 \\
\hline $\mathrm{H}$ & 0.8742278788 & 0.6644943331 & 0.0693414898 \\
\hline $\mathrm{H}$ & 0.5687117594 & 0.8709257519 & 0.9055557822 \\
\hline $\mathrm{H}$ & 0.9504966145 & 0.6839485330 & 0.6492195446 \\
\hline $\mathrm{H}$ & 0.3872205400 & 0.6735588591 & 0.4028998619 \\
\hline $\mathrm{H}$ & 0.7911808254 & 0.8307789879 & 0.1608065956 \\
\hline $\mathrm{H}$ & 0.7283998130 & 0.6672896631 & 0.2706904173 \\
\hline $\mathrm{H}$ & 0.6840613485 & 0.6268153153 & 0.1644756323 \\
\hline $\mathrm{H}$ & 0.7005758828 & 0.8938878152 & 0.0526672278 \\
\hline $\mathrm{H}$ & 0.9577532141 & 0.6591724358 & 0.8640530293 \\
\hline $\mathrm{H}$ & 0.4658993107 & 0.4394017740 & 0.0568116132 \\
\hline $\mathrm{H}$ & 0.7673419488 & 0.4590311417 & 0.7819589589 \\
\hline $\mathrm{H}$ & 0.0811775864 & 0.4389666750 & 0.7685523950 \\
\hline $\mathrm{H}$ & 0.3751502900 & 0.4498609667 & 0.2469258441 \\
\hline
\end{tabular}




\begin{tabular}{|c|c|c|c|}
\hline $\mathrm{H}$ & 0.9292084209 & 0.4578623770 & 0.8862473932 \\
\hline $\mathrm{H}$ & 0.5976291902 & 0.2964106397 & 0.6608524545 \\
\hline $\mathrm{H}$ & 0.6280545008 & 0.5362642058 & 0.6492034842 \\
\hline $\mathrm{H}$ & 0.4652182361 & 0.4472555307 & 0.8374922144 \\
\hline $\mathrm{H}$ & 0.8471661998 & 0.5894743401 & 0.3294066453 \\
\hline $\mathrm{H}$ & 0.7750603686 & 0.6481102422 & 0.4654484348 \\
\hline $\mathrm{H}$ & 0.6638640170 & 0.6213611182 & 0.6321216327 \\
\hline $\mathrm{H}$ & 0.9078703391 & 0.5009247784 & 0.3501336186 \\
\hline $\mathrm{H}$ & 0.8087173668 & 0.5025887289 & 0.2503148822 \\
\hline $\mathrm{H}$ & 0.7696788940 & 0.3847023451 & 0.2836203884 \\
\hline $\mathrm{H}$ & 0.8016482496 & 0.4306339316 & 0.1854858345 \\
\hline $\mathrm{H}$ & 0.6497588224 & 0.4295852344 & 0.0635483417 \\
\hline $\mathrm{H}$ & 0.0632877074 & 0.4230862650 & 0.5496024424 \\
\hline $\mathrm{H}$ & 0.9576642683 & 0.4266144471 & 0.3710916170 \\
\hline $\mathrm{H}$ & 0.8741328134 & 0.3836807236 & 0.4173235653 \\
\hline $\mathrm{H}$ & 0.4708261216 & 0.4508938840 & 0.4387042378 \\
\hline $\mathrm{H}$ & 0.4650964349 & 0.2130270917 & 0.8738144025 \\
\hline $\mathrm{H}$ & 0.4314498699 & 0.3399831886 & 0.3944963710 \\
\hline $\mathrm{H}$ & 0.5985662222 & 0.2871256645 & 0.0320100539 \\
\hline $\mathrm{H}$ & 0.6992686325 & 0.3006711491 & 0.2149640885 \\
\hline $\mathrm{H}$ & 0.1565506662 & 0.2608627292 & 0.1109147745 \\
\hline $\mathrm{H}$ & 0.2403722692 & 0.2613852870 & 0.2276921142 \\
\hline $\mathrm{H}$ & 0.3482554847 & 0.2716918150 & 0.7113908006 \\
\hline $\mathrm{H}$ & 0.2032722999 & 0.2798091608 & 0.5730360696 \\
\hline $\mathrm{H}$ & 0.2208283765 & 0.3733689720 & 0.0226606687 \\
\hline $\mathrm{H}$ & 0.1257721282 & 0.3355056669 & 0.0693414898 \\
\hline $\mathrm{H}$ & 0.4312882106 & 0.1290742181 & 0.9055557822 \\
\hline $\mathrm{H}$ & 0.0495033785 & 0.3160514960 & 0.6492195446 \\
\hline $\mathrm{H}$ & 0.6127794310 & 0.3264411109 & 0.4028998619 \\
\hline $\mathrm{H}$ & 0.2088191746 & 0.1692210120 & 0.1608065956 \\
\hline $\mathrm{H}$ & 0.2716001870 & 0.3327103659 & 0.2706904173 \\
\hline $\mathrm{H}$ & 0.3159386515 & 0.3731846847 & 0.1644756323 \\
\hline $\mathrm{H}$ & 0.2994241172 & 0.1061121628 & 0.0526672278 \\
\hline $\mathrm{H}$ & 0.0422468159 & 0.3408275642 & 0.8640530293 \\
\hline $\mathrm{H}$ & 0.9658993407 & 0.0605982260 & 0.9431883718 \\
\hline $\mathrm{H}$ & 0.2673419488 & 0.0409688473 & 0.2180410411 \\
\hline $\mathrm{H}$ & 0.5811775864 & 0.0610333320 & 0.2314475900 \\
\hline $\mathrm{H}$ & 0.8751502600 & 0.0501390293 & 0.7530741859 \\
\hline $\mathrm{H}$ & 0.4292083909 & 0.0421376080 & 0.1137525838 \\
\hline $\mathrm{H}$ & 0.0976291982 & 0.2035893603 & 0.3391475455 \\
\hline $\mathrm{H}$ & 0.1280545158 & 0.9637357942 & 0.3507965158 \\
\hline $\mathrm{H}$ & 0.9652182361 & 0.0527444693 & 0.1625078156 \\
\hline $\mathrm{H}$ & 0.3471661998 & 0.9105256599 & 0.6705933547 \\
\hline $\mathrm{H}$ & 0.2750603686 & 0.8518897578 & 0.5345515942 \\
\hline $\mathrm{H}$ & 0.1638640170 & 0.8786388818 & 0.3678783673 \\
\hline $\mathrm{H}$ & 0.4078703691 & 0.9990752216 & 0.6498663814 \\
\hline $\mathrm{H}$ & 0.3087173968 & 0.9974112711 & 0.7496851478 \\
\hline $\mathrm{H}$ & 0.2696788940 & 0.1152976399 & 0.7163786276 \\
\hline $\mathrm{H}$ & 0.3016482496 & 0.0693660534 & 0.8145141955 \\
\hline $\mathrm{H}$ & 0.1497588224 & 0.0704147656 & 0.9364516803 \\
\hline $\mathrm{H}$ & 0.5632877074 & 0.0769137200 & 0.4503975276 \\
\hline $\mathrm{H}$ & 0.4576642683 & 0.0733855449 & 0.6289084130 \\
\hline $\mathrm{H}$ & 0.3741328434 & 0.1163192684 & 0.5826774187 \\
\hline $\mathrm{H}$ & 0.9708261216 & 0.0491061270 & 0.5612957322 \\
\hline $\mathrm{H}$ & 0.9650964349 & 0.2869729083 & 0.1261855975 \\
\hline $\mathrm{H}$ & 0.9314498999 & 0.1600167964 & 0.6055036290 \\
\hline $\mathrm{H}$ & 0.0985662002 & 0.2128743355 & 0.9679899461 \\
\hline $\mathrm{H}$ & 0.1992686475 & 0.1993288509 & 0.7850359115 \\
\hline
\end{tabular}




\begin{tabular}{|c|c|c|c|}
\hline $\mathrm{H}$ & 0.6565506812 & 0.2391372558 & 0.8890852855 \\
\hline $\mathrm{H}$ & 0.7403723142 & 0.2386147130 & 0.7723078858 \\
\hline $\mathrm{H}$ & 0.8482564677 & 0.2283081710 & 0.2886091694 \\
\hline $\mathrm{H}$ & 0.7032722999 & 0.2201908392 & 0.4269639304 \\
\hline $\mathrm{H}$ & 0.7208283915 & 0.1266310130 & 0.9773393313 \\
\hline $\mathrm{H}$ & 0.6257721212 & 0.1644943481 & 0.9306584512 \\
\hline $\mathrm{H}$ & 0.9312881806 & 0.3709257819 & 0.0944442108 \\
\hline $\mathrm{H}$ & 0.5495033855 & 0.1839485040 & 0.3507804854 \\
\hline $\mathrm{H}$ & 0.1127794600 & 0.1735588891 & 0.5971001381 \\
\hline $\mathrm{H}$ & 0.7088191746 & 0.3307789879 & 0.8391933444 \\
\hline $\mathrm{H}$ & 0.7716001870 & 0.1672896341 & 0.7293085687 \\
\hline $\mathrm{H}$ & 0.8159386515 & 0.1268153153 & 0.8355243677 \\
\hline $\mathrm{H}$ & 0.7994241172 & 0.3938878442 & 0.9473327122 \\
\hline $\mathrm{H}$ & 0.5422467859 & 0.1591724508 & 0.1359469857 \\
\hline $\mathrm{H}$ & 0.0341006593 & 0.9394017440 & 0.9431883718 \\
\hline $\mathrm{H}$ & 0.7326580512 & 0.9590311417 & 0.2180410411 \\
\hline $\mathrm{H}$ & 0.4188224136 & 0.9389666750 & 0.2314475900 \\
\hline $\mathrm{H}$ & 0.1248497400 & 0.9498609667 & 0.7530741859 \\
\hline $\mathrm{H}$ & 0.5707915791 & 0.9578623770 & 0.1137525838 \\
\hline $\mathrm{H}$ & 0.9023708098 & 0.7964106697 & 0.3391475455 \\
\hline $\mathrm{H}$ & 0.8719454992 & 0.0362642058 & 0.3507965158 \\
\hline $\mathrm{H}$ & 0.0347817639 & 0.9472555307 & 0.1625078156 \\
\hline $\mathrm{H}$ & 0.6528338002 & 0.0894743401 & 0.6705933547 \\
\hline $\mathrm{H}$ & 0.7249396314 & 0.1481102422 & 0.5345515942 \\
\hline $\mathrm{H}$ & 0.8361359830 & 0.1213611782 & 0.3678783673 \\
\hline $\mathrm{H}$ & 0.5921296609 & 0.0009247784 & 0.6498663814 \\
\hline $\mathrm{H}$ & 0.6912826332 & 0.0025886699 & 0.7496851478 \\
\hline $\mathrm{H}$ & 0.7303211060 & 0.8847023751 & 0.7163786276 \\
\hline $\mathrm{H}$ & 0.6983517504 & 0.9306339316 & 0.8145141955 \\
\hline $\mathrm{H}$ & 0.8502411776 & 0.9295852344 & 0.9364516803 \\
\hline $\mathrm{H}$ & 0.4367122626 & 0.9230862650 & 0.4503975276 \\
\hline $\mathrm{H}$ & 0.5423357317 & 0.9266144771 & 0.6289084130 \\
\hline $\mathrm{H}$ & 0.6258671866 & 0.8836807536 & 0.5826774187 \\
\hline $\mathrm{H}$ & 0.0291739374 & 0.9508938540 & 0.5612957322 \\
\hline $\mathrm{H}$ & 0.0349036251 & 0.7130270627 & 0.1261855975 \\
\hline $\mathrm{H}$ & 0.0685501001 & 0.8399831886 & 0.6055036290 \\
\hline $\mathrm{H}$ & 0.9014337778 & 0.7871256645 & 0.9679899461 \\
\hline $\mathrm{H}$ & 0.8007313675 & 0.8006711491 & 0.7850359115 \\
\hline $\mathrm{H}$ & 0.3434493188 & 0.7608627292 & 0.8890852855 \\
\hline $\mathrm{H}$ & 0.2596276858 & 0.7613852870 & 0.7723078858 \\
\hline $\mathrm{H}$ & 0.1517445453 & 0.7716918150 & 0.2886091694 \\
\hline $\mathrm{H}$ & 0.2967277001 & 0.7798091608 & 0.4269639304 \\
\hline $\mathrm{H}$ & 0.2791716085 & 0.8733690020 & 0.9773393313 \\
\hline $\mathrm{H}$ & 0.3742278198 & 0.8355056669 & 0.9306584512 \\
\hline $\mathrm{H}$ & 0.0687118194 & 0.6290742481 & 0.0944442108 \\
\hline $\mathrm{H}$ & 0.4504966735 & 0.8160514670 & 0.3507804854 \\
\hline $\mathrm{H}$ & 0.8872205690 & 0.8264411409 & 0.5971001381 \\
\hline $\mathrm{H}$ & 0.2911807654 & 0.6692210121 & 0.8391933444 \\
\hline $\mathrm{H}$ & 0.2283998730 & 0.8327103369 & 0.7293085687 \\
\hline $\mathrm{H}$ & 0.1840612885 & 0.8731846847 & 0.8355243677 \\
\hline $\mathrm{H}$ & 0.2005758828 & 0.6061121848 & 0.9473327122 \\
\hline $\mathrm{H}$ & 0.4577532141 & 0.8408275642 & 0.1359469857 \\
\hline $\mathrm{F}$ & 0.8186723994 & 0.4834998811 & 0.0328581998 \\
\hline $\mathrm{F}$ & 0.7458988502 & 0.5501987144 & 0.1081670146 \\
\hline $\mathrm{F}$ & 0.8257725072 & 0.5527698329 & 0.9263590748 \\
\hline $\mathrm{F}$ & 0.6780300825 & 0.5126884745 & 0.9435133828 \\
\hline $\mathrm{F}$ & 0.1813276006 & 0.5165001489 & 0.0328581998 \\
\hline $\mathrm{F}$ & 0.2541011798 & 0.4498012856 & 0.1081670146 \\
\hline
\end{tabular}




\begin{tabular}{|c|c|c|c|}
\hline $\mathrm{F}$ & 0.1742274848 & 0.4472301381 & 0.9263590748 \\
\hline $\mathrm{F}$ & 0.3219698875 & 0.4873114955 & 0.9435133828 \\
\hline$F$ & 0.6813276006 & 0.9834998511 & 0.9671417742 \\
\hline $\mathrm{F}$ & 0.7541011498 & 0.0501987024 & 0.8918330004 \\
\hline $\mathrm{F}$ & 0.6742274928 & 0.0527698549 & 0.0736409252 \\
\hline $\mathrm{F}$ & 0.8219699175 & 0.0126884965 & 0.0564865582 \\
\hline$F$ & 0.3186723994 & 0.0165000889 & 0.9671417742 \\
\hline $\mathrm{F}$ & 0.2458988352 & 0.9498012856 & 0.8918330004 \\
\hline $\mathrm{F}$ & 0.3257725072 & 0.9472301671 & 0.0736409252 \\
\hline $\mathrm{F}$ & 0.1780300975 & 0.9873115255 & 0.0564865582 \\
\hline $\mathrm{F}$ & 0.4711579351 & 0.8419941792 & 0.5371395733 \\
\hline $\mathrm{F}$ & 0.4409674408 & 0.8414614261 & 0.7400352377 \\
\hline $\mathrm{F}$ & 0.5396571067 & 0.7845567390 & 0.6544265444 \\
\hline $\mathrm{F}$ & 0.3704573543 & 0.7854105811 & 0.6188737961 \\
\hline $\mathrm{F}$ & 0.5288420649 & 0.1580058208 & 0.5371395733 \\
\hline $\mathrm{F}$ & 0.5590325592 & 0.1585385889 & 0.7400352377 \\
\hline $\mathrm{F}$ & 0.4603428933 & 0.2154432910 & 0.6544265444 \\
\hline $\mathrm{F}$ & 0.6295426457 & 0.2145894489 & 0.6188737961 \\
\hline $\mathrm{F}$ & 0.0288420729 & 0.3419941792 & 0.4628604557 \\
\hline $\mathrm{F}$ & 0.0590325632 & 0.3414614261 & 0.2599647323 \\
\hline $\mathrm{F}$ & 0.9603428933 & 0.2845567090 & 0.3455734556 \\
\hline $\mathrm{F}$ & 0.1295426607 & 0.2854105511 & 0.3811262039 \\
\hline $\mathrm{F}$ & 0.9711579351 & 0.6580058208 & 0.4628604557 \\
\hline $\mathrm{F}$ & 0.9409674408 & 0.6585385739 & 0.2599647323 \\
\hline $\mathrm{F}$ & 0.0396571067 & 0.7154432610 & 0.3455734556 \\
\hline $\mathrm{F}$ & 0.8704573543 & 0.7145894189 & 0.3811262039 \\
\hline B & 0.7665297303 & 0.5249141499 & 0.0012392335 \\
\hline B & 0.2334702547 & 0.4750858501 & 0.0012392335 \\
\hline B & 0.7334702697 & 0.0249141369 & 0.9987607435 \\
\hline B & 0.2665297303 & 0.9750858501 & 0.9987607435 \\
\hline B & 0.4565698775 & 0.8129889310 & 0.6374016587 \\
\hline B & 0.5434291385 & 0.1870110990 & 0.6374016587 \\
\hline B & 0.0434301255 & 0.3129889010 & 0.3625983413 \\
\hline B & 0.9565708615 & 0.6870110690 & 0.3625983413 \\
\hline
\end{tabular}

1-LS

CELL_PARAMETERS (angstrom)
13.670034936
0.000000000
0.000000000
$0.000000000 \quad 29.260715478$
0.000000000
0.000000000
0.000000000
10.894842398

ATOMIC_POSITIONS (crystal)

$\begin{array}{ll}\text { Fe2 } & 0.2923316836 \\ \text { Fe2 } & 0.5973365205 \\ \text { Fe2 } & 0.7076683164 \\ \text { Fe2 } & 0.4026634795 \\ \text { Fe2 } & 0.2076683014 \\ \text { Fe2 } & 0.9026634795 \\ \text { Fe2 } & 0.7923316836 \\ \text { Fe2 } & 0.0973365205 \\ O & 0.3889461198 \\ O & 0.3178583751 \\ O & 0.5728225428 \\ O & 0.4977643495 \\ O & 0.6110538802 \\ O & 0.6821416539 \\ O & 0.4271774872 \\ O & 0.5022356505 \\ O & 0.1110538652\end{array}$

$\begin{array}{ll}0.5531414300 & 0.5301349480 \\ 0.6982459517 & 0.9793134468 \\ 0.4468586000 & 0.5301349480 \\ 0.3017540183 & 0.9793134468 \\ 0.0531414070 & 0.4698650230 \\ 0.1982459817 & 0.0206865452 \\ 0.9468585700 & 0.4698650230 \\ 0.8017540483 & 0.0206865452 \\ 0.5454129619 & 0.6582317102 \\ 0.6192187421 & 0.5639045652 \\ 0.6327804639 & 0.9445293663 \\ 0.7064590152 & 0.8540456533 \\ 0.4545870381 & 0.6582317102 \\ 0.3807812879 & 0.5639045652 \\ 0.3672195361 & 0.9445293663 \\ 0.2935410148 & 0.8540456533 \\ 0.0454129689 & 0.3417682898\end{array}$




\begin{tabular}{|c|c|c|c|}
\hline 0 & 0.1821416249 & 0.1192187271 & 0.4360954048 \\
\hline $\mathrm{O}$ & 0.9271774572 & 0.1327804779 & 0.0554706527 \\
\hline 0 & 1.0022356505 & 0.2064589852 & 0.1459543317 \\
\hline 0 & 0.8889461198 & 0.9545870381 & 0.3417682898 \\
\hline $\mathrm{O}$ & 0.8178583461 & 0.8807812580 & 0.4360954048 \\
\hline 0 & 0.0728225428 & 0.8672195361 & 0.0554706527 \\
\hline 0 & -0.0022355915 & 0.7935409848 & 0.1459543317 \\
\hline $\mathrm{N}$ & 0.3849274786 & 0.5574800428 & 0.3919456262 \\
\hline $\mathrm{N}$ & 0.2728284221 & 0.4881006342 & 0.5145578605 \\
\hline $\mathrm{N}$ & 0.1783771023 & 0.5554051773 & 0.6394019515 \\
\hline $\mathrm{N}$ & 0.1909616295 & 0.5586379490 & 0.3967869738 \\
\hline $\mathrm{N}$ & 0.6186436843 & 0.7627423380 & 0.0007821992 \\
\hline $\mathrm{N}$ & 0.5085667755 & 0.6936098678 & 0.1193541838 \\
\hline $\mathrm{N}$ & 0.7062751191 & 0.6958761888 & 0.8631625523 \\
\hline $\mathrm{N}$ & 0.7023534887 & 0.6901277975 & 0.1062608350 \\
\hline $\mathrm{N}$ & 0.6150725214 & 0.4425199572 & 0.3919456262 \\
\hline $\mathrm{N}$ & 0.7271715479 & 0.5118993658 & 0.5145578605 \\
\hline $\mathrm{N}$ & 0.8216229127 & 0.4445947937 & 0.6394019515 \\
\hline $\mathrm{N}$ & 0.8090383705 & 0.4413620810 & 0.3967869738 \\
\hline $\mathrm{N}$ & 0.3813563157 & 0.2372576320 & 0.0007821992 \\
\hline $\mathrm{N}$ & 0.4914331955 & 0.3063901322 & 0.1193541838 \\
\hline $\mathrm{N}$ & 0.2937248509 & 0.3041238112 & 0.8631625523 \\
\hline $\mathrm{N}$ & 0.2976464813 & 0.3098722325 & 0.1062608350 \\
\hline $\mathrm{N}$ & 0.1150725214 & 0.0574800428 & 0.6080543738 \\
\hline $\mathrm{N}$ & 0.2271715779 & 0.9881006342 & 0.4854421395 \\
\hline $\mathrm{N}$ & 0.3216229127 & 0.0554051953 & 0.3605980785 \\
\hline $\mathrm{N}$ & 0.3090383705 & 0.0586379190 & 0.6032129962 \\
\hline $\mathrm{N}$ & 0.8813563157 & 0.2627423680 & 0.9992178008 \\
\hline $\mathrm{N}$ & 0.9914332245 & 0.1936098828 & 0.8806458752 \\
\hline $\mathrm{N}$ & 0.7937248209 & 0.1958761738 & 0.1368374327 \\
\hline $\mathrm{N}$ & 0.7976465113 & 0.1901277825 & 0.8937391060 \\
\hline $\mathrm{N}$ & 0.8849274786 & 0.9425199572 & 0.6080543738 \\
\hline $\mathrm{N}$ & 0.7728284521 & 0.0118994258 & 0.4854421395 \\
\hline $\mathrm{N}$ & 0.6783770873 & 0.9445948227 & 0.3605980785 \\
\hline $\mathrm{N}$ & 0.6909616295 & 0.9413620510 & 0.6032129962 \\
\hline $\mathrm{N}$ & 0.1186436243 & 0.7372576620 & 0.9992178008 \\
\hline $\mathrm{N}$ & 0.0085668345 & 0.8063901322 & 0.8806458752 \\
\hline $\mathrm{N}$ & 0.2062751791 & 0.8041238112 & 0.1368374327 \\
\hline $\mathrm{N}$ & 0.2023534887 & 0.8098722025 & 0.8937391060 \\
\hline C & 0.4202197312 & 0.5826492067 & 0.7084276963 \\
\hline C & 0.4978487335 & 0.5626520171 & 0.1783299392 \\
\hline C & 0.2072956211 & 0.4743318309 & 0.4289106175 \\
\hline C & 0.1729034158 & 0.5418884245 & 0.7576796820 \\
\hline C & 0.3798028459 & 0.6254042556 & 0.6522251328 \\
\hline C & 0.0022006683 & 0.5616428443 & 0.7677827651 \\
\hline C & 0.5413052300 & 0.5545042257 & 0.2920141672 \\
\hline C & 0.0862015970 & 0.5446934107 & 0.8243802424 \\
\hline C & 0.4092941048 & 0.6681295592 & 0.6968736479 \\
\hline C & 0.3159444918 & 0.4562982569 & 0.5870427353 \\
\hline C & 0.5140609064 & 0.6265607996 & 0.8530685574 \\
\hline C & 0.4865831226 & 0.5839012671 & 0.8063768525 \\
\hline C & 0.1875188713 & 0.4282086989 & 0.4084661689 \\
\hline C & 0.2328955686 & 0.3954951474 & 0.4820605275 \\
\hline C & 0.2971191033 & 0.4099210237 & 0.5738920167 \\
\hline C & 0.1558378707 & 0.5118284656 & 0.3604229844 \\
\hline C & 0.2376447086 & 0.5819892086 & 0.2905351242 \\
\hline C & 0.3969794824 & 0.5698121355 & 0.1739466666 \\
\hline C & 0.0075625843 & 0.5749799529 & 0.6450007505 \\
\hline
\end{tabular}




\begin{tabular}{|c|c|c|c|}
\hline C & 0.1116144776 & 0.5865829521 & 0.4528420738 \\
\hline C & 0.3432487972 & 0.5683470804 & 0.2823676737 \\
\hline C & 0.4825774354 & 0.5514285811 & 0.3959416055 \\
\hline C & 0.4725116006 & 0.6693276546 & 0.7982784566 \\
\hline C & 0.0964205719 & 0.5715376639 & 0.5833918785 \\
\hline C & 0.5733103964 & 0.7957492910 & 0.9348366182 \\
\hline C & 0.6890319464 & 0.7748611780 & 0.0820903638 \\
\hline C & 0.5519461171 & 0.6787059034 & 0.2243206046 \\
\hline C & 0.4994878336 & 0.6737782714 & 0.3331072333 \\
\hline C & 0.4114308402 & 0.7015908601 & 0.1197555199 \\
\hline C & 0.3541108511 & 0.6952465156 & 0.2237529802 \\
\hline C & 0.7386634969 & 0.7361046640 & 0.1480942929 \\
\hline C & 0.7060466216 & 0.7088634628 & 0.7440923634 \\
\hline C & 0.7887927218 & 0.7046495594 & 0.6705803337 \\
\hline C & 0.7803674442 & 0.6630360906 & 0.0437016418 \\
\hline C & 0.7895807925 & 0.6786019332 & 0.9130228159 \\
\hline C & 0.5966316999 & 0.8417353929 & 0.9475516326 \\
\hline C & 0.8745775254 & 0.6869385425 & 0.7212981904 \\
\hline C & 0.3989157916 & 0.6819277002 & 0.3331426662 \\
\hline C & 0.7146614391 & 0.8204919799 & 0.1005475890 \\
\hline C & 0.6569171535 & 0.6644746799 & 0.2095896860 \\
\hline C & 0.6684899036 & 0.8544590127 & 0.0319925764 \\
\hline C & 0.8748568292 & 0.6739494934 & 0.8443054672 \\
\hline C & 0.5797802388 & 0.4173507933 & 0.7084276963 \\
\hline C & 0.5021512965 & 0.4373479829 & 0.1783299392 \\
\hline C & 0.7927043639 & 0.5256681691 & 0.4289106175 \\
\hline C & 0.8270965992 & 0.4581116055 & 0.7576796820 \\
\hline C & 0.6201971241 & 0.3745957444 & 0.6522251328 \\
\hline C & 0.9977993387 & 0.4383571257 & 0.7677827651 \\
\hline C & 0.4586947700 & 0.4454957743 & 0.2920141672 \\
\hline C & 0.9137984030 & 0.4553065893 & 0.8243802424 \\
\hline C & 0.5907058652 & 0.3318704108 & 0.6968736479 \\
\hline C & 0.6840555082 & 0.5437017731 & 0.5870427353 \\
\hline C & 0.4859390646 & 0.3734392004 & 0.8530685574 \\
\hline C & 0.5134168774 & 0.4160987029 & 0.8063768525 \\
\hline C & 0.8124811587 & 0.5717913011 & 0.4084661689 \\
\hline C & 0.7671044314 & 0.6045048526 & 0.4820605275 \\
\hline C & 0.7028808967 & 0.5900789463 & 0.5738920167 \\
\hline C & 0.8441621143 & 0.4881715044 & 0.3604229844 \\
\hline C & 0.7623552914 & 0.4180108214 & 0.2905351242 \\
\hline C & 0.6030205176 & 0.4301878645 & 0.1739466666 \\
\hline C & 0.9924374287 & 0.4250200471 & 0.6450007505 \\
\hline C & 0.8883855304 & 0.4134170479 & 0.4528420738 \\
\hline C & 0.6567512028 & 0.4316529486 & 0.2823676737 \\
\hline C & 0.5174225356 & 0.4485713889 & 0.3959416055 \\
\hline C & 0.5274883994 & 0.3306723154 & 0.7982784566 \\
\hline C & 0.9035793981 & 0.4284623361 & 0.5833918785 \\
\hline C & 0.4266896036 & 0.2042506940 & 0.9348366182 \\
\hline C & 0.3109680536 & 0.2251388220 & 0.0820903638 \\
\hline C & 0.4480538529 & 0.3212941266 & 0.2243206046 \\
\hline C & 0.5005121664 & 0.3262217586 & 0.3331072333 \\
\hline C & 0.5885691298 & 0.2984091099 & 0.1197555199 \\
\hline C & 0.6458891189 & 0.3047534844 & 0.2237529802 \\
\hline C & 0.2613364881 & 0.2638953360 & 0.1480942929 \\
\hline C & 0.2939533784 & 0.2911365372 & 0.7440923634 \\
\hline C & 0.2112072932 & 0.2953504406 & 0.6705803337 \\
\hline C & 0.2196325558 & 0.3369639394 & 0.0437016418 \\
\hline c & 0.2104192075 & 0.3213980378 & 0.9130228159 \\
\hline
\end{tabular}




\begin{tabular}{|c|c|c|c|}
\hline C & 0.4033683001 & 0.1582646361 & 0.9475516326 \\
\hline C & 0.1254224606 & 0.3130614875 & 0.7212981904 \\
\hline C & 0.6010842084 & 0.3180722998 & 0.3331426662 \\
\hline C & 0.2853385609 & 0.1795080201 & 0.1005475890 \\
\hline C & 0.3430828165 & 0.3355253201 & 0.2095896860 \\
\hline C & 0.3315101264 & 0.1455410023 & 0.0319925764 \\
\hline C & 0.1251431488 & 0.3260505066 & 0.8443054672 \\
\hline C & 0.0797802608 & 0.0826491917 & 0.2915723337 \\
\hline C & 0.0021512675 & 0.0626520171 & 0.8216700758 \\
\hline C & 0.2927043939 & 0.9743318309 & 0.5710894125 \\
\hline C & 0.3270965992 & 0.0418884055 & 0.2423203330 \\
\hline C & 0.1201971461 & 0.1254042706 & 0.3477748972 \\
\hline C & 0.4977993387 & 0.0616428703 & 0.2322172199 \\
\hline C & 0.9586947700 & 0.0545042107 & 0.7079858028 \\
\hline C & 0.4137984030 & 0.0446934217 & 0.1756197576 \\
\hline C & 0.0907058882 & 0.1681285752 & 0.3031263811 \\
\hline C & 0.1840555082 & 0.9562982269 & 0.4129572647 \\
\hline C & 0.9859390936 & 0.1265607996 & 0.1469314286 \\
\hline C & 0.0134168664 & 0.0839012901 & 0.1936231325 \\
\hline C & 0.3124811287 & 0.9282086989 & 0.5915338611 \\
\hline C & 0.2671044314 & 0.8954951474 & 0.5179394725 \\
\hline C & 0.2028809117 & 0.9099210537 & 0.4261080133 \\
\hline C & 0.3441621143 & 0.0118284956 & 0.6395770156 \\
\hline C & 0.2623552914 & 0.0819891856 & 0.7094649058 \\
\hline C & 0.1030205026 & 0.0698121425 & 0.8260543164 \\
\hline C & 0.4924374287 & 0.0749799459 & 0.3549992495 \\
\hline C & 0.3883855304 & 0.0865829371 & 0.5471578972 \\
\hline C & 0.1567512028 & 0.0683470584 & 0.7176323263 \\
\hline C & 0.0174225596 & 0.0514286071 & 0.6040583945 \\
\hline C & 0.0274883864 & 0.1693276696 & 0.2017215284 \\
\hline C & 0.4035794281 & 0.0715376639 & 0.4166081215 \\
\hline C & 0.9266896036 & 0.2957492910 & 0.0651634078 \\
\hline C & 0.8109680536 & 0.2748611780 & 0.9179096362 \\
\hline C & 0.9480538829 & 0.1787058734 & 0.7756793954 \\
\hline C & 1.0005121664 & 0.1737782414 & 0.6668927667 \\
\hline C & 0.0885691598 & 0.2015908901 & 0.8802445401 \\
\hline C & 0.1458891489 & 0.1952465156 & 0.7762470198 \\
\hline C & 0.7613365031 & 0.2361046790 & 0.8519057071 \\
\hline C & 0.7939533784 & 0.2088634478 & 0.2559076066 \\
\hline C & 0.7112072782 & 0.2046495594 & 0.3294196663 \\
\hline C & 0.7196325558 & 0.1630360756 & 0.9562983582 \\
\hline C & 0.7104192075 & 0.1786019482 & 0.0869771911 \\
\hline C & 0.9033683001 & 0.3417353639 & 0.0524483904 \\
\hline C & 0.6254224156 & 0.1869385125 & 0.2787018096 \\
\hline C & 0.1010842004 & 0.1819276852 & 0.6668573338 \\
\hline C & 0.7853385609 & 0.3204919799 & 0.8994524110 \\
\hline C & 0.8430827865 & 0.1644746649 & 0.7904103140 \\
\hline C & 0.8315100964 & 0.3544590127 & 0.9680074826 \\
\hline C & 0.6251431118 & 0.1739494784 & 0.1556945038 \\
\hline C & 0.9202197612 & 0.9173507933 & 0.2915723337 \\
\hline C & 0.9978487035 & 0.9373479829 & 0.8216700758 \\
\hline C & 0.7072956361 & 0.0256682291 & 0.5710894125 \\
\hline C & 0.6729034008 & 0.9581115755 & 0.2423203330 \\
\hline C & 0.8798028759 & 0.8745957444 & 0.3477748972 \\
\hline C & 0.5022006613 & 0.9383571557 & 0.2322172199 \\
\hline C & 0.0413052900 & 0.9454957743 & 0.7079858028 \\
\hline C & 0.5862015970 & 0.9553065893 & 0.1756197576 \\
\hline C & 0.9092941348 & 0.8318704408 & 0.3031263811 \\
\hline
\end{tabular}




\begin{tabular}{|c|c|c|c|}
\hline C & 0.8159444918 & 0.0437017131 & 0.4129572647 \\
\hline C & 0.0140609654 & 0.8734392004 & 0.1469314286 \\
\hline C & 0.9865831226 & 0.9160987329 & 0.1936231325 \\
\hline C & 0.6875188413 & 0.0717913011 & 0.5915338611 \\
\hline C & 0.7328955686 & 0.1045048526 & 0.5179394725 \\
\hline C & 0.7971191033 & 0.0900789463 & 0.4261080133 \\
\hline C & 0.6558378857 & 0.9881715344 & 0.6395770156 \\
\hline C & 0.7376447086 & 0.9180107914 & 0.7094649058 \\
\hline C & 0.8969794824 & 0.9301878645 & 0.8260543164 \\
\hline C & 0.5075625713 & 0.9250200471 & 0.3549992495 \\
\hline C & 0.6116144696 & 0.9134170479 & 0.5471578972 \\
\hline C & 0.8432487972 & 0.9316529196 & 0.7176323263 \\
\hline C & 0.9825774644 & 0.9485714189 & 0.6040583945 \\
\hline C & 0.9725116006 & 0.8306723454 & 0.2017215284 \\
\hline C & 0.5964206019 & 0.9284623361 & 0.4166081215 \\
\hline C & 0.0733104564 & 0.7042507090 & 0.0651634078 \\
\hline C & 0.1890319464 & 0.7251388220 & 0.9179096362 \\
\hline C & 0.0519461171 & 0.8212940966 & 0.7756793954 \\
\hline C & -0.0005121664 & 0.8262217286 & 0.6668927667 \\
\hline C & 0.9114308702 & 0.7984091399 & 0.8802445401 \\
\hline C & 0.8541108811 & 0.8047534844 & 0.7762470198 \\
\hline C & 0.2386634969 & 0.7638953360 & 0.8519057071 \\
\hline C & 0.2060466216 & 0.7911365372 & 0.2559076066 \\
\hline C & 0.2887927218 & 0.7953504406 & 0.3294196663 \\
\hline C & 0.2803674442 & 0.8369639094 & 0.9562983582 \\
\hline C & 0.2895807335 & 0.8213980668 & 0.0869771911 \\
\hline C & 0.0966317599 & 0.6582646071 & 0.0524483904 \\
\hline C & 0.3745775844 & 0.8130614575 & 0.2787018096 \\
\hline C & 0.8989157916 & 0.8180722998 & 0.6668573338 \\
\hline C & 0.2146613801 & 0.6795080201 & 0.8994524110 \\
\hline C & 0.1569172135 & 0.8355253201 & 0.7904103140 \\
\hline C & 0.1684899036 & 0.6455409873 & 0.9680074826 \\
\hline C & 0.3748568882 & 0.8260505066 & 0.1556945038 \\
\hline $\mathrm{H}$ & 0.5420279752 & 0.5635390856 & 0.0950401175 \\
\hline $\mathrm{H}$ & 0.2400783144 & 0.5283974167 & 0.7979575730 \\
\hline $\mathrm{H}$ & 0.9333001836 & 0.5636034442 & 0.8181089014 \\
\hline $\mathrm{H}$ & 0.6199048630 & 0.5491119894 & 0.2987874077 \\
\hline $\mathrm{H}$ & 0.0859281170 & 0.5329438157 & 0.9192427228 \\
\hline $\mathrm{H}$ & 0.3821832995 & 0.6999950446 & 0.6573063384 \\
\hline $\mathrm{H}$ & 0.3659937681 & 0.4695473815 & 0.6563037081 \\
\hline $\mathrm{H}$ & 0.5155903284 & 0.5524227371 & 0.8459266776 \\
\hline $\mathrm{H}$ & 0.1370880953 & 0.4177948179 & 0.3360929310 \\
\hline $\mathrm{H}$ & 0.2159086523 & 0.3594252416 & 0.4693818336 \\
\hline $\mathrm{H}$ & 0.3327026324 & 0.3857645942 & 0.6356596542 \\
\hline $\mathrm{H}$ & 0.0767936629 & 0.5095902804 & 0.3793301636 \\
\hline $\mathrm{H}$ & 0.1651091617 & 0.5069526779 & 0.2608976088 \\
\hline $\mathrm{H}$ & 0.2346728410 & 0.6189923522 & 0.3083292858 \\
\hline $\mathrm{H}$ & 0.1987664174 & 0.5751576373 & 0.2040419050 \\
\hline $\mathrm{H}$ & 0.3598476500 & 0.5762537130 & 0.0871055419 \\
\hline $\mathrm{H}$ & 0.9440698792 & 0.5884421871 & 0.5967205858 \\
\hline $\mathrm{H}$ & 0.0433559838 & 0.5854720337 & 0.3992060893 \\
\hline $\mathrm{H}$ & 0.1361987995 & 0.6223750218 & 0.4534782522 \\
\hline $\mathrm{H}$ & 0.5123352336 & 0.5433498996 & 0.4860210754 \\
\hline $\mathrm{H}$ & 0.5192455928 & 0.7839035993 & 0.8689170772 \\
\hline $\mathrm{H}$ & 0.5368069837 & 0.6625875052 & 0.4163576167 \\
\hline $\mathrm{H}$ & 0.3807107976 & 0.7129359093 & 0.0327308338 \\
\hline $\mathrm{H}$ & 0.2755843008 & 0.7013775471 & 0.2186897441 \\
\hline $\mathrm{H}$ & 0.8184744948 & 0.7381336478 & 0.1352484098 \\
\hline
\end{tabular}




\begin{tabular}{|c|c|c|c|}
\hline $\mathrm{H}$ & 0.7277338964 & 0.7393697642 & 0.2477081123 \\
\hline $\mathrm{H}$ & 0.6377349634 & 0.7234737753 & 0.7101113596 \\
\hline $\mathrm{H}$ & 0.7871084109 & 0.7157616070 & 0.5750667194 \\
\hline $\mathrm{H}$ & 0.7570549066 & 0.6270299369 & 0.0428686865 \\
\hline $\mathrm{H}$ & 0.8502012076 & 0.6647303164 & 0.0938447478 \\
\hline $\mathrm{H}$ & 0.5580858486 & 0.8665435500 & 0.8907334126 \\
\hline $\mathrm{H}$ & 0.9397880823 & 0.6834491539 & 0.6646744488 \\
\hline $\mathrm{H}$ & 0.3565246563 & 0.6769177303 & 0.4169317577 \\
\hline $\mathrm{H}$ & 0.7714202270 & 0.8290090264 & 0.1671501134 \\
\hline $\mathrm{H}$ & 0.6990410340 & 0.6685014264 & 0.2950417790 \\
\hline $\mathrm{H}$ & 0.6582524701 & 0.6279682015 & 0.1851502322 \\
\hline $\mathrm{H}$ & 0.6884700251 & 0.8902482647 & 0.0443095677 \\
\hline $\mathrm{H}$ & 0.9404230692 & 0.6601204403 & 0.8874844008 \\
\hline $\mathrm{H}$ & 0.4579720548 & 0.4364609444 & 0.0950401175 \\
\hline $\mathrm{H}$ & 0.7599217156 & 0.4716025833 & 0.7979575730 \\
\hline $\mathrm{H}$ & 0.0666998764 & 0.4363965558 & 0.8181089014 \\
\hline $\mathrm{H}$ & 0.3800951670 & 0.4508880106 & 0.2987874077 \\
\hline $\mathrm{H}$ & 0.9140718830 & 0.4670561843 & 0.9192427228 \\
\hline $\mathrm{H}$ & 0.6178167305 & 0.3000049554 & 0.6573063384 \\
\hline $\mathrm{H}$ & 0.6340062619 & 0.5304526485 & 0.6563037081 \\
\hline H & 0.4844097006 & 0.4475772929 & 0.8459266776 \\
\hline $\mathrm{H}$ & 0.8629119047 & 0.5822051821 & 0.3360929310 \\
\hline $\mathrm{H}$ & 0.7840913627 & 0.6405747284 & 0.4693818336 \\
\hline H & 0.6672973676 & 0.6142354348 & 0.6356596543 \\
\hline $\mathrm{H}$ & 0.9232063371 & 0.4904096896 & 0.3793301636 \\
\hline $\mathrm{H}$ & 0.8348908383 & 0.4930473221 & 0.2608976088 \\
\hline H & 0.7653271440 & 0.3810076768 & 0.3083292858 \\
\hline $\mathrm{H}$ & 0.8012335976 & 0.4248423927 & 0.2040419050 \\
\hline $\mathrm{H}$ & 0.6401523200 & 0.4237462870 & 0.0871055419 \\
\hline $\mathrm{H}$ & 0.0559301208 & 0.4115578129 & 0.5967205858 \\
\hline $\mathrm{H}$ & 0.9566440052 & 0.4145279953 & 0.3992060893 \\
\hline $\mathrm{H}$ & 0.8638012005 & 0.3776249782 & 0.4534782522 \\
\hline $\mathrm{H}$ & 0.4876647964 & 0.4566501004 & 0.4860210754 \\
\hline H & 0.4807544372 & 0.2160963857 & 0.8689170772 \\
\hline $\mathrm{H}$ & 0.4631930163 & 0.3374124948 & 0.4163576167 \\
\hline $\mathrm{H}$ & 0.6192892024 & 0.2870640607 & 0.0327308338 \\
\hline $\mathrm{H}$ & 0.7244156992 & 0.2986224529 & 0.2186897441 \\
\hline $\mathrm{H}$ & 0.1815255202 & 0.2618663822 & 0.1352484098 \\
\hline $\mathrm{H}$ & 0.2722661186 & 0.2606302068 & 0.2477081123 \\
\hline H & 0.3622650656 & 0.2765262247 & 0.7101113596 \\
\hline $\mathrm{H}$ & 0.2128916191 & 0.2842383930 & 0.5750667194 \\
\hline $\mathrm{H}$ & 0.2429451084 & 0.3729700921 & 0.0428686865 \\
\hline H & 0.1497987634 & 0.3352696836 & 0.0938447478 \\
\hline $\mathrm{H}$ & 0.4419141514 & 0.1334564350 & 0.8907334126 \\
\hline $\mathrm{H}$ & 0.0602118997 & 0.3165508461 & 0.6646744488 \\
\hline $\mathrm{H}$ & 0.6434753137 & 0.3230822397 & 0.4169317577 \\
\hline $\mathrm{H}$ & 0.2285797430 & 0.1709909436 & 0.1671501134 \\
\hline H & 0.3009589960 & 0.3314985736 & 0.2950417790 \\
\hline $\mathrm{H}$ & 0.3417474999 & 0.3720318285 & 0.1851502322 \\
\hline $\mathrm{H}$ & 0.3115299749 & 0.1097527113 & 0.0443095677 \\
\hline $\mathrm{H}$ & 0.0595769458 & 0.3398795887 & 0.8874844008 \\
\hline $\mathrm{H}$ & 0.9579720248 & 0.0635390626 & 0.9049598825 \\
\hline $\mathrm{H}$ & 0.2599216856 & 0.0283974097 & 0.2020423970 \\
\hline $\mathrm{H}$ & 0.5666998164 & 0.0636034442 & 0.1818911286 \\
\hline $\mathrm{H}$ & 0.8800951370 & 0.0491119974 & 0.7012125633 \\
\hline $\mathrm{H}$ & 0.4140718830 & 0.0329438077 & 0.0807572552 \\
\hline $\mathrm{H}$ & 0.1178167005 & 0.1999950446 & 0.3426946446 \\
\hline $\mathrm{H}$ & 0.1340062319 & 0.9695473515 & 0.3436963219 \\
\hline
\end{tabular}




\begin{tabular}{|c|c|c|c|}
\hline $\mathrm{H}$ & 0.9844096716 & 0.0524227181 & 0.1540733374 \\
\hline $\mathrm{H}$ & 0.3629119047 & 0.9177948179 & 0.6639070990 \\
\hline $\mathrm{H}$ & 0.2840913327 & 0.8594252716 & 0.5306181664 \\
\hline $\mathrm{H}$ & 0.1672973676 & 0.8857645652 & 0.3643403458 \\
\hline $\mathrm{H}$ & 0.4232063371 & 0.0095902964 & 0.6206698364 \\
\hline $\mathrm{H}$ & 0.3348908383 & 0.0069526879 & 0.7391023912 \\
\hline $\mathrm{H}$ & 0.2653271740 & 0.1189923302 & 0.6916707142 \\
\hline $\mathrm{H}$ & 0.3012335976 & 0.0751576143 & 0.7959580800 \\
\hline $\mathrm{H}$ & 0.1401523500 & 0.0762537210 & 0.9128944281 \\
\hline $\mathrm{H}$ & 0.5559301208 & 0.0884421871 & 0.4032794142 \\
\hline $\mathrm{H}$ & 0.4566440052 & 0.0854720117 & 0.6007939107 \\
\hline $\mathrm{H}$ & 0.3638012005 & 0.1223750218 & 0.5465217478 \\
\hline $\mathrm{H}$ & 0.9876647664 & 0.0433498966 & 0.5139789546 \\
\hline $\mathrm{H}$ & 0.9807544072 & 0.2839035993 & 0.1310829228 \\
\hline $\mathrm{H}$ & 0.9631930163 & 0.1625875202 & 0.5836423833 \\
\hline $\mathrm{H}$ & 0.1192891874 & 0.2129359243 & 0.9672691662 \\
\hline $\mathrm{H}$ & 0.2244157142 & 0.2013775471 & 0.7813102559 \\
\hline $\mathrm{H}$ & 0.6815255052 & 0.2381336328 & 0.8647515302 \\
\hline $\mathrm{H}$ & 0.7722661036 & 0.2393697782 & 0.7522918877 \\
\hline $\mathrm{H}$ & 0.8622650366 & 0.2234737753 & 0.2898886104 \\
\hline H & 0.7128916491 & 0.2157616070 & 0.4249332806 \\
\hline $\mathrm{H}$ & 0.7429450934 & 0.1270299149 & 0.9571313135 \\
\hline $\mathrm{H}$ & 0.6497987924 & 0.1647303014 & 0.9061551922 \\
\hline H & 0.9419141514 & 0.3665435500 & 0.1092676084 \\
\hline $\mathrm{H}$ & 0.5602119177 & 0.1834491539 & 0.3353255812 \\
\hline $\mathrm{H}$ & 0.1434753437 & 0.1769177603 & 0.5830682423 \\
\hline $\mathrm{H}$ & 0.7285797730 & 0.3290090564 & 0.8328498276 \\
\hline $\mathrm{H}$ & 0.8009589660 & 0.1685014414 & 0.7049582210 \\
\hline $\mathrm{H}$ & 0.8417474709 & 0.1279681715 & 0.8148497678 \\
\hline $\mathrm{H}$ & 0.8115299749 & 0.3902472817 & 0.9556903733 \\
\hline $\mathrm{H}$ & 0.5595769898 & 0.1601204253 & 0.1125155912 \\
\hline $\mathrm{H}$ & 0.0420279152 & 0.9364609144 & 0.9049598825 \\
\hline $\mathrm{H}$ & 0.7400782844 & 0.9716025833 & 0.2020423970 \\
\hline H & 0.4333001836 & 0.9363965558 & 0.1818911286 \\
\hline $\mathrm{H}$ & 0.1199048030 & 0.9508880106 & 0.7012125633 \\
\hline $\mathrm{H}$ & 0.5859281170 & 0.9670561843 & 0.0807572552 \\
\hline $\mathrm{H}$ & 0.8821832695 & 0.8000049554 & 0.3426946446 \\
\hline $\mathrm{H}$ & 0.8659937381 & 0.0304525895 & 0.3436963219 \\
\hline $\mathrm{H}$ & 0.0155902694 & 0.9475772629 & 0.1540733374 \\
\hline H & 0.6370880953 & 0.0822051821 & 0.6639070990 \\
\hline $\mathrm{H}$ & 0.7159086373 & 0.1405747284 & 0.5306181664 \\
\hline $\mathrm{H}$ & 0.8327026324 & 0.1142353758 & 0.3643403458 \\
\hline H & 0.5767936629 & 0.9904097196 & 0.6206698364 \\
\hline $\mathrm{H}$ & 0.6651091617 & 0.9930473221 & 0.7391023912 \\
\hline $\mathrm{H}$ & 0.7346738090 & 0.8810076478 & 0.6916707142 \\
\hline $\mathrm{H}$ & 0.6987664024 & 0.9248423627 & 0.7959580800 \\
\hline $\mathrm{H}$ & 0.8598476800 & 0.9237462870 & 0.9128944281 \\
\hline H & 0.4440699092 & 0.9115578129 & 0.4032794142 \\
\hline $\mathrm{H}$ & 0.5433559948 & 0.9145279663 & 0.6007939107 \\
\hline $\mathrm{H}$ & 0.6361987995 & 0.8776249782 & 0.5465217478 \\
\hline $\mathrm{H}$ & 0.0123352336 & 0.9566501004 & 0.5139789546 \\
\hline $\mathrm{H}$ & 0.0192455928 & 0.7160964007 & 0.1310829228 \\
\hline $\mathrm{H}$ & 0.0368069837 & 0.8374124948 & 0.5836423833 \\
\hline $\mathrm{H}$ & 0.8807107976 & 0.7870640907 & 0.9672691662 \\
\hline $\mathrm{H}$ & 0.7755843008 & 0.7986224529 & 0.7813102559 \\
\hline $\mathrm{H}$ & 0.3184744948 & 0.7618663522 & 0.8647515302 \\
\hline $\mathrm{H}$ & 0.2277338374 & 0.7606302358 & 0.7522918877 \\
\hline $\mathrm{H}$ & 0.1377349044 & 0.7765262247 & 0.2898886104 \\
\hline
\end{tabular}




\begin{tabular}{|c|c|c|c|}
\hline $\mathrm{H}$ & 0.2871083509 & 0.7842383930 & 0.4249332806 \\
\hline $\mathrm{H}$ & 0.2570549066 & 0.8729700631 & 0.9571313135 \\
\hline $\mathrm{H}$ & 0.3502012076 & 0.8352696836 & 0.9061551922 \\
\hline $\mathrm{H}$ & 0.0580859086 & 0.6334564500 & 0.1092676084 \\
\hline $\mathrm{H}$ & 0.4397880823 & 0.8165508461 & 0.3353255812 \\
\hline $\mathrm{H}$ & 0.8565246863 & 0.8230822697 & 0.5830682423 \\
\hline $\mathrm{H}$ & 0.2714202270 & 0.6709909736 & 0.8328498276 \\
\hline $\mathrm{H}$ & 0.1990410340 & 0.8314985736 & 0.7049582210 \\
\hline $\mathrm{H}$ & 0.1582525291 & 0.8720317985 & 0.8148497678 \\
\hline $\mathrm{H}$ & 0.1884700251 & 0.6097517353 & 0.9556903733 \\
\hline $\mathrm{H}$ & 0.4404230102 & 0.8398795597 & 0.1125155912 \\
\hline $\mathrm{F}$ & 0.8072106253 & 0.4877456149 & 0.0645838355 \\
\hline $\mathrm{F}$ & 0.7315205714 & 0.5538522127 & 0.1345921353 \\
\hline $\mathrm{F}$ & 0.8134847948 & 0.5545956502 & -0.0517944539 \\
\hline $\mathrm{F}$ & 0.6673923423 & 0.5143232961 & 0.9664394882 \\
\hline $\mathrm{F}$ & 0.1927893747 & 0.5122543851 & 0.0645838355 \\
\hline $\mathrm{F}$ & 0.2684794286 & 0.4461477873 & 0.1345921353 \\
\hline $\mathrm{F}$ & 0.1865152352 & 0.4454043498 & -0.0517944539 \\
\hline $\mathrm{F}$ & 0.3326076287 & 0.4856767039 & 0.9664394882 \\
\hline $\mathrm{F}$ & 0.6927893747 & 0.9877456149 & 0.9354161425 \\
\hline $\mathrm{F}$ & 0.7684794286 & 0.0538522017 & 0.8654078947 \\
\hline $\mathrm{F}$ & 0.6865152052 & 0.0545956572 & 1.0517944349 \\
\hline $\mathrm{F}$ & 0.8326076577 & 0.0143233081 & 0.0335605718 \\
\hline $\mathrm{F}$ & 0.3072106253 & 0.0122543851 & 0.9354161425 \\
\hline $\mathrm{F}$ & 0.2315205564 & 0.9461477873 & 0.8654078947 \\
\hline $\mathrm{F}$ & 0.3134847648 & 0.9454043498 & 1.0517944349 \\
\hline $\mathrm{F}$ & 0.1673923563 & 0.9856767039 & 0.0335605718 \\
\hline $\mathrm{F}$ & 0.4696874252 & 0.8383341964 & 0.5199055886 \\
\hline $\mathrm{F}$ & 0.4422619057 & 0.8426336940 & 0.7293256203 \\
\hline $\mathrm{F}$ & 0.5358746681 & 0.7832255941 & 0.6477439597 \\
\hline $\mathrm{F}$ & 0.3670443946 & 0.7856387296 & 0.6148090277 \\
\hline $\mathrm{F}$ & 0.5303125748 & 0.1616657746 & 0.5199055886 \\
\hline $\mathrm{F}$ & 0.5577371113 & 0.1573662910 & 0.7293256203 \\
\hline $\mathrm{F}$ & 0.4641253019 & 0.2167743909 & 0.6477439597 \\
\hline $\mathrm{F}$ & 0.6329555754 & 0.2143613004 & 0.6148090277 \\
\hline $\mathrm{F}$ & 0.0303125608 & 0.3383342254 & 0.4800944114 \\
\hline $\mathrm{F}$ & 0.0577380943 & 0.3426337240 & 0.2706743797 \\
\hline $\mathrm{F}$ & 0.9641253319 & 0.2832256241 & 0.3522560403 \\
\hline $\mathrm{F}$ & 0.1329555974 & 0.2856386996 & 0.3851909723 \\
\hline $\mathrm{F}$ & 0.9696874252 & 0.6616658036 & 0.4800944114 \\
\hline $\mathrm{F}$ & 0.9422628887 & 0.6573663060 & 0.2706743797 \\
\hline $\mathrm{F}$ & 0.0358746681 & 0.7167744059 & 0.3522560403 \\
\hline $\mathrm{F}$ & 0.8670444246 & 0.7143612704 & 0.3851909723 \\
\hline B & 0.7539295409 & 0.5278363865 & 0.0272196809 \\
\hline B & 0.2460704451 & 0.4721636135 & 0.0272196809 \\
\hline B & 0.7460704591 & 0.0278363865 & 0.9727803111 \\
\hline B & 0.2539295409 & 0.9721636135 & 0.9727803111 \\
\hline B & 0.4545020765 & 0.8120055701 & 0.6278066307 \\
\hline B & 0.5454978945 & 0.1879944449 & 0.6278066307 \\
\hline B & 0.0454979165 & 0.3120055401 & 0.3721933693 \\
\hline B & 0.9545021055 & 0.6879944299 & 0.3721933693 \\
\hline \\
\hline & & & \\
\hline \multicolumn{4}{|c|}{$\begin{array}{c}\text { CELL_PARAMETERS (angstrom) } \\
13.616418687 \\
0.000000000\end{array}$} \\
\hline 0.000000000 & 28.990617082 & 0.000000000 & \\
\hline 0.000000000 & 0.000000000 & 11.452984873 & \\
\hline
\end{tabular}




\begin{tabular}{|c|c|c|c|}
\hline Fe1 & 4.2262225620 & 15.9858202783 & 5.9796741113 \\
\hline $\mathrm{Fe} 1$ & 9.3901961256 & 13.0047968028 & 5.9796741113 \\
\hline Fe1 & 2.5819867823 & 1.4905117383 & 5.4733107623 \\
\hline Fe1 & 11.0344319053 & 27.5001053439 & 5.4733107623 \\
\hline Fe1 & 8.4421018108 & 20.3117146587 & 11.0698019687 \\
\hline Fe1 & 5.1743168758 & 8.6789024234 & 11.0698019687 \\
\hline Fe1 & 11.9825262191 & 5.8164061177 & 0.3831829038 \\
\hline $\mathrm{Fe} 1$ & 1.6338924675 & 23.1742109635 & 0.3831829038 \\
\hline 0 & 5.5746992882 & 15.7650599415 & 7.4839768091 \\
\hline 0 & 8.0417193983 & 13.2255571406 & 7.4839768091 \\
\hline 0 & 1.2335100551 & 1.2697514004 & 3.9690080633 \\
\hline $\mathrm{O}$ & 12.3829086325 & 27.7208656807 & 3.9690080633 \\
\hline 0 & 4.6801655404 & 17.9950503495 & 6.4101264767 \\
\hline 0 & 8.9362531471 & 10.9955667325 & 6.4101264767 \\
\hline 0 & 2.1280438038 & 3.4997418085 & 5.0428583958 \\
\hline 0 & 11.4883748837 & 25.4908752736 & 5.0428583958 \\
\hline 0 & 8.0050851292 & 18.3102967024 & 10.6539901138 \\
\hline 0 & 5.61133355574 & 10.6803203787 & 10.6539901138 \\
\hline 0 & 12.4195429006 & 3.8149881624 & 0.7989947598 \\
\hline 0 & 1.1968757859 & 25.1756289198 & 0.7989947598 \\
\hline 0 & 7.0161970420 & 20.5373033224 & 9.6483551618 \\
\hline 0 & 6.6002216446 & 8.4533137597 & 9.6483551618 \\
\hline 0 & 13.4084309889 & 6.0419947813 & 1.8046297107 \\
\hline 0 & 0.2079876987 & 22.9486222998 & 1.8046297107 \\
\hline 0 & -0.1303384666 & 16.5559090821 & 2.1919166995 \\
\hline 0 & 13.7467575343 & 12.4347079991 & 2.1919166995 \\
\hline 0 & 6.9385477850 & 2.0606005410 & 9.2610678316 \\
\hline 0 & 6.6778704966 & 26.9300165402 & 9.2610678316 \\
\hline $\mathrm{N}$ & 5.3867894329 & 16.2048917934 & 4.1669912273 \\
\hline $\mathrm{N}$ & 8.2296292547 & 12.7857252877 & 4.1669912273 \\
\hline $\mathrm{N}$ & 1.4214199114 & 1.7095832523 & 7.2859933038 \\
\hline $\mathrm{N}$ & 12.1949987762 & 27.2810338288 & 7.2859933038 \\
\hline $\mathrm{N}$ & 3.8779670881 & 13.8902892965 & 5.8534837227 \\
\hline $\mathrm{N}$ & 9.7384515984 & 15.1003269209 & 5.8534837227 \\
\hline $\mathrm{N}$ & 2.9302422552 & 28.3855987013 & 5.5995011508 \\
\hline $\mathrm{N}$ & 10.6861764314 & 0.6050192445 & 5.5995011508 \\
\hline $\mathrm{N}$ & 8.8922755260 & 22.3556844077 & 11.3547506410 \\
\hline $\mathrm{N}$ & 4.7241431606 & 6.6349326735 & 11.3547506410 \\
\hline $\mathrm{N}$ & 11.5323525048 & 7.8603758666 & 0.0982342326 \\
\hline $\mathrm{N}$ & 2.0840661827 & 21.1302412146 & 0.0982342326 \\
\hline $\mathrm{N}$ & 2.3962120261 & 16.0895509136 & 7.1162014473 \\
\hline $\mathrm{N}$ & 11.2202068644 & 12.9010661685 & 7.1162014473 \\
\hline $\mathrm{N}$ & 4.4119975211 & 1.5942423726 & 4.3367834252 \\
\hline $\mathrm{N}$ & 9.2044211664 & 27.3963747086 & 4.3367834252 \\
\hline $\mathrm{N}$ & 7.3667351872 & 20.0872314551 & 1.4657749376 \\
\hline $\mathrm{N}$ & 6.2496835004 & 8.9033856261 & 1.4657749376 \\
\hline $\mathrm{N}$ & 13.0578928437 & 5.5919229140 & 9.9872099349 \\
\hline$N$ & 0.5585258439 & 23.3986941672 & 9.9872099349 \\
\hline $\mathrm{N}$ & 10.1915336335 & 20.1790300498 & 9.8083558933 \\
\hline $\mathrm{N}$ & 3.4248850530 & 8.8115870314 & 9.8083558933 \\
\hline $\mathrm{N}$ & 10.2330943973 & 5.6837215097 & 1.6446289802 \\
\hline $\mathrm{N}$ & 3.3833242902 & 23.3068955724 & 1.6446289802 \\
\hline $\mathrm{N}$ & 2.6733140505 & 15.9473378521 & 4.3452189645 \\
\hline $\mathrm{N}$ & 10.9431048390 & 13.0432792291 & 4.3452189645 \\
\hline $\mathrm{N}$ & 4.1348954957 & 1.4520293110 & 7.1077655676 \\
\hline $\mathrm{N}$ & 9.4815231919 & 27.5385877701 & 7.1077655676 \\
\hline $\mathrm{N}$ & 10.1079178420 & 20.1033363529 & 1.1185223876 \\
\hline $\mathrm{N}$ & 3.5085008455 & 8.8872807292 & 1.1185223877 \\
\hline
\end{tabular}




\begin{tabular}{|c|c|c|c|}
\hline $\mathrm{N}$ & 10.3167101888 & 5.6080278119 & 10.3344624848 \\
\hline $\mathrm{N}$ & 3.2997084977 & 23.3825892693 & 10.3344624848 \\
\hline C & 5.9718000665 & 16.8633128311 & 8.0436241933 \\
\hline C & 7.6446186210 & 12.1273042500 & 8.0436241933 \\
\hline C & 0.8364092768 & 2.3680042900 & 3.4093606791 \\
\hline C & 12.7800094098 & 26.6226127911 & 3.4093606791 \\
\hline C & 6.7259965677 & 16.4254402901 & 1.7323316202 \\
\hline C & 6.8904221199 & 12.5651767911 & 1.7323316202 \\
\hline C & 13.6986314633 & 1.9301317490 & 9.7206534230 \\
\hline C & -0.0822127756 & 27.0604853322 & 9.7206534230 \\
\hline C & 3.0377740597 & 13.4908139083 & 4.8756616101 \\
\hline C & 10.5786446268 & 15.4998031729 & 4.8756616101 \\
\hline C & 3.7704352836 & 27.9861224493 & 6.5773232634 \\
\hline C & 9.8459834040 & 1.0044946328 & 6.5773232634 \\
\hline C & 2.2982442550 & 15.8977883667 & 8.4491257823 \\
\hline C & 11.3181740257 & 13.0928287154 & 8.4491257823 \\
\hline C & 4.5099650883 & 1.4024798257 & 3.0038590902 \\
\hline C & 9.1064540042 & 27.5881372555 & 3.0038590902 \\
\hline C & 5.4831573563 & 18.1398478360 & 7.3984945230 \\
\hline C & 8.1332613313 & 10.8507692452 & 7.3984945230 \\
\hline C & 1.3250519870 & 3.6445392949 & 4.0544903495 \\
\hline C & 12.2913666996 & 25.3460777863 & 4.0544903495 \\
\hline C & 13.5390982383 & 16.2095501490 & 8.3894302845 \\
\hline C & 0.0773203886 & 12.7810669331 & 8.3894302845 \\
\hline C & 6.8855297925 & 1.7142416079 & 3.0635545891 \\
\hline C & 6.7308888940 & 27.2763754732 & 3.0635545891 \\
\hline C & 7.4314875561 & 16.1788747393 & 2.9111635498 \\
\hline C & 6.1849311314 & 12.8117423428 & 2.9111635498 \\
\hline C & 12.9931404747 & 1.6835661982 & 8.5418216641 \\
\hline C & 0.6232782119 & 27.3070508829 & 8.5418216641 \\
\hline C & 1.0830484873 & 15.9552871959 & 9.1237810605 \\
\hline C & 12.5333706052 & 13.0353298853 & 9.1237810605 \\
\hline C & 5.7251608560 & 1.4599786548 & 2.3292038119 \\
\hline C & 7.8912574246 & 27.5306384263 & 2.3292038119 \\
\hline C & 5.8960569076 & 19.3922180625 & 7.8956565397 \\
\hline C & 7.7203613730 & 9.5983990196 & 7.8956565397 \\
\hline C & 0.9121524357 & 4.8969095215 & 3.5573283328 \\
\hline C & 12.7042666568 & 24.0937075596 & 3.5573283328 \\
\hline C & 4.3602522653 & 12.9843614929 & 6.7320324297 \\
\hline C & 9.2561660153 & 16.0062564529 & 6.7320324297 \\
\hline C & 2.4479570780 & 27.4796691703 & 4.7209524428 \\
\hline C & 11.1684620145 & 1.5109470482 & 4.7209524428 \\
\hline C & 7.2117807446 & 18.1631999747 & 9.6608513315 \\
\hline C & 6.4046379429 & 10.8274171065 & 9.6608513315 \\
\hline C & 13.2128472862 & 3.6678914336 & 1.7921335410 \\
\hline C & 0.4035714014 & 25.3227256475 & 1.7921335410 \\
\hline C & 6.8101666118 & 16.9134072921 & 9.1538300330 \\
\hline C & 6.8062524806 & 12.0772097891 & 9.1538300330 \\
\hline C & -0.0019572686 & 2.4180987520 & 2.2991548395 \\
\hline C & 13.6183755492 & 26.5725183301 & 2.2991548395 \\
\hline C & 2.7081503561 & 12.1429985649 & 4.7151893494 \\
\hline C & 10.9082681275 & 16.8476176535 & 4.7151893494 \\
\hline C & 4.1000587842 & 26.6383079697 & 6.7377951816 \\
\hline C & 9.5163599024 & 2.3523099761 & 6.7377951816 \\
\hline C & 3.2229208158 & 11.2034501398 & 5.6067232671 \\
\hline C & 10.3934980738 & 17.7871678060 & 5.6067232671 \\
\hline C & 3.5852887305 & 25.6987578162 & 5.8462619468 \\
\hline C & 10.0311299571 & 3.2918584012 & 5.8462619468 \\
\hline
\end{tabular}




\begin{tabular}{|c|c|c|c|}
\hline C & 8.3025906173 & 23.3717483580 & 10.6852345077 \\
\hline C & 5.3138280692 & 5.6188687241 & 10.6852345077 \\
\hline C & 12.1220374135 & 8.8764398169 & 0.7677503659 \\
\hline C & 1.4943812741 & 20.1141772642 & 0.7677503659 \\
\hline C & 4.0528011299 & 11.6318379309 & 6.6445496910 \\
\hline C & 9.5636175566 & 17.3587782866 & 6.6445496910 \\
\hline C & 2.7554082134 & 26.1271473356 & 4.8084351826 \\
\hline C & 10.8610104742 & 2.8634706102 & 4.8084351826 \\
\hline C & 9.8489986421 & 22.6251633175 & 0.8131004227 \\
\hline C & 3.7674200444 & 6.3654537636 & 0.8131004227 \\
\hline C & 10.5756293887 & 8.1298547775 & 10.6398844498 \\
\hline C & 3.0407892989 & 20.8607623046 & 10.6398844498 \\
\hline C & 8.0967843405 & 19.7323624706 & 2.5448246703 \\
\hline C & 5.5196343460 & 9.2582546105 & 2.5448246703 \\
\hline C & 12.3278436893 & 5.2370539305 & 8.9081602033 \\
\hline C & 1.2885749972 & 23.7535631516 & 8.9081602033 \\
\hline C & 7.5058124508 & 19.5720111294 & 3.8003516364 \\
\hline C & 6.1106062368 & 9.4186059527 & 3.8003516364 \\
\hline C & -0.6976031075 & 5.0767025884 & 7.6526332361 \\
\hline C & 14.3140217941 & 23.9139144937 & 7.6526332361 \\
\hline C & 2.4895279847 & 14.5303563176 & 3.9240854088 \\
\hline C & 11.1268904989 & 14.4602607645 & 3.9240854088 \\
\hline C & 4.3186811557 & 0.0350477765 & 7.5288994647 \\
\hline C & 9.2977375309 & 28.9555693047 & 7.5288994647 \\
\hline C & 3.2215918622 & 16.7331986640 & 3.2112025445 \\
\hline C & 10.3948264184 & 12.2574184172 & 3.2112025445 \\
\hline C & 3.5866174811 & 2.2378901239 & 8.2417823290 \\
\hline C & 10.0298016114 & 26.7527269582 & 8.2417823290 \\
\hline C & 5.3388099865 & 16.5632662365 & 1.7965310406 \\
\hline C & 8.2776087001 & 12.4273508446 & 1.7965310406 \\
\hline C & 1.4693993568 & 2.0679576964 & 9.6564541733 \\
\hline C & 12.1470193297 & 26.9226593857 & 9.6564541733 \\
\hline C & 6.0340005778 & 20.2353401118 & 1.5928079419 \\
\hline C & 7.5824181088 & 8.7552769704 & 1.5928079419 \\
\hline C & 0.7742087655 & 5.7400315707 & 9.8601769306 \\
\hline C & 12.8422099220 & 23.2505855114 & 9.8601769306 \\
\hline C & 0.0213734818 & 16.3695533940 & 7.0092101104 \\
\hline C & 13.5950453699 & 12.6210636872 & 7.0092101104 \\
\hline C & 6.7868360266 & 1.8742448529 & 4.4437747632 \\
\hline C & 6.8295826609 & 27.1163722282 & 4.4437747632 \\
\hline C & 1.4478606993 & 16.5598708549 & 4.9188098344 \\
\hline C & 12.1685577853 & 12.4307462272 & 4.9188098344 \\
\hline C & 5.3603484420 & 2.0645623138 & 6.5341750381 \\
\hline C & 8.2560702455 & 26.9260547683 & 6.5341750381 \\
\hline C & 4.6992803272 & 16.4693647478 & 3.0340820016 \\
\hline C & 8.9171383603 & 12.5212523333 & 3.0340820016 \\
\hline C & 2.1089290160 & 1.9740562068 & 8.4189032134 \\
\hline C & 11.5074896705 & 27.0165608744 & 8.4189032134 \\
\hline C & 5.3724641370 & 20.0358741286 & 2.7976976691 \\
\hline C & 8.2439549555 & 8.9547429525 & 2.7976976691 \\
\hline C & 1.4357452063 & 5.5405655886 & 8.6552872044 \\
\hline C & 12.1806730743 & 23.4500514936 & 8.6552872044 \\
\hline C & 6.7246952049 & 16.0654721291 & 4.1023127144 \\
\hline C & 6.8917238876 & 12.9251449531 & 4.1023127144 \\
\hline C & 0.0835141384 & 1.5701635880 & 7.3506721581 \\
\hline C & 13.5329041432 & 27.4204534941 & 7.3506721581 \\
\hline C & 10.5733938103 & 21.4705828671 & 1.4851284460 \\
\hline C & 3.0430248762 & 7.5200342140 & 1.4851284460 \\
\hline
\end{tabular}




\begin{tabular}{|c|c|c|c|}
\hline C & 9.8512342195 & 6.9752743270 & 9.9678564265 \\
\hline C & 3.7651844671 & 22.0153427551 & 9.9678564265 \\
\hline C & 6.6954302377 & 19.4392985423 & 9.0346276463 \\
\hline C & 6.9209884489 & 9.5513185388 & 9.0346276463 \\
\hline C & 0.1127791056 & 4.9439900012 & 2.4183572273 \\
\hline C & 13.5036395810 & 24.0466270799 & 2.4183572273 \\
\hline C & 1.2751392011 & 16.3027356245 & 6.3976044940 \\
\hline C & 12.3412793850 & 12.6878814577 & 6.3976044940 \\
\hline C & 5.5330700417 & 1.8074270834 & 5.0553803785 \\
\hline C & 8.0833486459 & 27.1831899977 & 5.0553803785 \\
\hline C & 10.2480801297 & 20.5257973189 & 8.5054703624 \\
\hline C & 3.3683385569 & 8.4648197622 & 8.5054703624 \\
\hline C & 10.1765479002 & 6.0304887789 & 2.9475145101 \\
\hline C & 3.4398707864 & 22.9601283033 & 2.9475145101 \\
\hline C & 11.4061919477 & 20.3736202796 & 7.7496028577 \\
\hline C & 2.2102267388 & 8.6169968026 & 7.7496028577 \\
\hline C & 9.0184360821 & 5.8783117385 & 3.7033820148 \\
\hline C & 4.5979826044 & 23.1123053437 & 3.7033820148 \\
\hline C & 11.1420124417 & 19.2999693180 & 0.4129381788 \\
\hline C & 2.4744062449 & 9.6906477631 & 0.4129381788 \\
\hline C & 9.2826155881 & 4.8046607780 & 11.0400466937 \\
\hline C & 4.3338030984 & 24.1859563042 & 11.0400466937 \\
\hline C & 11.2954304022 & 19.6973307063 & 10.4163007081 \\
\hline C & 2.3209882854 & 9.2932863758 & 10.4163007080 \\
\hline C & 9.1291976287 & 5.2020221652 & 1.0366841644 \\
\hline C & 4.4872210589 & 23.7885949169 & 1.0366841644 \\
\hline C & 8.6248344277 & 24.7031764999 & 10.9205711411 \\
\hline C & 4.9915842598 & 4.2874405823 & 10.9205711411 \\
\hline C & 11.7997936031 & 10.2078679588 & 0.5324137325 \\
\hline C & 1.8166250844 & 18.7827491233 & 0.5324137325 \\
\hline C & 12.5410304705 & 19.8362525892 & 8.3616512630 \\
\hline C & 1.0753882171 & 9.1543644919 & 8.3616512630 \\
\hline C & 7.8835975604 & 5.3409440491 & 3.0913336095 \\
\hline C & 5.7328211262 & 23.6496730330 & 3.0913336095 \\
\hline C & 6.1248340334 & 19.7156865713 & 3.9302058358 \\
\hline C & 7.4915846532 & 9.2749305108 & 3.9302058358 \\
\hline C & 0.6833753099 & 5.2203780303 & 7.5227790378 \\
\hline C & 12.9330433766 & 23.7702390509 & 7.5227790378 \\
\hline C & 10.2037215715 & 23.9433564165 & 1.1165926890 \\
\hline C & 3.4126971151 & 5.0472606656 & 1.1165926890 \\
\hline C & 10.2209064584 & 9.4480478755 & 10.3363921835 \\
\hline C & 3.3955122282 & 19.5425692057 & 10.3363921835 \\
\hline C & 9.5459050033 & 19.3834403601 & 2.2866522362 \\
\hline C & 4.0705136833 & 9.6071767220 & 2.2866522362 \\
\hline C & 10.8787230266 & 4.8881318190 & 9.1663326363 \\
\hline C & 2.7376956600 & 24.1024852621 & 9.1663326363 \\
\hline C & 9.5862340990 & 24.9921917298 & 0.4392557944 \\
\hline C & 4.0301845876 & 3.9984253523 & 0.4392557944 \\
\hline C & 10.8383939309 & 10.4968831887 & 11.0137290781 \\
\hline C & 2.7780247557 & 18.4937338924 & 11.0137290781 \\
\hline C & 12.4890168073 & 19.5083501569 & 9.7152604566 \\
\hline C & 1.1274018792 & 9.4822669242 & 9.7152604566 \\
\hline C & 7.9356112225 & 5.0130416158 & 1.7377244159 \\
\hline C & 5.6808074640 & 23.9775754653 & 1.7377244159 \\
\hline $\mathrm{H}$ & 7.2598597825 & 16.5197818432 & 0.7849223663 \\
\hline $\mathrm{H}$ & 6.3565589040 & 12.4708352389 & 0.7849223663 \\
\hline $\mathrm{H}$ & 13.1647682473 & 2.0244733021 & 10.6680625062 \\
\hline $\mathrm{H}$ & 0.4516504392 & 26.9661437800 & 10.6680625062 \\
\hline
\end{tabular}




\begin{tabular}{|c|c|c|c|}
\hline $\mathrm{H}$ & 3.2292328467 & 15.6692274716 & 8.9722689751 \\
\hline $\mathrm{H}$ & 10.3871856369 & 13.3213896096 & 8.9722689751 \\
\hline $\mathrm{H}$ & 3.5789762936 & 1.1739189305 & 2.4807158974 \\
\hline $\mathrm{H}$ & 10.0374423930 & 27.8166981506 & 2.4807158974 \\
\hline $\mathrm{H}$ & 12.5716958157 & 16.2543759705 & 8.8926710064 \\
\hline $\mathrm{H}$ & 1.0447228708 & 12.7362411116 & 8.8926710064 \\
\hline $\mathrm{H}$ & 7.8529322141 & 1.7590674294 & 2.5603138661 \\
\hline $\mathrm{H}$ & 5.7634864724 & 27.2315496517 & 2.5603138661 \\
\hline $\mathrm{H}$ & 8.5129795978 & 16.0566037425 & 2.8920207382 \\
\hline $\mathrm{H}$ & 5.1034390888 & 12.9340133386 & 2.8920207382 \\
\hline $\mathrm{H}$ & 11.9116484331 & 1.5612952024 & 8.5609644757 \\
\hline $\mathrm{H}$ & 1.7047702545 & 27.4293218797 & 8.5609644757 \\
\hline $\mathrm{H}$ & 1.0490252319 & 15.7751641445 & 10.1981338517 \\
\hline $\mathrm{H}$ & 12.5673934546 & 13.2154529377 & 10.1981338517 \\
\hline $\mathrm{H}$ & 5.7591841114 & 1.2798556034 & 1.2548510208 \\
\hline $\mathrm{H}$ & 7.8572345762 & 27.7107614778 & 1.2548510208 \\
\hline $\mathrm{H}$ & 5.5416319468 & 20.3182370642 & 7.4387453525 \\
\hline $\mathrm{H}$ & 8.0747871457 & 8.6723800179 & 7.4387453525 \\
\hline $\mathrm{H}$ & 1.2665773965 & 5.8229285231 & 4.0142395211 \\
\hline $\mathrm{H}$ & 12.3498408841 & 23.1676885580 & 4.0142395211 \\
\hline $\mathrm{H}$ & 5.0067337047 & 13.3825735044 & 7.5151361920 \\
\hline $\mathrm{H}$ & 8.6096849818 & 15.6080444415 & 7.5151361920 \\
\hline $\mathrm{H}$ & 1.8014756386 & 27.8778811808 & 3.9378486805 \\
\hline $\mathrm{H}$ & 11.8149430480 & 1.1127350367 & 3.9378486804 \\
\hline $\mathrm{H}$ & 7.1970437091 & 15.9969982099 & 9.6024543246 \\
\hline $\mathrm{H}$ & 6.4193749775 & 12.9936188712 & 9.6024543246 \\
\hline $\mathrm{H}$ & 13.2275843208 & 1.5016896689 & 1.8505305489 \\
\hline $\mathrm{H}$ & 0.3888343658 & 27.4889274123 & 1.8505305489 \\
\hline $\mathrm{H}$ & 2.0328929307 & 11.8447152591 & 3.9137783870 \\
\hline $\mathrm{H}$ & 11.5835259598 & 17.1459026867 & 3.9137783870 \\
\hline $\mathrm{H}$ & 4.7753166155 & 26.3400229355 & 7.5392068280 \\
\hline $\mathrm{H}$ & 8.8411020710 & 2.6505932820 & 7.5392068280 \\
\hline $\mathrm{H}$ & 2.9403187148 & 10.1554786317 & 5.5068048533 \\
\hline $\mathrm{H}$ & 10.6760999728 & 18.8351384505 & 5.5068048533 \\
\hline $\mathrm{H}$ & 3.8678906295 & 24.6507871717 & 5.9461803606 \\
\hline $\mathrm{H}$ & 9.7485280581 & 4.3398299094 & 5.9461803606 \\
\hline $\mathrm{H}$ & 7.5665792744 & 23.0814012339 & 9.9335110127 \\
\hline $\mathrm{H}$ & 6.0498394122 & 5.9092158482 & 9.9335110127 \\
\hline $\mathrm{H}$ & 12.8580487554 & 8.5860926928 & 1.5194738609 \\
\hline $\mathrm{H}$ & 0.7583699311 & 20.4045243883 & 1.5194738609 \\
\hline $\mathrm{H}$ & 4.4538383615 & 10.9395381736 & 7.3845152528 \\
\hline $\mathrm{H}$ & 9.1625803260 & 18.0510789075 & 7.3845152528 \\
\hline $\mathrm{H}$ & 2.3543709827 & 25.4348467147 & 4.0684696197 \\
\hline $\mathrm{H}$ & 11.2620477048 & 3.5557703675 & 4.0684696197 \\
\hline $\mathrm{H}$ & 8.1210862389 & 19.3039827224 & 4.6603473983 \\
\hline $\mathrm{H}$ & 5.4953324476 & 9.6866343597 & 4.6603473983 \\
\hline $\mathrm{H}$ & 12.3035417909 & 4.8086741813 & 6.7926374742 \\
\hline $\mathrm{H}$ & 1.3128768957 & 24.1819428998 & 6.7926374742 \\
\hline $\mathrm{H}$ & 1.4257531367 & 14.3231944613 & 3.7193916680 \\
\hline $\mathrm{H}$ & 12.1906655508 & 14.6674226198 & 3.7193916680 \\
\hline $\mathrm{H}$ & 5.3824562065 & -0.1721140787 & 7.7335928641 \\
\hline $\mathrm{H}$ & 8.2339624800 & 29.1627311610 & 7.7335928641 \\
\hline $\mathrm{H}$ & 2.9903608448 & 14.3850727048 & 2.9529769673 \\
\hline $\mathrm{H}$ & 10.6260582477 & 14.6055443774 & 2.9529769673 \\
\hline $\mathrm{H}$ & 3.8178484985 & -0.1102358363 & 8.5000079052 \\
\hline $\mathrm{H}$ & 9.7985697831 & 29.1008529173 & 8.5000079052 \\
\hline $\mathrm{H}$ & 3.0922637793 & 17.8014437319 & 3.4515457499 \\
\hline $\mathrm{H}$ & 10.5241545023 & 11.1891733503 & 3.4515457499 \\
\hline
\end{tabular}




\begin{tabular}{|c|c|c|c|}
\hline $\mathrm{H}$ & 3.7159455640 & 3.3061351908 & 8.0014387822 \\
\hline $\mathrm{H}$ & 9.9004735285 & 25.6844818913 & 8.0014387822 \\
\hline $\mathrm{H}$ & 2.6727532459 & 16.5296372041 & 2.2791236181 \\
\hline $\mathrm{H}$ & 10.9436654407 & 12.4609798771 & 2.2791236181 \\
\hline $\mathrm{H}$ & 4.1354560974 & 2.0343286640 & 9.1738612544 \\
\hline $\mathrm{H}$ & 9.4809625892 & 26.9562884181 & 9.1738612544 \\
\hline $\mathrm{H}$ & 4.7465848900 & 16.7304102286 & 0.8975723428 \\
\hline $\mathrm{H}$ & 8.8698337975 & 12.2602068535 & 0.8975723428 \\
\hline $\mathrm{H}$ & 2.0616244533 & 2.2351016875 & 10.5554121882 \\
\hline $\mathrm{H}$ & 11.5547942333 & 26.7555153946 & 10.5554121882 \\
\hline $\mathrm{H}$ & 5.4968089327 & 20.5059687252 & 0.6821276531 \\
\hline $\mathrm{H}$ & 8.1196097539 & 8.4846483559 & 0.6821276531 \\
\hline $\mathrm{H}$ & 1.3114004106 & 6.0106601842 & 10.7708572194 \\
\hline $\mathrm{H}$ & 12.3050182760 & 22.9799568970 & 10.7708572194 \\
\hline $\mathrm{H}$ & 12.7559924002 & 16.5639920191 & 6.4019265173 \\
\hline $\mathrm{H}$ & 0.8604261849 & 12.4266250630 & 6.4019265173 \\
\hline $\mathrm{H}$ & 7.6686356307 & 2.0686834781 & 5.0510583552 \\
\hline $\mathrm{H}$ & 5.9477830569 & 26.9219336041 & 5.0510583552 \\
\hline $\mathrm{H}$ & 0.5539196733 & 16.2490206015 & 4.3571577876 \\
\hline $\mathrm{H}$ & 13.0624992162 & 12.7415964806 & 4.3571577876 \\
\hline $\mathrm{H}$ & 6.2542898729 & 1.7537120605 & 7.0958274263 \\
\hline $\mathrm{H}$ & 7.3621288137 & 27.2369050207 & 7.0958274263 \\
\hline $\mathrm{H}$ & 1.5217654433 & 17.6511396514 & 4.7913392206 \\
\hline $\mathrm{H}$ & 12.0946536492 & 11.3394774308 & 4.7913392206 \\
\hline $\mathrm{H}$ & 5.2864439000 & 3.1558311103 & 6.6616459934 \\
\hline $\mathrm{H}$ & 8.3299743817 & 25.8347859708 & 6.6616459934 \\
\hline $\mathrm{H}$ & 4.2890124862 & 20.1443202805 & 2.8471521572 \\
\hline $\mathrm{H}$ & 9.3274062003 & 8.8462968016 & 2.8471521572 \\
\hline $\mathrm{H}$ & 2.5191968571 & 5.6490117394 & 8.6058327164 \\
\hline $\mathrm{H}$ & 11.0972218295 & 23.3416053417 & 8.6058327164 \\
\hline $\mathrm{H}$ & 7.2242506985 & 15.8533695471 & 5.0483879822 \\
\hline $\mathrm{H}$ & 6.3921679881 & 13.1372475350 & 5.0483879823 \\
\hline $\mathrm{H}$ & 13.2003773314 & 1.3580610061 & 6.4045968913 \\
\hline $\mathrm{H}$ & 0.4160413552 & 27.6325560751 & 6.4045968913 \\
\hline $\mathrm{H}$ & 11.6514767715 & 21.5640727114 & 1.2668376079 \\
\hline $\mathrm{H}$ & 1.9649419151 & 7.4265443708 & 1.2668376079 \\
\hline $\mathrm{H}$ & 8.7731512583 & 7.0687641703 & 10.1861472657 \\
\hline $\mathrm{H}$ & 4.8432674282 & 21.9218529108 & 10.1861472657 \\
\hline $\mathrm{H}$ & 10.5080864001 & 21.5951441838 & 2.5774345219 \\
\hline $\mathrm{H}$ & 3.1083322864 & 7.3954728983 & 2.5774345219 \\
\hline $\mathrm{H}$ & 9.9165416297 & 7.0998356427 & 8.8755503506 \\
\hline $\mathrm{H}$ & 3.6998770569 & 21.8907814384 & 8.8755503506 \\
\hline $\mathrm{H}$ & 9.3295280320 & 20.9443910991 & 8.0824150168 \\
\hline $\mathrm{H}$ & 4.2868906546 & 8.0462259830 & 8.0824150168 \\
\hline $\mathrm{H}$ & 11.0950999979 & 6.4490825580 & 3.3705698567 \\
\hline $\mathrm{H}$ & 2.5213186887 & 22.5415345241 & 3.3705698567 \\
\hline $\mathrm{H}$ & 11.4310396854 & 20.6764198867 & 6.7023216729 \\
\hline $\mathrm{H}$ & 2.1853790012 & 8.3141971954 & 6.7023216729 \\
\hline $\mathrm{H}$ & 8.9935883445 & 6.1811113456 & 4.7506631996 \\
\hline $\mathrm{H}$ & 4.6228303421 & 22.8095057355 & 4.7506631996 \\
\hline $\mathrm{H}$ & 10.8136530175 & 18.2480691264 & 0.4361320845 \\
\hline $\mathrm{H}$ & 2.8027656691 & 10.7425479548 & 0.4361320845 \\
\hline $\mathrm{H}$ & 9.6109750124 & 3.7527605853 & 11.0168527890 \\
\hline $\mathrm{H}$ & 4.0054436742 & 25.2378564958 & 11.0168527890 \\
\hline $\mathrm{H}$ & 12.1100742987 & 19.3513060935 & 0.9345675734 \\
\hline $\mathrm{H}$ & 1.5063443878 & 9.6393109886 & 0.9345675734 \\
\hline $\mathrm{H}$ & 8.3145537311 & 4.8559975525 & 10.5184172991 \\
\hline $\mathrm{H}$ & 5.3018649555 & 24.1346195296 & 10.5184172991 \\
\hline
\end{tabular}




\begin{tabular}{|c|c|c|c|}
\hline $\mathrm{H}$ & 8.1192510562 & 25.4980176199 & 10.3683603953 \\
\hline $\mathrm{H}$ & 5.4971676314 & 3.4925994613 & 10.3683603953 \\
\hline $\mathrm{H}$ & 12.3053769746 & 11.0027090798 & 1.0846244772 \\
\hline $\mathrm{H}$ & 1.3110417119 & 17.9879080024 & 1.0846244772 \\
\hline $\mathrm{H}$ & 13.4491339861 & 19.6812619372 & 7.7779126502 \\
\hline $\mathrm{H}$ & 0.1672847004 & 9.3093551439 & 7.7779126502 \\
\hline $\mathrm{H}$ & 6.9754940437 & 5.1859533962 & 3.6750722233 \\
\hline $\mathrm{H}$ & 6.6409246428 & 23.8046636850 & 3.6750722233 \\
\hline $\mathrm{H}$ & 5.6366433554 & 19.5538948870 & 4.8924982478 \\
\hline $\mathrm{H}$ & 7.9797757371 & 9.4367221941 & 4.8924982478 \\
\hline $\mathrm{H}$ & 1.1715659879 & 5.0585863460 & 6.5604866246 \\
\hline $\mathrm{H}$ & 12.4448522928 & 23.9320307352 & 6.5604866246 \\
\hline $\mathrm{H}$ & 10.9613939884 & 24.1346152983 & 1.8775501843 \\
\hline $\mathrm{H}$ & 2.6550246982 & 4.8560017838 & 1.8775501843 \\
\hline $\mathrm{H}$ & 9.4632340415 & 9.6393067572 & 9.5754346892 \\
\hline $\mathrm{H}$ & 4.1531846451 & 19.3513103239 & 9.5754346892 \\
\hline $\mathrm{H}$ & 10.1590693142 & 19.5468330733 & 3.1871535273 \\
\hline $\mathrm{H}$ & 3.4573493724 & 9.4437840079 & 3.1871535273 \\
\hline $\mathrm{H}$ & 10.2655587157 & 5.0515245322 & 8.2658313452 \\
\hline $\mathrm{H}$ & 3.3508599709 & 23.9390925489 & 8.2658313452 \\
\hline $\mathrm{H}$ & 9.5814582867 & 18.3050750174 & 2.0586260475 \\
\hline $\mathrm{H}$ & 4.0349604008 & 10.6855420647 & 2.0586260475 \\
\hline $\mathrm{H}$ & 10.8431697441 & 3.8097664763 & 9.3943588261 \\
\hline $\mathrm{H}$ & 2.7732489434 & 25.1808506048 & 9.3943588261 \\
\hline $\mathrm{H}$ & 9.8485347884 & 26.0279147714 & 0.6593250150 \\
\hline $\mathrm{H}$ & 3.7678838982 & 2.9627023098 & 0.6593250150 \\
\hline $\mathrm{H}$ & 10.5760932414 & 11.5326062303 & 10.7936598575 \\
\hline $\mathrm{H}$ & 3.0403254451 & 17.4580108508 & 10.7936598575 \\
\hline $\mathrm{H}$ & 13.3590093186 & 19.0982175537 & 10.2289082473 \\
\hline $\mathrm{H}$ & 0.2574093680 & 9.8923995285 & 10.2289082473 \\
\hline $\mathrm{H}$ & 7.0656187113 & 4.6029090126 & 1.2240766263 \\
\hline $\mathrm{H}$ & 6.5507999753 & 24.3877080685 & 1.2240766263 \\
\hline $\mathrm{H}$ & 12.9590804646 & 16.2946348603 & 1.4011584484 \\
\hline $\mathrm{H}$ & 0.6573380578 & 12.6959822218 & 1.4011584484 \\
\hline $\mathrm{H}$ & 7.4655475663 & 1.7993263192 & 10.0518265959 \\
\hline $\mathrm{H}$ & 6.1508711213 & 27.1912907619 & 10.0518265959 \\
\hline $\mathrm{H}$ & 13.1971892811 & 17.4634118861 & 2.4254526013 \\
\hline $\mathrm{H}$ & 0.4192298113 & 11.5272051951 & 2.4254526013 \\
\hline $\mathrm{H}$ & 7.2274387487 & 2.9681033450 & 9.0275319298 \\
\hline $\mathrm{H}$ & 6.3889795319 & 26.0225137361 & 9.0275319298 \\
\hline $\mathrm{F}$ & 10.7770045702 & 13.9636871309 & 0.3283700719 \\
\hline $\mathrm{F}$ & 2.8394141163 & 15.0269290876 & 0.3283700719 \\
\hline $\mathrm{F}$ & 9.6476234596 & 28.4589965356 & 11.1246148006 \\
\hline $\mathrm{F}$ & 3.9687952269 & 0.5316214102 & 11.1246148006 \\
\hline $\mathrm{F}$ & 10.0731057452 & 16.0372272099 & 1.1230582177 \\
\hline $\mathrm{F}$ & 3.5433129414 & 12.9533898713 & 1.1230582177 \\
\hline $\mathrm{F}$ & 10.3515222847 & 1.5419186688 & 10.3299266559 \\
\hline $\mathrm{F}$ & 3.2648964019 & 27.4486984123 & 10.3299266559 \\
\hline $\mathrm{F}$ & 6.5577425063 & 24.3477328493 & 6.0863234116 \\
\hline $\mathrm{F}$ & 7.0586761813 & 4.6428842318 & 6.0863234116 \\
\hline $\mathrm{F}$ & 0.2504668370 & 9.8524243093 & 5.3666614620 \\
\hline $\mathrm{F}$ & 13.3659518495 & 19.1381927729 & 5.3666614620 \\
\hline $\mathrm{F}$ & 12.0377530995 & 15.8815288392 & 11.3551676416 \\
\hline $\mathrm{F}$ & 1.5786655871 & 13.1090882419 & 11.3551676416 \\
\hline $\mathrm{F}$ & 8.3868749314 & 1.3862202982 & 0.0978172309 \\
\hline $\mathrm{F}$ & 5.2295437562 & 27.6043967830 & 0.0978172309 \\
\hline $\mathrm{F}$ & 6.1101047045 & 24.2915604939 & 8.3688249386 \\
\hline $\mathrm{F}$ & 7.5063139831 & 4.6990565873 & 8.3688249386 \\
\hline
\end{tabular}




\begin{tabular}{|c|c|c|c|}
\hline $\mathrm{F}$ & 0.6981046398 & 9.7962519528 & 3.0841599339 \\
\hline 12 & 2.9183140478 & 19.1943651284 & 3.0841599339 \\
\hline $\mathrm{F}$ & 9.9969641277 & 15.5649127620 & 10.2873915940 \\
\hline $\mathrm{F}$ & 3.6194545588 & 13.4257043192 & 10.2873915940 \\
\hline 10 & 0.4276639031 & 1.0696042209 & 1.1655932785 \\
\hline $\mathrm{F}$ & 3.1887547844 & 27.9210128602 & 1.1655932785 \\
\hline $\mathrm{F}$ & 7.5583906418 & 22.7323767754 & 7.4186568292 \\
\hline $\mathrm{F}$ & 6.0580280448 & 6.2582403057 & 7.4186568292 \\
\hline 12 & 2.8662373881 & 8.2370682343 & 4.0343280444 \\
\hline $\mathrm{F}$ & 0.7501812985 & 20.7535488468 & 4.0343280444 \\
\hline $\mathrm{F}$ & 5.2595213057 & 22.6415653366 & 6.9776256692 \\
\hline $\mathrm{F}$ & 8.3568969750 & 6.3490517445 & 6.9776256692 \\
\hline $\mathrm{F}$ & 1.5486880376 & 8.1462567966 & 4.4753592033 \\
\hline 12 & 2.0677310549 & 20.8443602856 & 4.4753592033 \\
\hline 10 & 0.7009546855 & 15.3544339428 & 0.0327642118 \\
\hline B & 2.9154640020 & 13.6361831393 & 0.0327642118 \\
\hline B & 9.7236733453 & 0.8591254017 & 11.4202206618 \\
\hline B & 3.8927453413 & 28.1314916794 & 11.4202206618 \\
\hline B & 6.3911723718 & 23.4838692668 & 7.1960559458 \\
\hline B & 7.2252463158 & 5.5067478143 & 7.1960559458 \\
\hline B & 0.4170369725 & 8.9885607267 & 4.2569289267 \\
\hline 13 & 3.1993817150 & 20.0020563554 & 4.2569289267 \\
\hline \multicolumn{4}{|c|}{$1 \cdot \mathrm{H}_{2} \mathrm{O}$ iS } \\
\hline \multicolumn{4}{|c|}{ CELL_PARAMETERS (angstrom) } \\
\hline 13.684690601 & 0.000000000 & 0.000000000 & \\
\hline 0.000000000 & 29.212834993 & 0.000000000 & \\
\hline 0.000000000 & 0.000000000 & 11.224293169 & \\
\hline \multicolumn{4}{|c|}{ ATOMIC_POSITIONS (angstrom) } \\
\hline Fe1 4 & 4.2481804578 & 16.2106250038 & 5.9021859358 \\
\hline Fe1 & 9.4365101440 & 13.0022099891 & 5.9021859358 \\
\hline Fe1 & 2.5941648436 & 1.6042075078 & 5.3221072337 \\
\hline Fe1 & 1.0905257582 & 27.6086274860 & 5.3221072337 \\
\hline $\mathrm{Fe} 2$ & 8.4919853086 & 20.4184881376 & 10.9090837504 \\
\hline $\mathrm{Fe} 2$ & 5.1927052922 & 8.7943468562 & 10.9090837503 \\
\hline $\mathrm{Fe} 2$ & 2.0350505926 & 5.8120706407 & 0.3152094181 \\
\hline $\mathrm{Fe} 2$ & 1.6496400082 & 23.4007643521 & 0.3152094181 \\
\hline $\mathrm{O}$ & 5.6231837511 & 15.9758245850 & 7.3951435942 \\
\hline $\mathrm{O}$ & 8.0615068497 & 13.2370104088 & 7.3951435942 \\
\hline $\mathrm{O}$ & 1.2191615493 & 1.3694070881 & 3.8291495742 \\
\hline $\mathrm{O}$ & 12.4655290525 & 27.8434279047 & 3.8291495743 \\
\hline $\mathrm{O}$ & 4.7105032427 & 18.2040395334 & 6.3374525546 \\
\hline $\mathrm{O}$ & 8.9741873590 & 11.0087954604 & 6.3374525546 \\
\hline $\mathrm{O}$ & 2.1318420587 & 3.5976220365 & 4.8868406139 \\
\hline $\mathrm{O}$ & 1.5528485431 & 25.6152129573 & 4.8868406139 \\
\hline $\mathrm{O}$ & 8.1233907326 & 18.5190415100 & 10.5295766305 \\
\hline $\mathrm{O}$ & 5.5612998681 & 10.6937934828 & 10.5295766305 \\
\hline $\mathrm{O}$ & 2.4036451685 & 3.9126240141 & 0.6947165390 \\
\hline $\mathrm{O}$ & 1.2810454323 & 25.3002109797 & 0.6947165390 \\
\hline $\mathrm{O}$ & 7.0875535800 & 20.6973558549 & 9.6016235982 \\
\hline $\mathrm{O}$ & 6.5971370208 & 8.5154791390 & 9.6016235982 \\
\hline $\mathrm{O}$ & 13.4394823222 & 6.0909383579 & 1.6226695703 \\
\hline $\mathrm{O}$ & 0.2452082796 & 23.1218966349 & 1.6226695703 \\
\hline $\mathrm{O}$ & 0.1868360602 & 16.7577103427 & 2.0928109913 \\
\hline $\mathrm{O}$ & 13.8715270440 & 12.4551246501 & 2.0928109913 \\
\hline $\mathrm{O}$ & 7.0291813356 & 2.1512928458 & 9.1314818426 \\
\hline $\mathrm{O}$ & 6.6555088582 & 27.0615421470 & 9.1314818426 \\
\hline $\mathrm{N}$ & 5.4201737936 & 16.3856365092 & 4.0897256002 \\
\hline
\end{tabular}




\begin{tabular}{|c|c|c|c|}
\hline $\mathrm{N}$ & 8.2645168082 & 12.8271984836 & 4.0897256002 \\
\hline $\mathrm{N}$ & 1.4221715078 & 1.7792190123 & 7.1345672337 \\
\hline $\mathrm{N}$ & 12.2625190939 & 27.4336159806 & 7.1345672337 \\
\hline $\mathrm{N}$ & 3.8968693382 & 14.1162797298 & 5.7613237020 \\
\hline $\mathrm{N}$ & 9.7878212626 & 15.0965543928 & 5.7613237020 \\
\hline $\mathrm{N}$ & 2.9454759622 & 28.7226980969 & 5.4629694675 \\
\hline $\mathrm{N}$ & 10.7392146386 & 0.4901377672 & 5.4629694675 \\
\hline $\mathrm{N}$ & 8.8117117206 & 22.2916284773 & 11.1993017799 \\
\hline $\mathrm{N}$ & 4.8729788801 & 6.9212065155 & 11.1993017799 \\
\hline $\mathrm{N}$ & 11.7153241815 & 7.6852109804 & 0.0249913896 \\
\hline $\mathrm{N}$ & 1.9693664203 & 21.5276240125 & 0.0249913896 \\
\hline $\mathrm{N}$ & 2.3980440063 & 16.3236715822 & 7.0126925627 \\
\hline $\mathrm{N}$ & 11.2866467994 & 12.8891634116 & 7.0126925627 \\
\hline $\mathrm{N}$ & 4.4443014990 & 1.7172540853 & 4.2116006058 \\
\hline $\mathrm{N}$ & 9.2403891027 & 27.4955809075 & 4.2116006058 \\
\hline $\mathrm{N}$ & 7.3488275969 & 20.2605968960 & 1.2598038986 \\
\hline $\mathrm{N}$ & 6.3358630049 & 8.9522380968 & 1.2598038986 \\
\hline $\mathrm{N}$ & 13.1782083053 & 5.6541793991 & 9.9644892699 \\
\hline $\mathrm{N}$ & 0.5064822965 & 23.5586555938 & 9.9644892699 \\
\hline $\mathrm{N}$ & 9.9077537841 & 20.3735687216 & 9.5639361609 \\
\hline $\mathrm{N}$ & 3.7769368167 & 8.8392662712 & 9.5639361609 \\
\hline $\mathrm{N}$ & 10.6192821181 & 5.7671512257 & 1.6603570086 \\
\hline $\mathrm{N}$ & 3.0654084837 & 23.4456837682 & 1.6603570086 \\
\hline $\mathrm{N}$ & 2.7076680133 & 16.1881636864 & 4.2488525788 \\
\hline $\mathrm{N}$ & 10.9770227914 & 13.0246713064 & 4.2488525788 \\
\hline $\mathrm{N}$ & 4.1346774910 & 1.5817461895 & 6.9754402561 \\
\hline $\mathrm{N}$ & 9.5500131107 & 27.6310888034 & 6.9754402561 \\
\hline $\mathrm{N}$ & 9.9867864869 & 20.1078778880 & 0.9774310782 \\
\hline $\mathrm{N}$ & 3.6979041149 & 9.1049571058 & 0.9774310782 \\
\hline $\mathrm{N}$ & 10.5402494153 & 5.5014603911 & 10.2468620902 \\
\hline $\mathrm{N}$ & 3.1444411855 & 23.7113746017 & 10.2468620902 \\
\hline C & 6.0366862537 & 17.0712211244 & 7.9461124320 \\
\hline C & 7.6480043481 & 12.1416138685 & 7.9461124321 \\
\hline C & 0.8056590467 & 2.4648036275 & 3.2781807364 \\
\hline C & 12.8790315541 & 26.7480313654 & 3.2781807364 \\
\hline C & 6.7718352831 & 16.5001875633 & 1.6575347118 \\
\hline C & 6.9128553186 & 12.7126474295 & 1.6575347118 \\
\hline C & 13.7552006191 & 1.8937700664 & 9.5667586240 \\
\hline C & -0.0705100173 & 27.3190649264 & 9.5667586240 \\
\hline C & 3.0703680301 & 13.7247991665 & 4.7691945436 \\
\hline C & 10.6143225707 & 15.4880358264 & 4.7691945436 \\
\hline C & 3.7719772703 & 28.3312166634 & 6.4550986259 \\
\hline C & 9.9127133314 & 0.8816183304 & 6.4550986259 \\
\hline C & 2.2772201320 & 16.1013297102 & 8.3381463150 \\
\hline C & 11.4074700608 & 13.1115052837 & 8.3381463150 \\
\hline C & 4.5651251684 & 1.4949122133 & 2.8861468534 \\
\hline C & 9.1195658404 & 27.7179227796 & 2.8861468534 \\
\hline C & 5.5211751708 & 18.3490500228 & 7.3259805283 \\
\hline C & 8.1635154310 & 10.8637849701 & 7.3259805283 \\
\hline C & 1.3211701296 & 3.7426325259 & 3.8983126401 \\
\hline C & 12.3635204712 & 25.4702024670 & 3.8983126401 \\
\hline C & 13.5796770012 & 16.3458362261 & 8.2334890170 \\
\hline C & 0.1050135396 & 12.8669987678 & 8.2334890170 \\
\hline C & 6.9473589010 & 1.7394187292 & 2.9908041525 \\
\hline C & 6.7373316998 & 27.4734162637 & 2.9908041525 \\
\hline C & 7.4630522869 & 16.2403876739 & 2.8416009037 \\
\hline C & 6.2216383148 & 12.9724473199 & 2.8416009037 \\
\hline C & 13.0639836152 & 1.6339701770 & 8.3826925994 \\
\hline
\end{tabular}




\begin{tabular}{|c|c|c|c|}
\hline C & 0.6207069856 & 27.5788648158 & 8.3826925994 \\
\hline C & 1.0464063798 & 16.1103838277 & 8.9874135552 \\
\hline C & 12.6382846289 & 13.1024511651 & 8.9874135552 \\
\hline C & 5.7959389205 & 1.5039663308 & 2.2368796133 \\
\hline C & 7.8887512723 & 27.7088686621 & 2.2368796133 \\
\hline C & 5.9208192042 & 19.5922389386 & 7.8423476377 \\
\hline C & 7.7638709886 & 9.6205960552 & 7.8423476377 \\
\hline C & 0.9215260962 & 4.9858214417 & 3.3819455307 \\
\hline C & 12.7631649125 & 24.2270135511 & 3.3819455307 \\
\hline C & 4.3923411542 & 13.1993150806 & 6.6210253565 \\
\hline C & 9.2923490387 & 16.0135207835 & 6.6210253565 \\
\hline C & 2.4500041462 & 27.8057317072 & 4.6032678119 \\
\hline C & 11.2346868625 & 1.4071024163 & 4.6032678119 \\
\hline C & 7.3102656496 & 18.3582281021 & 9.5399850398 \\
\hline C & 6.3744249522 & 10.8546068907 & 9.5399850398 \\
\hline C & 13.2167702526 & 3.7518106052 & 1.6843081287 \\
\hline C & 0.4679203492 & 25.4610243877 & 1.6843081287 \\
\hline C & 6.9186946379 & 17.1107695286 & 9.0295526499 \\
\hline C & 6.7659963708 & 12.1020654642 & 9.0295526499 \\
\hline C & -0.0763493376 & 2.5043520327 & 2.1947405186 \\
\hline C & 13.7610395303 & 26.7084829611 & 2.1947405186 \\
\hline C & 2.7677383075 & 12.3749646630 & 4.5749144922 \\
\hline C & 10.9169520893 & 16.8378694605 & 4.5749144922 \\
\hline C & 4.0746067889 & 26.9813830302 & 6.6493783417 \\
\hline C & 9.6100838119 & 2.2314528339 & 6.6493783417 \\
\hline C & 3.2961778481 & 11.4245104986 & 5.4468596383 \\
\hline C & 10.3885129567 & 17.7883253656 & 5.4468596383 \\
\hline C & 3.5461676563 & 26.0309271242 & 5.7774338647 \\
\hline C & 10.1385229455 & 3.1819069983 & 5.7774338647 \\
\hline C & 8.1544386042 & 23.2975640775 & 10.5725627218 \\
\hline C & 5.5302519966 & 5.9152709163 & 10.5725627218 \\
\hline C & 12.3725972980 & 8.6911465806 & 0.6517304477 \\
\hline C & 1.3120933038 & 20.5216884122 & 0.6517304477 \\
\hline C & 4.1116366403 & 11.8433843791 & 6.4996846108 \\
\hline C & 9.5730539605 & 17.3694497434 & 6.4996846108 \\
\hline C & 2.7307086601 & 26.4498027463 & 4.7246085587 \\
\hline C & 10.9539819417 & 2.7630331178 & 4.7246085587 \\
\hline C & 9.8096813559 & 22.5956448343 & 0.8406530348 \\
\hline C & 3.8750092449 & 6.6171901585 & 0.8406530348 \\
\hline C & 10.7173545463 & 7.9892273384 & 10.3836401337 \\
\hline C & 2.9673360555 & 21.2236076554 & 10.3836401337 \\
\hline C & 7.9881870662 & 19.7833165962 & 2.3605524845 \\
\hline C & 5.6965035345 & 9.4295183966 & 2.3605524845 \\
\hline C & 12.5388488349 & 5.1768991003 & 8.8637406850 \\
\hline C & 1.1458417658 & 24.0359358935 & 8.8637406850 \\
\hline C & 7.3237376009 & 19.6112162798 & 3.5730631031 \\
\hline C & 6.3609530008 & 9.6016187140 & 3.5730631031 \\
\hline C & -0.4813923006 & 5.0047987829 & 7.6512300653 \\
\hline C & 14.1660829014 & 24.2080362110 & 7.6512300653 \\
\hline C & 2.4998829403 & 14.7719750366 & 3.8370539193 \\
\hline C & 11.1848074565 & 14.4408599572 & 3.8370539193 \\
\hline C & 4.3424621561 & 0.1655575397 & 7.3872392502 \\
\hline C & 9.3422284447 & 29.0472774531 & 7.3872392502 \\
\hline C & 3.2773619044 & 16.9642503948 & 3.1190824404 \\
\hline C & 10.4073282884 & 12.2485845980 & 3.1190824404 \\
\hline C & 3.5649833960 & 2.3578328989 & 8.1052107292 \\
\hline C & 10.1197076127 & 26.8550020949 & 8.1052107292 \\
\hline C & 5.3929085681 & 16.7050067549 & 1.7125380007 \\
\hline
\end{tabular}




\begin{tabular}{|c|c|c|c|}
\hline C & 8.2917820326 & 12.5078282380 & 1.7125380006 \\
\hline C & 1.4494367322 & 2.0985892589 & 9.5117555024 \\
\hline C & 12.2352538685 & 27.1142457349 & 9.5117555024 \\
\hline C & 6.0258793597 & 20.5107734226 & 1.3340662803 \\
\hline C & 7.6588112411 & 8.7020615712 & 1.3340662803 \\
\hline C & 0.8164659407 & 5.9043559257 & 9.8902268882 \\
\hline C & 12.8682246611 & 23.3084790681 & 9.8902268882 \\
\hline C & 0.0182894947 & 16.5444537792 & 6.8599661249 \\
\hline C & 13.6664012721 & 12.6683812137 & 6.8599661249 \\
\hline C & 6.8240559717 & 1.9380362823 & 4.3643270446 \\
\hline C & 6.8606346301 & 27.2747987106 & 4.3643270446 \\
\hline C & 1.4880388637 & 16.8220540881 & 4.8087266228 \\
\hline C & 12.1966515341 & 12.3907809057 & 4.8087266228 \\
\hline C & 5.3543062337 & 2.2156365912 & 6.4155665457 \\
\hline C & 8.3303843681 & 26.9971984027 & 6.4155665457 \\
\hline C & 4.7468705249 & 16.6561480053 & 2.9491103442 \\
\hline C & 8.9378200769 & 12.5566869876 & 2.9491103442 \\
\hline C & 2.0954747755 & 2.0497305084 & 8.2751831599 \\
\hline C & 11.5892158252 & 27.1631044844 & 8.2751831599 \\
\hline C & 5.2928755186 & 20.2988861210 & 2.4959939736 \\
\hline C & 8.3918154901 & 8.9139488719 & 2.4959939736 \\
\hline C & 1.5494697818 & 5.6924686250 & 8.7282991959 \\
\hline C & 12.1352204111 & 23.5203663688 & 8.7282991959 \\
\hline C & 6.7503009838 & 16.1859296928 & 4.0328079072 \\
\hline C & 6.9343900249 & 13.0269053010 & 4.0328079072 \\
\hline C & 0.0920443165 & 1.5795121959 & 7.1914852613 \\
\hline C & 13.5926458773 & 27.6333227979 & 7.1914852613 \\
\hline C & 10.5456093239 & 21.4263567643 & 1.4364922563 \\
\hline C & 3.1390812769 & 7.7864782285 & 1.4364922563 \\
\hline C & 9.9814265773 & 6.8199392684 & 9.7878009122 \\
\hline C & 3.7032640235 & 22.3928957254 & 9.7878009122 \\
\hline C & 6.7562720351 & 19.6186091458 & 8.9616570870 \\
\hline C & 6.9284185657 & 9.5942258471 & 8.9616570870 \\
\hline C & 0.0860732653 & 5.0121916489 & 2.2626360825 \\
\hline C & 13.5986173355 & 24.2006433440 & 2.2626360825 \\
\hline C & 1.2859979010 & 16.5277552792 & 6.2756948276 \\
\hline C & 12.3986925988 & 12.6850797147 & 6.2756948276 \\
\hline C & 5.5563472984 & 1.9213377823 & 4.9485983409 \\
\hline C & 8.1283433034 & 27.2914972106 & 4.9485983409 \\
\hline C & 9.8523141535 & 20.8185435121 & 8.2882160316 \\
\hline C & 3.8323764473 & 8.3942914807 & 8.2882160316 \\
\hline C & 10.6747217476 & 6.2121260162 & 2.9360771368 \\
\hline C & 3.0099688531 & 23.0007089776 & 2.9360771368 \\
\hline C & 10.9346766026 & 20.6910617833 & 7.4223788033 \\
\hline C & 2.7500139982 & 8.5217732105 & 7.4223788033 \\
\hline C & 9.5923592986 & 6.0846442864 & 3.8019143652 \\
\hline C & 4.0923313022 & 23.1281907074 & 3.8019143652 \\
\hline C & 10.9861834263 & 19.3049058876 & 0.2097636749 \\
\hline C & 2.6985071745 & 9.9079291052 & 0.2097636749 \\
\hline C & 9.5408524749 & 4.6984883917 & 11.0145294935 \\
\hline C & 4.1438381259 & 24.5143466021 & 11.0145294935 \\
\hline C & 11.0559733529 & 19.8083682788 & 10.0213493696 \\
\hline C & 2.6287172489 & 9.4044667150 & 10.0213493696 \\
\hline C & 9.4710625492 & 5.2019507819 & 1.2029437988 \\
\hline C & 4.2136280525 & 24.0108842119 & 1.2029437988 \\
\hline C & 8.4650024034 & 24.6341386722 & 10.7949634375 \\
\hline C & 5.2196881983 & 4.5786963217 & 10.7949634375 \\
\hline C & 12.0620334987 & 10.0277211752 & 0.4293297320 \\
\hline
\end{tabular}




\begin{tabular}{|c|c|c|c|}
\hline C & 1.6226571030 & 19.1851138186 & 0.4293297320 \\
\hline C & 12.1117975411 & 20.0968785613 & 7.8817451236 \\
\hline C & 1.5728930607 & 9.1159564316 & 7.8817451236 \\
\hline C & 8.4152383611 & 5.4904610653 & 3.3425480449 \\
\hline C & 5.2694522397 & 23.7223739285 & 3.3425480448 \\
\hline C & 5.9524925887 & 19.8634129588 & 3.6465340226 \\
\hline C & 7.7321980120 & 9.3494220351 & 3.6465340226 \\
\hline C & 0.8898527117 & 5.2569954619 & 7.5777591469 \\
\hline C & 12.7948378891 & 23.9558395310 & 7.5777591469 \\
\hline C & 10.1529654436 & 23.9174672919 & 1.1240667230 \\
\hline C & 3.5317251571 & 5.2953677019 & 1.1240667230 \\
\hline C & 10.3740704575 & 9.3110497950 & 10.1002264455 \\
\hline C & 3.3106201433 & 19.9017851978 & 10.1002264455 \\
\hline C & 9.4051570186 & 19.3437079361 & 2.1172467579 \\
\hline C & 4.2795335821 & 9.8691270577 & 2.1172467579 \\
\hline C & 11.1218788825 & 4.7372904392 & 9.1070464106 \\
\hline C & 2.5628117183 & 24.4755445536 & 9.1070464106 \\
\hline C & 9.4774100882 & 24.9515353825 & 0.4791145041 \\
\hline C & 4.2072805125 & 4.2612996113 & 0.4791145041 \\
\hline C & 11.0496258129 & 10.3451178856 & 10.7451786644 \\
\hline C & 2.6350647878 & 18.8677171072 & 10.7451786644 \\
\hline C & 12.1751080585 & 19.6623320192 & 9.2055757650 \\
\hline C & 1.5095825423 & 9.5505029736 & 9.2055757650 \\
\hline C & 8.3519278427 & 5.0559145223 & 2.0187174035 \\
\hline C & 5.3327627581 & 24.1569204706 & 2.0187174035 \\
\hline $\mathrm{H}$ & 7.3112903991 & 16.5497378256 & 0.7111179545 \\
\hline $\mathrm{H}$ & 6.3734002016 & 12.6630971682 & 0.7111179545 \\
\hline $\mathrm{H}$ & 13.2157455020 & 1.9433203287 & 10.5131752139 \\
\hline $\mathrm{H}$ & 0.4689450987 & 27.2695146651 & 10.5131752139 \\
\hline $\mathrm{H}$ & 3.2022575790 & 15.8886995567 & 8.8780875975 \\
\hline $\mathrm{H}$ & 10.4824328178 & 13.3241354361 & 8.8780875975 \\
\hline $\mathrm{H}$ & 3.6400875174 & 1.2822820598 & 2.3462055710 \\
\hline $\mathrm{H}$ & 10.0446030834 & 27.9305529331 & 2.3462055710 \\
\hline $\mathrm{H}$ & 12.6010133761 & 16.3475249832 & 8.7162332626 \\
\hline $\mathrm{H}$ & 1.0836772247 & 12.8653100106 & 8.7162332627 \\
\hline $\mathrm{H}$ & 7.9260225250 & 1.7411074863 & 2.5080599058 \\
\hline $\mathrm{H}$ & 5.7586680757 & 27.4717275066 & 2.5080599058 \\
\hline $\mathrm{H}$ & 8.5369127842 & 16.0647210052 & 2.8274341043 \\
\hline $\mathrm{H}$ & 5.1477778166 & 13.1481139877 & 2.8274341043 \\
\hline $\mathrm{H}$ & 11.9901231180 & 1.4583035092 & 8.3968593988 \\
\hline $\mathrm{H}$ & 1.6945674838 & 27.7545314846 & 8.3968593988 \\
\hline $\mathrm{H}$ & 0.9965360486 & 15.9093259137 & 10.0571301651 \\
\hline $\mathrm{H}$ & 12.6881545522 & 13.3035090801 & 10.0571301650 \\
\hline $\mathrm{H}$ & 5.8458092518 & 1.3029084168 & 1.1671630034 \\
\hline $\mathrm{H}$ & 7.8388813500 & 27.9099265760 & 1.1671630034 \\
\hline $\mathrm{H}$ & 5.5545671371 & 20.5241404801 & 7.4073484576 \\
\hline $\mathrm{H}$ & 8.1301238716 & 8.6886945137 & 7.4073484576 \\
\hline $\mathrm{H}$ & 1.2877781633 & 5.9177229831 & 3.8169447119 \\
\hline $\mathrm{H}$ & 12.3969120295 & 23.2951120097 & 3.8169447119 \\
\hline $\mathrm{H}$ & 5.0282630801 & 13.5914238929 & 7.4155692790 \\
\hline $\mathrm{H}$ & 8.6564275206 & 15.6214119713 & 7.4155692790 \\
\hline $\mathrm{H}$ & 1.8140822203 & 28.1978405185 & 3.8087238895 \\
\hline $\mathrm{H}$ & 11.8706083805 & 1.0149936041 & 3.8087238895 \\
\hline $\mathrm{H}$ & 7.3266902222 & 16.1900089851 & 9.4493688726 \\
\hline $\mathrm{H}$ & 6.3580003786 & 13.0228260078 & 9.4493688726 \\
\hline $\mathrm{H}$ & 13.2003456789 & 1.5835914881 & 1.7749242969 \\
\hline $\mathrm{H}$ & 0.4843449218 & 27.6292435047 & 1.7749242969 \\
\hline $\mathrm{H}$ & 2.1036036530 & 12.0877163058 & 3.7599814492 \\
\hline
\end{tabular}




\begin{tabular}{|c|c|c|c|}
\hline $\mathrm{H}$ & 11.5810871527 & 17.1251195583 & 3.7599814492 \\
\hline $\mathrm{H}$ & 4.7387418513 & 26.6941329315 & 7.4643120550 \\
\hline $\mathrm{H}$ & 8.9459487494 & 2.5187011911 & 7.4643120550 \\
\hline $\mathrm{H}$ & 3.0356648112 & 10.3731913443 & 5.3243213941 \\
\hline $\mathrm{H}$ & 10.6490257906 & 18.8396436496 & 5.3243213941 \\
\hline $\mathrm{H}$ & 3.8066804902 & 24.9796088402 & 5.8999721089 \\
\hline $\mathrm{H}$ & 9.8780101116 & 4.2332261526 & 5.8999721089 \\
\hline $\mathrm{H}$ & 7.3899431142 & 22.9896537947 & 9.8611728926 \\
\hline $\mathrm{H}$ & 6.2947474866 & 6.2231811992 & 9.8611728926 \\
\hline $\mathrm{H}$ & 13.1370927870 & 8.3832362978 & 1.3631202769 \\
\hline $\mathrm{H}$ & 0.5475978138 & 20.8295986951 & 1.3631202769 \\
\hline $\mathrm{H}$ & 4.5231921325 & 11.1402560044 & 7.2236855950 \\
\hline $\mathrm{H}$ & 9.1614984693 & 18.0725789884 & 7.2236855950 \\
\hline $\mathrm{H}$ & 2.3191531689 & 25.7466735013 & 4.0006075735 \\
\hline $\mathrm{H}$ & 11.3655374329 & 3.4661614925 & 4.0006075735 \\
\hline $\mathrm{H}$ & 7.8722095880 & 19.2466970332 & 4.4426197421 \\
\hline $\mathrm{H}$ & 5.8124810128 & 9.9661379607 & 4.4426197421 \\
\hline $\mathrm{H}$ & 12.6548263132 & 4.6402795362 & 6.7816734264 \\
\hline $\mathrm{H}$ & 1.0298642876 & 24.5725554566 & 6.7816734264 \\
\hline $\mathrm{H}$ & 1.4265267053 & 14.5742850474 & 3.6779286672 \\
\hline $\mathrm{H}$ & 12.2581638965 & 14.6385499454 & 3.6779286672 \\
\hline $\mathrm{H}$ & 5.4158185951 & -0.0321324485 & 7.5463641677 \\
\hline $\mathrm{H}$ & 8.2688720057 & 29.2449674422 & 7.5463641677 \\
\hline $\mathrm{H}$ & 2.9608861594 & 14.6192260517 & 2.8481069069 \\
\hline $\mathrm{H}$ & 10.7238048494 & 14.5936089421 & 2.8481069069 \\
\hline $\mathrm{H}$ & 3.8814591410 & 0.0128085548 & 8.3761862616 \\
\hline $\mathrm{H}$ & 9.8032310528 & 29.2000264380 & 8.3761862616 \\
\hline $\mathrm{H}$ & 3.1776679675 & 18.0340813894 & 3.3663504162 \\
\hline $\mathrm{H}$ & 10.5070222263 & 11.1787536044 & 3.3663504163 \\
\hline $\mathrm{H}$ & 3.6646773329 & 3.4276638925 & 7.8579424186 \\
\hline $\mathrm{H}$ & 10.0200136759 & 25.7851711013 & 7.8579424186 \\
\hline $\mathrm{H}$ & 2.7256174030 & 16.7839033473 & 2.1829562928 \\
\hline $\mathrm{H}$ & 10.9590731977 & 12.4289316456 & 2.1829562928 \\
\hline $\mathrm{H}$ & 4.1167278973 & 2.1774858514 & 9.0413368756 \\
\hline $\mathrm{H}$ & 9.5679627034 & 27.0353491425 & 9.0413368756 \\
\hline $\mathrm{H}$ & 4.8119717215 & 16.8839850927 & 0.8080291871 \\
\hline $\mathrm{H}$ & 8.8727188803 & 12.3288499012 & 0.8080291871 \\
\hline $\mathrm{H}$ & 2.0303735789 & 2.2775675957 & 10.4162636467 \\
\hline $\mathrm{H}$ & 11.6543170218 & 26.9352673981 & 10.4162636467 \\
\hline $\mathrm{H}$ & 5.5663831677 & 20.8699101514 & 0.4147376130 \\
\hline $\mathrm{H}$ & 8.1183074331 & 8.3429248414 & 0.4147376130 \\
\hline $\mathrm{H}$ & 1.2759621327 & 6.2634926545 & 10.8095555555 \\
\hline $\mathrm{H}$ & 12.4087284681 & 22.9493423384 & 10.8095555555 \\
\hline $\mathrm{H}$ & 12.8298663977 & 16.7287237964 & 6.2370235748 \\
\hline $\mathrm{H}$ & 0.8548241011 & 12.4841111975 & 6.2370235748 \\
\hline $\mathrm{H}$ & 7.6971695045 & 2.1223062994 & 4.9872695937 \\
\hline $\mathrm{H}$ & 5.9875210973 & 27.0905286944 & 4.9872695936 \\
\hline $\mathrm{H}$ & 0.5971245984 & 16.5503576065 & 4.2226345502 \\
\hline $\mathrm{H}$ & 13.0875662064 & 12.6624773873 & 4.2226345502 \\
\hline $\mathrm{H}$ & 6.2452209060 & 1.9439401096 & 7.0016589529 \\
\hline $\mathrm{H}$ & 7.4394696948 & 27.2688948832 & 7.0016589529 \\
\hline $\mathrm{H}$ & 1.5987123538 & 17.9131111697 & 4.7091578993 \\
\hline $\mathrm{H}$ & 12.0859786550 & 11.2997238242 & 4.7091578993 \\
\hline $\mathrm{H}$ & 5.2436329466 & 3.3066936728 & 6.5151356038 \\
\hline $\mathrm{H}$ & 8.4410572472 & 25.9061413201 & 6.5151356038 \\
\hline $\mathrm{H}$ & 4.2200457673 & 20.4895877341 & 2.4938340228 \\
\hline $\mathrm{H}$ & 9.4646448334 & 8.7232472597 & 2.4938340228 \\
\hline $\mathrm{H}$ & 2.6222995331 & 5.8831702372 & 8.7304591467 \\
\hline
\end{tabular}




\begin{tabular}{|c|c|c|c|}
\hline $\mathrm{H}$ & 11.0623910677 & 23.3296647556 & 8.7304591467 \\
\hline $\mathrm{H}$ & 7.2370995732 & 15.9720424851 & 4.9852041793 \\
\hline $\mathrm{H}$ & 6.4475910276 & 13.2407925087 & 4.9852041793 \\
\hline $\mathrm{H}$ & 13.2899363280 & 1.3656249882 & 6.2390889902 \\
\hline $\mathrm{H}$ & 0.3947542728 & 27.8472100046 & 6.2390889902 \\
\hline $\mathrm{H}$ & 11.6139705600 & 21.4828706993 & 1.1737614464 \\
\hline $\mathrm{H}$ & 2.0707200408 & 7.7299642945 & 1.1737614464 \\
\hline $\mathrm{H}$ & 8.9130653412 & 6.8764532024 & 10.0505317231 \\
\hline $\mathrm{H}$ & 4.7716252596 & 22.3363817904 & 10.0505317231 \\
\hline $\mathrm{H}$ & 10.5277510058 & 21.4753309777 & 2.5350077096 \\
\hline $\mathrm{H}$ & 3.1569395950 & 7.7375040161 & 2.5350077096 \\
\hline $\mathrm{H}$ & 9.9992848953 & 6.8689134808 & 8.6892854589 \\
\hline $\mathrm{H}$ & 3.6854057054 & 22.3439215120 & 8.6892854589 \\
\hline $\mathrm{H}$ & 8.9150289557 & 21.2901561504 & 7.9864790933 \\
\hline $\mathrm{H}$ & 4.7696616451 & 7.9226788434 & 7.9864790933 \\
\hline $\mathrm{H}$ & 11.6120069455 & 6.6837386535 & 3.2378140762 \\
\hline $\mathrm{H}$ & 2.0726836553 & 22.5290963404 & 3.2378140762 \\
\hline $\mathrm{H}$ & 10.8672697833 & 21.0648542907 & 6.4003992883 \\
\hline $\mathrm{H}$ & 2.8174208175 & 8.1479807031 & 6.4003992883 \\
\hline $\mathrm{H}$ & 9.6597661179 & 6.4584367938 & 4.8238938801 \\
\hline $\mathrm{H}$ & 4.0249244829 & 22.7543981990 & 4.8238938801 \\
\hline $\mathrm{H}$ & 10.6236706094 & 18.2660491699 & 0.1881508920 \\
\hline $\mathrm{H}$ & 3.0610199914 & 10.9467858229 & 0.1881508920 \\
\hline $\mathrm{H}$ & 9.9033652917 & 3.6596316730 & 11.0361422775 \\
\hline $\mathrm{H}$ & 3.7813253090 & 25.5532033199 & 11.0361422775 \\
\hline $\mathrm{H}$ & 11.9713847772 & 19.3110674396 & 0.6975369496 \\
\hline $\mathrm{H}$ & 1.7133058236 & 9.9017675542 & 0.6975369496 \\
\hline $\mathrm{H}$ & 8.5556511240 & 4.7046499427 & 10.5267562189 \\
\hline $\mathrm{H}$ & 5.1290394768 & 24.5081850511 & 10.5267562189 \\
\hline $\mathrm{H}$ & 7.9232545262 & 25.4168569833 & 10.2606114750 \\
\hline $\mathrm{H}$ & 5.7614360756 & 3.7959780096 & 10.2606114750 \\
\hline $\mathrm{H}$ & 12.6037813760 & 10.8104394873 & 0.9636816935 \\
\hline $\mathrm{H}$ & 1.0809092248 & 18.4023955065 & 0.9636816935 \\
\hline $\mathrm{H}$ & 12.9603508410 & 19.9738634631 & 7.2089040508 \\
\hline $\mathrm{H}$ & 0.7243397597 & 9.2389715297 & 7.2089040508 \\
\hline $\mathrm{H}$ & 7.5666850601 & 5.3674459662 & 4.0153891187 \\
\hline $\mathrm{H}$ & 6.1180055406 & 23.8453890266 & 4.0153891187 \\
\hline $\mathrm{H}$ & 5.4111845588 & 19.6937780578 & 4.5799420763 \\
\hline $\mathrm{H}$ & 8.2735064499 & 9.5190569351 & 4.5799420763 \\
\hline $\mathrm{H}$ & 1.4311607416 & 5.0873605608 & 6.6443510922 \\
\hline $\mathrm{H}$ & 12.2535294512 & 24.1254744320 & 6.6443510922 \\
\hline $\mathrm{H}$ & 10.9516431760 & 24.1243159621 & 1.8381395919 \\
\hline $\mathrm{H}$ & 2.7330474248 & 5.0885190317 & 1.8381395919 \\
\hline $\mathrm{H}$ & 9.5753927252 & 9.5178984652 & 9.3861535776 \\
\hline $\mathrm{H}$ & 4.1092978756 & 19.6949365276 & 9.3861535776 \\
\hline $\mathrm{H}$ & 10.0308911537 & 19.4288521527 & 3.0179766892 \\
\hline $\mathrm{H}$ & 3.6537994471 & 9.7839828402 & 3.0179766892 \\
\hline H & 10.4961447475 & 4.8224346558 & 8.2063164793 \\
\hline $\mathrm{H}$ & 3.1885458533 & 24.3904003371 & 8.2063164793 \\
\hline $\mathrm{H}$ & 9.3832862264 & 18.2821760679 & 1.8240141052 \\
\hline $\mathrm{H}$ & 4.3014043754 & 10.9306589259 & 1.8240141052 \\
\hline $\mathrm{H}$ & 11.1437496758 & 3.6757585710 & 9.4002790643 \\
\hline $\mathrm{H}$ & 2.5409409260 & 25.5370764218 & 9.4002790643 \\
\hline $\mathrm{H}$ & 9.7367677622 & 25.9926370060 & 0.6746065944 \\
\hline $\mathrm{H}$ & 3.9479228385 & 3.2201979868 & 0.6746065944 \\
\hline $\mathrm{H}$ & 10.7902681389 & 11.3862195091 & 10.5496865741 \\
\hline $\mathrm{H}$ & 2.8944224618 & 17.8266154837 & 10.5496865741 \\
\hline $\mathrm{H}$ & 13.0798803956 & 19.2052842819 & 9.6091136877 \\
\hline
\end{tabular}




\begin{tabular}{|c|c|c|c|}
\hline $\mathrm{H}$ & 0.6048102052 & 10.0075507120 & 9.6091136877 \\
\hline $\mathrm{H}$ & 7.4471555056 & 4.5988667849 & 1.6151794818 \\
\hline $\mathrm{H}$ & 6.2375350952 & 24.6139682079 & 1.6151794818 \\
\hline $\mathrm{H}$ & 12.9666197376 & 16.4419447065 & 1.3257103513 \\
\hline $\mathrm{H}$ & 0.7180706982 & 12.7708902873 & 1.3257103513 \\
\hline $\mathrm{H}$ & 7.5604161646 & 1.8355272096 & 9.8985829855 \\
\hline $\mathrm{H}$ & 6.1242744372 & 27.3773077832 & 9.8985829855 \\
\hline $\mathrm{H}$ & 13.1637641561 & 17.6560888732 & 2.3018769297 \\
\hline $\mathrm{H}$ & 0.5209268526 & 11.5567461196 & 2.3018769297 \\
\hline $\mathrm{H}$ & 7.3632717450 & 3.0496713763 & 8.9224159042 \\
\hline $\mathrm{H}$ & 6.3214184478 & 26.1631636165 & 8.9224159042 \\
\hline $\mathrm{F}$ & 10.7740718079 & 14.0083811785 & 0.2806042471 \\
\hline $\mathrm{F}$ & 2.9106187929 & 15.2044529450 & 0.2806042471 \\
\hline $\mathrm{F}$ & 9.7529640932 & 28.6147995457 & 10.9436889214 \\
\hline $\mathrm{F}$ & 3.9317265075 & 0.5980363184 & 10.9436889214 \\
\hline $\mathrm{F}$ & 10.0945542553 & 16.0726400730 & 1.1147786770 \\
\hline $\mathrm{F}$ & 3.5901363454 & 13.1401949199 & 1.1147786770 \\
\hline $\mathrm{F}$ & 10.4324816458 & 1.4662225761 & 10.1095144925 \\
\hline $\mathrm{F}$ & 3.2522089549 & 27.7466124168 & 10.1095144925 \\
\hline $\mathrm{F}$ & 6.3962677892 & 24.6610114169 & 6.1290995576 \\
\hline $\mathrm{F}$ & 7.2884228125 & 4.5518235760 & 6.1290995576 \\
\hline $\mathrm{F}$ & 0.4460775111 & 10.0545939209 & 5.0951936120 \\
\hline $\mathrm{F}$ & 13.2386130896 & 19.1582410729 & 5.0951936120 \\
\hline $\mathrm{F}$ & 12.0365981033 & 15.9242420801 & 11.0832909024 \\
\hline $\mathrm{F}$ & 1.6480924974 & 13.2885929128 & 11.0832909024 \\
\hline $\mathrm{F}$ & 8.4904377988 & 1.3178245832 & 0.1410022661 \\
\hline $\mathrm{F}$ & 5.1942528030 & 27.8950104096 & 0.1410022661 \\
\hline $\mathrm{F}$ & 5.9310330293 & 24.4181357997 & 8.3908518246 \\
\hline $\mathrm{F}$ & 7.7536575725 & 4.7946991931 & 8.3908518246 \\
\hline $\mathrm{F}$ & 0.9113122721 & 9.8117183028 & 2.8334413438 \\
\hline $\mathrm{F}$ & 12.7733783297 & 19.4011166900 & 2.8334413438 \\
\hline $\mathrm{F}$ & 9.9746645600 & 15.6371530404 & 10.0488349825 \\
\hline $\mathrm{F}$ & 3.7100260408 & 13.5756819524 & 10.0488349825 \\
\hline $\mathrm{F}$ & 10.5523713422 & 1.0307355435 & 1.1754581860 \\
\hline $\mathrm{F}$ & 3.1323192596 & 28.1820994493 & 1.1754581860 \\
\hline $\mathrm{F}$ & 7.4320982736 & 22.9764294357 & 7.3370933099 \\
\hline $\mathrm{F}$ & 6.2525923272 & 6.2364055572 & 7.3370933099 \\
\hline $\mathrm{F}$ & 13.0949376276 & 8.3700119388 & 3.8871998596 \\
\hline $\mathrm{F}$ & 0.5897529732 & 20.8428230540 & 3.8871998596 \\
\hline $\mathrm{F}$ & 5.1443148235 & 22.8508817309 & 6.8738818103 \\
\hline $\mathrm{F}$ & 8.5403753693 & 6.3619532620 & 6.8738818103 \\
\hline $\mathrm{F}$ & 1.6980304769 & 8.2444642350 & 4.3504113581 \\
\hline $\mathrm{F}$ & 11.9866605318 & 20.9683707589 & 4.3504113581 \\
\hline B & 10.7014627907 & 15.4015867172 & 0.0061503399 \\
\hline B & 2.9832278111 & 13.8112482767 & 0.0061503399 \\
\hline B & 9.8255731115 & 0.7951692203 & 11.2181428297 \\
\hline B & 3.8591174893 & 28.4176657726 & 11.2181428297 \\
\hline B & 6.2443163429 & 23.7093477846 & 7.1653878701 \\
\hline B & 7.4403742588 & 5.5034872083 & 7.1653878701 \\
\hline B & 0.5980289584 & 9.1029302886 & 4.0589052983 \\
\hline B & 13.0866616433 & 20.1099047052 & 4.0589052983 \\
\hline
\end{tabular}

$\mathbf{1} \cdot \mathrm{H}_{2} \mathrm{O} \quad$-LS

CELL_PARAMETERS (angstrom)
13.725428592
0.000000000
0.000000000
$0.000000000 \quad 30.009455719$
0.000000000
0.000000000
0.000000000
10.847275717

ATOMIC_POSITIONS (angstrom) 


\begin{tabular}{|c|c|c|c|}
\hline $\mathrm{Fe} 2$ & 4.0729414261 & 16.6624297008 & 5.8175639198 \\
\hline $\mathrm{Fe} 2$ & 9.6524871665 & 13.3470260177 & 5.8175639198 \\
\hline $\mathrm{Fe} 2$ & 2.7897728707 & 1.6577018421 & 5.0297117974 \\
\hline $\mathrm{Fe} 2$ & 10.9356557219 & 28.3517538774 & 5.0297117974 \\
\hline $\mathrm{Fe} 2$ & 8.3179778697 & 20.9051705616 & 10.6237335552 \\
\hline $\mathrm{Fe} 2$ & 5.4074507219 & 9.1042851579 & 10.6237335552 \\
\hline $\mathrm{Fe} 2$ & 12.2701650177 & 5.9004427019 & 0.2235421610 \\
\hline $\mathrm{Fe} 2$ & 1.4552635739 & 24.1090130165 & 0.2235421610 \\
\hline 0 & 5.3996845482 & 16.4336200244 & 7.2013915361 \\
\hline 0 & 8.3257440434 & 13.5758356950 & 7.2013915361 \\
\hline 0 & 1.4630297476 & 1.4288921647 & 3.6458841800 \\
\hline 0 & 12.2623988450 & 28.5805635537 & 3.6458841800 \\
\hline 0 & 4.4475086731 & 18.5926772541 & 6.1508964148 \\
\hline 0 & 9.2779199195 & 11.4167784653 & 6.1508964148 \\
\hline 0 & 2.4152056237 & 3.5879493944 & 4.6963793014 \\
\hline 0 & 11.3102229689 & 26.4215063250 & 4.6963793014 \\
\hline $\mathrm{O}$ & 7.9434863469 & 19.0044075148 & 10.2782985386 \\
\hline $\mathrm{O}$ & 5.7819422447 & 11.0050482036 & 10.2782985386 \\
\hline 0 & 12.6446565405 & 3.9996796561 & 0.5689771786 \\
\hline 0 & 1.0807720511 & 26.0097760634 & 0.5689771786 \\
\hline 0 & 6.9657206861 & 21.1666134019 & 9.2506870875 \\
\hline 0 & 6.7597079055 & 8.8428423176 & 9.2506870875 \\
\hline 0 & 13.6224222023 & 6.1618855422 & 1.5965886287 \\
\hline 0 & 0.1030063903 & 23.8475701763 & 1.5965886287 \\
\hline 0 & -0.0942441267 & 17.4548247636 & 2.1890691177 \\
\hline 0 & 13.8196731023 & 12.5546309549 & 2.1890691177 \\
\hline 0 & 6.9569583974 & 2.4500969039 & 8.6582062751 \\
\hline 0 & 6.7684697860 & 27.5593588146 & 8.6582062751 \\
\hline $\mathrm{N}$ & 5.3184990989 & 16.7749979838 & 4.3011113576 \\
\hline $\mathrm{N}$ & 8.4069294937 & 13.2344577346 & 4.3011113576 \\
\hline $\mathrm{N}$ & 1.5442151979 & 1.7702701241 & 6.5461640352 \\
\hline $\mathrm{N}$ & 12.1812133947 & 28.2391855943 & 6.5461640352 \\
\hline $\mathrm{N}$ & 3.7749690660 & 14.7671457623 & 5.6570924462 \\
\hline $\mathrm{N}$ & 9.9504595256 & 15.2423090620 & 5.6570924462 \\
\hline $\mathrm{N}$ & 3.0877452298 & 29.7718745161 & 5.1901832710 \\
\hline $\mathrm{N}$ & 10.6376833618 & 0.2375820974 & 5.1901832710 \\
\hline $\mathrm{N}$ & 8.6615201127 & 22.7776485603 & 10.8976562446 \\
\hline $\mathrm{N}$ & 5.0639084789 & 7.2318071581 & 10.8976562446 \\
\hline $\mathrm{N}$ & 11.9266227757 & 7.7729207006 & -0.0503805274 \\
\hline $\mathrm{N}$ & 1.7988058169 & 22.2365350179 & -0.0503805274 \\
\hline $\mathrm{N}$ & 2.5324043397 & 16.7254865535 & 7.0346546719 \\
\hline $\mathrm{N}$ & 11.1930244575 & 13.2839691660 & 7.0346546719 \\
\hline $\mathrm{N}$ & 4.3303101617 & 1.7207586937 & 3.8126210443 \\
\hline $\mathrm{N}$ & 9.3951184309 & 28.2886970247 & 3.8126210443 \\
\hline $\mathrm{N}$ & 7.1141825736 & 20.8059069590 & 1.3097340501 \\
\hline $\mathrm{N}$ & 6.6112460190 & 9.2035487595 & 1.3097340501 \\
\hline $\mathrm{N}$ & 13.4739603148 & 5.8011790992 & 9.5375416661 \\
\hline$N$ & 0.2514682778 & 24.2082766192 & 9.5375416661 \\
\hline $\mathrm{N}$ & 9.7816438628 & 20.8093326620 & 9.3371534945 \\
\hline $\mathrm{N}$ & 3.9437847288 & 9.2001230564 & 9.3371534945 \\
\hline $\mathrm{N}$ & 10.8064990256 & 5.8046048033 & 1.5101222227 \\
\hline $\mathrm{N}$ & 2.9189295670 & 24.2048509161 & 1.5101222227 \\
\hline $\mathrm{N}$ & 2.6855132109 & 16.8519517580 & 4.3828198362 \\
\hline $\mathrm{N}$ & 11.0399155853 & 13.1575039604 & 4.3828198362 \\
\hline $\mathrm{N}$ & 4.1772012895 & 1.8472238983 & 6.4644555576 \\
\hline $\mathrm{N}$ & 9.5482273031 & 28.1622318201 & 6.4644555576 \\
\hline $\mathrm{N}$ & 9.7557094985 & 20.5923379290 & 1.1341814356 \\
\hline $\mathrm{N}$ & 3.9697190941 & 9.4171177904 & 1.1341814356 \\
\hline
\end{tabular}




\begin{tabular}{|c|c|c|c|}
\hline $\mathrm{N}$ & 10.8324333899 & 5.5876100693 & 9.7130942806 \\
\hline $\mathrm{N}$ & 2.8929952017 & 24.4218456491 & 9.7130942806 \\
\hline C & 5.8447077522 & 17.5281196737 & 7.7258513662 \\
\hline C & 7.8807208404 & 12.4813360447 & 7.7258513662 \\
\hline C & 1.0180065436 & 2.5233918140 & 3.1214243500 \\
\hline C & 12.7074220480 & 27.4860639044 & 3.1214243500 \\
\hline C & 6.8507350715 & 16.9843047861 & 1.9740050207 \\
\hline C & 6.8746935211 & 13.0251509324 & 1.9740050207 \\
\hline C & 13.7374078169 & 1.9795769263 & 8.8732708572 \\
\hline C & -0.0119792243 & 28.0298787921 & 8.8732708572 \\
\hline C & 2.8518882459 & 14.3860660388 & 4.7405951182 \\
\hline C & 10.8735403457 & 15.6233896796 & 4.7405951182 \\
\hline C & 4.0108260499 & 29.3907938986 & 6.1066805990 \\
\hline C & 9.7146025427 & 0.6186618209 & 6.1066805990 \\
\hline C & 2.4725984428 & 16.3212209997 & 8.3226797214 \\
\hline C & 11.2528297396 & 13.6882347198 & 8.3226797214 \\
\hline C & 4.3901158529 & 1.3164931399 & 2.5245959948 \\
\hline C & 9.3353131478 & 28.6929625785 & 2.5245959948 \\
\hline C & 5.3142632535 & 18.7759633204 & 7.0951225576 \\
\hline C & 8.4111653391 & 11.2334923980 & 7.0951225576 \\
\hline C & 1.5484510423 & 3.7712354607 & 3.7521531586 \\
\hline C & 12.1769775493 & 26.2382202578 & 3.7521531586 \\
\hline C & 13.8528237874 & 16.8399992213 & 8.4477893121 \\
\hline C & -0.1273952560 & 13.1694564982 & 8.4477893121 \\
\hline C & 6.7353191010 & 1.8352713615 & 2.3994864051 \\
\hline C & 6.9901094906 & 28.1741843569 & 2.3994864051 \\
\hline C & 7.4425786180 & 16.6432804801 & 3.1901165689 \\
\hline C & 6.2828499746 & 13.3661752394 & 3.1901165689 \\
\hline C & 13.1455642703 & 1.6385526203 & 7.6571594707 \\
\hline C & 0.5798643212 & 28.3709030981 & 7.6571594707 \\
\hline C & 1.2908242910 & 16.3719330000 & 9.0589118260 \\
\hline C & 12.4346047097 & 13.6375227185 & 9.0589118260 \\
\hline C & 5.5718900048 & 1.3672051402 & 1.7883638902 \\
\hline C & 8.1535381776 & 28.6422505782 & 1.7883638902 \\
\hline C & 5.7535324432 & 20.0283858587 & 7.5456440709 \\
\hline C & 7.9718957392 & 9.9810698608 & 7.5456440709 \\
\hline C & 1.1091818526 & 5.0236579989 & 3.3016316453 \\
\hline C & 12.6162471482 & 24.9857977195 & 3.3016316453 \\
\hline C & 4.3332432607 & 13.8271349245 & 6.4586847843 \\
\hline C & 9.3921849217 & 16.1823216890 & 6.4586847843 \\
\hline C & 2.5294710351 & 28.8318618902 & 4.3885909319 \\
\hline C & 11.1959579657 & 1.1775929352 & 4.3885909319 \\
\hline C & 7.1441285435 & 18.8229836666 & 9.2783893367 \\
\hline C & 6.5813000491 & 11.1864720519 & 9.2783893367 \\
\hline C & 13.4440143449 & 3.8182558068 & 1.5688863795 \\
\hline C & 0.2814142477 & 26.1911999116 & 1.5688863795 \\
\hline C & 6.7522346443 & 17.5723604933 & 8.7910954685 \\
\hline C & 6.9731943564 & 12.4370952252 & 8.7910954685 \\
\hline C & 0.1104796515 & 2.5676326345 & 2.0561802477 \\
\hline C & 13.6149485310 & 27.4418230849 & 2.0561802477 \\
\hline C & 2.5059069344 & 13.0472084944 & 4.5657605170 \\
\hline C & 11.2195214526 & 16.9622463310 & 4.5657605170 \\
\hline C & 4.3568071568 & 28.0519372482 & 6.2815148759 \\
\hline C & 9.3686214348 & 1.9575193653 & 6.2815148759 \\
\hline C & 3.0998962875 & 12.0778044501 & 5.3726249201 \\
\hline C & 10.6255325087 & 17.9316521634 & 5.3726249201 \\
\hline C & 3.7628182129 & 27.0825314148 & 5.4746511194 \\
\hline C & 9.9626103797 & 2.9269234096 & 5.4746511194 \\
\hline
\end{tabular}




\begin{tabular}{|c|c|c|c|}
\hline C & 8.0454736551 & 23.7878031468 & 10.2381861953 \\
\hline C & 5.6799549365 & 6.2216525726 & 10.2381861953 \\
\hline C & 12.5426692333 & 8.7830752871 & 0.6090895218 \\
\hline C & 1.1827593593 & 21.2263804313 & 0.6090895218 \\
\hline C & 4.0200444799 & 12.4788195089 & 6.3439812539 \\
\hline C & 9.7053841116 & 17.5306353155 & 6.3439812539 \\
\hline C & 2.8426698159 & 27.4835482626 & 4.5032944633 \\
\hline C & 10.8827587767 & 2.5259083508 & 4.5032944633 \\
\hline C & 9.6286890785 & 23.0761762663 & 0.9526417196 \\
\hline C & 4.0967395131 & 6.9332794521 & 0.9526417196 \\
\hline C & 10.9594538099 & 8.0714484076 & 9.8946339966 \\
\hline C & 2.7659747827 & 21.9380073118 & 9.8946339966 \\
\hline C & 7.6998273329 & 20.3449109752 & 2.4475119439 \\
\hline C & 6.0256012587 & 9.6645447432 & 2.4475119439 \\
\hline C & 12.8883155545 & 5.3401831165 & 8.3997637733 \\
\hline C & 0.8371130371 & 24.6692726029 & 8.3997637733 \\
\hline C & 6.9861231109 & 20.2208513421 & 3.6373362240 \\
\hline C & 6.7393054817 & 9.7886043774 & 3.6373362240 \\
\hline C & -0.1234088151 & 5.2161234824 & 7.2099394922 \\
\hline C & 13.8488374067 & 24.7933322371 & 7.2099394922 \\
\hline C & 2.2455865920 & 15.4884994539 & 3.9210335619 \\
\hline C & 11.4798417950 & 14.5209562655 & 3.9210335619 \\
\hline C & 4.6171274992 & 0.4837715942 & 6.9262421553 \\
\hline C & 9.1083010924 & 29.5256841242 & 6.9262421553 \\
\hline C & 3.3132002064 & 17.5918862444 & 3.2527976801 \\
\hline C & 10.4122279760 & 12.4175694741 & 3.2527976801 \\
\hline C & 3.5495140894 & 2.5871583857 & 7.5944780371 \\
\hline C & 10.1759149113 & 27.4222973338 & 7.5944780371 \\
\hline C & 5.4810045748 & 17.2520641006 & 1.9487302777 \\
\hline C & 8.2444240168 & 12.7573916178 & 1.9487302777 \\
\hline C & 1.3817097210 & 2.2473362419 & 8.8985457619 \\
\hline C & 12.3437188706 & 27.7621194776 & 8.8985457619 \\
\hline C & 5.7958584692 & 21.0914554327 & 1.3254770354 \\
\hline C & 7.9295701224 & 8.9180002868 & 1.3254770354 \\
\hline C & 1.0668558266 & 6.0867275730 & 9.5217986808 \\
\hline C & 12.6585727660 & 23.9227281465 & 9.5217986808 \\
\hline C & 0.1848119383 & 17.2404036376 & 7.1127353424 \\
\hline C & 13.5406168198 & 12.7690520809 & 7.1127353424 \\
\hline C & 6.6779025240 & 2.2356757778 & 3.7345403748 \\
\hline C & 7.0475260686 & 27.7737799406 & 3.7345403748 \\
\hline C & 1.5756364479 & 17.6218959452 & 5.0124180789 \\
\hline C & 12.1497919401 & 12.3875597743 & 5.0124180789 \\
\hline C & 5.2870776443 & 2.6171680854 & 5.8348576373 \\
\hline C & 8.4383509483 & 27.3922876340 & 5.8348576373 \\
\hline C & 4.7489809880 & 17.1701230708 & 3.1299232391 \\
\hline C & 8.9764476046 & 12.8393326476 & 3.1299232391 \\
\hline C & 2.1137333078 & 2.1653952111 & 7.7173528015 \\
\hline C & 11.6116952838 & 27.8440605073 & 7.7173528015 \\
\hline C & 5.0149184565 & 20.9330988143 & 2.4646728815 \\
\hline C & 8.7105105443 & 9.0763569041 & 2.4646728815 \\
\hline C & 1.8477958393 & 5.9283709556 & 8.3826028357 \\
\hline C & 11.8776323431 & 24.0810847639 & 8.3826028357 \\
\hline C & 6.6446390848 & 16.5356996669 & 4.3239781076 \\
\hline C & 7.0807899159 & 13.4737560526 & 4.3239781076 \\
\hline C & 0.2180752110 & 1.5309718071 & 6.5232976086 \\
\hline C & 13.5073529725 & 28.4784839123 & 6.5232976086 \\
\hline C & 10.3451793108 & 21.9049514527 & 1.5652669509 \\
\hline C & 3.3802492808 & 8.1045042658 & 1.5652669509 \\
\hline
\end{tabular}




\begin{tabular}{|c|c|c|c|}
\hline C & 10.2429635766 & 6.9002235939 & 9.2820087653 \\
\hline C & 3.4824650150 & 23.1092321255 & 9.2820087653 \\
\hline C & 6.6078872067 & 20.0733887406 & 8.6571018748 \\
\hline C & 7.1175413849 & 9.9360669778 & 8.6571018748 \\
\hline C & 0.2548270891 & 5.0686608809 & 2.1901738424 \\
\hline C & 13.4706015025 & 24.9407948376 & 2.1901738424 \\
\hline C & 1.3981104184 & 17.1739521367 & 6.4335143101 \\
\hline C & 12.3273180719 & 12.8355035828 & 6.4335143101 \\
\hline C & 5.4646037762 & 2.1692242769 & 4.4137614061 \\
\hline C & 8.2608248164 & 27.8402314415 & 4.4137614061 \\
\hline C & 9.7899216115 & 21.2368939196 & 8.0551042976 \\
\hline C & 3.9355069801 & 8.7725617988 & 8.0551042976 \\
\hline C & 10.7982212759 & 6.2321660609 & 2.7921714186 \\
\hline C & 2.9272073157 & 23.7772896586 & 2.7921714186 \\
\hline C & 10.9173808684 & 21.1068815971 & 7.2491039995 \\
\hline C & 2.8080477232 & 8.9025741223 & 7.2491039995 \\
\hline C & 9.6707620190 & 6.1021537374 & 3.5981717167 \\
\hline C & 4.0546665726 & 23.9073019820 & 3.5981717167 \\
\hline C & 10.7521930869 & 19.7423955450 & 0.4157390062 \\
\hline C & 2.9732355047 & 10.2670601734 & 0.4157390062 \\
\hline C & 9.8359498005 & 4.7376676863 & 10.4315367100 \\
\hline C & 3.8894787911 & 25.2717880332 & 10.4315367100 \\
\hline C & 10.9026497527 & 20.2434974547 & 9.8575112056 \\
\hline C & 2.8227788399 & 9.7659582647 & 9.8575112056 \\
\hline C & 9.6854931357 & 5.2387695950 & 0.9897645106 \\
\hline C & 4.0399354569 & 24.7706861244 & 0.9897645106 \\
\hline C & 8.3693567655 & 25.1218505090 & 10.4598265715 \\
\hline C & 5.3560718271 & 4.8876052104 & 10.4598265715 \\
\hline C & 12.2187861229 & 10.1171226493 & 0.3874491457 \\
\hline C & 1.5066424697 & 19.8923330701 & 0.3874491457 \\
\hline C & 12.0754692132 & 20.5386360967 & 7.7828587350 \\
\hline C & 1.6499593794 & 9.4708196217 & 7.7828587350 \\
\hline C & 8.5126736752 & 5.5339082380 & 3.0644169812 \\
\hline C & 5.2127549164 & 24.4755474815 & 3.0644169812 \\
\hline C & 5.6212955400 & 20.5119085458 & 3.6493207039 \\
\hline C & 8.1041330516 & 9.4975471736 & 3.6493207039 \\
\hline C & 1.2414187558 & 5.5071806861 & 7.1979550133 \\
\hline C & 12.4840098358 & 24.5022750323 & 7.1979550133 \\
\hline C & 9.9726122475 & 24.3941582343 & 1.2459915644 \\
\hline C & 3.7528163441 & 5.6152974851 & 1.2459915644 \\
\hline C & 10.6155306399 & 9.3894303746 & 9.6012841518 \\
\hline C & 3.1098979517 & 20.6200253438 & 9.6012841518 \\
\hline C & 9.1189778682 & 19.8775923830 & 2.2752578205 \\
\hline C & 4.6064507234 & 10.1318633364 & 2.2752578205 \\
\hline C & 11.4691650192 & 4.8728645233 & 8.5720178957 \\
\hline C & 2.2562635724 & 25.1365911951 & 8.5720178957 \\
\hline C & 9.3424908074 & 25.4327134951 & 0.5643954400 \\
\hline C & 4.3829377842 & 4.5767422244 & 0.5643954400 \\
\hline C & 11.2456520800 & 10.4279856353 & 10.2828802762 \\
\hline C & 2.4797765116 & 19.5814700831 & 10.2828802762 \\
\hline C & 12.0670236160 & 20.1061940731 & 9.1083092638 \\
\hline C & 1.6584049756 & 9.9032616454 & 9.1083092638 \\
\hline C & 8.5211192714 & 5.1014662133 & 1.7389664524 \\
\hline C & 5.2043093202 & 24.9079895051 & 1.7389664524 \\
\hline $\mathrm{H}$ & 7.4522794491 & 17.0576538094 & 1.0667408763 \\
\hline $\mathrm{H}$ & 6.2731491425 & 12.9518019100 & 1.0667408763 \\
\hline $\mathrm{H}$ & 13.1358634383 & 2.0529259497 & 9.7805348399 \\
\hline $\mathrm{H}$ & 0.5895651533 & 27.9565297698 & 9.7805348399 \\
\hline
\end{tabular}




\begin{tabular}{|c|c|c|c|}
\hline $\mathrm{H}$ & 3.3981836005 & 15.9371646691 & 8.7529182612 \\
\hline $\mathrm{H}$ & 10.3272447865 & 14.0722910493 & 8.7529182612 \\
\hline $\mathrm{H}$ & 3.4645304907 & 0.9324368094 & 2.0943574550 \\
\hline $\mathrm{H}$ & 10.2608981009 & 29.0770189090 & 2.0943574550 \\
\hline $\mathrm{H}$ & 12.9149650811 & 16.8692807197 & 9.0042520099 \\
\hline $\mathrm{H}$ & 0.8104635105 & 13.1401749997 & 9.0042520099 \\
\hline $\mathrm{H}$ & 7.6731778063 & 1.8645528600 & 1.8430237063 \\
\hline $\mathrm{H}$ & 6.0522507853 & 28.1449028584 & 1.8430237063 \\
\hline $\mathrm{H}$ & 8.5098426576 & 16.4357906817 & 3.2478201321 \\
\hline $\mathrm{H}$ & 5.2155859340 & 13.5736650367 & 3.2478201321 \\
\hline $\mathrm{H}$ & 12.0783002308 & 1.4310628230 & 7.5994559075 \\
\hline $\mathrm{H}$ & 1.6471283618 & 28.5783928964 & 7.5994559075 \\
\hline $\mathrm{H}$ & 1.2947072865 & 16.0317254332 & 10.0935394415 \\
\hline $\mathrm{H}$ & 12.4307213051 & 13.9777302862 & 10.0935394415 \\
\hline $\mathrm{H}$ & 5.5680070093 & 1.0269975735 & 0.7537362747 \\
\hline $\mathrm{H}$ & 8.1574215833 & 28.9824581449 & 0.7537362747 \\
\hline $\mathrm{H}$ & 5.4038571536 & 20.9577199246 & 7.0914771735 \\
\hline $\mathrm{H}$ & 8.3215718472 & 9.0517357948 & 7.0914771735 \\
\hline $\mathrm{H}$ & 1.4588571422 & 5.9529920649 & 3.7557985437 \\
\hline $\mathrm{H}$ & 12.2665710402 & 24.0564636535 & 3.7557985437 \\
\hline $\mathrm{H}$ & 5.0379568057 & 14.1990413659 & 7.2015599868 \\
\hline $\mathrm{H}$ & 8.6874717859 & 15.8104152476 & 7.2015599868 \\
\hline $\mathrm{H}$ & 1.8247574901 & 29.2037683305 & 3.6457157294 \\
\hline $\mathrm{H}$ & 11.9006711015 & 0.8056864939 & 3.6457157294 \\
\hline $\mathrm{H}$ & 7.1457068219 & 16.6547500637 & 9.2309292869 \\
\hline $\mathrm{H}$ & 6.5797217697 & 13.3547056547 & 9.2309292869 \\
\hline $\mathrm{H}$ & 13.4424360655 & 1.6500222040 & 1.6163464303 \\
\hline $\mathrm{H}$ & 0.2829925261 & 28.3594335145 & 1.6163464303 \\
\hline $\mathrm{H}$ & 1.7680356007 & 12.7842629680 & 3.8066929128 \\
\hline $\mathrm{H}$ & 11.9573931965 & 17.2251936454 & 3.8066929128 \\
\hline $\mathrm{H}$ & 5.0946788997 & 27.7889899327 & 7.0405831278 \\
\hline $\mathrm{H}$ & 8.6307496919 & 2.2204648917 & 7.0405831278 \\
\hline $\mathrm{H}$ & 2.8114398158 & 11.0321296452 & 5.2654503202 \\
\hline $\mathrm{H}$ & 10.9139887768 & 18.9773260743 & 5.2654503202 \\
\hline $\mathrm{H}$ & 4.0512744810 & 26.0368575038 & 5.5818257193 \\
\hline $\mathrm{H}$ & 9.6741541116 & 3.9725982146 & 5.5818257193 \\
\hline $\mathrm{H}$ & 7.2998500846 & 23.4875636318 & 9.5048991934 \\
\hline $\mathrm{H}$ & 6.4255785070 & 6.5218920877 & 9.5048991934 \\
\hline $\mathrm{H}$ & 13.2882928028 & 8.4828357720 & 1.3423765238 \\
\hline $\mathrm{H}$ & 0.4371357888 & 21.5266199464 & 1.3423765238 \\
\hline $\mathrm{H}$ & 4.4907769792 & 11.7658967382 & 7.0218629999 \\
\hline $\mathrm{H}$ & 9.2346516134 & 18.2435589803 & 7.0218629999 \\
\hline $\mathrm{H}$ & 2.3719373176 & 26.7706245979 & 3.8254127163 \\
\hline $\mathrm{H}$ & 11.3534912750 & 3.2388311216 & 3.8254127163 \\
\hline $\mathrm{H}$ & 7.4892109234 & 19.8686677965 & 4.5392421810 \\
\hline $\mathrm{H}$ & 6.2362176681 & 10.1407879230 & 4.5392421810 \\
\hline $\mathrm{H}$ & 13.0989319640 & 4.8639399367 & 6.3080335352 \\
\hline $\mathrm{H}$ & 0.6264966276 & 25.1455157817 & 6.3080335352 \\
\hline $\mathrm{H}$ & 1.1468468359 & 15.4298040757 & 3.9368415167 \\
\hline $\mathrm{H}$ & 12.5785817567 & 14.5796516428 & 3.9368415167 \\
\hline $\mathrm{H}$ & 5.7158674599 & 0.4250762170 & 6.9104338771 \\
\hline $\mathrm{H}$ & 8.0095611317 & 29.5843795025 & 6.9104338771 \\
\hline $\mathrm{H}$ & 2.5364880556 & 15.3577459904 & 2.8681100982 \\
\hline $\mathrm{H}$ & 11.1889409452 & 14.6517097290 & 2.8681100982 \\
\hline $\mathrm{H}$ & 4.3262262402 & 0.3530181307 & 7.9791656180 \\
\hline $\mathrm{H}$ & 9.3992019432 & 29.6564375877 & 7.9791656180 \\
\hline $\mathrm{H}$ & 3.2932585477 & 18.6623292204 & 3.5095519136 \\
\hline $\mathrm{H}$ & 10.4321696357 & 11.3471264990 & 3.5095519136 \\
\hline
\end{tabular}




\begin{tabular}{|c|c|c|c|}
\hline $\mathrm{H}$ & 3.5694557481 & 3.6576013608 & 7.3377234802 \\
\hline $\mathrm{H}$ & 10.1559732527 & 26.3518543587 & 7.3377234802 \\
\hline $\mathrm{H}$ & 2.7526562521 & 17.4549868610 & 2.3158831727 \\
\hline $\mathrm{H}$ & 10.9727723395 & 12.5544688575 & 2.3158831727 \\
\hline $\mathrm{H}$ & 4.1100580437 & 2.4502590023 & 8.5313925435 \\
\hline $\mathrm{H}$ & 9.6153705479 & 27.5591967172 & 8.5313925435 \\
\hline $\mathrm{H}$ & 4.9762184105 & 17.5232347322 & 1.0213322741 \\
\hline $\mathrm{H}$ & 8.7492101821 & 12.4862209872 & 1.0213322741 \\
\hline $\mathrm{H}$ & 1.8864958853 & 2.5185068725 & 9.8259431187 \\
\hline $\mathrm{H}$ & 11.8389327063 & 27.4909488469 & 9.8259431187 \\
\hline $\mathrm{H}$ & 5.3812554263 & 21.4392684074 & 0.3799511754 \\
\hline $\mathrm{H}$ & 8.3441731652 & 8.5701873110 & 0.3799511754 \\
\hline $\mathrm{H}$ & 1.4814588695 & 6.4345405477 & 10.4673245408 \\
\hline $\mathrm{H}$ & 12.2439697221 & 23.5749151708 & 10.4673245408 \\
\hline $\mathrm{H}$ & 13.0273767529 & 17.6058361234 & 6.5902340723 \\
\hline $\mathrm{H}$ & 0.6980517364 & 12.4036195960 & 6.5902340723 \\
\hline $\mathrm{H}$ & 7.5607661355 & 2.6011082637 & 4.2570416438 \\
\hline $\mathrm{H}$ & 6.1646624571 & 27.4083474558 & 4.2570416438 \\
\hline $\mathrm{H}$ & 0.6479541999 & 17.5395890898 & 4.4306965379 \\
\hline $\mathrm{H}$ & 13.0774745962 & 12.4698666296 & 4.4306965379 \\
\hline $\mathrm{H}$ & 6.2147603004 & 2.5348612301 & 6.4165795017 \\
\hline $\mathrm{H}$ & 7.5106682912 & 27.4745944883 & 6.4165795017 \\
\hline $\mathrm{H}$ & 1.8671156484 & 18.6828917610 & 5.0165839026 \\
\hline $\mathrm{H}$ & 11.8583133524 & 11.3265639584 & 5.0165839026 \\
\hline $\mathrm{H}$ & 4.9955986474 & 3.6781639013 & 5.8306921370 \\
\hline $\mathrm{H}$ & 8.7298295360 & 26.3312918171 & 5.8306921370 \\
\hline $\mathrm{H}$ & 3.9482072103 & 21.1515860513 & 2.4193076676 \\
\hline $\mathrm{H}$ & 9.7772213813 & 8.8578696681 & 2.4193076676 \\
\hline $\mathrm{H}$ & 2.9145070855 & 6.1468581916 & 8.4279680496 \\
\hline $\mathrm{H}$ & 10.8109215061 & 23.8625975268 & 8.4279680496 \\
\hline $\mathrm{H}$ & 7.0492292611 & 16.2423837459 & 5.2909482430 \\
\hline $\mathrm{H}$ & 6.6761993305 & 13.7670719735 & 5.2909482430 \\
\hline $\mathrm{H}$ & 13.5389136263 & 1.2376558862 & 5.5563274742 \\
\hline $\mathrm{H}$ & 0.1865149653 & 28.7717998322 & 5.5563274742 \\
\hline $\mathrm{H}$ & 11.4083991030 & 21.9339657532 & 1.2791320840 \\
\hline $\mathrm{H}$ & 2.3170294886 & 8.0754899663 & 1.2791320840 \\
\hline $\mathrm{H}$ & 9.1797437844 & 6.9292378934 & 9.5681436332 \\
\hline $\mathrm{H}$ & 4.5456848072 & 23.0802178250 & 9.5681436332 \\
\hline $\mathrm{H}$ & 10.3506966364 & 21.9759156954 & 2.6626545643 \\
\hline $\mathrm{H}$ & 3.3747319552 & 8.0335400241 & 2.6626545643 \\
\hline $\mathrm{H}$ & 10.2374462510 & 6.9711878357 & 8.1846211519 \\
\hline $\mathrm{H}$ & 3.4879823406 & 23.0382678828 & 8.1846211519 \\
\hline $\mathrm{H}$ & 8.8697568989 & 21.7065707270 & 7.7016624121 \\
\hline $\mathrm{H}$ & 4.8556716927 & 8.3028849924 & 7.7016624121 \\
\hline $\mathrm{H}$ & 11.7183859885 & 6.7018428673 & 3.1456133051 \\
\hline $\mathrm{H}$ & 2.0070426031 & 23.3076128521 & 3.1456133051 \\
\hline $\mathrm{H}$ & 10.9053163461 & 21.4701315878 & 6.2211783826 \\
\hline H & 2.8201122455 & 8.5393241316 & 6.2211783826 \\
\hline $\mathrm{H}$ & 9.6828265413 & 6.4654037281 & 4.6260973336 \\
\hline $\mathrm{H}$ & 4.0426020503 & 23.5440519904 & 4.6260973336 \\
\hline $\mathrm{H}$ & 10.3411516446 & 18.7234978180 & 0.3759592743 \\
\hline $\mathrm{H}$ & 3.3842769470 & 11.2859579004 & 0.3759592743 \\
\hline $\mathrm{H}$ & 10.2469912428 & 3.7187699583 & 10.4713164429 \\
\hline $\mathrm{H}$ & 3.4784373488 & 26.2906857601 & 10.4713164429 \\
\hline $\mathrm{H}$ & 11.7130786608 & 19.7075854936 & 0.9468851310 \\
\hline $\mathrm{H}$ & 2.0123499308 & 10.3018702258 & 0.9468851310 \\
\hline $\mathrm{H}$ & 8.8750642266 & 4.7028576339 & 9.9003905852 \\
\hline $\mathrm{H}$ & 4.8503643650 & 25.3065980856 & 9.9003905852 \\
\hline
\end{tabular}




\begin{tabular}{|c|c|c|c|}
\hline $\mathrm{H}$ & 7.8666140172 & 25.9068107744 & 9.8927750695 \\
\hline $\mathrm{H}$ & 5.8588145754 & 4.1026449440 & 9.8927750695 \\
\hline $\mathrm{H}$ & 12.7215288712 & 10.9020829157 & 0.9545006467 \\
\hline $\mathrm{H}$ & 1.0038997204 & 19.1073728038 & 0.9545006467 \\
\hline $\mathrm{H}$ & 12.9659652143 & 20.4364513179 & 7.1642130562 \\
\hline $\mathrm{H}$ & 0.7594633773 & 9.5730044005 & 7.1642130562 \\
\hline $\mathrm{H}$ & 7.6221776731 & 5.4317234582 & 3.6830626610 \\
\hline $\mathrm{H}$ & 6.1032509185 & 24.5777322603 & 3.6830626610 \\
\hline $\mathrm{H}$ & 5.0424421019 & 20.3835916691 & 4.5650115090 \\
\hline $\mathrm{H}$ & 8.6829868989 & 9.6258640493 & 4.5650115090 \\
\hline $\mathrm{H}$ & 1.8202721939 & 5.3788638094 & 6.2822642072 \\
\hline $\mathrm{H}$ & 11.9051559885 & 24.6305919090 & 6.2822642072 \\
\hline $\mathrm{H}$ & 10.7456384967 & 24.5984227892 & 1.9875721871 \\
\hline $\mathrm{H}$ & 2.9797900949 & 5.4110329302 & 1.9875721871 \\
\hline $\mathrm{H}$ & 9.8425043907 & 9.5936949295 & 8.8597035301 \\
\hline $\mathrm{H}$ & 3.8829242009 & 20.4157607890 & 8.8597035301 \\
\hline $\mathrm{H}$ & 9.7075879547 & 19.9842262016 & 3.2004841694 \\
\hline $\mathrm{H}$ & 4.0178406369 & 10.0252295168 & 3.2004841694 \\
\hline $\mathrm{H}$ & 10.8805549328 & 4.9794983419 & 7.6467915467 \\
\hline $\mathrm{H}$ & 2.8448736589 & 25.0299573765 & 7.6467915467 \\
\hline $\mathrm{H}$ & 9.0984590275 & 18.8062182199 & 2.0236642026 \\
\hline $\mathrm{H}$ & 4.6269695651 & 11.2032374995 & 2.0236642026 \\
\hline H & 11.4896838609 & 3.8014903602 & 8.8236115146 \\
\hline $\mathrm{H}$ & 2.2357447317 & 26.2079653582 & 8.8236115146 \\
\hline $\mathrm{H}$ & 9.6071789199 & 26.4703653770 & 0.7714447668 \\
\hline H & 4.1182496717 & 3.5390903414 & 0.7714447668 \\
\hline $\mathrm{H}$ & 10.9809639675 & 11.4656375173 & 10.0758309494 \\
\hline $\mathrm{H}$ & 2.7444646241 & 18.5438182011 & 10.0758309494 \\
\hline $\mathrm{H}$ & 12.9529367396 & 19.6635747195 & 9.5646427629 \\
\hline $\mathrm{H}$ & 0.7724918520 & 10.3458810000 & 9.5646427629 \\
\hline $\mathrm{H}$ & 7.6352061478 & 4.6588468598 & 1.2826329542 \\
\hline $\mathrm{H}$ & 6.0902224438 & 25.3506088586 & 1.2826329543 \\
\hline $\mathrm{H}$ & 13.0340575500 & 17.0771607626 & 1.5021529436 \\
\hline $\mathrm{H}$ & 0.6913708761 & 12.9322949568 & 1.5021529436 \\
\hline $\mathrm{H}$ & 7.5540853384 & 2.0724329029 & 9.3451229353 \\
\hline $\mathrm{H}$ & 6.1713432542 & 27.9370228155 & 9.3451229353 \\
\hline $\mathrm{H}$ & 13.2772567933 & 18.3499596876 & 2.3861335344 \\
\hline $\mathrm{H}$ & 0.4481722075 & 11.6594960308 & 2.3861335345 \\
\hline $\mathrm{H}$ & 7.3108860941 & 3.3452318279 & 8.4611418583 \\
\hline $\mathrm{H}$ & 6.4145420883 & 26.6642238905 & 8.4611418584 \\
\hline $\mathrm{F}$ & 10.6392658745 & 14.5617080258 & 0.6901483291 \\
\hline $\mathrm{F}$ & 3.0861627171 & 15.4477467996 & 0.6901483291 \\
\hline $\mathrm{F}$ & 9.9488770129 & 29.5664367796 & 10.1571273871 \\
\hline $\mathrm{F}$ & 3.7765515787 & 0.4430198339 & 10.1571273871 \\
\hline $\mathrm{F}$ & 10.0679175276 & 16.6598319334 & 1.5121327681 \\
\hline $\mathrm{F}$ & 3.6575110640 & 13.3496237850 & 1.5121327681 \\
\hline $\mathrm{F}$ & 10.5202253598 & 1.6551040737 & 9.3351429491 \\
\hline $\mathrm{F}$ & 3.2052032318 & 28.3543516447 & 9.3351429491 \\
\hline$F$ & 6.3817210194 & 25.2026840070 & 5.7896834093 \\
\hline $\mathrm{F}$ & 7.3437075732 & 4.8067717114 & 5.7896834093 \\
\hline $\mathrm{F}$ & 0.4809932764 & 10.1979561483 & 5.0575923079 \\
\hline $\mathrm{F}$ & 13.2444353152 & 19.8114995712 & 5.0575923079 \\
\hline $\mathrm{F}$ & 11.9642514450 & 16.4157259303 & 11.0507122761 \\
\hline $\mathrm{F}$ & 1.7611771465 & 13.5937297881 & 11.0507122760 \\
\hline $\mathrm{F}$ & 8.6238914433 & 1.4109980706 & -0.2034365598 \\
\hline$F$ & 5.1015371492 & 28.5984576479 & -0.2034365598 \\
\hline $\mathrm{F}$ & 5.9874777716 & 24.9098693296 & 8.0509370772 \\
\hline $\mathrm{F}$ & 7.7379508210 & 5.0995863888 & 8.0509370772 \\
\hline
\end{tabular}




\begin{tabular}{|c|c|c|c|}
\hline $\mathrm{F}$ & 0.8752365252 & 9.9051414699 & 2.7963386390 \\
\hline $\mathrm{F}$ & 12.8501920674 & 20.1043142485 & 2.7963386390 \\
\hline $\mathrm{F}$ & 9.8598890134 & 16.2009866834 & 10.0821325650 \\
\hline $\mathrm{F}$ & 3.8655395782 & 13.8084690350 & 10.0821325650 \\
\hline $\mathrm{F}$ & 10.7282538750 & 1.1962588237 & 0.7651431512 \\
\hline $\mathrm{F}$ & 2.9971747176 & 28.8131968947 & 0.7651431512 \\
\hline $\mathrm{F}$ & 7.5801326281 & 23.5974651765 & 6.9611250866 \\
\hline $\mathrm{F}$ & 6.1452959635 & 6.4119905419 & 6.9611250866 \\
\hline $\mathrm{F}$ & 13.0080102593 & 8.5927373168 & 3.8861506306 \\
\hline $\mathrm{F}$ & 0.7174183323 & 21.4167184017 & 3.8861506306 \\
\hline $\mathrm{F}$ & 5.3034279268 & 23.2842354533 & 6.5419373914 \\
\hline $\mathrm{F}$ & 8.4220002556 & 6.7252202651 & 6.5419373914 \\
\hline $\mathrm{F}$ & 1.5592863690 & 8.2795075947 & 4.3053383248 \\
\hline $\mathrm{F}$ & 12.1661426317 & 21.7299481248 & 4.3053383248 \\
\hline B & 10.6088722793 & 15.9527441277 & 0.3990161284 \\
\hline B & 3.1165563132 & 14.0567115917 & 0.3990161284 \\
\hline B & 9.9792706090 & 0.9480162680 & 10.4482595888 \\
\hline B & 3.7461579826 & 29.0614394504 & 10.4482595888 \\
\hline B & 6.3293352671 & 24.2282180739 & 6.8184954376 \\
\hline B & 7.3960933255 & 5.7812376445 & 6.8184954376 \\
\hline B & 0.5333790297 & 9.2234902152 & 4.0287802786 \\
\hline B & 13.1920495628 & 20.7859655042 & 4.0287802786 \\
\hline $1 \cdot \mathrm{CH}_{3} \mathrm{OH} \quad \mathrm{HS}$ & & & \\
\hline CELL_PARAMETE & ERS (angstrom) & & \\
\hline 13.742181521 & $1 \quad 0.000000000$ & 0.000000000 & \\
\hline 0.000000000 & 28.170361667 & 0.000000000 & \\
\hline 0.000000000 & 0.000000000 & 11.536218282 & \\
\hline ATOMIC_POSITIO & ONS (crystal) & & \\
\hline $\mathrm{Fe} 1$ & 0.1914942928 & 0.9489192703 & 0.5307394498 \\
\hline $\mathrm{Fe} 1$ & 0.3085057072 & 0.4489192703 & 0.4692605800 \\
\hline $\mathrm{Fe} 1$ & 0.3696594500 & 0.6991312960 & 0.0311738495 \\
\hline $\mathrm{Fe} 1$ & 0.6303405501 & 0.3008687040 & 0.0311738495 \\
\hline $\mathrm{Fe} 1$ & 0.6914943226 & 0.5510807297 & 0.4692605800 \\
\hline $\mathrm{Fe} 1$ & 0.8085056774 & 0.0510807297 & 0.5307394498 \\
\hline $\mathrm{Fe} 1$ & 0.1303405501 & 0.1991312960 & 0.9688261486 \\
\hline $\mathrm{Fe} 1$ & 0.8696594499 & 0.8008687040 & 0.9688261487 \\
\hline $\mathrm{N}$ & 0.8253219123 & 0.5557497806 & 0.3730996096 \\
\hline $\mathrm{N}$ & 0.1746780878 & 0.4442502194 & 0.3730996096 \\
\hline $\mathrm{N}$ & 0.6746780877 & 0.0557497806 & 0.6269003904 \\
\hline $\mathrm{N}$ & 0.3253219123 & 0.9442502194 & 0.6269003904 \\
\hline $\mathrm{N}$ & 0.8060293741 & 0.5508584076 & 0.6119946963 \\
\hline $\mathrm{N}$ & 0.1939706408 & 0.4491416222 & 0.6119946963 \\
\hline $\mathrm{N}$ & 0.6939706259 & 0.0508583778 & 0.3880053037 \\
\hline $\mathrm{N}$ & 0.3060293742 & 0.9491415924 & 0.3880053037 \\
\hline $\mathrm{N}$ & 0.6062991549 & 0.5588758984 & 0.6256533635 \\
\hline $\mathrm{N}$ & 0.3937008451 & 0.4411241314 & 0.6256533635 \\
\hline $\mathrm{N}$ & 0.8937008451 & 0.0588758686 & 0.3743466364 \\
\hline $\mathrm{N}$ & 0.1062991549 & 0.9411241016 & 0.3743466364 \\
\hline $\mathrm{N}$ & 0.7174685695 & 0.4771650211 & 0.4842276727 \\
\hline $\mathrm{N}$ & 0.2825314305 & 0.5228349789 & 0.4842276727 \\
\hline $\mathrm{N}$ & 0.7825314305 & 0.9771650211 & 0.5157723273 \\
\hline $\mathrm{N}$ & 0.2174685695 & 0.0228349789 & 0.5157723273 \\
\hline $\mathrm{N}$ & 0.2392064479 & 0.6950498733 & 0.1368621944 \\
\hline $\mathrm{N}$ & 0.7607935521 & 0.3049501565 & 0.1368621944 \\
\hline $\mathrm{N}$ & 0.2607935521 & 0.1950498435 & 0.8631378057 \\
\hline $\mathrm{N}$ & 0.7392064479 & 0.8049501267 & 0.8631378057 \\
\hline $\mathrm{N}$ & 0.2532095651 & 0.6962360728 & 0.8974785796 \\
\hline
\end{tabular}




\begin{tabular}{|c|c|c|c|}
\hline $\mathrm{N}$ & 0.7467904349 & 0.3037639272 & 0.8974785796 \\
\hline $\mathrm{N}$ & 0.2467904349 & 0.1962360728 & 0.1025213905 \\
\hline $\mathrm{N}$ & 0.7532095651 & 0.8037639272 & 0.1025213905 \\
\hline $\mathrm{N}$ & 0.3426083463 & 0.7728389589 & 0.0153445939 \\
\hline $\mathrm{N}$ & 0.6573916538 & 0.2271610262 & 0.0153445939 \\
\hline $\mathrm{N}$ & 0.1573916537 & 0.2728389589 & 0.9846554190 \\
\hline $\mathrm{N}$ & 0.8426083462 & 0.7271610411 & 0.9846554190 \\
\hline $\mathrm{N}$ & 0.4528753349 & 0.6919303230 & 0.8738972632 \\
\hline $\mathrm{N}$ & 0.5471246650 & 0.3080696769 & 0.8738972632 \\
\hline $\mathrm{N}$ & 0.0471246650 & 0.1919303230 & 0.1261027368 \\
\hline $\mathrm{N}$ & 0.9528753350 & 0.8080696770 & 0.1261027368 \\
\hline 0 & 0.5915956804 & 0.5422968248 & 0.3416211892 \\
\hline 0 & 0.4084042898 & 0.4577031752 & 0.3416211892 \\
\hline 0 & 0.9084043196 & 0.0422968248 & 0.6583788108 \\
\hline 0 & 0.0915957102 & 0.9577031752 & 0.6583788108 \\
\hline 0 & 0.8093461022 & 0.6866999135 & 0.3603730406 \\
\hline $\mathrm{O}$ & 0.1906539127 & 0.3133000865 & 0.3603730406 \\
\hline 0 & 0.6906538978 & 0.1866999135 & 0.6396269594 \\
\hline $\mathrm{O}$ & 0.3093461022 & 0.8133000865 & 0.6396269594 \\
\hline 0 & 0.6585462558 & 0.6229865129 & 0.4265215009 \\
\hline 0 & 0.3414537740 & 0.3770134871 & 0.4265215009 \\
\hline $\mathrm{O}$ & 0.8414537442 & 0.1229865129 & 0.5734785289 \\
\hline 0 & 0.1585462260 & 0.8770134871 & 0.5734785289 \\
\hline 0 & 0.4752630291 & 0.7088256788 & 0.1506745458 \\
\hline $\mathrm{O}$ & 0.5247369710 & 0.2911743510 & 0.1506745458 \\
\hline 0 & 0.0247369710 & 0.2088256490 & 0.8493254393 \\
\hline 0 & 0.9752630290 & 0.7911743212 & 0.8493254393 \\
\hline 0 & 0.4027454685 & 0.6292445319 & 0.0704971507 \\
\hline 0 & 0.5972545315 & 0.3707554681 & 0.0704971507 \\
\hline 0 & 0.0972545315 & 0.1292445319 & 0.9295028604 \\
\hline $\mathrm{O}$ & 0.9027454685 & 0.8707554681 & 0.9295028604 \\
\hline $\mathrm{H}$ & 0.7591035576 & 0.6629014508 & 0.3790869934 \\
\hline $\mathrm{H}$ & 0.2408964275 & 0.3370985491 & 0.3790869934 \\
\hline $\mathrm{H}$ & 0.7408964424 & 0.1629014509 & 0.6209130066 \\
\hline $\mathrm{H}$ & 0.2591035576 & 0.8370985492 & 0.6209130066 \\
\hline $\mathrm{H}$ & 0.7673861669 & 0.5370694305 & 0.2129298933 \\
\hline $\mathrm{H}$ & 0.2326138480 & 0.4629305695 & 0.2129298933 \\
\hline $\mathrm{H}$ & 0.7326138331 & 0.0370694305 & 0.7870701216 \\
\hline $\mathrm{H}$ & 0.2673861669 & 0.9629305695 & 0.7870701216 \\
\hline $\mathrm{H}$ & 0.8101899658 & 0.7013982723 & 0.5396102841 \\
\hline $\mathrm{H}$ & 0.1898100193 & 0.2986017277 & 0.5396102841 \\
\hline $\mathrm{H}$ & 0.6898100342 & 0.2013982723 & 0.4603897456 \\
\hline $\mathrm{H}$ & 0.3101899658 & 0.7986017277 & 0.4603897456 \\
\hline $\mathrm{H}$ & 0.7479764316 & 0.7442644956 & 0.4510936723 \\
\hline $\mathrm{H}$ & 0.2520235684 & 0.2557355043 & 0.4510936723 \\
\hline $\mathrm{H}$ & 0.7520235684 & 0.2442644956 & 0.5489062978 \\
\hline $\mathrm{H}$ & 0.2479764316 & 0.7557355043 & 0.5489062978 \\
\hline $\mathrm{H}$ & 0.8784528719 & 0.7398243173 & 0.4501503149 \\
\hline $\mathrm{H}$ & 0.1215471133 & 0.2601756529 & 0.4501503149 \\
\hline $\mathrm{H}$ & 0.6215471281 & 0.2398243471 & 0.5498496851 \\
\hline $\mathrm{H}$ & 0.3784528719 & 0.7601756827 & 0.5498496851 \\
\hline $\mathrm{H}$ & 0.9266018032 & 0.5419851563 & 0.1086989978 \\
\hline $\mathrm{H}$ & 0.0733981894 & 0.4580148437 & 0.1086989978 \\
\hline $\mathrm{H}$ & 0.5733981968 & 0.0419851563 & 0.8913010171 \\
\hline $\mathrm{H}$ & 0.4266018031 & 0.9580148437 & 0.8913010171 \\
\hline $\mathrm{H}$ & 0.0764303825 & 0.5640154065 & 0.2216456930 \\
\hline $\mathrm{H}$ & 0.9235695913 & 0.4359845936 & 0.2216456930 \\
\hline $\mathrm{H}$ & 0.4235696212 & 0.0640154064 & 0.7783543368 \\
\hline
\end{tabular}




\begin{tabular}{|c|c|c|c|}
\hline $\mathrm{H}$ & 0.5764304087 & 0.9359845936 & 0.7783543368 \\
\hline $\mathrm{H}$ & 0.0597602127 & 0.5794599751 & 0.4359635229 \\
\hline $\mathrm{H}$ & 0.9402398171 & 0.4205399951 & 0.4359635229 \\
\hline $\mathrm{H}$ & 0.4402397873 & 0.0794600050 & 0.5640364771 \\
\hline $\mathrm{H}$ & 0.5597601829 & 0.9205400248 & 0.5640364771 \\
\hline $\mathrm{H}$ & 0.9560615678 & 0.5732287030 & 0.6126127391 \\
\hline $\mathrm{H}$ & 0.0439384546 & 0.4267712970 & 0.6126127391 \\
\hline $\mathrm{H}$ & 0.5439384322 & 0.0732287030 & 0.3873872609 \\
\hline $\mathrm{H}$ & 0.4560615380 & 0.9267712970 & 0.3873872609 \\
\hline $\mathrm{H}$ & 0.8709270487 & 0.6159628393 & 0.5672784317 \\
\hline $\mathrm{H}$ & 0.1290729513 & 0.3840371309 & 0.5672784317 \\
\hline $\mathrm{H}$ & 0.6290729513 & 0.1159628691 & 0.4327215683 \\
\hline $\mathrm{H}$ & 0.3709270487 & 0.8840371607 & 0.4327215683 \\
\hline $\mathrm{H}$ & 0.7721033118 & 0.6132286055 & 0.7028192182 \\
\hline $\mathrm{H}$ & 0.2278966584 & 0.3867714244 & 0.7028192182 \\
\hline $\mathrm{H}$ & 0.7278966882 & 0.1132285756 & 0.2971807818 \\
\hline $\mathrm{H}$ & 0.2721033416 & 0.8867713946 & 0.2971807818 \\
\hline $\mathrm{H}$ & 0.8008232651 & 0.5646156387 & 0.7949924327 \\
\hline $\mathrm{H}$ & 0.1991767200 & 0.4353843910 & 0.7949924327 \\
\hline $\mathrm{H}$ & 0.6991767349 & 0.0646156090 & 0.2050075672 \\
\hline $\mathrm{H}$ & 0.3008232651 & 0.9353843612 & 0.2050075672 \\
\hline $\mathrm{H}$ & 0.6498912561 & 0.5678794875 & 0.9128057991 \\
\hline $\mathrm{H}$ & 0.3501087440 & 0.4321205125 & 0.9128057991 \\
\hline $\mathrm{H}$ & 0.8501087439 & 0.0678794875 & 0.0871942009 \\
\hline $\mathrm{H}$ & 0.1498912561 & 0.9321205125 & 0.0871942009 \\
\hline $\mathrm{H}$ & 0.4669045721 & 0.5627919658 & 0.9182653760 \\
\hline $\mathrm{H}$ & 0.5330954280 & 0.4372080342 & 0.9182653760 \\
\hline $\mathrm{H}$ & 0.0330954280 & 0.0627919658 & 0.0817346239 \\
\hline $\mathrm{H}$ & 0.9669045720 & 0.9372080342 & 0.0817346239 \\
\hline $\mathrm{H}$ & 0.3769179311 & 0.5544287034 & 0.7315141387 \\
\hline $\mathrm{H}$ & 0.6230820689 & 0.4455713264 & 0.7315141387 \\
\hline $\mathrm{H}$ & 0.1230820689 & 0.0544286736 & 0.2684858613 \\
\hline $\mathrm{H}$ & 0.8769179311 & 0.9455712966 & 0.2684858613 \\
\hline $\mathrm{H}$ & 0.4729153620 & 0.5502066567 & 0.5455474449 \\
\hline $\mathrm{H}$ & 0.5270846380 & 0.4497933433 & 0.5455474449 \\
\hline $\mathrm{H}$ & 0.0270846380 & 0.0502066567 & 0.4544525551 \\
\hline $\mathrm{H}$ & 0.9729153620 & 0.9497933433 & 0.4544525551 \\
\hline $\mathrm{H}$ & 0.9106751830 & 0.4959161161 & 0.6285147833 \\
\hline $\mathrm{H}$ & 0.0893248318 & 0.5040838541 & 0.6285147833 \\
\hline $\mathrm{H}$ & 0.5893248170 & -0.0040838541 & 0.3714852167 \\
\hline $\mathrm{H}$ & 0.4106751830 & 1.0040838839 & 0.3714852167 \\
\hline $\mathrm{H}$ & 0.8159974004 & 0.4932497361 & 0.7309133742 \\
\hline $\mathrm{H}$ & 0.1840026144 & 0.5067502341 & 0.7309133742 \\
\hline $\mathrm{H}$ & 0.6840025996 & -0.0067502341 & 0.2690866257 \\
\hline $\mathrm{H}$ & 0.3159974004 & 1.0067502639 & 0.2690866257 \\
\hline $\mathrm{H}$ & 0.8529744200 & 0.4045810345 & 0.6522520665 \\
\hline $\mathrm{H}$ & 0.1470255651 & 0.5954189654 & 0.6522520665 \\
\hline $\mathrm{H}$ & 0.6470255800 & 0.9045810345 & 0.3477479336 \\
\hline $\mathrm{H}$ & 0.3529744199 & 0.0954189654 & 0.3477479336 \\
\hline $\mathrm{H}$ & 0.7783801122 & 0.3441167890 & 0.5235740806 \\
\hline $\mathrm{H}$ & 0.2216198729 & 0.6558832110 & 0.5235740806 \\
\hline $\mathrm{H}$ & 0.7216198878 & 0.8441167890 & 0.4764259492 \\
\hline $\mathrm{H}$ & 0.2783801122 & 0.1558832110 & 0.4764259492 \\
\hline $\mathrm{H}$ & 0.6632313118 & 0.3711867764 & 0.3644845570 \\
\hline $\mathrm{H}$ & 0.3367686882 & 0.6288132235 & 0.3644845570 \\
\hline $\mathrm{H}$ & 0.8367686882 & 0.8711867764 & 0.6355154728 \\
\hline $\mathrm{H}$ & 0.1632313118 & 0.1288132235 & 0.6355154728 \\
\hline $\mathrm{H}$ & 0.6274347785 & 0.4581787766 & 0.3479317698 \\
\hline
\end{tabular}




\begin{tabular}{|c|c|c|c|}
\hline $\mathrm{H}$ & 0.3725652513 & 0.5418212234 & 0.3479317698 \\
\hline $\mathrm{H}$ & 0.8725652215 & 0.9581787766 & 0.6520682302 \\
\hline $\mathrm{H}$ & 0.1274347486 & 0.0418212234 & 0.6520682302 \\
\hline $\mathrm{H}$ & 0.5894757099 & 0.7037579829 & 0.3362154858 \\
\hline $\mathrm{H}$ & 0.4105242603 & 0.2962419873 & 0.3362154858 \\
\hline $\mathrm{H}$ & 0.9105242901 & 0.2037580127 & 0.6637844845 \\
\hline $\mathrm{H}$ & 0.0894757397 & 0.7962420171 & 0.6637844845 \\
\hline $\mathrm{H}$ & 0.4683950207 & 0.5478826883 & 0.1618856230 \\
\hline $\mathrm{H}$ & 0.5316049793 & 0.4521172819 & 0.1618856230 \\
\hline $\mathrm{H}$ & 0.0316049793 & 0.0478827181 & 0.8381143770 \\
\hline $\mathrm{H}$ & 0.9683950207 & 0.9521173117 & 0.8381143770 \\
\hline $\mathrm{H}$ & 0.2998839952 & 0.7173548080 & 0.2921222285 \\
\hline $\mathrm{H}$ & 0.7001160047 & 0.2826451622 & 0.2921222285 \\
\hline $\mathrm{H}$ & 0.2001160048 & 0.2173548378 & 0.7078777417 \\
\hline $\mathrm{H}$ & 0.7998839953 & 0.7826451920 & 0.7078777417 \\
\hline $\mathrm{H}$ & 0.1427016424 & 0.7092748693 & 0.4043405176 \\
\hline $\mathrm{H}$ & 0.8572983576 & 0.2907251009 & 0.4043405176 \\
\hline $\mathrm{H}$ & 0.3572983576 & 0.2092748992 & 0.5956595121 \\
\hline $\mathrm{H}$ & 0.6427016424 & 0.7907251306 & 0.5956595121 \\
\hline $\mathrm{H}$ & -0.0060259650 & 0.6807799054 & 0.2997784336 \\
\hline $\mathrm{H}$ & 1.0060259650 & 0.3192200946 & 0.2997784336 \\
\hline $\mathrm{H}$ & 0.5060259650 & 0.1807799054 & 0.7002215962 \\
\hline $\mathrm{H}$ & 0.4939740350 & 0.8192200946 & 0.7002215962 \\
\hline $\mathrm{H}$ & 0.0061718765 & 0.6638555339 & 0.0867360656 \\
\hline $\mathrm{H}$ & 0.9938281235 & 0.3361444661 & 0.0867360656 \\
\hline $\mathrm{H}$ & 0.4938281234 & 0.1638555339 & 0.9132639196 \\
\hline $\mathrm{H}$ & 0.5061718766 & 0.8361444661 & 0.9132639196 \\
\hline $\mathrm{H}$ & 0.1038215513 & 0.6737971909 & 0.9035714072 \\
\hline $\mathrm{H}$ & 0.8961784488 & 0.3262028091 & 0.9035714072 \\
\hline $\mathrm{H}$ & 0.3961784488 & 0.1737971909 & 0.0964285778 \\
\hline $\mathrm{H}$ & 0.6038215512 & 0.8262028091 & 0.0964285778 \\
\hline $\mathrm{H}$ & 0.1906858637 & 0.6311559187 & 0.9479619204 \\
\hline $\mathrm{H}$ & 0.8093141363 & 0.3688441111 & 0.9479619204 \\
\hline $\mathrm{H}$ & 0.3093141363 & 0.1311558889 & 0.0520380535 \\
\hline $\mathrm{H}$ & 0.6906858637 & 0.8688440813 & 0.0520380535 \\
\hline $\mathrm{H}$ & 0.2463130030 & 0.7528349694 & 0.7752060717 \\
\hline $\mathrm{H}$ & 0.7536869971 & 0.2471650306 & 0.7752060717 \\
\hline $\mathrm{H}$ & 0.2536869970 & 0.2528349694 & 0.2247939432 \\
\hline $\mathrm{H}$ & 0.7463130029 & 0.7471650306 & 0.2247939432 \\
\hline $\mathrm{H}$ & 0.1466483370 & 0.7502518212 & 0.8694974991 \\
\hline $\mathrm{H}$ & 0.8533516630 & 0.2497481490 & 0.8694974991 \\
\hline $\mathrm{H}$ & 0.3533516630 & 0.2502518510 & 0.1305025009 \\
\hline $\mathrm{H}$ & 0.6466483370 & 0.7497481788 & 0.1305025009 \\
\hline $\mathrm{H}$ & 0.1945608916 & 0.8413478782 & 0.8541066265 \\
\hline $\mathrm{H}$ & 0.8054391084 & 0.1586521516 & 0.8541066265 \\
\hline $\mathrm{H}$ & 0.3054391084 & 0.3413478484 & 0.1458934033 \\
\hline $\mathrm{H}$ & 0.6945608916 & 0.6586521218 & 0.1458934033 \\
\hline $\mathrm{H}$ & 0.2787574327 & 0.9051653437 & 0.9689288369 \\
\hline $\mathrm{H}$ & 0.7212425673 & 0.0948346711 & 0.9689288369 \\
\hline $\mathrm{H}$ & 0.2212425673 & 0.4051653437 & 0.0310711557 \\
\hline $\mathrm{H}$ & 0.7787574327 & 0.5948346562 & 0.0310711557 \\
\hline $\mathrm{H}$ & 0.4051356602 & 0.8808888321 & 0.1162479539 \\
\hline $\mathrm{H}$ & 0.5948643398 & 0.1191111679 & 0.1162479539 \\
\hline $\mathrm{H}$ & 0.0948643399 & 0.3808888321 & 0.8837520684 \\
\hline $\mathrm{H}$ & 0.9051356602 & 0.6191111679 & 0.8837520684 \\
\hline $\mathrm{H}$ & 0.4390490161 & 0.7943093649 & 0.1431466183 \\
\hline $\mathrm{H}$ & 0.5609509839 & 0.2056906500 & 0.1431466183 \\
\hline $\mathrm{H}$ & 0.0609509839 & 0.2943093649 & 0.8568534114 \\
\hline
\end{tabular}




\begin{tabular}{|c|c|c|c|}
\hline $\mathrm{H}$ & 0.9390490161 & 0.7056906351 & 0.8568534114 \\
\hline $\mathrm{H}$ & 0.2519853316 & 0.6775619125 & 0.7176362171 \\
\hline $\mathrm{H}$ & 0.7480146684 & 0.3224381173 & 0.7176362171 \\
\hline $\mathrm{H}$ & 0.2480146685 & 0.1775618828 & 0.2823638127 \\
\hline $\mathrm{H}$ & 0.7519853316 & 0.8224380874 & 0.2823638127 \\
\hline $\mathrm{H}$ & 0.2884543728 & 0.6320933664 & 0.8150110266 \\
\hline $\mathrm{H}$ & 0.7115456272 & 0.3679066038 & 0.8150110266 \\
\hline $\mathrm{H}$ & 0.2115456272 & 0.1320933961 & 0.1849890033 \\
\hline $\mathrm{H}$ & 0.7884543728 & 0.8679066336 & 0.1849890033 \\
\hline $\mathrm{H}$ & 0.4028479047 & 0.6673170995 & 0.5942057298 \\
\hline $\mathrm{H}$ & 0.5971520953 & 0.3326829005 & 0.5942057298 \\
\hline $\mathrm{H}$ & 0.0971520953 & 0.1673170995 & 0.4057942702 \\
\hline $\mathrm{H}$ & 0.9028479047 & 0.8326829005 & 0.4057942702 \\
\hline $\mathrm{H}$ & 0.5841531099 & 0.6743437987 & 0.5796908886 \\
\hline $\mathrm{H}$ & 0.4158468603 & 0.3256562013 & 0.5796908886 \\
\hline $\mathrm{H}$ & 0.9158468901 & 0.1743437987 & 0.4203091114 \\
\hline $\mathrm{H}$ & 0.0841531397 & 0.8256562013 & 0.4203091114 \\
\hline $\mathrm{H}$ & 0.6794689419 & 0.6925358428 & 0.7615771388 \\
\hline $\mathrm{H}$ & 0.3205310581 & 0.3074641274 & 0.7615771388 \\
\hline $\mathrm{H}$ & 0.8205310581 & 0.1925358726 & 0.2384228761 \\
\hline $\mathrm{H}$ & 0.1794689419 & 0.8074641572 & 0.2384228761 \\
\hline $\mathrm{H}$ & 0.5879950487 & 0.7044271098 & 0.9470371774 \\
\hline $\mathrm{H}$ & 0.4120049811 & 0.2955728902 & 0.9470371774 \\
\hline $\mathrm{H}$ & 0.9120049513 & 0.2044271098 & 0.0529628338 \\
\hline $\mathrm{H}$ & 0.0879950189 & 0.7955728903 & 0.0529628338 \\
\hline C & 0.8340657799 & 0.5471090419 & 0.2584969022 \\
\hline C & 0.1659342350 & 0.4528909283 & 0.2584969022 \\
\hline C & 0.6659342201 & 0.0471090717 & 0.7415030978 \\
\hline C & 0.3340657798 & 0.9528909581 & 0.7415030978 \\
\hline C & 0.8103609968 & 0.7195729244 & 0.4550857730 \\
\hline C & 0.1896389734 & 0.2804270756 & 0.4550857730 \\
\hline C & 0.6896390032 & 0.2195729244 & 0.5449141971 \\
\hline C & 0.3103610266 & 0.7804270756 & 0.5449141971 \\
\hline C & 0.9229205190 & 0.5499365981 & 0.2011125655 \\
\hline C & 0.0770795033 & 0.4500634317 & 0.2011125655 \\
\hline C & 0.5770794810 & 0.0499365683 & 0.7988874643 \\
\hline C & 0.4229204892 & 0.9500634019 & 0.7988874643 \\
\hline C & 1.0058755152 & 0.5617673768 & 0.2647646031 \\
\hline C & -0.0058754872 & 0.4382326232 & 0.2647646031 \\
\hline C & 0.4941244848 & 0.0617673768 & 0.7352353671 \\
\hline C & 0.5058754854 & 0.9382326232 & 0.7352353671 \\
\hline C & 0.9969662146 & 0.5697275023 & 0.3835826732 \\
\hline C & 0.0030337612 & 0.4302724977 & 0.3835826732 \\
\hline C & 0.5030337854 & 0.0697275023 & 0.6164173269 \\
\hline C & 0.4969662444 & 0.9302724977 & 0.6164173269 \\
\hline C & 0.9055018420 & 0.5664921773 & 0.4353898733 \\
\hline C & 0.0944981804 & 0.4335078227 & 0.4353898733 \\
\hline C & 0.5944981580 & 0.0664921773 & 0.5646101564 \\
\hline C & 0.4055018122 & 0.9335078228 & 0.5646101564 \\
\hline C & 0.8886437616 & 0.5779147883 & 0.5619291946 \\
\hline C & 0.1113562534 & 0.4220852117 & 0.5619291946 \\
\hline C & 0.6113562384 & 0.0779147884 & 0.4380708054 \\
\hline C & 0.3886437616 & 0.9220852117 & 0.4380708054 \\
\hline C & 0.7629709249 & 0.5746472938 & 0.7145680804 \\
\hline C & 0.2370290750 & 0.4253526764 & 0.7145680804 \\
\hline C & 0.7370290751 & 0.0746473236 & 0.2854319196 \\
\hline C & 0.2629709250 & 0.9253527062 & 0.2854319196 \\
\hline C & 0.6552869809 & 0.5648909612 & 0.7260858271 \\
\hline
\end{tabular}




\begin{tabular}{|c|c|c|c|}
\hline C & 0.3447130191 & 0.4351090388 & 0.7260858271 \\
\hline C & 0.8447130191 & 0.0648909612 & 0.2739141729 \\
\hline C & 0.1552869809 & 0.9351090387 & 0.2739141729 \\
\hline C & 0.6077362050 & 0.5654485780 & 0.8330536679 \\
\hline C & 0.3922637950 & 0.4345513922 & 0.8330536679 \\
\hline C & 0.8922637950 & 0.0654486078 & 0.1669463321 \\
\hline C & 0.1077362050 & 0.9345514220 & 0.1669463321 \\
\hline C & 0.5064540793 & 0.5620554077 & 0.8362097567 \\
\hline C & 0.4935459207 & 0.4379445923 & 0.8362097567 \\
\hline C & -0.0064540793 & 0.0620554077 & 0.1637902433 \\
\hline C & 1.0064540793 & 0.9379445923 & 0.1637902433 \\
\hline C & 0.4559301062 & 0.5574590782 & 0.7318750610 \\
\hline C & 0.5440698937 & 0.4425409516 & 0.7318750610 \\
\hline C & 0.0440698937 & 0.0574590484 & 0.2681249390 \\
\hline C & 0.9559301063 & 0.9425409218 & 0.2681249390 \\
\hline C & 0.5085558865 & 0.5552273343 & 0.6290884110 \\
\hline C & 0.4914441134 & 0.4447726359 & 0.6290884110 \\
\hline C & 0.9914441135 & 0.0552273641 & 0.3709115890 \\
\hline C & 0.0085558865 & 0.9447726657 & 0.3709115890 \\
\hline C & 0.8314204452 & 0.5007123705 & 0.6389381102 \\
\hline C & 0.1685795547 & 0.4992876593 & 0.6389381102 \\
\hline C & 0.6685795548 & 0.0007123407 & 0.3610618898 \\
\hline C & 0.3314204453 & 0.9992876295 & 0.3610618898 \\
\hline C & 0.7811216832 & 0.4632642834 & 0.5663502526 \\
\hline C & 0.2188782870 & 0.5367357166 & 0.5663502526 \\
\hline C & 0.7188783168 & 0.9632642834 & 0.4336497475 \\
\hline C & 0.2811217130 & 0.0367357166 & 0.4336497475 \\
\hline C & 0.8024074253 & 0.4153231707 & 0.5841399245 \\
\hline C & 0.1975925598 & 0.5846768293 & 0.5841399245 \\
\hline C & 0.6975925747 & 0.9153231707 & 0.4158600755 \\
\hline C & 0.3024074253 & 0.0846768293 & 0.4158600755 \\
\hline C & 0.7600490454 & 0.3814622222 & 0.5119556292 \\
\hline C & 0.2399509397 & 0.6185377778 & 0.5119556292 \\
\hline C & 0.7399509546 & 0.8814622222 & 0.4880443708 \\
\hline C & 0.2600490454 & 0.1185377778 & 0.4880443708 \\
\hline C & 0.6969024639 & 0.3962417522 & 0.4242490725 \\
\hline C & 0.3030975361 & 0.6037582478 & 0.4242490725 \\
\hline C & 0.8030975361 & 0.8962417521 & 0.5757509275 \\
\hline C & 0.1969024640 & 0.1037582478 & 0.5757509275 \\
\hline C & 0.6769429878 & 0.4443652364 & 0.4136924262 \\
\hline C & 0.3230569824 & 0.5556347637 & 0.4136924262 \\
\hline C & 0.8230570122 & 0.9443652363 & 0.5863075439 \\
\hline C & 0.1769430175 & 0.0556347636 & 0.5863075439 \\
\hline C & 0.5955902299 & 0.6266390774 & 0.3437341235 \\
\hline C & 0.4044097701 & 0.3733609226 & 0.3437341235 \\
\hline C & 0.9044097701 & 0.1266390774 & 0.6562658467 \\
\hline C & 0.0955902298 & 0.8733609226 & 0.6562658467 \\
\hline C & 0.5634322281 & 0.6703142395 & 0.2999785048 \\
\hline C & 0.4365678017 & 0.3296857605 & 0.2999785048 \\
\hline C & 0.9365677719 & 0.1703142395 & 0.7000214952 \\
\hline C & 0.0634321983 & 0.8296857605 & 0.7000214952 \\
\hline C & 0.5018027406 & 0.6705496833 & 0.2040353229 \\
\hline C & 0.4981972594 & 0.3294502869 & 0.2040353229 \\
\hline C & -0.0018027406 & 0.1705497132 & 0.7959647069 \\
\hline C & 1.0018027406 & 0.8294503166 & 0.7959647069 \\
\hline C & 0.4637184717 & 0.6247401887 & 0.1539545865 \\
\hline C & 0.5362815283 & 0.3752598113 & 0.1539545865 \\
\hline C & 0.0362815283 & 0.1247401887 & 0.8460454135 \\
\hline
\end{tabular}




\begin{tabular}{|c|c|c|c|}
\hline C & 0.9637184717 & 0.8752598113 & 0.8460454135 \\
\hline C & 0.4957765679 & 0.5810000277 & 0.1986132777 \\
\hline C & 0.5042234321 & 0.4189999723 & 0.1986132777 \\
\hline C & 0.0042234322 & 0.0810000277 & 0.8013867075 \\
\hline C & 0.9957765679 & 0.9189999723 & 0.8013867075 \\
\hline C & 0.5597610939 & 0.5806416790 & 0.2924360900 \\
\hline C & 0.4402388762 & 0.4193582912 & 0.2924360900 \\
\hline C & 0.9402389061 & 0.0806417088 & 0.7075639099 \\
\hline C & 0.0597611238 & 0.9193583210 & 0.7075639099 \\
\hline C & 0.2326944064 & 0.7050722706 & 0.2509510594 \\
\hline C & 0.7673055936 & 0.2949277294 & 0.2509510594 \\
\hline C & 0.2673055935 & 0.2050722706 & 0.7490489704 \\
\hline C & 0.7326944064 & 0.7949277294 & 0.7490489704 \\
\hline C & 0.1460176323 & 0.7006527402 & 0.3123592576 \\
\hline C & 0.8539823677 & 0.2993472598 & 0.3123592576 \\
\hline C & 0.3539823677 & 0.2006527402 & 0.6876407424 \\
\hline C & 0.6460176323 & 0.7993472598 & 0.6876407424 \\
\hline C & 0.0629199600 & 0.6851351155 & 0.2537767809 \\
\hline C & 0.9370800400 & 0.3148648845 & 0.2537767809 \\
\hline C & 0.4370800401 & 0.1851351155 & 0.7462231894 \\
\hline C & 0.5629199600 & 0.8148648845 & 0.7462231894 \\
\hline C & 0.0695193870 & 0.6758038246 & 0.1354019846 \\
\hline C & 0.9304806131 & 0.3241961456 & 0.1354019846 \\
\hline C & 0.4304806131 & 0.1758038544 & 0.8645979856 \\
\hline C & 0.5695193869 & 0.8241961754 & 0.8645979856 \\
\hline C & 0.1587226163 & 0.6813142843 & 0.0787893089 \\
\hline C & 0.8412773837 & 0.3186857156 & 0.0787893089 \\
\hline C & 0.3412773837 & 0.1813142843 & 0.9212106985 \\
\hline C & 0.6587226163 & 0.8186857156 & 0.9212106985 \\
\hline C & 0.1721248488 & 0.6692136293 & 0.9522137433 \\
\hline C & 0.8278751513 & 0.3307863707 & 0.9522137433 \\
\hline C & 0.3278751512 & 0.1692136293 & 0.0477862381 \\
\hline C & 0.6721248487 & 0.8307863707 & 0.0477862381 \\
\hline C & 0.2264267262 & 0.7460374292 & 0.8662093202 \\
\hline C & 0.7735732738 & 0.2539625708 & 0.8662093202 \\
\hline C & 0.2735732738 & 0.2460374292 & 0.1337906798 \\
\hline C & 0.7264267262 & 0.7539625708 & 0.1337906798 \\
\hline C & 0.2741008392 & 0.7848908037 & 0.9371994972 \\
\hline C & 0.7258991608 & 0.2151092112 & 0.9371994972 \\
\hline C & 0.2258991608 & 0.2848908037 & 0.0628005325 \\
\hline C & 0.7741008392 & 0.7151091963 & 0.0628005325 \\
\hline C & 0.2492188069 & 0.8324924395 & 0.9190745216 \\
\hline C & 0.7507811930 & 0.1675075307 & 0.9190745216 \\
\hline C & 0.2507811931 & 0.3324924693 & 0.0809254709 \\
\hline C & 0.7492188070 & 0.6675075605 & 0.0809254709 \\
\hline C & 0.2959237093 & 0.8677744327 & 0.9828240772 \\
\hline C & 0.7040762906 & 0.1322255822 & 0.9828240772 \\
\hline C & 0.2040762907 & 0.3677744327 & 0.0171759414 \\
\hline C & 0.7959237094 & 0.6322255673 & 0.0171759414 \\
\hline C & 0.3658489609 & 0.8547154146 & 0.0648280005 \\
\hline C & 0.6341510391 & 0.1452845705 & 0.0648280005 \\
\hline C & 0.1341510392 & 0.3547154146 & 0.9351720256 \\
\hline C & 0.8658489609 & 0.6452845854 & 0.9351720256 \\
\hline C & 0.3866964186 & 0.8068286303 & 0.0791021023 \\
\hline C & 0.6133035815 & 0.1931713697 & 0.0791021023 \\
\hline C & 0.1133035815 & 0.3068286303 & 0.9208978828 \\
\hline C & 0.8866964185 & 0.6931713697 & 0.9208978828 \\
\hline C & 0.2944420701 & 0.6704071150 & 0.7965149152 \\
\hline
\end{tabular}




\begin{tabular}{|c|c|c|c|}
\hline C & 0.7055579299 & 0.3295928850 & 0.7965149152 \\
\hline C & 0.2055579299 & 0.1704071150 & 0.2034850848 \\
\hline C & 0.7944420701 & 0.8295928851 & 0.2034850848 \\
\hline C & 0.4011216085 & 0.6810325644 & 0.7781147566 \\
\hline C & 0.5988783915 & 0.3189674058 & 0.7781147566 \\
\hline C & 0.0988783915 & 0.1810325942 & 0.2218852286 \\
\hline C & 0.9011216085 & 0.8189674356 & 0.2218852286 \\
\hline C & 0.4464135464 & 0.6756489984 & 0.6704924454 \\
\hline C & 0.5535864536 & 0.3243510016 & 0.6704924454 \\
\hline C & 0.0535864536 & 0.1756489984 & 0.3295075545 \\
\hline C & 0.9464135464 & 0.8243510016 & 0.3295075545 \\
\hline C & 0.5473399058 & 0.6795909831 & 0.6626037544 \\
\hline C & 0.4526600644 & 0.3204089871 & 0.6626037544 \\
\hline C & 0.9526600942 & 0.1795910130 & 0.3373962158 \\
\hline C & 0.0473399356 & 0.8204090168 & 0.3373962158 \\
\hline C & 0.6004099511 & 0.6894218661 & 0.7630177682 \\
\hline C & 0.3995900191 & 0.3105781637 & 0.7630177682 \\
\hline C & 0.8995900489 & 0.1894218363 & 0.2369822467 \\
\hline C & 0.1004099809 & 0.8105781339 & 0.2369822467 \\
\hline C & 0.5502988142 & 0.6960199065 & 0.8663902198 \\
\hline C & 0.4497012156 & 0.3039800935 & 0.8663902198 \\
\hline C & 0.9497011859 & 0.1960199065 & 0.1336097951 \\
\hline C & 0.0502987844 & 0.8039800935 & 0.1336097951 \\
\hline B & 0.0299236653 & 0.6862409371 & 0.6345323597 \\
\hline B & 0.9700763608 & 0.3137590629 & 0.6345323597 \\
\hline B & 0.4700763310 & 0.1862409371 & 0.3654676701 \\
\hline B & 0.5299236392 & 0.8137590629 & 0.3654676701 \\
\hline B & 0.2250242246 & 0.5253578971 & -0.0198372619 \\
\hline B & 0.7749757456 & 0.4746421029 & -0.0198372619 \\
\hline B & 0.2749757754 & 0.0253578971 & 1.0198372641 \\
\hline B & 0.7250242544 & 0.9746421030 & 1.0198372641 \\
\hline $\mathrm{F}$ & -0.0480821031 & 0.7167861294 & 0.6621952047 \\
\hline $\mathrm{F}$ & 1.0480820807 & 0.2832139004 & 0.6621952047 \\
\hline $\mathrm{F}$ & 0.5480821105 & 0.2167860996 & 0.3378047952 \\
\hline $\mathrm{F}$ & 0.4519179193 & 0.7832138706 & 0.3378047952 \\
\hline $\mathrm{F}$ & 0.1191610268 & 0.7125099464 & 0.6312614789 \\
\hline $\mathrm{F}$ & 0.8808389732 & 0.2874900834 & 0.6312614789 \\
\hline $\mathrm{F}$ & 0.3808389733 & 0.2125099166 & 0.3687385211 \\
\hline $\mathrm{F}$ & 0.6191610268 & 0.7874900536 & 0.3687385211 \\
\hline $\mathrm{F}$ & 0.0150928609 & 0.6647196551 & 0.5249844105 \\
\hline $\mathrm{F}$ & 0.9849071540 & 0.3352803449 & 0.5249844105 \\
\hline $\mathrm{F}$ & 0.4849071540 & 0.1647196551 & 0.4750155896 \\
\hline $\mathrm{F}$ & 0.5150928460 & 0.8352803449 & 0.4750155896 \\
\hline $\mathrm{F}$ & 0.0377050374 & 0.6500964894 & 0.7215534794 \\
\hline $\mathrm{F}$ & 0.9622949849 & 0.3499035404 & 0.7215534794 \\
\hline $\mathrm{F}$ & 0.4622949551 & 0.1500964595 & 0.2784465206 \\
\hline $\mathrm{F}$ & 0.5377050151 & 0.8499035107 & 0.2784465206 \\
\hline $\mathrm{F}$ & 0.1688040643 & 0.5554137339 & 0.0523107209 \\
\hline $\mathrm{F}$ & 0.8311959357 & 0.4445862661 & 0.0523107209 \\
\hline $\mathrm{F}$ & 0.3311959357 & 0.0554137339 & 0.9476892642 \\
\hline $\mathrm{F}$ & 0.6688040643 & 0.9445862661 & 0.9476892642 \\
\hline $\mathrm{F}$ & 0.2542197844 & 0.5511671320 & 0.8786021522 \\
\hline $\mathrm{F}$ & 0.7457801858 & 0.4488328978 & 0.8786021522 \\
\hline $\mathrm{F}$ & 0.2457802156 & 0.0511671022 & 0.1213978552 \\
\hline $\mathrm{F}$ & 0.7542198142 & 0.9488328680 & 0.1213978552 \\
\hline $\mathrm{F}$ & 0.3078871683 & 0.5081952433 & 0.0399719965 \\
\hline $\mathrm{F}$ & 0.6921128317 & 0.4918047866 & 0.0399719965 \\
\hline $\mathrm{F}$ & 0.1921128318 & 0.0081952135 & 0.9600280184 \\
\hline
\end{tabular}




$\begin{array}{llll}\text { F } & 0.8078871683 & 0.9918047567 & 0.9600280184 \\ \text { F } & 0.1671721933 & 0.4855095609 & 0.9438728387 \\ \text { F } & 0.8328278067 & 0.5144904391 & 0.9438728387 \\ \text { F } & 0.3328278067 & 0.9855095608 & 0.0561271370 \\ \text { F } & 0.6671721933 & 0.0144904391 & 0.0561271370\end{array}$

$1 \cdot \mathrm{CH}_{3} \mathrm{OH}-\mathrm{iS}$

CELL_PARAMETERS (angstrom)
$13.271541742 \quad 0.000000000$
0.000000000
$0.000000000 \quad 29.071636335$
0.000000000
0.000000000
0.000000000
11.566522199

ATOMIC_POSITIONS (crystal)

$\begin{array}{llll}\text { Fe1 } & 0.6890169755 & 0.5522968116 & 0.4765728158 \\ \text { Fe1 } & 0.3109830245 & 0.4477032184 & 0.4765728158 \\ \text { Fe1 } & 0.8109830245 & 0.0522967816 & 0.5234271842 \\ \text { Fe1 } & 0.1890169755 & 0.9477031884 & 0.5234271842 \\ \text { Fe2 } & 0.3869720401 & 0.6960021929 & 0.0250361477 \\ \text { Fe2 } & 0.6130279599 & 0.3039978071 & 0.0250361477 \\ \text { Fe2 } & 0.1130279599 & 0.1960021929 & 0.9749638633 \\ \text { Fe2 } & 0.8869720401 & 0.8039978071 & 0.9749638633 \\ \text { N } & 0.8264973986 & 0.5565338876 & 0.3770765868 \\ \mathrm{~N} & 0.1735026164 & 0.4434661124 & 0.3770765868 \\ \mathrm{~N} & 0.6735026014 & 0.0565338876 & 0.6229234132 \\ \mathrm{~N} & 0.3264973986 & 0.9434661124 & 0.6229234132 \\ \mathrm{~N} & 0.8106504639 & 0.5512102498 & 0.6170045019 \\ \mathrm{~N} & 0.1893495511 & 0.4487897212 & 0.6170045019 \\ \mathrm{~N} & 0.6893495361 & 0.0512102788 & 0.3829954981 \\ \mathrm{~N} & 0.3106504639 & 0.9487897502 & 0.3829954981 \\ \mathrm{~N} & 0.6065880463 & 0.5594856897 & 0.6374130308 \\ \mathrm{~N} & 0.3934119237 & 0.4405142803 & 0.6374130308 \\ \mathrm{~N} & 0.8934119537 & 0.0594857197 & 0.3625869692 \\ \mathrm{~N} & 0.1065880763 & 0.9405143103 & 0.3625869692 \\ \mathrm{~N} & 0.7159459115 & 0.4803607198 & 0.4888726544 \\ \mathrm{~N} & 0.2840540885 & 0.5196392802 & 0.4888726544 \\ \mathrm{~N} & 0.7840540884 & 0.9803607198 & 0.5111273156 \\ \mathrm{~N} & 0.2159459116 & 0.0196392802 & 0.5111273156 \\ \mathrm{~N} & 0.2769349268 & 0.6936817409 & 0.1377188667 \\ \mathrm{~N} & 0.7230650732 & 0.3063182291 & 0.1377188667 \\ \mathrm{~N} & 0.2230650732 & 0.1936817709 & 0.8622811633 \\ \mathrm{~N} & 0.7769349268 & 0.8063182591 & 0.8622811633 \\ \mathrm{~N} & 0.2765555610 & 0.6872776632 & 0.9098274921 \\ \mathrm{~N} & 0.7234444390 & 0.3127223368 & 0.9098274921 \\ \mathrm{~N} & 0.2234444390 & 0.1872776632 & 0.0901725009 \\ \mathrm{~N} & 0.7765555610 & 0.8127223368 & 0.0901725009 \\ \mathrm{~N} & 0.3656221416 & 0.7608267705 & 0.0041082400 \\ \mathrm{~N} & 0.6343778584 & 0.2391732145 & 0.0041082400 \\ \mathrm{~N} & 0.1343778584 & 0.2608267705 & -0.0041082400 \\ \mathrm{~N} & 0.8656221416 & 0.7391732295 & -0.0041082400 \\ \mathrm{~N} & 0.4754077497 & 0.6916308071 & 0.8895802841 \\ \mathrm{~N} & 0.5245922503 & 0.3083692219 & 0.8895802841 \\ \mathrm{~N} & 0.0245922503 & 0.1916307781 & 0.1104197309 \\ \mathrm{~N} & 0.9754077497 & 0.8083691929 & 0.1104197309 \\ \mathrm{O} & 0.5883902893 & 0.5432719564 & 0.3436827681 \\ \mathrm{O} & 0.4116096817 & 0.4567280436 & 0.3436827681 \\ \mathrm{O} & 0.9116097107 & 0.0432719564 & 0.6563172319 \\ \mathrm{O} & 0.0883903183 & 0.9567280436 & 0.6563172319 \\ \mathrm{O} & 0.2459501520 & 0.7682145029 & 0.4083326103 \\ \mathrm{O} & & 0.2317854971 & 0.4083326103 \\ & & & \\ & & & \end{array}$




\begin{tabular}{|c|c|c|c|}
\hline 0 & 0.7449498480 & 0.2682145029 & 0.5916673897 \\
\hline $\mathrm{O}$ & 0.2550501520 & 0.7317854970 & 0.5916673897 \\
\hline 0 & 0.6542748216 & 0.6202796277 & 0.4357286365 \\
\hline 0 & 0.3457251784 & 0.3797203723 & 0.4357286365 \\
\hline $\mathrm{O}$ & 0.8457251784 & 0.1202796277 & 0.5642713635 \\
\hline 0 & 0.1542748216 & 0.8797203723 & 0.5642713635 \\
\hline 0 & 0.4901015894 & 0.7049339527 & 0.1408463356 \\
\hline 0 & 0.5098984106 & 0.2950660773 & 0.1408463356 \\
\hline 0 & 0.0098984106 & 0.2049339227 & 0.8591536494 \\
\hline 0 & 0.9901015894 & 0.7950660473 & 0.8591536494 \\
\hline 0 & 0.4137651597 & 0.6301287745 & 0.0578320725 \\
\hline 0 & 0.5862348403 & 0.3698712255 & 0.0578320725 \\
\hline 0 & 0.0862348403 & 0.1301287745 & 0.9421679385 \\
\hline 0 & 0.9137651597 & 0.8698712255 & 0.9421679385 \\
\hline $\mathrm{H}$ & 0.6816974355 & 0.7629952266 & 0.4058462477 \\
\hline $\mathrm{H}$ & 0.3183025795 & 0.2370047584 & 0.4058462477 \\
\hline $\mathrm{H}$ & 0.8183025645 & 0.2629952266 & 0.5941537523 \\
\hline $\mathrm{H}$ & 0.1816974355 & 0.7370047734 & 0.5941537523 \\
\hline $\mathrm{H}$ & 0.7622162710 & 0.5412194168 & 0.2177462979 \\
\hline $\mathrm{H}$ & 0.2377837440 & 0.4587806122 & 0.2177462979 \\
\hline $\mathrm{H}$ & 0.7377837290 & 0.0412193878 & 0.7822537321 \\
\hline $\mathrm{H}$ & 0.2622162710 & 0.9587805832 & 0.7822537321 \\
\hline $\mathrm{H}$ & 0.7921379471 & 0.6983283308 & 0.3801638187 \\
\hline $\mathrm{H}$ & 0.2078620829 & 0.3016716692 & 0.3801638187 \\
\hline $\mathrm{H}$ & 0.7078620529 & 0.1983283308 & 0.6198362113 \\
\hline $\mathrm{H}$ & 0.2921379171 & 0.8016716692 & 0.6198362113 \\
\hline $\mathrm{H}$ & 0.7840135599 & 0.7150426885 & 0.5285863120 \\
\hline $\mathrm{H}$ & 0.2159864101 & 0.2849572815 & 0.5285863120 \\
\hline $\mathrm{H}$ & 0.7159864401 & 0.2150427185 & 0.4714137170 \\
\hline $\mathrm{H}$ & 0.2840135899 & 0.7849573115 & 0.4714137170 \\
\hline $\mathrm{H}$ & 0.8870333481 & 0.7344044593 & 0.4431981956 \\
\hline $\mathrm{H}$ & 0.1129666519 & 0.2655955407 & 0.4431981956 \\
\hline $\mathrm{H}$ & 0.6129666519 & 0.2344044593 & 0.5568018044 \\
\hline $\mathrm{H}$ & 0.3870333481 & 0.7655955407 & 0.5568018044 \\
\hline $\mathrm{H}$ & 0.9256405406 & 0.5433251126 & 0.1103888567 \\
\hline $\mathrm{H}$ & 0.0743594445 & 0.4566748874 & 0.1103888567 \\
\hline $\mathrm{H}$ & 0.5743594595 & 0.0433251126 & 0.8896111583 \\
\hline $\mathrm{H}$ & 0.4256405405 & 0.9566748874 & 0.8896111583 \\
\hline $\mathrm{H}$ & 0.0845361769 & 0.5606184321 & 0.2205690124 \\
\hline $\mathrm{H}$ & 0.9154637931 & 0.4393815679 & 0.2205690124 \\
\hline $\mathrm{H}$ & 0.4154638231 & 0.0606184321 & 0.7794310026 \\
\hline $\mathrm{H}$ & 0.5845362069 & 0.9393815679 & 0.7794310026 \\
\hline $\mathrm{H}$ & 0.0723797245 & 0.5738463038 & 0.4353209546 \\
\hline $\mathrm{H}$ & 0.9276202735 & 0.4261537253 & 0.4353209546 \\
\hline $\mathrm{H}$ & 0.4276202735 & 0.0738462747 & 0.5646790164 \\
\hline $\mathrm{H}$ & 0.5723797265 & 0.9261536963 & 0.5646790164 \\
\hline $\mathrm{H}$ & 0.9686539477 & 0.5674382309 & 0.6125052085 \\
\hline $\mathrm{H}$ & 0.0313460603 & 0.4325617691 & 0.6125052085 \\
\hline $\mathrm{H}$ & 0.5313460523 & 0.0674382309 & 0.3874947915 \\
\hline $\mathrm{H}$ & 0.4686539477 & 0.9325617691 & 0.3874947915 \\
\hline $\mathrm{H}$ & 0.8857937205 & 0.6120345472 & 0.5732573295 \\
\hline $\mathrm{H}$ & 0.1142062645 & 0.3879654528 & 0.5732573295 \\
\hline $\mathrm{H}$ & 0.6142062795 & 0.1120345472 & 0.4267426705 \\
\hline $\mathrm{H}$ & 0.3857937205 & 0.8879654528 & 0.4267426705 \\
\hline $\mathrm{H}$ & 0.7794948238 & 0.6137554834 & 0.7005533138 \\
\hline $\mathrm{H}$ & 0.2205051462 & 0.3862445466 & 0.7005533138 \\
\hline $\mathrm{H}$ & 0.7205051762 & 0.1137554534 & 0.2994466862 \\
\hline $\mathrm{H}$ & 0.2794948538 & 0.8862445166 & 0.2994466862 \\
\hline
\end{tabular}




\begin{tabular}{|c|c|c|c|}
\hline $\mathrm{H}$ & 0.8127272542 & 0.5688085649 & 0.7978309990 \\
\hline $\mathrm{H}$ & 0.1872727458 & 0.4311914351 & 0.7978309990 \\
\hline $\mathrm{H}$ & 0.6872727458 & 0.0688085649 & 0.2021690010 \\
\hline $\mathrm{H}$ & 0.3127272542 & 0.9311914351 & 0.2021690010 \\
\hline $\mathrm{H}$ & 0.6605701952 & 0.5711577574 & 0.9212198520 \\
\hline $\mathrm{H}$ & 0.3394298048 & 0.4288422126 & 0.9212198520 \\
\hline $\mathrm{H}$ & 0.8394298048 & 0.0711577874 & 0.0787801480 \\
\hline $\mathrm{H}$ & 0.1605701952 & 0.9288422426 & 0.0787801480 \\
\hline $\mathrm{H}$ & 0.4722833938 & 0.5609405441 & 0.9352960316 \\
\hline $\mathrm{H}$ & 0.5277166062 & 0.4390594559 & 0.9352960316 \\
\hline $\mathrm{H}$ & 0.0277166062 & 0.0609405441 & 0.0647039684 \\
\hline $\mathrm{H}$ & 0.9722833938 & 0.9390594559 & 0.0647039684 \\
\hline $\mathrm{H}$ & 0.3741274331 & 0.5494992509 & 0.7539576625 \\
\hline $\mathrm{H}$ & 0.6258725669 & 0.4505007491 & 0.7539576625 \\
\hline $\mathrm{H}$ & 0.1258725669 & 0.0494992509 & 0.2460423375 \\
\hline $\mathrm{H}$ & 0.8741274331 & 0.9505007491 & 0.2460423375 \\
\hline $\mathrm{H}$ & 0.4669014824 & 0.5482019744 & 0.5639202794 \\
\hline $\mathrm{H}$ & 0.5330985176 & 0.4517980256 & 0.5639202794 \\
\hline $\mathrm{H}$ & 0.0330985176 & 0.0482019744 & 0.4360797206 \\
\hline $\mathrm{H}$ & 0.9669014824 & 0.9517980256 & 0.4360797206 \\
\hline $\mathrm{H}$ & 0.9122180080 & 0.4957863173 & 0.6431376892 \\
\hline $\mathrm{H}$ & 0.0877820070 & 0.5042136827 & 0.6431376892 \\
\hline $\mathrm{H}$ & 0.5877819920 & -0.0042136827 & 0.3568623108 \\
\hline $\mathrm{H}$ & 0.4122180080 & 1.0042136827 & 0.3568623108 \\
\hline $\mathrm{H}$ & 0.8092968871 & 0.4963102834 & 0.7385034838 \\
\hline $\mathrm{H}$ & 0.1907031279 & 0.5036896876 & 0.7385034838 \\
\hline $\mathrm{H}$ & 0.6907031129 & -0.0036896876 & 0.2614965162 \\
\hline $\mathrm{H}$ & 0.3092968871 & 1.0036897166 & 0.2614965162 \\
\hline $\mathrm{H}$ & 0.8429313342 & 0.4088337858 & 0.6668535984 \\
\hline $\mathrm{H}$ & 0.1570686658 & 0.5911662142 & 0.6668535984 \\
\hline $\mathrm{H}$ & 0.6570686658 & 0.9088337858 & 0.3331464016 \\
\hline $\mathrm{H}$ & 0.3429313342 & 0.0911662142 & 0.3331464016 \\
\hline $\mathrm{H}$ & 0.7624080008 & 0.3504909750 & 0.5386951395 \\
\hline $\mathrm{H}$ & 0.2375919992 & 0.6495090250 & 0.5386951395 \\
\hline $\mathrm{H}$ & 0.7375919992 & 0.8504909750 & 0.4613048605 \\
\hline $\mathrm{H}$ & 0.2624080008 & 0.1495090250 & 0.4613048605 \\
\hline $\mathrm{H}$ & 0.6541697387 & 0.3777013036 & 0.3726146145 \\
\hline $\mathrm{H}$ & 0.3458302613 & 0.6222986964 & 0.3726146145 \\
\hline $\mathrm{H}$ & 0.8458302613 & 0.8777013036 & 0.6273853855 \\
\hline $\mathrm{H}$ & 0.1541697387 & 0.1222986964 & 0.6273853855 \\
\hline $\mathrm{H}$ & 0.6256524639 & 0.4626688168 & 0.3497724727 \\
\hline $\mathrm{H}$ & 0.3743475361 & 0.5373311832 & 0.3497724727 \\
\hline $\mathrm{H}$ & 0.8743475361 & 0.9626688168 & 0.6502275273 \\
\hline $\mathrm{H}$ & 0.1256524639 & 0.0373311832 & 0.6502275273 \\
\hline $\mathrm{H}$ & 0.5979058955 & 0.6991834026 & 0.3344802362 \\
\hline $\mathrm{H}$ & 0.4020941045 & 0.3008165684 & 0.3344802362 \\
\hline $\mathrm{H}$ & 0.9020941045 & 0.1991834316 & 0.6655197638 \\
\hline $\mathrm{H}$ & 0.0979058955 & 0.8008165974 & 0.6655197638 \\
\hline $\mathrm{H}$ & 0.4688553379 & 0.5498526111 & 0.1568088875 \\
\hline $\mathrm{H}$ & 0.5311446621 & 0.4501473889 & 0.1568088875 \\
\hline $\mathrm{H}$ & 0.0311446621 & 0.0498526111 & 0.8431910975 \\
\hline $\mathrm{H}$ & 0.9688553379 & 0.9501473889 & 0.8431910975 \\
\hline $\mathrm{H}$ & 0.3500755875 & 0.7217828723 & 0.2797490728 \\
\hline $\mathrm{H}$ & 0.6499244125 & 0.2782171277 & 0.2797490728 \\
\hline $\mathrm{H}$ & 0.1499244125 & 0.2217828723 & 0.7202509272 \\
\hline $\mathrm{H}$ & 0.8500755875 & 0.7782171277 & 0.7202509272 \\
\hline $\mathrm{H}$ & 0.2029177232 & 0.7116334545 & 0.4127100559 \\
\hline $\mathrm{H}$ & 0.7970822768 & 0.2883665745 & 0.4127100559 \\
\hline
\end{tabular}




\begin{tabular}{|c|c|c|c|}
\hline $\mathrm{H}$ & 0.2970822768 & 0.2116334255 & 0.5872899741 \\
\hline $\mathrm{H}$ & 0.7029177232 & 0.7883665455 & 0.5872899741 \\
\hline $\mathrm{H}$ & 0.0431911970 & 0.6774657097 & 0.3350605916 \\
\hline $\mathrm{H}$ & 0.9568088030 & 0.3225342903 & 0.3350605916 \\
\hline $\mathrm{H}$ & 0.4568088030 & 0.1774657097 & 0.6649394084 \\
\hline $\mathrm{H}$ & 0.5431911970 & 0.8225342903 & 0.6649394084 \\
\hline $\mathrm{H}$ & 0.0368484189 & 0.6552929442 & 0.1240707084 \\
\hline $\mathrm{H}$ & 0.9631515811 & 0.3447070558 & 0.1240707084 \\
\hline $\mathrm{H}$ & 0.4631515811 & 0.1552929442 & 0.8759292986 \\
\hline $\mathrm{H}$ & 0.5368484189 & 0.8447070558 & 0.8759292986 \\
\hline $\mathrm{H}$ & 0.1242951647 & 0.6623732692 & 0.9255159376 \\
\hline $\mathrm{H}$ & 0.8757048353 & 0.3376267008 & 0.9255159376 \\
\hline $\mathrm{H}$ & 0.3757048353 & 0.1623732992 & 0.0744840324 \\
\hline $\mathrm{H}$ & 0.6242951647 & 0.8376267308 & 0.0744840324 \\
\hline $\mathrm{H}$ & 0.2202547648 & 0.6238363907 & 0.9704723801 \\
\hline $\mathrm{H}$ & 0.7797452352 & 0.3761636093 & 0.9704723801 \\
\hline $\mathrm{H}$ & 0.2797452352 & 0.1238363907 & 0.0295276009 \\
\hline $\mathrm{H}$ & 0.7202547648 & 0.8761636093 & 0.0295276009 \\
\hline $\mathrm{H}$ & 0.2481512148 & 0.7356112959 & 0.7751176054 \\
\hline $\mathrm{H}$ & 0.7518487852 & 0.2643887041 & 0.7751176054 \\
\hline $\mathrm{H}$ & 0.2518487852 & 0.2356112959 & 0.2248824096 \\
\hline $\mathrm{H}$ & 0.7481512148 & 0.7643887041 & 0.2248824096 \\
\hline $\mathrm{H}$ & 0.1573635984 & 0.7362109264 & 0.8853363055 \\
\hline $\mathrm{H}$ & 0.8426364016 & 0.2637890736 & 0.8853363055 \\
\hline $\mathrm{H}$ & 0.3426364016 & 0.2362109264 & 0.1146636945 \\
\hline $\mathrm{H}$ & 0.6573635984 & 0.7637890736 & 0.1146636945 \\
\hline $\mathrm{H}$ & 0.2122337621 & 0.8272095762 & 0.8413683974 \\
\hline $\mathrm{H}$ & 0.7877662379 & 0.1727904238 & 0.8413683974 \\
\hline $\mathrm{H}$ & 0.2877662379 & 0.3272095762 & 0.1586316176 \\
\hline $\mathrm{H}$ & 0.7122337621 & 0.6727904238 & 0.1586316176 \\
\hline $\mathrm{H}$ & 0.3019995529 & 0.8894191006 & 0.9513821755 \\
\hline $\mathrm{H}$ & 0.6980004471 & 0.1105808844 & 0.9513821755 \\
\hline $\mathrm{H}$ & 0.1980004471 & 0.3894191006 & 0.0486178095 \\
\hline $\mathrm{H}$ & 0.8019995529 & 0.6105808994 & 0.0486178095 \\
\hline $\mathrm{H}$ & 0.4327410970 & 0.8661176042 & 0.0993036258 \\
\hline $\mathrm{H}$ & 0.5672589030 & 0.1338824258 & 0.0993036258 \\
\hline $\mathrm{H}$ & 0.0672589030 & 0.3661175742 & 0.9006963452 \\
\hline $\mathrm{H}$ & 0.9327410970 & 0.6338823958 & 0.9006963452 \\
\hline $\mathrm{H}$ & 0.4695561424 & 0.7825323751 & 0.1261236629 \\
\hline $\mathrm{H}$ & 0.5304438576 & 0.2174676549 & 0.1261236629 \\
\hline $\mathrm{H}$ & 0.0304438576 & 0.2825323451 & 0.8738763371 \\
\hline $\mathrm{H}$ & 0.9695561424 & 0.7174676249 & 0.8738763371 \\
\hline $\mathrm{H}$ & 0.2755773294 & 0.6645240822 & 0.7330154086 \\
\hline $\mathrm{H}$ & 0.7244226706 & 0.3354759478 & 0.7330154086 \\
\hline $\mathrm{H}$ & 0.2244226706 & 0.1645240522 & 0.2669845914 \\
\hline $\mathrm{H}$ & 0.7755773294 & 0.8354759178 & 0.2669845914 \\
\hline $\mathrm{H}$ & 0.3219605400 & 0.6244831456 & 0.8368953300 \\
\hline $\mathrm{H}$ & 0.6780394600 & 0.3755168544 & 0.8368953300 \\
\hline $\mathrm{H}$ & 0.1780394600 & 0.1244831456 & 0.1631046550 \\
\hline $\mathrm{H}$ & 0.8219605400 & 0.8755168544 & 0.1631046550 \\
\hline $\mathrm{H}$ & 0.4372581251 & 0.6598024298 & 0.6119788086 \\
\hline $\mathrm{H}$ & 0.5627418749 & 0.3401976002 & 0.6119788086 \\
\hline $\mathrm{H}$ & 0.0627418749 & 0.1598023998 & 0.3880211914 \\
\hline $\mathrm{H}$ & 0.9372581251 & 0.8401975702 & 0.3880211914 \\
\hline $\mathrm{H}$ & 0.6227183949 & 0.6748908208 & 0.6022165107 \\
\hline $\mathrm{H}$ & 0.3772816051 & 0.3251092092 & 0.6022165107 \\
\hline $\mathrm{H}$ & 0.8772816051 & 0.1748907908 & 0.3977835193 \\
\hline $\mathrm{H}$ & 0.1227183949 & 0.8251091792 & 0.3977835193 \\
\hline
\end{tabular}




\begin{tabular}{|c|c|c|c|}
\hline $\mathrm{H}$ & 0.7117426247 & 0.7011610203 & 0.7843859459 \\
\hline $\mathrm{H}$ & 0.2882573753 & 0.2988390087 & 0.7843859459 \\
\hline $\mathrm{H}$ & 0.7882573753 & 0.2011609913 & 0.2156140841 \\
\hline $\mathrm{H}$ & 0.2117426247 & 0.7988389797 & 0.2156140841 \\
\hline $\mathrm{H}$ & 0.6089683077 & 0.7127177257 & 0.9642122423 \\
\hline $\mathrm{H}$ & 0.3910316923 & 0.2872822743 & 0.9642122423 \\
\hline $\mathrm{H}$ & 0.8910316923 & 0.2127177257 & 0.0357877277 \\
\hline $\mathrm{H}$ & 0.1089683077 & 0.7872822743 & 0.0357877277 \\
\hline C & 0.8328911800 & 0.5490882060 & 0.2620929256 \\
\hline C & 0.1671088350 & 0.4509117940 & 0.2620929256 \\
\hline C & 0.6671088200 & 0.0490882060 & 0.7379070744 \\
\hline C & 0.3328911800 & 0.9509117940 & 0.7379070744 \\
\hline C & 0.8061110669 & 0.7266588204 & 0.4415845813 \\
\hline C & 0.1938889621 & 0.2733411796 & 0.4415845813 \\
\hline C & 0.6938889331 & 0.2266588204 & 0.5584154187 \\
\hline C & 0.3061110379 & 0.7733411796 & 0.5584154187 \\
\hline C & 0.9241168823 & 0.5504662307 & 0.2029072724 \\
\hline C & 0.0758831327 & 0.4495337393 & 0.2029072724 \\
\hline C & 0.5758831177 & 0.0504662607 & 0.7970927126 \\
\hline C & 0.4241168823 & 0.9495337693 & 0.7970927126 \\
\hline C & 1.0120772743 & 0.5596681362 & 0.2649841028 \\
\hline C & -0.0120772553 & 0.4403318938 & 0.2649841028 \\
\hline C & 0.4879227257 & 0.0596681062 & 0.7350158972 \\
\hline C & 0.5120772443 & 0.9403318638 & 0.7350158972 \\
\hline C & 1.0055697859 & 0.5664095091 & 0.3840121237 \\
\hline C & -0.0055697889 & 0.4335904909 & 0.3840121237 \\
\hline C & 0.4944302141 & 0.0664095091 & 0.6159878763 \\
\hline C & 0.5055697859 & 0.9335904909 & 0.6159878763 \\
\hline C & 0.9115608388 & 0.5645409652 & 0.4377541685 \\
\hline C & 0.0884391692 & 0.4354590048 & 0.4377541685 \\
\hline C & 0.5884391612 & 0.0645409952 & 0.5622458015 \\
\hline C & 0.4115608388 & 0.9354590348 & 0.5622458015 \\
\hline C & 0.8983198478 & 0.5747957182 & 0.5648802771 \\
\hline C & 0.1016801372 & 0.4252042818 & 0.5648802771 \\
\hline C & 0.6016801522 & 0.0747957182 & 0.4351197229 \\
\hline C & 0.3983198478 & 0.9252042818 & 0.4351197229 \\
\hline C & 0.7705456422 & 0.5767243389 & 0.7181603201 \\
\hline C & 0.2294543578 & 0.4232756311 & 0.7181603201 \\
\hline C & 0.7294543578 & 0.0767243689 & 0.2818396799 \\
\hline C & 0.2705456422 & 0.9232756611 & 0.2818396799 \\
\hline C & 0.6600004743 & 0.5667994348 & 0.7352223816 \\
\hline C & 0.3399995257 & 0.4332005652 & 0.7352223816 \\
\hline C & 0.8399995257 & 0.0667994348 & 0.2647776184 \\
\hline C & 0.1600004743 & 0.9332005652 & 0.2647776184 \\
\hline C & 0.6144183381 & 0.5671920904 & 0.8439383325 \\
\hline C & 0.3855816919 & 0.4328079386 & 0.8439383325 \\
\hline C & 0.8855816619 & 0.0671920614 & 0.1560616675 \\
\hline C & 0.1144183081 & 0.9328079096 & 0.1560616675 \\
\hline C & 0.5104216716 & 0.5610783562 & 0.8518037175 \\
\hline C & 0.4895783284 & 0.4389216438 & 0.8518037175 \\
\hline C & 0.9895783284 & 0.0610783562 & 0.1481962825 \\
\hline C & 0.0104216716 & 0.9389216438 & 0.1481962825 \\
\hline C & 0.4552430572 & 0.5547721567 & 0.7503551501 \\
\hline C & 0.5447569428 & 0.4452278433 & 0.7503551501 \\
\hline C & 0.0447569428 & 0.0547721567 & 0.2496448499 \\
\hline C & 0.9552430572 & 0.9452278433 & 0.2496448499 \\
\hline C & 0.5061190105 & 0.5539004861 & 0.6454460076 \\
\hline C & 0.4938809895 & 0.4460995439 & 0.6454460076 \\
\hline
\end{tabular}




\begin{tabular}{|c|c|c|c|}
\hline C & -0.0061190105 & 0.0539004561 & 0.3545539924 \\
\hline C & 1.0061190105 & 0.9460995139 & 0.3545539924 \\
\hline C & 0.8303445936 & 0.5023525199 & 0.6476079321 \\
\hline C & 0.1696553764 & 0.4976474801 & 0.6476079321 \\
\hline C & 0.6696554064 & 0.0023525199 & 0.3523920679 \\
\hline C & 0.3303446236 & 0.9976474801 & 0.3523920679 \\
\hline C & 0.7778944367 & 0.4664019894 & 0.5743212758 \\
\hline C & 0.2221055783 & 0.5335980106 & 0.5743212758 \\
\hline C & 0.7221055633 & 0.9664019894 & 0.4256787242 \\
\hline C & 0.2778944367 & 0.0335980106 & 0.4256787242 \\
\hline C & 0.7945392355 & 0.4196839404 & 0.5956099278 \\
\hline C & 0.2054607645 & 0.5803160596 & 0.5956099278 \\
\hline C & 0.7054607645 & 0.9196839404 & 0.4043900722 \\
\hline C & 0.2945392355 & 0.0803160596 & 0.4043900722 \\
\hline C & 0.7495129563 & 0.3870685693 & 0.5239172464 \\
\hline C & 0.2504870137 & 0.6129314307 & 0.5239172464 \\
\hline C & 0.7504870437 & 0.8870685693 & 0.4760827536 \\
\hline C & 0.2495129863 & 0.1129314307 & 0.4760827536 \\
\hline C & 0.6893045850 & 0.4019212688 & 0.4321872529 \\
\hline C & 0.3106954150 & 0.5980787312 & 0.4321872529 \\
\hline C & 0.8106954150 & 0.9019212688 & 0.5678127471 \\
\hline C & 0.1893045850 & 0.0980787312 & 0.5678127471 \\
\hline C & 0.6737105429 & 0.4488321864 & 0.4179368265 \\
\hline C & 0.3262894571 & 0.5511678136 & 0.4179368265 \\
\hline C & 0.8262894572 & 0.9488321864 & 0.5820631735 \\
\hline C & 0.1737105428 & 0.0511678136 & 0.5820631735 \\
\hline C & 0.5986253377 & 0.6246531633 & 0.3453550334 \\
\hline C & 0.4013746623 & 0.3753468067 & 0.3453550334 \\
\hline C & 0.9013746623 & 0.1246531933 & 0.6546449666 \\
\hline C & 0.0986253377 & 0.8753468367 & 0.6546449666 \\
\hline C & 0.5726837088 & 0.6670370316 & 0.2961556282 \\
\hline C & 0.4273162912 & 0.3329629384 & 0.2961556282 \\
\hline C & 0.9273162912 & 0.1670370616 & 0.7038443718 \\
\hline C & 0.0726837088 & 0.8329629684 & 0.7038443718 \\
\hline C & 0.5129468783 & 0.6677645222 & 0.1970256619 \\
\hline C & 0.4870531217 & 0.3322354778 & 0.1970256619 \\
\hline C & -0.0129468783 & 0.1677645222 & 0.8029743231 \\
\hline C & 1.0129468783 & 0.8322354778 & 0.8029743231 \\
\hline C & 0.4717448906 & 0.6244456094 & 0.1463517575 \\
\hline C & 0.5282551094 & 0.3755544206 & 0.1463517575 \\
\hline C & 0.0282551094 & 0.1244455794 & 0.8536482425 \\
\hline C & 0.9717448906 & 0.8755543906 & 0.8536482425 \\
\hline C & 0.4983910859 & 0.5816310329 & 0.1939416464 \\
\hline C & 0.5016089141 & 0.4183689671 & 0.1939416464 \\
\hline C & 0.0016089141 & 0.0816310329 & 0.8060583686 \\
\hline C & 0.9983910859 & 0.9183689671 & 0.8060583686 \\
\hline C & 0.5607342098 & 0.5806003120 & 0.2915447890 \\
\hline C & 0.4392657902 & 0.4193996880 & 0.2915447890 \\
\hline C & 0.9392657902 & 0.0806003120 & 0.7084552110 \\
\hline C & 0.0607342098 & 0.9193996880 & 0.7084552110 \\
\hline C & 0.2794416173 & 0.7066870787 & 0.2498052024 \\
\hline C & 0.7205583827 & 0.2933129213 & 0.2498052024 \\
\hline C & 0.2205583827 & 0.2066870787 & 0.7501947976 \\
\hline C & 0.7794416173 & 0.7933129213 & 0.7501947976 \\
\hline C & 0.1967580347 & 0.7013059999 & 0.3224669879 \\
\hline C & 0.8032419653 & 0.2986940001 & 0.3224669879 \\
\hline C & 0.3032419653 & 0.2013059999 & 0.6775330421 \\
\hline C & 0.6967580347 & 0.7986940001 & 0.6775330421 \\
\hline
\end{tabular}




\begin{tabular}{|c|c|c|c|}
\hline C & 0.1080375660 & 0.6824460792 & 0.2785597128 \\
\hline C & 0.8919624340 & 0.3175539208 & 0.2785597128 \\
\hline C & 0.3919624340 & 0.1824460792 & 0.7214402872 \\
\hline C & 0.6080375660 & 0.8175539208 & 0.7214402872 \\
\hline C & 0.1047049802 & 0.6699675894 & 0.1622627149 \\
\hline C & 0.8952950198 & 0.3300323806 & 0.1622627149 \\
\hline C & 0.3952950198 & 0.1699676194 & 0.8377373151 \\
\hline C & 0.6047049802 & 0.8300324106 & 0.8377373151 \\
\hline C & 0.1903169954 & 0.6757033040 & 0.0941159321 \\
\hline C & 0.8096830046 & 0.3242966960 & 0.0941159321 \\
\hline C & 0.3096830046 & 0.1757033040 & 0.9058840529 \\
\hline C & 0.6903169954 & 0.8242966960 & 0.9058840529 \\
\hline C & 0.1971124965 & 0.6602159496 & 0.9706876339 \\
\hline C & 0.8028875035 & 0.3397840504 & 0.9706876339 \\
\hline C & 0.3028875035 & 0.1602159496 & 0.0293123811 \\
\hline C & 0.6971124965 & 0.8397840504 & 0.0293123811 \\
\hline C & 0.2390204567 & 0.7334750626 & 0.8696751672 \\
\hline C & 0.7609795433 & 0.2665249374 & 0.8696751672 \\
\hline C & 0.2609795433 & 0.2334750626 & 0.1303248478 \\
\hline C & 0.7390204567 & 0.7665249374 & 0.1303248478 \\
\hline C & 0.2931965326 & 0.7727268282 & 0.9270205883 \\
\hline C & 0.7068034674 & 0.2272731568 & 0.9270205883 \\
\hline C & 0.2068034674 & 0.2727268282 & 0.0729793967 \\
\hline C & 0.7931965326 & 0.7272731718 & 0.0729793967 \\
\hline C & 0.2700583821 & 0.8187554602 & 0.9050375208 \\
\hline C & 0.7299416179 & 0.1812445248 & 0.9050375208 \\
\hline C & 0.2299416179 & 0.3187554602 & 0.0949624642 \\
\hline C & 0.7700583821 & 0.6812445398 & 0.0949624642 \\
\hline C & 0.3198703959 & 0.8532726228 & 0.9662928608 \\
\hline C & 0.6801296041 & 0.1467273772 & 0.9662928608 \\
\hline C & 0.1801296041 & 0.3532726228 & 0.0337071502 \\
\hline C & 0.8198703959 & 0.6467273772 & 0.0337071502 \\
\hline C & 0.3925506280 & 0.8406657392 & 0.0476919883 \\
\hline C & 0.6074493720 & 0.1593342458 & 0.0476919883 \\
\hline C & 0.1074493720 & 0.3406657392 & 0.9523080337 \\
\hline C & 0.8925506280 & 0.6593342608 & 0.9523080337 \\
\hline C & 0.4140494118 & 0.7943239532 & 0.0635454488 \\
\hline C & 0.5859505882 & 0.2056760618 & 0.0635454488 \\
\hline C & 0.0859505882 & 0.2943239532 & 0.9364545582 \\
\hline C & 0.9140494118 & 0.7056760468 & 0.9364545582 \\
\hline C & 0.3211618596 & 0.6610072129 & 0.8117352605 \\
\hline C & 0.6788381404 & 0.3389928171 & 0.8117352605 \\
\hline C & 0.1788381404 & 0.1610071829 & 0.1882647245 \\
\hline C & 0.8211618596 & 0.8389927871 & 0.1882647245 \\
\hline C & 0.4279842420 & 0.6760757167 & 0.7929956205 \\
\hline C & 0.5720157580 & 0.3239242533 & 0.7929956205 \\
\hline C & 0.0720157580 & 0.1760757467 & 0.2070043945 \\
\hline C & 0.9279842420 & 0.8239242833 & 0.2070043945 \\
\hline C & 0.4785482640 & 0.6713053226 & 0.6881416999 \\
\hline C & 0.5214517360 & 0.3286946774 & 0.6881416999 \\
\hline C & 0.0214517360 & 0.1713053226 & 0.3118583001 \\
\hline C & 0.9785482640 & 0.8286946774 & 0.3118583001 \\
\hline C & 0.5818089889 & 0.6800891261 & 0.6831177898 \\
\hline C & 0.4181910411 & 0.3199108449 & 0.6831177898 \\
\hline C & 0.9181910111 & 0.1800891551 & 0.3168821802 \\
\hline C & 0.0818089589 & 0.8199108739 & 0.3168821802 \\
\hline C & 0.6309197238 & 0.6944891807 & 0.7835607615 \\
\hline C & 0.3690802762 & 0.3055108193 & 0.7835607615 \\
\hline
\end{tabular}




\begin{tabular}{|c|c|c|c|}
\hline C & 0.8690802762 & 0.1944891807 & 0.2164392385 \\
\hline C & 0.1309197238 & 0.8055108193 & 0.2164392385 \\
\hline C & 0.5749429269 & 0.7007769626 & 0.8840419147 \\
\hline C & 0.4250571031 & 0.2992230374 & 0.8840419147 \\
\hline C & 0.9250570731 & 0.2007769626 & 0.1159580703 \\
\hline C & 0.0749428969 & 0.7992230374 & 0.1159580703 \\
\hline B & 0.0153433192 & 0.6845982268 & 0.6344545856 \\
\hline B & 0.9846566508 & 0.3154017442 & 0.6344545856 \\
\hline B & 0.4846566808 & 0.1845982558 & 0.3655454144 \\
\hline B & 0.5153433492 & 0.8154017732 & 0.3655454144 \\
\hline B & 0.2294789349 & 0.5225770203 & 0.9871060538 \\
\hline B & 0.7705210501 & 0.4774229797 & 0.9871060538 \\
\hline B & 0.2705210501 & 0.0225770203 & 0.0128939252 \\
\hline B & 0.7294789499 & 0.9774229797 & 0.0128939252 \\
\hline $\mathrm{F}$ & -0.0871783012 & 0.6842300912 & 0.6688385992 \\
\hline $\mathrm{F}$ & 1.0871783292 & 0.3157699088 & 0.6688385992 \\
\hline $\mathrm{F}$ & 0.5871782992 & 0.1842300912 & 0.3311613708 \\
\hline $\mathrm{F}$ & 0.4128216708 & 0.8157699088 & 0.3311613708 \\
\hline $\mathrm{F}$ & 0.0466696112 & 0.7309362275 & 0.6107800340 \\
\hline $\mathrm{F}$ & 0.9533303738 & 0.2690637425 & 0.6107800340 \\
\hline $\mathrm{F}$ & 0.4533303738 & 0.2309362575 & 0.3892199660 \\
\hline $\mathrm{F}$ & 0.5466696262 & 0.7690637725 & 0.3892199660 \\
\hline $\mathrm{F}$ & 0.0285068225 & 0.6582258059 & 0.5326090004 \\
\hline $\mathrm{F}$ & 0.9714931495 & 0.3417742231 & 0.5326090004 \\
\hline $\mathrm{F}$ & 0.4714931795 & 0.1582257769 & 0.4673909996 \\
\hline $\mathrm{F}$ & 0.5285068505 & 0.8417741941 & 0.4673909996 \\
\hline $\mathrm{F}$ & 0.0763546352 & 0.6663404711 & 0.7249459355 \\
\hline $\mathrm{F}$ & 0.9236453748 & 0.3336595289 & 0.7249459355 \\
\hline $\mathrm{F}$ & 0.4236453748 & 0.1663404711 & 0.2750540345 \\
\hline $\mathrm{F}$ & 0.5763546252 & 0.8336595289 & 0.2750540345 \\
\hline $\mathrm{F}$ & 0.1690275817 & 0.5499908701 & 0.0610583792 \\
\hline $\mathrm{F}$ & 0.8309724183 & 0.4500091299 & 0.0610583792 \\
\hline $\mathrm{F}$ & 0.3309724183 & 0.0499908701 & 0.9389416208 \\
\hline $\mathrm{F}$ & 0.6690275817 & 0.9500091299 & 0.9389416208 \\
\hline $\mathrm{F}$ & 0.2484245332 & 0.5479797008 & 0.8827205127 \\
\hline $\mathrm{F}$ & 0.7515754668 & 0.4520203292 & 0.8827205127 \\
\hline $\mathrm{F}$ & 0.2515754668 & 0.0479796708 & 0.1172795023 \\
\hline $\mathrm{F}$ & 0.7484245332 & 0.9520202992 & 0.1172795023 \\
\hline $\mathrm{F}$ & 0.3214626074 & 0.5110636065 & 0.0414762722 \\
\hline $\mathrm{F}$ & 0.6785373926 & 0.4889364225 & 0.0414762722 \\
\hline $\mathrm{F}$ & 0.1785373926 & 0.0110635775 & 0.9585237428 \\
\hline $\mathrm{F}$ & 0.8214626074 & 0.9889363935 & 0.9585237428 \\
\hline $\mathrm{F}$ & 0.1767006032 & 0.4812037261 & 0.9572341342 \\
\hline $\mathrm{F}$ & 0.8232993818 & 0.5187962739 & 0.9572341342 \\
\hline $\mathrm{F}$ & 0.3232993818 & 0.9812037261 & 0.0427658688 \\
\hline $\mathrm{F}$ & 0.6767006182 & 0.0187962739 & 0.0427658688 \\
\hline
\end{tabular}

\section{$1 \cdot \mathrm{CH}_{3} \mathrm{OH}$-LS}

CELL_PARAMETERS (angstrom)
13.669179776
0.000000000
0.000000000
$0.000000000 \quad 29.171931184$
0.000000000
0.000000000
0.000000000
11.022639120

ATOMIC_POSITIONS (angstrom)

$\begin{array}{ll}\mathrm{Fe} 2 & 2.8292810910 \\ \mathrm{Fe} 2 & 4.0053087964 \\ \mathrm{Fe} 2 & 5.3179122007 \\ \mathrm{Fe} 2 & 8.3512675761 \\ \mathrm{Fe} 2 & 9.6638713867\end{array}$

$\begin{array}{rr}27.5936712051 & 5.9824970699 \\ 13.0077056131 & 5.0401423782 \\ 20.3400989804 & 0.2296099049 \\ 8.8318322026 & 0.2296099048 \\ 16.1642255699 & 5.0401423782\end{array}$




\begin{tabular}{|c|c|c|c|}
\hline $\mathrm{Fe} 2$ & 10.8398982775 & 1.5782599789 & 5.9824970699 \\
\hline $\mathrm{Fe} 2$ & 1.5166776877 & 5.7541333894 & 10.7930291951 \\
\hline Fe2 & 12.1525020882 & 23.4177977946 & 10.7930291951 \\
\hline $\mathrm{N}$ & 11.2234530698 & 16.2239716027 & 3.8484346083 \\
\hline $\mathrm{N}$ & 2.4457267070 & 12.9479595803 & 3.8484346083 \\
\hline $\mathrm{N}$ & 9.2803165944 & 1.6380060117 & 7.1742045117 \\
\hline $\mathrm{N}$ & 4.3888631814 & 27.5339251723 & 7.1742045117 \\
\hline $\mathrm{N}$ & 11.0503017965 & 16.3198489532 & 6.4969357906 \\
\hline $\mathrm{N}$ & 2.6188781830 & 12.8520830995 & 6.4969357906 \\
\hline $\mathrm{N}$ & 9.4534678677 & 1.7338824924 & 4.5257033294 \\
\hline $\mathrm{N}$ & 4.2157119091 & 27.4380478218 & 4.5257033294 \\
\hline $\mathrm{N}$ & 8.4020094136 & 16.2989138989 & 6.5536702905 \\
\hline $\mathrm{N}$ & 5.2671703632 & 12.8730181539 & 6.5536702905 \\
\hline $\mathrm{N}$ & 12.1017602506 & 1.7129474381 & 4.4689688286 \\
\hline $\mathrm{N}$ & 1.5674195252 & 27.4589828761 & 4.4689688285 \\
\hline $\mathrm{N}$ & 9.9259503919 & 14.2661092482 & 5.2136622814 \\
\hline $\mathrm{N}$ & 3.7432293839 & 14.9058219357 & 5.2136622814 \\
\hline $\mathrm{N}$ & 10.5778192723 & 28.8520748393 & 5.8089768386 \\
\hline $\mathrm{N}$ & 3.0913605045 & 0.3198563437 & 5.8089768386 \\
\hline $\mathrm{N}$ & 3.7956446330 & 20.2255884885 & 1.4642943188 \\
\hline $\mathrm{N}$ & 9.8735351429 & 8.9463435653 & 1.4642943188 \\
\hline $\mathrm{N}$ & 3.0389452554 & 5.6396220267 & 9.5583448012 \\
\hline $\mathrm{N}$ & 10.6302345213 & 23.5323082875 & 9.5583448012 \\
\hline $\mathrm{N}$ & 3.9114274564 & 20.1407729198 & 9.8381548275 \\
\hline $\mathrm{N}$ & 9.7577523194 & 9.0311582642 & 9.8381548275 \\
\hline $\mathrm{N}$ & 2.9231624310 & 5.5548073278 & 1.1844839634 \\
\hline $\mathrm{N}$ & 10.7460173448 & 23.6171238562 & 1.1844839635 \\
\hline $\mathrm{N}$ & 5.0186253427 & 22.2343285181 & 0.0699819148 \\
\hline $\mathrm{N}$ & 8.6505544341 & 6.9376022310 & 0.0699819148 \\
\hline $\mathrm{N}$ & 1.8159645457 & 7.6483629261 & 10.9526573473 \\
\hline $\mathrm{N}$ & 11.8532152301 & 21.5235682569 & 10.9526573473 \\
\hline $\mathrm{N}$ & 6.5475628797 & 20.2555714450 & 9.7264604250 \\
\hline $\mathrm{N}$ & 7.1216168961 & 8.9163597380 & 9.7264604250 \\
\hline $\mathrm{N}$ & 0.2870270077 & 5.6696058530 & 1.2961786950 \\
\hline $\mathrm{N}$ & 13.3821527681 & 23.5023253300 & 1.2961786950 \\
\hline 0 & 8.3425820509 & 15.9201748516 & 3.6534781653 \\
\hline 0 & 5.3265973177 & 13.2517563314 & 3.6534781654 \\
\hline 0 & 12.1611876133 & 1.3342092596 & 7.3691609546 \\
\hline 0 & 1.5079925698 & 27.8377219233 & 7.3691609546 \\
\hline 0 & 11.3049307896 & 19.9640998946 & 4.0688190340 \\
\hline 0 & 2.3642491899 & 9.2078312884 & 4.0688190340 \\
\hline 0 & 9.1988388737 & 5.3781343036 & 6.9538200860 \\
\hline 0 & 4.4703409022 & 23.7937968804 & 6.9538200860 \\
\hline 0 & 9.2842363834 & 18.1024095539 & 4.6562852755 \\
\hline 0 & 4.3849437997 & 11.0695216291 & 4.6562852755 \\
\hline 0 & 11.2195332808 & 3.5164439619 & 6.3663541726 \\
\hline 0 & 2.4496460877 & 25.6554872210 & 6.3663541726 \\
\hline 0 & 6.6419666328 & 20.5809052724 & 1.5962934254 \\
\hline 0 & 7.0272131440 & 8.5910267804 & 1.5962934254 \\
\hline 0 & 0.1926232556 & 5.9949388106 & 9.4263455305 \\
\hline 0 & 13.4765565202 & 23.1769915035 & 9.4263455305 \\
\hline 0 & 5.6980271725 & 18.4170247035 & 0.5746810878 \\
\hline 0 & 7.9711526034 & 10.7549064805 & 0.5746810878 \\
\hline 0 & 1.1365627150 & 3.8310591115 & 10.4479581553 \\
\hline 0 & 12.5326170609 & 25.3408720724 & 10.4479581553 \\
\hline $\mathrm{H}$ & 10.6034874931 & 19.2903326940 & 4.2455632699 \\
\hline $\mathrm{H}$ & 3.0656920791 & 9.8815984890 & 4.2455632699 \\
\hline $\mathrm{H}$ & 9.9002821712 & 4.7043671030 & 6.7770758501 \\
\hline
\end{tabular}




\begin{tabular}{|c|c|c|c|}
\hline $\mathrm{H}$ & 3.7688976056 & 24.4675640809 & 6.7770758501 \\
\hline $\mathrm{H}$ & 10.3507765782 & 15.4980957660 & 2.1066796960 \\
\hline $\mathrm{H}$ & 3.3184034022 & 13.6738354170 & 2.1066796961 \\
\hline $\mathrm{H}$ & 10.1529930860 & 0.9121301750 & 8.9159595880 \\
\hline $\mathrm{H}$ & 3.5161866899 & 28.2598010089 & 8.9159595880 \\
\hline $\mathrm{H}$ & 11.3478656548 & 20.4647306276 & 6.1216400017 \\
\hline $\mathrm{H}$ & 2.3213139184 & 8.7072005564 & 6.1216400017 \\
\hline $\mathrm{H}$ & 9.1559040095 & 5.8787650356 & 4.9009994463 \\
\hline $\mathrm{H}$ & 4.5132757664 & 23.2931661474 & 4.9009994464 \\
\hline $\mathrm{H}$ & 10.2900019043 & 21.4966394845 & 5.0922502690 \\
\hline $\mathrm{H}$ & 3.3791778716 & 7.6752916985 & 5.0922502690 \\
\hline $\mathrm{H}$ & 10.2137677600 & 6.9106738925 & 5.9303885219 \\
\hline $\mathrm{H}$ & 3.4554120159 & 22.2612572905 & 5.9303885219 \\
\hline $\mathrm{H}$ & 12.0765202924 & 21.6179752544 & 5.0003227024 \\
\hline $\mathrm{H}$ & 1.5926592808 & 7.5539550597 & 5.0003227024 \\
\hline $\mathrm{H}$ & 8.4272493718 & 7.0320105313 & 6.0223164176 \\
\hline $\mathrm{H}$ & 5.2419304040 & 22.1399215215 & 6.0223164176 \\
\hline $\mathrm{H}$ & 12.4515161826 & 15.6106063415 & 0.7744233632 \\
\hline $\mathrm{H}$ & 1.2176634914 & 13.5613248425 & 0.7744233632 \\
\hline $\mathrm{H}$ & 8.0522534816 & 1.0246407495 & 10.2482159208 \\
\hline $\mathrm{H}$ & 5.6169262942 & 28.1472904335 & 10.2482159208 \\
\hline $\mathrm{H}$ & 0.8996653280 & 16.4195470267 & 1.8856321184 \\
\hline $\mathrm{H}$ & 12.7695140910 & 12.7523841573 & 1.8856321184 \\
\hline $\mathrm{H}$ & 5.9349246108 & 1.8335814347 & 9.1370073297 \\
\hline $\mathrm{H}$ & 7.7342555733 & 27.3383497493 & 9.1370073297 \\
\hline $\mathrm{H}$ & 0.7861698755 & 17.0911533025 & 4.3159875179 \\
\hline $\mathrm{H}$ & 12.8830103076 & 12.0807770117 & 4.3159875179 \\
\hline $\mathrm{H}$ & 6.0484200119 & 2.5051885803 & 6.7066516021 \\
\hline $\mathrm{H}$ & 7.6207593566 & 26.6667434725 & 6.7066516021 \\
\hline $\mathrm{H}$ & 13.1061866548 & 16.9907691397 & 6.4739990441 \\
\hline $\mathrm{H}$ & 0.5629934274 & 12.1811620442 & 6.4739990441 \\
\hline $\mathrm{H}$ & 7.3975830094 & 2.4048035477 & 4.5486400759 \\
\hline $\mathrm{H}$ & 6.2715963591 & 26.7671276362 & 4.5486400759 \\
\hline $\mathrm{H}$ & 11.9125848282 & 18.1469720031 & 5.8885188113 \\
\hline $\mathrm{H}$ & 1.7565949476 & 11.0249583111 & 5.8885188112 \\
\hline $\mathrm{H}$ & 8.5911848360 & 3.5610072799 & 5.1341203087 \\
\hline $\mathrm{H}$ & 5.0779949408 & 25.6109247729 & 5.1341203087 \\
\hline $\mathrm{H}$ & 10.4489918099 & 18.1225095997 & 7.3890878760 \\
\hline $\mathrm{H}$ & 3.2201875587 & 11.0494224541 & 7.3890878760 \\
\hline $\mathrm{H}$ & 10.0547778543 & 3.5365431379 & 3.6335512440 \\
\hline $\mathrm{H}$ & 3.6144023288 & 25.6353871763 & 3.6335512440 \\
\hline $\mathrm{H}$ & 10.9558483350 & 16.8902697207 & 8.5755579557 \\
\hline $\mathrm{H}$ & 2.7133312381 & 12.2816623321 & 8.5755579557 \\
\hline $\mathrm{H}$ & 9.5479213292 & 2.3043032599 & 2.4470811633 \\
\hline $\mathrm{H}$ & 4.1212584466 & 26.8676270542 & 2.4470811633 \\
\hline $\mathrm{H}$ & 8.7726679947 & 16.8747606263 & 9.8673323667 \\
\hline $\mathrm{H}$ & 4.8965117821 & 12.2971705567 & 9.8673323667 \\
\hline $\mathrm{H}$ & 11.7311016696 & 2.2887950343 & 1.1553067533 \\
\hline $\mathrm{H}$ & 1.9380781063 & 26.8831361486 & 1.1553067533 \\
\hline $\mathrm{H}$ & 6.2850913255 & 16.4619841612 & 9.8127025720 \\
\hline $\mathrm{H}$ & 7.3840884513 & 12.7099470218 & 9.8127025720 \\
\hline $\mathrm{H}$ & 0.5494985629 & 1.8760185702 & 1.2099365469 \\
\hline $\mathrm{H}$ & 13.1196812130 & 27.2959126138 & 1.2099365469 \\
\hline $\mathrm{H}$ & 5.2043267746 & 16.0125073848 & 7.6066995158 \\
\hline $\mathrm{H}$ & 8.4648530012 & 13.1594246679 & 7.6066995158 \\
\hline $\mathrm{H}$ & 1.6302631128 & 1.4265409240 & 3.4159396042 \\
\hline $\mathrm{H}$ & 12.0389166630 & 27.7453893901 & 3.4159396042 \\
\hline $\mathrm{H}$ & 6.6542376810 & 15.8496362217 & 5.5522269341 \\
\hline
\end{tabular}




\begin{tabular}{|c|c|c|c|}
\hline $\mathrm{H}$ & 7.0149420948 & 13.3222949612 & 5.5522269341 \\
\hline $\mathrm{H}$ & 0.1803522074 & 1.2636706298 & 5.4704121858 \\
\hline $\mathrm{H}$ & 13.4888275694 & 27.9082605532 & 5.4704121859 \\
\hline $\mathrm{H}$ & 12.5659000914 & 14.8551751923 & 6.8286781533 \\
\hline $\mathrm{H}$ & 1.1032798880 & 14.3167551219 & 6.8286781533 \\
\hline $\mathrm{H}$ & 7.9378695728 & 0.2692104691 & 4.1939609667 \\
\hline $\mathrm{H}$ & 5.7313102030 & 28.9027215836 & 4.1939609667 \\
\hline $\mathrm{H}$ & 11.2603994251 & 14.8223109806 & 8.0167996914 \\
\hline $\mathrm{H}$ & 2.4087805544 & 14.3496193335 & 8.0167996914 \\
\hline $\mathrm{H}$ & 9.2433702392 & 0.2363462575 & 3.0058394276 \\
\hline $\mathrm{H}$ & 4.4258095367 & 28.9355857953 & 3.0058394276 \\
\hline $\mathrm{H}$ & 11.7269683448 & 12.1968652627 & 7.1956337479 \\
\hline $\mathrm{H}$ & 1.9422112274 & 16.9750659202 & 7.1956337479 \\
\hline $\mathrm{H}$ & 8.7768013195 & 26.7828308547 & 3.8270053720 \\
\hline $\mathrm{H}$ & 4.8923784564 & 2.3891003283 & 3.8270053720 \\
\hline $\mathrm{H}$ & 10.6488428304 & 10.4959434716 & 5.7363560713 \\
\hline $\mathrm{H}$ & 3.0203367418 & 18.6759877124 & 5.7363560713 \\
\hline $\mathrm{H}$ & 9.8549268338 & 25.0819090636 & 5.2862833778 \\
\hline $\mathrm{H}$ & 3.8142529420 & 4.0900221204 & 5.2862833778 \\
\hline $\mathrm{H}$ & 9.1057931463 & 11.2797945557 & 3.8821733806 \\
\hline $\mathrm{H}$ & 4.5633866296 & 17.8921366273 & 3.8821733806 \\
\hline $\mathrm{H}$ & 11.3979765179 & 25.8657601476 & 7.1404660675 \\
\hline $\mathrm{H}$ & 2.2712032579 & 3.3061710353 & 7.1404660675 \\
\hline $\mathrm{H}$ & 8.6819275689 & 13.7322076014 & 3.6414988988 \\
\hline $\mathrm{H}$ & 4.9872526142 & 15.4397235816 & 3.6414988988 \\
\hline $\mathrm{H}$ & 11.8218420953 & 28.3181731934 & 7.3811402212 \\
\hline $\mathrm{H}$ & 1.8473372732 & 0.8537579906 & 7.3811402212 \\
\hline $\mathrm{H}$ & 8.2668507413 & 20.4345784166 & 3.6943899942 \\
\hline $\mathrm{H}$ & 5.4023286272 & 8.7373518975 & 3.6943899942 \\
\hline $\mathrm{H}$ & 12.2369189229 & 5.8486136945 & 7.3282487977 \\
\hline $\mathrm{H}$ & 1.4322612612 & 23.3233183593 & 7.3282487977 \\
\hline $\mathrm{H}$ & 6.5837386042 & 16.0729022461 & 1.6227221397 \\
\hline $\mathrm{H}$ & 7.0854411726 & 13.0990280681 & 1.6227221397 \\
\hline $\mathrm{H}$ & 0.2508512842 & 1.4869375240 & 9.3999169802 \\
\hline $\mathrm{H}$ & 13.4183284917 & 27.6849945288 & 9.3999169803 \\
\hline $\mathrm{H}$ & 4.6997774785 & 20.9396952261 & 3.1886948264 \\
\hline $\mathrm{H}$ & 8.9694022973 & 8.2322350881 & 3.1886948264 \\
\hline $\mathrm{H}$ & 2.1348124099 & 6.3537305039 & 7.8339439645 \\
\hline $\mathrm{H}$ & 11.5343673669 & 22.8182015499 & 7.8339439645 \\
\hline $\mathrm{H}$ & 2.6180096395 & 20.6988882395 & 4.5939580624 \\
\hline $\mathrm{H}$ & 11.0511701373 & 8.4730420746 & 4.5939580624 \\
\hline $\mathrm{H}$ & 4.2165802489 & 6.1129235173 & 6.4286813857 \\
\hline $\mathrm{H}$ & 9.4525995269 & 23.0590085354 & 6.4286813857 \\
\hline $\mathrm{H}$ & 0.5199684441 & 19.8617192084 & 3.5205690563 \\
\hline $\mathrm{H}$ & 13.1492113327 & 9.3102119755 & 3.5205690563 \\
\hline $\mathrm{H}$ & 6.3146214443 & 5.2757536164 & 7.5020703928 \\
\hline $\mathrm{H}$ & 7.3545583315 & 23.8961775675 & 7.5020703928 \\
\hline $\mathrm{H}$ & 0.5749257282 & 19.2743036413 & 1.0734381663 \\
\hline $\mathrm{H}$ & 13.0942540476 & 9.8976275427 & 1.0734381663 \\
\hline $\mathrm{H}$ & 6.2596641592 & 4.6883380493 & 9.9492007897 \\
\hline $\mathrm{H}$ & 7.4095156166 & 24.4835931337 & 9.9492007896 \\
\hline $\mathrm{H}$ & 1.8734275891 & 19.4359236776 & 9.8998911024 \\
\hline $\mathrm{H}$ & 11.7957521877 & 9.7360075063 & 9.8998911024 \\
\hline $\mathrm{H}$ & 4.9611622993 & 4.8499580856 & 1.1227478525 \\
\hline $\mathrm{H}$ & 8.7080174765 & 24.3219730983 & 1.1227478525 \\
\hline $\mathrm{H}$ & 3.1083731657 & 18.2924928182 & 10.4568986362 \\
\hline $\mathrm{H}$ & 10.5608066102 & 10.8794392346 & 10.4568986363 \\
\hline $\mathrm{H}$ & 3.7262167227 & 3.7065263574 & 0.5657401957 \\
\hline
\end{tabular}




\begin{tabular}{|c|c|c|c|}
\hline $\mathrm{H}$ & 9.9429630541 & 25.4654039568 & 0.5657401957 \\
\hline $\mathrm{H}$ & 3.7510977531 & 21.6611887088 & 8.3413827247 \\
\hline $\mathrm{H}$ & 9.9180820237 & 7.5107424742 & 8.3413827247 \\
\hline $\mathrm{H}$ & 3.0834921353 & 7.0752231168 & 2.6812565593 \\
\hline $\mathrm{H}$ & 10.5856876405 & 22.0967080662 & 2.6812565593 \\
\hline $\mathrm{H}$ & 2.3614616699 & 21.5639785388 & 9.3995773948 \\
\hline $\mathrm{H}$ & 11.3077181060 & 7.6079517754 & 9.3995773948 \\
\hline $\mathrm{H}$ & 4.4731282176 & 6.9780138166 & 1.6230617251 \\
\hline $\mathrm{H}$ & 9.1960515583 & 22.1939182362 & 1.6230617252 \\
\hline $\mathrm{H}$ & 2.9305583232 & 24.2139661262 & 9.3353617094 \\
\hline $\mathrm{H}$ & 10.7386214536 & 4.9579659266 & 9.3353617094 \\
\hline $\mathrm{H}$ & 3.9040315652 & 9.6279996654 & 1.6872777396 \\
\hline $\mathrm{H}$ & 9.7651482107 & 19.5439306488 & 1.6872777397 \\
\hline $\mathrm{H}$ & 4.0320411223 & 25.9712453455 & 10.7507516967 \\
\hline $\mathrm{H}$ & 9.6371386536 & 3.2006862724 & 10.7507516967 \\
\hline $\mathrm{H}$ & 2.8025487661 & 11.3852797535 & 0.2718873412 \\
\hline $\mathrm{H}$ & 10.8666310107 & 17.7866514295 & 0.2718873412 \\
\hline $\mathrm{H}$ & 5.8135510481 & 25.2320425521 & 1.3835446751 \\
\hline $\mathrm{H}$ & 7.8556287277 & 3.9398886319 & 1.3835446751 \\
\hline $\mathrm{H}$ & 1.0210388402 & 10.6460769601 & 9.6390946910 \\
\hline $\mathrm{H}$ & 12.6481409366 & 18.5258542229 & 9.6390946910 \\
\hline $\mathrm{H}$ & 6.3522802659 & 22.8121327514 & 1.5520735861 \\
\hline $\mathrm{H}$ & 7.3168995099 & 6.3597988675 & 1.5520735861 \\
\hline $\mathrm{H}$ & 0.4823096225 & 8.2261671594 & 9.4705658620 \\
\hline $\mathrm{H}$ & 13.1868701543 & 20.9457640246 & 9.4705658620 \\
\hline $\mathrm{H}$ & 3.9723954557 & 19.5143669947 & 7.7826434372 \\
\hline $\mathrm{H}$ & 9.6967843201 & 9.6575650581 & 7.7826434372 \\
\hline $\mathrm{H}$ & 2.8621944327 & 4.9284005339 & 3.2399960109 \\
\hline $\mathrm{H}$ & 10.8069853441 & 24.2435297802 & 3.2399960109 \\
\hline $\mathrm{H}$ & 4.5263515069 & 18.3232360010 & 8.9816001836 \\
\hline $\mathrm{H}$ & 9.1428282699 & 10.8486943131 & 8.9816001836 \\
\hline $\mathrm{H}$ & 2.3082383815 & 3.7372712778 & 2.0410392655 \\
\hline $\mathrm{H}$ & 11.3609413944 & 25.4346607749 & 2.0410392655 \\
\hline $\mathrm{H}$ & 6.1981877319 & 19.3550395619 & 6.4843233341 \\
\hline $\mathrm{H}$ & 7.4709920439 & 9.8168916211 & 6.4843233341 \\
\hline $\mathrm{H}$ & 0.6364021565 & 4.7690739709 & 4.5383157859 \\
\hline $\mathrm{H}$ & 13.0327776203 & 24.4028572131 & 4.5383157859 \\
\hline $\mathrm{H}$ & 8.6583301901 & 19.8132265358 & 6.4950026597 \\
\hline $\mathrm{H}$ & 5.0108491785 & 9.3587046472 & 6.4950026597 \\
\hline $\mathrm{H}$ & 11.8454394742 & 5.2272609438 & 4.5276364603 \\
\hline $\mathrm{H}$ & 1.8237407099 & 23.9446702392 & 4.5276364603 \\
\hline $\mathrm{H}$ & 9.7365264337 & 20.5360746395 & 8.6691740404 \\
\hline $\mathrm{H}$ & 3.9326533431 & 8.6358556747 & 8.6691740404 \\
\hline $\mathrm{H}$ & 10.7672432306 & 5.9501099173 & 2.3534652437 \\
\hline $\mathrm{H}$ & 2.9019365453 & 23.2218221355 & 2.3534652437 \\
\hline $\mathrm{H}$ & 8.2809960988 & 20.8508769728 & 10.6804608963 \\
\hline $\mathrm{H}$ & 5.3881840853 & 8.3210542111 & 10.6804608963 \\
\hline $\mathrm{H}$ & 12.2227735655 & 6.2649113809 & 0.3421783468 \\
\hline $\mathrm{H}$ & 1.4464058031 & 22.9070198031 & 0.3421783468 \\
\hline C & 11.2808841457 & 15.8599319907 & 2.5489266775 \\
\hline C & 2.3882958338 & 13.3119983235 & 2.5489266775 \\
\hline C & 9.2228855186 & 1.2739672675 & 8.4737124425 \\
\hline C & 4.4462942573 & 27.8979647852 & 8.4737124425 \\
\hline C & 11.2338804279 & 20.9240145531 & 5.1237198796 \\
\hline C & 2.4352989417 & 8.2479166309 & 5.1237198796 \\
\hline C & 9.2698892364 & 6.3380489611 & 5.8989189114 \\
\hline C & 4.3992909467 & 22.8338822219 & 5.8989189114 \\
\hline C & 12.4629639478 & 15.9287372165 & 1.8161959167 \\
\hline
\end{tabular}




\begin{tabular}{|c|c|c|c|}
\hline C & 1.2062161335 & 13.2431948362 & 1.8161959167 \\
\hline C & 8.0408057164 & 1.3427707558 & 9.2064435314 \\
\hline C & 5.6283736531 & 27.8291595584 & 9.2064435314 \\
\hline C & 13.6288503779 & 16.3745862186 & 2.4386126365 \\
\hline C & 0.0403297804 & 12.7973449644 & 2.4386126365 \\
\hline C & 6.8749192863 & 1.7886206276 & 8.5840261545 \\
\hline C & 6.7942600823 & 27.3833105563 & 8.5840261545 \\
\hline C & 13.5737646405 & 16.7323486074 & 3.7860184267 \\
\hline C & 0.0954148051 & 12.4395825766 & 3.7860184267 \\
\hline C & 6.9300050237 & 2.1463830154 & 7.2366206932 \\
\hline C & 6.7391751594 & 27.0255481676 & 7.2366206933 \\
\hline C & 12.3607994313 & 16.6493752375 & 4.4620909760 \\
\hline C & 1.3083806509 & 12.5225559465 & 4.4620909760 \\
\hline C & 8.1429702329 & 2.0634096455 & 6.5605484721 \\
\hline C & 5.5262091356 & 27.1085215384 & 6.5605484721 \\
\hline C & 12.1771029330 & 17.0799653388 & 5.8893207813 \\
\hline C & 1.4920770475 & 12.0919658441 & 5.8893207813 \\
\hline C & 8.3266667313 & 2.4939997478 & 5.1333183387 \\
\hline C & 5.3425130446 & 26.6779314361 & 5.1333183387 \\
\hline C & 10.4169948454 & 17.0483103739 & 7.6293362284 \\
\hline C & 3.2521849304 & 12.1236199403 & 7.6293362284 \\
\hline C & 10.0867748188 & 2.4623456517 & 3.3933028916 \\
\hline C & 3.5824049580 & 26.7095864020 & 3.3933028916 \\
\hline C & 8.9783797561 & 16.6361960202 & 7.7399875048 \\
\hline C & 4.6908000207 & 12.5357351637 & 7.7399875048 \\
\hline C & 11.5253899081 & 2.0502304282 & 3.2826516152 \\
\hline C & 2.1437898677 & 27.1217007547 & 3.2826516152 \\
\hline C & 8.2562940322 & 16.6737070348 & 8.9289621455 \\
\hline C & 5.4128857446 & 12.4982232793 & 8.9289621455 \\
\hline C & 12.2474756321 & 2.0877423127 & 2.0936769745 \\
\hline C & 1.4217041438 & 27.0841897401 & 2.0936769745 \\
\hline C & 6.8801267778 & 16.4421164702 & 8.8985303495 \\
\hline C & 6.7890529980 & 12.7298147127 & 8.8985303495 \\
\hline C & -0.0455368904 & 1.8561508783 & 2.1241087705 \\
\hline C & 13.7147166662 & 27.3157803047 & 2.1241087705 \\
\hline C & 6.2772004077 & 16.1865936742 & 7.6677387262 \\
\hline C & 7.3919793681 & 12.9853383786 & 7.6677387262 \\
\hline C & 0.5573894797 & 1.6006272124 & 3.3549003938 \\
\hline C & 13.1117902961 & 27.5713031008 & 3.3549003938 \\
\hline C & 7.0691965596 & 16.1007050907 & 6.5265900500 \\
\hline C & 6.5999832163 & 13.0712252235 & 6.5265900500 \\
\hline C & 13.4345731047 & 1.5147403685 & 4.4960490700 \\
\hline C & 0.2346066712 & 27.6571916843 & 4.4960490700 \\
\hline C & 11.4737964971 & 14.9462420130 & 6.9436264291 \\
\hline C & 2.1953832788 & 14.2256900398 & 6.9436264291 \\
\hline C & 9.0299731672 & 0.3602755522 & 4.0790126909 \\
\hline C & 4.6392066096 & 28.8116547620 & 4.0790126909 \\
\hline C & 10.7996315662 & 13.8561784295 & 6.1647507890 \\
\hline C & 2.8695478024 & 15.3157527545 & 6.1647507890 \\
\hline C & 9.7041380980 & 28.4421440215 & 4.8578883310 \\
\hline C & 3.9650420861 & 0.7297871625 & 4.8578883310 \\
\hline C & 11.0557916096 & 12.5049826648 & 6.3924092590 \\
\hline C & 2.6133879626 & 16.6669485181 & 6.3924092590 \\
\hline C & 9.4479780546 & 27.0909482568 & 4.6302298610 \\
\hline C & 4.2212017212 & 2.0809829262 & 4.6302298610 \\
\hline C & 10.4393174873 & 11.5528915558 & 5.5819639743 \\
\hline C & 3.2298620849 & 17.6190396282 & 5.5819639742 \\
\hline C & 10.0644521770 & 26.1388571468 & 5.4406751458 \\
\hline
\end{tabular}




\begin{tabular}{|c|c|c|c|}
\hline C & 3.6047275998 & 3.0330740362 & 5.4406751458 \\
\hline C & 9.5876190561 & 11.9822215244 & 4.5626075891 \\
\hline C & 4.0815607198 & 17.1897096595 & 4.5626075891 \\
\hline C & 10.9161506082 & 26.5681871155 & 6.4600315309 \\
\hline C & 2.7530291686 & 2.6037440675 & 6.4600315309 \\
\hline C & 9.3472116933 & 13.3404068461 & 4.4100937099 \\
\hline C & 4.3219676753 & 15.8315243378 & 4.4100937099 \\
\hline C & 11.1565579710 & 27.9263724371 & 6.6125450810 \\
\hline C & 2.5126222121 & 1.2455587458 & 6.6125450810 \\
\hline C & 8.3777479935 & 18.2574172961 & 3.7279586452 \\
\hline C & 5.2914317823 & 10.9145138879 & 3.7279586451 \\
\hline C & 12.1260216707 & 3.6714517041 & 7.2946801468 \\
\hline C & 1.5431581051 & 25.5004794799 & 7.2946801468 \\
\hline C & 7.9196694121 & 19.4909434082 & 3.2706652711 \\
\hline C & 5.7495107710 & 9.6809877747 & 3.2706652711 \\
\hline C & 12.5841002521 & 4.9049778162 & 7.7519738489 \\
\hline C & 1.0850791164 & 24.2669533668 & 7.7519738489 \\
\hline C & 7.0347915676 & 19.5002737604 & 2.1790032343 \\
\hline C & 6.6343882083 & 9.6716565537 & 2.1790032343 \\
\hline C & -0.2002016792 & 4.9143090383 & 8.8436362138 \\
\hline C & 13.8693814560 & 24.2576230145 & 8.8436362137 \\
\hline C & 6.5118589233 & 18.2427787228 & 1.5666213614 \\
\hline C & 7.1573208525 & 10.9291524611 & 1.5666213614 \\
\hline C & 0.3227309641 & 3.6568131309 & 9.4560177586 \\
\hline C & 13.3464488117 & 25.5151180531 & 9.4560177586 \\
\hline C & 6.9419335092 & 17.0058421698 & 2.0613486361 \\
\hline C & 6.7272462667 & 12.1660890131 & 2.0613486361 \\
\hline C & -0.1073436208 & 2.4198765779 & 8.9612903199 \\
\hline C & 13.7765233976 & 26.7520546051 & 8.9612903199 \\
\hline C & 7.8606818161 & 16.9966907839 & 3.1222986876 \\
\hline C & 5.8084975524 & 12.1752395302 & 3.1222986876 \\
\hline C & 12.6430878481 & 2.4107260618 & 7.9003404314 \\
\hline C & 1.0260923360 & 26.7612059920 & 7.9003404314 \\
\hline C & 3.7652330888 & 20.5543007568 & 2.7750605977 \\
\hline C & 9.9039466870 & 8.6176304262 & 2.7750605977 \\
\hline C & 3.0693567986 & 5.9683351648 & 8.2475788504 \\
\hline C & 10.5998229772 & 23.2035960182 & 8.2475788504 \\
\hline C & 2.6093460928 & 20.4251711340 & 3.5398399698 \\
\hline C & 11.0598336831 & 8.7467600499 & 3.5398399698 \\
\hline C & 4.2252437956 & 5.8392055421 & 7.4827991502 \\
\hline C & 9.4439359812 & 23.3327256419 & 7.4827991501 \\
\hline C & 1.4374185186 & 19.9609297293 & 2.9395335858 \\
\hline C & 12.2317612572 & 9.2110014546 & 2.9395335858 \\
\hline C & 5.3971713698 & 5.3749641373 & 8.0831052061 \\
\hline C & 8.2720084070 & 23.7969670466 & 8.0831052061 \\
\hline C & 1.4670414163 & 19.6350321611 & 1.5857703587 \\
\hline C & 12.2021383605 & 9.5368981530 & 1.5857703587 \\
\hline C & 5.3675484721 & 5.0490674389 & 9.4368684322 \\
\hline C & 8.3016313037 & 24.1228646138 & 9.4368684322 \\
\hline C & 2.6582072246 & 19.7673074526 & 0.8766436633 \\
\hline C & 11.0109725512 & 9.4046237304 & 0.8766436633 \\
\hline C & 4.1763826628 & 5.1813418606 & 10.1459955387 \\
\hline C & 9.4927971130 & 23.9905893224 & 10.1459955387 \\
\hline C & 2.8119173453 & 19.3536783835 & 10.4686525795 \\
\hline C & 10.8572624315 & 9.8182527995 & 10.4686525795 \\
\hline C & 4.0226725431 & 4.7677127915 & 0.5539863355 \\
\hline C & 9.6465072327 & 24.4042183914 & 0.5539863355 \\
\hline C & 3.4610364794 & 21.5079528878 & 9.3899153746 \\
\hline
\end{tabular}




\begin{tabular}{|c|c|c|c|}
\hline C & 10.2081432965 & 7.6639782961 & 9.3899153746 \\
\hline C & 3.3735534081 & 6.9219872959 & 1.6327237454 \\
\hline C & 10.2956263678 & 22.2499438872 & 1.6327237454 \\
\hline C & 4.0642667630 & 22.6119437870 & 10.2105595578 \\
\hline C & 9.6049130138 & 6.5599878309 & 10.2105595578 \\
\hline C & 2.7703231254 & 8.0259781950 & 0.8120798902 \\
\hline C & 10.8988566505 & 21.1459529879 & 0.8120798902 \\
\hline C & 3.6942572975 & 23.9492075102 & 10.0654124177 \\
\hline C & 9.9749224783 & 5.2227228040 & 10.0654124177 \\
\hline C & 3.1403325909 & 9.3632427870 & 0.9572266192 \\
\hline C & 10.5288471859 & 19.8086892658 & 0.9572266192 \\
\hline C & 4.3097752892 & 24.9209160358 & 10.8510338119 \\
\hline C & 9.3594044866 & 4.2510155831 & 10.8510338119 \\
\hline C & 2.5248145992 & 10.3349504438 & 0.1716055131 \\
\hline C & 11.1443651776 & 18.8369807402 & 0.1716055131 \\
\hline C & 5.2909891526 & 24.5241217291 & 0.7401965562 \\
\hline C & 8.3781906232 & 4.6478090200 & 0.7401965562 \\
\hline C & 1.5436007358 & 9.9381561371 & 10.2824428508 \\
\hline C & 12.1255790410 & 19.2337750459 & 10.2824428508 \\
\hline C & 5.6193322694 & 23.1774364976 & 0.8357873159 \\
\hline C & 8.0498475075 & 5.9944946853 & 0.8357873159 \\
\hline C & 1.2152576190 & 8.5914709057 & 10.1868516400 \\
\hline C & 12.4539221568 & 20.5804602773 & 10.1868516400 \\
\hline C & 4.5418268676 & 19.3920734574 & 8.7149756484 \\
\hline C & 9.1273529083 & 9.7798577265 & 8.7149756484 \\
\hline C & 2.2927630199 & 4.8061078655 & 2.3076634716 \\
\hline C & 11.3764167560 & 24.3658233185 & 2.3076634716 \\
\hline C & 5.9718813492 & 19.8174509141 & 8.5744916520 \\
\hline C & 7.6972984277 & 9.3544794001 & 8.5744916520 \\
\hline C & 0.8627085392 & 5.2314861919 & 2.4481473039 \\
\hline C & 12.8064712366 & 23.9404458609 & 2.4481473039 \\
\hline C & 6.6997405707 & 19.6875685041 & 7.3941661245 \\
\hline C & 6.9694392051 & 9.4843626798 & 7.3941661245 \\
\hline C & 0.1348493168 & 5.1016029122 & 3.6284729945 \\
\hline C & 13.5343304591 & 24.0703282718 & 3.6284729945 \\
\hline C & 8.0707525500 & 19.9430122579 & 7.4053142963 \\
\hline C & 5.5984268186 & 9.2289180562 & 7.4053142963 \\
\hline C & 12.4330171142 & 5.3570475358 & 3.6173244946 \\
\hline C & 1.2361630689 & 23.8148845170 & 3.6173244946 \\
\hline C & 8.6667611636 & 20.3409787521 & 8.6025056192 \\
\hline C & 5.0024182050 & 8.8309533007 & 8.6025056192 \\
\hline C & 11.8370085007 & 5.7550122903 & 2.4201336649 \\
\hline C & 1.8321716834 & 23.4169180239 & 2.4201336649 \\
\hline C & 7.8714152319 & 20.5121155130 & 9.7306225901 \\
\hline C & 5.7977649512 & 8.6598156710 & 9.7306225901 \\
\hline C & 12.6323544323 & 5.9261499210 & 1.2920166939 \\
\hline C & 1.0368249372 & 23.2457812630 & 1.2920166939 \\
\hline B & 0.7648627888 & 19.9701749449 & 6.9531171859 \\
\hline B & 12.9043173439 & 9.2017562390 & 6.9531171859 \\
\hline B & 6.0697270482 & 5.3842093530 & 4.0695222622 \\
\hline B & 7.5994523204 & 23.7877218300 & 4.0695222622 \\
\hline B & 3.3846302154 & 15.3278559334 & -0.4296804031 \\
\hline B & 10.2845491541 & 13.8440752506 & -0.4296804031 \\
\hline B & 3.4499596730 & 0.7418903414 & 11.4523195481 \\
\hline B & 10.2192205101 & 28.4300408425 & 11.4523195482 \\
\hline $\mathrm{F}$ & -0.1010532831 & 21.0452385577 & 7.2448854658 \\
\hline $\mathrm{F}$ & 13.7702327535 & 8.1266934961 & 7.2448854658 \\
\hline $\mathrm{F}$ & 6.9356432724 & 6.4592720959 & 3.7777536532 \\
\hline
\end{tabular}




$\begin{array}{lr}\text { F } & 6.7335369108 \\ F & 2.1086093747 \\ F & 11.5605704011 \\ F & 4.7259805137 \\ F & 8.9431992631 \\ F & 0.4107121630 \\ F & 13.2584678174 \\ F & 6.4238779290 \\ F & 7.2453018468 \\ F & 0.7064827682 \\ F & 12.9626973131 \\ F & 6.1281070174 \\ F & 7.5410723511 \\ F & 2.5389237030 \\ F & 11.1302560728 \\ F & 4.2956661854 \\ F & 9.3735135914 \\ F & 3.6809774780 \\ F & 9.9882018906 \\ F & 3.1536124105 \\ F & 10.5155677736 \\ F & 4.5722409666 \\ F & 9.0969388092 \\ F & 2.2623489218 \\ F & 11.4068308550 \\ F & 2.6957606507 \\ F & 10.9734191252 \\ F & 4.1388292368 \\ F & 9.5303505390\end{array}$

22.7126582183

3.7777536532

20.4636946649

6.8572412207

8.7082373878

5.8777282041

23.2942021101

19.3561337543

9.8157974297

4.7701681623

24.4017630217

18.9932862851

10.1786457676

4.4073198234

24.7646104908

16.0839483871

13.0879827969

1.4979827951

27.6739483889

16.1008015051

13.0711305477

1.5148350443

27.6570952699

14.9631552665

14.2087767872

0.3771888048

28.7947415094

14.1332155511

15.0387156328

28.7191811421

0.4527500408

6.8572412207

4.1653978993

4.1653978993

5.7300217027

5.7300217027

5.2926174173

5.2926174173

7.9970926331

7.9970926331

3.0255464869

3.0255464869

0.4238999451

0.4238999451

10.5987390108

10.5987390108

9.4270110524

9.4270110524

1.5956281496

1.5956281496

0.2423302075

0.2423302075

10.7803090765

10.7803090765

10.1753289236

10.1753289236

0.8473099293

0.8473099293

$\mathbf{2} \cdot \mathbf{2} \mathrm{CH}_{3} \mathrm{OH}-\mathrm{HS}$

CELL_PARAMETERS (angstrom)
9.104161657
0.209083665
4.654548092
9.750391564
0.030202162
4.017781348
$-0.944425355$
$-0.250009471$
13.442240741

ATOMIC_POSITIONS (angstrom)

$\begin{array}{lrrr}\mathrm{Fe} 1 & 9.1392174592 & 1.4602931548 & 4.3213138446 \\ \mathrm{Fe} 1 & 8.6372734995 & 7.5547564281 & 8.9011195942 \\ \mathrm{~F} & 5.6006024985 & 4.8593279647 & 1.2971703887 \\ \mathrm{~F} & 12.1758885377 & 4.1557219235 & 11.9252628423 \\ \mathrm{~F} & 4.1556728881 & 5.2558186671 & 3.0800765231 \\ \mathrm{~F} & 13.6208182092 & 3.7592312065 & 10.1423569082 \\ \mathrm{~F} & 3.7726731483 & 6.2695720625 & 1.0269954517 \\ \mathrm{~F} & 14.0038178879 & 2.7454778257 & 12.1954377793 \\ \mathrm{~F} & 5.5826988112 & 6.9359806632 & 2.3449092112 \\ \mathrm{~F} & 12.1937923462 & 2.0790691968 & 10.8775244205 \\ \mathrm{O} & 8.9596815875 & -0.2445842065 & 5.4471875869 \\ \mathrm{O} & 8.8168096679 & 9.2596341333 & 7.7752462430 \\ \mathrm{O} & 7.1821611114 & 1.6836537545 & 5.0924973130 \\ \mathrm{O} & 10.5943299663 & 7.3313958001 & 8.1299365264 \\ \mathrm{O} & 10.3742336398 & 6.8656284934 & 2.2299467812 \\ \mathrm{O} & 7.4022574575 & 2.1494213803 & 10.9924866501 \\ \mathrm{~N} & 10.1075045435 & 2.6806170542 & 5.8573384463 \\ \mathrm{~N} & 7.6689865528 & 6.3344328194 & 7.3650949840 \\ \mathrm{~N} & 9.4246292666 & 3.4447060644 & 3.2890178117 \\ \mathrm{~N} & 8.3518616910 & 5.5703435185 & 9.9334156271 \\ \mathrm{~N} & 8.2701800264 & 1.1635815223 & 2.3611516354 \\ \mathrm{~N} & 9.5063109312 & 7.8514680605 & 10.8612818034\end{array}$




\begin{tabular}{|c|c|c|c|}
\hline $\mathrm{N}$ & 1.9930008692 & 0.9650075260 & 3.5070583311 \\
\hline $\mathrm{N}$ & 15.7834901577 & 8.0500422027 & 9.7153751039 \\
\hline $\mathrm{C}$ & 12.4988111378 & 9.3855184407 & 5.8436861795 \\
\hline $\mathrm{C}$ & 5.2776799639 & -0.3704685581 & 7.3787472506 \\
\hline $\mathrm{C}$ & 2.7761113936 & -0.0979496179 & 3.7848949249 \\
\hline $\mathrm{C}$ & 15.0003796853 & 9.1129994548 & 9.4375385074 \\
\hline $\mathrm{C}$ & 2.5171628458 & 2.0308380931 & 2.8648016972 \\
\hline $\mathrm{C}$ & 15.2593281118 & 6.9842114898 & 10.3576317416 \\
\hline $\mathrm{C}$ & 6.8260497715 & 0.7376675251 & 5.8756954120 \\
\hline $\mathrm{C}$ & 10.9504414806 & 8.2773823927 & 7.3467384171 \\
\hline $\mathrm{C}$ & 7.3680341407 & 2.2570174000 & 0.4274646842 \\
\hline $\mathrm{C}$ & 10.4084569110 & 6.7580324843 & 12.7949685961 \\
\hline $\mathrm{C}$ & 3.8666540268 & 2.0643808441 & 2.5034822184 \\
\hline $\mathrm{C}$ & 13.9098368811 & 6.9506689129 & 10.7189508159 \\
\hline $\mathrm{C}$ & 12.6789919568 & 9.7175534736 & 1.5444913291 \\
\hline $\mathrm{C}$ & 5.0974991302 & -0.7025037594 & 11.6779423053 \\
\hline $\mathrm{C}$ & 10.3034021750 & 3.9778868260 & 5.5214216870 \\
\hline $\mathrm{C}$ & 7.4730889212 & 5.0371630476 & 7.7010117443 \\
\hline $\mathrm{C}$ & 10.6936612314 & 3.3762277118 & 2.5116881462 \\
\hline $\mathrm{C}$ & 7.0828298659 & 5.6388221618 & 10.7107452851 \\
\hline $\mathrm{C}$ & 7.9526358608 & 2.2954155525 & 1.6913128819 \\
\hline $\mathrm{C}$ & 9.8238553058 & 6.7196344660 & 11.5311205458 \\
\hline $\mathrm{C}$ & 8.2416185807 & 3.5927612671 & 2.4109735049 \\
\hline $\mathrm{C}$ & 9.5348722567 & 5.4222883440 & 10.8114595332 \\
\hline $\mathrm{C}$ & 10.7793321526 & 2.1718883493 & 6.9070861294 \\
\hline $\mathrm{C}$ & 6.9971588743 & 6.8431613794 & 6.3153473056 \\
\hline $\mathrm{C}$ & 12.2012601781 & 8.3304472825 & 6.7014905522 \\
\hline $\mathrm{C}$ & 5.5752308142 & 0.6846023728 & 6.5209428847 \\
\hline $\mathrm{C}$ & 4.1235283898 & -0.1392091729 & 3.4492575275 \\
\hline $\mathrm{C}$ & 13.6529628126 & 9.1542592677 & 9.7731758982 \\
\hline $\mathrm{C}$ & 12.0850141752 & 9.6044348242 & 0.2915033959 \\
\hline $\mathrm{C}$ & 5.6914771462 & -0.5893847606 & 12.9309304305 \\
\hline $\mathrm{C}$ & 11.1163233557 & 0.0728497145 & 13.2899520859 \\
\hline $\mathrm{C}$ & 6.6601677116 & 8.9422000048 & -0.0675185183 \\
\hline $\mathrm{C}$ & 11.1783819836 & 4.7960133136 & 6.2330347190 \\
\hline $\mathrm{C}$ & 6.5981091136 & 4.2190365600 & 6.9893987113 \\
\hline $\mathrm{C}$ & 11.6565070991 & 2.9308201926 & 7.6792199472 \\
\hline $\mathrm{C}$ & 6.1199839972 & 6.0842296810 & 5.5432134831 \\
\hline $\mathrm{C}$ & 11.8549698973 & 4.2700992666 & 7.3367137920 \\
\hline $\mathrm{C}$ & 5.9215213377 & 4.7449508977 & 5.8857196319 \\
\hline $\mathrm{C}$ & 4.6735308316 & 0.9691539923 & 2.8022594363 \\
\hline $\mathrm{C}$ & 13.1029604195 & 8.0458959255 & 10.4201743939 \\
\hline $\mathrm{C}$ & 9.4843501463 & 4.4724195344 & 4.3567968728 \\
\hline $\mathrm{C}$ & 8.2921410897 & 4.5426306299 & 8.8656365510 \\
\hline $\mathrm{C}$ & 9.4784351901 & 6.9324459108 & 1.1171828744 \\
\hline $\mathrm{C}$ & 8.2980559663 & 2.0826039491 & 12.1052507562 \\
\hline $\mathrm{H}$ & 6.9296622049 & 8.8187077576 & 4.0289608423 \\
\hline $\mathrm{H}$ & 10.8468289075 & 0.1963418695 & 9.1934729953 \\
\hline $\mathrm{H}$ & 11.1617797176 & 2.2444140912 & 13.3527352543 \\
\hline $\mathrm{H}$ & 6.6147113032 & 6.7706354769 & -0.1303016070 \\
\hline $\mathrm{H}$ & 4.2800829561 & 2.9455403318 & 2.0116040291 \\
\hline $\mathrm{H}$ & 13.4964080811 & 6.0695095555 & 11.2108292019 \\
\hline $\mathrm{H}$ & 12.9860886543 & 8.8392971588 & 2.1136805846 \\
\hline $\mathrm{H}$ & 4.7904024073 & 0.1757526424 & 11.1087528476 \\
\hline $\mathrm{H}$ & 11.2389132136 & 4.3293263359 & 2.5878561192 \\
\hline $\mathrm{H}$ & 6.5375778826 & 4.6857235377 & 10.6345773122 \\
\hline $\mathrm{H}$ & 10.4346283611 & 3.2568621417 & 1.4459015643 \\
\hline $\mathrm{H}$ & 7.3418627848 & 5.7581880431 & 11.7765315586 \\
\hline
\end{tabular}




$\begin{array}{lrrr}\mathrm{H} & 8.3499531239 & 4.4141846427 & 1.6858474663 \\ \mathrm{H} & 9.4265377736 & 4.6008649538 & 11.5365857722 \\ \mathrm{H} & 7.3765309012 & 3.8384404585 & 3.0473501291 \\ \mathrm{H} & 10.3999602747 & 5.1766097195 & 10.1750830944 \\ \mathrm{H} & 10.6081639643 & 1.1121849511 & 7.1079574036 \\ \mathrm{H} & 7.1683272024 & 7.9028650684 & 6.1144760240 \\ \mathrm{H} & 12.9374834756 & 7.5453906381 & 6.8789087957 \\ \mathrm{H} & 4.8390074820 & 1.4696589458 & 6.3435246431 \\ \mathrm{H} & 9.3819400599 & 8.7301056576 & 3.4197008394 \\ \mathrm{H} & 8.3945510017 & 0.2849441426 & 9.8027325938 \\ \mathrm{H} & 11.9116644651 & 8.6149182863 & -0.1269573066 \\ \mathrm{H} & 5.8648264883 & 0.4001314599 & 13.3493904909 \\ \mathrm{H} & 10.6719425842 & 0.0227002211 & 12.2964750457 \\ \mathrm{H} & 7.1045486067 & 8.9923497113 & 0.9259585841 \\ \mathrm{H} & 11.3241667211 & 5.8305753445 & 5.9217330679 \\ \mathrm{H} & 6.4523243762 & 3.1844745291 & 7.3007003635 \\ \mathrm{H} & 12.1736897503 & 2.4809839955 & 8.5259309831 \\ \mathrm{H} & 5.6028012072 & 6.5340655873 & 4.6965024556 \\ \mathrm{H} & 12.5318074366 & 4.8911324966 & 7.9247521166 \\ \mathrm{H} & 5.2446836607 & 4.1239173780 & 5.2976813137 \\ \mathrm{H} & 5.7285153302 & 0.9810654293 & 2.5395578353 \\ \mathrm{H} & 12.0479757417 & 8.0339845314 & 10.6828753938 \\ \mathrm{H} & 11.2859103779 & 6.7468899354 & 1.8798857308 \\ \mathrm{H} & 6.4905807794 & 2.2681599246 & 11.3425479008 \\ \mathrm{H} & 9.8681074506 & 5.4307396088 & 3.9683573362 \\ \mathrm{H} & 7.9083835265 & 3.5843102921 & 9.2540756945 \\ \mathrm{H} & 8.4526497751 & 4.6351342743 & 4.7094656304 \\ \mathrm{H} & 9.3238414423 & 4.3799155711 & 8.5129682016 \\ \mathrm{H} & 8.4552320583 & 6.8679423813 & 1.5145140565 \\ \mathrm{H} & 9.3212591591 & 2.1471074641 & 11.7079197754 \\ \mathrm{H} & 9.6274392412 & 6.1022752993 & 0.4063256448 \\ \mathrm{H} & 8.1490517960 & 2.9127745879 & 12.8161075862 \\ \mathrm{H} & 9.5715058802 & 7.8875461900 & 0.5678436976 \\ \mathrm{H} & 8.2049852761 & 1.1275036699 & 12.6545899341 \\ \mathrm{~B} & 4.7958661743 & 5.8259774833 & 1.9486408430 \\ \mathrm{~B} & 12.9806248019 & 3.1890724186 & 11.2737921866\end{array}$

2・2 $\mathrm{CH}_{3} \mathrm{OH}$-iS

CELL_PARAMETERS (angstrom)

$\begin{array}{rrr}9.022927488 & 0.132459381 & 0.043976832 \\ 4.527829499 & 9.801656584 & -0.073066490 \\ 4.014322960 & -0.762691605 & 13.221030134\end{array}$

ATOMIC POSITIONS (angstrom)

$\begin{array}{lr}\text { Fe1 } & 8.9924297487 \\ \text { Fe2 } & 8.4706298697 \\ \text { F } & 5.4703534674 \\ \text { F } & 12.0994030425 \\ \text { F } & 4.0659629747 \\ \text { F } & 13.4550035550 \\ \text { F } & 3.6239089386 \\ \text { F } & 13.9651115278 \\ \text { F } & 5.4568006585 \\ \text { F } & 12.1000394027 \\ \text { O } & 8.8239591242 \\ \text { O } & 8.6287816763 \\ \text { O } & 7.0872120851 \\ \text { O } & 10.2695918759 \\ \text { O } & 10.2043137265\end{array}$

1.4389091936

4.3591690914

8.9678743478

1.2921814429

4.9158184473

11.8938942654

4.3239584002

5.2666494322

3.9897968425

6.3115811570

2.9484138372

6.9740950845

2.2712152724

$-0.2948872661$

9.2111987236

1.6723860124

7.2194475197

6.9609479592

3.1181807637

10.0270892369

1.0911682464

12.0354448990

2.3792858430

10.8014808884

5.4443543272

8.0353149461

5.1840541867

8.1819517800

2.3836433561 


\begin{tabular}{|c|c|c|c|}
\hline O & 7.3732651884 & 2.2107645890 & 10.7393502525 \\
\hline $\mathrm{N}$ & 9.9663229053 & 2.6863339240 & 5.8558322936 \\
\hline $\mathrm{N}$ & 7.5524929731 & 6.5831871291 & 7.4938178524 \\
\hline $\mathrm{N}$ & 9.2814473067 & 3.4052208649 & 3.2820909882 \\
\hline$N$ & 8.3043302875 & 5.7406900235 & 9.8882846228 \\
\hline $\mathrm{N}$ & 8.1267109229 & 1.1086510167 & 2.4101820386 \\
\hline $\mathrm{N}$ & 9.3218760509 & 8.0353954351 & 10.6566175806 \\
\hline $\mathrm{N}$ & 1.9145029645 & 0.9967082892 & 3.5063018891 \\
\hline $\mathrm{N}$ & 15.7431302552 & 8.0161497308 & 9.7172104827 \\
\hline C & 12.2363349828 & 9.3713713531 & 6.0212983205 \\
\hline C & 5.1942349176 & -0.4492429215 & 7.4253031116 \\
\hline C & 2.6939517606 & -0.0655413374 & 3.7960459159 \\
\hline C & 15.0281691481 & 9.1271734578 & 9.4270913783 \\
\hline C & 2.4393513409 & 2.0486564470 & 2.8413470979 \\
\hline C & 15.1618806677 & 7.0112544096 & 10.4154737608 \\
\hline C & 6.7217984834 & 0.7032531688 & 5.9487516948 \\
\hline C & 10.6771289539 & 8.2105914847 & 7.4624164525 \\
\hline C & 7.2409134076 & 2.1694148142 & 0.4507327543 \\
\hline C & 10.2870736416 & 7.1319953573 & 12.6693913294 \\
\hline C & 3.7869100395 & 2.0702238153 & 2.4744767508 \\
\hline C & 13.8243956142 & 7.0816622517 & 10.8086592186 \\
\hline C & 12.4417028748 & 9.7049703729 & 1.7760137486 \\
\hline C & 4.9598660639 & -0.5212727462 & 11.2202135702 \\
\hline C & 10.1941629653 & 3.9692618655 & 5.4854033602 \\
\hline C & 7.4557570596 & 5.2359383311 & 7.6745902312 \\
\hline C & 10.5350913576 & 3.3108114321 & 2.4842536416 \\
\hline C & 7.0343479611 & 5.7139390762 & 10.6972624864 \\
\hline C & 7.8061244317 & 2.2294072388 & 1.7209652733 \\
\hline C & 9.7219330965 & 6.9721693956 & 11.4106853189 \\
\hline C & 8.0827700509 & 3.5373892644 & 2.4237676787 \\
\hline C & 9.5072845465 & 5.6369076895 & 10.7586628798 \\
\hline C & 10.6306886532 & 2.1875011734 & 6.9141749141 \\
\hline C & 6.8622059294 & 7.1510915943 & 6.4828466896 \\
\hline C & 11.9240410182 & 8.2654015399 & 6.8156312484 \\
\hline C & 5.4967090742 & 0.6585326290 & 6.6307086084 \\
\hline C & 4.0393801748 & -0.1182614265 & 3.4565177889 \\
\hline C & 13.6936023765 & 9.2604112012 & 9.7863355313 \\
\hline C & 11.8776004737 & 9.5708034770 & 0.5109491159 \\
\hline C & 5.5329227395 & -0.2940777168 & 12.4705207613 \\
\hline C & 11.0196820130 & 0.1571325911 & 13.0975525185 \\
\hline C & 6.4481771663 & 9.1853269841 & -0.0480933244 \\
\hline C & 11.1049052169 & 4.7777443180 & 6.1610840432 \\
\hline C & 6.6599256623 & 4.4388573446 & 6.8585863851 \\
\hline C & 11.5413000415 & 2.9392140764 & 7.6526572146 \\
\hline C & 6.0630218059 & 6.4096556329 & 5.6148382995 \\
\hline C & 11.7825715728 & 4.2596513721 & 7.2670458654 \\
\hline C & 5.9579066386 & 5.0303368253 & 5.8047453043 \\
\hline C & 4.5904465263 & 0.9769906205 & 2.7898527633 \\
\hline C & 13.0818054588 & 8.2141585360 & 10.4794449263 \\
\hline C & 9.3716417682 & 4.4569253701 & 4.3228016152 \\
\hline C & 8.2856634967 & 4.7127459600 & 8.8073661538 \\
\hline C & 9.2466252436 & 7.0166895341 & 1.3227145146 \\
\hline C & 8.2727316605 & 2.1916126367 & 11.8517813612 \\
\hline $\mathrm{H}$ & 6.7208419003 & 8.9143512068 & 4.2344681881 \\
\hline $\mathrm{H}$ & 11.0508633885 & 0.0984035488 & 8.9673575964 \\
\hline $\mathrm{H}$ & 11.0262381329 & 2.3305074827 & 13.1432050426 \\
\hline $\mathrm{H}$ & 6.5486085219 & 7.0130185136 & 0.0241946586 \\
\hline $\mathrm{H}$ & 4.2018301449 & 2.9416803243 & 1.9662346859 \\
\hline
\end{tabular}




\begin{tabular}{|c|c|c|c|}
\hline $\mathrm{H}$ & 13.3669260700 & 6.2457230449 & 11.3374947969 \\
\hline $\mathrm{H}$ & 12.7498435255 & 8.8368740678 & 2.3602572267 \\
\hline $\mathrm{H}$ & 4.6018128283 & 0.2945053836 & 10.5926374307 \\
\hline $\mathrm{H}$ & 11.0877258222 & 4.2620617656 & 2.5275746004 \\
\hline $\mathrm{H}$ & 6.5002946031 & 4.7673702692 & 10.5363482943 \\
\hline $\mathrm{H}$ & 10.2595277370 & 3.1630384722 & 1.4265785616 \\
\hline $\mathrm{H}$ & 7.2991751044 & 5.7477940889 & 11.7673325772 \\
\hline $\mathrm{H}$ & 8.1684132007 & 4.3539718121 & 1.6895096633 \\
\hline $\mathrm{H}$ & 9.4277571645 & 4.8311862967 & 11.5033844751 \\
\hline $\mathrm{H}$ & 7.2267594899 & 3.7779091514 & 3.0745850590 \\
\hline $\mathrm{H}$ & 10.3688822119 & 5.4130239460 & 10.1119633948 \\
\hline $\mathrm{H}$ & 10.4199647459 & 1.1422181455 & 7.1494982686 \\
\hline $\mathrm{H}$ & 6.9537452709 & 8.2347090817 & 6.4046831239 \\
\hline $\mathrm{H}$ & 12.6457046514 & 7.4566393887 & 6.9345997892 \\
\hline $\mathrm{H}$ & 4.7821310545 & 1.4764710737 & 6.5302053313 \\
\hline $\mathrm{H}$ & 9.1700976942 & 8.8059665635 & 3.6196232149 \\
\hline $\mathrm{H}$ & 8.6109759448 & 0.3636425019 & 9.6083049097 \\
\hline $\mathrm{H}$ & 11.7372074854 & 8.5756008125 & 0.0937893633 \\
\hline $\mathrm{H}$ & 5.6275198036 & 0.7312042534 & 12.8213587078 \\
\hline $\mathrm{H}$ & 10.5929883984 & 0.0901321546 & 12.0976554507 \\
\hline $\mathrm{H}$ & 6.8769695240 & 9.3312320508 & 0.9435280414 \\
\hline $\mathrm{H}$ & 11.2744306299 & 5.7987672860 & 5.8179979088 \\
\hline $\mathrm{H}$ & 6.5995239403 & 3.3666909108 & 7.0420571253 \\
\hline $\mathrm{H}$ & 12.0471598216 & 2.5026660364 & 8.5124990000 \\
\hline $\mathrm{H}$ & 5.5215337285 & 6.9079415437 & 4.8111352129 \\
\hline $\mathrm{H}$ & 12.4902679212 & 4.8688643185 & 7.8302107091 \\
\hline $\mathrm{H}$ & 5.3312517584 & 4.4314521396 & 5.1424397854 \\
\hline $\mathrm{H}$ & 5.6436595316 & 0.9788066184 & 2.5235296458 \\
\hline $\mathrm{H}$ & 12.0321174440 & 8.2827232910 & 10.7583798364 \\
\hline $\mathrm{H}$ & 11.0908420613 & 6.7999933131 & 1.9887616889 \\
\hline $\mathrm{H}$ & 6.4676566981 & 2.3658942538 & 11.0895031649 \\
\hline $\mathrm{H}$ & 9.7677420991 & 5.4002992010 & 3.9104484677 \\
\hline $\mathrm{H}$ & 7.9292586549 & 3.7389101073 & 9.1807550047 \\
\hline $\mathrm{H}$ & 8.3483416660 & 4.6472444773 & 4.6844284240 \\
\hline $\mathrm{H}$ & 9.3232869405 & 4.5979827461 & 8.4568009231 \\
\hline $\mathrm{H}$ & 8.2461204878 & 6.9581833133 & 1.7757157420 \\
\hline $\mathrm{H}$ & 9.2942710296 & 2.2579370396 & 11.4505361402 \\
\hline $\mathrm{H}$ & 9.3568703225 & 6.1793684709 & 0.6133300397 \\
\hline H & 8.1118480956 & 3.0401604919 & 12.5382880003 \\
\hline H & 9.3124033732 & 7.9653537795 & 0.7584882528 \\
\hline $\mathrm{H}$ & 8.1905973888 & 1.2537263947 & 12.4314029810 \\
\hline B & 4.6738801022 & 5.8622284099 & 1.9826781097 \\
\hline B & 12.8864495032 & 3.3879278304 & 11.1791461386 \\
\hline & LS & & \\
\hline \multicolumn{4}{|c|}{ CELL_PARAMETERS (angstrom) } \\
\hline & $99 \quad 0.035322087$ & 0.039093286 & \\
\hline & 9.813383909 & 0.083875196 & \\
\hline & $61-0.599845278$ & 13.092252820 & \\
\hline \multicolumn{4}{|c|}{ ATOMIC_POSITIONS (angstrom) } \\
\hline $\mathrm{Fe}$ & 8.9336391812 & 1.5795793460 & 4.2432214420 \\
\hline $\mathrm{Fe}$ & 8.3524124605 & 7.6692810795 & 8.9719998573 \\
\hline $\mathrm{F}$ & 5.3337488361 & 4.8989063923 & 1.3075474306 \\
\hline $\mathrm{F}$ & 11.9523028754 & 4.3499543353 & 11.9076736761 \\
\hline $\mathrm{F}$ & 4.0144040925 & 5.1565678037 & 3.2132399446 \\
\hline $\mathrm{F}$ & 13.2716476795 & 4.0922929145 & 10.0019813572 \\
\hline $\mathrm{F}$ & 3.4309203512 & 6.2261318102 & 1.2393864170 \\
\hline $\mathrm{F}$ & 13.8551313604 & 3.0227289174 & 11.9758346897 \\
\hline
\end{tabular}




\begin{tabular}{|c|c|c|c|}
\hline $\mathrm{F}$ & 5.3044908597 & 6.9268927716 & 2.4478006865 \\
\hline $\mathrm{F}$ & 11.9815609717 & 2.3219679381 & 10.7674208104 \\
\hline $\mathrm{O}$ & 8.7755223628 & -0.1319556396 & 5.1844573174 \\
\hline $\mathrm{O}$ & 8.5105295637 & 9.3808164217 & 8.0307643754 \\
\hline 0 & 7.1496977306 & 1.8773284059 & 5.0747186222 \\
\hline 0 & 10.1363540289 & 7.3715320017 & 8.1405030674 \\
\hline $\mathrm{O}$ & 9.9805888326 & 6.9520897963 & 2.5752742434 \\
\hline $\mathrm{O}$ & 7.3054629394 & 2.2967709219 & 10.6399470584 \\
\hline $\mathrm{N}$ & 9.8654842132 & 2.5278856207 & 5.7000462041 \\
\hline $\mathrm{N}$ & 7.4205675578 & 6.7209750975 & 7.5151750967 \\
\hline $\mathrm{N}$ & 9.0961274372 & 3.3423940932 & 3.3003505699 \\
\hline $\mathrm{N}$ & 8.1899242035 & 5.9064663324 & 9.9148707294 \\
\hline $\mathrm{N}$ & 8.0809100462 & 1.0392330520 & 2.5554275999 \\
\hline $\mathrm{N}$ & 9.2051415945 & 8.2096273735 & 10.6597936995 \\
\hline $\mathrm{N}$ & 1.7405946672 & 1.1641738655 & 3.4735008714 \\
\hline $\mathrm{N}$ & 15.5454570386 & 8.0846867069 & 9.7417204292 \\
\hline C & 12.0634116574 & 9.5367039070 & 5.9568691871 \\
\hline C & 5.2226401187 & -0.2878431798 & 7.2583521138 \\
\hline C & 2.4553059798 & 0.0527458603 & 3.7583647374 \\
\hline C & 14.8307457749 & 9.1961148209 & 9.4568565641 \\
\hline C & 2.3130815840 & 2.1594431147 & 2.7554590530 \\
\hline C & 14.9729700567 & 7.0894173108 & 10.4597622463 \\
\hline C & 6.7480396933 & 0.8858556655 & 5.8008762494 \\
\hline C & 10.5380122301 & 8.3630051076 & 7.4143454422 \\
\hline C & 7.1542671772 & 1.9363267656 & 0.5211732666 \\
\hline C & 10.1317845497 & 7.3125339595 & 12.6940478882 \\
\hline C & 3.6431666067 & 2.0794259448 & 2.3398035324 \\
\hline C & 13.6428849813 & 7.1694346455 & 10.8754173779 \\
\hline C & 12.3181522271 & 9.6079823549 & 2.1397523111 \\
\hline C & 4.9678995381 & -0.3591217921 & 11.0754691835 \\
\hline C & 9.9714365122 & 3.8703440272 & 5.4931934254 \\
\hline C & 7.3146152588 & 5.3785166910 & 7.7220278765 \\
\hline C & 10.3548238140 & 3.3609939911 & 2.4748765756 \\
\hline C & 6.9312279581 & 5.8878667271 & 10.7403447262 \\
\hline C & 7.6866330157 & 2.0990892410 & 1.7926967546 \\
\hline C & 9.5994188215 & 7.1497716230 & 11.4225245484 \\
\hline C & 7.8828967141 & 3.4356491322 & 2.4452709931 \\
\hline C & 9.4031548078 & 5.8132113113 & 10.7699499160 \\
\hline C & 10.5509139961 & 1.9772208195 & 6.7220728458 \\
\hline C & 6.7351377098 & 7.2716397528 & 6.4931484548 \\
\hline C & 11.7554743675 & 8.4044700395 & 6.7223911801 \\
\hline C & 5.5305773057 & 0.8443904590 & 6.4928301199 \\
\hline C & 3.7822897589 & -0.0910999085 & 3.3780514788 \\
\hline C & 13.5037621118 & 9.3399608494 & 9.8371698250 \\
\hline C & 11.7812129142 & 9.3772528289 & 0.8738522168 \\
\hline C & 5.5048390744 & -0.1283919090 & 12.3413694771 \\
\hline C & 10.9838037152 & 0.0456368940 & 13.1013152841 \\
\hline C & 6.3022480306 & 9.2032236724 & 0.1139061455 \\
\hline C & 10.7859054804 & 4.6758864757 & 6.2815766827 \\
\hline C & 6.5001462916 & 4.5729742425 & 6.9336446181 \\
\hline C & 11.3622184014 & 2.7293964550 & 7.5689290618 \\
\hline C & 5.9238333696 & 6.5194642631 & 5.6462922391 \\
\hline C & 11.4899638007 & 4.1010165726 & 7.3418595377 \\
\hline C & 5.7960881006 & 5.1478444382 & 5.8733617666 \\
\hline C & 4.3858834677 & 0.9461408464 & 2.6655505577 \\
\hline C & 12.9001684548 & 8.3027199267 & 10.5496711350 \\
\hline C & 9.1313582649 & 4.3827819517 & 4.3651634600 \\
\hline C & 8.1546936374 & 4.8660790591 & 8.8500578443 \\
\hline
\end{tabular}




\begin{tabular}{|c|c|c|c|}
\hline C & 9.0326951677 & 6.9896039787 & 1.5039485293 \\
\hline C & 8.2533566627 & 2.2592567309 & 11.7112729666 \\
\hline $\mathrm{H}$ & 6.2868974982 & 9.1005192472 & 4.3908694804 \\
\hline $\mathrm{H}$ & 10.9991542939 & 0.1483412333 & 8.8243522098 \\
\hline $\mathrm{H}$ & 10.8604023144 & 2.2170796780 & 13.0331359240 \\
\hline $\mathrm{H}$ & 6.4256493887 & 7.0317807381 & 0.1820855784 \\
\hline $\mathrm{H}$ & 4.0949077724 & 2.9094351640 & 1.7965131970 \\
\hline $\mathrm{H}$ & 13.1911439402 & 6.3394255626 & 11.4187079097 \\
\hline $\mathrm{H}$ & 12.6713310229 & 8.7941418978 & 2.7723101877 \\
\hline $\mathrm{H}$ & 4.6147207155 & 0.4547187475 & 10.4429111125 \\
\hline $\mathrm{H}$ & 10.8959187968 & 4.3048507152 & 2.6279778919 \\
\hline $\mathrm{H}$ & 6.3901329742 & 4.9440100030 & 10.5872434099 \\
\hline $\mathrm{H}$ & 10.0796164580 & 3.3264482165 & 1.4077678933 \\
\hline $\mathrm{H}$ & 7.2064353552 & 5.9224128071 & 11.8074531178 \\
\hline $\mathrm{H}$ & 7.9417066523 & 4.2448899769 & 1.7020512593 \\
\hline $\mathrm{H}$ & 9.3443449290 & 5.0039704571 & 11.5131698449 \\
\hline $\mathrm{H}$ & 7.0266232916 & 3.6443010390 & 3.1043349314 \\
\hline $\mathrm{H}$ & 10.2594285514 & 5.6045599803 & 10.1108861778 \\
\hline $\mathrm{H}$ & 10.4403446341 & 0.8978269503 & 6.8281040771 \\
\hline $\mathrm{H}$ & 6.8457072030 & 8.3510339147 & 6.3871172260 \\
\hline $\mathrm{H}$ & 12.4605473881 & 7.5748017350 & 6.7847926197 \\
\hline $\mathrm{H}$ & 4.8255042526 & 1.6740586916 & 6.4304286796 \\
\hline $\mathrm{H}$ & 8.7110637576 & 8.8179334416 & 3.7148135302 \\
\hline $\mathrm{H}$ & 8.5749879808 & 0.4309272026 & 9.5004077710 \\
\hline $\mathrm{H}$ & 11.7142813744 & 8.3536093299 & 0.5123103884 \\
\hline $\mathrm{H}$ & 5.5717702587 & 0.8952512535 & 12.7029106681 \\
\hline $\mathrm{H}$ & 10.5822701193 & -0.1027789915 & 12.0992820607 \\
\hline $\mathrm{H}$ & 6.7037817437 & 9.3516397741 & 1.1159394369 \\
\hline $\mathrm{H}$ & 10.8580773944 & 5.7417545395 & 6.0658714957 \\
\hline $\mathrm{H}$ & 6.4279743776 & 3.5071061786 & 7.1493498061 \\
\hline $\mathrm{H}$ & 11.8932319585 & 2.2496860374 & 8.3903455961 \\
\hline $\mathrm{H}$ & 5.3928196821 & 6.9991743881 & 4.8248757032 \\
\hline $\mathrm{H}$ & 12.1301506681 & 4.7035504986 & 7.9869375032 \\
\hline $\mathrm{H}$ & 5.1559011039 & 4.5453102206 & 5.2282837977 \\
\hline $\mathrm{H}$ & 5.4286580821 & 0.8681081765 & 2.3673066292 \\
\hline $\mathrm{H}$ & 11.8573936631 & 8.3807526240 & 10.8479144781 \\
\hline $\mathrm{H}$ & 10.8657281407 & 6.7677371545 & 2.1888498517 \\
\hline $\mathrm{H}$ & 6.4203236907 & 2.4811235552 & 11.0263716453 \\
\hline $\mathrm{H}$ & 9.4858765838 & 5.3522685012 & 3.9781439354 \\
\hline $\mathrm{H}$ & 7.8001750694 & 3.8965922339 & 9.2370769762 \\
\hline $\mathrm{H}$ & 8.0997011650 & 4.5066038302 & 4.7280843395 \\
\hline $\mathrm{H}$ & 9.1863507258 & 4.7422568700 & 8.4871373525 \\
\hline $\mathrm{H}$ & 8.0282971403 & 6.9298077927 & 1.9475343838 \\
\hline $\mathrm{H}$ & 9.2577547506 & 2.3190529075 & 11.2676873082 \\
\hline $\mathrm{H}$ & 9.1564046539 & 6.1457818307 & 0.8042171778 \\
\hline $\mathrm{H}$ & 8.1296470587 & 3.1030788959 & 12.4110039289 \\
\hline $\mathrm{H}$ & 9.1014341121 & 7.9318169532 & 0.9298799964 \\
\hline $\mathrm{H}$ & 8.1846177183 & 1.3170437565 & 12.2853415005 \\
\hline B & 4.5416246733 & 5.7975364097 & 2.0633586051 \\
\hline B & 12.7444269788 & 3.4513243264 & 11.1518623055 \\
\hline $2-\mathrm{HS}$ & & & \\
\hline \multicolumn{4}{|c|}{ CELL_PARAMETERS (angstrom) } \\
\hline 8.109822677 & $7 \quad-0.009972200$ & 0.055175848 & \\
\hline-3.289587985 & $5 \quad 10.004662098$ & 0.015142858 & \\
\hline-1.355199734 & -2.318877168 & 13.506759190 & \\
\hline \multicolumn{4}{|c|}{ ATOMIC_POSITIONS (crystal) } \\
\hline Fe1 & 0.5512174943 & 0.3125216852 & 0.8238859535 \\
\hline
\end{tabular}




\begin{tabular}{|c|c|c|c|}
\hline Fe1 & 0.4487825057 & 0.6874782848 & 0.1761139465 \\
\hline $\mathrm{O}$ & 0.6742333723 & 0.4886421803 & 0.9190753638 \\
\hline $\mathrm{O}$ & 0.3257666277 & 0.5113578197 & 0.0809246362 \\
\hline 0 & 0.2117784410 & 0.7161749503 & 0.1297250610 \\
\hline 0 & 0.7882215440 & 0.2838250497 & 0.8702749320 \\
\hline $\mathrm{N}$ & 0.4180985537 & 0.1719367212 & 0.9149010401 \\
\hline $\mathrm{N}$ & 0.5819014763 & 0.8280632488 & 0.0850989599 \\
\hline $\mathrm{N}$ & 0.6375902658 & 0.3664691769 & 0.6847935982 \\
\hline $\mathrm{N}$ & 0.3624097342 & 0.6335308231 & 0.3152064018 \\
\hline $\mathrm{N}$ & 0.4253054815 & 0.1169386907 & 0.7178470988 \\
\hline $\mathrm{N}$ & 0.5746945185 & 0.8830612953 & 0.2821529012 \\
\hline $\mathrm{N}$ & 0.3157120379 & 0.3461323216 & 0.7650511482 \\
\hline $\mathrm{N}$ & 0.6842879321 & 0.6538676484 & 0.2349488518 \\
\hline $\mathrm{F}$ & 0.6463869086 & 0.7945807685 & 0.6924112739 \\
\hline $\mathrm{F}$ & 0.3536130914 & 0.2054192315 & 0.3075887261 \\
\hline $\mathrm{F}$ & 0.8556574248 & 0.0070416939 & 0.7435720285 \\
\hline $\mathrm{F}$ & 0.1443425752 & 0.9929582921 & 0.2564279715 \\
\hline $\mathrm{F}$ & 0.9428694538 & 0.8207103995 & 0.6950563844 \\
\hline $\mathrm{F}$ & 0.0571305462 & 0.1792896005 & 0.3049436156 \\
\hline $\mathrm{F}$ & 0.8034034753 & 0.9083313728 & 0.5778072715 \\
\hline $\mathrm{F}$ & 0.1965965247 & 0.0916686272 & 0.4221927285 \\
\hline C & 0.8313819345 & 0.5018449332 & 0.9596267391 \\
\hline C & 0.1686180655 & 0.4981550668 & 0.0403732609 \\
\hline C & 0.1057426598 & 0.6166625955 & 0.0678375287 \\
\hline C & 0.8942573332 & 0.3833374045 & 0.9321624643 \\
\hline C & 0.3638650267 & 0.0389105646 & 0.8750648299 \\
\hline C & 0.6361350033 & 0.9610894424 & 0.1249351701 \\
\hline C & 0.9394315737 & 0.6144736691 & 0.0258053552 \\
\hline C & 0.0605684263 & 0.3855263309 & 0.9741946438 \\
\hline C & 0.3766647783 & 0.2094858314 & 0.0041549885 \\
\hline C & 0.6233352217 & 0.7905141536 & 0.9958450105 \\
\hline C & 0.2158688834 & 0.2475249421 & 0.6908480562 \\
\hline C & 0.7841311166 & 0.7524750579 & 0.3091519438 \\
\hline C & 0.6939468264 & 0.4936648515 & 0.6645746863 \\
\hline C & 0.3060531736 & 0.5063351485 & 0.3354253137 \\
\hline C & 0.5929145186 & 0.2605850603 & 0.6095069790 \\
\hline C & 0.4070854814 & 0.7394149687 & 0.3904930210 \\
\hline C & 0.5992865500 & 0.2795700619 & 0.5115259487 \\
\hline C & 0.4007134500 & 0.7204299091 & 0.4884740513 \\
\hline C & 0.2809347564 & 0.1160503020 & 0.0579410895 \\
\hline C & 0.7190652136 & 0.8839497060 & 0.9420589075 \\
\hline C & 0.7078215105 & 0.5204257935 & 0.5686232470 \\
\hline C & 0.2921784895 & 0.4795742065 & 0.4313767530 \\
\hline C & 0.4200489456 & 0.0067435877 & 0.7757149771 \\
\hline C & 0.5799510244 & 0.9932563943 & 0.2242850229 \\
\hline C & 0.2457141104 & 0.1123824856 & 0.6797542342 \\
\hline C & 0.7542858896 & 0.8876175214 & 0.3202457658 \\
\hline C & 0.2814855172 & 0.4638052357 & 0.7816995163 \\
\hline C & 0.7185144528 & 0.5361947643 & 0.2183004837 \\
\hline C & 0.2248295163 & 0.9785890368 & 0.0174427096 \\
\hline C & 0.7751704987 & 0.0214109632 & 0.9825572884 \\
\hline C & 0.6569689465 & 0.4112869637 & 0.4908022034 \\
\hline C & 0.3430310535 & 0.5887130363 & 0.5091977666 \\
\hline C & 0.5388486544 & 0.1222105885 & 0.6394288075 \\
\hline C & 0.4611513456 & 0.8777893965 & 0.3605711925 \\
\hline C & 0.0817176561 & 0.2661729290 & 0.6293432098 \\
\hline C & 0.9182823369 & 0.7338270420 & 0.3706567902 \\
\hline C & 0.2654397771 & 0.9398032239 & 0.9243549863 \\
\hline
\end{tabular}




$\begin{array}{llll}\mathrm{C} & 0.7345602519 & 0.0601967761 & 0.0756450137 \\ \mathrm{C} & 0.1480751305 & 0.4885812281 & 0.7246338696 \\ \mathrm{C} & 0.8519248695 & 0.5114187719 & 0.2753661304 \\ \mathrm{C} & 0.0482714075 & 0.3882916491 & 0.6461627110 \\ \mathrm{C} & 0.9517285775 & 0.6117083509 & 0.3538372890 \\ \mathrm{H} & 0.8953092480 & 0.7001803525 & 0.0479875531 \\ \mathrm{H} & 0.1046907520 & 0.2998196475 & 0.9520124439 \\ \mathrm{H} & 0.4186680366 & 0.3184789927 & 0.0311095883 \\ \mathrm{H} & 0.5813319934 & 0.6815210073 & 0.9688904077 \\ \mathrm{H} & 0.7269669202 & 0.5760679488 & 0.7272962418 \\ \mathrm{H} & 0.2730330798 & 0.4239320512 & 0.2727037582 \\ \mathrm{H} & 0.5532807199 & 0.1930308618 & 0.4518391050 \\ \mathrm{H} & 0.4467192801 & 0.8069691082 & 0.5481608950 \\ \mathrm{H} & 0.2513723269 & 0.1503179294 & 0.1302541135 \\ \mathrm{H} & 0.7486276441 & 0.8496820856 & 0.8697458785 \\ \mathrm{H} & 0.7539323193 & 0.6250869011 & 0.5561817085 \\ \mathrm{H} & 0.2460676807 & 0.3749130989 & 0.4438182915 \\ \mathrm{H} & 0.5534407109 & 0.0007131384 & 0.7869996829 \\ \mathrm{H} & 0.4465592891 & 0.9992868896 & 0.2130003171 \\ \mathrm{H} & 0.3322984468 & 0.9072183073 & 0.7358525082 \\ \mathrm{H} & 0.6677015232 & 0.0927816927 & 0.2641474918 \\ \mathrm{H} & 0.1526754011 & 0.0486037435 & 0.7215092851 \\ \mathrm{H} & 0.8473245839 & 0.9513962785 & 0.2784907149 \\ \mathrm{H} & 0.2090057177 & 0.0628773648 & 0.6017611126 \\ \mathrm{H} & 0.7909942823 & 0.9371226572 & 0.3982388874 \\ \mathrm{H} & 0.3663160282 & 0.5392821946 & 0.8426715625 \\ \mathrm{H} & 0.6336840018 & 0.4607178054 & 0.1573284375 \\ \mathrm{H} & 0.1516508411 & 0.9031505982 & 0.0587148218 \\ \mathrm{H} & 0.8483491739 & 0.0968494018 & 0.9412851742 \\ \mathrm{H} & 0.6585975643 & 0.4277414086 & 0.4143739879 \\ \mathrm{H} & 0.3414024357 & 0.5722585914 & 0.5856260121 \\ \mathrm{H} & 0.4729626183 & 0.0438843815 & 0.5742634181 \\ \mathrm{H} & 0.5270374117 & 0.9561156185 & 0.4257365819 \\ \mathrm{H} & 0.6575385151 & 0.1023453465 & 0.6693646383 \\ \mathrm{H} & 0.3424614849 & 0.8976546605 & 0.3306353617 \\ \mathrm{H} & 0.0062454268 & 0.1851511507 & 0.5690983835 \\ \mathrm{H} & 0.9937545752 & 0.8148488793 & 0.4309016165 \\ \mathrm{H} & 0.2204119698 & 0.8334572949 & 0.8896756845 \\ \mathrm{H} & 0.7795880452 & 0.1665427051 & 0.1103243155 \\ \mathrm{H} & 0.1240101536 & 0.5849236054 & 0.7403989705 \\ \mathrm{H} & 0.8759898464 & 0.4150763946 & 0.2596010295 \\ \mathrm{H} & 0.9469953270 & 0.4062111684 & 0.5977054494 \\ \mathrm{H} & 0.0530046730 & 0.5937888616 & 0.4022945506 \\ \mathrm{~B} & 0.8123662385 & 0.8824500584 & 0.6769687205 \\ \mathrm{~B} & 0.1876337615 & 0.1175499416 & 0.3230312795 \\ \mathrm{2} & & & \end{array}$

2 -IS

CELL_PARAMETERS (angstrom)

$\begin{array}{rrr}8.684885593 & -0.028100733 & -0.540197911 \\ -3.519860297 & 9.396207881 & 0.583764863 \\ -2.426031283 & -1.721251010 & 13.280158002\end{array}$

ATOMIC_POSITIONS (angstrom)

$\begin{array}{lc}\text { Fe1 } & 1.8062708582 \\ \text { Fe2 } & 1.0653931842 \\ \mathrm{O} & 2.0934042480 \\ \mathrm{O} & 0.8211594168 \\ \mathrm{O} & -0.7651833070 \\ \mathrm{O} & 3.7425806527\end{array}$

1.6335796900

10.7432712338

6.1725866230

2.7778188193

3.1337772388

4.6922599071

12.1045275680

6.6146932554

1.5301233398

2.2035740220

1.2235974981

11.3538846466 


\begin{tabular}{|c|c|c|c|}
\hline$N$ & 0.9470586526 & 0.1126464052 & 11.9849918912 \\
\hline $\mathrm{N}$ & 1.8504682630 & 7.4039977870 & 1.4756002154 \\
\hline $\mathrm{N}$ & 2.5039052875 & 2.3954778350 & 8.8590445977 \\
\hline $\mathrm{N}$ & 0.3606321600 & 5.2718134947 & 4.3688009278 \\
\hline$N$ & 1.4157592040 & -0.0828938448 & 9.3064827958 \\
\hline $\mathrm{N}$ & 1.3165865020 & 7.7171016330 & 4.0300347357 \\
\hline $\mathrm{N}$ & -0.1614157730 & 2.1431102743 & 10.0907345013 \\
\hline $\mathrm{N}$ & 2.8377899071 & 5.6420277430 & 3.3007427406 \\
\hline $\mathrm{F}$ & 1.0715478012 & 6.3567843476 & 9.5061882064 \\
\hline $\mathrm{F}$ & 1.6597503764 & 1.2277960943 & 3.7819962085 \\
\hline $\mathrm{F}$ & 5.5465149490 & -1.0518759750 & 9.6413764705 \\
\hline $\mathrm{F}$ & -2.8203444555 & 8.6312769332 & 3.6724747101 \\
\hline $\mathrm{F}$ & 3.3759685791 & 6.6810325819 & 9.3151055501 \\
\hline $\mathrm{F}$ & -0.6610349030 & 0.9751411821 & 3.8122753728 \\
\hline $\mathrm{F}$ & 1.9158867618 & 7.8715661908 & 7.9498334803 \\
\hline $\mathrm{F}$ & 0.6582111626 & -0.1786953505 & 5.3431044839 \\
\hline C & 3.2742425667 & 3.1495995775 & 12.6523943414 \\
\hline C & -0.3436522639 & 4.6690388691 & 0.9544130140 \\
\hline C & -1.2599736270 & 5.7705265690 & 1.3644350197 \\
\hline C & 4.2114652967 & 2.0508736123 & 12.2187086982 \\
\hline C & 0.7971901517 & -1.1164524909 & 11.4411914146 \\
\hline C & 1.8784985875 & 8.7017776233 & 1.8857826629 \\
\hline C & 6.1164609856 & 5.8146447274 & 0.3096601129 \\
\hline C & -3.1797572627 & 1.9971255730 & 13.3000493709 \\
\hline C & 2.7728525642 & 2.1172178935 & -0.1255802200 \\
\hline C & 0.0419879182 & 5.3485445436 & 13.6048502258 \\
\hline C & -0.7225731316 & 1.2964272991 & 9.2034463036 \\
\hline C & 3.4628515635 & 6.4479557576 & 4.1905509460 \\
\hline C & 2.6646317283 & 3.7032325533 & 8.5824984347 \\
\hline C & 0.2015237399 & 3.9461646697 & 4.5573355952 \\
\hline C & 2.6481657609 & 1.4805388897 & 7.8717494894 \\
\hline C & 0.1204939141 & 6.1220205701 & 5.4068650435 \\
\hline C & 2.9541420252 & 1.8626688371 & 6.5679763261 \\
\hline C & -0.2319648905 & 5.6556626169 & 6.6677023577 \\
\hline C & 2.0095682668 & 1.1851075987 & 0.5661235686 \\
\hline C & 0.6873033048 & 6.2892146086 & 12.8075216565 \\
\hline C & 2.9939151190 & 4.1547791599 & 7.3072068992 \\
\hline C & -0.1927045945 & 3.4181078232 & 5.7860423473 \\
\hline C & 1.5132236410 & -1.3145799519 & 10.1239256475 \\
\hline C & 1.1622559884 & 8.9372330712 & 3.1873269643 \\
\hline C & 0.0582299468 & 0.0906699399 & 8.7211670292 \\
\hline C & 2.6846352554 & 7.6473021060 & 4.6566440504 \\
\hline C & -0.8531195511 & 3.2232794673 & 10.5127449681 \\
\hline C & 3.4812558878 & 4.5543167338 & 2.8186125938 \\
\hline C & -1.6595032008 & 9.3075259990 & 0.6005606061 \\
\hline C & 4.2326447052 & -1.7734160577 & 12.6349969317 \\
\hline C & 3.1322948501 & 3.2177322447 & 6.2839368056 \\
\hline C & -0.3902754799 & 4.2816087732 & 6.8608145303 \\
\hline C & 2.4898992750 & 0.0397997119 & 8.2949288140 \\
\hline C & 0.2382743535 & 7.5742738767 & 5.0466172276 \\
\hline C & -2.0127249937 & 1.5161013259 & 8.7140350699 \\
\hline C & 4.7621306825 & 6.1792517910 & 4.6221787431 \\
\hline C & -3.4795245485 & 7.2949067502 & 12.6571676150 \\
\hline C & 6.0417173338 & 0.2909216951 & 0.5604590409 \\
\hline C & -2.1390372077 & 3.5001797558 & 10.0713504273 \\
\hline C & 4.7817033188 & 4.2457730289 & 3.1900667237 \\
\hline C & -2.7227273292 & 2.6280156363 & 9.1504712699 \\
\hline C & 5.4299280328 & 5.0710036716 & 4.1107314390 \\
\hline
\end{tabular}




\begin{tabular}{|c|c|c|c|}
\hline $\mathrm{H}$ & 5.4327525482 & 6.5897148499 & 0.6561794818 \\
\hline $\mathrm{H}$ & -2.5075302532 & 1.2004608769 & 12.9798644661 \\
\hline $\mathrm{H}$ & 2.9029737687 & 3.1397496102 & 0.2334306328 \\
\hline $\mathrm{H}$ & 0.0275576086 & 4.2883314776 & 13.3534952851 \\
\hline $\mathrm{H}$ & 2.5219283815 & 4.4005155767 & 9.4081474538 \\
\hline $\mathrm{H}$ & 0.4077284343 & 3.3066706000 & 3.7009321483 \\
\hline $\mathrm{H}$ & 3.0052046560 & 1.1179479834 & 5.7755917967 \\
\hline $\mathrm{H}$ & -0.3401223867 & 6.3541043668 & 7.4962318923 \\
\hline $\mathrm{H}$ & 1.5479060717 & 1.4491931684 & 1.5164750775 \\
\hline $\mathrm{H}$ & 1.1655873948 & 5.9764235256 & 11.8792698285 \\
\hline $\mathrm{H}$ & 3.1210979952 & 5.2231340915 & 7.1384076668 \\
\hline $\mathrm{H}$ & -0.3128277714 & 2.3400231833 & 5.8802729822 \\
\hline $\mathrm{H}$ & 2.5812849147 & -1.5022937195 & 10.3178244689 \\
\hline $\mathrm{H}$ & 0.0863688001 & 9.0681315376 & 2.9964943477 \\
\hline $\mathrm{H}$ & -2.4114259157 & 7.2012949463 & 10.1765084575 \\
\hline $\mathrm{H}$ & 5.0556961689 & 0.4450045207 & 3.1140654916 \\
\hline $\mathrm{H}$ & -0.5460100967 & -0.8071158339 & 8.9293748248 \\
\hline $\mathrm{H}$ & 3.2461948872 & 8.5605248437 & 4.4071969915 \\
\hline $\mathrm{H}$ & 0.1422361026 & 0.1479176976 & 7.6227073097 \\
\hline $\mathrm{H}$ & 2.5842854426 & 7.6304507479 & 5.7538119855 \\
\hline $\mathrm{H}$ & -0.3304835654 & 3.8725852260 & 11.2152749344 \\
\hline $\mathrm{H}$ & 2.9014502437 & 3.9459809440 & 2.1282985684 \\
\hline $\mathrm{H}$ & -2.2434546318 & 8.5579229171 & 1.1304819112 \\
\hline $\mathrm{H}$ & 4.7209453203 & -1.0150483463 & 12.0216836239 \\
\hline $\mathrm{H}$ & 3.3598729851 & 3.5305055839 & 5.2665791550 \\
\hline $\mathrm{H}$ & -0.6565777573 & 3.8991089925 & 7.8443118345 \\
\hline $\mathrm{H}$ & 2.3036941498 & -0.5964169416 & 7.4125272239 \\
\hline $\mathrm{H}$ & 0.4240470400 & 8.1939521559 & 5.9383237567 \\
\hline $\mathrm{H}$ & 3.4323587196 & -0.2878030354 & 8.7630907218 \\
\hline $\mathrm{H}$ & -0.7053064685 & 7.8938424572 & 4.5763954646 \\
\hline $\mathrm{H}$ & -2.4540901402 & 0.8024441732 & 8.0168902697 \\
\hline $\mathrm{H}$ & 5.2473100086 & 6.8623703093 & 5.3194032318 \\
\hline $\mathrm{H}$ & -3.6141563928 & 6.3195776947 & 12.1875346648 \\
\hline $\mathrm{H}$ & 6.0761549207 & 1.3158957461 & 0.9310903711 \\
\hline $\mathrm{H}$ & -2.6704308036 & 4.3796379735 & 10.4364112387 \\
\hline $\mathrm{H}$ & 5.2769813863 & 3.3714095254 & 2.7668360895 \\
\hline $\mathrm{H}$ & 4.9659489338 & 2.7954009026 & 8.2267702538 \\
\hline $\mathrm{H}$ & -2.2398871751 & 4.8754848838 & 4.9673535065 \\
\hline B & 2.0988478015 & 7.3139118591 & 9.2607904512 \\
\hline B & 0.5867207375 & 0.3120935868 & 3.9957202484 \\
\hline \multirow{2}{*}{\multicolumn{4}{|c|}{$\begin{array}{l}\text { 2- } \quad \text { LS } \\
\text { CELL_PARAMETERS (angstrom) }\end{array}$}} \\
\hline & & & \\
\hline \multicolumn{4}{|c|}{$\begin{array}{ll}-5.571951934 & -0.029618889\end{array}$} \\
\hline-3.446885498 & 39.022965891 & 0.502962301 & \\
\hline-2.603473756 & -1.802505867 & 13.028325592 & \\
\hline \multicolumn{4}{|c|}{ ATOMIC_POSITIONS (angstrom) } \\
\hline $\mathrm{Fe} 2$ & 1.5113925800 & 1.3664191496 & 10.2393350323 \\
\hline $\mathrm{Fe} 2$ & 1.0102001008 & 5.8244219860 & 2.6258841800 \\
\hline 0 & 1.7938490253 & 2.8555325777 & 11.4763916952 \\
\hline 0 & 0.7277435527 & 4.3353088272 & 1.3888275331 \\
\hline 0 & -0.7964765567 & 6.3174945916 & 2.0376949776 \\
\hline 0 & 3.3180692375 & 0.8733465431 & 10.8275242347 \\
\hline $\mathrm{N}$ & 0.7070925555 & 0.1563417935 & 11.5416187994 \\
\hline $\mathrm{N}$ & 1.8145001761 & 7.0344992075 & 1.3236004054 \\
\hline $\mathrm{N}$ & 2.2150205076 & 2.2381423664 & 8.6363779296 \\
\hline $\mathrm{N}$ & 0.3065722758 & 4.9526984999 & 4.2288412686 \\
\hline $\mathrm{N}$ & 1.2379555525 & -0.1880334341 & 8.9994734522 \\
\hline
\end{tabular}




\begin{tabular}{|c|c|c|c|}
\hline $\mathrm{N}$ & 1.2836373836 & 7.3788745688 & 3.8657457402 \\
\hline $\mathrm{N}$ & -0.2557138806 & 1.9219630182 & 9.7166067824 \\
\hline $\mathrm{N}$ & 2.7773065613 & 5.2688781175 & 3.1486124308 \\
\hline $\mathrm{F}$ & 0.8750998750 & 6.1990110530 & 9.3531880095 \\
\hline $\mathrm{F}$ & 1.6464928058 & 0.9918300825 & 3.5120312028 \\
\hline $\mathrm{F}$ & 5.4240277211 & -0.8678839467 & 9.4375765710 \\
\hline $\mathrm{F}$ & -2.9024352470 & 8.0587249548 & 3.4276426540 \\
\hline $\mathrm{F}$ & 3.2137936681 & 6.2960472615 & 9.3135430536 \\
\hline $\mathrm{F}$ & -0.6922007320 & 0.8947938732 & 3.5516761388 \\
\hline $\mathrm{F}$ & 1.9608749668 & 7.4387294850 & 7.7214446606 \\
\hline $\mathrm{F}$ & 0.5607177399 & -0.2478884168 & 5.1437745479 \\
\hline C & 2.9493023566 & 2.8431474733 & 12.0663699056 \\
\hline C & -0.4277096758 & 4.3476936623 & 0.7988493067 \\
\hline C & -1.3079398628 & 5.4941391546 & 1.1858305585 \\
\hline C & 3.8295325435 & 1.6967019810 & 11.6793886537 \\
\hline C & 0.6697818425 & -1.1463567994 & 11.1513071861 \\
\hline C & 1.8518107999 & 8.3371980350 & 1.7139120318 \\
\hline C & 5.9819317113 & 5.5976807303 & -0.0328374518 \\
\hline C & -3.4603390306 & 1.5931604053 & 12.8980566641 \\
\hline C & 2.6798264814 & 2.3188163477 & -0.3531720746 \\
\hline C & -0.1582340560 & 4.8720247879 & 13.2183913067 \\
\hline C & -0.8902606453 & 1.1153158392 & 8.8354501323 \\
\hline C & 3.4118533516 & 6.0755255644 & 4.0297690850 \\
\hline C & 2.3587080971 & 3.5613309638 & 8.4319895073 \\
\hline C & 0.1628846863 & 3.6295099025 & 4.4332296900 \\
\hline C & 2.4464786034 & 1.3787659832 & 7.6045371836 \\
\hline C & 0.0751140784 & 5.8120751523 & 5.2606820286 \\
\hline C & 2.7972451826 & 1.8329865963 & 6.3399066689 \\
\hline C & -0.2756525019 & 5.3578545384 & 6.5253125443 \\
\hline C & 2.0140446098 & 1.3996570627 & 0.4495916166 \\
\hline C & 0.5075480192 & 5.7911842066 & 12.4156276031 \\
\hline C & 2.7449475872 & 4.0811387148 & 7.1968568101 \\
\hline C & -0.2233549064 & 3.1097024199 & 5.6683624021 \\
\hline C & 1.3773975883 & -1.4052364551 & 9.8502895541 \\
\hline C & 1.1441951505 & 8.5960774391 & 3.0149296497 \\
\hline C & -0.1315218997 & -0.1006832496 & 8.3796475189 \\
\hline C & 2.6531146568 & 7.2915245185 & 4.4855716910 \\
\hline C & -0.8876212397 & 3.0194998758 & 10.1924228150 \\
\hline C & 3.4092138177 & 4.1713415282 & 2.6727964132 \\
\hline C & -1.4542312067 & 9.0803639365 & 0.5711446157 \\
\hline C & 3.9758240433 & -1.8895225414 & 12.2940745913 \\
\hline C & 2.9524035040 & 3.2065363303 & 6.1329968328 \\
\hline C & -0.4308109260 & 3.9843050737 & 6.7322223945 \\
\hline C & 2.3148406541 & -0.0690057607 & 7.9775587340 \\
\hline C & 0.2067520267 & 7.2598468963 & 4.8876604782 \\
\hline C & -2.1861513751 & 1.3947367545 & 8.4032112462 \\
\hline C & 4.7077440558 & 5.7961043802 & 4.4620079670 \\
\hline C & -3.4277452173 & 6.9069852848 & 12.4095693911 \\
\hline C & 5.9493379624 & 0.2838556824 & 0.4556498118 \\
\hline C & -2.1889343939 & 3.3335069289 & 9.8273569905 \\
\hline C & 4.7105273045 & 3.8573339370 & 3.0378621969 \\
\hline C & -2.8462008641 & 2.5072877054 & 8.9143963003 \\
\hline C & 5.3677935516 & 4.6835531613 & 3.9508229044 \\
\hline $\mathrm{H}$ & 5.3333050559 & 6.4186744432 & 0.2726542535 \\
\hline $\mathrm{H}$ & -2.8117123751 & 0.7721666924 & 12.5925649588 \\
\hline $\mathrm{H}$ & 2.7056588549 & 3.3825671200 & -0.1192975539 \\
\hline $\mathrm{H}$ & -0.1840660714 & 3.8082737463 & 12.9845167512 \\
\hline $\mathrm{H}$ & 2.1536787772 & 4.2007274919 & 9.2868411461 \\
\hline
\end{tabular}




\begin{tabular}{lrcr} 
H & 0.3679139036 & 2.9901136437 & \\
$\mathrm{H}$ & 2.9030270299 & 1.1258521277 & 5.5783780662 \\
$\mathrm{H}$ & -0.3814342205 & 6.0649889261 & 7.34663444760 \\
$\mathrm{H}$ & 1.5135344891 & 1.7386907554 & 1.3543923677 \\
$\mathrm{H}$ & 1.0080580890 & 5.4521506486 & 11.5108268605 \\
$\mathrm{H}$ & 2.8515616704 & 5.1594806710 & 7.0908827463 \\
$\mathrm{H}$ & -0.3299689897 & 2.0313604645 & 5.7743364659 \\
$\mathrm{H}$ & 2.4522263756 & -1.5551674952 & 10.0341473311 \\
$\mathrm{H}$ & 0.0693662046 & 8.7460088956 & 2.8310718958 \\
$\mathrm{H}$ & -2.4700437663 & 6.7194006487 & 9.8518075970 \\
$\mathrm{H}$ & 4.9916364979 & 0.4714403523 & 3.0134116078 \\
$\mathrm{H}$ & -0.7073829425 & -0.9993789137 & 8.6456202676 \\
$\mathrm{H}$ & 3.2289757259 & 8.1902197801 & 4.2195989307 \\
$\mathrm{H}$ & -0.0362520667 & -0.0927753933 & 7.2826917597 \\
$\mathrm{H}$ & 2.5578449773 & 7.2836162591 & 5.5825274277 \\
$\mathrm{H}$ & -0.3020043501 & 3.6358818853 & 10.8695595295 \\
$\mathrm{H}$ & 2.8235970309 & 3.5549592502 & 1.9956596827 \\
$\mathrm{H}$ & -1.9559387144 & 8.3396800236 & 1.1936959420 \\
$\mathrm{H}$ & 4.4775315479 & -1.1488386205 & 11.6715232654 \\
$\mathrm{H}$ & 3.2233675323 & 3.5788527711 & 5.1482638419 \\
$\mathrm{H}$ & -0.7017746710 & 3.6119881489 & 7.7169549668 \\
$\mathrm{H}$ & 2.1145323494 & -0.6923473665 & 7.0905034523 \\
$\mathrm{H}$ & 0.4070603054 & 7.8831885686 & 5.7747157637 \\
$\mathrm{H}$ & 3.2538415304 & -0.3949008653 & 8.4512954452 \\
$\mathrm{H}$ & -0.7322489016 & 7.5857421356 & 4.4139237756 \\
$\mathrm{H}$ & -2.6781601119 & 0.7136468061 & 7.7107620859 \\
$\mathrm{H}$ & 5.1997528757 & 6.4771941948 & 5.1544571173 \\
$\mathrm{H}$ & -3.4678412534 & 5.8758430990 & 12.0572817081 \\
$\mathrm{H}$ & 5.9894341387 & 1.3149981704 & 0.8079374918 \\
$\mathrm{H}$ & -2.6763641713 & 4.2144443954 & 10.2468241927 \\
$\mathrm{H}$ & 5.1979569792 & 2.9763967398 & 2.6183950097 \\
$\mathrm{H}$ & 4.7127491792 & 2.7058996637 & 7.9338552400 \\
$\mathrm{H}$ & -2.1911567225 & 4.4849414718 & 4.9313639896 \\
$\mathrm{~B}$ & 2.0095999907 & 7.0255798089 & 9.0998613233 \\
$\mathrm{~B}$ & 0.5119928950 & 0.1652611243 & 3.7653578678 \\
3 & & & \\
& & & \\
\hline
\end{tabular}

3•4DMF HS

CELL_PARAMETERS (angstrom)
$9.008913058-0.001424998$
0.148046797
$3.707526831 \quad 11.546182329$
0.324882711
3.909660970
2.345800336
13.962817220

ATOMIC_POSITIONS (angstrom)

$\begin{array}{lr}\text { Fe1 } & 6.6846479446 \\ \text { Fe1 } & 9.9414530306 \\ \mathrm{O} & 7.5083577399 \\ \mathrm{O} & 9.1177431237 \\ \mathrm{O} & 7.1141349391 \\ \mathrm{O} & 9.5119659255 \\ \mathrm{O} & 4.4859350535 \\ \mathrm{O} & 12.1401658926 \\ \mathrm{O} & 3.6246544865 \\ \mathrm{O} & 13.0014464545 \\ \mathrm{~N} & 4.6934830056 \\ \mathrm{~N} & 11.9326182132 \\ \mathrm{~N} & 6.4230078822 \\ \mathrm{~N} & 10.2030928660 \\ \mathrm{~N} & 5.8579453588 \\ \mathrm{~N} & 10.7681551204\end{array}$

$\begin{array}{rr}4.3550245125 & 4.6864539840 \\ 9.5355332248 & 9.7492931603 \\ 6.3038689548 & 4.7279205895 \\ 7.5866884382 & 9.7078265451 \\ 4.5511148808 & 6.6731231962 \\ 9.3394425122 & 7.7626239374 \\ 8.6194151392 & 1.0411878702 \\ 5.2711425815 & 13.3945591693 \\ 1.9811849048 & 7.6403640591 \\ 11.9093730204 & 6.7953826762 \\ 5.2240551446 & 4.5131990606 \\ 8.6665029367 & 9.9225480945 \\ 4.5185222952 & 2.4822541712 \\ 9.3720350278 & 11.9534925472 \\ 2.4363696158 & 4.3154080226 \\ 11.4541877073 & 10.1203386914\end{array}$




\begin{tabular}{|c|c|c|c|}
\hline $\mathrm{N}$ & 8.6152085545 & 3.6735825715 & 3.9395773903 \\
\hline $\mathrm{N}$ & 8.0108924197 & 10.2169751657 & 10.4961697530 \\
\hline $\mathrm{N}$ & 4.1449777014 & 10.7491608101 & 1.8567175076 \\
\hline $\mathrm{N}$ & 12.4811231248 & 3.1413969766 & 12.5790289129 \\
\hline $\mathrm{N}$ & 3.2655222295 & 3.9222159905 & 8.8441035640 \\
\hline $\mathrm{N}$ & 13.3605782741 & 9.9683413325 & 5.5916431504 \\
\hline C & 3.7736872872 & 5.2845068945 & 5.4944981290 \\
\hline C & 12.8524135045 & 8.6060507736 & 8.9412485970 \\
\hline C & 11.5171383890 & 5.8255297157 & 5.4482760416 \\
\hline C & 5.1089625542 & 8.0650278814 & 8.9874702726 \\
\hline C & 11.1864067638 & 6.3136471403 & 4.1820280357 \\
\hline C & 5.4396938930 & 7.5769105269 & 10.2537186881 \\
\hline C & 3.1198696227 & 6.2304774041 & 3.0108363729 \\
\hline C & 13.5062310341 & 7.6600802630 & 11.4249103508 \\
\hline C & 4.3767101074 & 5.6838197963 & 3.2828447454 \\
\hline C & 12.2493909366 & 8.2067381799 & 11.1529017860 \\
\hline C & 5.4720259131 & 5.6330240801 & 2.2443043923 \\
\hline C & 11.1540749447 & 8.2575335870 & 12.1914423348 \\
\hline C & 5.9122623136 & 3.2167562227 & 1.9680704486 \\
\hline C & 10.7138386617 & 10.6738015145 & 12.4676766947 \\
\hline C & 5.0723096602 & 0.9309526154 & 2.6271177578 \\
\hline C & 11.5537909604 & 12.9596050008 & 11.8086291710 \\
\hline C & 8.5187224397 & 11.5093746605 & 3.9213797301 \\
\hline C & 8.1073784464 & 2.3811830937 & 10.5143670004 \\
\hline C & 8.7811841481 & 11.7994564865 & 5.2626027356 \\
\hline C & 7.8449164081 & 2.0911010734 & 9.1731439840 \\
\hline C & 5.5905335293 & 1.5034114279 & 5.2536957372 \\
\hline C & 11.0355676249 & 12.3871463253 & 9.1820509977 \\
\hline C & 5.6050817996 & 2.1644076957 & 3.0162618055 \\
\hline C & 11.0210188449 & 11.7261501436 & 11.4194849230 \\
\hline C & 7.7802721192 & 4.8321014489 & 1.9659417581 \\
\hline C & 8.8458286803 & 9.0584561833 & 12.4698047614 \\
\hline C & 8.8475266876 & 4.0193065967 & 2.6529758874 \\
\hline C & 7.7785743971 & 9.8712514846 & 11.7827712656 \\
\hline C & 10.0409192587 & 3.6846145968 & 2.0137247846 \\
\hline C & 6.5851815467 & 10.2059427612 & 12.4220221414 \\
\hline C & 2.0040745974 & 2.9820171538 & 2.5733019080 \\
\hline C & 14.6220262614 & 10.9085405134 & 11.8624448191 \\
\hline C & 10.7699889363 & 2.6246232713 & 4.0486751427 \\
\hline C & 5.8561120389 & 11.2659344659 & 10.3870720005 \\
\hline C & 9.5531386842 & 2.9884435589 & 4.6201970882 \\
\hline C & 7.0729620630 & 10.9021137651 & 9.8155496292 \\
\hline C & 7.9148667154 & 6.6597558368 & 5.8842465631 \\
\hline C & 8.7112341433 & 7.2308018303 & 8.5515001650 \\
\hline C & 7.6806818187 & 5.6711990609 & 7.0051870589 \\
\hline C & 8.9454191506 & 8.2193589504 & 7.4305596778 \\
\hline C & 8.5529582830 & 7.8928553048 & 6.1361518154 \\
\hline C & 8.0731426913 & 5.9977024324 & 8.2995953279 \\
\hline C & 12.1941710891 & 11.7306207971 & 2.4818809038 \\
\hline C & 4.4319296288 & 2.1599367251 & 11.9538654059 \\
\hline C & 3.7390919903 & 9.5181914925 & 1.4671819791 \\
\hline C & 12.8870087801 & 4.3723661221 & 12.9685644366 \\
\hline C & 5.5499069706 & 11.1219545485 & 1.8257488389 \\
\hline C & 11.0761939738 & 2.7686032407 & 12.6099980992 \\
\hline C & 11.3133285545 & 4.8908349076 & 9.4964497106 \\
\hline C & 5.3127721937 & 8.9997224154 & 4.9392970078 \\
\hline C & 11.8806078904 & 2.8275376784 & 8.2897650251 \\
\hline C & 4.7454927248 & 11.0630198167 & 6.1459816941 \\
\hline
\end{tabular}




\begin{tabular}{|c|c|c|c|}
\hline C & 4.6774374042 & 4.2091392227 & 9.0543054344 \\
\hline C & 11.9486636443 & 9.6814186165 & 5.3814412997 \\
\hline $\mathrm{H}$ & 4.0803343165 & 4.8739657801 & 6.4575136766 \\
\hline $\mathrm{H}$ & 12.5457667927 & 9.0165919570 & 7.9782334698 \\
\hline $\mathrm{H}$ & 10.8029745969 & 5.8612514727 & 6.2721214336 \\
\hline $\mathrm{H}$ & 5.8231260103 & 8.0293061244 & 8.1636248751 \\
\hline $\mathrm{H}$ & 10.2088161894 & 6.7539193105 & 3.9864879457 \\
\hline $\mathrm{H}$ & 6.4172848034 & 7.1366383567 & 10.4492587846 \\
\hline $\mathrm{H}$ & 2.8892721836 & 6.5913004816 & 2.0085319748 \\
\hline $\mathrm{H}$ & 13.7368286926 & 7.2992571155 & 12.4272143383 \\
\hline $\mathrm{H}$ & 5.0252483423 & 5.5667162018 & 1.2395484302 \\
\hline $\mathrm{H}$ & 11.6008523466 & 8.3238410862 & 13.1961980795 \\
\hline $\mathrm{H}$ & 6.0337035507 & 6.5805236696 & 2.2915497877 \\
\hline $\mathrm{H}$ & 10.5923973663 & 7.3100340326 & 12.1441971480 \\
\hline $\mathrm{H}$ & 6.6386105742 & 2.8055714328 & 1.2465662323 \\
\hline $\mathrm{H}$ & 9.9874903976 & 11.0849864414 & 13.1891807082 \\
\hline $\mathrm{H}$ & 5.0015578350 & 3.3934097762 & 1.3732404560 \\
\hline $\mathrm{H}$ & 11.6245425859 & 10.4971475118 & 13.0625060494 \\
\hline $\mathrm{H}$ & 4.8731786489 & 0.7348260247 & 1.5716420504 \\
\hline $\mathrm{H}$ & 11.7529218137 & 13.1557313918 & 12.8641044586 \\
\hline $\mathrm{H}$ & 8.1318515570 & 10.5344292425 & 3.6230930082 \\
\hline $\mathrm{H}$ & 8.4942492460 & 3.3561282526 & 10.8126537140 \\
\hline $\mathrm{H}$ & 8.6114495324 & 11.0720350033 & 6.0579944271 \\
\hline $\mathrm{H}$ & 8.0146512098 & 2.8185225938 & 8.3777518848 \\
\hline $\mathrm{H}$ & 5.7777787656 & 1.7952843115 & 6.2874258628 \\
\hline $\mathrm{H}$ & 10.8483221753 & 12.0952736138 & 8.1483208715 \\
\hline $\mathrm{H}$ & 7.8481333232 & 4.6660402842 & 0.8810753718 \\
\hline $\mathrm{H}$ & 8.7779673366 & 9.2245169863 & 13.5546710342 \\
\hline $\mathrm{H}$ & 7.9742726545 & 5.8961524123 & 2.1664744283 \\
\hline $\mathrm{H}$ & 8.6518284303 & 7.9944056690 & 12.2692727246 \\
\hline $\mathrm{H}$ & 10.2064277291 & 3.9683990359 & 0.9755995012 \\
\hline $\mathrm{H}$ & 6.4196730763 & 9.9221583222 & 13.4601474248 \\
\hline $\mathrm{H}$ & 2.9304044653 & 2.7009500378 & 2.0729005467 \\
\hline $\mathrm{H}$ & 13.6956965100 & 11.1896076994 & 12.3628465966 \\
\hline $\mathrm{H}$ & 2.4918921747 & 2.0732167204 & 4.4924563232 \\
\hline $\mathrm{H}$ & 14.1342087388 & 11.8173411188 & 9.9432904097 \\
\hline $\mathrm{H}$ & 9.3043137910 & 2.7049370849 & 5.6445836550 \\
\hline $\mathrm{H}$ & 7.3217868955 & 11.1856203411 & 8.7911626511 \\
\hline $\mathrm{H}$ & 8.7429016812 & 8.5676823078 & 5.2994938279 \\
\hline $\mathrm{H}$ & 7.8831992931 & 5.3228754294 & 9.1362533164 \\
\hline $\mathrm{H}$ & 11.1853236082 & 11.3026302696 & 2.4402769341 \\
\hline $\mathrm{H}$ & 5.4407774492 & 2.5879277257 & 11.9954702165 \\
\hline $\mathrm{H}$ & 8.5180002112 & 1.0907732910 & 1.5344797494 \\
\hline $\mathrm{H}$ & 8.1081007640 & 12.7997844462 & 12.9012673949 \\
\hline $\mathrm{H}$ & 12.4160041442 & 12.0194885862 & 3.5213819396 \\
\hline $\mathrm{H}$ & 4.2100966766 & 1.8710691107 & 10.9143645817 \\
\hline $\mathrm{H}$ & 11.6542340070 & 9.3756565825 & 1.7219400689 \\
\hline $\mathrm{H}$ & 4.9718669332 & 4.5149014113 & 12.7138065651 \\
\hline $\mathrm{H}$ & 5.7356601316 & 11.9180249186 & 1.0885835923 \\
\hline $\mathrm{H}$ & 10.8904407377 & 1.9725328948 & 13.3471629849 \\
\hline $\mathrm{H}$ & 6.1498384512 & 10.2384040825 & 1.5779993985 \\
\hline $\mathrm{H}$ & 10.4762623517 & 3.6521534136 & 12.8577473248 \\
\hline $\mathrm{H}$ & 2.1432791982 & -0.0457638033 & 2.4892993554 \\
\hline $\mathrm{H}$ & 14.4828216596 & 13.9363214704 & 11.9464473717 \\
\hline $\mathrm{H}$ & 10.2956402370 & 4.5803875347 & 9.2298544442 \\
\hline $\mathrm{H}$ & 6.3304606217 & 9.3101701324 & 5.2058922839 \\
\hline $\mathrm{H}$ & 11.4962742983 & 5.8832557518 & 9.0550666218 \\
\hline $\mathrm{H}$ & 5.1298264255 & 8.0073019154 & 5.3806801041 \\
\hline
\end{tabular}




\begin{tabular}{|c|c|c|c|}
\hline $\mathrm{H}$ & 11.3776749662 & 4.9871192005 & 10.5902019970 \\
\hline $\mathrm{H}$ & 5.2484259160 & 8.9034381225 & 3.8455447226 \\
\hline $\mathrm{H}$ & 10.7733951935 & 2.7531329952 & 8.2016561022 \\
\hline $\mathrm{H}$ & 5.8527054642 & 11.1374246720 & 6.2340906225 \\
\hline $\mathrm{H}$ & 4.8926520706 & 4.3494209517 & 10.1223907318 \\
\hline $\mathrm{H}$ & 11.7334489221 & 9.5411367164 & 4.3133559975 \\
\hline $\mathrm{H}$ & 4.9668910252 & 5.1350490779 & 8.5337507166 \\
\hline $\mathrm{H}$ & 11.6592098091 & 8.7555089344 & 5.9019960189 \\
\hline $\mathrm{H}$ & 5.2732639241 & 3.3797556052 & 8.6604730644 \\
\hline $\mathrm{H}$ & 11.3528368789 & 10.5108018898 & 5.7752736579 \\
\hline $\mathrm{F}$ & 9.0429555062 & 9.7506193886 & 0.8077359658 \\
\hline $\mathrm{F}$ & 7.5831452350 & 4.1399382085 & 13.6280103450 \\
\hline $\mathrm{F}$ & 9.4207132710 & 7.4585581866 & 0.9163661590 \\
\hline $\mathrm{F}$ & 7.2053875145 & 6.4319994363 & 13.5193803086 \\
\hline $\mathrm{F}$ & 9.7473369318 & 8.7853882247 & 2.8031248806 \\
\hline $\mathrm{F}$ & 6.8787638095 & 5.1051693724 & 11.6326214312 \\
\hline $\mathrm{F}$ & 7.5961648249 & 8.3912423853 & 2.0205367967 \\
\hline $\mathrm{F}$ & 9.0299360339 & 5.4993152819 & 12.4152099314 \\
\hline B & 8.9452291898 & 8.5933332341 & 1.6398075413 \\
\hline B & 7.6808716388 & 5.2972244156 & 12.7959390819 \\
\hline \multicolumn{4}{|c|}{$\begin{array}{l}\text { 3•4DMF -iS } \\
\text { CELL PARAMETERS (angstrom) }\end{array}$} \\
\hline \multicolumn{4}{|c|}{ CELL_PARAMETERS (angstrom) } \\
\hline & $74 \quad-0.009740739$ & 0.060307150 & \\
\hline & 11.418306140 & 0.152016258 & \\
\hline & 2.161224580 & 13.904163208 & \\
\hline \multicolumn{4}{|c|}{ ATOMIC_POSITIONS (crystal) } \\
\hline $\mathrm{Fe}$ & 0.4735923440 & 0.3211472738 & 0.3247430129 \\
\hline $\mathrm{Fe}$ & 0.5253089086 & 0.6744219039 & 0.6967664861 \\
\hline 0 & 0.4952485941 & 0.4881433773 & 0.3294704518 \\
\hline 0 & 0.5068059955 & 0.5184114429 & 0.6912613938 \\
\hline 0 & 0.4633579126 & 0.3050906774 & 0.4675997608 \\
\hline 0 & 0.5411430751 & 0.6980963107 & 0.5565386829 \\
\hline 0 & 0.1695590637 & 0.7353421303 & 0.0557641476 \\
\hline 0 & 0.8243815849 & 0.2638553222 & 0.9461332442 \\
\hline 0 & 0.1386669314 & 0.0515624538 & 0.5304249968 \\
\hline 0 & 0.8548186864 & 0.9519732518 & 0.4601800823 \\
\hline $\mathrm{N}$ & 0.2249461095 & 0.3982623908 & 0.3139207391 \\
\hline $\mathrm{N}$ & 0.7548035596 & 0.6077447458 & 0.6932599648 \\
\hline $\mathrm{N}$ & 0.4910941296 & 0.3632183586 & 0.1661423051 \\
\hline $\mathrm{N}$ & 0.5047257330 & 0.6376570433 & 0.8389332797 \\
\hline $\mathrm{N}$ & 0.4598875934 & 0.1565057124 & 0.3022976209 \\
\hline $\mathrm{N}$ & 0.5397699229 & 0.8284504405 & 0.7054391809 \\
\hline $\mathrm{N}$ & 0.7308377992 & 0.2718875543 & 0.2700791737 \\
\hline $\mathrm{N}$ & 0.2908174585 & 0.7278574610 & 0.7283845542 \\
\hline $\mathrm{N}$ & 0.0362315665 & 0.9114071811 & 0.1122384686 \\
\hline $\mathrm{N}$ & 0.9597462085 & 0.0886379483 & 0.8884537957 \\
\hline $\mathrm{N}$ & 0.0075015243 & 0.2003421216 & 0.6218314395 \\
\hline $\mathrm{N}$ & 0.9972520575 & 0.7977957062 & 0.3760526341 \\
\hline C & 0.0972211835 & 0.3906793284 & 0.3862631722 \\
\hline C & 0.8766985055 & 0.6190878684 & 0.6173441734 \\
\hline C & 0.9408615345 & 0.4398168720 & 0.3734719332 \\
\hline C & 0.0359460975 & 0.5676824554 & 0.6239734250 \\
\hline C & 0.9158019665 & 0.4985043051 & 0.2818798557 \\
\hline C & 0.0706422139 & 0.5036897092 & 0.7123101741 \\
\hline C & 0.0478855203 & 0.5047052363 & 0.2065380347 \\
\hline C & 0.9448010935 & 0.4933725533 & 0.7912283079 \\
\hline C & 0.2016876421 & 0.4539120529 & 0.2248885825 \\
\hline
\end{tabular}




\begin{tabular}{|c|c|c|c|}
\hline C & 0.7879749937 & 0.5454535799 & 0.7794736270 \\
\hline C & 0.3501996607 & 0.4630972954 & 0.1481962967 \\
\hline C & 0.6434904156 & 0.5347705584 & 0.8578833777 \\
\hline C & 0.4941068014 & 0.2547920821 & 0.1316583971 \\
\hline C & 0.5058507288 & 0.7442042144 & 0.8760908648 \\
\hline C & 0.4652614681 & 0.0452991170 & 0.1841560422 \\
\hline C & 0.5307287820 & 0.9550545634 & 0.8137315435 \\
\hline C & 0.4470446365 & 0.9480673846 & 0.2562356987 \\
\hline C & 0.5513609419 & 0.0461521271 & 0.7360451167 \\
\hline C & 0.4342664653 & 0.9568957762 & 0.3520761693 \\
\hline C & 0.5676263447 & 0.0262632677 & 0.6427108971 \\
\hline C & 0.4410295331 & 0.0627504002 & 0.3720391914 \\
\hline C & 0.5612008972 & 0.9165567861 & 0.6302071278 \\
\hline C & 0.4718349514 & 0.1489823191 & 0.2093614823 \\
\hline C & 0.5254247744 & 0.8469799041 & 0.7962719418 \\
\hline C & 0.6435589630 & 0.3986539916 & 0.1270465501 \\
\hline C & 0.3490640809 & 0.6052302459 & 0.8776387659 \\
\hline C & 0.7753809238 & 0.3189224902 & 0.1764926272 \\
\hline C & 0.2281624135 & 0.6848318930 & 0.8208621182 \\
\hline C & 0.9359257673 & 0.2980314817 & 0.1295453575 \\
\hline C & 0.0637263940 & 0.7105998240 & 0.8572155760 \\
\hline C & 0.0523828767 & 0.2269918226 & 0.1805661778 \\
\hline C & 0.9613510725 & 0.7816484088 & 0.7967741098 \\
\hline C & 1.0060114349 & 0.1783569593 & 0.2770874505 \\
\hline C & 0.0258337302 & 0.8265474294 & 0.7022394496 \\
\hline C & 0.8434226377 & 0.2029602169 & 0.3191697102 \\
\hline C & 0.1915845816 & 0.7983123804 & 0.6706939823 \\
\hline C & 0.4993794538 & 0.5005792703 & 0.4140363405 \\
\hline C & 0.5020456554 & 0.5058824268 & 0.6066957972 \\
\hline C & 0.4796499153 & 0.3969649212 & 0.4931942167 \\
\hline C & 0.5227180751 & 0.6085781329 & 0.5287563170 \\
\hline C & 0.5220374309 & 0.6027672442 & 0.4340996564 \\
\hline C & 0.4800571432 & 0.4031445794 & 0.5874562530 \\
\hline C & 0.8825917390 & 0.9917511914 & 0.1453201741 \\
\hline C & 0.1142137952 & 0.0084328628 & 0.8566248803 \\
\hline C & 0.0438624095 & 0.8089230684 & 0.0852986186 \\
\hline C & 0.9505993453 & 0.1907154290 & 0.9166552577 \\
\hline C & 0.1807235347 & 0.9436719969 & 0.1100894604 \\
\hline C & 0.8162442189 & 0.0560220929 & 0.8889153755 \\
\hline C & 0.8567218544 & 0.2818531032 & 0.6579452617 \\
\hline C & 0.1521755444 & 0.7194359046 & 0.3417242246 \\
\hline C & 1.0146180093 & 0.1202720477 & 0.5675331188 \\
\hline C & -0.0173407707 & 0.8840497659 & 0.4263271988 \\
\hline C & 0.1527713186 & 0.2126320505 & 0.6405743705 \\
\hline C & 0.8566336624 & 0.7770141776 & 0.3582141895 \\
\hline $\mathrm{H}$ & 0.1239778593 & 0.3431457351 & 0.4560109692 \\
\hline $\mathrm{H}$ & 0.8415810700 & 0.6714888472 & 0.5509491345 \\
\hline $\mathrm{H}$ & 0.8404594957 & 0.4324210457 & 0.4340049014 \\
\hline $\mathrm{H}$ & 0.1313217904 & 0.5786259375 & 0.5609047676 \\
\hline $\mathrm{H}$ & 0.7951463851 & 0.5389149441 & 0.2688581509 \\
\hline $\mathrm{H}$ & 0.1937092565 & 0.4623374596 & 0.7205217636 \\
\hline $\mathrm{H}$ & 0.0323412803 & 0.5489256066 & 0.1340249877 \\
\hline $\mathrm{H}$ & 0.9677204020 & 0.4449749664 & 0.8614388736 \\
\hline $\mathrm{H}$ & 0.3262231001 & 0.4689541992 & 0.0766119395 \\
\hline $\mathrm{H}$ & 0.6682920694 & 0.5286874019 & 0.9291552657 \\
\hline $\mathrm{H}$ & 0.3777149487 & 0.5464309745 & 0.1485275365 \\
\hline $\mathrm{H}$ & 0.6129164397 & 0.4528061471 & 0.8572796266 \\
\hline $\mathrm{H}$ & 0.6079060484 & 0.2282469943 & 0.0799616510 \\
\hline
\end{tabular}




\begin{tabular}{|c|c|c|c|}
\hline $\mathrm{H}$ & 0.3952889384 & 0.7693958194 & 0.9304103746 \\
\hline $\mathrm{H}$ & 0.4013834132 & 0.2769663659 & 0.0888210729 \\
\hline $\mathrm{H}$ & 0.6036703017 & 0.7199546432 & 0.9152036138 \\
\hline $\mathrm{H}$ & 0.4754550200 & 0.0409820265 & 0.1086034722 \\
\hline $\mathrm{H}$ & 0.5180076053 & 0.9673237304 & 0.8876221991 \\
\hline $\mathrm{H}$ & 0.4451878358 & 0.8659718523 & 0.2370685737 \\
\hline $\mathrm{H}$ & 0.5523460320 & 0.1317651027 & 0.7491396304 \\
\hline $\mathrm{H}$ & 0.4200393591 & 0.8834600837 & 0.4110522821 \\
\hline $\mathrm{H}$ & 0.5840358418 & 0.0947622316 & 0.5797803707 \\
\hline $\mathrm{H}$ & 0.4285699486 & 0.0752244935 & 0.4457442155 \\
\hline $\mathrm{H}$ & 0.5749839936 & 0.8954161863 & 0.5591557857 \\
\hline $\mathrm{H}$ & 0.6832158126 & 0.3982766315 & 0.0489306481 \\
\hline $\mathrm{H}$ & 0.3048931984 & 0.6110040356 & 0.9547216034 \\
\hline $\mathrm{H}$ & 0.6216751707 & 0.4895812609 & 0.1400549299 \\
\hline $\mathrm{H}$ & 0.3691361155 & 0.5132970728 & 0.8677928856 \\
\hline $\mathrm{H}$ & 0.9693188914 & 0.3365291415 & 0.0540827255 \\
\hline $\mathrm{H}$ & 0.0164589360 & 0.6752652557 & 0.9316375353 \\
\hline $\mathrm{H}$ & 0.1778670043 & 0.2088893141 & 0.1438511355 \\
\hline $\mathrm{H}$ & 0.8322836958 & 0.8027392049 & 0.8241677621 \\
\hline $\mathrm{H}$ & 0.0928824711 & 0.1221055184 & 0.3198465718 \\
\hline $\mathrm{H}$ & 0.9501857527 & 0.8829503385 & 0.6527340064 \\
\hline $\mathrm{H}$ & 0.8007895477 & 0.1649043871 & 0.3937418633 \\
\hline $\mathrm{H}$ & 0.2481965351 & 0.8329220056 & 0.5981887981 \\
\hline $\mathrm{H}$ & 0.5410107196 & 0.6752997616 & 0.3750387578 \\
\hline $\mathrm{H}$ & 0.4620344329 & 0.3304550444 & 0.6465603193 \\
\hline $\mathrm{H}$ & 0.7862109239 & 0.9553022926 & 0.1422739061 \\
\hline $\mathrm{H}$ & 0.2097967252 & 0.0459076906 & 0.8593765930 \\
\hline $\mathrm{H}$ & 0.8707666736 & 0.0796113998 & 0.0995384836 \\
\hline $\mathrm{H}$ & 0.1264067789 & 0.9210600363 & 0.9031963042 \\
\hline $\mathrm{H}$ & 0.8701587988 & 1.0029262706 & 0.2201602651 \\
\hline $\mathrm{H}$ & 0.1277614210 & -0.0042099822 & 0.7820732244 \\
\hline $\mathrm{H}$ & 0.9241963114 & 0.7956935661 & 0.0927265863 \\
\hline $\mathrm{H}$ & 0.0700313881 & 0.2038629494 & 0.9103799140 \\
\hline $\mathrm{H}$ & 0.1928100958 & 1.0233215011 & 0.0561585414 \\
\hline $\mathrm{H}$ & 0.8044104176 & -0.0244814213 & 0.9418314596 \\
\hline $\mathrm{H}$ & 0.2848493560 & 0.8692256622 & 0.0930933572 \\
\hline $\mathrm{H}$ & 0.7113923273 & 0.1298438832 & 0.9062583235 \\
\hline $\mathrm{H}$ & 0.1754795037 & -0.0367329171 & 0.1811863429 \\
\hline $\mathrm{H}$ & 0.8227402775 & 1.0377783433 & 0.8171053884 \\
\hline $\mathrm{H}$ & 0.7585844921 & 0.2645896642 & 0.6365207148 \\
\hline $\mathrm{H}$ & 0.2464240005 & 0.7415935455 & 0.3633799666 \\
\hline $\mathrm{H}$ & 0.8594605875 & 0.3745316690 & 0.6278498061 \\
\hline $\mathrm{H}$ & 0.1545402919 & 0.6262180443 & 0.3729313552 \\
\hline $\mathrm{H}$ & 0.8317226986 & 0.2720754965 & 0.7371040399 \\
\hline $\mathrm{H}$ & 0.1794030794 & 0.7271978185 & 0.2626228239 \\
\hline $\mathrm{H}$ & 0.8946951961 & 0.1207952120 & 0.5592201462 \\
\hline $\mathrm{H}$ & 0.0996882465 & 0.8894842928 & 0.4348151663 \\
\hline $\mathrm{H}$ & 0.1358159158 & 0.2194739145 & 0.7167041012 \\
\hline $\mathrm{H}$ & 0.8698041580 & 0.7768210705 & 0.2810994673 \\
\hline $\mathrm{H}$ & 0.1856136271 & 0.2928898722 & 0.5964442033 \\
\hline $\mathrm{H}$ & 0.8391952559 & 0.6902692633 & 0.3976372402 \\
\hline $\mathrm{H}$ & 0.2510595072 & 0.1352243110 & 0.6226744793 \\
\hline $\mathrm{H}$ & 0.7513747584 & 0.8469878104 & 0.3825019377 \\
\hline $\mathrm{F}$ & 0.6445401520 & 0.8419454549 & 0.0312842396 \\
\hline $\mathrm{F}$ & 0.3500889689 & 0.1594744809 & 0.9670563332 \\
\hline $\mathrm{F}$ & 0.7718518419 & 0.6406336826 & 0.0387006951 \\
\hline $\mathrm{F}$ & 0.2285423832 & 0.3617670141 & 0.9573381075 \\
\hline $\mathrm{F}$ & 0.7086431049 & 0.7291124213 & 0.1753884759 \\
\hline
\end{tabular}




\begin{tabular}{|c|c|c|}
\hline F & 0.2895355986 & 0.271600 \\
\hline $\mathrm{F}$ & 0.5063434457 & 0.702089 \\
\hline $\mathrm{F}$ & 0.4937273768 & $0.2956 \epsilon$ \\
\hline B & 0.6572864383 & 0.7282 \\
\hline B & 0.3412697241 & 0.2724 \\
\hline 3•4DMF & & \\
\hline \multicolumn{3}{|c|}{ CELL_PARAMETERS (angstrom) } \\
\hline 8.936202075 & -0.030161120 & 0.00557911 \\
\hline 3.641590456 & 11.399372180 & 0.0021054 \\
\hline 3.654861547 & 1.998579243 & 13.8013941 \\
\hline
\end{tabular}

ATOMIC_POSITIONS (angstrom)

\begin{tabular}{|c|c|c|c|}
\hline $\mathrm{Fe} 2$ & 6.5674068559 & 4.3803806083 & 4.2332964315 \\
\hline $\mathrm{Fe} 2$ & 9.6652473307 & 8.9874097551 & 9.5757827264 \\
\hline 0 & 7.3375828554 & 6.1836720066 & 4.3710484862 \\
\hline 0 & 8.8950712216 & 7.1841180171 & 9.4380306716 \\
\hline 0 & 6.8626071249 & 4.3860261834 & 6.1776980631 \\
\hline 0 & 9.3700469530 & 8.9817638403 & 7.6313810937 \\
\hline 0 & 4.4164760484 & 8.4957700932 & 0.7495506128 \\
\hline 0 & 11.8161781109 & 4.8720202563 & 13.0595284414 \\
\hline 0 & 3.3978297538 & 1.5775330714 & 7.3517637174 \\
\hline 0 & 12.8348244046 & 11.7902574872 & 6.4573150291 \\
\hline $\mathrm{N}$ & 4.7631896819 & 5.1511484182 & 4.2821531239 \\
\hline $\mathrm{N}$ & 11.4694647452 & 8.2166422846 & 9.5269260331 \\
\hline $\mathrm{N}$ & 6.3484732196 & 4.5023469806 & 2.2608618051 \\
\hline $\mathrm{N}$ & 9.8841807494 & 8.8654429834 & 11.5482169413 \\
\hline $\mathrm{N}$ & 5.8398298214 & 2.6001999916 & 4.0747942807 \\
\hline $\mathrm{N}$ & 10.3928238808 & 10.7675899733 & 9.7342844656 \\
\hline $\mathrm{N}$ & 8.3554750058 & 3.6959112765 & 3.7894571126 \\
\hline $\mathrm{N}$ & 7.8771791798 & 9.6718790870 & 10.0196220443 \\
\hline $\mathrm{N}$ & 4.0691941424 & 10.6314873244 & 1.5473305889 \\
\hline $\mathrm{N}$ & 12.1634599074 & 2.7363031044 & 12.2617478488 \\
\hline $\mathrm{N}$ & 3.0555650256 & 3.4887433187 & 8.6015081769 \\
\hline $\mathrm{N}$ & 13.1770887008 & 9.8790466461 & 5.2075705694 \\
\hline C & 3.9100992167 & 5.1778991423 & 5.3261792563 \\
\hline C & 12.3225547945 & 8.1898911626 & 8.4828994891 \\
\hline C & 11.5761438791 & 5.7093636118 & 5.2362157616 \\
\hline C & 4.6565102890 & 7.6584266316 & 8.5728625726 \\
\hline C & 11.1652297152 & 6.2465049531 & 4.0155910023 \\
\hline C & 5.0674241621 & 7.1212853513 & 9.7934877431 \\
\hline C & 3.1028675438 & 6.2337867892 & 2.9236622524 \\
\hline C & 13.1297863335 & 7.1340035152 & 10.8854164929 \\
\hline C & 4.3680045627 & 5.6755533349 & 3.0911674553 \\
\hline C & 11.8646497010 & 7.6922372783 & 10.7179110852 \\
\hline C & 5.4123304633 & 5.6394349126 & 2.0134056324 \\
\hline C & 10.8203236133 & 7.7283553911 & 11.7956731130 \\
\hline C & 5.8016758099 & 3.2099319476 & 1.7217378745 \\
\hline C & 10.4309783767 & 10.1578584158 & 12.0873412824 \\
\hline C & 5.0504271713 & 0.9277843315 & 2.5409765363 \\
\hline C & 11.1822266671 & 12.4400059180 & 11.2681024152 \\
\hline C & 8.4715712582 & 11.4455209402 & 3.6021561011 \\
\hline C & 7.7610828463 & 1.9222694495 & 10.2069226454 \\
\hline C & 8.7514532169 & 11.8678761368 & 4.9030471414 \\
\hline C & 7.4812005610 & 1.4999140619 & 8.9060316039 \\
\hline C & 5.6143082915 & 1.7483157483 & 5.1001655587 \\
\hline C & 10.6183460789 & 11.6194746394 & 8.7089131880 \\
\hline C & 5.5560568896 & 2.2001480134 & 2.8083034300 \\
\hline C & 10.6765969751 & 11.1676424611 & 11.0007753163 \\
\hline
\end{tabular}

0.8218161674

0.1179671048

0.8795036069

0.0910611217

0.9060761646

CELL_PARAMETERS (angstrom)

$\begin{array}{lll}3.641590456 & 11.399372180 & 0.002105445\end{array}$

$\begin{array}{lll}3.654861547 & 1.998579243 & 13.801394184\end{array}$ 


\begin{tabular}{|c|c|c|c|}
\hline C & 7.7189119540 & 4.7858892479 & 1.7374495324 \\
\hline C & 8.5137420682 & 8.5819010260 & 12.0716290079 \\
\hline C & 8.7215316585 & 3.9856835230 & 2.5119877431 \\
\hline C & 7.5111226357 & 9.3821071802 & 11.2970914138 \\
\hline C & 9.9555905146 & 3.5961461014 & 1.9995283443 \\
\hline C & 6.2770635078 & 9.7716438924 & 11.8095506073 \\
\hline C & 1.9012236434 & 2.9291719711 & 2.8191847763 \\
\hline C & 14.3314304342 & 10.4386183327 & 10.9898939692 \\
\hline C & 10.4564534175 & 2.5920350554 & 4.1307291758 \\
\hline C & 5.7762007690 & 10.7757553081 & 9.6783499811 \\
\hline C & 9.2009166357 & 3.0006032381 & 4.5760534654 \\
\hline C & 7.0317373324 & 10.3671867269 & 9.2330252800 \\
\hline C & 7.7409944048 & 6.4866317740 & 5.5567965793 \\
\hline C & 8.4916596729 & 6.8811585298 & 8.2522821671 \\
\hline C & 7.4613644021 & 5.4529077850 & 6.6042621660 \\
\hline C & 8.7712897842 & 7.9148828585 & 7.2048165796 \\
\hline C & 8.3941118276 & 7.6865103624 & 5.8773586018 \\
\hline C & 7.8385423579 & 5.6812800010 & 7.9317205551 \\
\hline C & 12.0356523658 & 11.5920827205 & 1.9909029857 \\
\hline C & 4.1970015791 & 1.7757074495 & 11.8181753494 \\
\hline C & 3.6651443701 & 9.4057022513 & 1.1435913305 \\
\hline C & 12.5675096249 & 3.9620880076 & 12.6654871071 \\
\hline C & 5.4773424540 & 10.9922098307 & 1.5695272858 \\
\hline C & 10.7553117050 & 2.3755805888 & 12.2395516659 \\
\hline C & 11.0449263311 & 4.4466171454 & 9.1007016383 \\
\hline C & 5.1877276380 & 8.9211728185 & 4.7083771081 \\
\hline C & 11.5868804502 & 2.4016576999 & 7.8589765355 \\
\hline C & 4.6457733863 & 10.9661324346 & 5.9501022098 \\
\hline C & 4.4678897660 & 3.7114794121 & 8.8733049408 \\
\hline C & 11.7647644994 & 9.6563110611 & 4.9357738048 \\
\hline $\mathrm{H}$ & 4.2831056661 & 4.7259099851 & 6.2446748296 \\
\hline $\mathrm{H}$ & 11.9495486534 & 8.6418803779 & 7.5644043283 \\
\hline $\mathrm{H}$ & 10.9162390259 & 5.7155674000 & 6.1049993982 \\
\hline $\mathrm{H}$ & 5.3164148090 & 7.6522228445 & 7.7040789358 \\
\hline $\mathrm{H}$ & 10.1774108414 & 6.6916133561 & 3.8988995082 \\
\hline $\mathrm{H}$ & 6.0552433692 & 6.6761769472 & 9.9101792384 \\
\hline $\mathrm{H}$ & 2.8104705754 & 6.6342214939 & 1.9531913575 \\
\hline $\mathrm{H}$ & 13.4221835263 & 6.7335687497 & 11.8558869767 \\
\hline $\mathrm{H}$ & 4.9471609638 & 5.5677563710 & 1.0193759316 \\
\hline $\mathrm{H}$ & 11.2854929498 & 7.8000335631 & 12.7897026086 \\
\hline $\mathrm{H}$ & 5.9919550448 & 6.5750512613 & 2.0468989891 \\
\hline $\mathrm{H}$ & 10.2406990873 & 6.7927390723 & 11.7621799626 \\
\hline $\mathrm{H}$ & 6.4902107950 & 2.8088629984 & 0.9603002256 \\
\hline $\mathrm{H}$ & 9.7424433909 & 10.5589275050 & 12.8487787261 \\
\hline $\mathrm{H}$ & 4.8616056317 & 3.4089078544 & 1.1848251340 \\
\hline $\mathrm{H}$ & 11.3710480161 & 9.9588820806 & 12.6242534060 \\
\hline $\mathrm{H}$ & 4.8401561293 & 0.6336751052 & 1.5112197022 \\
\hline $\mathrm{H}$ & 11.3924975593 & 12.7341149567 & 12.2978588379 \\
\hline $\mathrm{H}$ & 8.1041944709 & 10.4383112074 & 3.4025921406 \\
\hline $\mathrm{H}$ & 8.1284595519 & 2.9294789264 & 10.4064866049 \\
\hline $\mathrm{H}$ & 8.5838941070 & 11.2220367576 & 5.7652045348 \\
\hline $\mathrm{H}$ & 7.6487598618 & 2.1457534864 & 8.0438738003 \\
\hline $\mathrm{H}$ & 5.8401057086 & 2.1343903912 & 6.0928499668 \\
\hline $\mathrm{H}$ & 10.3925484498 & 11.2334001673 & 7.7162287787 \\
\hline $\mathrm{H}$ & 7.8026179364 & 4.5612134380 & 0.6655815748 \\
\hline $\mathrm{H}$ & 8.4300359500 & 8.8065764812 & 13.1434968628 \\
\hline $\mathrm{H}$ & 7.9177287186 & 5.8551154945 & 1.8957087359 \\
\hline $\mathrm{H}$ & 8.3149255755 & 7.5126752087 & 11.9133704210 \\
\hline
\end{tabular}




\begin{tabular}{|c|c|c|c|}
\hline $\mathrm{H}$ & 10.2236021195 & 3.8381604501 & 0.9712029752 \\
\hline $\mathrm{H}$ & 6.0090519030 & 9.5296295437 & 12.8378759765 \\
\hline $\mathrm{H}$ & 2.8709204141 & 2.6151855722 & 2.4331868217 \\
\hline $\mathrm{H}$ & 13.3617337724 & 10.7526047912 & 11.3758923352 \\
\hline $\mathrm{H}$ & 2.1761067460 & 2.0724054879 & 4.8013485799 \\
\hline $\mathrm{H}$ & 14.0565473855 & 11.2953849857 & 9.0077301666 \\
\hline $\mathrm{H}$ & 8.8427879730 & 2.7622645545 & 5.5771275260 \\
\hline $\mathrm{H}$ & 7.3898659410 & 10.6055255206 & 8.2319508080 \\
\hline $\mathrm{H}$ & 8.6127435664 & 8.4126174660 & 5.0920830320 \\
\hline $\mathrm{H}$ & 7.6199106191 & 4.9551728974 & 8.7169961259 \\
\hline $\mathrm{H}$ & 11.0270844601 & 11.1692098523 & 1.9153439517 \\
\hline $\mathrm{H}$ & 5.2055698072 & 2.1985807660 & 11.8937352052 \\
\hline $\mathrm{H}$ & 8.4523899266 & 1.0962631313 & 1.3650410913 \\
\hline $\mathrm{H}$ & 7.7802642600 & 12.2715272322 & 12.4440380665 \\
\hline $\mathrm{H}$ & 12.2226976524 & 11.8835179587 & 3.0362455753 \\
\hline $\mathrm{H}$ & 4.0099563908 & 1.4842723792 & 10.7728329650 \\
\hline $\mathrm{H}$ & 11.5034413830 & 9.2496611693 & 1.2093653724 \\
\hline $\mathrm{H}$ & 4.7292127760 & 4.1181294593 & 12.5997132716 \\
\hline $\mathrm{H}$ & 5.6928151313 & 11.7942010735 & 0.8469909342 \\
\hline $\mathrm{H}$ & 10.5398389588 & 1.5735893782 & 12.9620876584 \\
\hline $\mathrm{H}$ & 6.0785514186 & 10.1070664025 & 1.3303061723 \\
\hline $\mathrm{H}$ & 10.1541026042 & 3.2607237323 & 12.4787725742 \\
\hline $\mathrm{H}$ & 2.1064045158 & -0.0438306608 & 2.5704439096 \\
\hline $\mathrm{H}$ & 14.1262495609 & 13.4116209645 & 11.2386348359 \\
\hline $\mathrm{H}$ & 10.0277123023 & 4.1705319910 & 8.7965929472 \\
\hline $\mathrm{H}$ & 6.2049417754 & 9.1972583127 & 5.0124857992 \\
\hline $\mathrm{H}$ & 11.2686368118 & 5.4419548229 & 8.6858696859 \\
\hline $\mathrm{H}$ & 4.9640171320 & 7.9258354813 & 5.1232090604 \\
\hline $\mathrm{H}$ & 11.0799285326 & 4.5200744338 & 10.1976412618 \\
\hline $\mathrm{H}$ & 5.1527255693 & 8.8477155297 & 3.6114374837 \\
\hline $\mathrm{H}$ & 10.4833260743 & 2.3546786049 & 7.7317498547 \\
\hline $\mathrm{H}$ & 5.7493278040 & 11.0131116995 & 6.0773288916 \\
\hline $\mathrm{H}$ & 4.6303906488 & 3.9071699732 & 9.9410561120 \\
\hline $\mathrm{H}$ & 11.6022635618 & 9.4606203311 & 3.8680226336 \\
\hline $\mathrm{H}$ & 4.8471068786 & 4.5823075452 & 8.3163526994 \\
\hline $\mathrm{H}$ & 11.3855471738 & 8.7854830997 & 5.4927260470 \\
\hline $\mathrm{H}$ & 5.0299801325 & 2.8230074346 & 8.5682434023 \\
\hline $\mathrm{H}$ & 11.2026738904 & 10.5447826992 & 5.2408353431 \\
\hline $\mathrm{F}$ & 9.0224967010 & 9.6546600017 & 0.4964726897 \\
\hline $\mathrm{F}$ & 7.2101572668 & 3.7131302423 & 13.3126056444 \\
\hline $\mathrm{F}$ & 9.4014025857 & 7.3622572565 & 0.5495771888 \\
\hline $\mathrm{F}$ & 6.8312514234 & 6.0055330094 & 13.2595013002 \\
\hline $\mathrm{F}$ & 9.6601690214 & 8.6298062835 & 2.4868839065 \\
\hline $\mathrm{F}$ & 6.5724849464 & 4.7379839605 & 11.3221944286 \\
\hline $\mathrm{F}$ & 7.5385097106 & 8.2602244976 & 1.6190022263 \\
\hline $\mathrm{F}$ & 8.6941443670 & 5.1075658062 & 12.1900765202 \\
\hline B & 8.9003221994 & 8.4754052157 & 1.2924175420 \\
\hline B & 7.3323318500 & 4.8923850731 & 12.5166611009 \\
\hline & & & \\
\hline \multicolumn{4}{|c|}{ CELL_PARAMETERS (angstrom) } \\
\hline & $6 \quad 0.055640599$ & 0.294909035 & \\
\hline & 9.268802352 & -0.042551753 & \\
\hline & 4.108978271 & 13.655925761 & \\
\hline \multicolumn{4}{|c|}{ ATOMIC_POSITIONS (angstrom) } \\
\hline $\mathrm{Fe}$ & 4.3259278771 & 8.1110589320 & 9.3220743274 \\
\hline $\mathrm{Fe}$ & 7.4012618235 & 5.3223622900 & 4.5862087159 \\
\hline 0 & 9.3559747806 & 6.1142845662 & 4.6017399703 \\
\hline
\end{tabular}




\begin{tabular}{|c|c|c|c|}
\hline 0 & 2.3712150096 & 7.3191369330 & 9.3065430740 \\
\hline $\mathrm{O}$ & 4.0192820018 & 7.8563036247 & 7.3140944782 \\
\hline 0 & 7.7079078275 & 5.5771175981 & 6.5941885696 \\
\hline $\mathrm{N}$ & 4.4213168421 & 8.3650016968 & 11.5436634463 \\
\hline $\mathrm{N}$ & 7.3058726049 & 5.0684195235 & 2.3646195872 \\
\hline $\mathrm{N}$ & 6.3085478781 & 8.9016768115 & 9.4830842433 \\
\hline $\mathrm{N}$ & 5.4186415690 & 4.5317444088 & 4.4251987902 \\
\hline $\mathrm{N}$ & 3.6439682771 & 10.1876495020 & 9.6050001558 \\
\hline $\mathrm{N}$ & 8.0832214245 & 3.2457717199 & 4.3032828875 \\
\hline $\mathrm{N}$ & 4.9896992216 & 6.1648735059 & 10.0715101353 \\
\hline $\mathrm{N}$ & 6.7374902506 & 7.2685479902 & 3.8367728979 \\
\hline $\mathrm{F}$ & 7.5323000855 & 6.2802911593 & 13.6137534453 \\
\hline $\mathrm{F}$ & 4.1948896287 & 7.1531302000 & 0.2945295974 \\
\hline $\mathrm{F}$ & 9.1888167330 & 7.6370104809 & 12.7230504759 \\
\hline $\mathrm{F}$ & 2.5383729676 & 5.7964107410 & 1.1852325674 \\
\hline $\mathrm{F}$ & 8.2188935111 & 5.9730491914 & 11.4133375841 \\
\hline $\mathrm{F}$ & 3.5082961905 & 7.4603720306 & 2.4949454592 \\
\hline $\mathrm{F}$ & 9.6467475414 & 5.4028996142 & 13.1637692687 \\
\hline $\mathrm{F}$ & 2.0804421466 & 8.0305214703 & 0.7445137752 \\
\hline C & 2.8811179279 & 7.3124991350 & 6.9922058482 \\
\hline C & 8.8460719014 & 6.1209220878 & 6.9160771995 \\
\hline C & 9.7758597996 & 6.4400935133 & 5.7609571018 \\
\hline C & 1.9513300911 & 6.9933278322 & 8.1473263526 \\
\hline C & 2.4923762013 & 7.0081839177 & 5.6937048847 \\
\hline C & 9.2348134301 & 6.4252371825 & 8.2145777516 \\
\hline C & 6.6910064542 & 9.1664646337 & 10.7512609920 \\
\hline C & 5.0361832464 & 4.2669565882 & 3.1570220504 \\
\hline C & 3.4831200599 & 9.4477552485 & 11.9126632125 \\
\hline C & 8.2440696407 & 3.9856659734 & 1.9956198308 \\
\hline C & 7.1161829847 & 9.2611679993 & 8.4646007522 \\
\hline C & 4.6110067169 & 4.1722532227 & 5.4436822911 \\
\hline C & 3.4498542829 & 10.5568589069 & 10.8921395952 \\
\hline C & 8.2773356088 & 2.8765623163 & 3.0161434547 \\
\hline C & 4.0081804895 & 7.0562056447 & 12.1007575738 \\
\hline C & 7.7190089838 & 6.3772158524 & 1.8075254595 \\
\hline C & 5.8357973802 & 8.6702694295 & 11.8899201116 \\
\hline C & 5.8913925759 & 4.7631517941 & 2.0183629405 \\
\hline C & 7.8875923525 & 9.8315155136 & 11.0266092537 \\
\hline C & 3.8395973491 & 3.6019057084 & 2.8816737897 \\
\hline C & 3.1219834014 & 11.8701323943 & 11.2425089281 \\
\hline C & 8.6052062524 & 1.5632888273 & 2.6657741135 \\
\hline C & 3.5037120687 & 11.1137117322 & 8.6367574718 \\
\hline C & 8.2234774417 & 2.3197094885 & 5.2715255649 \\
\hline C & 4.7049063743 & 5.9350136109 & 11.3726091163 \\
\hline C & 7.0222830990 & 7.4984078852 & 2.5356739170 \\
\hline C & 8.3257853621 & 9.9107753183 & 8.6687250046 \\
\hline C & 3.4014043395 & 3.5226459036 & 5.2395580387 \\
\hline C & 5.6198691155 & 5.2137233526 & 9.3598086754 \\
\hline C & 6.1073205599 & 8.2196975934 & 4.5484743692 \\
\hline C & 2.0827321429 & 3.5472952568 & 10.2766689704 \\
\hline C & 9.6444573958 & 9.8861259651 & 3.6316140674 \\
\hline C & 2.2757557016 & 3.1600917967 & 8.9480312663 \\
\hline C & 9.4514342223 & 10.2733294267 & 4.9602517847 \\
\hline C & 5.0228971940 & 4.7300935115 & 11.9928660831 \\
\hline C & 6.7042925202 & 8.7033278489 & 1.9154169596 \\
\hline C & 8.7095274768 & 10.2157396027 & 9.9747882594 \\
\hline C & 3.0176622248 & 3.2176816192 & 3.9334947840 \\
\hline C & 5.9601386731 & 3.9820950350 & 9.9093784731 \\
\hline
\end{tabular}




\begin{tabular}{|c|c|c|c|}
\hline C & 5.7670510284 & 9.4513261870 & 3.9989045702 \\
\hline C & 5.6496404409 & 3.7366449713 & 11.2460664368 \\
\hline C & 6.0775492796 & 9.6967764583 & 2.6622166056 \\
\hline $\mathrm{H}$ & 3.1381082889 & 7.2148664740 & 4.8382461520 \\
\hline $\mathrm{H}$ & 8.5890813550 & 6.2185548698 & 9.0700372939 \\
\hline $\mathrm{H}$ & 3.7065865010 & 9.8502386157 & 12.9126569087 \\
\hline $\mathrm{H}$ & 8.0206031372 & 3.5831826058 & 0.9956261325 \\
\hline $\mathrm{H}$ & 11.0062663039 & 9.0555775608 & 12.2379796191 \\
\hline $\mathrm{H}$ & 0.7209231587 & 4.3778436595 & 1.6703034160 \\
\hline $\mathrm{H}$ & 6.7629036116 & 9.0100188497 & 7.4623958058 \\
\hline $\mathrm{H}$ & 4.9642858354 & 4.4234023706 & 6.4458872287 \\
\hline $\mathrm{H}$ & 2.9205292556 & 6.9570006720 & 11.9626147390 \\
\hline $\mathrm{H}$ & 8.8066603564 & 6.4764202736 & 1.9456683033 \\
\hline $\mathrm{H}$ & 4.2156839184 & 6.9886503927 & 13.1836216734 \\
\hline $\mathrm{H}$ & 7.5115057569 & 6.4447705523 & 0.7246613712 \\
\hline $\mathrm{H}$ & 6.3084028153 & 7.7431866348 & 12.2536957753 \\
\hline $\mathrm{H}$ & 5.4187871660 & 5.6902348646 & 1.6545872756 \\
\hline $\mathrm{H}$ & 5.8824591766 & 9.3843828995 & 12.7256429422 \\
\hline $\mathrm{H}$ & 5.8447305250 & 4.0490383224 & 1.1826401011 \\
\hline $\mathrm{H}$ & 8.1550894960 & 10.0388939568 & 12.0624793331 \\
\hline $\mathrm{H}$ & 3.5721002056 & 3.3945272651 & 1.8458037102 \\
\hline $\mathrm{H}$ & 11.5451181198 & 12.2028153462 & 12.5872146347 \\
\hline $\mathrm{H}$ & 0.1820715106 & 1.2306058753 & 1.3210684062 \\
\hline $\mathrm{H}$ & 3.6853738525 & 10.7577608079 & 7.6185078268 \\
\hline $\mathrm{H}$ & 8.0418161036 & 2.6756604156 & 6.2897752253 \\
\hline $\mathrm{H}$ & 8.9582192970 & 10.1719865925 & 7.8222625031 \\
\hline $\mathrm{H}$ & 2.7689704036 & 3.2614346294 & 6.0860205402 \\
\hline $\mathrm{H}$ & 5.8835475013 & 5.4718892870 & 8.3308502492 \\
\hline $\mathrm{H}$ & 5.8436421741 & 7.9615316581 & 5.5774327954 \\
\hline $\mathrm{H}$ & 10.3168180119 & 4.6220359303 & 10.8213155711 \\
\hline $\mathrm{H}$ & 1.4103714352 & 8.8113852899 & 3.0869674635 \\
\hline $\mathrm{H}$ & 2.1334849223 & 3.8797165895 & 8.1403676654 \\
\hline $\mathrm{H}$ & 9.5937049060 & 9.5537046333 & 5.7679153813 \\
\hline $\mathrm{H}$ & 4.7876181000 & 4.5648694264 & 13.0446005552 \\
\hline $\mathrm{H}$ & 6.9395716268 & 8.8685520714 & 0.8636824869 \\
\hline $\mathrm{H}$ & 9.6309564494 & 10.7593143207 & 10.1682150271 \\
\hline $\mathrm{H}$ & 2.0962332522 & 2.6741069012 & 3.7400680163 \\
\hline $\mathrm{H}$ & 6.4792654658 & 3.2411459471 & 9.3016630538 \\
\hline $\mathrm{H}$ & 5.2479242547 & 10.1922754825 & 4.6066199886 \\
\hline $\mathrm{H}$ & 6.7607114006 & 12.0624949091 & 11.6844137488 \\
\hline $\mathrm{H}$ & 4.9664783095 & 1.3709264162 & 2.2238692930 \\
\hline B & 8.6435630239 & 6.3089653080 & 12.7138079382 \\
\hline B & 3.0836266776 & 7.1244559140 & 1.1944751041 \\
\hline \multicolumn{4}{|c|}{$3-I S$} \\
\hline \multicolumn{4}{|c|}{ CELL_PARAMETERS (angstrom) } \\
\hline & 550.081148050 & 0.092470812 & \\
\hline & 9.146051562 & 0.052386789 & \\
\hline & 4.239489111 & 13.558391855 & \\
\hline \multicolumn{4}{|c|}{ ATOMIC_POSITIONS (angstrom) } \\
\hline $\mathrm{Fe}$ & 3.9677171184 & 8.1868295323 & 9.2234638150 \\
\hline $\mathrm{Fe}$ & 7.5515951198 & 5.2182780427 & 4.2033631785 \\
\hline 0 & 9.3702489526 & 5.9585418485 & 4.2366837177 \\
\hline 0 & 2.0361103730 & 7.4345481281 & 9.1536923089 \\
\hline 0 & 3.7781762744 & 7.8667532952 & 7.2208754220 \\
\hline 0 & 7.6882213815 & 5.4655275034 & 6.1398072138 \\
\hline $\mathrm{N}$ & 4.1285958629 & 8.4355334100 & 11.4446125707 \\
\hline $\mathrm{N}$ & 7.4246601934 & 5.0680723051 & 2.2190401315 \\
\hline
\end{tabular}




\begin{tabular}{|c|c|c|c|}
\hline $\mathrm{N}$ & 5.9446815112 & 8.9658149730 & 9.3326152279 \\
\hline $\mathrm{N}$ & 5.7735476224 & 4.4959962675 & 4.2382158824 \\
\hline $\mathrm{N}$ & 3.2727224705 & 10.2279943385 & 9.5147870501 \\
\hline$N$ & 8.2360749976 & 3.3794992760 & 4.1185447351 \\
\hline $\mathrm{N}$ & 4.6399490876 & 6.2454200131 & 9.9290980226 \\
\hline $\mathrm{N}$ & 6.8630974088 & 7.0255811169 & 3.8436253055 \\
\hline $\mathrm{F}$ & 7.3304767340 & 6.3659925507 & 13.4290254978 \\
\hline $\mathrm{F}$ & 4.2294252013 & 7.1016457348 & 0.2975949260 \\
\hline $\mathrm{F}$ & 9.0227751731 & 7.6679010292 & 12.5178478467 \\
\hline $\mathrm{F}$ & 2.5352998016 & 5.7626484880 & 1.1392239111 \\
\hline $\mathrm{F}$ & 7.9697617381 & 6.0557315750 & 11.2104685192 \\
\hline $\mathrm{F}$ & 3.5829360809 & 7.3200091536 & 2.5187483168 \\
\hline $\mathrm{F}$ & 9.4005431177 & 5.4147865641 & 12.9374073692 \\
\hline $\mathrm{F}$ & 2.1532147681 & 8.0306550428 & 0.8235076634 \\
\hline C & 2.6634895314 & 7.2855054048 & 6.8724382521 \\
\hline C & 8.7688051168 & 6.0923973819 & 6.5066747671 \\
\hline C & 9.7406221612 & 6.3555330418 & 5.4058659566 \\
\hline C & 1.6727258397 & 7.0431948725 & 7.9849208661 \\
\hline C & 2.3640618163 & 6.8929937708 & 5.5702127147 \\
\hline C & 9.0578866738 & 6.4924425904 & 7.8122665300 \\
\hline C & 6.3895330727 & 9.2003336080 & 10.5847141737 \\
\hline C & 5.2064400918 & 4.2788611321 & 3.0279084213 \\
\hline C & 3.1872419121 & 9.5117308625 & 11.8321452540 \\
\hline C & 8.4019392843 & 4.0228767641 & 1.8040250311 \\
\hline C & 6.7152332270 & 9.3092685292 & 8.2808460517 \\
\hline C & 5.0992255196 & 4.1557753912 & 5.3579887782 \\
\hline C & 3.1151276531 & 10.6121529948 & 10.8024173089 \\
\hline C & 8.4794691533 & 2.9497224834 & 2.8486834954 \\
\hline C & 3.7411142753 & 7.1176399902 & 12.0020089757 \\
\hline C & 7.7917193065 & 6.4222911791 & 1.7117575085 \\
\hline C & 5.5492149268 & 8.7535911613 & 11.7565625411 \\
\hline C & 6.0091442655 & 4.7235467688 & 1.8425587335 \\
\hline C & 7.6285876482 & 9.8032471447 & 10.8109417329 \\
\hline C & 3.9385941364 & 3.7108218986 & 2.9118105339 \\
\hline C & 2.7911197421 & 11.9289726067 & 11.1424507436 \\
\hline C & 8.8525388372 & 1.6354950957 & 2.5724635360 \\
\hline C & 3.0969179243 & 11.1359981906 & 8.5361057609 \\
\hline C & 8.3948849020 & 2.5086340328 & 5.1379902285 \\
\hline C & 4.4260216292 & 6.0092394716 & 11.2423296155 \\
\hline C & 7.0929640180 & 7.4384421070 & 2.5681281289 \\
\hline C & 7.9580957736 & 9.9053437448 & 8.4350169523 \\
\hline C & 3.8288736297 & 3.6010022801 & 5.3089726852 \\
\hline C & 5.2494549093 & 5.3089897238 & 9.1825336691 \\
\hline C & 6.1893371815 & 7.8346992008 & 4.6835273595 \\
\hline C & 1.6934483528 & 3.7137192282 & 10.0720549496 \\
\hline C & 9.9195681831 & 9.8891955576 & 3.6830103360 \\
\hline C & 1.8473991929 & 3.3081098710 & 8.7430905526 \\
\hline C & 9.6956087693 & 10.3397515067 & 4.9855241566 \\
\hline C & 4.8008433179 & 4.8104774802 & 11.8413336739 \\
\hline C & 6.7011808501 & 8.6933936445 & 2.1199551111 \\
\hline C & 8.4179696803 & 10.1656251610 & 9.7264601251 \\
\hline C & 3.2389416556 & 3.3693430201 & 4.0653799846 \\
\hline C & 5.6511584975 & 4.0869344495 & 9.7099897824 \\
\hline C & 5.7577329388 & 9.0998805100 & 4.2956817490 \\
\hline C & 5.4158247864 & 3.8352831307 & 11.0609264660 \\
\hline C & 6.0329913840 & 9.5401905303 & 3.0023803256 \\
\hline $\mathrm{H}$ & 3.0658536963 & 7.0514854317 & 4.7501516196 \\
\hline $\mathrm{H}$ & 8.3606101676 & 6.3124961843 & 8.6331139923 \\
\hline
\end{tabular}




\begin{tabular}{|c|c|c|c|}
\hline $\mathrm{H}$ & 3.4315449129 & 9.9246140840 & 12.8226493320 \\
\hline $\mathrm{H}$ & 8.1591188098 & 3.6114081587 & 0.8137408833 \\
\hline $\mathrm{H}$ & 10.7999363827 & 9.1329549372 & 11.9844166196 \\
\hline $\mathrm{H}$ & 0.7737368105 & 4.4315320578 & 1.6571309188 \\
\hline $\mathrm{H}$ & 6.3007567901 & 9.0911051791 & 7.2954772308 \\
\hline $\mathrm{H}$ & 5.6217931250 & 4.3559174232 & 6.2923788241 \\
\hline $\mathrm{H}$ & 2.6502619448 & 7.0111517695 & 11.8933884099 \\
\hline $\mathrm{H}$ & 8.8810670112 & 6.5365039130 & 1.8125223238 \\
\hline $\mathrm{H}$ & 3.9803392214 & 7.0410803123 & 13.0776293818 \\
\hline $\mathrm{H}$ & 7.5268327294 & 6.5495023956 & 0.6484161220 \\
\hline $\mathrm{H}$ & 6.0288573748 & 7.8474504000 & 12.1630377082 \\
\hline $\mathrm{H}$ & 5.5303442123 & 5.6222625918 & 1.4222697172 \\
\hline $\mathrm{H}$ & 5.6046726217 & 9.5096165615 & 12.5547723228 \\
\hline $\mathrm{H}$ & 5.9923833851 & 3.9607762756 & 1.0514919170 \\
\hline $\mathrm{H}$ & 7.9592278482 & 9.9759046086 & 11.8348459341 \\
\hline $\mathrm{H}$ & 3.5117880999 & 3.5580822410 & 1.9216183364 \\
\hline $\mathrm{H}$ & 11.3164518702 & 12.3052966404 & 12.2819232844 \\
\hline $\mathrm{H}$ & 0.3789044888 & 1.2272474427 & 1.4486539042 \\
\hline $\mathrm{H}$ & 3.2387926558 & 10.7614179503 & 7.5182167138 \\
\hline $\mathrm{H}$ & 8.1861275141 & 2.9151735870 & 6.1297960984 \\
\hline $\mathrm{H}$ & 8.5501683067 & 10.1583909510 & 7.5584210458 \\
\hline $\mathrm{H}$ & 3.3152671494 & 3.3631601454 & 6.2388769358 \\
\hline $\mathrm{H}$ & 5.4254505839 & 5.5738104974 & 8.1374452206 \\
\hline $\mathrm{H}$ & 5.9854184213 & 7.4298828951 & 5.6752876545 \\
\hline $\mathrm{H}$ & 10.0152348768 & 4.8175764692 & 10.4063281759 \\
\hline $\mathrm{H}$ & 1.6236450073 & 8.7832310289 & 3.3953094166 \\
\hline $\mathrm{H}$ & 1.6792296604 & 4.0131224385 & 7.9273399348 \\
\hline $\mathrm{H}$ & 9.8390010634 & 9.6639497279 & 5.8303155744 \\
\hline $\mathrm{H}$ & 4.6198824737 & 4.6363651871 & 12.9017462199 \\
\hline $\mathrm{H}$ & 6.9031312233 & 9.0043576041 & 1.0944452927 \\
\hline $\mathrm{H}$ & 9.3745783832 & 10.6572133023 & 9.8841505216 \\
\hline $\mathrm{H}$ & 2.2481829153 & 2.9253210267 & 3.9961543801 \\
\hline $\mathrm{H}$ & 6.1544953002 & 3.3570987690 & 9.0759150671 \\
\hline $\mathrm{H}$ & 5.1933327140 & 9.7145527263 & 4.9978036708 \\
\hline $\mathrm{H}$ & 6.6060842569 & 12.0454476512 & 11.5784471251 \\
\hline $\mathrm{H}$ & 4.8231173933 & 1.3770872948 & 2.6106194472 \\
\hline B & 8.4253863843 & 6.3582932080 & 12.5083081453 \\
\hline B & 3.1268096710 & 7.0695661269 & 1.2101145980 \\
\hline \multicolumn{4}{|c|}{ 3-LS } \\
\hline \multicolumn{4}{|c|}{ CELL_PARAMETERS (angstrom) } \\
\hline & $0 \quad 0.051119258$ & -0.004011009 & \\
\hline & 9.061255097 & 0.054252932 & \\
\hline & 4.217775781 & 13.344909942 & \\
\hline
\end{tabular}

\begin{tabular}{|c|c|c|c|}
\hline \multicolumn{4}{|c|}{ ATOMIC_POSITIONS (angstrom) } \\
\hline $\mathrm{Fe} 2$ & 3.8248890794 & 8.1495495888 & 9.2624183965 \\
\hline $\mathrm{Fe} 2$ & 7.5872966255 & 5.1806005472 & 4.1327334695 \\
\hline 0 & 9.4169359039 & 5.9164287811 & 4.1808780511 \\
\hline $\mathrm{O}$ & 1.9952498917 & 7.4137216260 & 9.2142738164 \\
\hline 0 & 3.6941797753 & 7.9018754696 & 7.3221084176 \\
\hline 0 & 7.7180060603 & 5.4282746672 & 6.0730434483 \\
\hline $\mathrm{N}$ & 3.9527867297 & 8.3054496934 & 11.2505715796 \\
\hline $\mathrm{N}$ & 7.4593987180 & 5.0247004411 & 2.1445802855 \\
\hline $\mathrm{N}$ & 5.6092310144 & 8.8632990500 & 9.2346214473 \\
\hline $\mathrm{N}$ & 5.8029544333 & 4.4668510845 & 4.1605304178 \\
\hline $\mathrm{N}$ & 3.1446358070 & 9.9909912061 & 9.3434114085 \\
\hline $\mathrm{N}$ & 8.2675498989 & 3.3391589300 & 4.0517404574 \\
\hline $\mathrm{N}$ & 4.5025571043 & 6.3366816647 & 9.6265730151 \\
\hline
\end{tabular}




\begin{tabular}{|c|c|c|c|}
\hline $\mathrm{N}$ & 6.9096283687 & 6.9934687395 & 3.7685788526 \\
\hline $\mathrm{F}$ & 7.1913470332 & 6.2571914982 & 13.1420043715 \\
\hline $\mathrm{F}$ & 4.2208386853 & 7.0729587721 & 0.2531474952 \\
\hline $\mathrm{F}$ & 8.9194185627 & 7.5375457076 & 12.2745265365 \\
\hline $\mathrm{F}$ & 2.4927671423 & 5.7926044285 & 1.1206253295 \\
\hline $\mathrm{F}$ & 7.8241205767 & 5.9981358774 & 10.9156202541 \\
\hline $\mathrm{F}$ & 3.5880651292 & 7.3320142587 & 2.4795316119 \\
\hline $\mathrm{F}$ & 9.2348240014 & 5.2627150704 & 12.6182732111 \\
\hline $\mathrm{F}$ & 2.1773616909 & 8.0674349313 & 0.7768786540 \\
\hline C & 2.6179261525 & 7.2813421718 & 6.9445884497 \\
\hline C & 8.7942596831 & 6.0488079650 & 6.4505634162 \\
\hline C & 9.7800450844 & 6.3127398557 & 5.3533655957 \\
\hline C & 1.6321407992 & 7.0174104070 & 8.0417866679 \\
\hline C & 2.3492924963 & 6.8875783957 & 5.6306604843 \\
\hline C & 9.0628931527 & 6.4425716154 & 7.7644909839 \\
\hline C & 6.1822232305 & 9.0686592575 & 10.4434046334 \\
\hline C & 5.2299624744 & 4.2614908785 & 2.9517472315 \\
\hline C & 2.9733417222 & 9.3513789234 & 11.6586244549 \\
\hline C & 8.4388439827 & 3.9787712126 & 1.7365274111 \\
\hline C & 6.2840428317 & 9.2014810293 & 8.1151476478 \\
\hline C & 5.1281428742 & 4.1286691068 & 5.2800042181 \\
\hline C & 2.8993203856 & 10.4228819968 & 10.6117453419 \\
\hline C & 8.5128655133 & 2.9072681404 & 2.7834065239 \\
\hline C & 3.5842316719 & 6.9527977346 & 11.7589458645 \\
\hline C & 7.8279538021 & 6.3773526706 & 1.6362060032 \\
\hline C & 5.3681372158 & 8.6489242280 & 11.6310667031 \\
\hline C & 6.0440487484 & 4.6812259095 & 1.7640851628 \\
\hline C & 7.4618506334 & 9.6113223640 & 10.5564717076 \\
\hline C & 3.9503350726 & 3.7188277721 & 2.8386801584 \\
\hline C & 2.5263193715 & 11.7376878499 & 10.8852011906 \\
\hline C & 8.8858662859 & 1.5924622859 & 2.5099506754 \\
\hline C & 2.9890751889 & 10.8599271889 & 8.3228254209 \\
\hline C & 8.4231103232 & 2.4702229461 & 5.0723264452 \\
\hline C & 4.2873474199 & 5.9358687660 & 10.9080392930 \\
\hline C & 7.1248380541 & 7.3942816382 & 2.4871125747 \\
\hline C & 7.5620272310 & 9.7379024836 & 8.1599413792 \\
\hline C & 3.8501584750 & 3.5922476525 & 5.2352104867 \\
\hline C & 5.1618320930 & 5.5169887106 & 8.7866899989 \\
\hline C & 6.2503535866 & 7.8131611558 & 4.6084618654 \\
\hline C & 1.4910140063 & 3.5670930266 & 9.7705564888 \\
\hline C & 9.9211715344 & 9.7630571095 & 3.6245953773 \\
\hline C & 1.7186129617 & 3.1147955680 & 8.4694610838 \\
\hline C & 9.6935729698 & 10.2153545694 & 4.9256907821 \\
\hline C & 4.6952442202 & 4.6908234521 & 11.3677862282 \\
\hline C & 6.7169414984 & 8.6393268193 & 2.0273656385 \\
\hline C & 8.1629165600 & 9.9478830383 & 9.4020596481 \\
\hline C & 3.2492691459 & 3.3822670978 & 3.9930922179 \\
\hline C & 5.6045365877 & 4.2584946648 & 9.1832598751 \\
\hline C & 5.8076491182 & 9.0716554712 & 4.2118919908 \\
\hline C & 5.3574184286 & 3.8373545150 & 10.4881910516 \\
\hline C & 6.0547672963 & 9.4927958241 & 2.9069608156 \\
\hline $\mathrm{H}$ & 3.0614804316 & 7.0657782116 & 4.8230663569 \\
\hline $\mathrm{H}$ & 8.3507052016 & 6.2643720496 & 8.5720859068 \\
\hline $\mathrm{H}$ & 3.2104953981 & 9.7664095409 & 12.6488103965 \\
\hline $\mathrm{H}$ & 8.2016902435 & 3.5637405948 & 0.7463414695 \\
\hline $\mathrm{H}$ & 10.6441763466 & 8.9110841875 & 11.7048809269 \\
\hline $\mathrm{H}$ & 0.7680091169 & 4.4190659471 & 1.6902709392 \\
\hline $\mathrm{H}$ & 5.7553177056 & 9.0154881618 & 7.1823129880 \\
\hline
\end{tabular}




\begin{tabular}{|c|c|c|c|}
\hline $\mathrm{H}$ & 5.6568677421 & 4.3146619728 & 6.2128388781 \\
\hline $\mathrm{H}$ & 2.4953334536 & 6.8382439564 & 11.6519691971 \\
\hline $\mathrm{H}$ & 8.9168521617 & 6.4919059096 & 1.7431826672 \\
\hline $\mathrm{H}$ & 3.8430401881 & 6.8266225524 & 12.8240132215 \\
\hline $\mathrm{H}$ & 7.5691454916 & 6.5035273129 & 0.5711386428 \\
\hline $\mathrm{H}$ & 5.8404093149 & 7.7575896343 & 12.0752109660 \\
\hline $\mathrm{H}$ & 5.5717766745 & 5.5725607729 & 1.3199409014 \\
\hline $\mathrm{H}$ & 5.3808535792 & 9.4292878533 & 12.4054506754 \\
\hline $\mathrm{H}$ & 6.0313321267 & 3.9008622827 & 0.9897011906 \\
\hline $\mathrm{H}$ & 7.8972214225 & 9.7507292488 & 11.5449890462 \\
\hline $\mathrm{H}$ & 3.5149642834 & 3.5794208873 & 1.8501628197 \\
\hline $\mathrm{H}$ & 11.0344670273 & 12.1191130202 & 11.9106140823 \\
\hline $\mathrm{H}$ & 0.3777186064 & 1.2110371155 & 1.4845377837 \\
\hline $\mathrm{H}$ & 3.1983398241 & 10.4534892940 & 7.3313386395 \\
\hline $\mathrm{H}$ & 8.2138461401 & 2.8766608436 & 6.0638132264 \\
\hline $\mathrm{H}$ & 8.0705052719 & 9.9780392905 & 7.2278633866 \\
\hline $\mathrm{H}$ & 3.3416804330 & 3.3521108456 & 6.1672884794 \\
\hline $\mathrm{H}$ & 5.3359613954 & 5.9091329318 & 7.7843731619 \\
\hline $\mathrm{H}$ & 6.0762242843 & 7.4210169336 & 5.6107787024 \\
\hline $\mathrm{H}$ & 9.8291072045 & 4.6435403600 & 9.9591608038 \\
\hline $\mathrm{H}$ & 1.5830782432 & 8.6866097745 & 3.4359910623 \\
\hline $\mathrm{H}$ & 1.5812666819 & 3.7869258396 & 7.6209492804 \\
\hline $\mathrm{H}$ & 9.8309191526 & 9.5432242972 & 5.7742025845 \\
\hline $\mathrm{H}$ & 4.5098299049 & 4.3933547386 & 12.3997328858 \\
\hline $\mathrm{H}$ & 6.9023558264 & 8.9367956671 & 0.9954189817 \\
\hline $\mathrm{H}$ & 9.1622217457 & 10.3729140432 & 9.4697762759 \\
\hline $\mathrm{H}$ & 2.2499639602 & 2.9572360928 & 3.9253755900 \\
\hline $\mathrm{H}$ & 6.1557040360 & 3.6309166560 & 8.4822140189 \\
\hline $\mathrm{H}$ & 5.2564816889 & 9.6992336830 & 4.9129378482 \\
\hline $\mathrm{H}$ & 6.5555093384 & 11.9250091513 & 10.8920681548 \\
\hline $\mathrm{H}$ & 4.8566763760 & 1.4051410858 & 2.5030837107 \\
\hline B & 8.2876686781 & 6.2457689904 & 12.2220126999 \\
\hline B & 3.1245170278 & 7.0843811457 & 1.1731391650 \\
\hline $4 \quad-H S$ & & & \\
\hline \multicolumn{4}{|c|}{ CELL_PARAMETERS (angstrom) } \\
\hline 16.346427453 & 0.000000000 & 0.061643650 & \\
\hline 0.000000000 & 15.289238313 & 0.000000000 & \\
\hline-1.843607128 & 0.000000000 & 11.393899001 & \\
\hline \multicolumn{4}{|c|}{ ATOMIC_POSITIONS (crystal) } \\
\hline Fe1 & 0.3106489495 & 0.0000000000 & 0.1777262201 \\
\hline Fe1 & 0.6893510505 & 0.0000000000 & 0.8222737649 \\
\hline $\mathrm{Fe} 1$ & 0.8106842101 & 0.5000000000 & 0.1778018844 \\
\hline $\mathrm{Fe} 1$ & 0.1893157899 & 0.5000000000 & 0.8221981006 \\
\hline 0 & 0.4382754203 & 0.0000000000 & 0.2066717450 \\
\hline 0 & 0.5617245797 & 0.0000000000 & 0.7933282700 \\
\hline 0 & 0.9383155798 & 0.5000000000 & 0.2067056368 \\
\hline 0 & 0.0616844202 & 0.5000000000 & 0.7932943782 \\
\hline 0 & 0.6645903551 & 0.0000000000 & 0.9942742890 \\
\hline 0 & 0.3354096449 & 0.0000000000 & 0.0057257130 \\
\hline 0 & 0.1645824937 & 0.5000000000 & 0.9942101466 \\
\hline 0 & 0.8354175063 & 0.5000000000 & 0.0057898554 \\
\hline $\mathrm{N}$ & 0.1721249185 & 0.0000000000 & 0.1569853962 \\
\hline $\mathrm{N}$ & 0.8278751115 & 0.0000000000 & 0.8430146038 \\
\hline $\mathrm{N}$ & 0.6721550914 & 0.5000000000 & 0.1570532735 \\
\hline $\mathrm{N}$ & 0.3278448786 & 0.5000000000 & 0.8429467265 \\
\hline $\mathrm{N}$ & 0.2974158254 & 0.0000000000 & 0.3571986187 \\
\hline $\mathrm{N}$ & 0.7025841746 & 0.0000000000 & 0.6428013513 \\
\hline
\end{tabular}




\begin{tabular}{|c|c|c|c|}
\hline $\mathrm{N}$ & 0.7974330670 & 0.5000000000 & 0.3572661310 \\
\hline $\mathrm{N}$ & 0.2025669330 & 0.5000000000 & 0.6427338390 \\
\hline $\mathrm{N}$ & 0.2804285749 & 0.8625312075 & 0.1660739136 \\
\hline $\mathrm{N}$ & 0.7195714251 & 0.1374687925 & 0.8339260564 \\
\hline $\mathrm{N}$ & 0.7195714251 & 0.8625312075 & 0.8339260564 \\
\hline $\mathrm{N}$ & 0.2804285749 & 0.1374687925 & 0.1660739136 \\
\hline $\mathrm{N}$ & 0.7804619024 & 0.3625257665 & 0.1661650215 \\
\hline $\mathrm{N}$ & 0.2195380976 & 0.6374742335 & 0.8338349485 \\
\hline $\mathrm{N}$ & 0.2195380976 & 0.3625257665 & 0.8338349485 \\
\hline $\mathrm{N}$ & 0.7804619024 & 0.6374742335 & 0.1661650215 \\
\hline C & 0.2187601202 & 0.0000000000 & 0.3790996900 \\
\hline C & 0.7812398648 & 0.0000000000 & 0.6209003100 \\
\hline C & 0.7187764047 & 0.5000000000 & 0.3791649587 \\
\hline C & 0.2812235953 & 0.5000000000 & 0.6208350413 \\
\hline C & 0.5871787825 & 0.0000000000 & 1.0021673715 \\
\hline C & 0.4128212175 & 0.0000000000 & -0.0021673855 \\
\hline C & 0.0871753696 & 0.5000000000 & 1.0021331645 \\
\hline C & 0.9128246304 & 0.5000000000 & -0.0021331785 \\
\hline C & 0.2013619769 & 0.0000000000 & 0.4940911020 \\
\hline C & 0.7986380381 & 0.0000000000 & 0.5059088680 \\
\hline C & 0.7013799907 & 0.5000000000 & 0.4941574318 \\
\hline C & 0.2986200093 & 0.5000000000 & 0.5058425382 \\
\hline C & 0.4718409057 & 0.0000000000 & 0.1118207566 \\
\hline C & 0.5281590653 & 0.0000000000 & 0.8881792434 \\
\hline C & 0.9718624067 & 0.5000000000 & 0.1118376148 \\
\hline C & 0.0281376223 & 0.5000000000 & 0.8881623852 \\
\hline C & 0.1482667477 & 0.0000000000 & 0.2767355435 \\
\hline C & 0.8517332673 & 0.0000000000 & 0.7232644565 \\
\hline C & 0.6482853292 & 0.5000000000 & 0.2767959635 \\
\hline C & 0.3517146708 & 0.5000000000 & 0.7232040365 \\
\hline C & 0.5567783710 & 0.0000000000 & 0.1097269470 \\
\hline C & 0.4432216290 & 0.0000000000 & 0.8902730750 \\
\hline C & 0.0567959034 & 0.5000000000 & 0.1097090057 \\
\hline C & 0.9432040966 & 0.5000000000 & 0.8902910163 \\
\hline C & 0.3607371441 & 0.0000000000 & 0.4475103408 \\
\hline C & 0.6392628859 & 0.0000000000 & 0.5524896592 \\
\hline C & 0.8607547203 & 0.5000000000 & 0.4475762613 \\
\hline C & 0.1392452497 & 0.5000000000 & 0.5524237387 \\
\hline C & 0.3481903858 & 0.0000000000 & 0.5636513921 \\
\hline C & 0.6518096142 & 0.0000000000 & 0.4363486079 \\
\hline C & 0.8482093090 & 0.5000000000 & 0.5637174051 \\
\hline C & 0.1517906910 & 0.5000000000 & 0.4362825949 \\
\hline C & 0.2667960112 & 0.0000000000 & 0.5873452965 \\
\hline C & 0.7332039888 & 0.0000000000 & 0.4126547035 \\
\hline C & 0.7668149389 & 0.5000000000 & 0.5874101035 \\
\hline C & 0.2331850611 & 0.5000000000 & 0.4125898965 \\
\hline C & 0.1999409467 & 0.8430754944 & 0.1293275050 \\
\hline C & 0.8000590533 & 0.1569245056 & 0.8706725100 \\
\hline C & 0.8000590533 & 0.8430754944 & 0.8706725100 \\
\hline C & 0.1999409467 & 0.1569245056 & 0.1293275050 \\
\hline C & 0.6999744336 & 0.3430719220 & 0.1294079066 \\
\hline C & 0.3000255664 & 0.6569280780 & 0.8705921084 \\
\hline C & 0.3000255664 & 0.3430719220 & 0.8705921084 \\
\hline C & 0.6999744336 & 0.6569280780 & 0.1294079066 \\
\hline C & 0.1443988905 & 0.9196428244 & 0.0897997314 \\
\hline C & 0.8556011395 & 0.0803571756 & 0.9102002466 \\
\hline C & 0.8556011395 & 0.9196428244 & 0.9102002466 \\
\hline C & 0.1443988905 & 0.0803571756 & 0.0897997314 \\
\hline
\end{tabular}




\begin{tabular}{|c|c|c|c|}
\hline C & 0.6444373733 & 0.4196416825 & 0.0898648948 \\
\hline C & 0.3555625967 & 0.5803583175 & 0.9101350832 \\
\hline C & 0.3555625967 & 0.4196416825 & 0.9101350832 \\
\hline C & 0.6444373733 & 0.5803583175 & 0.0898648948 \\
\hline C & 0.1719543901 & 0.7566339896 & 0.1222879705 \\
\hline C & 0.8280455809 & 0.2433660104 & 0.8777120365 \\
\hline C & 0.8280455809 & 0.7566339896 & 0.8777120365 \\
\hline C & 0.1719543901 & 0.2433660104 & 0.1222879705 \\
\hline C & 0.6719834423 & 0.2566320097 & 0.1223666674 \\
\hline C & 0.3280165867 & 0.7433679903 & 0.8776333396 \\
\hline C & 0.3280165867 & 0.2566320097 & 0.8776333396 \\
\hline C & 0.6719834423 & 0.7433679903 & 0.1223666674 \\
\hline C & 0.2285314768 & 0.6890354805 & 0.1528273400 \\
\hline C & 0.7714685382 & 0.3109645195 & 0.8471726450 \\
\hline C & 0.7714685382 & 0.6890354805 & 0.8471726450 \\
\hline C & 0.2285314768 & 0.3109645195 & 0.1528273400 \\
\hline C & 0.7285564249 & 0.1890291350 & 0.1529053918 \\
\hline C & 0.2714435751 & 0.8109708650 & 0.8470945932 \\
\hline C & 0.2714435751 & 0.1890291350 & 0.8470945932 \\
\hline C & 0.7285564249 & 0.8109708650 & 0.1529053918 \\
\hline C & 0.3114396178 & 0.7097570017 & 0.1922324049 \\
\hline C & 0.6885603822 & 0.2902429983 & 0.8077675951 \\
\hline C & 0.6885603822 & 0.7097570017 & 0.8077675951 \\
\hline C & 0.3114396178 & 0.2902429983 & 0.1922324049 \\
\hline C & 0.8114641757 & 0.2097472042 & 0.1923178534 \\
\hline C & 0.1885358243 & 0.7902527958 & 0.8076821466 \\
\hline C & 0.1885358243 & 0.2097472042 & 0.8076821466 \\
\hline C & 0.8114641757 & 0.7902527958 & 0.1923178534 \\
\hline C & 0.3346738416 & 0.7972581107 & 0.1976630842 \\
\hline C & 0.6653261584 & 0.2027418893 & 0.8023369158 \\
\hline C & 0.6653261584 & 0.7972581107 & 0.8023369158 \\
\hline C & 0.3346738416 & 0.2027418893 & 0.1976630842 \\
\hline C & 0.8347025114 & 0.2972487572 & 0.1977566091 \\
\hline C & 0.1652974886 & 0.7027512428 & 0.8022433909 \\
\hline C & 0.1652974886 & 0.2972487572 & 0.8022433909 \\
\hline C & 0.8347025114 & 0.7027512428 & 0.1977566091 \\
\hline $\mathrm{H}$ & 0.1373475165 & 0.0000000000 & 0.5107344202 \\
\hline $\mathrm{H}$ & 0.8626524985 & 0.0000000000 & 0.4892655798 \\
\hline $\mathrm{H}$ & 0.6373658934 & 0.5000000000 & 0.5108029040 \\
\hline $\mathrm{H}$ & 0.3626341066 & 0.5000000000 & 0.4891970960 \\
\hline $\mathrm{H}$ & 0.1084354253 & 0.9430873498 & 0.2854330552 \\
\hline $\mathrm{H}$ & 0.8915645827 & 0.0569126502 & 0.7145669148 \\
\hline $\mathrm{H}$ & 0.8915645827 & 0.9430873498 & 0.7145669148 \\
\hline $\mathrm{H}$ & 0.1084354253 & 0.0569126502 & 0.2854330552 \\
\hline $\mathrm{H}$ & 0.6084530901 & 0.4430866624 & 0.2854874318 \\
\hline $\mathrm{H}$ & 0.3915469099 & 0.5569133376 & 0.7145125382 \\
\hline $\mathrm{H}$ & 0.3915469099 & 0.4430866624 & 0.7145125382 \\
\hline $\mathrm{H}$ & 0.6084530901 & 0.5569133376 & 0.2854874318 \\
\hline $\mathrm{H}$ & 0.6007882944 & 0.0000000000 & 0.1909430443 \\
\hline $\mathrm{H}$ & 0.3992117056 & 0.0000000000 & 0.8090569847 \\
\hline $\mathrm{H}$ & 0.1008191595 & 0.5000000000 & 0.1909115384 \\
\hline $\mathrm{H}$ & 0.8991808405 & 0.5000000000 & 0.8090884906 \\
\hline $\mathrm{H}$ & 0.4225737197 & 0.0000000000 & 0.4227407623 \\
\hline $\mathrm{H}$ & 0.5774262803 & 0.0000000000 & 0.5772592377 \\
\hline $\mathrm{H}$ & 0.9225910158 & 0.5000000000 & 0.4228051649 \\
\hline $\mathrm{H}$ & 0.0774089842 & 0.5000000000 & 0.5771948351 \\
\hline $\mathrm{H}$ & 0.4017459905 & 0.0000000000 & 0.6330616366 \\
\hline $\mathrm{H}$ & 0.5982540395 & 0.0000000000 & 0.3669383634 \\
\hline
\end{tabular}




\begin{tabular}{|c|c|c|c|}
\hline $\mathrm{H}$ & 0.9017662081 & 0.5000000000 & 0.6331258795 \\
\hline $\mathrm{H}$ & 0.0982337619 & 0.5000000000 & 0.3668741205 \\
\hline $\mathrm{H}$ & 0.2531103720 & 0.0000000000 & 0.6770084702 \\
\hline $\mathrm{H}$ & 0.7468895990 & 0.0000000000 & 0.3229915298 \\
\hline $\mathrm{H}$ & 0.7531286842 & 0.5000000000 & 0.6770733075 \\
\hline $\mathrm{H}$ & 0.2468713448 & 0.5000000000 & 0.3229266925 \\
\hline $\mathrm{H}$ & 0.0791947371 & 0.9045029911 & 0.0948556859 \\
\hline $\mathrm{H}$ & 0.9208052709 & 0.0954970089 & 0.9051443211 \\
\hline $\mathrm{H}$ & 0.9208052709 & 0.9045029911 & 0.9051443211 \\
\hline $\mathrm{H}$ & 0.0791947371 & 0.0954970089 & 0.0948556859 \\
\hline $\mathrm{H}$ & 0.5792299449 & 0.4045033507 & 0.0949009475 \\
\hline $\mathrm{H}$ & 0.4207700551 & 0.5954966493 & 0.9050990595 \\
\hline $\mathrm{H}$ & 0.4207700551 & 0.4045033507 & 0.9050990595 \\
\hline $\mathrm{H}$ & 0.5792299449 & 0.5954966493 & 0.0949009475 \\
\hline $\mathrm{H}$ & 0.1486678420 & 0.9324624480 & -0.0029919312 \\
\hline $\mathrm{H}$ & 0.8513321430 & 0.0675375520 & 1.0029919092 \\
\hline $\mathrm{H}$ & 0.8513321430 & 0.9324624480 & 1.0029919092 \\
\hline $\mathrm{H}$ & 0.1486678420 & 0.0675375520 & -0.0029919312 \\
\hline $\mathrm{H}$ & 0.6487195195 & 0.4324603377 & -0.0029234234 \\
\hline $\mathrm{H}$ & 0.3512804805 & 0.5675396623 & 1.0029234014 \\
\hline $\mathrm{H}$ & 0.3512804805 & 0.4324603377 & 1.0029234014 \\
\hline $\mathrm{H}$ & 0.6487195195 & 0.5675396623 & -0.0029234234 \\
\hline $\mathrm{H}$ & 0.1065505344 & 0.7430472299 & 0.0920182330 \\
\hline $\mathrm{H}$ & 0.8934494806 & 0.2569527701 & 0.9079817530 \\
\hline $\mathrm{H}$ & 0.8934494806 & 0.7430472299 & 0.9079817530 \\
\hline $\mathrm{H}$ & 0.1065505344 & 0.2569527701 & 0.0920182330 \\
\hline $\mathrm{H}$ & 0.6065797436 & 0.2430497109 & 0.0920887249 \\
\hline $\mathrm{H}$ & 0.3934202564 & 0.7569502891 & 0.9079112611 \\
\hline $\mathrm{H}$ & 0.3934202564 & 0.2430497109 & 0.9079112611 \\
\hline $\mathrm{H}$ & 0.6065797436 & 0.7569502891 & 0.0920887249 \\
\hline $\mathrm{H}$ & 0.2090187297 & 0.6207642479 & 0.1444912925 \\
\hline $\mathrm{H}$ & 0.7909812703 & 0.3792357521 & 0.8555087215 \\
\hline $\mathrm{H}$ & 0.7909812703 & 0.6207642479 & 0.8555087215 \\
\hline $\mathrm{H}$ & 0.2090187297 & 0.3792357521 & 0.1444912925 \\
\hline $\mathrm{H}$ & 0.7090424291 & 0.1207579295 & 0.1445619826 \\
\hline $\mathrm{H}$ & 0.2909575709 & 0.8792420705 & 0.8554380314 \\
\hline $\mathrm{H}$ & 0.2909575709 & 0.1207579295 & 0.8554380314 \\
\hline $\mathrm{H}$ & 0.7090424291 & 0.8792420705 & 0.1445619826 \\
\hline $\mathrm{H}$ & 0.3575717291 & 0.6590103961 & 0.2165159477 \\
\hline $\mathrm{H}$ & 0.6424282709 & 0.3409896039 & 0.7834840373 \\
\hline $\mathrm{H}$ & 0.6424282709 & 0.6590103961 & 0.7834840373 \\
\hline $\mathrm{H}$ & 0.3575717291 & 0.3409896039 & 0.2165159477 \\
\hline $\mathrm{H}$ & 0.8575956160 & 0.1589999065 & 0.2165973909 \\
\hline $\mathrm{H}$ & 0.1424043840 & 0.8410000935 & 0.7834025941 \\
\hline $\mathrm{H}$ & 0.1424043840 & 0.1589999065 & 0.7834025941 \\
\hline $\mathrm{H}$ & 0.8575956160 & 0.8410000935 & 0.2165973909 \\
\hline $\mathrm{H}$ & 0.3987096601 & 0.8172392509 & 0.2273635667 \\
\hline $\mathrm{H}$ & 0.6012903399 & 0.1827607491 & 0.7726364033 \\
\hline $\mathrm{H}$ & 0.6012903399 & 0.8172392509 & 0.7726364033 \\
\hline $\mathrm{H}$ & 0.3987096601 & 0.1827607491 & 0.2273635667 \\
\hline $\mathrm{H}$ & 0.8987395196 & 0.3172246528 & 0.2274594010 \\
\hline $\mathrm{H}$ & 0.1012604804 & 0.6827753472 & 0.7725405690 \\
\hline $\mathrm{H}$ & 0.1012604804 & 0.3172246528 & 0.7725405690 \\
\hline $\mathrm{H}$ & 0.8987395196 & 0.6827753472 & 0.2274594010 \\
\hline $\mathrm{F}$ & 0.8451613192 & 0.0000000000 & 0.1940166713 \\
\hline $\mathrm{F}$ & 0.1548386658 & 0.0000000000 & 0.8059833287 \\
\hline $\mathrm{F}$ & 0.3451576356 & 0.5000000000 & 0.1939737168 \\
\hline $\mathrm{F}$ & 0.6548423644 & 0.5000000000 & 0.8060262832 \\
\hline
\end{tabular}




$\begin{array}{llll}\text { F } & 0.9525789703 & 0.0000000000 & 0.3527949972 \\ \text { F } & 0.0474210297 & 0.0000000000 & 0.6472049728 \\ \text { F } & 0.4525718185 & 0.5000000000 & 0.3527558570 \\ F & 0.5474281815 & 0.5000000000 & 0.6472441130 \\ \text { F } & 0.9665405412 & 0.9238110374 & 0.1825771368 \\ F & 0.0334594548 & 0.0761889626 & 0.8174228932 \\ F & 0.0334594548 & 0.9238110374 & 0.8174228932 \\ F & 0.9665405412 & 0.0761889626 & 0.1825771368 \\ \text { F } & 0.4665380599 & 0.4238109589 & 0.1825404597 \\ F & 0.5334619401 & 0.5761890411 & 0.8174595703 \\ F & 0.5334619401 & 0.4238109589 & 0.8174595703 \\ F & 0.4665380599 & 0.5761890411 & 0.1825404597 \\ B & 0.9331499336 & 0.0000000000 & 0.2296473734 \\ B & 0.0668500884 & 0.0000000000 & 0.7703526116 \\ \text { B } & 0.4331462737 & 0.5000000000 & 0.2296069957 \\ \text { B } & 0.5668536963 & 0.5000000000 & 0.7703929893\end{array}$

4-iS

CELL_PARAMETERS (angstrom)

$\begin{array}{rrr}15.995713813 & 0.000000000 & 0.083792922 \\ 0.000000000 & 15.288407089 & 0.000000000 \\ -1.789739737 & 0.000000000 & 11.340066742\end{array}$

ATOMIC_POSITIONS (crystal)

\begin{tabular}{|c|c|c|c|}
\hline Fe1 & 0.3194408687 & 0.0000000000 & 0.1789469370 \\
\hline $\mathrm{Fe} 2$ & 0.6945983859 & 0.0000000000 & 0.8143851516 \\
\hline Fe1 & 0.8194468187 & 0.5000000000 & 0.1789277882 \\
\hline $\mathrm{Fe} 2$ & 0.1946194935 & 0.5000000000 & 0.8143806599 \\
\hline $\mathrm{O}$ & 0.4500910037 & 0.0000000000 & 0.2054997532 \\
\hline $\mathrm{O}$ & 0.5711180104 & 0.0000000000 & 0.7871784582 \\
\hline 0 & 0.9501042509 & 0.5000000000 & 0.2054849785 \\
\hline 0 & 0.0711430561 & 0.5000000000 & 0.7871756201 \\
\hline $\mathrm{O}$ & 0.6773836637 & 0.0000000000 & 0.9814391176 \\
\hline $\mathrm{O}$ & 0.3421153980 & 0.0000000000 & 0.0069365869 \\
\hline $\mathrm{O}$ & 0.1774055106 & 0.5000000000 & 0.9814359580 \\
\hline 0 & 0.8421347299 & 0.5000000000 & 0.0069222517 \\
\hline $\mathrm{N}$ & 0.1785213255 & 0.0000000000 & 0.1583534575 \\
\hline $\mathrm{N}$ & 0.8206100344 & 0.0000000000 & 0.8450912217 \\
\hline $\mathrm{N}$ & 0.6785278358 & 0.5000000000 & 0.1583609807 \\
\hline $\mathrm{N}$ & 0.3206375679 & 0.5000000000 & 0.8450957922 \\
\hline $\mathrm{N}$ & 0.3056130716 & 0.0000000000 & 0.3590417199 \\
\hline $\mathrm{N}$ & 0.7052791911 & 0.0000000000 & 0.6497137683 \\
\hline $\mathrm{N}$ & 0.8056350712 & 0.5000000000 & 0.3590341599 \\
\hline $\mathrm{N}$ & 0.2053120410 & 0.5000000000 & 0.6497149151 \\
\hline $\mathrm{N}$ & 0.2884897078 & 0.8619189299 & 0.1687077288 \\
\hline $\mathrm{N}$ & 0.7082937879 & 0.1273922802 & 0.8199937734 \\
\hline $\mathrm{N}$ & 0.7082937879 & 0.8726077198 & 0.8199937734 \\
\hline $\mathrm{N}$ & 0.2884897078 & 0.1380810701 & 0.1687077288 \\
\hline $\mathrm{N}$ & 0.7884853578 & 0.3619115505 & 0.1686933133 \\
\hline$N$ & 0.2083200627 & 0.6273950533 & 0.8199929694 \\
\hline $\mathrm{N}$ & 0.2083200627 & 0.3726049467 & 0.8199929694 \\
\hline$N$ & 0.7884853578 & 0.6380884495 & 0.1686933133 \\
\hline C & 0.2252294130 & 0.0000000000 & 0.3812854496 \\
\hline C & 0.7854645414 & 0.0000000000 & 0.6251360293 \\
\hline C & 0.7252546672 & 0.5000000000 & 0.3812900814 \\
\hline C & 0.2854981537 & 0.5000000000 & 0.6251399173 \\
\hline C & 0.5985788534 & 0.0000000000 & 0.9942942927 \\
\hline C & 0.4210713750 & 0.0000000000 & -0.0039257320 \\
\hline C & 0.0986004324 & 0.5000000000 & 0.9942921548 \\
\hline
\end{tabular}




\begin{tabular}{|c|c|c|c|}
\hline C & 0.9210905654 & 0.5000000000 & -0.0039373508 \\
\hline C & 0.2073117784 & 0.0000000000 & 0.4969715634 \\
\hline C & 0.8025739526 & 0.0000000000 & 0.5094365433 \\
\hline C & 0.7073457031 & 0.5000000000 & 0.4969810376 \\
\hline C & 0.3026082022 & 0.5000000000 & 0.5094401071 \\
\hline C & 0.4828879744 & 0.0000000000 & 0.1086199162 \\
\hline C & 0.5372295331 & 0.0000000000 & 0.8831233518 \\
\hline C & 0.9829057141 & 0.5000000000 & 0.1086102436 \\
\hline C & 0.0372520788 & 0.5000000000 & 0.8831196503 \\
\hline C & 0.1535876334 & 0.0000000000 & 0.2785237180 \\
\hline C & 0.8543336500 & 0.0000000000 & 0.7295346594 \\
\hline C & 0.6536047557 & 0.5000000000 & 0.2785378221 \\
\hline C & 0.3543644761 & 0.5000000000 & 0.7295413463 \\
\hline C & 0.5696654219 & 0.0000000000 & 0.1040332776 \\
\hline C & 0.4504753017 & 0.0000000000 & 0.8871276127 \\
\hline C & 0.0696849426 & 0.5000000000 & 0.1040297940 \\
\hline C & 0.9504971410 & 0.5000000000 & 0.8871171918 \\
\hline C & 0.3701915265 & 0.0000000000 & 0.4493395693 \\
\hline C & 0.6400149139 & 0.0000000000 & 0.5598040775 \\
\hline C & 0.8702185004 & 0.5000000000 & 0.4493250796 \\
\hline C & 0.1400495814 & 0.5000000000 & 0.5598034335 \\
\hline C & 0.3572363298 & 0.0000000000 & 0.5660718399 \\
\hline C & 0.6524804973 & 0.0000000000 & 0.4427135190 \\
\hline C & 0.8572720712 & 0.5000000000 & 0.5660615454 \\
\hline C & 0.1525148675 & 0.5000000000 & 0.4427130306 \\
\hline C & 0.2740724818 & 0.0000000000 & 0.5902871300 \\
\hline C & 0.7352147435 & 0.0000000000 & 0.4169392254 \\
\hline C & 0.7741113876 & 0.5000000000 & 0.5902894577 \\
\hline C & 0.2352500131 & 0.5000000000 & 0.4169416218 \\
\hline C & 0.2061700452 & 0.8430251692 & 0.1323024814 \\
\hline C & 0.7873023327 & 0.1538122222 & 0.8675726680 \\
\hline C & 0.7873023327 & 0.8461877778 & 0.8675726680 \\
\hline C & 0.2061700452 & 0.1569748308 & 0.1323024814 \\
\hline C & 0.7061622288 & 0.3430247846 & 0.1323021745 \\
\hline C & 0.2873302810 & 0.6538128246 & 0.8675697907 \\
\hline C & 0.2873302810 & 0.3461871754 & 0.8675697907 \\
\hline C & 0.7061622288 & 0.6569752154 & 0.1323021745 \\
\hline C & 0.1504991461 & 0.9197231606 & 0.0906887477 \\
\hline C & 0.8454513824 & 0.0808261075 & 0.9146884217 \\
\hline C & 0.8454513824 & 0.9191738925 & 0.9146884217 \\
\hline C & 0.1504991461 & 0.0802768394 & 0.0906887477 \\
\hline C & 0.6504968764 & 0.4197269806 & 0.0906961076 \\
\hline C & 0.3454760563 & 0.5808267673 & 0.9146922928 \\
\hline C & 0.3454760563 & 0.4191732327 & 0.9146922928 \\
\hline C & 0.6504968764 & 0.5802730194 & 0.0906961076 \\
\hline C & 0.1764817390 & 0.7570181976 & 0.1284010075 \\
\hline C & 0.8092828255 & 0.2420168567 & 0.8756704508 \\
\hline C & 0.8092828255 & 0.7579831433 & 0.8756704508 \\
\hline C & 0.1764817390 & 0.2429818024 & 0.1284010075 \\
\hline C & 0.6764633572 & 0.2570226333 & 0.1284064039 \\
\hline C & 0.3093132762 & 0.7420175987 & 0.8756630336 \\
\hline C & 0.3093132762 & 0.2579824013 & 0.8756630336 \\
\hline C & 0.6764633572 & 0.7429773667 & 0.1284064039 \\
\hline C & 0.2332700657 & 0.6892884332 & 0.1626261061 \\
\hline C & 0.7485496960 & 0.3050279923 & 0.8356785427 \\
\hline C & 0.7485496960 & 0.6949720077 & 0.8356785427 \\
\hline C & 0.2332700657 & 0.3107115668 & 0.1626261061 \\
\hline C & 0.7332450442 & 0.1892859561 & 0.1626233014 \\
\hline
\end{tabular}




\begin{tabular}{|c|c|c|c|}
\hline C & 0.2485814895 & 0.8050288625 & 0.8356726600 \\
\hline C & 0.2485814895 & 0.1949711375 & 0.8356726600 \\
\hline C & 0.7332450442 & 0.8107140439 & 0.1626233014 \\
\hline C & 0.3179714019 & 0.7093965031 & 0.2020196832 \\
\hline C & 0.6677073214 & 0.2774997619 & 0.7862014446 \\
\hline C & 0.6677073214 & 0.7225002381 & 0.7862014446 \\
\hline C & 0.3179714019 & 0.2906034969 & 0.2020196832 \\
\hline C & 0.8179508192 & 0.2093836481 & 0.2020004981 \\
\hline C & 0.1677361659 & 0.7775045147 & 0.7862020877 \\
\hline C & 0.1677361659 & 0.2224954853 & 0.7862020877 \\
\hline C & 0.8179508192 & 0.7906163519 & 0.2020004981 \\
\hline C & 0.3429149532 & 0.7964926004 & 0.2034056678 \\
\hline C & 0.6500998925 & 0.1883797607 & 0.7795488431 \\
\hline C & 0.6500998925 & 0.8116202393 & 0.7795488431 \\
\hline C & 0.3429149532 & 0.2035073996 & 0.2034056678 \\
\hline C & 0.8429045981 & 0.2964781032 & 0.2033803183 \\
\hline C & 0.1501270111 & 0.6883845673 & 0.7795519826 \\
\hline C & 0.1501270111 & 0.3116154327 & 0.7795519826 \\
\hline C & 0.8429045981 & 0.7035218968 & 0.2033803183 \\
\hline $\mathrm{H}$ & 0.1418923689 & 0.0000000000 & 0.5139973264 \\
\hline $\mathrm{H}$ & 0.8678065163 & 0.0000000000 & 0.4918094489 \\
\hline $\mathrm{H}$ & 0.6419287272 & 0.5000000000 & 0.5140164706 \\
\hline $\mathrm{H}$ & 0.3678408573 & 0.5000000000 & 0.4918140663 \\
\hline $\mathrm{H}$ & 0.1128803901 & 0.9430894260 & 0.2873324147 \\
\hline $\mathrm{H}$ & 0.8956707937 & 0.0570163768 & 0.7257772763 \\
\hline $\mathrm{H}$ & 0.8956707937 & 0.9429836232 & 0.7257772763 \\
\hline $\mathrm{H}$ & 0.1128803901 & 0.0569105740 & 0.2873324147 \\
\hline $\mathrm{H}$ & 0.6128994043 & 0.4430887883 & 0.2873527781 \\
\hline $\mathrm{H}$ & 0.3957015428 & 0.5570164116 & 0.7257851106 \\
\hline $\mathrm{H}$ & 0.3957015428 & 0.4429835884 & 0.7257851106 \\
\hline $\mathrm{H}$ & 0.6128994043 & 0.5569112117 & 0.2873527781 \\
\hline $\mathrm{H}$ & 0.6150613489 & 0.0000000000 & 0.1850013167 \\
\hline $\mathrm{H}$ & 0.4046284762 & 0.0000000000 & 0.8068360074 \\
\hline $\mathrm{H}$ & 0.1150775222 & 0.5000000000 & 0.1850015061 \\
\hline $\mathrm{H}$ & 0.9046533580 & 0.5000000000 & 0.8068232462 \\
\hline $\mathrm{H}$ & 0.4333561416 & 0.0000000000 & 0.4240639371 \\
\hline $\mathrm{H}$ & 0.5773496004 & 0.0000000000 & 0.5862358379 \\
\hline $\mathrm{H}$ & 0.9333805207 & 0.5000000000 & 0.4240406136 \\
\hline $\mathrm{H}$ & 0.0773836359 & 0.5000000000 & 0.5862344755 \\
\hline $\mathrm{H}$ & 0.4118429429 & 0.0000000000 & 0.6355000005 \\
\hline $\mathrm{H}$ & 0.5975496686 & 0.0000000000 & 0.3735480984 \\
\hline $\mathrm{H}$ & 0.9118832826 & 0.5000000000 & 0.6354827052 \\
\hline $\mathrm{H}$ & 0.0975840340 & 0.5000000000 & 0.3735474622 \\
\hline $\mathrm{H}$ & 0.2600885845 & 0.0000000000 & 0.6805243624 \\
\hline $\mathrm{H}$ & 0.7486030287 & 0.0000000000 & 0.3264811001 \\
\hline $\mathrm{H}$ & 0.7601348878 & 0.5000000000 & 0.6805303231 \\
\hline $\mathrm{H}$ & 0.2486398623 & 0.5000000000 & 0.3264845201 \\
\hline $\mathrm{H}$ & 0.0835454979 & 0.9052112294 & 0.0941508064 \\
\hline $\mathrm{H}$ & 0.9125014982 & 0.0970301753 & 0.9148430578 \\
\hline $\mathrm{H}$ & 0.9125014982 & 0.9029698247 & 0.9148430578 \\
\hline $\mathrm{H}$ & 0.0835454979 & 0.0947887706 & 0.0941508064 \\
\hline $\mathrm{H}$ & 0.5835429195 & 0.4052208955 & 0.0941628649 \\
\hline $\mathrm{H}$ & 0.4125268472 & 0.5970297224 & 0.9148524872 \\
\hline $\mathrm{H}$ & 0.4125268472 & 0.4029702776 & 0.9148524872 \\
\hline $\mathrm{H}$ & 0.5835429195 & 0.5947791045 & 0.0941628649 \\
\hline $\mathrm{H}$ & 0.1565423940 & 0.9322656251 & -0.0021058011 \\
\hline $\mathrm{H}$ & 0.8364722988 & 0.0676626166 & 1.0062263819 \\
\hline $\mathrm{H}$ & 0.8364722988 & 0.9323373834 & 1.0062263819 \\
\hline
\end{tabular}




\begin{tabular}{|c|c|c|c|}
\hline $\mathrm{H}$ & 0.1565423940 & 0.0677343749 & -0.0021058011 \\
\hline $\mathrm{H}$ & 0.6565375578 & 0.4322699428 & -0.0020990266 \\
\hline $\mathrm{H}$ & 0.3364927809 & 0.5676655538 & 1.0062290817 \\
\hline $\mathrm{H}$ & 0.3364927809 & 0.4323344462 & 1.0062290817 \\
\hline $\mathrm{H}$ & 0.6565375578 & 0.5677300572 & -0.0020990266 \\
\hline $\mathrm{H}$ & 0.1096829527 & 0.7439231831 & 0.0979589623 \\
\hline $\mathrm{H}$ & 0.8733130840 & 0.2606938348 & 0.9143257798 \\
\hline $\mathrm{H}$ & 0.8733130840 & 0.7393061652 & 0.9143257798 \\
\hline $\mathrm{H}$ & 0.1096829527 & 0.2560768169 & 0.0979589623 \\
\hline $\mathrm{H}$ & 0.6096614010 & 0.2439362117 & 0.0979755485 \\
\hline $\mathrm{H}$ & 0.3733440122 & 0.7606945371 & 0.9143158213 \\
\hline $\mathrm{H}$ & 0.3733440122 & 0.2393054629 & 0.9143158213 \\
\hline $\mathrm{H}$ & 0.6096614010 & 0.7560637883 & 0.0979755485 \\
\hline $\mathrm{H}$ & 0.2124648749 & 0.6212961327 & 0.1574968449 \\
\hline $\mathrm{H}$ & 0.7633531447 & 0.3746412362 & 0.8440636060 \\
\hline $\mathrm{H}$ & 0.7633531447 & 0.6253587638 & 0.8440636060 \\
\hline $\mathrm{H}$ & 0.2124648749 & 0.3787038673 & 0.1574968449 \\
\hline $\mathrm{H}$ & 0.7124314236 & 0.1212961448 & 0.1575004778 \\
\hline $\mathrm{H}$ & 0.2633867033 & 0.8746419342 & 0.8440569671 \\
\hline $\mathrm{H}$ & 0.2633867033 & 0.1253580658 & 0.8440569671 \\
\hline $\mathrm{H}$ & 0.7124314236 & 0.8787038552 & 0.1575004778 \\
\hline $\mathrm{H}$ & 0.3642768165 & 0.6584959867 & 0.2290743396 \\
\hline $\mathrm{H}$ & 0.6182837927 & 0.3245161175 & 0.7549955450 \\
\hline $\mathrm{H}$ & 0.6182837927 & 0.6754838825 & 0.7549955450 \\
\hline $\mathrm{H}$ & 0.3642768165 & 0.3415040133 & 0.2290743396 \\
\hline $\mathrm{H}$ & 0.8642526461 & 0.1584788806 & 0.2290488713 \\
\hline $\mathrm{H}$ & 0.1183126660 & 0.8245221762 & 0.7550005126 \\
\hline $\mathrm{H}$ & 0.1183126660 & 0.1754778238 & 0.7550005126 \\
\hline $\mathrm{H}$ & 0.8642526461 & 0.8415211194 & 0.2290488713 \\
\hline $\mathrm{H}$ & 0.4084879486 & 0.8159488102 & 0.2325340076 \\
\hline $\mathrm{H}$ & 0.5881005049 & 0.1630528732 & 0.7420168868 \\
\hline $\mathrm{H}$ & 0.5881005049 & 0.8369471268 & 0.7420168868 \\
\hline $\mathrm{H}$ & 0.4084879486 & 0.1840511898 & 0.2325340076 \\
\hline $\mathrm{H}$ & 0.9084816816 & 0.3159252330 & 0.2324973084 \\
\hline $\mathrm{H}$ & 0.0881255988 & 0.6630595058 & 0.7420233673 \\
\hline $\mathrm{H}$ & 0.0881255988 & 0.3369404942 & 0.7420233673 \\
\hline $\mathrm{H}$ & 0.9084816816 & 0.6840747670 & 0.2324973084 \\
\hline $\mathrm{F}$ & 0.8464553310 & 0.0000000000 & 0.1978171962 \\
\hline $\mathrm{F}$ & 0.1579960463 & 0.0000000000 & 0.8109016885 \\
\hline $\mathrm{F}$ & 0.3464651822 & 0.5000000000 & 0.1977976188 \\
\hline $\mathrm{F}$ & 0.6580115456 & 0.5000000000 & 0.8109030331 \\
\hline $\mathrm{F}$ & 0.9586819667 & 0.0000000000 & 0.3528863302 \\
\hline $\mathrm{F}$ & 0.0470469726 & 0.0000000000 & 0.6544580293 \\
\hline $\mathrm{F}$ & 0.4586844301 & 0.5000000000 & 0.3528774735 \\
\hline $\mathrm{F}$ & 0.5470654401 & 0.5000000000 & 0.6544558968 \\
\hline $\mathrm{F}$ & 0.9696285547 & 0.9238683090 & 0.1805464002 \\
\hline $\mathrm{F}$ & 0.0342103255 & 0.0759784099 & 0.8265449700 \\
\hline $\mathrm{F}$ & 0.0342103255 & 0.9240215901 & 0.8265449700 \\
\hline $\mathrm{F}$ & 0.9696285547 & 0.0761316910 & 0.1805464002 \\
\hline $\mathrm{F}$ & 0.4696403451 & 0.4238680078 & 0.1805418235 \\
\hline $\mathrm{F}$ & 0.5342249231 & 0.5759776704 & 0.8265426134 \\
\hline $\mathrm{F}$ & 0.5342249231 & 0.4240223296 & 0.8265426134 \\
\hline $\mathrm{F}$ & 0.4696403451 & 0.5761319922 & 0.1805418235 \\
\hline B & 0.9365665884 & 0.0000000000 & 0.2293177681 \\
\hline B & 0.0680800519 & 0.0000000000 & 0.7782662483 \\
\hline B & 0.4365755703 & 0.5000000000 & 0.2293082163 \\
\hline B & 0.5680965744 & 0.5000000000 & 0.7782644688 \\
\hline
\end{tabular}




$\begin{array}{ccr}\text { CELL_PARAMETERS (angstrom) } & \\ 15.662089800 & 0.000000000 & 0.156566250 \\ 0.000000000 & 15.265743511 & 0.000000000 \\ -1.696776731 & 0.000000000 & 11.317114102\end{array}$

\begin{tabular}{|c|c|c|c|}
\hline \multicolumn{4}{|c|}{ ATOMIC_POSITIONS (crystal) } \\
\hline Fe2 & 0.3134048485 & 0.0000000000 & 0.1874544822 \\
\hline $\mathrm{Fe} 2$ & 0.6865951515 & 0.0000000000 & 0.8125455028 \\
\hline $\mathrm{Fe} 2$ & 0.8134025449 & 0.5000000000 & 0.1874494993 \\
\hline $\mathrm{Fe} 2$ & 0.1865974551 & 0.5000000000 & 0.8125504857 \\
\hline 0 & 0.4398340578 & 0.0000000000 & 0.2110802246 \\
\hline 0 & 0.5601659422 & 0.0000000000 & 0.7889197904 \\
\hline 0 & 0.9398174980 & 0.5000000000 & 0.2110631523 \\
\hline 0 & 0.0601825020 & 0.5000000000 & 0.7889368627 \\
\hline 0 & 0.6702565043 & 0.0000000000 & 0.9802328419 \\
\hline 0 & 0.3297434957 & 0.0000000000 & 0.0197671601 \\
\hline 0 & 0.1702638422 & 0.5000000000 & 0.9802547666 \\
\hline 0 & 0.8297361578 & 0.5000000000 & 0.0197452354 \\
\hline $\mathrm{N}$ & 0.1852161883 & 0.0000000000 & 0.1576979805 \\
\hline $\mathrm{N}$ & 0.8147838417 & 0.0000000000 & 0.8423020195 \\
\hline $\mathrm{N}$ & 0.6852002682 & 0.5000000000 & 0.1577066926 \\
\hline $\mathrm{N}$ & 0.3147997018 & 0.5000000000 & 0.8422933074 \\
\hline $\mathrm{N}$ & 0.3017463739 & 0.0000000000 & 0.3524494921 \\
\hline $\mathrm{N}$ & 0.6982536261 & 0.0000000000 & 0.6475504779 \\
\hline $\mathrm{N}$ & 0.8017543428 & 0.5000000000 & 0.3524338873 \\
\hline $\mathrm{N}$ & 0.1982456572 & 0.5000000000 & 0.6475660827 \\
\hline $\mathrm{N}$ & 0.2993644416 & 0.8724797257 & 0.1833302892 \\
\hline $\mathrm{N}$ & 0.7006355584 & 0.1275202743 & 0.8166696808 \\
\hline $\mathrm{N}$ & 0.7006355584 & 0.8724797257 & 0.8166696808 \\
\hline $\mathrm{N}$ & 0.2993644416 & 0.1275202743 & 0.1833302892 \\
\hline $\mathrm{N}$ & 0.7993408356 & 0.3724726646 & 0.1833178283 \\
\hline $\mathrm{N}$ & 0.2006591644 & 0.6275273354 & 0.8166821417 \\
\hline $\mathrm{N}$ & 0.2006591644 & 0.3724726646 & 0.8166821417 \\
\hline $\mathrm{N}$ & 0.7993408356 & 0.6275273354 & 0.1833178283 \\
\hline C & 0.2197623412 & 0.0000000000 & 0.3777300592 \\
\hline C & 0.7802376438 & 0.0000000000 & 0.6222699408 \\
\hline C & 0.7197769122 & 0.5000000000 & 0.3777374175 \\
\hline C & 0.2802230878 & 0.5000000000 & 0.6222625825 \\
\hline C & 0.5902176245 & 0.0000000000 & 0.9961803406 \\
\hline C & 0.4097823755 & 0.0000000000 & 0.0038196454 \\
\hline C & 0.0902231589 & 0.5000000000 & 0.9961965271 \\
\hline C & 0.9097768411 & 0.5000000000 & 0.0038034589 \\
\hline C & 0.2017660146 & 0.0000000000 & 0.4938487405 \\
\hline C & 0.7982340004 & 0.0000000000 & 0.5061512295 \\
\hline C & 0.7017981679 & 0.5000000000 & 0.4938634546 \\
\hline C & 0.2982018321 & 0.5000000000 & 0.5061365154 \\
\hline C & 0.4735250092 & 0.0000000000 & 0.1137596815 \\
\hline C & 0.5264749618 & 0.0000000000 & 0.8862403185 \\
\hline C & 0.9735143383 & 0.5000000000 & 0.1137478597 \\
\hline C & 0.0264856907 & 0.5000000000 & 0.8862521403 \\
\hline C & 0.1500496472 & 0.0000000000 & 0.2735649810 \\
\hline C & 0.8499503678 & 0.0000000000 & 0.7264350190 \\
\hline C & 0.6500512122 & 0.5000000000 & 0.2735867373 \\
\hline C & 0.3499487878 & 0.5000000000 & 0.7264132627 \\
\hline C & 0.5620408384 & 0.0000000000 & 0.1071363464 \\
\hline C & 0.4379591616 & 0.0000000000 & 0.8928636756 \\
\hline C & 0.0620334010 & 0.5000000000 & 0.1071424819 \\
\hline C & 0.9379665990 & 0.5000000000 & 0.8928575401 \\
\hline
\end{tabular}




\begin{tabular}{|c|c|c|c|}
\hline C & 0.3678863125 & 0.0000000000 & 0.4419770304 \\
\hline C & 0.6321137175 & 0.0000000000 & 0.5580229696 \\
\hline C & 0.8679073358 & 0.5000000000 & 0.4419451268 \\
\hline C & 0.1320926342 & 0.5000000000 & 0.5580548732 \\
\hline C & 0.3545945225 & 0.0000000000 & 0.5594080456 \\
\hline C & 0.6454054775 & 0.0000000000 & 0.4405919544 \\
\hline C & 0.8546324360 & 0.5000000000 & 0.5593832061 \\
\hline C & 0.1453675640 & 0.5000000000 & 0.4406167939 \\
\hline C & 0.2700655010 & 0.0000000000 & 0.5859696119 \\
\hline C & 0.7299344990 & 0.0000000000 & 0.4140303881 \\
\hline C & 0.7701104226 & 0.5000000000 & 0.5859689775 \\
\hline C & 0.2298895774 & 0.5000000000 & 0.4140310225 \\
\hline C & 0.2188482010 & 0.8460471582 & 0.1366955106 \\
\hline C & 0.7811517990 & 0.1539528418 & 0.8633045044 \\
\hline C & 0.7811517990 & 0.8460471582 & 0.8633045044 \\
\hline C & 0.2188482010 & 0.1539528418 & 0.1366955106 \\
\hline C & 0.7188180016 & 0.3460447838 & 0.1367067730 \\
\hline C & 0.2811819984 & 0.6539552162 & 0.8632932420 \\
\hline C & 0.2811819984 & 0.3460447838 & 0.8632932420 \\
\hline C & 0.7188180016 & 0.6539552162 & 0.1367067730 \\
\hline C & 0.1604518410 & 0.9190371437 & 0.0880742795 \\
\hline C & 0.8395481890 & 0.0809628563 & 0.9119256985 \\
\hline C & 0.8395481890 & 0.9190371437 & 0.9119256985 \\
\hline C & 0.1604518410 & 0.0809628563 & 0.0880742795 \\
\hline C & 0.6604240878 & 0.4190370866 & 0.0880885184 \\
\hline C & 0.3395758822 & 0.5809629134 & 0.9119114596 \\
\hline C & 0.3395758822 & 0.4190370866 & 0.9119114596 \\
\hline C & 0.6604240878 & 0.5809629134 & 0.0880885184 \\
\hline C & 0.1956474858 & 0.7579148009 & 0.1319508065 \\
\hline C & 0.8043524852 & 0.2420851991 & 0.8680492005 \\
\hline C & 0.8043524852 & 0.7579148009 & 0.8680492005 \\
\hline C & 0.1956474858 & 0.2420851991 & 0.1319508065 \\
\hline C & 0.6956059197 & 0.2579157823 & 0.1319784861 \\
\hline C & 0.3043941093 & 0.7420842177 & 0.8680215209 \\
\hline C & 0.3043941093 & 0.2579157823 & 0.8680215209 \\
\hline C & 0.6956059197 & 0.7420842177 & 0.1319784861 \\
\hline C & 0.2566164925 & 0.6950290488 & 0.1751211469 \\
\hline C & 0.7433835225 & 0.3049709512 & 0.8248788381 \\
\hline C & 0.7433835225 & 0.6950290488 & 0.8248788381 \\
\hline C & 0.2566164925 & 0.3049709512 & 0.1751211469 \\
\hline C & 0.7565729347 & 0.1950246016 & 0.1751402366 \\
\hline C & 0.2434270653 & 0.8049753984 & 0.8248597484 \\
\hline C & 0.2434270653 & 0.1950246016 & 0.8248597484 \\
\hline C & 0.7565729347 & 0.8049753984 & 0.1751402366 \\
\hline C & 0.3389466060 & 0.7225239019 & 0.2237009817 \\
\hline C & 0.6610533940 & 0.2774760981 & 0.7762990183 \\
\hline C & 0.6610533940 & 0.7225239019 & 0.7762990183 \\
\hline C & 0.3389466060 & 0.2774760981 & 0.2237009817 \\
\hline C & 0.8389106912 & 0.2225124438 & 0.2236932492 \\
\hline C & 0.1610893088 & 0.7774875562 & 0.7763067508 \\
\hline C & 0.1610893088 & 0.2225124438 & 0.7763067508 \\
\hline C & 0.8389106912 & 0.7774875562 & 0.2236932492 \\
\hline C & 0.3578587656 & 0.8115872651 & 0.2263116215 \\
\hline C & 0.6421412344 & 0.1884127349 & 0.7736883785 \\
\hline C & 0.6421412344 & 0.8115872651 & 0.7736883785 \\
\hline C & 0.3578587656 & 0.1884127349 & 0.2263116215 \\
\hline C & 0.8578340349 & 0.3115743629 & 0.2262878068 \\
\hline C & 0.1421659651 & 0.6884256371 & 0.7737121932 \\
\hline
\end{tabular}




\begin{tabular}{|c|c|c|c|}
\hline C & 0.1421659651 & 0.3115743629 & 0.7737121932 \\
\hline C & 0.8578340349 & 0.6884256371 & 0.2262878068 \\
\hline $\mathrm{H}$ & 0.1351276700 & 0.0000000000 & 0.5121028799 \\
\hline $\mathrm{H}$ & 0.8648723450 & 0.0000000000 & 0.4878971201 \\
\hline $\mathrm{H}$ & 0.6351646854 & 0.5000000000 & 0.5121374173 \\
\hline $\mathrm{H}$ & 0.3648353146 & 0.5000000000 & 0.4878625827 \\
\hline $\mathrm{H}$ & 0.1078588421 & 0.9428928738 & 0.2776155605 \\
\hline $\mathrm{H}$ & 0.8921411659 & 0.0571071262 & 0.7223844095 \\
\hline $\mathrm{H}$ & 0.8921411659 & 0.9428928738 & 0.7223844095 \\
\hline $\mathrm{H}$ & 0.1078588421 & 0.0571071262 & 0.2776155605 \\
\hline $\mathrm{H}$ & 0.6078623120 & 0.4428923917 & 0.2776482654 \\
\hline $\mathrm{H}$ & 0.3921376880 & 0.5571076083 & 0.7223517046 \\
\hline $\mathrm{H}$ & 0.3921376880 & 0.4428923917 & 0.7223517046 \\
\hline $\mathrm{H}$ & 0.6078623120 & 0.5571076083 & 0.2776482654 \\
\hline $\mathrm{H}$ & 0.6090898237 & 0.0000000000 & 0.1867302875 \\
\hline $\mathrm{H}$ & 0.3909101763 & 0.0000000000 & 0.8132697415 \\
\hline $\mathrm{H}$ & 0.1090723227 & 0.5000000000 & 0.1867472533 \\
\hline $\mathrm{H}$ & 0.8909276773 & 0.5000000000 & 0.8132527757 \\
\hline $\mathrm{H}$ & 0.4319622686 & 0.0000000000 & 0.4149693695 \\
\hline $\mathrm{H}$ & 0.5680377314 & 0.0000000000 & 0.5850306305 \\
\hline $\mathrm{H}$ & 0.9319756608 & 0.5000000000 & 0.4149170993 \\
\hline $\mathrm{H}$ & 0.0680243392 & 0.5000000000 & 0.5850829007 \\
\hline $\mathrm{H}$ & 0.4102790518 & 0.0000000000 & 0.6282704356 \\
\hline $\mathrm{H}$ & 0.5897209782 & 0.0000000000 & 0.3717295644 \\
\hline $\mathrm{H}$ & 0.9103246985 & 0.5000000000 & 0.6282331610 \\
\hline $\mathrm{H}$ & 0.0896752715 & 0.5000000000 & 0.3717668390 \\
\hline $\mathrm{H}$ & 0.2560704268 & 0.0000000000 & 0.6767753948 \\
\hline $\mathrm{H}$ & 0.7439295442 & 0.0000000000 & 0.3232246052 \\
\hline $\mathrm{H}$ & 0.7561286458 & 0.5000000000 & 0.6767808215 \\
\hline $\mathrm{H}$ & 0.2438713832 & 0.5000000000 & 0.3232191785 \\
\hline $\mathrm{H}$ & 0.0918335729 & 0.9032383342 & 0.0869713108 \\
\hline $\mathrm{H}$ & 0.9081664351 & 0.0967616658 & 0.9130286962 \\
\hline $\mathrm{H}$ & 0.9081664351 & 0.9032383342 & 0.9130286962 \\
\hline $\mathrm{H}$ & 0.0918335729 & 0.0967616658 & 0.0869713108 \\
\hline $\mathrm{H}$ & 0.5918056070 & 0.4032439941 & 0.0869960097 \\
\hline $\mathrm{H}$ & 0.4081943930 & 0.5967560059 & 0.9130039973 \\
\hline $\mathrm{H}$ & 0.4081943930 & 0.4032439941 & 0.9130039973 \\
\hline $\mathrm{H}$ & 0.5918056070 & 0.5967560059 & 0.0869960097 \\
\hline $\mathrm{H}$ & 0.1712643544 & 0.9319631961 & -0.0032855884 \\
\hline $\mathrm{H}$ & 0.8287356306 & 0.0680368039 & 1.0032855664 \\
\hline $\mathrm{H}$ & 0.8287356306 & 0.9319631961 & 1.0032855664 \\
\hline $\mathrm{H}$ & 0.1712643544 & 0.0680368039 & -0.0032855884 \\
\hline $\mathrm{H}$ & 0.6712314044 & 0.4319589881 & -0.0032746574 \\
\hline $\mathrm{H}$ & 0.3287685956 & 0.5680410119 & 1.0032746354 \\
\hline $\mathrm{H}$ & 0.3287685956 & 0.4319589881 & 1.0032746354 \\
\hline $\mathrm{H}$ & 0.6712314044 & 0.5680410119 & -0.0032746574 \\
\hline $\mathrm{H}$ & 0.1304907746 & 0.7392444913 & 0.0936841122 \\
\hline $\mathrm{H}$ & 0.8695092404 & 0.2607555087 & 0.9063158738 \\
\hline $\mathrm{H}$ & 0.8695092404 & 0.7392444913 & 0.9063158738 \\
\hline $\mathrm{H}$ & 0.1304907746 & 0.2607555087 & 0.0936841122 \\
\hline $\mathrm{H}$ & 0.6304435606 & 0.2392508144 & 0.0937291338 \\
\hline $\mathrm{H}$ & 0.3695564394 & 0.7607491856 & 0.9062708522 \\
\hline $\mathrm{H}$ & 0.3695564394 & 0.2392508144 & 0.9062708522 \\
\hline $\mathrm{H}$ & 0.6304435606 & 0.7607491856 & 0.0937291338 \\
\hline $\mathrm{H}$ & 0.2410024431 & 0.6253997397 & 0.1699988493 \\
\hline $\mathrm{H}$ & 0.7589975569 & 0.3746002603 & 0.8300011647 \\
\hline $\mathrm{H}$ & 0.7589975569 & 0.6253997397 & 0.8300011647 \\
\hline $\mathrm{H}$ & 0.2410024431 & 0.3746002603 & 0.1699988493 \\
\hline
\end{tabular}




\begin{tabular}{|c|c|c|c|}
\hline $\mathrm{H}$ & 0.7409504981 & 0.1253971617 & 0.1700289573 \\
\hline $\mathrm{H}$ & 0.2590495019 & 0.8746028383 & 0.8299710567 \\
\hline $\mathrm{H}$ & 0.2590495019 & 0.1253971617 & 0.8299710567 \\
\hline $\mathrm{H}$ & 0.7409504981 & 0.8746028383 & 0.1700289573 \\
\hline $\mathrm{H}$ & 0.3885241021 & 0.6754737668 & 0.2572616178 \\
\hline $\mathrm{H}$ & 0.6114758979 & 0.3245262332 & 0.7427383672 \\
\hline $\mathrm{H}$ & 0.6114758979 & 0.6754737668 & 0.7427383672 \\
\hline $\mathrm{H}$ & 0.3885241021 & 0.3245262332 & 0.2572616178 \\
\hline $\mathrm{H}$ & 0.8884879642 & 0.1754594237 & 0.2572447359 \\
\hline $\mathrm{H}$ & 0.1115120358 & 0.8245405763 & 0.7427552491 \\
\hline $\mathrm{H}$ & 0.1115120358 & 0.1754594237 & 0.7427552491 \\
\hline $\mathrm{H}$ & 0.8884879642 & 0.8245405763 & 0.2572447359 \\
\hline $\mathrm{H}$ & 0.4210948183 & 0.8369195540 & 0.2628181056 \\
\hline $\mathrm{H}$ & 0.5789051817 & 0.1630804460 & 0.7371818644 \\
\hline $\mathrm{H}$ & 0.5789051817 & 0.8369195540 & 0.7371818644 \\
\hline $\mathrm{H}$ & 0.4210948183 & 0.1630804460 & 0.2628181056 \\
\hline $\mathrm{H}$ & 0.9210763014 & 0.3369005653 & 0.2627729053 \\
\hline $\mathrm{H}$ & 0.0789236986 & 0.6630994347 & 0.7372270647 \\
\hline $\mathrm{H}$ & 0.0789236986 & 0.3369005653 & 0.7372270647 \\
\hline $\mathrm{H}$ & 0.9210763014 & 0.6630994347 & 0.2627729053 \\
\hline $\mathrm{F}$ & 0.8421670556 & 0.0000000000 & 0.1900734068 \\
\hline $\mathrm{F}$ & 0.1578329294 & 0.0000000000 & 0.8099265932 \\
\hline $\mathrm{F}$ & 0.3421639803 & 0.5000000000 & 0.1900838986 \\
\hline $\mathrm{F}$ & 0.6578360197 & 0.5000000000 & 0.8099161014 \\
\hline $\mathrm{F}$ & 0.9570991667 & 0.0000000000 & 0.3422114880 \\
\hline $\mathrm{F}$ & 0.0429008333 & 0.0000000000 & 0.6577884820 \\
\hline $\mathrm{F}$ & 0.4570922318 & 0.5000000000 & 0.3422274661 \\
\hline $\mathrm{F}$ & 0.5429077682 & 0.5000000000 & 0.6577725039 \\
\hline $\mathrm{F}$ & 0.9677161976 & 0.9239478387 & 0.1685817098 \\
\hline $\mathrm{F}$ & 0.0322837984 & 0.0760521613 & 0.8314183202 \\
\hline $\mathrm{F}$ & 0.0322837984 & 0.9239478387 & 0.8314183202 \\
\hline $\mathrm{F}$ & 0.9677161976 & 0.0760521613 & 0.1685817098 \\
\hline $\mathrm{F}$ & 0.4677146405 & 0.4239482257 & 0.1685986173 \\
\hline $\mathrm{F}$ & 0.5322853595 & 0.5760517743 & 0.8314014127 \\
\hline $\mathrm{F}$ & 0.5322853595 & 0.4239482257 & 0.8314014127 \\
\hline $\mathrm{F}$ & 0.4677146405 & 0.5760517743 & 0.1685986173 \\
\hline B & 0.9340264008 & 0.0000000000 & 0.2182994743 \\
\hline B & 0.0659736212 & 0.0000000000 & 0.7817005107 \\
\hline B & 0.4340222268 & 0.5000000000 & 0.2183155578 \\
\hline B & 0.5659777432 & 0.5000000000 & 0.7816844272 \\
\hline
\end{tabular}

4-2Et ${ }_{2} \mathrm{O} \quad-\mathrm{HS}$

CELL_PARAMETERS (angstrom)

$\begin{array}{rrr}16.046192014 & 0.000000000 & -0.241745162 \\ 0.000000000 & 15.337508647 & 0.000000000 \\ -2.029230046 & 0.000000000 & 11.409381149\end{array}$

ATOMIC_POSITIONS (crystal)

$\begin{array}{llll}\text { Fe1 } & 0.3192261374 & 0.0000000000 & 0.1900948463 \\ \text { Fe1 } & 0.6807738626 & 0.0000000000 & 0.8099051827 \\ \text { Fe1 } & 0.8192302758 & 0.5000000000 & 0.1900921837 \\ \text { Fe1 } & 0.1807697242 & 0.5000000000 & 0.8099078453 \\ O & 0.4491070955 & 0.0000000000 & 0.2120678039 \\ O & 0.5508928755 & 0.0000000000 & 0.7879321961 \\ O & 0.9491098763 & 0.5000000000 & 0.2120690467 \\ O & 0.0508901527 & 0.5000000000 & 0.7879309533 \\ O & 0.6658192067 & 0.0000000000 & 0.9846834147 \\ O & 0.3341807933 & 0.0000000000 & 0.0153165573 \\ O & 0.1658157678 & 0.5000000000 & 0.9846815271\end{array}$




\begin{tabular}{|c|c|c|c|}
\hline 0 & 0.8341842322 & 0.5000000000 & 0.0153184449 \\
\hline 0 & 0.5000000000 & 0.7553850764 & 0.5000000000 \\
\hline 0 & 0.5000000000 & 0.2446149236 & 0.5000000000 \\
\hline 0 & 0.0000000000 & 0.2554773635 & 0.5000000000 \\
\hline 0 & 0.0000000000 & 0.7445226365 & 0.5000000000 \\
\hline $\mathrm{N}$ & 0.1777478674 & 0.0000000000 & 0.1576087287 \\
\hline $\mathrm{N}$ & 0.8222521626 & 0.0000000000 & 0.8423912863 \\
\hline $\mathrm{N}$ & 0.6777584888 & 0.5000000000 & 0.1575920657 \\
\hline $\mathrm{N}$ & 0.3222414812 & 0.5000000000 & 0.8424079493 \\
\hline $\mathrm{N}$ & 0.3041606735 & 0.0000000000 & 0.3662065491 \\
\hline $\mathrm{N}$ & 0.6958392965 & 0.0000000000 & 0.6337934209 \\
\hline $\mathrm{N}$ & 0.8041657514 & 0.5000000000 & 0.3661995266 \\
\hline $\mathrm{N}$ & 0.1958342786 & 0.5000000000 & 0.6338004434 \\
\hline $\mathrm{N}$ & 0.2876632553 & 0.8622290137 & 0.1794954916 \\
\hline $\mathrm{N}$ & 0.7123367147 & 0.1377709863 & 0.8205044944 \\
\hline $\mathrm{N}$ & 0.7123367147 & 0.8622290137 & 0.8205044944 \\
\hline $\mathrm{N}$ & 0.2876632553 & 0.1377709863 & 0.1794954916 \\
\hline $\mathrm{N}$ & 0.7876696633 & 0.3622223736 & 0.1794982208 \\
\hline $\mathrm{N}$ & 0.2123303667 & 0.6377776264 & 0.8205017652 \\
\hline $\mathrm{N}$ & 0.2123303667 & 0.3622223736 & 0.8205017652 \\
\hline $\mathrm{N}$ & 0.7876696633 & 0.6377776264 & 0.1794982208 \\
\hline C & 0.2230112356 & 0.0000000000 & 0.3817874439 \\
\hline C & 0.7769887494 & 0.0000000000 & 0.6182125561 \\
\hline C & 0.7230135807 & 0.5000000000 & 0.3817712767 \\
\hline C & 0.2769864193 & 0.5000000000 & 0.6182287233 \\
\hline C & 0.5880407979 & 0.0000000000 & 0.9969304705 \\
\hline C & 0.4119592021 & 0.0000000000 & 0.0030695005 \\
\hline C & 0.0880380273 & 0.5000000000 & 0.9969306471 \\
\hline C & 0.9119619727 & 0.5000000000 & 0.0030693239 \\
\hline C & 0.2037088747 & 0.0000000000 & 0.4945536849 \\
\hline C & 0.7962911253 & 0.0000000000 & 0.5054463451 \\
\hline C & 0.7037040299 & 0.5000000000 & 0.4945316106 \\
\hline C & 0.2962959701 & 0.5000000000 & 0.5054684194 \\
\hline C & 0.4777306157 & 0.0000000000 & 0.1144233610 \\
\hline C & 0.5222693843 & 0.0000000000 & 0.8855766240 \\
\hline C & 0.9777330351 & 0.5000000000 & 0.1144250925 \\
\hline $\mathrm{C}$ & 0.0222669649 & 0.5000000000 & 0.8855748925 \\
\hline C & 0.1520198549 & 0.0000000000 & 0.2748182083 \\
\hline C & 0.8479801751 & 0.0000000000 & 0.7251817917 \\
\hline C & 0.6520255816 & 0.5000000000 & 0.2747976259 \\
\hline C & 0.3479743884 & 0.5000000000 & 0.7252023741 \\
\hline C & 0.5633298840 & 0.0000000000 & 0.1070828939 \\
\hline C & 0.4366701160 & 0.0000000000 & 0.8929171061 \\
\hline C & 0.0633317706 & 0.5000000000 & 0.1070853861 \\
\hline C & 0.9366682294 & 0.5000000000 & 0.8929146139 \\
\hline C & 0.3679879207 & 0.0000000000 & 0.4604359167 \\
\hline C & 0.6320121093 & 0.0000000000 & 0.5395640833 \\
\hline C & 0.8679874589 & 0.5000000000 & 0.4604351872 \\
\hline C & 0.1320125111 & 0.5000000000 & 0.5395648128 \\
\hline C & 0.3536652732 & 0.0000000000 & 0.5746823041 \\
\hline C & 0.6463346968 & 0.0000000000 & 0.4253176959 \\
\hline C & 0.8536565484 & 0.5000000000 & 0.5746777042 \\
\hline C & 0.1463434816 & 0.5000000000 & 0.4253222958 \\
\hline C & 0.2697852523 & 0.0000000000 & 0.5919868593 \\
\hline C & 0.7302147777 & 0.0000000000 & 0.4080131407 \\
\hline C & 0.7697742922 & 0.5000000000 & 0.5919720862 \\
\hline C & 0.2302256778 & 0.5000000000 & 0.4080279138 \\
\hline C & 0.2061708910 & 0.8433442406 & 0.1318253984 \\
\hline
\end{tabular}




\begin{tabular}{|c|c|c|c|}
\hline C & 0.7938291390 & 0.1566557594 & 0.8681746316 \\
\hline C & 0.7938291390 & 0.8433442406 & 0.8681746316 \\
\hline C & 0.2061708910 & 0.1566557594 & 0.1318253984 \\
\hline C & 0.7061775732 & 0.3433404310 & 0.1318209135 \\
\hline C & 0.2938223968 & 0.6566595690 & 0.8681791165 \\
\hline C & 0.2938223968 & 0.3433404310 & 0.8681791165 \\
\hline C & 0.7061775732 & 0.6566595690 & 0.1318209135 \\
\hline C & 0.1501487445 & 0.9200765491 & 0.0885949836 \\
\hline C & 0.8498512705 & 0.0799234509 & 0.9114050384 \\
\hline C & 0.8498512705 & 0.9200765491 & 0.9114050384 \\
\hline C & 0.1501487445 & 0.0799234509 & 0.0885949836 \\
\hline C & 0.6501610037 & 0.4200758746 & 0.0885789818 \\
\hline C & 0.3498389963 & 0.5799241254 & 0.9114210402 \\
\hline C & 0.3498389963 & 0.4200758746 & 0.9114210402 \\
\hline C & 0.6501610037 & 0.5799241254 & 0.0885789818 \\
\hline C & 0.1774073366 & 0.7573405219 & 0.1180905881 \\
\hline C & 0.8225926634 & 0.2426594781 & 0.8819094119 \\
\hline C & 0.8225926634 & 0.7573405219 & 0.8819094119 \\
\hline C & 0.1774073366 & 0.2426594781 & 0.1180905881 \\
\hline C & 0.6774111330 & 0.2573384043 & 0.1180911400 \\
\hline C & 0.3225888670 & 0.7426615957 & 0.8819088600 \\
\hline C & 0.3225888670 & 0.2573384043 & 0.8819088600 \\
\hline C & 0.6774111330 & 0.7426615957 & 0.1180911400 \\
\hline C & 0.2342445048 & 0.6895260573 & 0.1541128282 \\
\hline C & 0.7657555252 & 0.3104739427 & 0.8458871718 \\
\hline C & 0.7657555252 & 0.6895260573 & 0.8458871718 \\
\hline C & 0.2342445048 & 0.3104739427 & 0.1541128282 \\
\hline C & 0.7342443046 & 0.1895225626 & 0.1541283616 \\
\hline C & 0.2657556654 & 0.8104774374 & 0.8458716384 \\
\hline C & 0.2657556654 & 0.1895225626 & 0.8458716384 \\
\hline C & 0.7342443046 & 0.8104774374 & 0.1541283616 \\
\hline C & 0.3177779471 & 0.7096767179 & 0.2063130120 \\
\hline C & 0.6822220529 & 0.2903232821 & 0.7936869730 \\
\hline C & 0.6822220529 & 0.7096767179 & 0.7936869730 \\
\hline C & 0.3177779471 & 0.2903232821 & 0.2063130120 \\
\hline C & 0.8177772774 & 0.2096682080 & 0.2063352745 \\
\hline C & 0.1822227226 & 0.7903317920 & 0.7936647105 \\
\hline C & 0.1822227226 & 0.2096682080 & 0.7936647105 \\
\hline C & 0.8177772774 & 0.7903317920 & 0.2063352745 \\
\hline C & 0.3419338140 & 0.7967389566 & 0.2175933697 \\
\hline C & 0.6580661860 & 0.2032610434 & 0.7824066153 \\
\hline C & 0.6580661860 & 0.7967389566 & 0.7824066153 \\
\hline C & 0.3419338140 & 0.2032610434 & 0.2175933697 \\
\hline C & 0.8419363284 & 0.2967315081 & 0.2176072328 \\
\hline C & 0.1580636716 & 0.7032684919 & 0.7823927522 \\
\hline C & 0.1580636716 & 0.2967315081 & 0.7823927522 \\
\hline C & 0.8419363284 & 0.7032684919 & 0.2176072328 \\
\hline C & 0.4361996017 & 0.7028213242 & 0.5374869292 \\
\hline C & 0.5638004283 & 0.2971786758 & 0.4625130708 \\
\hline C & 0.5638004283 & 0.7028213242 & 0.4625130708 \\
\hline C & 0.4361996017 & 0.2971786758 & 0.5374869292 \\
\hline C & 0.9362038937 & 0.2029127554 & 0.5374950599 \\
\hline C & 0.0637960763 & 0.7970872446 & 0.4625049401 \\
\hline C & 0.0637960763 & 0.2029127554 & 0.4625049401 \\
\hline C & 0.9362038937 & 0.7970872446 & 0.5374950599 \\
\hline C & 0.3708902167 & 0.7621386654 & 0.5752716185 \\
\hline C & 0.6291097833 & 0.2378613346 & 0.4247283815 \\
\hline C & 0.6291097833 & 0.7621386654 & 0.4247283815 \\
\hline
\end{tabular}




\begin{tabular}{|c|c|c|c|}
\hline C & 0.3708902167 & 0.2378613346 & 0.5752716185 \\
\hline C & 0.8708963441 & 0.2622254172 & 0.5752915072 \\
\hline C & 0.1291036559 & 0.7377745828 & 0.4247084928 \\
\hline C & 0.1291036559 & 0.2622254172 & 0.4247084928 \\
\hline C & 0.8708963441 & 0.7377745828 & 0.5752915072 \\
\hline $\mathrm{H}$ & 0.1377029694 & 0.0000000000 & 0.5060588728 \\
\hline $\mathrm{H}$ & 0.8622970606 & 0.0000000000 & 0.4939411272 \\
\hline $\mathrm{H}$ & 0.6376954400 & 0.5000000000 & 0.5060258618 \\
\hline $\mathrm{H}$ & 0.3623045300 & 0.5000000000 & 0.4939741382 \\
\hline $\mathrm{H}$ & 0.1110586083 & 0.9432640663 & 0.2804640196 \\
\hline $\mathrm{H}$ & 0.8889413837 & 0.0567359337 & 0.7195360104 \\
\hline $\mathrm{H}$ & 0.8889413837 & 0.9432640663 & 0.7195360104 \\
\hline $\mathrm{H}$ & 0.1110586083 & 0.0567359337 & 0.2804640196 \\
\hline $\mathrm{H}$ & 0.6110639332 & 0.4432638348 & 0.2804402211 \\
\hline $\mathrm{H}$ & 0.3889360668 & 0.5567361652 & 0.7195598089 \\
\hline $\mathrm{H}$ & 0.3889360668 & 0.4432638348 & 0.7195598089 \\
\hline $\mathrm{H}$ & 0.6110639332 & 0.5567361652 & 0.2804402211 \\
\hline $\mathrm{H}$ & 0.6125061125 & 0.0000000000 & 0.1859261248 \\
\hline $\mathrm{H}$ & 0.3874938875 & 0.0000000000 & 0.8140738902 \\
\hline $\mathrm{H}$ & 0.1125094430 & 0.5000000000 & 0.1859276838 \\
\hline $\mathrm{H}$ & 0.8874905570 & 0.5000000000 & 0.8140723312 \\
\hline $\mathrm{H}$ & 0.4318433187 & 0.0000000000 & 0.4407405325 \\
\hline $\mathrm{H}$ & 0.5681566813 & 0.0000000000 & 0.5592594975 \\
\hline $\mathrm{H}$ & 0.9318453993 & 0.5000000000 & 0.4407484280 \\
\hline $\mathrm{H}$ & 0.0681546007 & 0.5000000000 & 0.5592516020 \\
\hline $\mathrm{H}$ & 0.4081372517 & 0.0000000000 & 0.6470101494 \\
\hline $\mathrm{H}$ & 0.5918627783 & 0.0000000000 & 0.3529898506 \\
\hline $\mathrm{H}$ & 0.9081253507 & 0.5000000000 & 0.6470102718 \\
\hline $\mathrm{H}$ & 0.0918746193 & 0.5000000000 & 0.3529897282 \\
\hline $\mathrm{H}$ & 0.2544609318 & 0.0000000000 & 0.6798874179 \\
\hline $\mathrm{H}$ & 0.7455390682 & 0.0000000000 & 0.3201125821 \\
\hline $\mathrm{H}$ & 0.7544434495 & 0.5000000000 & 0.6798691579 \\
\hline $\mathrm{H}$ & 0.2455565505 & 0.5000000000 & 0.3201308421 \\
\hline $\mathrm{H}$ & 0.0829994382 & 0.9050882465 & 0.0884804897 \\
\hline $\mathrm{H}$ & 0.9170005468 & 0.0949117535 & 0.9115195033 \\
\hline $\mathrm{H}$ & 0.9170005468 & 0.9050882465 & 0.9115195033 \\
\hline $\mathrm{H}$ & 0.0829994382 & 0.0949117535 & 0.0884804897 \\
\hline $\mathrm{H}$ & 0.5830099511 & 0.4050913161 & 0.0884568467 \\
\hline $\mathrm{H}$ & 0.4169900489 & 0.5949086839 & 0.9115431463 \\
\hline $\mathrm{H}$ & 0.4169900489 & 0.4050913161 & 0.9115431463 \\
\hline $\mathrm{H}$ & 0.5830099511 & 0.5949086839 & 0.0884568467 \\
\hline $\mathrm{H}$ & 0.1559013890 & 0.9330289212 & -0.0030189899 \\
\hline $\mathrm{H}$ & 0.8440986110 & 0.0669710788 & 1.0030190169 \\
\hline $\mathrm{H}$ & 0.8440986110 & 0.9330289212 & 1.0030190169 \\
\hline $\mathrm{H}$ & 0.1559013890 & 0.0669710788 & -0.0030189899 \\
\hline $\mathrm{H}$ & 0.6559205168 & 0.4330252136 & -0.0030329060 \\
\hline $\mathrm{H}$ & 0.3440794832 & 0.5669747864 & 1.0030329330 \\
\hline $\mathrm{H}$ & 0.3440794832 & 0.4330252136 & 1.0030329330 \\
\hline $\mathrm{H}$ & 0.6559205168 & 0.5669747864 & -0.0030329060 \\
\hline $\mathrm{H}$ & 0.1112286451 & 0.7443050001 & 0.0788078414 \\
\hline $\mathrm{H}$ & 0.8887713549 & 0.2556949999 & 0.9211921436 \\
\hline $\mathrm{H}$ & 0.8887713549 & 0.7443050001 & 0.9211921436 \\
\hline $\mathrm{H}$ & 0.1112286451 & 0.2556949999 & 0.0788078414 \\
\hline $\mathrm{H}$ & 0.6112341269 & 0.2443027881 & 0.0788012312 \\
\hline $\mathrm{H}$ & 0.3887658731 & 0.7556972119 & 0.9211987538 \\
\hline $\mathrm{H}$ & 0.3887658731 & 0.2443027881 & 0.9211987538 \\
\hline $\mathrm{H}$ & 0.6112341269 & 0.7556972119 & 0.0788012312 \\
\hline $\mathrm{H}$ & 0.2143573274 & 0.6216324384 & 0.1411072741 \\
\hline
\end{tabular}




\begin{tabular}{|c|c|c|c|}
\hline $\mathrm{H}$ & 0.7856426576 & 0.3783675616 & 0.8588927109 \\
\hline $\mathrm{H}$ & 0.7856426576 & 0.6216324384 & 0.8588927109 \\
\hline $\mathrm{H}$ & 0.2143573274 & 0.3783675616 & 0.1411072741 \\
\hline $\mathrm{H}$ & 0.7143545642 & 0.1216292371 & 0.1411271644 \\
\hline $\mathrm{H}$ & 0.2856454358 & 0.8783707629 & 0.8588728206 \\
\hline $\mathrm{H}$ & 0.2856454358 & 0.1216292371 & 0.8588728206 \\
\hline $\mathrm{H}$ & 0.7143545642 & 0.8783707629 & 0.1411271644 \\
\hline $\mathrm{H}$ & 0.3639955140 & 0.6587590323 & 0.2358805466 \\
\hline $\mathrm{H}$ & 0.6360045160 & 0.3412409677 & 0.7641194234 \\
\hline $\mathrm{H}$ & 0.6360045160 & 0.6587590323 & 0.7641194234 \\
\hline $\mathrm{H}$ & 0.3639955140 & 0.3412409677 & 0.2358805466 \\
\hline $\mathrm{H}$ & 0.8639905250 & 0.1587490503 & 0.2359128894 \\
\hline $\mathrm{H}$ & 0.1360094450 & 0.8412509497 & 0.7640870806 \\
\hline $\mathrm{H}$ & 0.1360094450 & 0.1587490503 & 0.7640870806 \\
\hline $\mathrm{H}$ & 0.8639905250 & 0.8412509497 & 0.2359128894 \\
\hline $\mathrm{H}$ & 0.4064193636 & 0.8158536495 & 0.2584288262 \\
\hline $\mathrm{H}$ & 0.5935806364 & 0.1841463505 & 0.7415711738 \\
\hline $\mathrm{H}$ & 0.5935806364 & 0.8158536495 & 0.7415711738 \\
\hline $\mathrm{H}$ & 0.4064193636 & 0.1841463505 & 0.2584288262 \\
\hline $\mathrm{H}$ & 0.9064215946 & 0.3158454080 & 0.2584457211 \\
\hline $\mathrm{H}$ & 0.0935784054 & 0.6841545920 & 0.7415542789 \\
\hline $\mathrm{H}$ & 0.0935784054 & 0.3158454080 & 0.7415542789 \\
\hline $\mathrm{H}$ & 0.9064215946 & 0.6841545920 & 0.2584457211 \\
\hline $\mathrm{H}$ & 0.4062764898 & 0.6597525388 & 0.4645387346 \\
\hline $\mathrm{H}$ & 0.5937235102 & 0.3402474612 & 0.5354612654 \\
\hline $\mathrm{H}$ & 0.5937235102 & 0.6597525388 & 0.5354612654 \\
\hline $\mathrm{H}$ & 0.4062764898 & 0.3402474612 & 0.4645387346 \\
\hline $\mathrm{H}$ & 0.9062768689 & 0.1598441270 & 0.4645485164 \\
\hline $\mathrm{H}$ & 0.0937231311 & 0.8401558730 & 0.5354514836 \\
\hline $\mathrm{H}$ & 0.0937231311 & 0.1598441270 & 0.5354514836 \\
\hline $\mathrm{H}$ & 0.9062768689 & 0.8401558730 & 0.4645485164 \\
\hline $\mathrm{H}$ & 0.4661573118 & 0.6598256464 & 0.6104760742 \\
\hline $\mathrm{H}$ & 0.5338426882 & 0.3401743536 & 0.3895239258 \\
\hline $\mathrm{H}$ & 0.5338426882 & 0.6598256464 & 0.3895239258 \\
\hline $\mathrm{H}$ & 0.4661573118 & 0.3401743536 & 0.6104760742 \\
\hline $\mathrm{H}$ & 0.9661663566 & 0.1599150311 & 0.6104801131 \\
\hline $\mathrm{H}$ & 0.0338336434 & 0.8400849689 & 0.3895198869 \\
\hline $\mathrm{H}$ & 0.0338336434 & 0.1599150311 & 0.3895198869 \\
\hline $\mathrm{H}$ & 0.9661663566 & 0.8400849689 & 0.6104801131 \\
\hline $\mathrm{H}$ & 0.3404762416 & 0.8039342504 & 0.5022584457 \\
\hline $\mathrm{H}$ & 0.6595237884 & 0.1960657496 & 0.4977415543 \\
\hline $\mathrm{H}$ & 0.6595237884 & 0.8039342504 & 0.4977415543 \\
\hline $\mathrm{H}$ & 0.3404762416 & 0.1960657496 & 0.5022584457 \\
\hline $\mathrm{H}$ & 0.8404743792 & 0.3040194821 & 0.5022821187 \\
\hline $\mathrm{H}$ & 0.1595255908 & 0.6959805179 & 0.4977178813 \\
\hline $\mathrm{H}$ & 0.1595255908 & 0.3040194821 & 0.4977178813 \\
\hline $\mathrm{H}$ & 0.8404743792 & 0.6959805179 & 0.5022821187 \\
\hline $\mathrm{H}$ & 0.3210914716 & 0.7233852573 & 0.6045010061 \\
\hline $\mathrm{H}$ & 0.6789084984 & 0.2766147427 & 0.3954989939 \\
\hline $\mathrm{H}$ & 0.6789084984 & 0.7233852573 & 0.3954989939 \\
\hline $\mathrm{H}$ & 0.3210914716 & 0.2766147427 & 0.6045010061 \\
\hline $\mathrm{H}$ & 0.8211034148 & 0.2234682756 & 0.6045287733 \\
\hline $\mathrm{H}$ & 0.1788966152 & 0.7765317244 & 0.3954712267 \\
\hline $\mathrm{H}$ & 0.1788966152 & 0.2234682756 & 0.3954712267 \\
\hline $\mathrm{H}$ & 0.8211034148 & 0.7765317244 & 0.6045287733 \\
\hline $\mathrm{H}$ & 0.4001474480 & 0.8044716934 & 0.6484108709 \\
\hline $\mathrm{H}$ & 0.5998525820 & 0.1955283066 & 0.3515891291 \\
\hline $\mathrm{H}$ & 0.5998525820 & 0.8044716934 & 0.3515891291 \\
\hline
\end{tabular}




\begin{tabular}{|c|c|c|c|}
\hline $\mathrm{H}$ & 0.4001474480 & 0.1955283066 & 0.6484108709 \\
\hline $\mathrm{H}$ & 0.9001561202 & 0.3045596572 & 0.6484277992 \\
\hline $\mathrm{H}$ & 0.0998438498 & 0.6954403428 & 0.3515722008 \\
\hline $\mathrm{H}$ & 0.0998438498 & 0.3045596572 & 0.3515722008 \\
\hline $\mathrm{H}$ & 0.9001561202 & 0.6954403428 & 0.6484277992 \\
\hline $\mathrm{F}$ & 0.8443248622 & 0.0000000000 & 0.1916491469 \\
\hline $\mathrm{F}$ & 0.1556751228 & 0.0000000000 & 0.8083508381 \\
\hline $\mathrm{F}$ & 0.3443291425 & 0.5000000000 & 0.1916445909 \\
\hline $\mathrm{F}$ & 0.6556708575 & 0.5000000000 & 0.8083553941 \\
\hline $\mathrm{F}$ & 0.9559844764 & 0.0000000000 & 0.3523537861 \\
\hline $\mathrm{F}$ & 0.0440155306 & 0.0000000000 & 0.6476462439 \\
\hline $\mathrm{F}$ & 0.4559928960 & 0.5000000000 & 0.3523411693 \\
\hline $\mathrm{F}$ & 0.5440071040 & 0.5000000000 & 0.6476588607 \\
\hline $\mathrm{F}$ & 0.9682692577 & 0.9241399300 & 0.1825304315 \\
\hline $\mathrm{F}$ & 0.0317307273 & 0.0758600700 & 0.8174695685 \\
\hline $\mathrm{F}$ & 0.0317307273 & 0.9241399300 & 0.8174695685 \\
\hline $\mathrm{F}$ & 0.9682692577 & 0.0758600700 & 0.1825304315 \\
\hline $\mathrm{F}$ & 0.4682719444 & 0.4241402120 & 0.1825162399 \\
\hline $\mathrm{F}$ & 0.5317280556 & 0.5758597880 & 0.8174837601 \\
\hline $\mathrm{F}$ & 0.5317280556 & 0.4241402120 & 0.8174837601 \\
\hline $\mathrm{F}$ & 0.4682719444 & 0.5758597880 & 0.1825162399 \\
\hline B & 0.9345409617 & 0.0000000000 & 0.2283469034 \\
\hline B & 0.0654590453 & 0.0000000000 & 0.7716531116 \\
\hline B & 0.4345456011 & 0.5000000000 & 0.2283348037 \\
\hline B & 0.5654543989 & 0.5000000000 & 0.7716652113 \\
\hline \multirow{2}{*}{\multicolumn{4}{|c|}{$\begin{array}{l}\mathbf{4} \mathbf{\bullet} \mathbf{E t}_{2} \mathbf{O} \quad \text {-iS } \\
\text { CELL_PARAMETERS (angstrom) }\end{array}$}} \\
\hline & & & \\
\hline 15.863085189 & 0.000000000 & \multicolumn{2}{|l|}{-0.107448670} \\
\hline 0.000000000 & 15.330729118 & \multicolumn{2}{|l|}{0.000000000} \\
\hline-1.909312511 & 0.000000000 & \multicolumn{2}{|l|}{11.334555182} \\
\hline \multicolumn{4}{|c|}{ ATOMIC_POSITIONS (crystal) } \\
\hline $\mathrm{Fe} 1$ & 0.3246528190 & 0.0000000000 & 0.1858442828 \\
\hline Fe2 & 0.6915507531 & 0.0000000000 & 0.8115798551 \\
\hline Fe1 & 0.8246250028 & 0.5000000000 & 0.1858675049 \\
\hline $\mathrm{Fe} 2$ & 0.1915319715 & 0.5000000000 & 0.8115951820 \\
\hline 0 & 0.4561293568 & 0.0000000000 & 0.2090560531 \\
\hline $\mathrm{O}$ & 0.5662096725 & 0.0000000000 & 0.7857308138 \\
\hline 0 & 0.9561120455 & 0.5000000000 & 0.2090741250 \\
\hline 0 & 0.0661798123 & 0.5000000000 & 0.7857360115 \\
\hline 0 & 0.6781474464 & 0.0000000000 & 0.9789671102 \\
\hline 0 & 0.3419803796 & 0.0000000000 & 0.0120327906 \\
\hline 0 & 0.1781204299 & 0.5000000000 & 0.9789682886 \\
\hline 0 & 0.8419585552 & 0.5000000000 & 0.0120601369 \\
\hline 0 & 0.4987645152 & 0.7548574802 & 0.5002718773 \\
\hline 0 & 0.4987645152 & 0.2451425198 & 0.5002718773 \\
\hline 0 & -0.0012286868 & 0.2547648665 & 0.5002695309 \\
\hline 0 & -0.0012286868 & 0.7452351335 & 0.5002695309 \\
\hline $\mathrm{N}$ & 0.1822493178 & 0.0000000000 & 0.1576759399 \\
\hline $\mathrm{N}$ & 0.8188593978 & 0.0000000000 & 0.8450973290 \\
\hline $\mathrm{N}$ & 0.6822274218 & 0.5000000000 & 0.1577068422 \\
\hline $\mathrm{N}$ & 0.3188341798 & 0.5000000000 & 0.8451224632 \\
\hline $\mathrm{N}$ & 0.3099602746 & 0.0000000000 & 0.3640195769 \\
\hline $\mathrm{N}$ & 0.7010137381 & 0.0000000000 & 0.6470928686 \\
\hline $\mathrm{N}$ & 0.8099395038 & 0.5000000000 & 0.3640530357 \\
\hline $\mathrm{N}$ & 0.2009987297 & 0.5000000000 & 0.6471016737 \\
\hline $\mathrm{N}$ & 0.2928032414 & 0.8619127083 & 0.1766545518 \\
\hline $\mathrm{N}$ & 0.7053914105 & 0.1270518026 & 0.8166268273 \\
\hline
\end{tabular}




\begin{tabular}{|c|c|c|c|}
\hline $\mathrm{N}$ & 0.7053914105 & 0.8729481974 & 0.8166268273 \\
\hline $\mathrm{N}$ & 0.2928032414 & 0.1380872917 & 0.1766545518 \\
\hline $\mathrm{N}$ & 0.7927712792 & 0.3619048221 & 0.1766877938 \\
\hline $\mathrm{N}$ & 0.2053721391 & 0.6270605051 & 0.8166318465 \\
\hline $\mathrm{N}$ & 0.2053721391 & 0.3729394949 & 0.8166318465 \\
\hline $\mathrm{N}$ & 0.7927712792 & 0.6380951779 & 0.1766877938 \\
\hline C & 0.2282887698 & 0.0000000000 & 0.3823342076 \\
\hline C & 0.7820177605 & 0.0000000000 & 0.6244152279 \\
\hline C & 0.7282668821 & 0.5000000000 & 0.3823663590 \\
\hline C & 0.2820066876 & 0.5000000000 & 0.6244383936 \\
\hline C & 0.5992070656 & 0.0000000000 & 0.9933246443 \\
\hline C & 0.4210642883 & 0.0000000000 & -0.0007556717 \\
\hline C & 0.0991806021 & 0.5000000000 & 0.9933292537 \\
\hline C & 0.9210407267 & 0.5000000000 & -0.0007372919 \\
\hline C & 0.2093567168 & 0.0000000000 & 0.4967986504 \\
\hline C & 0.7987528255 & 0.0000000000 & 0.5091669626 \\
\hline C & 0.7093316671 & 0.5000000000 & 0.4968298736 \\
\hline C & 0.2987547678 & 0.5000000000 & 0.5091968576 \\
\hline C & 0.4862816969 & 0.0000000000 & 0.1109146168 \\
\hline C & 0.5346533885 & 0.0000000000 & 0.8827672268 \\
\hline C & 0.9862611399 & 0.5000000000 & 0.1109306751 \\
\hline C & 0.0346250090 & 0.5000000000 & 0.8827735000 \\
\hline C & 0.1564568059 & 0.0000000000 & 0.2765362658 \\
\hline C & 0.8521547803 & 0.0000000000 & 0.7302963794 \\
\hline C & 0.6564355244 & 0.5000000000 & 0.2765679557 \\
\hline C & 0.3521374202 & 0.5000000000 & 0.7303273621 \\
\hline C & 0.5733418766 & 0.0000000000 & 0.1042430426 \\
\hline C & 0.4475103371 & 0.0000000000 & 0.8890541407 \\
\hline C & 0.0733199175 & 0.5000000000 & 0.1042502615 \\
\hline C & 0.9474824325 & 0.5000000000 & 0.8890689316 \\
\hline C & 0.3747401636 & 0.0000000000 & 0.4568514573 \\
\hline C & 0.6346612581 & 0.0000000000 & 0.5557418608 \\
\hline C & 0.8747164899 & 0.5000000000 & 0.4568887061 \\
\hline C & 0.1346540907 & 0.5000000000 & 0.5557400318 \\
\hline C & 0.3607609196 & 0.0000000000 & 0.5725593302 \\
\hline C & 0.6467312957 & 0.0000000000 & 0.4389682923 \\
\hline C & 0.8607346969 & 0.5000000000 & 0.5725959106 \\
\hline C & 0.1467364641 & 0.5000000000 & 0.4389714757 \\
\hline C & 0.2763883167 & 0.0000000000 & 0.5928819432 \\
\hline C & 0.7302091413 & 0.0000000000 & 0.4151766327 \\
\hline C & 0.7763612549 & 0.5000000000 & 0.5929160774 \\
\hline C & 0.2302186374 & 0.5000000000 & 0.4151958653 \\
\hline C & 0.2102154491 & 0.8432975228 & 0.1323775239 \\
\hline C & 0.7851825751 & 0.1534001811 & 0.8675430284 \\
\hline C & 0.7851825751 & 0.8465998189 & 0.8675430284 \\
\hline C & 0.2102154491 & 0.1567024772 & 0.1323775239 \\
\hline C & 0.7101843914 & 0.3432958093 & 0.1323962219 \\
\hline C & 0.2851636304 & 0.6534028343 & 0.8675578229 \\
\hline C & 0.2851636304 & 0.3465971657 & 0.8675578229 \\
\hline C & 0.7101843914 & 0.6567041907 & 0.1323962219 \\
\hline C & 0.1543151902 & 0.9200670785 & 0.0888267253 \\
\hline C & 0.8442939108 & 0.0805613713 & 0.9154215593 \\
\hline C & 0.8442939108 & 0.9194386287 & 0.9154215593 \\
\hline C & 0.1543151902 & 0.0799329215 & 0.0888267253 \\
\hline C & 0.6542900806 & 0.4200705599 & 0.0888529223 \\
\hline C & 0.3442675762 & 0.5805609427 & 0.9154481116 \\
\hline C & 0.3442675762 & 0.4194390573 & 0.9154481116 \\
\hline C & 0.6542900806 & 0.5799294401 & 0.0888529223 \\
\hline
\end{tabular}




\begin{tabular}{|c|c|c|c|}
\hline C & 0.1802617057 & 0.7575285051 & 0.1227480311 \\
\hline C & 0.8072683825 & 0.2413193044 & 0.8776090791 \\
\hline C & 0.8072683825 & 0.7586806956 & 0.8776090791 \\
\hline C & 0.1802617057 & 0.2424714949 & 0.1227480311 \\
\hline C & 0.6802254390 & 0.2575298239 & 0.1227423094 \\
\hline C & 0.3072617321 & 0.7413198780 & 0.8776205243 \\
\hline C & 0.3072617321 & 0.2586801220 & 0.8776205243 \\
\hline C & 0.6802254390 & 0.7424701761 & 0.1227423094 \\
\hline C & 0.2370225251 & 0.6896615184 & 0.1598013476 \\
\hline C & 0.7459421553 & 0.3041630826 & 0.8353955750 \\
\hline C & 0.7459421553 & 0.6958369174 & 0.8353955750 \\
\hline C & 0.2370225251 & 0.3103384816 & 0.1598013476 \\
\hline C & 0.7369831724 & 0.1896571568 & 0.1597778093 \\
\hline C & 0.2459485522 & 0.8041717217 & 0.8353913244 \\
\hline C & 0.2459485522 & 0.1958282783 & 0.8353913244 \\
\hline C & 0.7369831724 & 0.8103428432 & 0.1597778093 \\
\hline C & 0.3216625825 & 0.7095227127 & 0.2084335203 \\
\hline C & 0.6646706479 & 0.2766662357 & 0.7813888482 \\
\hline C & 0.6646706479 & 0.7233337643 & 0.7813888482 \\
\hline C & 0.3216625825 & 0.2904772873 & 0.2084335203 \\
\hline C & 0.8216231010 & 0.2095085111 & 0.2084233316 \\
\hline C & 0.1646777841 & 0.7766829408 & 0.7813695852 \\
\hline C & 0.1646777841 & 0.2233170592 & 0.7813695852 \\
\hline C & 0.8216231010 & 0.7904914889 & 0.2084233316 \\
\hline C & 0.3470409012 & 0.7964054861 & 0.2153406150 \\
\hline C & 0.6467709973 & 0.1878155672 & 0.7730548342 \\
\hline C & 0.6467709973 & 0.8121844328 & 0.7730548342 \\
\hline C & 0.3470409012 & 0.2035945139 & 0.2153406150 \\
\hline C & 0.8470043135 & 0.2963914101 & 0.2153616720 \\
\hline C & 0.1467648677 & 0.6878338451 & 0.7730426810 \\
\hline C & 0.1467648677 & 0.3121661549 & 0.7730426810 \\
\hline C & 0.8470043135 & 0.7036085899 & 0.2153616720 \\
\hline C & 0.4335907447 & 0.7014448064 & 0.5358770071 \\
\hline C & 0.5626069149 & 0.2965986040 & 0.4579135910 \\
\hline C & 0.5626069149 & 0.7034013960 & 0.4579135910 \\
\hline C & 0.4335907447 & 0.2985551936 & 0.5358770071 \\
\hline C & 0.9335977416 & 0.2013439385 & 0.5358618946 \\
\hline C & 0.0626167716 & 0.7966862262 & 0.4579108370 \\
\hline C & 0.0626167716 & 0.2033137738 & 0.4579108370 \\
\hline C & 0.9335977416 & 0.7986560615 & 0.5358618946 \\
\hline C & 0.3686740051 & 0.7598222997 & 0.5794862774 \\
\hline C & 0.6299295345 & 0.2361934742 & 0.4242577532 \\
\hline C & 0.6299295345 & 0.7638065258 & 0.4242577532 \\
\hline C & 0.3686740051 & 0.2401777003 & 0.5794862774 \\
\hline C & 0.8686863184 & 0.2597161604 & 0.5794929254 \\
\hline C & 0.1299182288 & 0.7362804499 & 0.4242024580 \\
\hline C & 0.1299182288 & 0.2637195501 & 0.4242024580 \\
\hline C & 0.8686863184 & 0.7402838396 & 0.5794929254 \\
\hline $\mathrm{H}$ & 0.1429280052 & 0.0000000000 & 0.5106247599 \\
\hline $\mathrm{H}$ & 0.8646426014 & 0.0000000000 & 0.4932168083 \\
\hline $\mathrm{H}$ & 0.6429022086 & 0.5000000000 & 0.5106540703 \\
\hline $\mathrm{H}$ & 0.3646482432 & 0.5000000000 & 0.4932612036 \\
\hline $\mathrm{H}$ & 0.1151944231 & 0.9432415572 & 0.2834465812 \\
\hline $\mathrm{H}$ & 0.8938744875 & 0.0568683418 & 0.7274526375 \\
\hline $\mathrm{H}$ & 0.8938744875 & 0.9431316582 & 0.7274526375 \\
\hline $\mathrm{H}$ & 0.1151944231 & 0.0567584428 & 0.2834465812 \\
\hline $\mathrm{H}$ & 0.6151734690 & 0.4432413352 & 0.2834786190 \\
\hline $\mathrm{H}$ & 0.3938578420 & 0.5568686773 & 0.7274878282 \\
\hline
\end{tabular}




\begin{tabular}{|c|c|c|c|}
\hline $\mathrm{H}$ & 0.3938578420 & 0.4431313227 & 0.7274878282 \\
\hline $\mathrm{H}$ & 0.6151734690 & 0.5567586648 & 0.2834786190 \\
\hline $\mathrm{H}$ & 0.6213584936 & 0.0000000000 & 0.1844062130 \\
\hline $\mathrm{H}$ & 0.3989223852 & 0.0000000000 & 0.8097465500 \\
\hline $\mathrm{H}$ & 0.1213408601 & 0.5000000000 & 0.1844089028 \\
\hline $\mathrm{H}$ & 0.8988906066 & 0.5000000000 & 0.8097655556 \\
\hline $\mathrm{H}$ & 0.4389353282 & 0.0000000000 & 0.4347788105 \\
\hline $\mathrm{H}$ & 0.5714491768 & 0.0000000000 & 0.5806472418 \\
\hline $\mathrm{H}$ & 0.9389128540 & 0.5000000000 & 0.4348199497 \\
\hline $\mathrm{H}$ & 0.0714381727 & 0.5000000000 & 0.5806330325 \\
\hline $\mathrm{H}$ & 0.4158254978 & 0.0000000000 & 0.6438363315 \\
\hline $\mathrm{H}$ & 0.5908164389 & 0.0000000000 & 0.3686623902 \\
\hline $\mathrm{H}$ & 0.9157991964 & 0.5000000000 & 0.6438734760 \\
\hline $\mathrm{H}$ & 0.0908270156 & 0.5000000000 & 0.3686575678 \\
\hline $\mathrm{H}$ & 0.2614838800 & 0.0000000000 & 0.6821619566 \\
\hline $\mathrm{H}$ & 0.7434181689 & 0.0000000000 & 0.3250729586 \\
\hline $\mathrm{H}$ & 0.7614549543 & 0.5000000000 & 0.6821951709 \\
\hline $\mathrm{H}$ & 0.2434367202 & 0.5000000000 & 0.3250968204 \\
\hline $\mathrm{H}$ & 0.0864112756 & 0.9055527704 & 0.0893644697 \\
\hline $\mathrm{H}$ & 0.9120073723 & 0.0969198224 & 0.9166806093 \\
\hline $\mathrm{H}$ & 0.9120073723 & 0.9030801776 & 0.9166806093 \\
\hline $\mathrm{H}$ & 0.0864112756 & 0.0944472296 & 0.0893644697 \\
\hline $\mathrm{H}$ & 0.5863851039 & 0.4055612696 & 0.0893867310 \\
\hline $\mathrm{H}$ & 0.4119828271 & 0.5969164430 & 0.9167144259 \\
\hline $\mathrm{H}$ & 0.4119828271 & 0.4030835570 & 0.9167144259 \\
\hline $\mathrm{H}$ & 0.5863851039 & 0.5944387304 & 0.0893867310 \\
\hline $\mathrm{H}$ & 0.1609465293 & 0.9327481914 & -0.0034126076 \\
\hline $\mathrm{H}$ & 0.8360109551 & 0.0671991900 & 1.0067956662 \\
\hline $\mathrm{H}$ & 0.8360109551 & 0.9328008100 & 1.0067956662 \\
\hline $\mathrm{H}$ & 0.1609465293 & 0.0672518086 & -0.0034126076 \\
\hline $\mathrm{H}$ & 0.6609247792 & 0.4327554996 & -0.0033843355 \\
\hline $\mathrm{H}$ & 0.3359787062 & 0.5672006323 & 1.0068204384 \\
\hline $\mathrm{H}$ & 0.3359787062 & 0.4327993677 & 1.0068204384 \\
\hline $\mathrm{H}$ & 0.6609247792 & 0.5672445004 & -0.0033843355 \\
\hline $\mathrm{H}$ & 0.1132551274 & 0.7447361950 & 0.0860598715 \\
\hline $\mathrm{H}$ & 0.8719074194 & 0.2599090918 & 0.9190092763 \\
\hline $\mathrm{H}$ & 0.8719074194 & 0.7400909082 & 0.9190092763 \\
\hline $\mathrm{H}$ & 0.1132551274 & 0.2552638050 & 0.0860598715 \\
\hline $\mathrm{H}$ & 0.6132193548 & 0.2447434608 & 0.0860448452 \\
\hline $\mathrm{H}$ & 0.3719015127 & 0.7599008472 & 0.9190291170 \\
\hline $\mathrm{H}$ & 0.3719015127 & 0.2400991528 & 0.9190291170 \\
\hline $\mathrm{H}$ & 0.6132193548 & 0.7552565392 & 0.0860448452 \\
\hline $\mathrm{H}$ & 0.2161437721 & 0.6218747185 & 0.1505274219 \\
\hline $\mathrm{H}$ & 0.7608232232 & 0.3735689811 & 0.8447940661 \\
\hline $\mathrm{H}$ & 0.7608232232 & 0.6264310189 & 0.8447940661 \\
\hline $\mathrm{H}$ & 0.2161437721 & 0.3781252815 & 0.1505274219 \\
\hline $\mathrm{H}$ & 0.7161027767 & 0.1218716601 & 0.1504800724 \\
\hline $\mathrm{H}$ & 0.2608389666 & 0.8735754979 & 0.8447881087 \\
\hline $\mathrm{H}$ & 0.2608389666 & 0.1264245021 & 0.8447881087 \\
\hline $\mathrm{H}$ & 0.7161027767 & 0.8781283399 & 0.1504800724 \\
\hline $\mathrm{H}$ & 0.3678053623 & 0.6584871570 & 0.2384425898 \\
\hline $\mathrm{H}$ & 0.6148927631 & 0.3234692482 & 0.7476575665 \\
\hline $\mathrm{H}$ & 0.6148927631 & 0.6765307518 & 0.7476575665 \\
\hline $\mathrm{H}$ & 0.3678053623 & 0.3415128430 & 0.2384425898 \\
\hline $\mathrm{H}$ & 0.8677631009 & 0.1584678358 & 0.2384196442 \\
\hline $\mathrm{H}$ & 0.1149086910 & 0.8234903326 & 0.7476222142 \\
\hline $\mathrm{H}$ & 0.1149086910 & 0.1765096674 & 0.7476222142 \\
\hline $\mathrm{H}$ & 0.8677631009 & 0.8415321642 & 0.2384196442 \\
\hline
\end{tabular}




\begin{tabular}{|c|c|c|c|}
\hline $\mathrm{H}$ & 0.4124570504 & 0.8153583319 & 0.2531795606 \\
\hline $\mathrm{H}$ & 0.5845401428 & 0.1630613339 & 0.7306365350 \\
\hline $\mathrm{H}$ & 0.5845401428 & 0.8369386661 & 0.7306365350 \\
\hline $\mathrm{H}$ & 0.4124570504 & 0.1846416681 & 0.2531795606 \\
\hline $\mathrm{H}$ & 0.9124194826 & 0.3153373598 & 0.2532162797 \\
\hline $\mathrm{H}$ & 0.0845334010 & 0.6630899711 & 0.7306110076 \\
\hline $\mathrm{H}$ & 0.0845334010 & 0.3369100289 & 0.7306110076 \\
\hline $\mathrm{H}$ & 0.9124194826 & 0.6846626402 & 0.2532162797 \\
\hline $\mathrm{H}$ & 0.4027179273 & 0.6612545012 & 0.4603252282 \\
\hline $\mathrm{H}$ & 0.5914218308 & 0.3429345766 & 0.5275287501 \\
\hline $\mathrm{H}$ & 0.5914218308 & 0.6570654234 & 0.5275287501 \\
\hline $\mathrm{H}$ & 0.4027179273 & 0.3387454988 & 0.4603252282 \\
\hline $\mathrm{H}$ & 0.9027219111 & 0.1611680849 & 0.4602973939 \\
\hline $\mathrm{H}$ & 0.0914527303 & 0.8430019183 & 0.5275395310 \\
\hline $\mathrm{H}$ & 0.0914527303 & 0.1569980817 & 0.5275395310 \\
\hline $\mathrm{H}$ & 0.9027219111 & 0.8388319151 & 0.4602973939 \\
\hline $\mathrm{H}$ & 0.4630777771 & 0.6555633826 & 0.6056686058 \\
\hline $\mathrm{H}$ & 0.5323073512 & 0.3362642292 & 0.3814884368 \\
\hline $\mathrm{H}$ & 0.5323073512 & 0.6637357708 & 0.3814884368 \\
\hline $\mathrm{H}$ & 0.4630777771 & 0.3444366174 & 0.6056686058 \\
\hline $\mathrm{H}$ & 0.9630858801 & 0.1554514435 & 0.6056372879 \\
\hline $\mathrm{H}$ & 0.0323138294 & 0.8363739705 & 0.3815110390 \\
\hline $\mathrm{H}$ & 0.0323138294 & 0.1636260295 & 0.3815110390 \\
\hline $\mathrm{H}$ & 0.9630858801 & 0.8445485565 & 0.6056372879 \\
\hline $\mathrm{H}$ & 0.3387771224 & 0.8044234451 & 0.5094838701 \\
\hline $\mathrm{H}$ & 0.6600577169 & 0.1972780466 & 0.5004962570 \\
\hline $\mathrm{H}$ & 0.6600577169 & 0.8027219534 & 0.5004962570 \\
\hline $\mathrm{H}$ & 0.3387771224 & 0.1955765549 & 0.5094838701 \\
\hline $\mathrm{H}$ & 0.8387947330 & 0.3043328308 & 0.5095061515 \\
\hline $\mathrm{H}$ & 0.1600467963 & 0.6973362512 & 0.5004124552 \\
\hline $\mathrm{H}$ & 0.1600467963 & 0.3026637488 & 0.5004124552 \\
\hline $\mathrm{H}$ & 0.8387947330 & 0.6956671692 & 0.5095061515 \\
\hline $\mathrm{H}$ & 0.3178024924 & 0.7204319811 & 0.6074444741 \\
\hline $\mathrm{H}$ & 0.6802820864 & 0.2739629137 & 0.3923655607 \\
\hline $\mathrm{H}$ & 0.6802820864 & 0.7260370863 & 0.3923655607 \\
\hline $\mathrm{H}$ & 0.3178024924 & 0.2795680189 & 0.6074444741 \\
\hline $\mathrm{H}$ & 0.8178098730 & 0.2203262087 & 0.6074385196 \\
\hline $\mathrm{H}$ & 0.1802750806 & 0.7740496318 & 0.3923203221 \\
\hline $\mathrm{H}$ & 0.1802750806 & 0.2259503682 & 0.3923203221 \\
\hline $\mathrm{H}$ & 0.8178098730 & 0.7796737913 & 0.6074385196 \\
\hline $\mathrm{H}$ & 0.3987933703 & 0.7992611664 & 0.6553039102 \\
\hline $\mathrm{H}$ & 0.6019050272 & 0.1910397280 & 0.3537584773 \\
\hline $\mathrm{H}$ & 0.6019050272 & 0.8089602720 & 0.3537584773 \\
\hline $\mathrm{H}$ & 0.3987933703 & 0.2007388336 & 0.6553039102 \\
\hline $\mathrm{H}$ & 0.8988106810 & 0.2991403155 & 0.6553229687 \\
\hline $\mathrm{H}$ & 0.1018758817 & 0.6911514824 & 0.3536813049 \\
\hline $\mathrm{H}$ & 0.1018758817 & 0.3088485176 & 0.3536813049 \\
\hline $\mathrm{H}$ & 0.8988106810 & 0.7008596845 & 0.6553229687 \\
\hline $\mathrm{F}$ & 0.8466156410 & 0.0000000000 & 0.1977071784 \\
\hline $\mathrm{F}$ & 0.1589744991 & 0.0000000000 & 0.8093210377 \\
\hline $\mathrm{F}$ & 0.3466180782 & 0.5000000000 & 0.1977526783 \\
\hline$F$ & 0.6589529667 & 0.5000000000 & 0.8093365065 \\
\hline $\mathrm{F}$ & 0.9610328130 & 0.0000000000 & 0.3543318619 \\
\hline $\mathrm{F}$ & 0.0453057031 & 0.0000000000 & 0.6517100227 \\
\hline $\mathrm{F}$ & 0.4610404523 & 0.5000000000 & 0.3543719548 \\
\hline $\mathrm{F}$ & 0.5452774323 & 0.5000000000 & 0.6517316132 \\
\hline $\mathrm{F}$ & 0.9710372417 & 0.9241661305 & 0.1819627889 \\
\hline $\mathrm{F}$ & 0.0341999116 & 0.0757499488 & 0.8238858769 \\
\hline
\end{tabular}




$\begin{array}{llll}\text { F } & 0.0341999116 & 0.9242500512 & 0.8238858769 \\ \text { F } & 0.9710372417 & 0.0758338695 & 0.1819627889 \\ \text { F } & 0.4710373821 & 0.4241663863 & 0.1819999129 \\ \text { F } & 0.5341807339 & 0.5757508203 & 0.8239099297 \\ \text { F } & 0.5341807339 & 0.4242491797 & 0.8239099297 \\ \text { F } & 0.4710373821 & 0.5758336137 & 0.1819999129 \\ \text { B } & 0.9377488691 & 0.0000000000 & 0.2299857493 \\ \text { B } & 0.0679943295 & 0.0000000000 & 0.7762298505 \\ \text { B } & 0.4377513150 & 0.5000000000 & 0.2300273769 \\ \text { B } & 0.5679721618 & 0.5000000000 & 0.7762502601\end{array}$

4-2 $\mathrm{Et}_{2} \mathrm{O}-\mathrm{LS}$

CELL_PARAMETERS (angstrom)

$\begin{array}{lll}15.646603959 & 0.000000000 & 0.029417057\end{array}$

$\begin{array}{lll}0.000000000 & 15.321873231 & 0.000000000\end{array}$

$\begin{array}{lll}-1.782072909 & 0.000000000 & 11.293222011\end{array}$

ATOMIC_POSITIONS (crystal)

\begin{tabular}{|c|c|c|c|}
\hline Fe2 & 0.3152194395 & 0.0000000000 & 0.1882120897 \\
\hline Fe2 & 0.6847805605 & 0.0000000000 & 0.8117879393 \\
\hline $\mathrm{Fe} 2$ & 0.8152229953 & 0.5000000000 & 0.1882224587 \\
\hline $\mathrm{Fe} 2$ & 0.1847770047 & 0.5000000000 & 0.8117775703 \\
\hline $\mathrm{O}$ & 0.4420880415 & 0.0000000000 & 0.2117380549 \\
\hline $\mathrm{O}$ & 0.5579119295 & 0.0000000000 & 0.7882619451 \\
\hline $\mathrm{O}$ & 0.9421051785 & 0.5000000000 & 0.2117528843 \\
\hline $\mathrm{O}$ & 0.0578948505 & 0.5000000000 & 0.7882471157 \\
\hline $\mathrm{O}$ & 0.6702486226 & 0.0000000000 & 0.9802329144 \\
\hline $\mathrm{O}$ & 0.3297513774 & 0.0000000000 & 0.0197670576 \\
\hline $\mathrm{O}$ & 0.1702430269 & 0.5000000000 & 0.9802027040 \\
\hline $\mathrm{O}$ & 0.8297569731 & 0.5000000000 & 0.0197972680 \\
\hline $\mathrm{O}$ & 0.5000000000 & 0.7543182009 & 0.5000000000 \\
\hline $\mathrm{O}$ & 0.5000000000 & 0.2456817991 & 0.5000000000 \\
\hline $\mathrm{O}$ & 0.0000000000 & 0.2543530755 & 0.5000000000 \\
\hline $\mathrm{O}$ & 0.0000000000 & 0.7456469245 & 0.5000000000 \\
\hline $\mathrm{N}$ & 0.1865957722 & 0.0000000000 & 0.1569760342 \\
\hline $\mathrm{N}$ & 0.8134042578 & 0.0000000000 & 0.8430239808 \\
\hline$N$ & 0.6866010013 & 0.5000000000 & 0.1569699313 \\
\hline $\mathrm{N}$ & 0.3133989687 & 0.5000000000 & 0.8430300837 \\
\hline$N$ & 0.3048305325 & 0.0000000000 & 0.3534936332 \\
\hline$N$ & 0.6951694375 & 0.0000000000 & 0.6465063368 \\
\hline$N$ & 0.8048257528 & 0.5000000000 & 0.3534972345 \\
\hline$N$ & 0.1951742772 & 0.5000000000 & 0.6465027355 \\
\hline$N$ & 0.3010381986 & 0.8729339125 & 0.1838865635 \\
\hline$N$ & 0.6989617714 & 0.1270660875 & 0.8161134225 \\
\hline $\mathrm{N}$ & 0.6989617714 & 0.8729339125 & 0.8161134225 \\
\hline$N$ & 0.3010381986 & 0.1270660875 & 0.1838865635 \\
\hline$N$ & 0.8010298461 & 0.3729222450 & 0.1838873975 \\
\hline $\mathrm{N}$ & 0.1989701839 & 0.6270777550 & 0.8161125885 \\
\hline$N$ & 0.1989701839 & 0.3729222450 & 0.8161125885 \\
\hline $\mathrm{N}$ & 0.8010298461 & 0.6270777550 & 0.1838873975 \\
\hline C & 0.2227961557 & 0.0000000000 & 0.3779023711 \\
\hline C & 0.7772038293 & 0.0000000000 & 0.6220976289 \\
\hline C & 0.7227895573 & 0.5000000000 & 0.3778979989 \\
\hline C & 0.2772104427 & 0.5000000000 & 0.6221020011 \\
\hline C & 0.5902775486 & 0.0000000000 & 0.9962224503 \\
\hline C & 0.4097224514 & 0.0000000000 & 0.0037775207 \\
\hline C & 0.0902755102 & 0.5000000000 & 0.9962080761 \\
\hline C & 0.9097244898 & 0.5000000000 & 0.0037918949 \\
\hline C & 0.2054798709 & 0.0000000000 & 0.4940422674 \\
\hline
\end{tabular}




\begin{tabular}{|c|c|c|c|}
\hline C & 0.7945201291 & 0.0000000000 & 0.5059577626 \\
\hline C & 0.7054671753 & 0.5000000000 & 0.4940338571 \\
\hline C & 0.2945328247 & 0.5000000000 & 0.5059661729 \\
\hline C & 0.4747033085 & 0.0000000000 & 0.1141777298 \\
\hline C & 0.5252966915 & 0.0000000000 & 0.8858222552 \\
\hline C & 0.9747133517 & 0.5000000000 & 0.1141858918 \\
\hline C & 0.0252866483 & 0.5000000000 & 0.8858140932 \\
\hline C & 0.1522966546 & 0.0000000000 & 0.2727974749 \\
\hline C & 0.8477033754 & 0.0000000000 & 0.7272025251 \\
\hline C & 0.6522953073 & 0.5000000000 & 0.2727871332 \\
\hline C & 0.3477046627 & 0.5000000000 & 0.7272128668 \\
\hline C & 0.5632904821 & 0.0000000000 & 0.1074809738 \\
\hline C & 0.4367095179 & 0.0000000000 & 0.8925190262 \\
\hline C & 0.0632987571 & 0.5000000000 & 0.1074754834 \\
\hline C & 0.9367012429 & 0.5000000000 & 0.8925245166 \\
\hline C & 0.3716122589 & 0.0000000000 & 0.4439379589 \\
\hline C & 0.6283877711 & 0.0000000000 & 0.5560620411 \\
\hline C & 0.8716038522 & 0.5000000000 & 0.4439448677 \\
\hline C & 0.1283961178 & 0.5000000000 & 0.5560551323 \\
\hline C & 0.3589318007 & 0.0000000000 & 0.5614132622 \\
\hline C & 0.6410681693 & 0.0000000000 & 0.4385867378 \\
\hline C & 0.8589183881 & 0.5000000000 & 0.5614183553 \\
\hline C & 0.1410816419 & 0.5000000000 & 0.4385816447 \\
\hline C & 0.2744658906 & 0.0000000000 & 0.5871191173 \\
\hline C & 0.7255341394 & 0.0000000000 & 0.4128808827 \\
\hline C & 0.7744501702 & 0.5000000000 & 0.5871156299 \\
\hline C & 0.2255497998 & 0.5000000000 & 0.4128843701 \\
\hline C & 0.2203350515 & 0.8466067801 & 0.1348715074 \\
\hline C & 0.7796649785 & 0.1533932199 & 0.8651285226 \\
\hline C & 0.7796649785 & 0.8466067801 & 0.8651285226 \\
\hline C & 0.2203350515 & 0.1533932199 & 0.1348715074 \\
\hline C & 0.7203277269 & 0.3466055591 & 0.1348524845 \\
\hline C & 0.2796722431 & 0.6533944409 & 0.8651475455 \\
\hline C & 0.2796722431 & 0.3466055591 & 0.8651475455 \\
\hline C & 0.7203277269 & 0.6533944409 & 0.1348524845 \\
\hline C & 0.1611867913 & 0.9194260599 & 0.0868227240 \\
\hline C & 0.8388132237 & 0.0805739401 & 0.9131772980 \\
\hline C & 0.8388132237 & 0.9194260599 & 0.9131772980 \\
\hline C & 0.1611867913 & 0.0805739401 & 0.0868227240 \\
\hline C & 0.6611860327 & 0.4194297543 & 0.0868134593 \\
\hline C & 0.3388139673 & 0.5805702457 & 0.9131865627 \\
\hline C & 0.3388139673 & 0.4194297543 & 0.9131865627 \\
\hline C & 0.6611860327 & 0.5805702457 & 0.0868134593 \\
\hline C & 0.1974212804 & 0.7587744273 & 0.1272421451 \\
\hline C & 0.8025787196 & 0.2412255727 & 0.8727578549 \\
\hline C & 0.8025787196 & 0.7587744273 & 0.8727578549 \\
\hline C & 0.1974212804 & 0.2412255727 & 0.1272421451 \\
\hline C & 0.6974012957 & 0.2587763823 & 0.1271891030 \\
\hline C & 0.3025987043 & 0.7412236177 & 0.8728108970 \\
\hline C & 0.3025987043 & 0.2587763823 & 0.8728108970 \\
\hline C & 0.6974012957 & 0.7412236177 & 0.1271891030 \\
\hline C & 0.2588609830 & 0.6959417312 & 0.1700825825 \\
\hline C & 0.7411390470 & 0.3040582688 & 0.8299174175 \\
\hline C & 0.7411390470 & 0.6959417312 & 0.8299174175 \\
\hline C & 0.2588609830 & 0.3040582688 & 0.1700825825 \\
\hline C & 0.7588270315 & 0.1959291608 & 0.1700206109 \\
\hline C & 0.2411729385 & 0.8040708392 & 0.8299793891 \\
\hline C & 0.2411729385 & 0.1959291608 & 0.8299793891 \\
\hline
\end{tabular}




\begin{tabular}{|c|c|c|c|}
\hline C & 0.7588270315 & 0.8040708392 & 0.1700206109 \\
\hline C & 0.3410953068 & 0.7233638727 & 0.2217585477 \\
\hline C & 0.6589046932 & 0.2766361273 & 0.7782414373 \\
\hline C & 0.6589046932 & 0.7233638727 & 0.7782414373 \\
\hline C & 0.3410953068 & 0.2766361273 & 0.2217585477 \\
\hline C & 0.8410621877 & 0.2233369580 & 0.2217139671 \\
\hline C & 0.1589378123 & 0.7766630420 & 0.7782860179 \\
\hline C & 0.1589378123 & 0.2233369580 & 0.7782860179 \\
\hline C & 0.8410621877 & 0.7766630420 & 0.2217139671 \\
\hline C & 0.3598128443 & 0.8121641161 & 0.2276763671 \\
\hline C & 0.6401871557 & 0.1878358839 & 0.7723236179 \\
\hline C & 0.6401871557 & 0.8121641161 & 0.7723236179 \\
\hline C & 0.3598128443 & 0.1878358839 & 0.2276763671 \\
\hline C & 0.8597922831 & 0.3121354163 & 0.2276625003 \\
\hline C & 0.1402077169 & 0.6878645837 & 0.7723374847 \\
\hline C & 0.1402077169 & 0.3121354163 & 0.7723374847 \\
\hline C & 0.8597922831 & 0.6878645837 & 0.2276625003 \\
\hline C & 0.4345073879 & 0.7019031564 & 0.5403060810 \\
\hline C & 0.5654926421 & 0.2980968436 & 0.4596939190 \\
\hline C & 0.5654926421 & 0.7019031564 & 0.4596939190 \\
\hline C & 0.4345073879 & 0.2980968436 & 0.5403060810 \\
\hline C & 0.9345028421 & 0.2019416232 & 0.5403012552 \\
\hline C & 0.0654971279 & 0.7980583768 & 0.4596987448 \\
\hline C & 0.0654971279 & 0.2019416232 & 0.4596987448 \\
\hline C & 0.9345028421 & 0.7980583768 & 0.5403012552 \\
\hline C & 0.3676078773 & 0.7615901968 & 0.5803636749 \\
\hline C & 0.6323921227 & 0.2384098032 & 0.4196363251 \\
\hline C & 0.6323921227 & 0.7615901968 & 0.4196363251 \\
\hline C & 0.3676078773 & 0.2384098032 & 0.5803636749 \\
\hline C & 0.8676015116 & 0.2616394800 & 0.5803333285 \\
\hline C & 0.1323984884 & 0.7383605200 & 0.4196666715 \\
\hline C & 0.1323984884 & 0.2616394800 & 0.4196666715 \\
\hline C & 0.8676015116 & 0.7383605200 & 0.5803333285 \\
\hline $\mathrm{H}$ & 0.1387870491 & 0.0000000000 & 0.5114175323 \\
\hline $\mathrm{H}$ & 0.8612129809 & 0.0000000000 & 0.4885824677 \\
\hline $\mathrm{H}$ & 0.6387714497 & 0.5000000000 & 0.5113979358 \\
\hline $\mathrm{H}$ & 0.3612285203 & 0.5000000000 & 0.4886020642 \\
\hline $\mathrm{H}$ & 0.1100782526 & 0.9430999119 & 0.2763876162 \\
\hline $\mathrm{H}$ & 0.8899217394 & 0.0569000881 & 0.7236124138 \\
\hline $\mathrm{H}$ & 0.8899217394 & 0.9430999119 & 0.7236124138 \\
\hline $\mathrm{H}$ & 0.1100782526 & 0.0569000881 & 0.2763876162 \\
\hline $\mathrm{H}$ & 0.6100753173 & 0.4431005902 & 0.2763698899 \\
\hline $\mathrm{H}$ & 0.3899246827 & 0.5568994098 & 0.7236301401 \\
\hline $\mathrm{H}$ & 0.3899246827 & 0.4431005902 & 0.7236301401 \\
\hline $\mathrm{H}$ & 0.6100753173 & 0.5568994098 & 0.2763698899 \\
\hline $\mathrm{H}$ & 0.6112940827 & 0.0000000000 & 0.1873113211 \\
\hline $\mathrm{H}$ & 0.3887059173 & 0.0000000000 & 0.8126886939 \\
\hline $\mathrm{H}$ & 0.1112964497 & 0.5000000000 & 0.1873124467 \\
\hline $\mathrm{H}$ & 0.8887035503 & 0.5000000000 & 0.8126875683 \\
\hline $\mathrm{H}$ & 0.4356744074 & 0.0000000000 & 0.4178130708 \\
\hline $\mathrm{H}$ & 0.5643255926 & 0.0000000000 & 0.5821869592 \\
\hline $\mathrm{H}$ & 0.9356673823 & 0.5000000000 & 0.4178233722 \\
\hline $\mathrm{H}$ & 0.0643326177 & 0.5000000000 & 0.5821766578 \\
\hline $\mathrm{H}$ & 0.4152021722 & 0.0000000000 & 0.6308771278 \\
\hline $\mathrm{H}$ & 0.5847978578 & 0.0000000000 & 0.3691228722 \\
\hline $\mathrm{H}$ & 0.9151876645 & 0.5000000000 & 0.6308850705 \\
\hline $\mathrm{H}$ & 0.0848123055 & 0.5000000000 & 0.3691149295 \\
\hline $\mathrm{H}$ & 0.2609267250 & 0.0000000000 & 0.6779535722 \\
\hline
\end{tabular}




\begin{tabular}{|c|c|c|c|}
\hline $\mathrm{H}$ & 0.7390732750 & 0.0000000000 & 0.3220464278 \\
\hline $\mathrm{H}$ & 0.7609050570 & 0.5000000000 & 0.6779463622 \\
\hline $\mathrm{H}$ & 0.2390949430 & 0.5000000000 & 0.3220536378 \\
\hline $\mathrm{H}$ & 0.0925387475 & 0.9034042177 & 0.0858789625 \\
\hline $\mathrm{H}$ & 0.9074612375 & 0.0965957823 & 0.9141210305 \\
\hline $\mathrm{H}$ & 0.9074612375 & 0.9034042177 & 0.9141210305 \\
\hline $\mathrm{H}$ & 0.0925387475 & 0.0965957823 & 0.0858789625 \\
\hline $\mathrm{H}$ & 0.5925380210 & 0.4034142273 & 0.0858773605 \\
\hline $\mathrm{H}$ & 0.4074619790 & 0.5965857727 & 0.9141226325 \\
\hline $\mathrm{H}$ & 0.4074619790 & 0.4034142273 & 0.9141226325 \\
\hline $\mathrm{H}$ & 0.5925380210 & 0.5965857727 & 0.0858773605 \\
\hline $\mathrm{H}$ & 0.1706612294 & 0.9326406825 & -0.0048425262 \\
\hline $\mathrm{H}$ & 0.8293387706 & 0.0673593175 & 1.0048425532 \\
\hline $\mathrm{H}$ & 0.8293387706 & 0.9326406825 & 1.0048425532 \\
\hline $\mathrm{H}$ & 0.1706612294 & 0.0673593175 & -0.0048425262 \\
\hline $\mathrm{H}$ & 0.6706517492 & 0.4326457427 & -0.0048547736 \\
\hline $\mathrm{H}$ & 0.3293482508 & 0.5673542573 & 1.0048548006 \\
\hline $\mathrm{H}$ & 0.3293482508 & 0.4326457427 & 1.0048548006 \\
\hline $\mathrm{H}$ & 0.6706517492 & 0.5673542573 & -0.0048547736 \\
\hline $\mathrm{H}$ & 0.1320590824 & 0.7402974045 & 0.0873122005 \\
\hline H & 0.8679409176 & 0.2597025955 & 0.9126877845 \\
\hline $\mathrm{H}$ & 0.8679409176 & 0.7402974045 & 0.9126877845 \\
\hline $\mathrm{H}$ & 0.1320590824 & 0.2597025955 & 0.0873122005 \\
\hline H & 0.6320377806 & 0.2403157944 & 0.0872443331 \\
\hline $\mathrm{H}$ & 0.3679622194 & 0.7596842056 & 0.9127556519 \\
\hline $\mathrm{H}$ & 0.3679622194 & 0.2403157944 & 0.9127556519 \\
\hline $\mathrm{H}$ & 0.6320377806 & 0.7596842056 & 0.0872443331 \\
\hline $\mathrm{H}$ & 0.2434166584 & 0.6265404635 & 0.1631052015 \\
\hline $\mathrm{H}$ & 0.7565833266 & 0.3734595365 & 0.8368947835 \\
\hline $\mathrm{H}$ & 0.7565833266 & 0.6265404635 & 0.8368947835 \\
\hline $\mathrm{H}$ & 0.2434166584 & 0.3734595365 & 0.1631052015 \\
\hline $\mathrm{H}$ & 0.7433718502 & 0.1265310874 & 0.1630221877 \\
\hline $\mathrm{H}$ & 0.2566281498 & 0.8734689126 & 0.8369777973 \\
\hline H & 0.2566281498 & 0.1265310874 & 0.8369777973 \\
\hline $\mathrm{H}$ & 0.7433718502 & 0.8734689126 & 0.1630221877 \\
\hline $\mathrm{H}$ & 0.3909947824 & 0.6764833623 & 0.2555163979 \\
\hline $\mathrm{H}$ & 0.6090052476 & 0.3235166377 & 0.7444835721 \\
\hline $\mathrm{H}$ & 0.6090052476 & 0.6764833623 & 0.7444835721 \\
\hline $\mathrm{H}$ & 0.3909947824 & 0.3235166377 & 0.2555163979 \\
\hline H & 0.8909536955 & 0.1764471672 & 0.2554669919 \\
\hline $\mathrm{H}$ & 0.1090462745 & 0.8235528328 & 0.7445329781 \\
\hline $\mathrm{H}$ & 0.1090462745 & 0.1764471672 & 0.7445329781 \\
\hline H & 0.8909536955 & 0.8235528328 & 0.2554669919 \\
\hline $\mathrm{H}$ & 0.4227627594 & 0.8368650569 & 0.2685028786 \\
\hline $\mathrm{H}$ & 0.5772372406 & 0.1631349431 & 0.7314971214 \\
\hline $\mathrm{H}$ & 0.5772372406 & 0.8368650569 & 0.7314971214 \\
\hline $\mathrm{H}$ & 0.4227627594 & 0.1631349431 & 0.2685028786 \\
\hline H & 0.9227451687 & 0.3368209831 & 0.2685018912 \\
\hline $\mathrm{H}$ & 0.0772548313 & 0.6631790169 & 0.7314981088 \\
\hline $\mathrm{H}$ & 0.0772548313 & 0.3368209831 & 0.7314981088 \\
\hline $\mathrm{H}$ & 0.9227451687 & 0.6631790169 & 0.2685018912 \\
\hline $\mathrm{H}$ & 0.4044707107 & 0.6586307919 & 0.4678647546 \\
\hline H & 0.5955292893 & 0.3413692081 & 0.5321352454 \\
\hline $\mathrm{H}$ & 0.5955292893 & 0.6586307919 & 0.5321352454 \\
\hline $\mathrm{H}$ & 0.4044707107 & 0.3413692081 & 0.4678647546 \\
\hline $\mathrm{H}$ & 0.9044711018 & 0.1586637537 & 0.4678627300 \\
\hline $\mathrm{H}$ & 0.0955288982 & 0.8413362463 & 0.5321372700 \\
\hline $\mathrm{H}$ & 0.0955288982 & 0.1586637537 & 0.5321372700 \\
\hline
\end{tabular}




\begin{tabular}{|c|c|c|c|}
\hline $\mathrm{H}$ & 0.9044711018 & 0.8413362463 & 0.4678627300 \\
\hline $\mathrm{H}$ & 0.4643523002 & 0.6588949481 & 0.6132172318 \\
\hline $\mathrm{H}$ & 0.5356476998 & 0.3411050519 & 0.3867827682 \\
\hline $\mathrm{H}$ & 0.5356476998 & 0.6588949481 & 0.3867827682 \\
\hline $\mathrm{H}$ & 0.4643523002 & 0.3411050519 & 0.6132172318 \\
\hline $\mathrm{H}$ & 0.9643407411 & 0.1589396241 & 0.6132243528 \\
\hline $\mathrm{H}$ & 0.0356592589 & 0.8410603759 & 0.3867756472 \\
\hline $\mathrm{H}$ & 0.0356592589 & 0.1589396241 & 0.3867756472 \\
\hline $\mathrm{H}$ & 0.9643407411 & 0.8410603759 & 0.6132243528 \\
\hline $\mathrm{H}$ & 0.3381464020 & 0.8038960394 & 0.5075564514 \\
\hline $\mathrm{H}$ & 0.6618536280 & 0.1961039606 & 0.4924435486 \\
\hline $\mathrm{H}$ & 0.6618536280 & 0.8038960394 & 0.4924435486 \\
\hline $\mathrm{H}$ & 0.3381464020 & 0.1961039606 & 0.5075564514 \\
\hline $\mathrm{H}$ & 0.8381514822 & 0.3039396402 & 0.5075132362 \\
\hline $\mathrm{H}$ & 0.1618484878 & 0.6960603598 & 0.4924867638 \\
\hline $\mathrm{H}$ & 0.1618484878 & 0.3039396402 & 0.4924867638 \\
\hline $\mathrm{H}$ & 0.8381514822 & 0.6960603598 & 0.5075132362 \\
\hline $\mathrm{H}$ & 0.3158753327 & 0.7231163392 & 0.6105771538 \\
\hline $\mathrm{H}$ & 0.6841246373 & 0.2768836608 & 0.3894228462 \\
\hline $\mathrm{H}$ & 0.6841246373 & 0.7231163392 & 0.3894228462 \\
\hline $\mathrm{H}$ & 0.3158753327 & 0.2768836608 & 0.6105771538 \\
\hline $\mathrm{H}$ & 0.8158610293 & 0.2231756541 & 0.6105433976 \\
\hline $\mathrm{H}$ & 0.1841390007 & 0.7768243459 & 0.3894566024 \\
\hline $\mathrm{H}$ & 0.1841390007 & 0.2231756541 & 0.3894566024 \\
\hline $\mathrm{H}$ & 0.8158610293 & 0.7768243459 & 0.6105433976 \\
\hline $\mathrm{H}$ & 0.3966420122 & 0.8035097382 & 0.6539639115 \\
\hline $\mathrm{H}$ & 0.6033580178 & 0.1964902618 & 0.3460360885 \\
\hline $\mathrm{H}$ & 0.6033580178 & 0.8035097382 & 0.3460360885 \\
\hline $\mathrm{H}$ & 0.3966420122 & 0.1964902618 & 0.6539639115 \\
\hline $\mathrm{H}$ & 0.8966324606 & 0.3035640730 & 0.6539291491 \\
\hline $\mathrm{H}$ & 0.1033675094 & 0.6964359270 & 0.3460708509 \\
\hline $\mathrm{H}$ & 0.1033675094 & 0.3035640730 & 0.3460708509 \\
\hline $\mathrm{H}$ & 0.8966324606 & 0.6964359270 & 0.6539291491 \\
\hline $\mathrm{F}$ & 0.8428755723 & 0.0000000000 & 0.1930414658 \\
\hline $\mathrm{F}$ & 0.1571244127 & 0.0000000000 & 0.8069585192 \\
\hline $\mathrm{F}$ & 0.3428667609 & 0.5000000000 & 0.1930568988 \\
\hline $\mathrm{F}$ & 0.6571332391 & 0.5000000000 & 0.8069430862 \\
\hline $\mathrm{F}$ & 0.9588480622 & 0.0000000000 & 0.3466550428 \\
\hline $\mathrm{F}$ & 0.0411519448 & 0.0000000000 & 0.6533449872 \\
\hline $\mathrm{F}$ & 0.4588477934 & 0.5000000000 & 0.3466593672 \\
\hline $\mathrm{F}$ & 0.5411522066 & 0.5000000000 & 0.6533406628 \\
\hline $\mathrm{F}$ & 0.9686374069 & 0.9242839307 & 0.1727446737 \\
\hline $\mathrm{F}$ & 0.0313625781 & 0.0757160693 & 0.8272553263 \\
\hline $\mathrm{F}$ & 0.0313625781 & 0.9242839307 & 0.8272553263 \\
\hline $\mathrm{F}$ & 0.9686374069 & 0.0757160693 & 0.1727446737 \\
\hline $\mathrm{F}$ & 0.4686253364 & 0.4242836717 & 0.1727459867 \\
\hline $\mathrm{F}$ & 0.5313746636 & 0.5757163283 & 0.8272540133 \\
\hline $\mathrm{F}$ & 0.5313746636 & 0.4242836717 & 0.8272540133 \\
\hline $\mathrm{F}$ & 0.4686253364 & 0.5757163283 & 0.1727459867 \\
\hline B & 0.9349335961 & 0.0000000000 & 0.2220564697 \\
\hline B & 0.0650664109 & 0.0000000000 & 0.7779435453 \\
\hline B & 0.4349255075 & 0.5000000000 & 0.2220620352 \\
\hline B & 0.5650744925 & 0.5000000000 & 0.7779379798 \\
\hline \multicolumn{4}{|c|}{ 4•2acetone $\quad-H S$} \\
\hline \multicolumn{4}{|c|}{ CELL_PARAMETERS (angstrom) } \\
\hline & $4 \quad-0.287137642$ & 0.388606748 & \\
\hline & 11.682234223 & 0.204465842 & \\
\hline & 2.161346153 & 13.570655047 & \\
\hline
\end{tabular}




\begin{tabular}{|c|c|c|c|}
\hline \multicolumn{4}{|c|}{ ATOMIC_POSITIONS (crystal) } \\
\hline Fe1 & 0.9702434686 & 0.3027639295 & 0.3201679040 \\
\hline Fe1 & 0.0297565314 & 0.6972360415 & 0.6798320960 \\
\hline 0 & 0.9474426974 & 0.2963849835 & 0.4666951869 \\
\hline 0 & 0.0525573026 & 0.7036150165 & 0.5333047830 \\
\hline 0 & 0.0160125098 & 0.4693287450 & 0.3156817046 \\
\hline 0 & 0.9839874902 & 0.5306712850 & 0.6843182954 \\
\hline 0 & 0.5401537617 & 0.1548163164 & 0.5766635283 \\
\hline 0 & 0.4598462083 & 0.8451836536 & 0.4233364717 \\
\hline $\mathrm{N}$ & 0.7272037080 & 0.3786263097 & 0.3007412239 \\
\hline $\mathrm{N}$ & 0.2727962920 & 0.6213736903 & 0.6992587761 \\
\hline $\mathrm{N}$ & 0.0103856644 & 0.3273776127 & 0.1548401920 \\
\hline $\mathrm{N}$ & 0.9896143356 & 0.6726224173 & 0.8451597930 \\
\hline $\mathrm{N}$ & 0.9281105497 & 0.1360383025 & 0.3121461319 \\
\hline $\mathrm{N}$ & 0.0718894503 & 0.8639616975 & 0.6878538681 \\
\hline $\mathrm{N}$ & 0.2378333847 & 0.2410992029 & 0.2655057666 \\
\hline $\mathrm{N}$ & 0.7621666153 & 0.7589007971 & 0.7344942634 \\
\hline C & 0.5818153542 & 0.3761700926 & 0.3741519905 \\
\hline $\mathrm{C}$ & 0.4181846458 & 0.6238298774 & 0.6258480095 \\
\hline C & 0.4293910297 & 0.4291408613 & 0.3571416242 \\
\hline C & 0.5706089703 & 0.5708591687 & 0.6428583758 \\
\hline C & 0.4261826686 & 0.4838447026 & 0.2602915726 \\
\hline C & 0.5738173014 & 0.5161552974 & 0.7397084274 \\
\hline C & 0.5765073718 & 0.4831136453 & 0.1837614236 \\
\hline C & 0.4234926282 & 0.5168863847 & 0.8162385614 \\
\hline C & 0.7258344411 & 0.4311916471 & 0.2067301951 \\
\hline C & 0.2741655589 & 0.5688083829 & 0.7932698049 \\
\hline C & 0.8953621062 & 0.4325247294 & 0.1300999466 \\
\hline C & 0.1046378938 & 0.5674752706 & 0.8699000834 \\
\hline C & -0.0357898253 & 0.2230580011 & 0.1351010431 \\
\hline C & 1.0357898253 & 0.7769420129 & 0.8648989419 \\
\hline C & 0.9589481456 & 0.1184242399 & 0.2174489582 \\
\hline C & 0.0410518544 & 0.8815757601 & 0.7825510418 \\
\hline C & 0.9714621324 & 0.0081183981 & 0.1968399722 \\
\hline C & 0.0285378676 & 0.9918816119 & 0.8031599978 \\
\hline C & 0.9471764281 & 0.9149609557 & 0.2759602057 \\
\hline C & 0.0528235719 & 0.0850390593 & 0.7240398243 \\
\hline C & 0.9120722735 & 0.9340148205 & 0.3737086456 \\
\hline C & 0.0879277265 & 0.0659851795 & 0.6262913244 \\
\hline C & 0.9043909908 & 0.0458087938 & 0.3886717487 \\
\hline C & 0.0956090092 & 0.9541912141 & 0.6113282813 \\
\hline C & 0.1895552865 & 0.3403281682 & 0.1069730794 \\
\hline C & 0.8104447135 & 0.6596718318 & 0.8930268906 \\
\hline C & 0.2996796445 & 0.2576526720 & 0.1645830177 \\
\hline C & 0.7003203555 & 0.7423473280 & 0.8354169823 \\
\hline C & 0.4578718495 & 0.2052242685 & 0.1179722038 \\
\hline C & 0.5421281505 & 0.7947757165 & 0.8820277812 \\
\hline C & 0.5554039758 & 0.1345841002 & 0.1769609440 \\
\hline C & 0.4445960242 & 0.8654158998 & 0.8230390560 \\
\hline C & 0.4930983014 & 0.1193029131 & 0.2809299424 \\
\hline C & 0.5069016986 & 0.8806970719 & 0.7190700576 \\
\hline C & 0.3333773353 & 0.1740190704 & 0.3219772225 \\
\hline C & 0.6666226647 & 0.8259809446 & 0.6780227775 \\
\hline C & 0.0075231516 & 0.4896888550 & 0.4014713930 \\
\hline C & 0.9924768484 & 0.5103111750 & 0.5985286370 \\
\hline C & 0.9707733893 & 0.3908773360 & 0.4880240954 \\
\hline C & 0.0292266107 & 0.6091226640 & 0.5119759346 \\
\hline
\end{tabular}




\begin{tabular}{|c|c|c|c|}
\hline C & 0.9652481785 & 0.4041411485 & 0.5833194523 \\
\hline C & 0.0347518215 & 0.5958588515 & 0.4166805477 \\
\hline C & 0.5843357865 & 0.1592561955 & 0.7337321161 \\
\hline C & 0.4156642135 & 0.8407438335 & 0.2662678839 \\
\hline C & 0.4979412157 & 0.1997635936 & 0.6519170245 \\
\hline C & 0.5020587543 & 0.8002364064 & 0.3480829755 \\
\hline C & 0.3583997758 & 0.2994249712 & 0.6657177684 \\
\hline C & 0.6416002242 & 0.7005750588 & 0.3342822316 \\
\hline $\mathrm{H}$ & 0.5899816280 & 0.3286862170 & 0.4476775155 \\
\hline $\mathrm{H}$ & 0.4100183720 & 0.6713137530 & 0.5523224545 \\
\hline $\mathrm{H}$ & 0.3150648074 & 0.4266164158 & 0.4190651188 \\
\hline $\mathrm{H}$ & 0.6849351626 & 0.5733835842 & 0.5809348812 \\
\hline $\mathrm{H}$ & 0.3086387588 & 0.5258264180 & 0.2442340769 \\
\hline $\mathrm{H}$ & 0.6913612712 & 0.4741735820 & 0.7557659531 \\
\hline $\mathrm{H}$ & 0.5778507406 & 0.5216909090 & 0.1067057348 \\
\hline $\mathrm{H}$ & 0.4221492594 & 0.4783090910 & 0.8932942502 \\
\hline $\mathrm{H}$ & 0.8806662544 & 0.4405837557 & 0.0547954284 \\
\hline $\mathrm{H}$ & 0.1193337456 & 0.5594162733 & 0.9452045796 \\
\hline $\mathrm{H}$ & 0.9495389728 & 0.5081105037 & 0.1325554559 \\
\hline $\mathrm{H}$ & 0.0504610272 & 0.4918894963 & 0.8674445441 \\
\hline $\mathrm{H}$ & 0.0456924560 & 0.2007847096 & 0.0633234188 \\
\hline $\mathrm{H}$ & 0.9543075440 & 0.7992152904 & 0.9366765812 \\
\hline $\mathrm{H}$ & 0.8371242018 & 0.2455243383 & 0.1249286143 \\
\hline $\mathrm{H}$ & 0.1628757982 & 0.7544756917 & 0.8750713777 \\
\hline $\mathrm{H}$ & 0.0011843993 & -0.0051026383 & 0.1197478302 \\
\hline $\mathrm{H}$ & 0.9988156007 & 1.0051026593 & 0.8802521398 \\
\hline $\mathrm{H}$ & 0.9588033242 & 0.8283909424 & 0.2605526176 \\
\hline $\mathrm{H}$ & 0.0411966758 & 0.1716090876 & 0.7394473824 \\
\hline $\mathrm{H}$ & 0.8966164005 & 0.8625865339 & 0.4369224018 \\
\hline $\mathrm{H}$ & 0.1033835995 & 0.1374134590 & 0.5630776282 \\
\hline $\mathrm{H}$ & 0.8768748474 & 0.0663683716 & 0.4633414330 \\
\hline $\mathrm{H}$ & 0.1231251526 & 0.9336316514 & 0.5366585670 \\
\hline $\mathrm{H}$ & 0.2281024224 & 0.3282650117 & 0.0281921704 \\
\hline $\mathrm{H}$ & 0.7718975776 & 0.6717349883 & 0.9718078376 \\
\hline $\mathrm{H}$ & 0.2062080326 & 0.4292744070 & 0.1072847846 \\
\hline $\mathrm{H}$ & 0.7937919674 & 0.5707255930 & 0.8927152004 \\
\hline $\mathrm{H}$ & 0.5057633988 & 0.2198104204 & 0.0366223223 \\
\hline $\mathrm{H}$ & 0.4942366012 & 0.7801895796 & 0.9633776927 \\
\hline $\mathrm{H}$ & 0.6785559984 & 0.0923392217 & 0.1408242375 \\
\hline $\mathrm{H}$ & 0.3214440016 & 0.9076607633 & 0.8591757915 \\
\hline $\mathrm{H}$ & 0.5641452589 & 0.0646427165 & 0.3301831936 \\
\hline $\mathrm{H}$ & 0.4358547411 & 0.9353573125 & 0.6698168064 \\
\hline $\mathrm{H}$ & 0.2784373303 & 0.1640050265 & 0.4028865701 \\
\hline $\mathrm{H}$ & 0.7215626697 & 0.8359949735 & 0.5971134299 \\
\hline $\mathrm{H}$ & 0.9346174121 & 0.3352103096 & 0.6475739456 \\
\hline $\mathrm{H}$ & 0.0653825879 & 0.6647897204 & 0.3524260544 \\
\hline $\mathrm{H}$ & 0.4936944592 & 0.1255405790 & 0.8038934639 \\
\hline $\mathrm{H}$ & 0.5063055408 & 0.8744594140 & 0.1961065361 \\
\hline $\mathrm{H}$ & 0.6298919590 & 0.2314095530 & 0.7492657336 \\
\hline $\mathrm{H}$ & 0.3701080410 & 0.7685904770 & 0.2507342664 \\
\hline $\mathrm{H}$ & 0.6895357516 & 0.0916173406 & 0.7136708817 \\
\hline $\mathrm{H}$ & 0.3104642484 & 0.9083826894 & 0.2863291183 \\
\hline $\mathrm{H}$ & 0.2885491225 & 0.3115217586 & 0.6102203140 \\
\hline $\mathrm{H}$ & 0.7114508775 & 0.6884782414 & 0.3897796860 \\
\hline $\mathrm{H}$ & 0.4139913813 & 0.3786462458 & 0.6563680821 \\
\hline $\mathrm{H}$ & 0.5860085887 & 0.6213537242 & 0.3436319179 \\
\hline $\mathrm{H}$ & 0.2715487614 & 0.2883652718 & 0.7416535984 \\
\hline $\mathrm{H}$ & 0.7284512686 & 0.7116347432 & 0.2583464016 \\
\hline
\end{tabular}




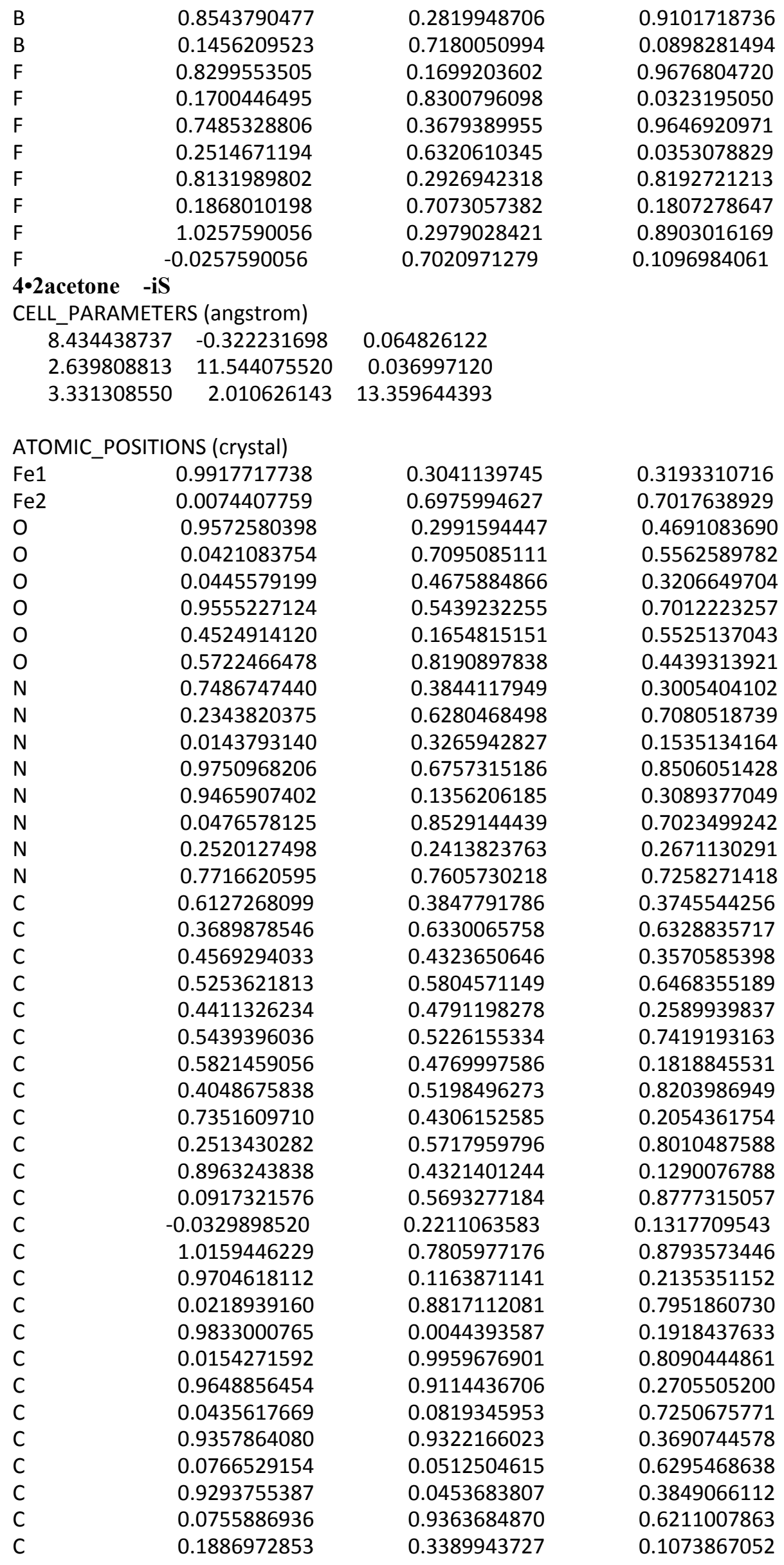




\begin{tabular}{|c|c|c|c|}
\hline C & 0.7984353391 & 0.6617564538 & 0.8907862026 \\
\hline C & 0.3033809111 & 0.2558095601 & 0.1660007175 \\
\hline C & 0.6958845121 & 0.7435621846 & 0.8246191586 \\
\hline C & 0.4559689535 & 0.2014247885 & 0.1201858426 \\
\hline C & 0.5350963649 & 0.7948059929 & 0.8584122963 \\
\hline C & 0.5585128802 & 0.1311182197 & 0.1802643340 \\
\hline C & 0.4484415179 & 0.8634454526 & 0.7885689571 \\
\hline C & 0.5074113807 & 0.1189998522 & 0.2843983288 \\
\hline C & 0.5237559983 & 0.8770492509 & 0.6870085500 \\
\hline C & 0.3527965804 & 0.1757135992 & 0.3248503517 \\
\hline C & 0.6862248267 & 0.8248222430 & 0.6586749573 \\
\hline C & 0.0245131231 & 0.4911390414 & 0.4095589002 \\
\hline C & 0.9744290218 & 0.5199640370 & 0.6122665607 \\
\hline C & 0.9732497621 & 0.3958365105 & 0.4945303052 \\
\hline C & 0.0255925764 & 0.6144387588 & 0.5285284657 \\
\hline C & 0.9474421793 & 0.4136116271 & 0.5934382166 \\
\hline C & 0.0499936023 & 0.5975525208 & 0.4292267517 \\
\hline C & 0.6126597831 & 0.1582843901 & 0.6750121157 \\
\hline C & 0.3895420290 & 0.8382896224 & 0.3319864885 \\
\hline C & 0.4659714176 & 0.2016809511 & 0.6270151490 \\
\hline C & 0.5473463810 & 0.7920690769 & 0.3682287362 \\
\hline C & 0.3368071534 & 0.2924158850 & 0.6742146544 \\
\hline C & 0.6721402421 & 0.7102737259 & 0.3078375308 \\
\hline $\mathrm{H}$ & 0.6321670132 & 0.3447478192 & 0.4492321665 \\
\hline $\mathrm{H}$ & 0.3476703239 & 0.6813877088 & 0.5608728159 \\
\hline $\mathrm{H}$ & 0.3499355475 & 0.4317159669 & 0.4191985518 \\
\hline $\mathrm{H}$ & 0.6306806170 & 0.5866284067 & 0.5837782803 \\
\hline $\mathrm{H}$ & 0.3206383096 & 0.5168349229 & 0.2428858818 \\
\hline $\mathrm{H}$ & 0.6645284834 & 0.4805986467 & 0.7555288331 \\
\hline $\mathrm{H}$ & 0.5728655553 & 0.5107261641 & 0.1043140175 \\
\hline $\mathrm{H}$ & 0.4162966778 & 0.4784075729 & 0.8961742868 \\
\hline $\mathrm{H}$ & 0.8738696154 & 0.4395502174 & 0.0531255764 \\
\hline $\mathrm{H}$ & 0.1124624529 & 0.5635049006 & 0.9537573127 \\
\hline $\mathrm{H}$ & 0.9504008288 & 0.5086372033 & 0.1329781077 \\
\hline $\mathrm{H}$ & 0.0372750529 & 0.4930167298 & 0.8740463663 \\
\hline $\mathrm{H}$ & 0.0410843623 & 0.1983731974 & 0.0596876257 \\
\hline $\mathrm{H}$ & 0.9320474931 & 0.8028320824 & 0.9488972160 \\
\hline $\mathrm{H}$ & 0.8391607897 & 0.2430779699 & 0.1209563932 \\
\hline $\mathrm{H}$ & 0.1393010848 & 0.7580831332 & 0.8971188149 \\
\hline $\mathrm{H}$ & 0.0083043216 & -0.0099163258 & 0.1141539731 \\
\hline $\mathrm{H}$ & 0.9879754334 & 1.0177036585 & 0.8845219715 \\
\hline $\mathrm{H}$ & 0.9754939535 & 0.8237030604 & 0.2545067214 \\
\hline $\mathrm{H}$ & 0.0378804949 & 0.1716767124 & 0.7348194209 \\
\hline $\mathrm{H}$ & 0.9223242099 & 0.8612257320 & 0.4321803600 \\
\hline $\mathrm{H}$ & 0.0987308142 & 0.1167776235 & 0.5626713182 \\
\hline $\mathrm{H}$ & 0.9093848688 & 0.0670736020 & 0.4600531228 \\
\hline $\mathrm{H}$ & 0.0964801404 & 0.9068394396 & 0.5490402859 \\
\hline $\mathrm{H}$ & 0.2201812911 & 0.3260810497 & 0.0281353686 \\
\hline $\mathrm{H}$ & 0.7575824393 & 0.6754558705 & 0.9696464682 \\
\hline $\mathrm{H}$ & 0.2059189186 & 0.4286378008 & 0.1090311794 \\
\hline $\mathrm{H}$ & 0.7856666205 & 0.5716573598 & 0.8876965326 \\
\hline $\mathrm{H}$ & 0.4959138785 & 0.2142420310 & 0.0387291937 \\
\hline $\mathrm{H}$ & 0.4768706386 & 0.7811409029 & 0.9379144705 \\
\hline $\mathrm{H}$ & 0.6772638308 & 0.0871591204 & 0.1448262969 \\
\hline $\mathrm{H}$ & 0.3232359872 & 0.9058787258 & 0.8140252042 \\
\hline $\mathrm{H}$ & 0.5838021426 & 0.0661081365 & 0.3344934321 \\
\hline $\mathrm{H}$ & 0.4607483302 & 0.9283286567 & 0.6294400494 \\
\hline $\mathrm{H}$ & 0.3085107320 & 0.1695526862 & 0.4059771036 \\
\hline
\end{tabular}




\begin{tabular}{|c|c|c|c|}
\hline $\mathrm{H}$ & 0.7506908041 & 0.8330184795 & 0.5803387027 \\
\hline $\mathrm{H}$ & 0.9079760786 & 0.3466966986 & 0.6562526664 \\
\hline $\mathrm{H}$ & 0.0896232707 & 0.6645315583 & 0.3664462359 \\
\hline $\mathrm{H}$ & 0.5731195311 & 0.1069272102 & 0.7494991557 \\
\hline $\mathrm{H}$ & 0.4157338589 & 0.8818001022 & 0.2534512121 \\
\hline $\mathrm{H}$ & 0.6645110004 & 0.2307607807 & 0.6887017573 \\
\hline H & 0.3248744584 & 0.7657556509 & 0.3317879947 \\
\hline $\mathrm{H}$ & 0.7075991236 & 0.1034198615 & 0.6288851694 \\
\hline $\mathrm{H}$ & 0.3093605311 & 0.8989961945 & 0.3793121409 \\
\hline $\mathrm{H}$ & 0.2345514889 & 0.3170790565 & 0.6349416663 \\
\hline $\mathrm{H}$ & 0.7746501448 & 0.6724500589 & 0.3451473509 \\
\hline $\mathrm{H}$ & 0.3930609873 & 0.3698989232 & 0.6721724637 \\
\hline $\mathrm{H}$ & 0.6126366274 & 0.6409983219 & 0.2951631935 \\
\hline $\mathrm{H}$ & 0.2887825257 & 0.2611150288 & 0.7541199389 \\
\hline H & 0.7215802678 & 0.7566519690 & 0.2328104665 \\
\hline B & 0.8256158502 & 0.2822831817 & 0.9068440061 \\
\hline B & 0.1825550869 & 0.7149423665 & 0.0959368679 \\
\hline $\mathrm{F}$ & 0.8039414096 & 0.1722349502 & 0.9680889906 \\
\hline $\mathrm{F}$ & 0.2080250979 & 0.8229114370 & 0.0319901077 \\
\hline $\mathrm{F}$ & 0.7254010090 & 0.3732618056 & 0.9574449997 \\
\hline $\mathrm{F}$ & 0.2796578993 & 0.6212667176 & 0.0473617349 \\
\hline $\mathrm{F}$ & 0.7780572018 & 0.2862725452 & 0.8151698515 \\
\hline $\mathrm{F}$ & 0.2295960030 & 0.7129435735 & 0.1872536930 \\
\hline $\mathrm{F}$ & 0.9947275477 & 0.2975568599 & 0.8886264544 \\
\hline $\mathrm{F}$ & 0.0123298878 & 0.7021176190 & 0.1143675716 \\
\hline \multicolumn{4}{|l|}{ 4•2acetone LS } \\
\hline \multicolumn{4}{|c|}{ CELL_PARAMETERS (angstrom) } \\
\hline 8.452983575 & -0.273434596 & 0.022675251 & \\
\hline 2.704578361 & 11.450741343 & -0.177060363 & \\
\hline 3.278851249 & 1.774255774 & 13.146035116 & \\
\hline \multicolumn{4}{|c|}{ ATOMIC_POSITIONS (crystal) } \\
\hline Fe2 & 0.9901977902 & 0.3071427503 & 0.3015872421 \\
\hline $\mathrm{Fe} 2$ & 0.0098022098 & 0.6928572207 & 0.6984127579 \\
\hline 0 & 0.9599933334 & 0.2960907333 & 0.4489267763 \\
\hline O & 0.0400066666 & 0.7039092667 & 0.5510731937 \\
\hline 0 & 0.0364419744 & 0.4625021710 & 0.3056439734 \\
\hline 0 & 0.9635580256 & 0.5374978590 & 0.6943560266 \\
\hline 0 & 0.4239529046 & 0.1622170294 & 0.5553821730 \\
\hline 0 & 0.5760470654 & 0.8377829406 & 0.4446178270 \\
\hline $\mathrm{N}$ & 0.7608994909 & 0.3760411546 & 0.2983718433 \\
\hline $\mathrm{N}$ & 0.2391005091 & 0.6239588454 & 0.7016281567 \\
\hline $\mathrm{N}$ & 0.0181739877 & 0.3272606929 & 0.1508669986 \\
\hline $\mathrm{N}$ & 0.9818260123 & 0.6727393371 & 0.8491329864 \\
\hline $\mathrm{N}$ & 0.9539686798 & 0.1508184923 & 0.2981341600 \\
\hline $\mathrm{N}$ & 0.0460313202 & 0.8491815077 & 0.7018658400 \\
\hline $\mathrm{N}$ & 0.2272209102 & 0.2438189682 & 0.2735661271 \\
\hline $\mathrm{N}$ & 0.7727790898 & 0.7561810318 & 0.7264339029 \\
\hline C & 0.6282261002 & 0.3717886136 & 0.3755331989 \\
\hline C & 0.3717738998 & 0.6282113564 & 0.6244668011 \\
\hline C & 0.4694395576 & 0.4215794620 & 0.3632467534 \\
\hline C & 0.5305604424 & 0.5784205680 & 0.6367532466 \\
\hline C & 0.4463802174 & 0.4754488198 & 0.2675613931 \\
\hline C & 0.5536197526 & 0.5245511802 & 0.7324386069 \\
\hline C & 0.5836126898 & 0.4781233652 & 0.1870050235 \\
\hline C & 0.4163873102 & 0.5218766648 & 0.8129949615 \\
\hline C & 0.7396352254 & 0.4297416726 & 0.2050248865 \\
\hline C & 0.2603647746 & 0.5702583574 & 0.7949751135 \\
\hline
\end{tabular}




\begin{tabular}{|c|c|c|c|}
\hline C & 0.8977094666 & 0.4334841702 & 0.1265934071 \\
\hline C & 0.1022905334 & 0.5665158298 & 0.8734066229 \\
\hline C & -0.0207518538 & 0.2214552517 & 0.1199289930 \\
\hline C & 1.0207518537 & 0.7785447623 & 0.8800709920 \\
\hline C & 0.9763405120 & 0.1210258689 & 0.2034215104 \\
\hline C & 0.0236594880 & 0.8789741311 & 0.7965784896 \\
\hline C & 0.9823696101 & 0.0063524775 & 0.1871225864 \\
\hline C & 0.0176303899 & 0.9936475325 & 0.8128773836 \\
\hline C & 0.9575097248 & 0.9209091254 & 0.2706251475 \\
\hline C & 0.0424902752 & 0.0790908896 & 0.7293748825 \\
\hline C & 0.9290410355 & 0.9525767144 & 0.3680963308 \\
\hline C & 0.0709589645 & 0.0474232856 & 0.6319036392 \\
\hline C & 0.9302917414 & 0.0676946912 & 0.3789670911 \\
\hline C & 0.0697082586 & 0.9323053168 & 0.6210329389 \\
\hline C & 0.1934748310 & 0.3411501633 & 0.1088041110 \\
\hline C & 0.8065251690 & 0.6588498367 & 0.8911958590 \\
\hline C & 0.2996590817 & 0.2593437255 & 0.1730624453 \\
\hline C & 0.7003409183 & 0.7406562745 & 0.8269375547 \\
\hline C & 0.4602664444 & 0.2065894448 & 0.1361056670 \\
\hline C & 0.5397335556 & 0.7934105402 & 0.8638943180 \\
\hline C & 0.5505008684 & 0.1379391452 & 0.2044648286 \\
\hline C & 0.4494991316 & 0.8620608548 & 0.7955351714 \\
\hline C & 0.4787484537 & 0.1259644301 & 0.3078217840 \\
\hline C & 0.5212515463 & 0.8740355549 & 0.6921782160 \\
\hline C & 0.3161029901 & 0.1797016313 & 0.3393404735 \\
\hline C & 0.6838970099 & 0.8202983837 & 0.6606595265 \\
\hline C & 0.0204426922 & 0.4867219590 & 0.3966527891 \\
\hline C & 0.9795573078 & 0.5132780710 & 0.6033472409 \\
\hline C & 0.9756734652 & 0.3910984198 & 0.4795630885 \\
\hline C & 0.0243265348 & 0.6089015802 & 0.5204369415 \\
\hline C & 0.9552051624 & 0.4067494462 & 0.5812513355 \\
\hline C & 0.0447948376 & 0.5932505538 & 0.4187486645 \\
\hline C & 0.6289341484 & 0.1587222487 & 0.6507855537 \\
\hline C & 0.3710658516 & 0.8412777803 & 0.3492144463 \\
\hline C & 0.4606522271 & 0.1975482493 & 0.6257179271 \\
\hline C & 0.5393477429 & 0.8024517507 & 0.3742820729 \\
\hline C & 0.3408461926 & 0.2829979948 & 0.6896499820 \\
\hline C & 0.6591538074 & 0.7170020352 & 0.3103500180 \\
\hline $\mathrm{H}$ & 0.6535516298 & 0.3262624271 & 0.4478133999 \\
\hline $\mathrm{H}$ & 0.3464483702 & 0.6737375429 & 0.5521865701 \\
\hline $\mathrm{H}$ & 0.3656412425 & 0.4164020777 & 0.4278894620 \\
\hline $\mathrm{H}$ & 0.6343587275 & 0.5835979223 & 0.5721105380 \\
\hline $\mathrm{H}$ & 0.3238267005 & 0.5149146655 & 0.2551226063 \\
\hline $\mathrm{H}$ & 0.6761733295 & 0.4850853345 & 0.7448774237 \\
\hline $\mathrm{H}$ & 0.5686588713 & 0.5172601128 & 0.1108930362 \\
\hline $\mathrm{H}$ & 0.4313411287 & 0.4827398872 & 0.8891069488 \\
\hline $\mathrm{H}$ & 0.8760182168 & 0.4390961739 & 0.0495297169 \\
\hline $\mathrm{H}$ & 0.1239817832 & 0.5609038551 & 0.9504702911 \\
\hline $\mathrm{H}$ & 0.9493410627 & 0.5103909618 & 0.1326359100 \\
\hline $\mathrm{H}$ & 0.0506589373 & 0.4896090382 & 0.8673640900 \\
\hline $\mathrm{H}$ & 0.0631359398 & 0.1979580228 & 0.0487934675 \\
\hline $\mathrm{H}$ & 0.9368640602 & 0.8020419772 & 0.9512065325 \\
\hline $\mathrm{H}$ & 0.8554435090 & 0.2439529224 & 0.1024876805 \\
\hline $\mathrm{H}$ & 0.1445564910 & 0.7560471076 & 0.8975123115 \\
\hline $\mathrm{H}$ & 0.0070318558 & -0.0160249570 & 0.1100038814 \\
\hline $\mathrm{H}$ & 0.9929681442 & 1.0160249780 & 0.8899960886 \\
\hline $\mathrm{H}$ & 0.9619673198 & 0.8309328907 & 0.2590469558 \\
\hline $\mathrm{H}$ & 0.0380326802 & 0.1690671393 & 0.7409530442 \\
\hline
\end{tabular}




$\begin{array}{llll}\text { H } & 0.9089703180 & 0.8877071073 & 0.4347533832 \\ \mathrm{H} & 0.0910296820 & 0.1122928857 & 0.5652466468 \\ \mathrm{H} & 0.9125997523 & 0.0975849276 & 0.4527383436 \\ \mathrm{H} & 0.0874002477 & 0.9024150954 & 0.5472616564 \\ \mathrm{H} & 0.2324577840 & 0.3267290625 & 0.0282488463 \\ \mathrm{H} & 0.7675422160 & 0.6732709375 & 0.9717511617 \\ \mathrm{H} & 0.2045233004 & 0.4316586946 & 0.1138090680 \\ \mathrm{H} & 0.7954766996 & 0.5683413054 & 0.8861909170 \\ \mathrm{H} & 0.5157544613 & 0.2191548107 & 0.0552937369 \\ \mathrm{H} & 0.4842455386 & 0.7808451893 & 0.9447062781 \\ \mathrm{H} & 0.6757880845 & 0.0945769071 & 0.1764456336 \\ \mathrm{H} & 0.3242119155 & 0.9054230779 & 0.8235543954 \\ \mathrm{H} & 0.5446569135 & 0.0752096836 & 0.3645593410 \\ \mathrm{H} & 0.4553430865 & 0.9247903454 & 0.6354406590 \\ \mathrm{H} & 0.2546805921 & 0.1729126042 & 0.4192668298 \\ \mathrm{H} & 0.7453194079 & 0.8270873958 & 0.5807331702 \\ \mathrm{H} & 0.9194462223 & 0.3389342048 & 0.6429823679 \\ \mathrm{H} & 0.0805537777 & 0.6610658252 & 0.3570176321 \\ \mathrm{H} & 0.6228254005 & 0.1440449866 & 0.7330758510 \\ \mathrm{H} & 0.3771745995 & 0.8559550064 & 0.2669241490 \\ \mathrm{H} & 0.7024170866 & 0.2295134828 & 0.6210022625 \\ \mathrm{H} & 0.2975829134 & 0.7704865472 & 0.3789977375 \\ \mathrm{H} & 0.6928515797 & 0.0803987863 & 0.6156137487 \\ \mathrm{H} & 0.3071484203 & 0.9196012437 & 0.3843862513 \\ \mathrm{H} & 0.2346902854 & 0.3214882592 & 0.6552626284 \\ \mathrm{H} & 0.7653097146 & 0.6785117408 & 0.3447373716 \\ \mathrm{H} & 0.4027209658 & 0.3515738401 & 0.7019557432 \\ \mathrm{H} & 0.5972790042 & 0.6484261299 & 0.2980442568 \\ \mathrm{H} & 0.2955990661 & 0.2375355358 & 0.7656001799 \\ \mathrm{H} & 0.7044009639 & 0.7624644792 & 0.2343998201 \\ \mathrm{~B} & 0.8117766391 & 0.2864354450 & 0.9008176198 \\ \mathrm{~B} & 0.1882233609 & 0.7135645250 & 0.0991824032 \\ \mathrm{~F} & 0.7874904052 & 0.1793722369 & 0.9643858372 \\ \mathrm{~F} & 0.2125095948 & 0.8206277331 & 0.0356141398 \\ \mathrm{~F} & 0.7137344063 & 0.3821251173 & 0.9513218411 \\ \mathrm{~F} & 0.2862655937 & 0.6178749127 & 0.0486781389 \\ \mathrm{~F} & 0.7643287814 & 0.2867920269 & 0.8082505143 \\ \mathrm{~F} & 0.2356712186 & 0.7132079431 & 0.1917494717 \\ \mathrm{~F} & 0.9817899020 & 0.2977374708 & 0.8823522410 \\ \mathrm{~F} & 0.0182100980 & 0.7022624992 & 0.1176477820 \\ \mathrm{H} & & & \end{array}$

5•Et ${ }_{2} \mathrm{O} \cdot \mathrm{MeCN}$ HS

CELL_PARAMETERS (angstrom)

$\begin{array}{rrr}18.948887087 & 0.000000000 & -0.130728733 \\ 0.000000000 & 12.783025767 & 0.000000000 \\ -4.594793818 & 0.000000000 & 20.782125063\end{array}$

ATOMIC_POSITIONS (crystal)

$\begin{array}{llll}\text { Fe1 } & 0.0105852967 & 0.4865575382 & 0.7157627904 \\ \text { Fe1 } & 0.9894147033 & 0.5134424618 & 0.2842372096 \\ \text { Fe1 } & 0.4894147033 & 0.9865575382 & 0.7842372096 \\ \text { Fe1 } & 0.5105852967 & 0.0134424618 & 0.2157627904 \\ \text { Fe1 } & 0.5966361812 & 0.4988503456 & 0.7427662412 \\ \text { Fe1 } & 0.4033638188 & 0.5011496244 & 0.2572337588 \\ \text { Fe1 } & 0.9033638188 & 0.9988503756 & 0.7572337588 \\ \text { Fe1 } & 0.0966361812 & 0.0011496544 & 0.2427662412 \\ \text { F } & 0.2373567099 & 0.2832582435 & 0.5379460222 \\ \text { F } & 0.7626433041 & 0.7167417265 & 0.4620539778 \\ \text { F } & 0.2626433041 & 0.7832582735 & 0.9620539778\end{array}$




\begin{tabular}{|c|c|c|c|}
\hline $\mathrm{F}$ & 0.7373566959 & 0.2167417565 & 0.0379460222 \\
\hline $\mathrm{F}$ & 0.3301818710 & 0.3956128710 & 0.5261751322 \\
\hline $\mathrm{F}$ & 0.6698181290 & 0.6043871290 & 0.4738248678 \\
\hline $\mathrm{F}$ & 0.1698181290 & 0.8956128710 & 0.9738248678 \\
\hline $\mathrm{F}$ & 0.8301818710 & 0.1043871290 & 0.0261751322 \\
\hline $\mathrm{F}$ & 0.2375944031 & 0.4556507966 & 0.5726283775 \\
\hline $\mathrm{F}$ & 0.7624055819 & 0.5443492334 & 0.4273716225 \\
\hline $\mathrm{F}$ & 0.2624055819 & 0.9556507666 & 0.9273716225 \\
\hline $\mathrm{F}$ & 0.7375944181 & 0.0443492034 & 0.0726283775 \\
\hline $\mathrm{F}$ & 0.7865298113 & 0.0375119406 & 0.3593802169 \\
\hline $\mathrm{F}$ & 0.2134701887 & 0.9624880474 & 0.6406197831 \\
\hline $\mathrm{F}$ & 0.7134701887 & 0.5375119526 & 0.1406197831 \\
\hline $\mathrm{F}$ & 0.2865298113 & 0.4624880474 & 0.8593802169 \\
\hline $\mathrm{F}$ & 0.7974391877 & 0.2041676175 & 0.4039974844 \\
\hline $\mathrm{F}$ & 0.2025608123 & 0.7958323825 & 0.5960025156 \\
\hline $\mathrm{F}$ & 0.7025608123 & 0.7041676175 & 0.0960025156 \\
\hline $\mathrm{F}$ & 0.2974391877 & 0.2958323825 & 0.9039974844 \\
\hline $\mathrm{F}$ & 0.8190972789 & 0.1781168103 & 0.3020679524 \\
\hline $\mathrm{F}$ & 0.1809027211 & 0.8218832197 & 0.6979320776 \\
\hline $\mathrm{F}$ & 0.6809027211 & 0.6781167803 & 0.1979320476 \\
\hline $\mathrm{F}$ & 0.3190972789 & 0.3218831897 & 0.8020679224 \\
\hline $\mathrm{F}$ & 0.8999878813 & 0.1128355988 & 0.3920353150 \\
\hline $\mathrm{F}$ & 0.1000121187 & 0.8871643862 & 0.6079646850 \\
\hline $\mathrm{F}$ & 0.6000121187 & 0.6128356138 & 0.1079646850 \\
\hline $\mathrm{F}$ & 0.3999878813 & 0.3871643862 & 0.8920353150 \\
\hline $\mathrm{F}$ & 0.3222861475 & 0.3381521141 & 0.6284928116 \\
\hline $\mathrm{F}$ & 0.6777138525 & 0.6618478859 & 0.3715071884 \\
\hline $\mathrm{F}$ & 0.1777138525 & 0.8381521141 & 0.8715071884 \\
\hline $\mathrm{F}$ & 0.8222861475 & 0.1618478859 & 0.1284928116 \\
\hline 0 & 0.9253706538 & 0.5976478063 & 0.7251162234 \\
\hline $\mathrm{O}$ & 0.0746293462 & 0.4023521937 & 0.2748837766 \\
\hline 0 & 0.5746293462 & 0.0976478063 & 0.7748837766 \\
\hline 0 & 0.4253706538 & 0.9023521937 & 0.2251162234 \\
\hline 0 & 0.9258206814 & 0.3926470571 & 0.7266926649 \\
\hline 0 & 0.0741793186 & 0.6073529429 & 0.2733073351 \\
\hline 0 & 0.5741793186 & 0.8926470571 & 0.7733073351 \\
\hline $\mathrm{O}$ & 0.4258206814 & 0.1073529429 & 0.2266926649 \\
\hline 0 & 0.6782608749 & 0.5990136243 & 0.7359504379 \\
\hline 0 & 0.3217391251 & 0.4009863757 & 0.2640495621 \\
\hline 0 & 0.8217391251 & 0.0990136243 & 0.7640495621 \\
\hline 0 & 0.1782608749 & 0.9009863757 & 0.2359504379 \\
\hline $\mathrm{O}$ & 0.6866223034 & 0.3958980898 & 0.7594787140 \\
\hline 0 & 0.3133776966 & 0.6041019102 & 0.2405212860 \\
\hline 0 & 0.8133776966 & 0.8958980898 & 0.7405212860 \\
\hline 0 & 0.1866223034 & 0.1041019102 & 0.2594787140 \\
\hline 0 & 0.7248893313 & 0.2474851626 & 0.5738196206 \\
\hline 0 & 0.2751106687 & 0.7525148674 & 0.4261803794 \\
\hline 0 & 0.7751106687 & 0.7474851326 & 0.9261803794 \\
\hline 0 & 0.2248893313 & 0.2525148374 & 0.0738196206 \\
\hline $\mathrm{N}$ & 1.0043764167 & 0.5634883044 & 0.6248532181 \\
\hline $\mathrm{N}$ & -0.0043764167 & 0.4365116956 & 0.3751467819 \\
\hline $\mathrm{N}$ & 0.4956235833 & 0.0634883044 & 0.8751467819 \\
\hline $\mathrm{N}$ & 0.5043764167 & 0.9365116956 & 0.1248532181 \\
\hline $\mathrm{N}$ & 0.5084452201 & 0.3864023071 & 0.7521468441 \\
\hline $\mathrm{N}$ & 0.4915547799 & 0.6135976929 & 0.2478531559 \\
\hline $\mathrm{N}$ & 0.9915547799 & 0.8864023071 & 0.7478531559 \\
\hline $\mathrm{N}$ & 0.0084452201 & 0.1135976929 & 0.2521468441 \\
\hline $\mathrm{N}$ & 0.1309388413 & 0.5300230145 & 0.7152372087 \\
\hline
\end{tabular}




\begin{tabular}{|c|c|c|c|}
\hline $\mathrm{N}$ & 0.8690611587 & 0.4699769855 & 0.2847627913 \\
\hline $\mathrm{N}$ & 0.3690611587 & 0.0300230145 & 0.7847627913 \\
\hline $\mathrm{N}$ & 0.6309388413 & 0.9699769855 & 0.2152372087 \\
\hline $\mathrm{N}$ & 0.6019036316 & 0.4981327380 & 0.8447423857 \\
\hline $\mathrm{N}$ & 0.3980963684 & 0.5018672620 & 0.1552576143 \\
\hline $\mathrm{N}$ & 0.8980963684 & 0.9981327380 & 0.6552576143 \\
\hline $\mathrm{N}$ & 0.1019036316 & 0.0018672620 & 0.3447423857 \\
\hline $\mathrm{N}$ & 0.5697503097 & 0.4305023747 & 0.6478506000 \\
\hline $\mathrm{N}$ & 0.4302496903 & 0.5694975953 & 0.3521494000 \\
\hline $\mathrm{N}$ & 0.9302496903 & 0.9305024047 & 0.8521494000 \\
\hline $\mathrm{N}$ & 0.0697503097 & 0.0694976253 & 0.1478506000 \\
\hline $\mathrm{N}$ & 0.5030957417 & 0.5968739798 & 0.7095533136 \\
\hline $\mathrm{N}$ & 0.4969042583 & 0.4031260202 & 0.2904466864 \\
\hline $\mathrm{N}$ & 0.9969042583 & 0.0968739798 & 0.7904466864 \\
\hline $\mathrm{N}$ & 0.0030957417 & 0.9031260202 & 0.2095533136 \\
\hline $\mathrm{N}$ & 0.0596767323 & 0.5256199643 & 0.8132656202 \\
\hline $\mathrm{N}$ & 0.9403232677 & 0.4743800357 & 0.1867343798 \\
\hline $\mathrm{N}$ & 0.4403232677 & 0.0256199643 & 0.6867343798 \\
\hline $\mathrm{N}$ & 0.5596767323 & 0.9743800357 & 0.3132656202 \\
\hline $\mathrm{N}$ & 0.0573535776 & 0.3396412523 & 0.6907927590 \\
\hline $\mathrm{N}$ & 0.9426464224 & 0.6603587477 & 0.3092072410 \\
\hline $\mathrm{N}$ & 0.4426464224 & 0.8396412523 & 0.8092072410 \\
\hline $\mathrm{N}$ & 0.5573535776 & 0.1603587477 & 0.1907927590 \\
\hline $\mathrm{N}$ & 0.9138048646 & 0.3307395606 & 0.9198653521 \\
\hline $\mathrm{N}$ & 0.0861951354 & 0.6692604094 & 0.0801346479 \\
\hline $\mathrm{N}$ & 0.5861951354 & 0.8307395906 & 0.5801346479 \\
\hline $\mathrm{N}$ & 0.4138048646 & 0.1692604394 & 0.4198653521 \\
\hline C & 0.7439963930 & 0.4382209922 & 0.7483217738 \\
\hline C & 0.2560036070 & 0.5617790078 & 0.2516782263 \\
\hline C & 0.7560036070 & 0.9382209922 & 0.7516782263 \\
\hline C & 0.2439963930 & 0.0617790078 & 0.2483217738 \\
\hline C & 0.8659637703 & 0.5548263801 & 0.7307097958 \\
\hline C & 0.1340362297 & 0.4451736199 & 0.2692902042 \\
\hline C & 0.6340362297 & 0.0548263801 & 0.7692902042 \\
\hline C & 0.3659637703 & 0.9451736199 & 0.2307097958 \\
\hline C & 0.1294779605 & 0.3404142883 & 0.6938558695 \\
\hline C & 0.8705220395 & 0.6595857417 & 0.3061441305 \\
\hline C & 0.3705220395 & 0.8404142583 & 0.8061441305 \\
\hline C & 0.6294779605 & 0.1595857117 & 0.1938558695 \\
\hline C & 0.1249424231 & 0.5735252939 & 0.8262103033 \\
\hline C & 0.8750575769 & 0.4264747061 & 0.1737896967 \\
\hline C & 0.3750575769 & 0.0735252939 & 0.6737896967 \\
\hline C & 0.6249424231 & 0.9264747061 & 0.3262103033 \\
\hline C & 0.0183104503 & 0.2565368003 & 0.6637404160 \\
\hline C & 0.9816895497 & 0.7434631997 & 0.3362595840 \\
\hline C & 0.4816895497 & 0.7565368003 & 0.8362595840 \\
\hline C & 0.5183104503 & 0.2434631997 & 0.1637404160 \\
\hline C & 0.7406449408 & 0.5555824128 & 0.7381669074 \\
\hline C & 0.2593550592 & 0.4444175872 & 0.2618330926 \\
\hline C & 0.7593550592 & 0.0555824128 & 0.7618330926 \\
\hline C & 0.2406449408 & 0.9444175872 & 0.2381669074 \\
\hline C & 0.8069292793 & 0.3825466572 & 0.7448317865 \\
\hline C & 0.1930707207 & 0.6174533128 & 0.2551682135 \\
\hline C & 0.6930707207 & 0.8825466872 & 0.7551682135 \\
\hline C & 0.3069292793 & 0.1174533428 & 0.2448317865 \\
\hline C & 0.8024701835 & 0.6104620267 & 0.7325499958 \\
\hline C & 0.1975298165 & 0.3895379733 & 0.2674500042 \\
\hline C & 0.6975298165 & 0.1104620267 & 0.7674500042 \\
\hline
\end{tabular}




\begin{tabular}{|c|c|c|c|}
\hline C & 0.3024701835 & 0.8895379733 & 0.2325499958 \\
\hline C & 0.1706806080 & 0.4303455760 & 0.7298727572 \\
\hline C & 0.8293193920 & 0.5696544540 & 0.2701272428 \\
\hline C & 0.3293193920 & 0.9303455460 & 0.7701272428 \\
\hline C & 0.6706806080 & 0.0696544240 & 0.2298727572 \\
\hline C & 0.4393804812 & 0.5482846352 & 0.7087212750 \\
\hline C & 0.5606195488 & 0.4517153648 & 0.2912787250 \\
\hline C & 0.0606195188 & 0.0482846352 & 0.7912787250 \\
\hline C & 0.9393804512 & 0.9517153648 & 0.2087212750 \\
\hline C & 0.0652285065 & 0.5922668298 & 0.6065332295 \\
\hline C & 0.9347714935 & 0.4077331702 & 0.3934667705 \\
\hline C & 0.4347714935 & 0.0922668298 & 0.8934667705 \\
\hline C & 0.5652285065 & 0.9077331702 & 0.1065332295 \\
\hline C & 0.4421152818 & 0.4505245181 & 0.7485775886 \\
\hline C & 0.5578847482 & 0.5494754529 & 0.2514224114 \\
\hline C & 0.0578847182 & 0.9505245471 & 0.7514224114 \\
\hline C & 0.9421152518 & 0.0494754819 & 0.2485775886 \\
\hline C & 0.5025767996 & 0.6874514232 & 0.6770428884 \\
\hline C & 0.4974232004 & 0.3125485768 & 0.3229571116 \\
\hline C & 0.9974232004 & 0.1874514232 & 0.8229571116 \\
\hline C & 0.0025767996 & 0.8125485768 & 0.1770428884 \\
\hline C & 0.8667959269 & 0.4368116006 & 0.7340466997 \\
\hline C & 0.1332040731 & 0.5631884294 & 0.2659533003 \\
\hline C & 0.6332040731 & 0.9368115706 & 0.7659533003 \\
\hline C & 0.3667959269 & 0.0631883994 & 0.2340466997 \\
\hline C & 0.0492501687 & 0.1726290538 & 0.6378748636 \\
\hline C & 0.9507498313 & 0.8273709312 & 0.3621251364 \\
\hline C & 0.4507498313 & 0.6726290688 & 0.8621251364 \\
\hline C & 0.5492501687 & 0.3273709312 & 0.1378748636 \\
\hline C & 0.1539338247 & 0.6051553661 & 0.7685081931 \\
\hline C & 0.8460661753 & 0.3948446339 & 0.2314918069 \\
\hline C & 0.3460661753 & 0.1051553661 & 0.7314918069 \\
\hline C & 0.6539338247 & 0.8948446339 & 0.2685081931 \\
\hline C & 0.1371792858 & 0.5721140392 & 0.6519963872 \\
\hline C & 0.8628207142 & 0.4278859608 & 0.3480036128 \\
\hline C & 0.3628207142 & 0.0721140392 & 0.8480036128 \\
\hline C & 0.6371792858 & 0.9278859608 & 0.1519963872 \\
\hline C & 0.5663201642 & 0.4188255183 & 0.8663154523 \\
\hline C & 0.4336798358 & 0.5811744818 & 0.1336845477 \\
\hline C & 0.9336798358 & 0.9188255182 & 0.6336845477 \\
\hline C & 0.0663201642 & 0.0811744818 & 0.3663154523 \\
\hline C & 0.5316254932 & 0.3382980381 & 0.8168908840 \\
\hline C & 0.4683745068 & 0.6617019619 & 0.1831091160 \\
\hline C & 0.9683745068 & 0.8382980381 & 0.6831091160 \\
\hline C & 0.0316254932 & 0.1617019619 & 0.3168908840 \\
\hline C & 0.0626233168 & 0.6380894054 & 0.5463427368 \\
\hline C & 0.9373766832 & 0.3619105946 & 0.4536572632 \\
\hline C & 0.4373766832 & 0.1380894054 & 0.9536572632 \\
\hline C & 0.5626233168 & 0.8619105946 & 0.0463427368 \\
\hline C & 0.4939146471 & 0.3086601465 & 0.5785593568 \\
\hline C & 0.5060853529 & 0.6913398535 & 0.4214406432 \\
\hline C & 1.0060853529 & 0.8086601465 & 0.9214406432 \\
\hline C & -0.0060853529 & 0.1913398535 & 0.0785593568 \\
\hline C & 0.4387747402 & 0.7334096860 & 0.6432291500 \\
\hline C & 0.5612252298 & 0.2665903140 & 0.3567708500 \\
\hline C & 0.0612252598 & 0.2334096860 & 0.8567708500 \\
\hline C & 0.9387747702 & 0.7665903140 & 0.1432291500 \\
\hline C & 0.5935557498 & 0.4668112292 & 0.5968203953 \\
\hline
\end{tabular}




\begin{tabular}{|c|c|c|c|}
\hline C & 0.4064442502 & 0.5331887708 & 0.4031796047 \\
\hline C & 0.9064442502 & 0.9668112292 & 0.9031796047 \\
\hline C & 0.0935557498 & 0.0331887708 & 0.0968203953 \\
\hline C & 0.5633497011 & 0.4112062662 & 0.9311280971 \\
\hline C & 0.4366502989 & 0.5887937338 & 0.0688719029 \\
\hline C & 0.9366502989 & 0.9112062662 & 0.5688719029 \\
\hline C & 0.0633497011 & 0.0887937338 & 0.4311280971 \\
\hline C & 0.5698350499 & 0.4265944188 & 0.5350970335 \\
\hline C & 0.4301649501 & 0.5734055812 & 0.4649029665 \\
\hline C & 0.9301649501 & 0.9265944188 & 0.9649029665 \\
\hline C & 0.0698350499 & 0.0734055812 & 0.0350970335 \\
\hline C & 0.0629692275 & 0.5171353402 & 0.9264104409 \\
\hline C & 0.9370307725 & 0.4828646598 & 0.0735895591 \\
\hline C & 0.4370307725 & 0.0171353402 & 0.5735895591 \\
\hline C & 0.5629692275 & 0.9828646598 & 0.4264104409 \\
\hline C & 0.1310816517 & 0.5644029188 & 0.9399518693 \\
\hline C & 0.8689183483 & 0.4355970812 & 0.0600481307 \\
\hline C & 0.3689183483 & 0.0644029188 & 0.5600481307 \\
\hline C & 0.6310816517 & 0.9355970812 & 0.4399518693 \\
\hline C & 0.9959311529 & 0.6528281497 & 0.5042588108 \\
\hline C & 0.0040688471 & 0.3471718503 & 0.4957411892 \\
\hline C & 0.5040688471 & 0.1528281497 & 0.9957411892 \\
\hline C & 0.4959311529 & 0.8471718503 & 0.0042588108 \\
\hline C & 0.5188762148 & 0.3459460589 & 0.5259532819 \\
\hline C & 0.4811237852 & 0.6540539411 & 0.4740467181 \\
\hline C & 0.9811237852 & 0.8459460589 & 0.9740467181 \\
\hline C & 0.0188762148 & 0.1540539411 & 0.0259532819 \\
\hline C & 0.5984797548 & 0.4863016640 & 0.9744981914 \\
\hline C & 0.4015202452 & 0.5136983660 & 0.0255018086 \\
\hline C & 0.9015202452 & 0.9863016340 & 0.5255018086 \\
\hline C & 0.0984797548 & 0.0136983360 & 0.4744981914 \\
\hline C & 0.6361749053 & 0.5670143024 & 0.9521406353 \\
\hline C & 0.3638250947 & 0.4329856976 & 0.0478593647 \\
\hline C & 0.8638250947 & 0.0670143024 & 0.5478593647 \\
\hline C & 0.1361749053 & 0.9329856976 & 0.4521406353 \\
\hline C & 0.3729067952 & 0.6843551219 & 0.6428126873 \\
\hline C & 0.6270932048 & 0.3156448781 & 0.3571873127 \\
\hline C & 0.1270932048 & 0.1843551219 & 0.8571873127 \\
\hline C & 0.8729067952 & 0.8156448781 & 0.1428126873 \\
\hline C & 0.4999100191 & 0.3092903119 & 0.6988530410 \\
\hline C & 0.5000899809 & 0.6907096581 & 0.3011469590 \\
\hline C & 1.0000899809 & 0.8092903419 & 0.8011469590 \\
\hline C & -0.0000899809 & 0.1907096881 & 0.1988530410 \\
\hline C & 0.1622371787 & 0.5937028754 & 0.8892523319 \\
\hline C & 0.8377628213 & 0.4062971246 & 0.1107476681 \\
\hline C & 0.3377628213 & 0.0937028754 & 0.6107476681 \\
\hline C & 0.6622371787 & 0.9062971246 & 0.3892523319 \\
\hline C & 0.0289053019 & 0.4994839541 & 0.8624516014 \\
\hline C & 0.9710946981 & 0.5005160459 & 0.1375483986 \\
\hline C & 0.4710946981 & -0.0005160459 & 0.6375483986 \\
\hline C & 0.5289053019 & 1.0005160459 & 0.3624516014 \\
\hline C & 0.1639161988 & 0.2600929975 & 0.6680669963 \\
\hline C & 0.8360838012 & 0.7399070025 & 0.3319330037 \\
\hline C & 0.3360838012 & 0.7600929975 & 0.8319330037 \\
\hline C & 0.6639161988 & 0.2399070025 & 0.1680669963 \\
\hline C & 0.3735141915 & 0.5898243613 & 0.6756534486 \\
\hline C & 0.6264858385 & 0.4101756387 & 0.3243465514 \\
\hline C & 0.1264858085 & 0.0898243613 & 0.8243465514 \\
\hline
\end{tabular}




\begin{tabular}{|c|c|c|c|}
\hline C & 0.8735141615 & 0.9101756387 & 0.1756534486 \\
\hline C & 0.5209049351 & 0.3521142405 & 0.6393456449 \\
\hline C & 0.4790950649 & 0.6478857595 & 0.3606543551 \\
\hline C & 0.9790950649 & 0.8521142405 & 0.8606543551 \\
\hline C & 0.0209049351 & 0.1478857595 & 0.1393456449 \\
\hline C & 0.1232897911 & 0.1746652362 & 0.6396154685 \\
\hline C & 0.8767102089 & 0.8253347788 & 0.3603845315 \\
\hline C & 0.3767102089 & 0.6746652212 & 0.8603845315 \\
\hline C & 0.6232897911 & 0.3253347788 & 0.1396154685 \\
\hline C & 0.9395672086 & 0.5806793390 & 0.5842761744 \\
\hline C & 0.0604327914 & 0.4193206610 & 0.4157238256 \\
\hline C & 0.5604327914 & 0.0806793390 & 0.9157238256 \\
\hline C & 0.4395672086 & 0.9193206610 & 0.0842761744 \\
\hline C & 0.9326876287 & 0.6239775796 & 0.5234337869 \\
\hline C & 0.0673123713 & 0.3760224204 & 0.4765662131 \\
\hline C & 0.5673123713 & 0.1239775796 & 0.9765662131 \\
\hline C & 0.4326876287 & 0.8760224204 & 0.0234337869 \\
\hline C & 0.6366474203 & 0.5702327864 & 0.8868819063 \\
\hline C & 0.3633525797 & 0.4297672136 & 0.1131180937 \\
\hline C & 0.8633525797 & 0.0702327864 & 0.6131180937 \\
\hline C & 0.1366474203 & 0.9297672136 & 0.3868819063 \\
\hline C & 0.7793263363 & 0.2687271942 & 0.9008428677 \\
\hline C & 0.2206736637 & 0.7312728358 & 0.0991571323 \\
\hline C & 0.7206736637 & 0.7687271642 & 0.5991571323 \\
\hline C & 0.2793263363 & 0.2312728058 & 0.4008428677 \\
\hline C & 0.8538706500 & 0.3025280648 & 0.9109937028 \\
\hline C & 0.1461293500 & 0.6974719352 & 0.0890062972 \\
\hline C & 0.6461293500 & 0.8025280648 & 0.5890062972 \\
\hline C & 0.3538706500 & 0.1974719352 & 0.4109937028 \\
\hline C & 0.6528753757 & 0.1032984293 & 0.5938879273 \\
\hline C & 0.3471246243 & 0.8967015847 & 0.4061120727 \\
\hline C & 0.8471246243 & 0.6032984153 & 0.9061120727 \\
\hline C & 0.1528753757 & 0.3967015847 & 0.0938879273 \\
\hline C & 0.7959198317 & 0.4000468074 & 0.5647955710 \\
\hline C & 0.2040801683 & 0.5999531926 & 0.4352044290 \\
\hline C & 0.7040801683 & 0.9000468074 & 0.9352044290 \\
\hline C & 0.2959198317 & 0.0999531926 & 0.0647955710 \\
\hline C & 0.7436972539 & 0.3197406227 & 0.5287484401 \\
\hline C & 0.2563027461 & 0.6802593773 & 0.4712515599 \\
\hline C & 0.7563027461 & 0.8197406227 & 0.9712515599 \\
\hline C & 0.2436972539 & 0.1802593773 & 0.0287484401 \\
\hline C & 0.6748296318 & 0.1700310014 & 0.5426805227 \\
\hline C & 0.3251703682 & 0.8299689836 & 0.4573194773 \\
\hline C & 0.8251703682 & 0.6700310164 & 0.9573194773 \\
\hline C & 0.1748296318 & 0.3299689836 & 0.0426805227 \\
\hline $\mathrm{H}$ & 0.9607740444 & 0.2591203652 & 0.6638580717 \\
\hline $\mathrm{H}$ & 0.0392259556 & 0.7408796348 & 0.3361419283 \\
\hline $\mathrm{H}$ & 0.5392259556 & 0.7591203652 & 0.8361419283 \\
\hline $\mathrm{H}$ & 0.4607740444 & 0.2408796348 & 0.1638580717 \\
\hline $\mathrm{H}$ & 0.8080877106 & 0.2973337047 & 0.7483916955 \\
\hline $\mathrm{H}$ & 0.1919122894 & 0.7026662953 & 0.2516083045 \\
\hline $\mathrm{H}$ & 0.6919122894 & 0.7973337047 & 0.7516083045 \\
\hline $\mathrm{H}$ & 0.3080877106 & 0.2026662953 & 0.2483916955 \\
\hline $\mathrm{H}$ & 0.8022808295 & 0.6957809563 & 0.7302426020 \\
\hline $\mathrm{H}$ & 0.1977191705 & 0.3042190437 & 0.2697573980 \\
\hline $\mathrm{H}$ & 0.6977191705 & 0.1957809563 & 0.7697573980 \\
\hline $\mathrm{H}$ & 0.3022808295 & 0.8042190437 & 0.2302426020 \\
\hline $\mathrm{H}$ & 0.1777773471 & 0.4148064334 & 0.7817898843 \\
\hline
\end{tabular}




\begin{tabular}{|c|c|c|c|}
\hline $\mathrm{H}$ & 0.8222226529 & 0.5851935366 & 0.2182101157 \\
\hline $\mathrm{H}$ & 0.3222226529 & 0.9148064634 & 0.7182101157 \\
\hline $\mathrm{H}$ & 0.6777773471 & 0.0851935666 & 0.2817898843 \\
\hline $\mathrm{H}$ & 0.2256368667 & 0.4348209500 & 0.7208127830 \\
\hline $\mathrm{H}$ & 0.7743631333 & 0.5651790500 & 0.2791872170 \\
\hline $\mathrm{H}$ & 0.2743631333 & 0.9348209500 & 0.7791872170 \\
\hline $\mathrm{H}$ & 0.7256368667 & 0.0651790500 & 0.2208127830 \\
\hline $\mathrm{H}$ & 0.4409185196 & 0.4744329859 & 0.7980434564 \\
\hline $\mathrm{H}$ & 0.5590814504 & 0.5255669851 & 0.2019565436 \\
\hline $\mathrm{H}$ & 0.0590814804 & 0.9744330149 & 0.7019565436 \\
\hline $\mathrm{H}$ & 0.9409185496 & 0.0255670141 & 0.2980434564 \\
\hline $\mathrm{H}$ & 0.3931615552 & 0.4032704163 & 0.7311101357 \\
\hline $\mathrm{H}$ & 0.6068384748 & 0.5967295837 & 0.2688898643 \\
\hline $\mathrm{H}$ & 0.1068384448 & 0.9032704163 & 0.7688898643 \\
\hline $\mathrm{H}$ & 0.8931615252 & 0.0967295837 & 0.2311101357 \\
\hline $\mathrm{H}$ & 0.5551155730 & 0.7234203058 & 0.6786692566 \\
\hline $\mathrm{H}$ & 0.4448844270 & 0.2765796942 & 0.3213307434 \\
\hline $\mathrm{H}$ & 0.9448844270 & 0.2234203058 & 0.8213307434 \\
\hline $\mathrm{H}$ & 0.0551155730 & 0.7765796942 & 0.1786692566 \\
\hline $\mathrm{H}$ & 0.0154517482 & 0.1073381741 & 0.6161652238 \\
\hline $\mathrm{H}$ & 0.9845482518 & 0.8926618259 & 0.3838347762 \\
\hline $\mathrm{H}$ & 0.4845482518 & 0.6073381741 & 0.8838347762 \\
\hline $\mathrm{H}$ & 0.5154517482 & 0.3926618259 & 0.1161652238 \\
\hline $\mathrm{H}$ & 0.1327507341 & 0.6828828738 & 0.7516121629 \\
\hline $\mathrm{H}$ & 0.8672492659 & 0.3171171262 & 0.2483878371 \\
\hline $\mathrm{H}$ & 0.3672492659 & 0.1828828738 & 0.7483878371 \\
\hline $\mathrm{H}$ & 0.6327507341 & 0.8171171262 & 0.2516121629 \\
\hline $\mathrm{H}$ & 0.2133973487 & 0.6125824754 & 0.7823161528 \\
\hline $\mathrm{H}$ & 0.7866026513 & 0.3874175246 & 0.2176838472 \\
\hline $\mathrm{H}$ & 0.2866026513 & 0.1125824754 & 0.7176838472 \\
\hline $\mathrm{H}$ & 0.7133973487 & 0.8874175246 & 0.2823161528 \\
\hline $\mathrm{H}$ & 0.1689250686 & 0.6450842626 & 0.6578878636 \\
\hline $\mathrm{H}$ & 0.8310749314 & 0.3549157374 & 0.3421121364 \\
\hline $\mathrm{H}$ & 0.3310749314 & 0.1450842626 & 0.8421121364 \\
\hline $\mathrm{H}$ & 0.6689250686 & 0.8549157374 & 0.1578878636 \\
\hline $\mathrm{H}$ & 0.1674977287 & 0.5166760201 & 0.6283847095 \\
\hline $\mathrm{H}$ & 0.8325022713 & 0.4833239799 & 0.3716152905 \\
\hline $\mathrm{H}$ & 0.3325022713 & 1.0166760201 & 0.8716152905 \\
\hline $\mathrm{H}$ & 0.6674977287 & -0.0166760201 & 0.1283847095 \\
\hline $\mathrm{H}$ & 0.5713544661 & 0.2766763429 & 0.8137720271 \\
\hline $\mathrm{H}$ & 0.4286455339 & 0.7233236571 & 0.1862279729 \\
\hline $\mathrm{H}$ & 0.9286455339 & 0.7766763429 & 0.6862279729 \\
\hline $\mathrm{H}$ & 0.0713544661 & 0.2233236571 & 0.3137720271 \\
\hline $\mathrm{H}$ & 0.4860998021 & 0.3005847043 & 0.8326110543 \\
\hline $\mathrm{H}$ & 0.5139001679 & 0.6994153257 & 0.1673889457 \\
\hline $\mathrm{H}$ & 0.0139001979 & 0.8005846743 & 0.6673889457 \\
\hline $\mathrm{H}$ & 0.9860998321 & 0.1994152957 & 0.3326110542 \\
\hline $\mathrm{H}$ & 0.1129374068 & 0.6620913964 & 0.5337952140 \\
\hline $\mathrm{H}$ & 0.8870625932 & 0.3379086036 & 0.4662047860 \\
\hline $\mathrm{H}$ & 0.3870625932 & 0.1620913964 & 0.9662047860 \\
\hline $\mathrm{H}$ & 0.6129374069 & 0.8379086036 & 0.0337952140 \\
\hline $\mathrm{H}$ & 0.4530158208 & 0.2473627211 & 0.5725728536 \\
\hline $\mathrm{H}$ & 0.5469841492 & 0.7526373089 & 0.4274271464 \\
\hline $\mathrm{H}$ & 0.0469841792 & 0.7473626911 & 0.9274271464 \\
\hline $\mathrm{H}$ & 0.9530158508 & 0.2526372789 & 0.0725728536 \\
\hline $\mathrm{H}$ & 0.4413887846 & 0.8062006512 & 0.6173460954 \\
\hline $\mathrm{H}$ & 0.5586112455 & 0.1937993488 & 0.3826539046 \\
\hline $\mathrm{H}$ & 0.0586112155 & 0.3062006512 & 0.8826539046 \\
\hline
\end{tabular}




\begin{tabular}{|c|c|c|c|}
\hline $\mathrm{H}$ & 0.9413887546 & 0.6937993488 & 0.1173460954 \\
\hline $\mathrm{H}$ & 0.6325792778 & 0.5308859425 & 0.6064587476 \\
\hline $\mathrm{H}$ & 0.3674207222 & 0.4691140575 & 0.3935412524 \\
\hline $\mathrm{H}$ & 0.8674207222 & 0.0308859425 & 0.8935412524 \\
\hline $\mathrm{H}$ & 0.1325792778 & 0.9691140575 & 0.1064587476 \\
\hline $\mathrm{H}$ & 0.5322646568 & 0.3483298637 & 0.9464984101 \\
\hline $\mathrm{H}$ & 0.4677353432 & 0.6516701063 & 0.0535015899 \\
\hline $\mathrm{H}$ & 0.9677353432 & 0.8483298937 & 0.5535015899 \\
\hline $\mathrm{H}$ & 0.0322646568 & 0.1516701363 & 0.4464984101 \\
\hline $\mathrm{H}$ & 0.5903553179 & 0.4599506117 & 0.4955122965 \\
\hline $\mathrm{H}$ & 0.4096446821 & 0.5400493883 & 0.5044876735 \\
\hline $\mathrm{H}$ & 0.9096446821 & 0.9599506117 & 0.0044877035 \\
\hline $\mathrm{H}$ & 0.0903553179 & 0.0400493883 & 0.9955123265 \\
\hline $\mathrm{H}$ & 0.0365132645 & 0.4928151372 & 0.9644862070 \\
\hline $\mathrm{H}$ & 0.9634867355 & 0.5071848628 & 0.0355137930 \\
\hline $\mathrm{H}$ & 0.4634867355 & -0.0071848628 & 0.5355137930 \\
\hline $\mathrm{H}$ & 0.5365132645 & 1.0071848628 & 0.4644862070 \\
\hline $\mathrm{H}$ & 0.1595420548 & 0.5785412586 & 0.9895748788 \\
\hline $\mathrm{H}$ & 0.8404579452 & 0.4214587414 & 0.0104251212 \\
\hline $\mathrm{H}$ & 0.3404579452 & 0.0785412586 & 0.5104251212 \\
\hline $\mathrm{H}$ & 0.6595420548 & 0.9214587414 & 0.4895748788 \\
\hline $\mathrm{H}$ & 0.9929391802 & 0.6876249990 & 0.4569795143 \\
\hline $\mathrm{H}$ & 0.0070608198 & 0.3123750010 & 0.5430204857 \\
\hline $\mathrm{H}$ & 0.5070608198 & 0.1876249990 & 0.0430204857 \\
\hline $\mathrm{H}$ & 0.4929391802 & 0.8123750010 & 0.9569795143 \\
\hline $\mathrm{H}$ & 0.4977217673 & 0.3127724651 & 0.4782589348 \\
\hline $\mathrm{H}$ & 0.5022782327 & 0.6872275059 & 0.5217410652 \\
\hline $\mathrm{H}$ & 1.0022782327 & 0.8127724941 & 0.0217410652 \\
\hline $\mathrm{H}$ & -0.0022782327 & 0.1872275349 & 0.9782589348 \\
\hline $\mathrm{H}$ & 0.5958257257 & 0.4842248552 & 0.0251115231 \\
\hline $\mathrm{H}$ & 0.4041742743 & 0.5157751448 & 0.9748884769 \\
\hline $\mathrm{H}$ & 0.9041742743 & 0.9842248552 & 0.4748884769 \\
\hline $\mathrm{H}$ & 0.0958257257 & 0.0157751448 & 0.5251115231 \\
\hline $\mathrm{H}$ & 0.6641090041 & 0.6268196520 & 0.9849648928 \\
\hline $\mathrm{H}$ & 0.3358909959 & 0.3731803480 & 0.0150351072 \\
\hline $\mathrm{H}$ & 0.8358909959 & 0.1268196520 & 0.5150351072 \\
\hline $\mathrm{H}$ & 0.1641090041 & 0.8731803480 & 0.4849648928 \\
\hline $\mathrm{H}$ & 0.3217671379 & 0.7185942960 & 0.6172574045 \\
\hline $\mathrm{H}$ & 0.6782328921 & 0.2814057040 & 0.3827425955 \\
\hline $\mathrm{H}$ & 0.1782328621 & 0.2185942960 & 0.8827425955 \\
\hline $\mathrm{H}$ & 0.8217671079 & 0.7814057040 & 0.1172574045 \\
\hline $\mathrm{H}$ & 0.5352474307 & 0.2420202686 & 0.7163776120 \\
\hline $\mathrm{H}$ & 0.4647525693 & 0.7579797163 & 0.2836223880 \\
\hline $\mathrm{H}$ & 0.9647525693 & 0.7420202837 & 0.7836223880 \\
\hline $\mathrm{H}$ & 0.0352474307 & 0.2579797164 & 0.2163776120 \\
\hline $\mathrm{H}$ & 0.4438968609 & 0.2798252458 & 0.6855254819 \\
\hline $\mathrm{H}$ & 0.5561031691 & 0.7201747542 & 0.3144745181 \\
\hline $\mathrm{H}$ & 0.0561031391 & 0.7798252458 & 0.8144745181 \\
\hline $\mathrm{H}$ & 0.9438968309 & 0.2201747542 & 0.1855254819 \\
\hline $\mathrm{H}$ & 0.2147914707 & 0.6319951591 & 0.8985456451 \\
\hline $\mathrm{H}$ & 0.7852085293 & 0.3680048409 & 0.1014543549 \\
\hline $\mathrm{H}$ & 0.2852085293 & 0.1319951591 & 0.6014543549 \\
\hline $\mathrm{H}$ & 0.7147914707 & 0.8680048409 & 0.3985456451 \\
\hline $\mathrm{H}$ & 0.9760391246 & 0.4616664442 & 0.8496495269 \\
\hline $\mathrm{H}$ & 0.0239608754 & 0.5383335858 & 0.1503504731 \\
\hline $\mathrm{H}$ & 0.5239608754 & 0.9616664142 & 0.6503504731 \\
\hline $\mathrm{H}$ & 0.4760391246 & 0.0383335558 & 0.3496495269 \\
\hline $\mathrm{H}$ & 0.2217541187 & 0.2662824205 & 0.6700499965 \\
\hline
\end{tabular}




\begin{tabular}{|c|c|c|c|}
\hline $\mathrm{H}$ & 0.7782458813 & 0.7337176095 & 0.3299500035 \\
\hline $\mathrm{H}$ & 0.2782458813 & 0.7662823905 & 0.8299500035 \\
\hline $\mathrm{H}$ & 0.7217541187 & 0.2337175795 & 0.1700499965 \\
\hline $\mathrm{H}$ & 0.3235512578 & 0.5469596790 & 0.6746600247 \\
\hline $\mathrm{H}$ & 0.6764487422 & 0.4530403210 & 0.3253399753 \\
\hline $\mathrm{H}$ & 0.1764487422 & 0.0469596790 & 0.8253399753 \\
\hline $\mathrm{H}$ & 0.8235512578 & 0.9530403210 & 0.1746600247 \\
\hline $\mathrm{H}$ & 0.1492917529 & 0.1100492114 & 0.6201090594 \\
\hline $\mathrm{H}$ & 0.8507082471 & 0.8899507666 & 0.3798909406 \\
\hline $\mathrm{H}$ & 0.3507082471 & 0.6100492334 & 0.8798909406 \\
\hline $\mathrm{H}$ & 0.6492917529 & 0.3899507966 & 0.1201090594 \\
\hline $\mathrm{H}$ & 0.8923007803 & 0.5585112521 & 0.6024410787 \\
\hline $\mathrm{H}$ & 0.1076992197 & 0.4414887479 & 0.3975589213 \\
\hline $\mathrm{H}$ & 0.6076992197 & 0.0585112521 & 0.8975589213 \\
\hline $\mathrm{H}$ & 0.3923007803 & 0.9414887479 & 0.1024410787 \\
\hline $\mathrm{H}$ & 0.8791098055 & 0.6357845409 & 0.4924757714 \\
\hline $\mathrm{H}$ & 0.1208901945 & 0.3642154591 & 0.5075242286 \\
\hline $\mathrm{H}$ & 0.6208901945 & 0.1357845409 & 1.0075242286 \\
\hline $\mathrm{H}$ & 0.3791098055 & 0.8642154591 & -0.0075242286 \\
\hline $\mathrm{H}$ & 0.6654385669 & 0.6318337331 & 0.8671873587 \\
\hline $\mathrm{H}$ & 0.3345614331 & 0.3681662669 & 0.1328126413 \\
\hline $\mathrm{H}$ & 0.8345614331 & 0.1318337331 & 0.6328126413 \\
\hline $\mathrm{H}$ & 0.1654385669 & 0.8681662669 & 0.3671873587 \\
\hline $\mathrm{H}$ & 0.7628914574 & 0.2634563379 & 0.9472122115 \\
\hline $\mathrm{H}$ & 0.2371085426 & 0.7365436621 & 0.0527877885 \\
\hline $\mathrm{H}$ & 0.7371085426 & 0.7634563379 & 0.5527877885 \\
\hline $\mathrm{H}$ & 0.2628914574 & 0.2365436621 & 0.4472122115 \\
\hline $\mathrm{H}$ & 0.7733037510 & 0.1907979407 & 0.8784163439 \\
\hline $\mathrm{H}$ & 0.2266962490 & 0.8092020293 & 0.1215836561 \\
\hline $\mathrm{H}$ & 0.7266962490 & 0.6907979707 & 0.6215836561 \\
\hline $\mathrm{H}$ & 0.2733037510 & 0.3092020593 & 0.3784163439 \\
\hline $\mathrm{H}$ & 0.7439869733 & 0.3232683880 & 0.8681457615 \\
\hline $\mathrm{H}$ & 0.2560130267 & 0.6767316120 & 0.1318542385 \\
\hline $\mathrm{H}$ & 0.7560130267 & 0.8232683880 & 0.6318542385 \\
\hline $\mathrm{H}$ & 0.2439869733 & 0.1767316120 & 0.3681457615 \\
\hline $\mathrm{H}$ & 0.6284875676 & 0.1519110548 & 0.6259967087 \\
\hline $\mathrm{H}$ & 0.3715124324 & 0.8480889592 & 0.3740032913 \\
\hline $\mathrm{H}$ & 0.8715124324 & 0.6519110408 & 0.8740032913 \\
\hline $\mathrm{H}$ & 0.1284875676 & 0.3480889592 & 0.1259967087 \\
\hline $\mathrm{H}$ & 0.6999830644 & 0.0631740614 & 0.6235778999 \\
\hline $\mathrm{H}$ & 0.3000169356 & 0.9368259536 & 0.3764221001 \\
\hline $\mathrm{H}$ & 0.8000169356 & 0.5631740464 & 0.8764221001 \\
\hline $\mathrm{H}$ & 0.1999830644 & 0.4368259536 & 0.1235778999 \\
\hline $\mathrm{H}$ & 0.6134137665 & 0.0433952950 & 0.5720884881 \\
\hline $\mathrm{H}$ & 0.3865862335 & 0.9566047080 & 0.4279115119 \\
\hline $\mathrm{H}$ & 0.8865862335 & 0.5433952920 & 0.9279115119 \\
\hline $\mathrm{H}$ & 0.1134137665 & 0.4566047080 & 0.0720884881 \\
\hline $\mathrm{H}$ & 0.7717050098 & 0.4424337858 & 0.5997350720 \\
\hline $\mathrm{H}$ & 0.2282949902 & 0.5575661842 & 0.4002649280 \\
\hline $\mathrm{H}$ & 0.7282949902 & 0.9424338158 & 0.9002649280 \\
\hline $\mathrm{H}$ & 0.2717050098 & 0.0575662142 & 0.0997350720 \\
\hline $\mathrm{H}$ & 0.8086178510 & 0.4564944462 & 0.5300417675 \\
\hline $\mathrm{H}$ & 0.1913821490 & 0.5435055538 & 0.4699582325 \\
\hline $\mathrm{H}$ & 0.6913821490 & 0.9564944462 & 0.9699582325 \\
\hline $\mathrm{H}$ & 0.3086178510 & 0.0435055538 & 0.0300417675 \\
\hline $\mathrm{H}$ & 0.8462681878 & 0.3631795146 & 0.5913196619 \\
\hline $\mathrm{H}$ & 0.1537318122 & 0.6368204554 & 0.4086803381 \\
\hline $\mathrm{H}$ & 0.6537318122 & 0.8631795446 & 0.9086803381 \\
\hline
\end{tabular}




$\begin{array}{llll}\mathrm{H} & 0.3462681878 & 0.1368204854 & 0.0913196619 \\ \mathrm{H} & 0.7677238443 & 0.2772738467 & 0.4933333981 \\ \mathrm{H} & 0.2322761557 & 0.7227261823 & 0.5066666019 \\ \mathrm{H} & 0.7322761557 & 0.7772738177 & 0.0066666019 \\ \mathrm{H} & 0.2677238443 & 0.2227261533 & 0.9933333981 \\ \mathrm{H} & 0.6943247162 & 0.3588646049 & 0.5012752172 \\ \mathrm{H} & 0.3056752838 & 0.6411353951 & 0.4987247828 \\ \mathrm{H} & 0.8056752838 & 0.8588646049 & 0.9987247828 \\ \mathrm{H} & 0.1943247162 & 0.1411353951 & 0.0012752172 \\ \mathrm{H} & 0.6268147921 & 0.2085559941 & 0.5121687397 \\ \mathrm{H} & 0.3731852079 & 0.7914439769 & 0.4878312603 \\ \mathrm{H} & 0.8731852079 & 0.7085560231 & 0.9878312603 \\ \mathrm{H} & 0.1268147921 & 0.2914440059 & 0.0121687397 \\ \mathrm{H} & 0.6999247087 & 0.1219792531 & 0.5100807216 \\ \mathrm{H} & 0.3000752913 & 0.8780207539 & 0.4899192784 \\ \mathrm{H} & 0.8000752913 & 0.6219792461 & 0.9899192784 \\ \mathrm{H} & 0.1999247087 & 0.3780207539 & 0.0100807216 \\ \mathrm{~B} & 0.2824987291 & 0.3686053319 & 0.5660978738 \\ \mathrm{~B} & 0.7175012409 & 0.6313946681 & 0.4339021262 \\ \mathrm{~B} & 0.2175012709 & 0.8686053319 & 0.9339021262 \\ \mathrm{~B} & 0.7824987591 & 0.1313946681 & 0.0660978738 \\ \mathrm{~B} & 0.8256807869 & 0.1330233483 & 0.3643208242 \\ \mathrm{~B} & 0.1743192131 & 0.8669766667 & 0.6356791758 \\ \mathrm{~B} & 0.6743192131 & 0.6330233333 & 0.1356791758 \\ \mathrm{~B} & 0.3256807869 & 0.3669766667 & 0.8643208242\end{array}$

$5 \cdot \mathrm{Et}_{2} \mathrm{O} \cdot \mathrm{MeCN}$ iS

$\begin{array}{ccc}\text { CELL_PARAMETERS (angstrom) } & \\ 18.933526301 & 0.000000000 & 0.009505904 \\ 0.000000000 & 13.028777900 & 0.000000000 \\ -4.561394189 & 0.000000000 & 20.264360064\end{array}$

$\begin{array}{llll}\text { ATOMIC_POSITIONS (crystal) } & & \\ \text { Fe1 } & 0.5040686230 & 0.9633156466 & 0.2041494664 \\ \text { Fe1 } & 0.4959313770 & 0.0366843314 & 0.7958505186 \\ \text { Fe1 } & 0.9959313770 & 0.4633156766 & 0.2958505186 \\ \text { Fe1 } & 0.0040686230 & 0.5366843534 & 0.7041494814 \\ \text { Fe2 } & 0.0945634276 & 0.9632281182 & 0.2257360439 \\ \text { Fe2 } & 0.9054365424 & 0.0367719078 & 0.7742639411 \\ \text { Fe2 } & 0.4054365724 & 0.4632280882 & 0.2742639411 \\ \text { Fe2 } & 0.5945634576 & 0.5367718818 & 0.7257360589 \\ O & 0.4194461701 & 0.0701703614 & 0.2191494924 \\ O & 0.5805538589 & 0.9298296686 & 0.7808504926 \\ O & 0.0805538299 & 0.5701703314 & 0.2808504926 \\ O & 0.9194461411 & 0.4298296386 & 0.7191495074 \\ O & 0.1703549152 & 0.0640306600 & 0.2252175438 \\ O & 0.8296451138 & 0.9359693330 & 0.7747824422 \\ O & 0.3296450848 & 0.5640306670 & 0.2747824422 \\ O & 0.6703548862 & 0.4359693330 & 0.7252175578 \\ O & 0.4198949562 & 0.8690309441 & 0.2155562910 \\ O & 0.5801050728 & 0.1309690409 & 0.7844437240 \\ O & 0.0801050438 & 0.3690309441 & 0.2844437240 \\ O & 0.9198949272 & 0.6309690559 & 0.7155562760 \\ O & 0.1771283945 & 0.8668687223 & 0.2408066232 \\ O & 0.8228716195 & 0.1331312627 & 0.7591933618 \\ O & 0.3228716195 & 0.3668687223 & 0.2591933618 \\ O & 0.6771283805 & 0.6331312777 & 0.7408066382 \\ O & 0.2285524072 & 0.7175833622 & 0.0605671258 \\ O & 0.7714476068 & 0.2824166678 & 0.9394328522\end{array}$




\begin{tabular}{|c|c|c|c|}
\hline 0 & 0.2714476068 & 0.2175833322 & 0.4394328822 \\
\hline $\mathrm{O}$ & 0.7285523932 & 0.7824166378 & 0.5605671478 \\
\hline $\mathrm{N}$ & 0.6219853236 & 0.0109616071 & 0.1983106659 \\
\hline $\mathrm{N}$ & 0.3780146764 & 0.9890384099 & 0.8016893491 \\
\hline $\mathrm{N}$ & 0.8780146764 & 0.5109615901 & 0.3016893491 \\
\hline $\mathrm{N}$ & 0.1219853236 & 0.4890383799 & 0.6983106509 \\
\hline $\mathrm{N}$ & 0.0140272560 & 0.0577304308 & 0.2046230441 \\
\hline $\mathrm{N}$ & 0.9859727600 & 0.9422695582 & 0.7953769409 \\
\hline $\mathrm{N}$ & 0.4859727300 & 0.5577304418 & 0.2953769409 \\
\hline $\mathrm{N}$ & 0.5140272400 & 0.4422695582 & 0.7046230591 \\
\hline $\mathrm{N}$ & 0.5553335264 & 0.0123507763 & 0.3017402699 \\
\hline $\mathrm{N}$ & 0.4446664736 & 0.9876492377 & 0.6982597001 \\
\hline $\mathrm{N}$ & 0.9446664736 & 0.5123507623 & 0.1982597301 \\
\hline $\mathrm{N}$ & 0.0553335264 & 0.4876492377 & 0.8017402999 \\
\hline $\mathrm{N}$ & 0.0791147292 & 0.9274080747 & 0.1321902335 \\
\hline $\mathrm{N}$ & 0.9208852638 & 0.0725919183 & 0.8678097515 \\
\hline $\mathrm{N}$ & 0.4208852638 & 0.4274080747 & 0.3678097515 \\
\hline $\mathrm{N}$ & 0.5791147362 & 0.5725919253 & 0.6321902485 \\
\hline $\mathrm{N}$ & 0.4913937834 & 0.0450024355 & 0.1117789914 \\
\hline $\mathrm{N}$ & 0.5086062166 & 0.9549975905 & 0.8882210006 \\
\hline $\mathrm{N}$ & 0.0086062166 & 0.5450024095 & 0.3882210006 \\
\hline $\mathrm{N}$ & 0.9913937834 & 0.4549975605 & 0.6117789994 \\
\hline $\mathrm{N}$ & 0.1002273144 & 0.9737212143 & 0.3203115091 \\
\hline $\mathrm{N}$ & 0.8997726706 & 0.0262787877 & 0.6796884909 \\
\hline $\mathrm{N}$ & 0.3997726706 & 0.4737212143 & 0.1796884909 \\
\hline $\mathrm{N}$ & 0.6002273294 & 0.5262787857 & 0.8203115091 \\
\hline $\mathrm{N}$ & 0.0195664242 & 0.8562330516 & 0.2282771031 \\
\hline $\mathrm{N}$ & 0.9804335678 & 0.1437669624 & 0.7717228819 \\
\hline $\mathrm{N}$ & 0.4804335678 & 0.3562330516 & 0.2717228819 \\
\hline $\mathrm{N}$ & 0.5195664322 & 0.6437669484 & 0.7282771181 \\
\hline $\mathrm{N}$ & 0.5551693052 & 0.8198980117 & 0.1828991222 \\
\hline $\mathrm{N}$ & 0.4448306948 & 0.1801019883 & 0.8171008628 \\
\hline $\mathrm{N}$ & 0.9448306948 & 0.3198980117 & 0.3171008628 \\
\hline $\mathrm{N}$ & 0.0551693052 & 0.6801019883 & 0.6828991372 \\
\hline $\mathrm{N}$ & 0.4102911723 & 0.8194279711 & 0.4140766764 \\
\hline $\mathrm{N}$ & 0.5897088277 & 0.1805720429 & 0.5859233236 \\
\hline $\mathrm{N}$ & 0.0897088277 & 0.3194279711 & 0.0859233236 \\
\hline $\mathrm{N}$ & 0.9102911723 & 0.6805720289 & 0.9140766764 \\
\hline C & 0.2361567510 & 0.9094079915 & 0.2325550824 \\
\hline C & 0.7638432340 & 0.0905920085 & 0.7674448876 \\
\hline C & 0.2638432340 & 0.4094079915 & 0.2674449176 \\
\hline C & 0.7361567660 & 0.5905920085 & 0.7325551124 \\
\hline C & 0.2332561200 & 0.0239939544 & 0.2265493080 \\
\hline C & 0.7667438500 & 0.9760060496 & 0.7734506920 \\
\hline C & 0.2667438800 & 0.5239939504 & 0.2734506920 \\
\hline C & 0.7332561500 & 0.4760060496 & 0.7265493080 \\
\hline C & 0.3594815756 & 0.0270418551 & 0.2224124082 \\
\hline C & 0.6405184544 & 0.9729581159 & 0.7775875918 \\
\hline C & 0.1405184244 & 0.5270418841 & 0.2775875918 \\
\hline C & 0.8594815456 & 0.4729581449 & 0.7224124082 \\
\hline C & 0.0191648618 & 0.1583246670 & 0.1902734244 \\
\hline C & 0.9808351612 & 0.8416753180 & 0.8097265756 \\
\hline C & 0.4808351312 & 0.6583246820 & 0.3097265756 \\
\hline C & 0.5191648388 & 0.3416753180 & 0.6902734244 \\
\hline C & 0.5505264718 & 0.0791237571 & 0.0920673040 \\
\hline C & 0.4494735282 & 0.9208762429 & 0.9079327260 \\
\hline C & 0.9494735282 & 0.5791237571 & 0.4079326960 \\
\hline C & 0.0505264718 & 0.4208762429 & 0.5920672740 \\
\hline
\end{tabular}




\begin{tabular}{|c|c|c|c|}
\hline C & 0.9474136588 & 0.0157997358 & 0.1992483150 \\
\hline C & 0.0525863452 & 0.9842002812 & 0.8007516700 \\
\hline C & 0.5525863412 & 0.5157997188 & 0.3007516700 \\
\hline C & 0.4474136588 & 0.4842002512 & 0.6992483300 \\
\hline C & 0.5263985326 & 0.9938347380 & 0.3544300352 \\
\hline C & 0.4736014674 & 0.0061652610 & 0.6455699358 \\
\hline C & 0.9736014674 & 0.4938347380 & 0.1455699648 \\
\hline C & 0.0263985326 & 0.5061652620 & 0.8544300642 \\
\hline C & 0.6236418157 & 0.0456008631 & 0.1309834708 \\
\hline C & 0.3763581843 & 0.9543991179 & 0.8690165142 \\
\hline C & 0.8763581843 & 0.5456008821 & 0.3690165142 \\
\hline C & 0.1236418157 & 0.4543991479 & 0.6309834858 \\
\hline C & 0.6169638628 & 0.0680550966 & 0.3098429524 \\
\hline C & 0.3830361372 & 0.9319449184 & 0.6901570776 \\
\hline C & 0.8830361372 & 0.5680550816 & 0.1901570476 \\
\hline C & 0.1169638628 & 0.4319449184 & 0.8098429224 \\
\hline C & 0.2994914292 & 0.8555659120 & 0.2300917213 \\
\hline C & 0.7005086008 & 0.1444341030 & 0.7699082487 \\
\hline C & 0.2005085708 & 0.3555659120 & 0.2699082787 \\
\hline C & 0.7994913992 & 0.6444340880 & 0.7300917513 \\
\hline C & 0.3602820353 & 0.9109204605 & 0.2225612077 \\
\hline C & 0.6397179947 & 0.0890795325 & 0.7774387923 \\
\hline C & 0.1397179647 & 0.4109204605 & 0.2774387923 \\
\hline C & 0.8602820053 & 0.5890795395 & 0.7225612077 \\
\hline C & 0.2955483566 & 0.0799890047 & 0.2244815169 \\
\hline C & 0.7044516724 & 0.9200109953 & 0.7755184691 \\
\hline C & 0.2044516434 & 0.5799890047 & 0.2755184691 \\
\hline C & 0.7955483276 & 0.4200109953 & 0.7244815309 \\
\hline C & 0.5192523579 & 0.7323382860 & 0.1619937623 \\
\hline C & 0.4807476421 & 0.2676617140 & 0.8380062377 \\
\hline C & 0.9807476421 & 0.2323382860 & 0.3380062377 \\
\hline C & 0.0192523579 & 0.7676617140 & 0.6619937623 \\
\hline C & 0.6273916406 & 0.8230690496 & 0.1867899186 \\
\hline C & 0.3726083594 & 0.1769309204 & 0.8132100814 \\
\hline C & 0.8726083594 & 0.3230690796 & 0.3132100814 \\
\hline C & 0.1273916406 & 0.6769309504 & 0.6867899186 \\
\hline C & 0.6662069325 & 0.9178714541 & 0.2168589765 \\
\hline C & 0.3337930675 & 0.0821285599 & 0.7831410535 \\
\hline C & 0.8337930675 & 0.4178714541 & 0.2831410235 \\
\hline C & 0.1662069325 & 0.5821285459 & 0.7168589465 \\
\hline C & 0.6432523402 & 0.0923485881 & 0.2479800792 \\
\hline C & 0.3567476598 & 0.9076514189 & 0.7520199208 \\
\hline C & 0.8567476598 & 0.5923485811 & 0.2520199208 \\
\hline C & 0.1432523402 & 0.4076514189 & 0.7479800792 \\
\hline C & 0.0221651709 & 0.8032662504 & 0.0521871354 \\
\hline C & 0.9778348271 & 0.1967337796 & 0.9478128606 \\
\hline C & 0.4778348271 & 0.3032662204 & 0.4478128606 \\
\hline C & 0.5221651729 & 0.6967337496 & 0.5521871394 \\
\hline C & 0.9582953244 & 0.2195766372 & 0.1702069452 \\
\hline C & 0.0417046786 & 0.7804233778 & 0.8297930848 \\
\hline C & 0.5417046756 & 0.7195766222 & 0.3297930548 \\
\hline C & 0.4582953244 & 0.2804233778 & 0.6702069152 \\
\hline C & 0.1007843006 & 0.9800887994 & 0.0840010081 \\
\hline C & 0.8992157074 & 0.0199112016 & 0.9159989769 \\
\hline C & 0.3992157074 & 0.4800887994 & 0.4159989769 \\
\hline C & 0.6007842926 & 0.5199112006 & 0.5840010231 \\
\hline C & 0.8895289056 & 0.1769764532 & 0.1645708820 \\
\hline C & 0.1104710874 & 0.8230235318 & 0.8354291030 \\
\hline
\end{tabular}




\begin{tabular}{|c|c|c|c|}
\hline C & 0.6104710944 & 0.6769764682 & 0.3354291030 \\
\hline C & 0.3895289056 & 0.3230235318 & 0.6645708970 \\
\hline C & 0.0672168542 & 0.8944542442 & 0.3446917035 \\
\hline C & 0.9327831688 & 0.1055457418 & 0.6553082965 \\
\hline C & 0.4327831388 & 0.3944542442 & 0.1553082965 \\
\hline C & 0.5672168312 & 0.6055457558 & 0.8446917035 \\
\hline C & 0.5535046324 & 0.6460065728 & 0.1436607431 \\
\hline C & 0.4464953676 & 0.3539934272 & 0.8563392419 \\
\hline C & 0.9464953676 & 0.1460065728 & 0.3563392419 \\
\hline C & 0.0535046324 & 0.8539934272 & 0.6436607581 \\
\hline C & 0.0400488026 & 0.8107538891 & 0.2957158705 \\
\hline C & 0.9599511944 & 0.1892461259 & 0.7042841005 \\
\hline C & 0.4599511944 & 0.3107538891 & 0.2042841295 \\
\hline C & 0.5400488056 & 0.6892461109 & 0.7957158995 \\
\hline C & 0.1314895344 & 0.0488676461 & 0.3617689489 \\
\hline C & 0.8685104806 & 0.9511323239 & 0.6382310211 \\
\hline C & 0.3685104806 & 0.5488676761 & 0.1382310511 \\
\hline C & 0.6314895194 & 0.4511323539 & 0.8617689789 \\
\hline C & 0.5447431487 & 0.1405877063 & 0.0359966597 \\
\hline C & 0.4552568513 & 0.8594123237 & 0.9640033553 \\
\hline C & 0.9552568513 & 0.6405876763 & 0.4640033553 \\
\hline C & 0.0447431487 & 0.3594122937 & 0.5359966447 \\
\hline C & 0.0412176461 & 0.8388870298 & 0.1167867842 \\
\hline C & 0.9587823539 & 0.1611129402 & 0.8832132158 \\
\hline C & 0.4587823539 & 0.3388870598 & 0.3832132158 \\
\hline C & 0.5412176461 & 0.6611129702 & 0.6167867842 \\
\hline C & 0.8842835234 & 0.0732978343 & 0.1792834825 \\
\hline C & 0.1157164766 & 0.9267021877 & 0.8207165175 \\
\hline C & 0.6157164766 & 0.5732978123 & 0.3207165175 \\
\hline C & 0.3842835234 & 0.4267021587 & 0.6792834825 \\
\hline C & 0.6521811930 & 0.1043533010 & 0.3716784775 \\
\hline C & 0.3478188070 & 0.8956466690 & 0.6283215525 \\
\hline C & 0.8478188070 & 0.6043533310 & 0.1283215225 \\
\hline C & 0.1521811930 & 0.3956466990 & 0.8716784475 \\
\hline C & 0.6647811208 & 0.7405311128 & 0.1674536909 \\
\hline C & 0.3352188792 & 0.2594688872 & 0.8325463091 \\
\hline C & 0.8352188792 & 0.2405311128 & 0.3325463091 \\
\hline C & 0.1647811208 & 0.7594688872 & 0.6674536909 \\
\hline C & 0.0447021615 & 0.8578424941 & 0.0024948821 \\
\hline C & 0.9552978315 & 0.1421575059 & 0.9975051419 \\
\hline C & 0.4552978315 & 0.3578424941 & 0.4975051129 \\
\hline C & 0.5447021685 & 0.6421575059 & 0.5024948581 \\
\hline C & 0.4765278445 & 0.1652365811 & 0.9988840426 \\
\hline C & 0.5234721855 & 0.8347634189 & 0.0011159504 \\
\hline C & 0.0234721555 & 0.6652365811 & 0.5011159574 \\
\hline C & 0.9765278145 & 0.3347634189 & 0.4988840426 \\
\hline C & 0.4251220977 & 0.0706288079 & 0.0759835522 \\
\hline C & 0.5748779023 & 0.9293711771 & 0.9240164248 \\
\hline C & 0.0748779023 & 0.5706288229 & 0.4240164548 \\
\hline C & 0.9251220977 & 0.4293711771 & 0.5759835752 \\
\hline C & 0.5584250528 & 0.0281692501 & 0.4174137732 \\
\hline C & 0.4415749472 & 0.9718307539 & 0.5825862268 \\
\hline C & 0.9415749472 & 0.5281692461 & 0.0825862268 \\
\hline C & 0.0584250528 & 0.4718307539 & 0.9174137732 \\
\hline C & 0.0853777209 & 0.9470651740 & 0.0187197625 \\
\hline C & 0.9146223091 & 0.0529348450 & 0.9812802185 \\
\hline C & 0.4146222791 & 0.4470651440 & 0.4812802485 \\
\hline C & 0.5853776909 & 0.5529348260 & 0.5187197815 \\
\hline
\end{tabular}




\begin{tabular}{|c|c|c|c|}
\hline C & 0.6276430380 & 0.6503622739 & 0.1456396273 \\
\hline C & 0.3723569620 & 0.3496377551 & 0.8543603877 \\
\hline C & 0.8723569620 & 0.1503622449 & 0.3543603877 \\
\hline C & 0.1276430380 & 0.8496377261 & 0.6456396123 \\
\hline C & 0.9456696556 & 0.9043560999 & 0.2167186352 \\
\hline C & 0.0543303184 & 0.0956439151 & 0.7832813798 \\
\hline C & 0.5543303444 & 0.4043560999 & 0.2832813798 \\
\hline C & 0.4456696856 & 0.5956439001 & 0.7167186202 \\
\hline C & 0.4152246637 & 0.1295673005 & 0.0190880172 \\
\hline C & 0.5847753363 & 0.8704327145 & 0.9809120048 \\
\hline C & 0.0847753363 & 0.6295672855 & 0.4809119748 \\
\hline C & 0.9152246637 & 0.3704327145 & 0.5190879952 \\
\hline C & 0.0251736489 & 0.7819795631 & 0.1749868396 \\
\hline C & 0.9748263341 & 0.2180204519 & 0.8250131304 \\
\hline C & 0.4748263641 & 0.2819795631 & 0.3250131604 \\
\hline C & 0.5251736659 & 0.7180204369 & 0.6749868696 \\
\hline C & 0.6225275683 & 0.0843955245 & 0.4261319639 \\
\hline C & 0.3774724317 & 0.9156044975 & 0.5738680061 \\
\hline C & 0.8774724317 & 0.5843955025 & 0.0738680361 \\
\hline C & 0.1225275683 & 0.4156044675 & 0.9261319939 \\
\hline C & 0.1308262320 & 0.0479461893 & 0.4286492833 \\
\hline C & 0.8691737530 & 0.9520538107 & 0.5713507457 \\
\hline C & 0.3691737530 & 0.5479461893 & 0.0713507167 \\
\hline C & 0.6308262470 & 0.4520538107 & 0.9286492543 \\
\hline C & 0.0625132783 & 0.8909669371 & 0.4106356246 \\
\hline C & 0.9374866917 & 0.1090330329 & 0.5893643754 \\
\hline C & 0.4374867217 & 0.3909669671 & 0.0893643754 \\
\hline C & 0.5625133083 & 0.6090330629 & 0.9106356246 \\
\hline C & 0.0953120856 & 0.9682571866 & 0.4535175727 \\
\hline C & 0.9046879064 & 0.0317427974 & 0.5464824273 \\
\hline C & 0.4046879064 & 0.4682572156 & 0.0464824273 \\
\hline C & 0.5953120936 & 0.5317428134 & 0.9535175727 \\
\hline C & 0.1580043060 & 0.5813899798 & 0.0903259819 \\
\hline C & 0.8419956940 & 0.4186100202 & 0.9096740481 \\
\hline C & 0.3419956940 & 0.0813899798 & 0.4096740181 \\
\hline C & 0.6580043060 & 0.9186100202 & 0.5903259519 \\
\hline C & 0.1793720632 & 0.6376717882 & 0.0337119506 \\
\hline C & 0.8206279068 & 0.3623282118 & 0.9662880724 \\
\hline C & 0.3206279368 & 0.1376717882 & 0.4662880424 \\
\hline C & 0.6793720932 & 0.8623282118 & 0.5337119276 \\
\hline C & 0.2964776767 & 0.8659292105 & 0.0442885431 \\
\hline C & 0.7035222943 & 0.1340708195 & 0.9557114869 \\
\hline C & 0.2035223233 & 0.3659291805 & 0.4557114569 \\
\hline C & 0.7964777057 & 0.6340707895 & 0.5442885131 \\
\hline C & 0.2457131314 & 0.7829068681 & 0.0106540334 \\
\hline C & 0.7542868836 & 0.2170931470 & 0.9893459876 \\
\hline C & 0.2542868836 & 0.2829068681 & 0.4893459576 \\
\hline C & 0.7457131164 & 0.7170931319 & 0.5106540124 \\
\hline C & 0.3482784820 & 0.8039681421 & 0.4056380822 \\
\hline C & 0.6517215470 & 0.1960318729 & 0.5943619178 \\
\hline C & 0.1517215180 & 0.3039681421 & 0.0943619178 \\
\hline C & 0.8482784530 & 0.6960318579 & 0.9056380822 \\
\hline C & 0.2712268701 & 0.7861064387 & 0.3963141096 \\
\hline C & 0.7287731299 & 0.2138935613 & 0.6036859204 \\
\hline C & 0.2287731299 & 0.2861064387 & 0.1036858904 \\
\hline C & 0.7712268701 & 0.7138935613 & 0.8963140796 \\
\hline $\mathrm{H}$ & 0.0737744203 & 0.1881902952 & 0.1951827210 \\
\hline $\mathrm{H}$ & 0.9262255567 & 0.8118096898 & 0.8048172940 \\
\hline
\end{tabular}




\begin{tabular}{|c|c|c|c|}
\hline $\mathrm{H}$ & 0.4262255867 & 0.6881903102 & 0.3048172940 \\
\hline $\mathrm{H}$ & 0.5737744433 & 0.3118096898 & 0.6951827060 \\
\hline $\mathrm{H}$ & 0.4759840173 & 0.9501262571 & 0.3452910194 \\
\hline $\mathrm{H}$ & 0.5240159827 & 0.0498737389 & 0.6547089806 \\
\hline $\mathrm{H}$ & 0.0240159827 & 0.4501262571 & 0.1547089806 \\
\hline $\mathrm{H}$ & 0.9759840173 & 0.5498737429 & 0.8452910194 \\
\hline $\mathrm{H}$ & 0.6423327270 & 0.9812503291 & 0.1045776369 \\
\hline $\mathrm{H}$ & 0.3576672730 & 0.0187496469 & 0.8954223701 \\
\hline $\mathrm{H}$ & 0.8576672730 & 0.4812503581 & 0.3954223701 \\
\hline $\mathrm{H}$ & 0.1423327270 & 0.5187496709 & 0.6045776299 \\
\hline $\mathrm{H}$ & 0.6633837730 & 0.1077994607 & 0.1318316986 \\
\hline $\mathrm{H}$ & 0.3366162270 & 0.8922005323 & 0.8681682864 \\
\hline $\mathrm{H}$ & 0.8366162270 & 0.6077994677 & 0.3681682864 \\
\hline $\mathrm{H}$ & 0.1633837730 & 0.3922005323 & 0.6318317136 \\
\hline $\mathrm{H}$ & 0.3005257002 & 0.7718673145 & 0.2315539233 \\
\hline $\mathrm{H}$ & 0.6994743298 & 0.2281326565 & 0.7684460917 \\
\hline $\mathrm{H}$ & 0.1994742998 & 0.2718673435 & 0.2684460917 \\
\hline $\mathrm{H}$ & 0.8005256702 & 0.7281326855 & 0.7315539083 \\
\hline $\mathrm{H}$ & 0.2947256455 & 0.1636869243 & 0.2243052506 \\
\hline $\mathrm{H}$ & 0.7052743545 & 0.8363130907 & 0.7756947344 \\
\hline $\mathrm{H}$ & 0.2052743545 & 0.6636869093 & 0.2756947344 \\
\hline $\mathrm{H}$ & 0.7947256455 & 0.3363130907 & 0.7243052656 \\
\hline $\mathrm{H}$ & 0.4614320874 & 0.7334119962 & 0.1609791783 \\
\hline $\mathrm{H}$ & 0.5385678826 & 0.2665880038 & 0.8390208217 \\
\hline $\mathrm{H}$ & 0.0385679126 & 0.2334119962 & 0.3390208217 \\
\hline $\mathrm{H}$ & 0.9614321174 & 0.7665880038 & 0.6609791783 \\
\hline $\mathrm{H}$ & 0.6767238410 & 0.9091203849 & 0.2708784129 \\
\hline $\mathrm{H}$ & 0.3232761590 & 0.0908796081 & 0.7291215871 \\
\hline $\mathrm{H}$ & 0.8232761590 & 0.4091203849 & 0.2291215871 \\
\hline $\mathrm{H}$ & 0.1767238410 & 0.5908796151 & 0.7708784129 \\
\hline $\mathrm{H}$ & 0.7195767039 & 0.9242658109 & 0.2043276321 \\
\hline $\mathrm{H}$ & 0.2804232961 & 0.0757341741 & 0.7956723529 \\
\hline $\mathrm{H}$ & 0.7804232961 & 0.4242658109 & 0.2956723529 \\
\hline $\mathrm{H}$ & 0.2195767039 & 0.5757341891 & 0.7043276471 \\
\hline $\mathrm{H}$ & 0.6185691621 & 0.1648042893 & 0.2265470372 \\
\hline $\mathrm{H}$ & 0.3814308379 & 0.8351957407 & 0.7734529328 \\
\hline $\mathrm{H}$ & 0.8814308379 & 0.6648042593 & 0.2734529628 \\
\hline $\mathrm{H}$ & 0.1185691621 & 0.3351957107 & 0.7265470672 \\
\hline $\mathrm{H}$ & 0.7024353290 & 0.1049146227 & 0.2597782845 \\
\hline $\mathrm{H}$ & 0.2975646710 & 0.8950853623 & 0.7402217455 \\
\hline $\mathrm{H}$ & 0.7975646710 & 0.6049146377 & 0.2402217155 \\
\hline $\mathrm{H}$ & 0.2024353290 & 0.3950853623 & 0.7597782545 \\
\hline $\mathrm{H}$ & 0.9892162258 & 0.7343757805 & 0.0406555286 \\
\hline $\mathrm{H}$ & 0.0107837852 & 0.2656242195 & 0.9593444564 \\
\hline $\mathrm{H}$ & 0.5107837742 & 0.2343757805 & 0.4593444564 \\
\hline $\mathrm{H}$ & 0.4892162258 & 0.7656242195 & 0.5406555436 \\
\hline $\mathrm{H}$ & 0.9654551148 & 0.2998699057 & 0.1589165905 \\
\hline $\mathrm{H}$ & 0.0345448892 & 0.7001300643 & 0.8410834095 \\
\hline $\mathrm{H}$ & 0.5345448852 & 0.7998699357 & 0.3410834095 \\
\hline $\mathrm{H}$ & 0.4654551148 & 0.2001300943 & 0.6589165905 \\
\hline $\mathrm{H}$ & 0.1309059784 & 0.0505360745 & 0.0990613923 \\
\hline $\mathrm{H}$ & 0.8690940516 & 0.9494639065 & 0.9009385787 \\
\hline $\mathrm{H}$ & 0.3690940216 & 0.5505360935 & 0.4009386077 \\
\hline $\mathrm{H}$ & 0.6309059484 & 0.4494639365 & 0.5990614213 \\
\hline $\mathrm{H}$ & 0.8407035663 & 0.2233189815 & 0.1493694770 \\
\hline $\mathrm{H}$ & 0.1592964477 & 0.7766810035 & 0.8506305230 \\
\hline $\mathrm{H}$ & 0.6592964337 & 0.7233189965 & 0.3506305230 \\
\hline $\mathrm{H}$ & 0.3407035663 & 0.2766810035 & 0.6493694770 \\
\hline
\end{tabular}




\begin{tabular}{|c|c|c|c|}
\hline $\mathrm{H}$ & 0.5223084465 & 0.5767712780 & 0.1276113143 \\
\hline $\mathrm{H}$ & 0.4776915535 & 0.4232287220 & 0.8723886707 \\
\hline $\mathrm{H}$ & 0.9776915535 & 0.0767712780 & 0.3723886707 \\
\hline $\mathrm{H}$ & 0.0223084465 & 0.9232287220 & 0.6276113293 \\
\hline $\mathrm{H}$ & 0.0836961256 & 0.7549371710 & 0.2959448827 \\
\hline $\mathrm{H}$ & 0.9163038514 & 0.2450628590 & 0.7040550873 \\
\hline $\mathrm{H}$ & 0.4163038814 & 0.2549371410 & 0.2040551173 \\
\hline $\mathrm{H}$ & 0.5836961486 & 0.7450628290 & 0.7959449127 \\
\hline $\mathrm{H}$ & -0.0051504491 & 0.7689243044 & 0.3089945583 \\
\hline $\mathrm{H}$ & 1.0051504640 & 0.2310756956 & 0.6910054717 \\
\hline $\mathrm{H}$ & 0.5051504341 & 0.2689243044 & 0.1910054417 \\
\hline $\mathrm{H}$ & 0.4948495360 & 0.7310756956 & 0.8089945283 \\
\hline $\mathrm{H}$ & 0.1576138128 & 0.1101762904 & 0.3398244861 \\
\hline $\mathrm{H}$ & 0.8423861722 & 0.8898236876 & 0.6601755439 \\
\hline $\mathrm{H}$ & 0.3423861722 & 0.6101763124 & 0.1601755139 \\
\hline $\mathrm{H}$ & 0.6576138278 & 0.3898237166 & 0.8398244561 \\
\hline $\mathrm{H}$ & 0.5936506998 & 0.1694540994 & 0.0227457248 \\
\hline $\mathrm{H}$ & 0.4063493002 & 0.8305459306 & 0.9772542832 \\
\hline $\mathrm{H}$ & 0.9063493002 & 0.6694540694 & 0.4772542832 \\
\hline $\mathrm{H}$ & 0.0936506998 & 0.3305459006 & 0.5227457168 \\
\hline $\mathrm{H}$ & 0.8319776402 & 0.0357179225 & 0.1745633884 \\
\hline $\mathrm{H}$ & 0.1680223308 & 0.9642820995 & 0.8254366116 \\
\hline $\mathrm{H}$ & 0.6680223598 & 0.5357179005 & 0.3254366116 \\
\hline $\mathrm{H}$ & 0.3319776692 & 0.4642820695 & 0.6745633884 \\
\hline $\mathrm{H}$ & 0.7018426814 & 0.1489284858 & 0.3778154014 \\
\hline $\mathrm{H}$ & 0.2981573186 & 0.8510714992 & 0.6221845686 \\
\hline $\mathrm{H}$ & 0.7981573186 & 0.6489285008 & 0.1221845986 \\
\hline $\mathrm{H}$ & 0.2018426814 & 0.3510714992 & 0.8778154314 \\
\hline $\mathrm{H}$ & 0.7224525014 & 0.7489495474 & 0.1695129628 \\
\hline $\mathrm{H}$ & 0.2775474986 & 0.2510504826 & 0.8304870222 \\
\hline $\mathrm{H}$ & 0.7775474986 & 0.2489495174 & 0.3304870222 \\
\hline $\mathrm{H}$ & 0.2224525014 & 0.7510504526 & 0.6695129778 \\
\hline $\mathrm{H}$ & 0.0296471695 & 0.8309698701 & 0.9516298111 \\
\hline $\mathrm{H}$ & 0.9703528415 & 0.1690301449 & 0.0483701969 \\
\hline $\mathrm{H}$ & 0.4703528415 & 0.3309698701 & 0.5483701889 \\
\hline $\mathrm{H}$ & 0.5296471585 & 0.6690301299 & 0.4516298111 \\
\hline $\mathrm{H}$ & 0.4709038812 & 0.2135515469 & 0.9552198550 \\
\hline $\mathrm{H}$ & 0.5290961188 & 0.7864484531 & 0.0447801720 \\
\hline $\mathrm{H}$ & 0.0290961188 & 0.7135515469 & 0.5447801450 \\
\hline $\mathrm{H}$ & 0.9709038812 & 0.2864484531 & 0.4552198250 \\
\hline $\mathrm{H}$ & 0.3789832552 & 0.0431897951 & 0.0944876456 \\
\hline $\mathrm{H}$ & 0.6210167158 & 0.9568101979 & 0.9055123834 \\
\hline $\mathrm{H}$ & 0.1210167448 & 0.5431898021 & 0.4055123544 \\
\hline $\mathrm{H}$ & 0.8789832842 & 0.4568101979 & 0.5944876166 \\
\hline $\mathrm{H}$ & 0.5332596789 & 0.0107737550 & 0.4585442315 \\
\hline $\mathrm{H}$ & 0.4667403211 & 0.9892262270 & 0.5414557685 \\
\hline $\mathrm{H}$ & 0.9667403211 & 0.5107737730 & 0.0414557685 \\
\hline $\mathrm{H}$ & 0.0332596789 & 0.4892262570 & 0.9585442315 \\
\hline $\mathrm{H}$ & 0.1042013405 & 0.9926961039 & 0.9818145520 \\
\hline $\mathrm{H}$ & 0.8957986365 & 0.0073039071 & 0.0181854330 \\
\hline $\mathrm{H}$ & 0.3957986665 & 0.4926961039 & 0.5181854480 \\
\hline $\mathrm{H}$ & 0.6042013635 & 0.5073038961 & 0.4818145520 \\
\hline $\mathrm{H}$ & 0.6556573102 & 0.5839226376 & 0.1312694317 \\
\hline $\mathrm{H}$ & 0.3443426898 & 0.4160773924 & 0.8687305383 \\
\hline $\mathrm{H}$ & 0.8443426898 & 0.0839226076 & 0.3687305683 \\
\hline $\mathrm{H}$ & 0.1556573102 & 0.9160773624 & 0.6312694617 \\
\hline $\mathrm{H}$ & 0.9239213648 & 0.8963735519 & 0.2614345020 \\
\hline $\mathrm{H}$ & 0.0760786422 & 0.1036264261 & 0.7385654980 \\
\hline
\end{tabular}




\begin{tabular}{|c|c|c|c|}
\hline $\mathrm{H}$ & 0.5760786352 & 0.3963735819 & 0.2385654980 \\
\hline $\mathrm{H}$ & 0.4239213648 & 0.6036264481 & 0.7614345020 \\
\hline $\mathrm{H}$ & 0.9082920111 & 0.8635430829 & 0.1769033479 \\
\hline $\mathrm{H}$ & 0.0917079669 & 0.1364569021 & 0.8230966221 \\
\hline $\mathrm{H}$ & 0.5917079889 & 0.3635430829 & 0.3230966521 \\
\hline $\mathrm{H}$ & 0.4082920401 & 0.6364569171 & 0.6769033779 \\
\hline $\mathrm{H}$ & 0.3608271927 & 0.1495172228 & 0.9924189462 \\
\hline $\mathrm{H}$ & 0.6391727783 & 0.8504827472 & 0.0075810498 \\
\hline $\mathrm{H}$ & 0.1391728073 & 0.6495172528 & 0.5075810538 \\
\hline $\mathrm{H}$ & 0.8608272217 & 0.3504827772 & 0.4924189462 \\
\hline $\mathrm{H}$ & 0.9759448771 & 0.7349240441 & 0.1604199294 \\
\hline $\mathrm{H}$ & 0.0240551159 & 0.2650759859 & 0.8395800706 \\
\hline $\mathrm{H}$ & 0.5240551229 & 0.2349240141 & 0.3395800706 \\
\hline $\mathrm{H}$ & 0.4759448771 & 0.7650759559 & 0.6604199294 \\
\hline $\mathrm{H}$ & 0.0706005914 & 0.7300685164 & 0.1947990603 \\
\hline $\mathrm{H}$ & 0.9293994236 & 0.2699314836 & 0.8052009247 \\
\hline $\mathrm{H}$ & 0.4293994236 & 0.2300685164 & 0.3052009247 \\
\hline $\mathrm{H}$ & 0.5706005764 & 0.7699314836 & 0.6947990753 \\
\hline $\mathrm{H}$ & 0.6488628515 & 0.1139562694 & 0.4744317203 \\
\hline $\mathrm{H}$ & 0.3511371485 & 0.8860437456 & 0.5255682497 \\
\hline $\mathrm{H}$ & 0.8511371485 & 0.6139562544 & 0.0255682797 \\
\hline $\mathrm{H}$ & 0.1488628515 & 0.3860437456 & 0.9744317503 \\
\hline $\mathrm{H}$ & 0.1573133857 & 0.1092487906 & 0.4606269754 \\
\hline $\mathrm{H}$ & 0.8426866443 & 0.8907511944 & 0.5393730246 \\
\hline $\mathrm{H}$ & 0.3426866143 & 0.6092488056 & 0.0393730246 \\
\hline $\mathrm{H}$ & 0.6573133557 & 0.3907511944 & 0.9606269754 \\
\hline $\mathrm{H}$ & 0.0329713636 & 0.8283299717 & 0.4273825848 \\
\hline $\mathrm{H}$ & 0.9670286254 & 0.1716700433 & 0.5726173852 \\
\hline $\mathrm{H}$ & 0.4670286254 & 0.3283299717 & 0.0726174152 \\
\hline $\mathrm{H}$ & 0.5329713746 & 0.6716700283 & 0.9273826148 \\
\hline $\mathrm{H}$ & 0.0933312027 & 0.9675963518 & 0.5055855710 \\
\hline $\mathrm{H}$ & 0.9066688273 & 0.0324036522 & 0.4944144290 \\
\hline $\mathrm{H}$ & 0.4066687973 & 0.4675963518 & 0.9944144290 \\
\hline $\mathrm{H}$ & 0.5933311727 & 0.5324036482 & 0.0055855710 \\
\hline $\mathrm{H}$ & 0.1189949747 & 0.5199948768 & 0.0721535341 \\
\hline $\mathrm{H}$ & 0.8810050023 & 0.4800050932 & 0.9278464509 \\
\hline $\mathrm{H}$ & 0.3810050323 & 0.0199949068 & 0.4278464509 \\
\hline $\mathrm{H}$ & 0.6189949977 & 0.9800051232 & 0.5721535491 \\
\hline $\mathrm{H}$ & 0.1334394865 & 0.6346929577 & 0.1201065646 \\
\hline $\mathrm{H}$ & 0.8665604835 & 0.3653070423 & 0.8798934354 \\
\hline $\mathrm{H}$ & 0.3665605135 & 0.1346929577 & 0.3798934354 \\
\hline $\mathrm{H}$ & 0.6334395165 & 0.8653070423 & 0.6201065646 \\
\hline $\mathrm{H}$ & 0.2054406767 & 0.5464345085 & 0.1230311498 \\
\hline $\mathrm{H}$ & 0.7945593383 & 0.4535654915 & 0.8769688502 \\
\hline $\mathrm{H}$ & 0.2945593383 & 0.0464345085 & 0.3769688502 \\
\hline $\mathrm{H}$ & 0.7054406617 & 0.9535654915 & 0.6230311498 \\
\hline $\mathrm{H}$ & 0.2050545134 & 0.5850121926 & 0.0035857993 \\
\hline $\mathrm{H}$ & 0.7949455156 & 0.4149878074 & 0.9964141857 \\
\hline $\mathrm{H}$ & 0.2949454866 & 0.0850121926 & 0.4964141857 \\
\hline $\mathrm{H}$ & 0.7050544844 & 0.9149878074 & 0.5035858143 \\
\hline $\mathrm{H}$ & 0.1310699465 & 0.6708944707 & 0.0001472927 \\
\hline $\mathrm{H}$ & 0.8689300385 & 0.3291055593 & 0.9998527363 \\
\hline $\mathrm{H}$ & 0.3689300385 & 0.1708944407 & 0.4998527063 \\
\hline $\mathrm{H}$ & 0.6310699615 & 0.8291055293 & 0.5001472637 \\
\hline $\mathrm{H}$ & 0.3080220623 & 0.9203819743 & 0.0075811945 \\
\hline $\mathrm{H}$ & 0.6919779377 & 0.0796180187 & 0.9924188255 \\
\hline $\mathrm{H}$ & 0.1919779377 & 0.4203819743 & 0.4924187965 \\
\hline $\mathrm{H}$ & 0.8080220623 & 0.5796180257 & 0.5075811745 \\
\hline
\end{tabular}




\begin{tabular}{|c|c|c|c|}
\hline $\mathrm{H}$ & 0.3475717433 & 0.8330230655 & 0.0723244260 \\
\hline $\mathrm{H}$ & 0.6524282867 & 0.1669769345 & 0.9276755880 \\
\hline $\mathrm{H}$ & 0.1524282567 & 0.3330230655 & 0.4276755880 \\
\hline $\mathrm{H}$ & 0.8475717133 & 0.6669769345 & 0.5723244120 \\
\hline $\mathrm{H}$ & 0.2713551435 & 0.9095256147 & 0.0786290643 \\
\hline $\mathrm{H}$ & 0.7286448565 & 0.0904743923 & 0.9213709657 \\
\hline $\mathrm{H}$ & 0.2286448565 & 0.4095256147 & 0.4213709357 \\
\hline $\mathrm{H}$ & 0.7713551435 & 0.5904743853 & 0.5786290343 \\
\hline $\mathrm{H}$ & 0.1953364649 & 0.8174048222 & 0.9814108244 \\
\hline $\mathrm{H}$ & 0.8046635501 & 0.1825951628 & 0.0185891886 \\
\hline $\mathrm{H}$ & 0.3046635501 & 0.3174048222 & 0.5185891756 \\
\hline $\mathrm{H}$ & 0.6953364499 & 0.6825951778 & 0.4814108244 \\
\hline $\mathrm{H}$ & 0.2701793584 & 0.7374171426 & 0.9760344261 \\
\hline $\mathrm{H}$ & 0.7298206416 & 0.2625828574 & 0.0239655739 \\
\hline $\mathrm{H}$ & 0.2298206416 & 0.2374171426 & 0.5239655739 \\
\hline $\mathrm{H}$ & 0.7701793584 & 0.7625828574 & 0.4760344261 \\
\hline $\mathrm{H}$ & 0.2506705441 & 0.8220718097 & 0.4364845731 \\
\hline $\mathrm{H}$ & 0.7493294559 & 0.1779281603 & 0.5635154269 \\
\hline $\mathrm{H}$ & 0.2493294559 & 0.3220718397 & 0.0635154269 \\
\hline $\mathrm{H}$ & 0.7506705441 & 0.6779281903 & 0.9364845731 \\
\hline $\mathrm{H}$ & 0.2606291778 & 0.7033483872 & 0.3982378122 \\
\hline $\mathrm{H}$ & 0.7393707922 & 0.2966515838 & 0.6017622178 \\
\hline $\mathrm{H}$ & 0.2393708222 & 0.2033484162 & 0.1017621878 \\
\hline $\mathrm{H}$ & 0.7606292078 & 0.7966516128 & 0.8982377822 \\
\hline $\mathrm{H}$ & 0.2424110314 & 0.8174547371 & 0.3486741599 \\
\hline $\mathrm{H}$ & 0.7575889686 & 0.1825452929 & 0.6513258401 \\
\hline $\mathrm{H}$ & 0.2575889686 & 0.3174547071 & 0.1513258401 \\
\hline $\mathrm{H}$ & 0.7424110314 & 0.6825452629 & 0.8486741599 \\
\hline $\mathrm{F}$ & 0.2543280072 & 0.2393140957 & 0.9588610803 \\
\hline $\mathrm{F}$ & 0.7456719928 & 0.7606859193 & 0.0411389007 \\
\hline $\mathrm{F}$ & 0.2456719928 & 0.7393140807 & 0.5411389197 \\
\hline $\mathrm{F}$ & 0.7543280072 & 0.2606859193 & 0.4588611103 \\
\hline $\mathrm{F}$ & 0.2410525353 & 0.0835636734 & 0.9032008983 \\
\hline $\mathrm{F}$ & 0.7589474797 & 0.9164363116 & 0.0967991097 \\
\hline $\mathrm{F}$ & 0.2589474797 & 0.5835636884 & 0.5967991017 \\
\hline $\mathrm{F}$ & 0.7410525203 & 0.4164363116 & 0.4032008983 \\
\hline $\mathrm{F}$ & 0.1409081692 & 0.1616951602 & 0.9303690025 \\
\hline $\mathrm{F}$ & 0.8590918018 & 0.8383048698 & 0.0696309905 \\
\hline $\mathrm{F}$ & 0.3590918308 & 0.6616951302 & 0.5696309975 \\
\hline $\mathrm{F}$ & 0.6409081982 & 0.3383048398 & 0.4303690025 \\
\hline $\mathrm{F}$ & 0.2309173919 & 0.0911789198 & 0.0128112356 \\
\hline $\mathrm{F}$ & 0.7690826381 & 0.9088210802 & 0.9871887484 \\
\hline $\mathrm{F}$ & 0.2690826081 & 0.5911789198 & 0.4871887784 \\
\hline $\mathrm{F}$ & 0.7309173619 & 0.4088210802 & 0.5128112516 \\
\hline $\mathrm{F}$ & 0.2715793224 & 0.7178624881 & 0.8418530072 \\
\hline $\mathrm{F}$ & 0.7284206776 & 0.2821374829 & 0.1581469778 \\
\hline $\mathrm{F}$ & 0.2284206776 & 0.2178625171 & 0.6581469928 \\
\hline $\mathrm{F}$ & 0.7715793224 & 0.7821375119 & 0.3418530072 \\
\hline $\mathrm{F}$ & 0.3201541082 & 0.5705871914 & 0.8047597157 \\
\hline $\mathrm{F}$ & 0.6798458918 & 0.4294128386 & 0.1952402843 \\
\hline $\mathrm{F}$ & 0.1798458918 & 0.0705871614 & 0.6952402843 \\
\hline$F$ & 0.8201541082 & 0.9294128086 & 0.3047597157 \\
\hline $\mathrm{F}$ & 0.3956845913 & 0.7035132933 & 0.8515311950 \\
\hline $\mathrm{F}$ & 0.6043154087 & 0.2964866767 & 0.1484688200 \\
\hline $\mathrm{F}$ & 0.1043154087 & 0.2035133233 & 0.6484688050 \\
\hline $\mathrm{F}$ & 0.8956845913 & 0.7964867067 & 0.3515311950 \\
\hline $\mathrm{F}$ & 0.3383582892 & 0.6025716122 & 0.9172193259 \\
\hline $\mathrm{F}$ & 0.6616416808 & 0.3974283878 & 0.0827806811 \\
\hline
\end{tabular}




\begin{tabular}{|c|c|c|c|}
\hline $\mathrm{F}$ & 0.1616417108 & 0.1025716122 & 0.5827806741 \\
\hline $\mathrm{F}$ & 0.8383583192 & 0.8974283878 & 0.4172193259 \\
\hline B & 0.2164277677 & 0.1434749427 & 0.9514411397 \\
\hline B & 0.7835722473 & 0.8565250723 & 0.0485588533 \\
\hline B & 0.2835722473 & 0.6434749277 & 0.5485588603 \\
\hline B & 0.7164277527 & 0.3565250723 & 0.4514411397 \\
\hline B & 0.3310180993 & 0.6482894461 & 0.8541231914 \\
\hline B & 0.6689819007 & 0.3517105239 & 0.1458768236 \\
\hline B & 0.1689819007 & 0.1482894761 & 0.6458768086 \\
\hline B & 0.8310180993 & 0.8517105539 & 0.3541231914 \\
\hline $5 \cdot \mathrm{Et}_{2} \mathrm{O} \cdot \mathrm{MeCN}$ & $-\mathbf{L S}$ & & \\
\hline \multicolumn{4}{|c|}{ CELL_PARAMETERS (angstrom) } \\
\hline 18.233227397 & 0.000000000 & 0.356503415 & \\
\hline 0.000000000 & 13.522772197 & 0.000000000 & \\
\hline-4.022043278 & 0.000000000 & 20.199606979 & \\
\hline
\end{tabular}

ATOMIC_POSITIONS (crystal)

$\begin{array}{llll}\text { Fe2 } & 0.5133422507 & 0.9829980130 & 0.2076872237 \\ \mathrm{Fe} 2 & 0.4866577492 & 0.0170019651 & 0.7923127612 \\ \mathrm{Fe} 2 & 0.9866577492 & 0.4829980430 & 0.2923127612 \\ \mathrm{Fe} 2 & 0.0133422507 & 0.5170019871 & 0.7076872387 \\ \mathrm{Fe} 2 & 0.0861140766 & 0.9857689847 & 0.2278575306 \\ \mathrm{Fe} 2 & 0.9138858934 & 0.0142310413 & 0.7721424542 \\ \mathrm{Fe} 2 & 0.4138859234 & 0.4857689546 & 0.2721424543 \\ \mathrm{Fe} 2 & 0.5861141066 & 0.5142310153 & 0.7278575458 \\ \mathrm{O} & 0.4216200656 & 0.1047753858 & 0.2190108484 \\ \mathrm{O} & 0.5783799634 & 0.8952246442 & 0.7809891366 \\ \mathrm{O} & 0.0783799343 & 0.6047753558 & 0.2809891365 \\ \mathrm{O} & 0.9216200366 & 0.3952246142 & 0.7190108634 \\ \mathrm{O} & 0.1621683125 & 0.0868926039 & 0.2248900762 \\ \mathrm{O} & 0.8378317165 & 0.9131073890 & 0.7751099098 \\ \mathrm{O} & 0.3378316875 & 0.5868926110 & 0.2751099100 \\ \mathrm{O} & 0.6621682835 & 0.4131073890 & 0.7248900900 \\ \mathrm{O} & 0.4227522830 & 0.9072307715 & 0.2114080500 \\ \mathrm{O} & 0.5772477461 & 0.0927692135 & 0.7885919651 \\ \mathrm{O} & 0.0772477172 & 0.4072307715 & 0.2885919651 \\ \mathrm{O} & 0.9227522539 & 0.5927692285 & 0.7114080349 \\ \mathrm{O} & 0.1735570160 & 0.8976969971 & 0.2452899943 \\ \mathrm{O} & 0.8264429980 & 0.1023029879 & 0.7547099907 \\ \mathrm{O} & 0.3264429980 & 0.3976969971 & 0.2547099907 \\ \mathrm{O} & 0.6735570020 & 0.6023030029 & 0.7452900093 \\ \mathrm{O} & 0.2266925391 & 0.7136613938 & 0.0633230235 \\ \mathrm{O} & 0.7733074748 & 0.2863386362 & 0.9366769546 \\ \mathrm{O} & 0.2733074748 & 0.2136613638 & 0.4366769844 \\ \mathrm{O} & 0.7266925251 & 0.7863386062 & 0.5633230456 \\ \mathrm{~N} & 0.6218102331 & 0.0438346083 & 0.2108580922 \\ \mathrm{~N} & 0.3781897669 & 0.9561654087 & 0.7891419228 \\ \mathrm{~N} & 0.8781897669 & 0.5438345913 & 0.2891419228 \\ \mathrm{~N} & 0.1218102330 & 0.4561653786 & 0.7108580772 \\ \mathrm{~N} & 0.0009374674 & 0.0718255817 & 0.2041291002 \\ \mathrm{~N} & 0.9990625486 & 0.9281744073 & 0.7958708848 \\ \mathrm{~N} & 0.4990625186 & 0.5718255927 & 0.2958708848 \\ \mathrm{~N} & 0.5009374514 & 0.4281744073 & 0.7041291152 \\ \mathrm{~N} & 0.5445113829 & 0.0030451826 & 0.3059454957 \\ \mathrm{~N} & 0.4554886171 & 0.9969548314 & 0.6940544743 \\ \mathrm{~N} & 0.9554886171 & 0.5030451686 & 0.1940545043 \\ \mathrm{~N} & 0.0445113829 & 0.4969548314 & 0.8059455257 \\ \mathrm{~N} & 0.0766792678 & 0.9457655602 & 0.1350672912 \\ & & & \\ & & & \end{array}$




\begin{tabular}{|c|c|c|c|}
\hline $\mathrm{N}$ & 0.9233207252 & 0.0542344328 & 0.8649326938 \\
\hline $\mathrm{N}$ & 0.4233207252 & 0.4457655603 & 0.3649326938 \\
\hline $\mathrm{N}$ & 0.5766792748 & 0.5542344398 & 0.6350673062 \\
\hline $\mathrm{N}$ & 0.4970203039 & 0.0336828339 & 0.1148736145 \\
\hline $\mathrm{N}$ & 0.5029796963 & 0.9663171921 & 0.8851263775 \\
\hline $\mathrm{N}$ & 0.0029796962 & 0.5336828080 & 0.3851263775 \\
\hline $\mathrm{N}$ & 0.9970203037 & 0.4663171620 & 0.6148736225 \\
\hline $\mathrm{N}$ & 0.0870865379 & 0.9986372172 & 0.3221971375 \\
\hline $\mathrm{N}$ & 0.9129134471 & 0.0013627849 & 0.6778028625 \\
\hline $\mathrm{N}$ & 0.4129134471 & 0.4986372171 & 0.1778028625 \\
\hline $\mathrm{N}$ & 0.5870865529 & 0.5013627828 & 0.8221971375 \\
\hline $\mathrm{N}$ & 0.0114723544 & 0.8784928045 & 0.2327037230 \\
\hline $\mathrm{N}$ & 0.9885276376 & 0.1215072094 & 0.7672962621 \\
\hline $\mathrm{N}$ & 0.4885276376 & 0.3784928046 & 0.2672962620 \\
\hline $\mathrm{N}$ & 0.5114723624 & 0.6215071955 & 0.7327037380 \\
\hline $\mathrm{N}$ & 0.5673074394 & 0.8610759618 & 0.1920813831 \\
\hline$N$ & 0.4326925607 & 0.1389240382 & 0.8079186019 \\
\hline $\mathrm{N}$ & 0.9326925607 & 0.3610759618 & 0.3079186019 \\
\hline $\mathrm{N}$ & 0.0673074392 & 0.6389240383 & 0.6920813981 \\
\hline $\mathrm{N}$ & 0.3939173706 & 0.8013957302 & 0.4115595401 \\
\hline $\mathrm{N}$ & 0.6060826294 & 0.1986042838 & 0.5884404599 \\
\hline $\mathrm{N}$ & 0.1060826294 & 0.3013957303 & 0.0884404599 \\
\hline $\mathrm{N}$ & 0.8939173706 & 0.6986042697 & 0.9115595401 \\
\hline C & 0.2334363068 & 0.9409796110 & 0.2340686058 \\
\hline C & 0.7665636782 & 0.0590203891 & 0.7659313642 \\
\hline C & 0.2665636782 & 0.4409796109 & 0.2659313942 \\
\hline C & 0.7334363218 & 0.5590203890 & 0.7340686358 \\
\hline C & 0.2281216253 & 0.0509798445 & 0.2258058602 \\
\hline C & 0.7718783447 & 0.9490201595 & 0.7741941398 \\
\hline C & 0.2718783747 & 0.5509798405 & 0.2741941398 \\
\hline C & 0.7281216553 & 0.4490201595 & 0.7258058602 \\
\hline C & 0.3597769856 & 0.0599290900 & 0.2207687372 \\
\hline C & 0.6402230445 & 0.9400708810 & 0.7792312628 \\
\hline C & 0.1402230144 & 0.5599291190 & 0.2792312628 \\
\hline C & 0.8597769555 & 0.4400709100 & 0.7207687372 \\
\hline C & 0.0035426130 & 0.1687312042 & 0.1881951404 \\
\hline C & 0.9964574100 & 0.8312687808 & 0.8118048596 \\
\hline C & 0.4964573801 & 0.6687312192 & 0.3118048596 \\
\hline C & 0.5035425900 & 0.3312687808 & 0.6881951404 \\
\hline C & 0.5526240561 & 0.0922666941 & 0.1001403552 \\
\hline C & 0.4473759440 & 0.9077333059 & 0.8998596748 \\
\hline C & 0.9473759438 & 0.5922666942 & 0.3998596447 \\
\hline C & 0.0526240561 & 0.4077333058 & 0.6001403252 \\
\hline C & 0.9335581280 & 0.0269707001 & 0.1978656702 \\
\hline C & 0.0664418760 & 0.9730293169 & 0.8021343148 \\
\hline C & 0.5664418720 & 0.5269706831 & 0.3021343148 \\
\hline C & 0.4335581280 & 0.4730292869 & 0.6978656852 \\
\hline C & 0.5120202490 & 0.9643828484 & 0.3541425407 \\
\hline C & 0.4879797510 & 0.0356171506 & 0.6458574304 \\
\hline C & 0.9879797510 & 0.4643828484 & 0.1458574593 \\
\hline C & 0.0120202490 & 0.5356171516 & 0.8541425696 \\
\hline C & 0.6274379515 & 0.0852646984 & 0.1446940691 \\
\hline C & 0.3725620482 & 0.9147352826 & 0.8553059159 \\
\hline C & 0.8725620484 & 0.5852647174 & 0.3553059159 \\
\hline C & 0.1274379518 & 0.4147353126 & 0.6446940841 \\
\hline C & 0.5987527946 & 0.0728285517 & 0.3222569700 \\
\hline C & 0.4012472054 & 0.9271714633 & 0.6777430600 \\
\hline C & 0.9012472054 & 0.5728285367 & 0.1777430300 \\
\hline
\end{tabular}




\begin{tabular}{|c|c|c|c|}
\hline C & 0.0987527946 & 0.4271714633 & 0.8222569400 \\
\hline C & 0.2997364269 & 0.8922187982 & 0.2300921508 \\
\hline C & 0.7002636031 & 0.1077812168 & 0.7699078192 \\
\hline C & 0.2002635731 & 0.3922187982 & 0.2699078493 \\
\hline C & 0.7997363969 & 0.6077812018 & 0.7300921808 \\
\hline C & 0.3614271340 & 0.9482349233 & 0.2204818409 \\
\hline C & 0.6385728960 & 0.0517650697 & 0.7795181592 \\
\hline C & 0.1385728658 & 0.4482349233 & 0.2795181591 \\
\hline C & 0.8614271042 & 0.5517650767 & 0.7204818409 \\
\hline C & 0.2915766409 & 0.1074924991 & 0.2220770464 \\
\hline C & 0.7084233881 & 0.8925075008 & 0.7779229395 \\
\hline C & 0.2084233592 & 0.6074924991 & 0.2779229396 \\
\hline C & 0.7915766119 & 0.3925075009 & 0.7220770604 \\
\hline C & 0.5331535908 & 0.7753861186 & 0.1701043079 \\
\hline C & 0.4668464092 & 0.2246138815 & 0.8298956921 \\
\hline C & 0.9668464093 & 0.2753861186 & 0.3298956921 \\
\hline C & 0.0331535909 & 0.7246138815 & 0.6701043079 \\
\hline C & 0.6428107418 & 0.8657459665 & 0.2005376509 \\
\hline C & 0.3571892583 & 0.1342540034 & 0.7994623491 \\
\hline C & 0.8571892583 & 0.3657459965 & 0.2994623491 \\
\hline C & 0.1428107418 & 0.6342540334 & 0.7005376509 \\
\hline C & 0.6755140258 & 0.9609228570 & 0.2294005100 \\
\hline C & 0.3244859743 & 0.0390771570 & 0.7705995200 \\
\hline C & 0.8244859743 & 0.4609228569 & 0.2705994900 \\
\hline C & 0.1755140257 & 0.5390771430 & 0.7294004800 \\
\hline C & 0.6290982554 & 0.1172692546 & 0.2650406372 \\
\hline C & 0.3709017446 & 0.8827307524 & 0.7349593628 \\
\hline C & 0.8709017446 & 0.6172692476 & 0.2349593628 \\
\hline C & 0.1290982554 & 0.3827307525 & 0.7650406372 \\
\hline C & 0.0272859872 & 0.8175048509 & 0.0591302012 \\
\hline C & 0.9727140108 & 0.1824951791 & 0.9408697949 \\
\hline C & 0.4727140108 & 0.3175048209 & 0.4408697948 \\
\hline C & 0.5272859892 & 0.6824951491 & 0.5591302052 \\
\hline C & 0.9392678883 & 0.2230896720 & 0.1664003854 \\
\hline C & 0.0607321147 & 0.7769103430 & 0.8335996446 \\
\hline C & 0.5607321117 & 0.7230896570 & 0.3335996146 \\
\hline C & 0.4392678883 & 0.2769103430 & 0.6664003554 \\
\hline C & 0.0994772563 & 0.9951265882 & 0.0852081893 \\
\hline C & 0.9005227517 & 0.0048734129 & 0.9147917958 \\
\hline C & 0.4005227517 & 0.4951265881 & 0.4147917958 \\
\hline C & 0.5994772483 & 0.5048734118 & 0.5852082041 \\
\hline C & 0.8697695882 & 0.1772940360 & 0.1596766971 \\
\hline C & 0.1302304048 & 0.8227059490 & 0.8403232879 \\
\hline C & 0.6302304118 & 0.6772940511 & 0.3403232879 \\
\hline C & 0.3697695882 & 0.3227059489 & 0.6596767121 \\
\hline C & 0.0547876262 & 0.9213312217 & 0.3487249093 \\
\hline C & 0.9452123968 & 0.0786687643 & 0.6512750907 \\
\hline C & 0.4452123669 & 0.4213312217 & 0.1512750907 \\
\hline C & 0.5547876031 & 0.5786687783 & 0.8487249093 \\
\hline C & 0.5728665209 & 0.6930282771 & 0.1555195673 \\
\hline C & 0.4271334789 & 0.3069717228 & 0.8444804177 \\
\hline C & 0.9271334792 & 0.1930282771 & 0.3444804177 \\
\hline C & 0.0728665208 & 0.8069717229 & 0.6555195823 \\
\hline C & 0.0284005650 & 0.8388106360 & 0.3013261031 \\
\hline C & 0.9715994320 & 0.1611893790 & 0.6986738679 \\
\hline C & 0.4715994320 & 0.3388106360 & 0.1986738969 \\
\hline C & 0.5284005680 & 0.6611893640 & 0.8013261321 \\
\hline C & 0.1172529989 & 0.0728035758 & 0.3622146178 \\
\hline
\end{tabular}




\begin{tabular}{|c|c|c|c|}
\hline C & 0.8827470161 & 0.9271963942 & 0.6377853521 \\
\hline C & 0.3827470161 & 0.5728036058 & 0.1377853822 \\
\hline C & 0.6172529838 & 0.4271964242 & 0.8622146479 \\
\hline C & 0.5415785363 & 0.1542443260 & 0.0452424564 \\
\hline C & 0.4584214636 & 0.8457557041 & 0.9547575586 \\
\hline C & 0.9584214636 & 0.6542442960 & 0.4547575585 \\
\hline C & 0.0415785363 & 0.3457556740 & 0.5452424414 \\
\hline C & 0.0420734338 & 0.8570813298 & 0.1224218557 \\
\hline C & 0.9579265662 & 0.1429186402 & 0.8775781443 \\
\hline C & 0.4579265662 & 0.3570813599 & 0.3775781443 \\
\hline C & 0.5420734338 & 0.6429186702 & 0.6224218557 \\
\hline C & 0.8673314125 & 0.0772848625 & 0.1750668752 \\
\hline C & 0.1326685874 & 0.9227151595 & 0.8249331248 \\
\hline C & 0.6326685874 & 0.5772848405 & 0.3249331247 \\
\hline C & 0.3673314125 & 0.4227151305 & 0.6750668752 \\
\hline C & 0.6222889828 & 0.1031051506 & 0.3871463322 \\
\hline C & 0.3777110173 & 0.8968948194 & 0.6128536978 \\
\hline C & 0.8777110173 & 0.6031051806 & 0.1128536678 \\
\hline C & 0.1222889828 & 0.3968948494 & 0.8871463022 \\
\hline C & 0.6854861593 & 0.7863569767 & 0.1858281584 \\
\hline C & 0.3145138406 & 0.2136430233 & 0.8141718416 \\
\hline C & 0.8145138406 & 0.2863569767 & 0.3141718416 \\
\hline C & 0.1854861594 & 0.7136430233 & 0.6858281584 \\
\hline C & 0.0507763699 & 0.8687256660 & 0.0077108696 \\
\hline C & 0.9492236231 & 0.1312743340 & 0.9922891543 \\
\hline C & 0.4492236231 & 0.3687256660 & 0.4922891253 \\
\hline C & 0.5507763769 & 0.6312743340 & 0.5077108456 \\
\hline C & 0.4724463261 & 0.1553967853 & 1.0036806275 \\
\hline C & 0.5275537038 & 0.8446032147 & -0.0036806346 \\
\hline C & 0.0275536739 & 0.6553967853 & 0.4963193724 \\
\hline C & 0.9724462962 & 0.3446032147 & 0.5036806275 \\
\hline C & 0.4300905913 & 0.0358823332 & 0.0744247906 \\
\hline C & 0.5699094086 & 0.9641176518 & 0.9255751864 \\
\hline C & 0.0699094086 & 0.5358823482 & 0.4255752164 \\
\hline C & 0.9300905914 & 0.4641176519 & 0.5744248136 \\
\hline C & 0.5331269025 & -0.0088704814 & 0.4199292360 \\
\hline C & 0.4668730975 & 1.0088704855 & 0.5800707640 \\
\hline C & 0.9668730975 & 0.4911295145 & 0.0800707640 \\
\hline C & 0.0331269025 & 0.5088704853 & 0.9199292360 \\
\hline C & 0.0882510292 & 0.9583034547 & 0.0211189136 \\
\hline C & 0.9117490008 & 0.0416965643 & 0.9788810673 \\
\hline C & 0.4117489709 & 0.4583034247 & 0.4788810973 \\
\hline C & 0.5882509992 & 0.5416965453 & 0.5211189326 \\
\hline C & 0.6503185442 & 0.6986293083 & 0.1628035349 \\
\hline C & 0.3496814558 & 0.3013707207 & 0.8371964801 \\
\hline C & 0.8496814558 & 0.1986292793 & 0.3371964801 \\
\hline C & 0.1503185443 & 0.8013706918 & 0.6628035199 \\
\hline C & 0.9340345229 & 0.9204269852 & 0.2178301758 \\
\hline C & 0.0659654510 & 0.0795730298 & 0.7821698391 \\
\hline C & 0.5659654771 & 0.4204269852 & 0.2821698391 \\
\hline C & 0.4340345530 & 0.5795730148 & 0.7178301607 \\
\hline C & 0.4157604841 & 0.0947183787 & 0.0183600722 \\
\hline C & 0.5842395160 & 0.9052816363 & 0.9816399498 \\
\hline C & 0.0842395159 & 0.5947183637 & 0.4816399199 \\
\hline C & 0.9157604841 & 0.4052816363 & 0.5183600502 \\
\hline C & 0.0243331444 & 0.8045808142 & 0.1820784885 \\
\hline C & 0.9756668386 & 0.1954192008 & 0.8179214815 \\
\hline C & 0.4756668686 & 0.3045808142 & 0.3179215115 \\
\hline
\end{tabular}




\begin{tabular}{|c|c|c|c|}
\hline C & 0.5243331614 & 0.6954191858 & 0.6820785185 \\
\hline C & 0.5889349170 & 0.0616513214 & 0.4368190497 \\
\hline C & 0.4110650830 & 0.9383487006 & 0.5631809203 \\
\hline C & 0.9110650831 & 0.5616512994 & 0.0631809503 \\
\hline C & 0.0889349169 & 0.4383486706 & 0.9368190797 \\
\hline C & 0.1164275281 & 0.0725368098 & 0.4296325339 \\
\hline C & 0.8835724569 & 0.9274631902 & 0.5703674951 \\
\hline C & 0.3835724569 & 0.5725368097 & 0.0703674660 \\
\hline C & 0.6164275431 & 0.4274631903 & 0.9296325048 \\
\hline C & 0.0505682009 & 0.9178122987 & 0.4155827051 \\
\hline C & 0.9494317692 & 0.0821876713 & 0.5844172950 \\
\hline C & 0.4494317991 & 0.4178123287 & 0.0844172949 \\
\hline C & 0.5505682308 & 0.5821877013 & 0.9155827050 \\
\hline C & 0.0823632797 & 0.9940804824 & 0.4569067789 \\
\hline C & 0.9176367124 & 0.0059195016 & 0.5430932211 \\
\hline C & 0.4176367124 & 0.4940805114 & 0.0430932211 \\
\hline C & 0.5823632876 & 0.5059195176 & 0.9569067790 \\
\hline C & 0.1669603914 & 0.5741782828 & 0.1017246147 \\
\hline C & 0.8330396087 & 0.4258217172 & 0.8982754153 \\
\hline C & 0.3330396087 & 0.0741782828 & 0.3982753853 \\
\hline C & 0.6669603913 & 0.9258217171 & 0.6017245847 \\
\hline C & 0.1807916244 & 0.6304157005 & 0.0415241573 \\
\hline C & 0.8192083456 & 0.3695842995 & 0.9584758657 \\
\hline C & 0.3192083757 & 0.1304157005 & 0.4584758357 \\
\hline C & 0.6807916544 & 0.8695842995 & 0.5415241343 \\
\hline C & 0.2866227956 & 0.8617698107 & 0.0372896940 \\
\hline C & 0.7133771755 & 0.1382302193 & 0.9627103361 \\
\hline C & 0.2133772045 & 0.3617697807 & 0.4627103060 \\
\hline C & 0.7866228245 & 0.6382301893 & 0.5372896639 \\
\hline C & 0.2383582859 & 0.7754776704 & 0.0096691047 \\
\hline C & 0.7616417291 & 0.2245223446 & 0.9903309162 \\
\hline C & 0.2616417291 & 0.2754776704 & 0.4903308863 \\
\hline C & 0.7383582709 & 0.7245223296 & 0.5096690837 \\
\hline C & 0.3291942294 & 0.8028461983 & 0.4062803718 \\
\hline C & 0.6708057996 & 0.1971538166 & 0.5937196281 \\
\hline C & 0.1708057705 & 0.3028461984 & 0.0937196281 \\
\hline C & 0.8291942004 & 0.6971538016 & 0.9062803719 \\
\hline C & 0.2489545110 & 0.8065514408 & 0.4005337170 \\
\hline C & 0.7510454890 & 0.1934485592 & 0.5994663131 \\
\hline C & 0.2510454890 & 0.3065514408 & 0.0994662832 \\
\hline C & 0.7489545110 & 0.6934485592 & 0.9005336869 \\
\hline $\mathrm{H}$ & 0.0590279521 & 0.2008769441 & 0.1929433562 \\
\hline $\mathrm{H}$ & 0.9409720249 & 0.7991230410 & 0.8070566588 \\
\hline $\mathrm{H}$ & 0.4409720549 & 0.7008769590 & 0.3070566588 \\
\hline $\mathrm{H}$ & 0.5590279751 & 0.2991230410 & 0.6929433412 \\
\hline $\mathrm{H}$ & 0.4676604383 & 0.9108273127 & 0.3388784914 \\
\hline $\mathrm{H}$ & 0.5323395617 & 0.0891726833 & 0.6611215086 \\
\hline $\mathrm{H}$ & 0.0323395616 & 0.4108273127 & 0.1611215086 \\
\hline $\mathrm{H}$ & 0.9676604383 & 0.5891726874 & 0.8388784914 \\
\hline $\mathrm{H}$ & 0.6628797306 & 1.0353036371 & 0.1213329836 \\
\hline $\mathrm{H}$ & 0.3371202694 & -0.0353036611 & 0.8786670234 \\
\hline $\mathrm{H}$ & 0.8371202695 & 0.5353036661 & 0.3786670234 \\
\hline $\mathrm{H}$ & 0.1628797306 & 0.4646963629 & 0.6213329766 \\
\hline $\mathrm{H}$ & 0.6548154888 & 0.1580503734 & 0.1498395536 \\
\hline $\mathrm{H}$ & 0.3451845111 & 0.8419496196 & 0.8501604314 \\
\hline $\mathrm{H}$ & 0.8451845111 & 0.6580503803 & 0.3501604314 \\
\hline $\mathrm{H}$ & 0.1548154888 & 0.3419496197 & 0.6498395686 \\
\hline $\mathrm{H}$ & 0.3033737606 & 0.8117342179 & 0.2324354700 \\
\hline
\end{tabular}




\begin{tabular}{|c|c|c|c|}
\hline $\mathrm{H}$ & 0.6966262694 & 0.1882657531 & 0.7675645450 \\
\hline $\mathrm{H}$ & 0.1966262394 & 0.3117342469 & 0.2675645449 \\
\hline $\mathrm{H}$ & 0.8033737306 & 0.6882657822 & 0.7324354551 \\
\hline $\mathrm{H}$ & 0.2883895873 & 0.1880241423 & 0.2212181401 \\
\hline $\mathrm{H}$ & 0.7116104127 & 0.8119758727 & 0.7787818449 \\
\hline $\mathrm{H}$ & 0.2116104126 & 0.6880241273 & 0.2787818449 \\
\hline $\mathrm{H}$ & 0.7883895873 & 0.3119758727 & 0.7212181550 \\
\hline $\mathrm{H}$ & 0.4726727322 & 0.7756316459 & 0.1650657677 \\
\hline $\mathrm{H}$ & 0.5273272378 & 0.2243683541 & 0.8349342323 \\
\hline $\mathrm{H}$ & 0.0273272677 & 0.2756316460 & 0.3349342323 \\
\hline $\mathrm{H}$ & 0.9726727622 & 0.7243683541 & 0.6650657676 \\
\hline $\mathrm{H}$ & 0.6861501530 & 0.9533760817 & 0.2834712435 \\
\hline $\mathrm{H}$ & 0.3138498470 & 0.0466239113 & 0.7165287565 \\
\hline $\mathrm{H}$ & 0.8138498469 & 0.4533760817 & 0.2165287565 \\
\hline $\mathrm{H}$ & 0.1861501531 & 0.5466239183 & 0.7834712435 \\
\hline $\mathrm{H}$ & 0.7294992092 & 0.9765904429 & 0.2147903990 \\
\hline $\mathrm{H}$ & 0.2705007907 & 0.0234095422 & 0.7852095860 \\
\hline $\mathrm{H}$ & 0.7705007908 & 0.4765904429 & 0.2852095860 \\
\hline $\mathrm{H}$ & 0.2294992094 & 0.5234095570 & 0.7147904140 \\
\hline $\mathrm{H}$ & 0.5960512589 & 0.1826174540 & 0.2462526261 \\
\hline H & 0.4039487412 & 0.8173825760 & 0.7537473439 \\
\hline $\mathrm{H}$ & 0.9039487412 & 0.6826174239 & 0.2537473739 \\
\hline $\mathrm{H}$ & 0.0960512589 & 0.3173825460 & 0.7462526561 \\
\hline H & 0.6871853279 & 0.1420307811 & 0.2804072310 \\
\hline $\mathrm{H}$ & 0.3128146721 & 0.8579692038 & 0.7195927990 \\
\hline $\mathrm{H}$ & 0.8128146721 & 0.6420307961 & 0.2195927690 \\
\hline $\mathrm{H}$ & 0.1871853279 & 0.3579692040 & 0.7804072010 \\
\hline $\mathrm{H}$ & 0.9965073747 & 0.7483204424 & 0.0501771429 \\
\hline $\mathrm{H}$ & 0.0034926363 & 0.2516795576 & 0.9498228421 \\
\hline $\mathrm{H}$ & 0.5034926253 & 0.2483204424 & 0.4498228421 \\
\hline $\mathrm{H}$ & 0.4965073747 & 0.7516795576 & 0.5501771578 \\
\hline $\mathrm{H}$ & 0.9436343150 & 0.3008032643 & 0.1543091153 \\
\hline $\mathrm{H}$ & 0.0563656890 & 0.6991967056 & 0.8456908847 \\
\hline H & 0.5563656850 & 0.8008032944 & 0.3456908847 \\
\hline H & 0.4436343150 & 0.1991967356 & 0.6543091152 \\
\hline $\mathrm{H}$ & 0.1268800134 & 0.0659580195 & 0.0976952608 \\
\hline $\mathrm{H}$ & 0.8731200165 & 0.9340419615 & 0.9023047103 \\
\hline $\mathrm{H}$ & 0.3731199866 & 0.5659580385 & 0.4023047392 \\
\hline $\mathrm{H}$ & 0.6268799835 & 0.4340419915 & 0.5976952896 \\
\hline H & 0.8190363344 & 0.2195651588 & 0.1427785350 \\
\hline $\mathrm{H}$ & 0.1809636796 & 0.7804348262 & 0.8572214650 \\
\hline $\mathrm{H}$ & 0.6809636656 & 0.7195651738 & 0.3572214650 \\
\hline H & 0.3190363344 & 0.2804348262 & 0.6427785350 \\
\hline $\mathrm{H}$ & 0.5432741185 & 0.6252508068 & 0.1384375457 \\
\hline $\mathrm{H}$ & 0.4567258818 & 0.3747491932 & 0.8615624392 \\
\hline $\mathrm{H}$ & 0.9567258816 & 0.1252508068 & 0.3615624393 \\
\hline $\mathrm{H}$ & 0.0432741184 & 0.8747491932 & 0.6384375607 \\
\hline H & 0.0727001126 & 0.7829895636 & 0.3032659094 \\
\hline $\mathrm{H}$ & 0.9272998643 & 0.2170104665 & 0.6967340607 \\
\hline $\mathrm{H}$ & 0.4272998944 & 0.2829895335 & 0.1967340906 \\
\hline $\mathrm{H}$ & 0.5727001356 & 0.7170104365 & 0.8032659394 \\
\hline $\mathrm{H}$ & -0.0201015376 & 0.8009529286 & 0.3152745100 \\
\hline H & 1.0201015526 & 0.1990470713 & 0.6847255200 \\
\hline $\mathrm{H}$ & 0.5201015226 & 0.3009529285 & 0.1847254901 \\
\hline $\mathrm{H}$ & 0.4798984474 & 0.6990470713 & 0.8152744801 \\
\hline $\mathrm{H}$ & 0.1432658693 & 0.1322397332 & 0.3385757705 \\
\hline $\mathrm{H}$ & 0.8567341158 & 0.8677602447 & 0.6614242594 \\
\hline $\mathrm{H}$ & 0.3567341158 & 0.6322397552 & 0.1614242293 \\
\hline
\end{tabular}




\begin{tabular}{|c|c|c|c|}
\hline $\mathrm{H}$ & 0.6432658842 & 0.3677602738 & 0.8385757407 \\
\hline $\mathrm{H}$ & 0.5867952609 & 0.2022657957 & 0.0365166189 \\
\hline $\mathrm{H}$ & 0.4132047391 & 0.7977342343 & 0.9634833890 \\
\hline $\mathrm{H}$ & 0.9132047391 & 0.7022657657 & 0.4634833891 \\
\hline $\mathrm{H}$ & 0.0867952609 & 0.2977342043 & 0.5365166110 \\
\hline $\mathrm{H}$ & 0.8149656672 & 0.0366848184 & 0.1681314369 \\
\hline $\mathrm{H}$ & 0.1850343038 & 0.9633152036 & 0.8318685631 \\
\hline $\mathrm{H}$ & 0.6850343328 & 0.5366847964 & 0.3318685630 \\
\hline $\mathrm{H}$ & 0.3149656962 & 0.4633151736 & 0.6681314370 \\
\hline $\mathrm{H}$ & 0.6660209081 & 0.1586796750 & 0.3989637167 \\
\hline $\mathrm{H}$ & 0.3339790918 & 0.8413203099 & 0.6010362533 \\
\hline $\mathrm{H}$ & 0.8339790919 & 0.6586796901 & 0.1010362833 \\
\hline $\mathrm{H}$ & 0.1660209080 & 0.3413203098 & 0.8989637467 \\
\hline $\mathrm{H}$ & 0.7456594664 & 0.7949885506 & 0.1923524688 \\
\hline $\mathrm{H}$ & 0.2543405336 & 0.2050114794 & 0.8076475163 \\
\hline $\mathrm{H}$ & 0.7543405335 & 0.2949885205 & 0.3076475163 \\
\hline $\mathrm{H}$ & 0.2456594665 & 0.7050114494 & 0.6923524838 \\
\hline $\mathrm{H}$ & 0.0387672253 & 0.8393436901 & 0.9576443871 \\
\hline $\mathrm{H}$ & 0.9612327857 & 0.1606563249 & 0.0423556209 \\
\hline $\mathrm{H}$ & 0.4612327856 & 0.3393436901 & 0.5423556129 \\
\hline $\mathrm{H}$ & 0.5387672143 & 0.6606563099 & 0.4576443870 \\
\hline $\mathrm{H}$ & 0.4625761163 & 0.2035937960 & 0.9605748726 \\
\hline $\mathrm{H}$ & 0.5374238839 & 0.7964062040 & 0.0394251542 \\
\hline $\mathrm{H}$ & 0.0374238838 & 0.7035937960 & 0.5394251274 \\
\hline $\mathrm{H}$ & 0.9625761162 & 0.2964062040 & 0.4605748427 \\
\hline $\mathrm{H}$ & 0.3871103518 & -0.0107317820 & 0.0889407851 \\
\hline $\mathrm{H}$ & 0.6128896192 & 1.0107317751 & 0.9110592440 \\
\hline $\mathrm{H}$ & 0.1128896481 & 0.4892682250 & 0.4110592149 \\
\hline $\mathrm{H}$ & 0.8871103808 & 0.5107317750 & 0.5889407560 \\
\hline $\mathrm{H}$ & 0.5057986932 & -0.0449394406 & 0.4564894252 \\
\hline $\mathrm{H}$ & 0.4942013068 & 1.0449394226 & 0.5435105748 \\
\hline $\mathrm{H}$ & 0.9942013068 & 0.4550605774 & 0.0435105748 \\
\hline $\mathrm{H}$ & 0.0057986932 & 0.5449394525 & 0.9564894252 \\
\hline $\mathrm{H}$ & 0.1075213339 & 1.0014408763 & 0.9827311168 \\
\hline $\mathrm{H}$ & 0.8924786431 & -0.0014408653 & 0.0172688684 \\
\hline $\mathrm{H}$ & 0.3924786731 & 0.5014408763 & 0.5172688834 \\
\hline $\mathrm{H}$ & 0.6075213569 & 0.4985591237 & 0.4827311166 \\
\hline $\mathrm{H}$ & 0.6818349280 & 0.6350233623 & 0.1504800415 \\
\hline $\mathrm{H}$ & 0.3181650720 & 0.3649766677 & 0.8495199284 \\
\hline $\mathrm{H}$ & 0.8181650721 & 0.1350233323 & 0.3495199585 \\
\hline $\mathrm{H}$ & 0.1818349280 & 0.8649766377 & 0.6504800716 \\
\hline $\mathrm{H}$ & 0.9077856566 & 0.9140190663 & 0.2618087147 \\
\hline $\mathrm{H}$ & 0.0922143505 & 0.0859809117 & 0.7381912854 \\
\hline $\mathrm{H}$ & 0.5922143435 & 0.4140190963 & 0.2381912854 \\
\hline $\mathrm{H}$ & 0.4077856566 & 0.5859809337 & 0.7618087147 \\
\hline $\mathrm{H}$ & 0.8999307333 & 0.8774514785 & 0.1782543126 \\
\hline $\mathrm{H}$ & 0.1000692446 & 0.1225485065 & 0.8217456574 \\
\hline $\mathrm{H}$ & 0.6000692667 & 0.3774514785 & 0.3217456873 \\
\hline $\mathrm{H}$ & 0.3999307624 & 0.6225485215 & 0.6782543427 \\
\hline $\mathrm{H}$ & 0.3607863213 & 0.0935247254 & 0.9875714073 \\
\hline $\mathrm{H}$ & 0.6392136496 & 0.9064752446 & 0.0124285888 \\
\hline $\mathrm{H}$ & 0.1392136787 & 0.5935247554 & 0.5124285927 \\
\hline $\mathrm{H}$ & 0.8607863503 & 0.4064752746 & 0.4875714074 \\
\hline $\mathrm{H}$ & 0.9764160891 & 0.7545961985 & 0.1684576658 \\
\hline $\mathrm{H}$ & 0.0235839039 & 0.2454038316 & 0.8315423342 \\
\hline $\mathrm{H}$ & 0.5235839109 & 0.2545961684 & 0.3315423342 \\
\hline $\mathrm{H}$ & 0.4764160890 & 0.7454038016 & 0.6684576658 \\
\hline $\mathrm{H}$ & 0.0725054520 & 0.7592738825 & 0.2039484698 \\
\hline
\end{tabular}




\begin{tabular}{|c|c|c|c|}
\hline $\mathrm{H}$ & 0.9274945630 & 0.2407261175 & 0.7960515152 \\
\hline $\mathrm{H}$ & 0.4274945630 & 0.2592738825 & 0.2960515152 \\
\hline $\mathrm{H}$ & 0.5725054370 & 0.7407261174 & 0.7039484849 \\
\hline $\mathrm{H}$ & 0.6062823177 & 0.0853177565 & 0.4878155783 \\
\hline $\mathrm{H}$ & 0.3937176822 & 0.9146822585 & 0.5121843918 \\
\hline $\mathrm{H}$ & 0.8937176823 & 0.5853177414 & 0.0121844217 \\
\hline $\mathrm{H}$ & 0.1062823177 & 0.4146822586 & 0.9878156082 \\
\hline $\mathrm{H}$ & 0.1424528535 & 0.1327612939 & 0.4604010230 \\
\hline $\mathrm{H}$ & 0.8575471766 & 0.8672386911 & 0.5395989770 \\
\hline $\mathrm{H}$ & 0.3575471466 & 0.6327613089 & 0.0395989771 \\
\hline $\mathrm{H}$ & 0.6424528235 & 0.3672386911 & 0.9604010229 \\
\hline $\mathrm{H}$ & 0.0223841426 & 0.8558098792 & 0.4344699960 \\
\hline $\mathrm{H}$ & 0.9776158464 & 0.1441901358 & 0.5655299741 \\
\hline $\mathrm{H}$ & 0.4776158464 & 0.3558098792 & 0.0655300041 \\
\hline $\mathrm{H}$ & 0.5223841537 & 0.6441901208 & 0.9344700259 \\
\hline $\mathrm{H}$ & 0.0809869723 & 0.9932592821 & 0.5096443957 \\
\hline $\mathrm{H}$ & 0.9190130577 & 0.0067407219 & 0.4903556042 \\
\hline $\mathrm{H}$ & 0.4190130275 & 0.4932592821 & 0.9903556043 \\
\hline $\mathrm{H}$ & 0.5809869424 & 0.5067407179 & 0.0096443957 \\
\hline $\mathrm{H}$ & 0.1314012264 & 0.5094387945 & 0.0876585076 \\
\hline $\mathrm{H}$ & 0.8685987506 & 0.4905611755 & 0.9123414774 \\
\hline $\mathrm{H}$ & 0.3685987805 & 0.0094388245 & 0.4123414774 \\
\hline $\mathrm{H}$ & 0.6314012494 & 0.9905612055 & 0.5876585226 \\
\hline $\mathrm{H}$ & 0.1395924523 & 0.6215947177 & 0.1336180491 \\
\hline $\mathrm{H}$ & 0.8604075177 & 0.3784052823 & 0.8663819508 \\
\hline $\mathrm{H}$ & 0.3604075477 & 0.1215947176 & 0.3663819508 \\
\hline $\mathrm{H}$ & 0.6395924823 & 0.8784052823 & 0.6336180493 \\
\hline $\mathrm{H}$ & 0.2196632977 & 0.5481527511 & 0.1310062868 \\
\hline $\mathrm{H}$ & 0.7803367174 & 0.4518472490 & 0.8689937132 \\
\hline $\mathrm{H}$ & 0.2803367173 & 0.0481527511 & 0.3689937132 \\
\hline $\mathrm{H}$ & 0.7196632826 & 0.9518472489 & 0.6310062868 \\
\hline $\mathrm{H}$ & 0.2082503991 & 0.5832028969 & 0.0088091017 \\
\hline $\mathrm{H}$ & 0.7917496300 & 0.4167971032 & 0.9911908833 \\
\hline $\mathrm{H}$ & 0.2917496010 & 0.0832028969 & 0.4911908834 \\
\hline $\mathrm{H}$ & 0.7082503701 & 0.9167971032 & 0.5088091167 \\
\hline $\mathrm{H}$ & 0.1275736714 & 0.6560554329 & 0.0115893722 \\
\hline $\mathrm{H}$ & 0.8724263136 & 0.3439445971 & 0.9884106567 \\
\hline $\mathrm{H}$ & 0.3724263136 & 0.1560554029 & 0.4884106268 \\
\hline $\mathrm{H}$ & 0.6275736864 & 0.8439445671 & 0.5115893433 \\
\hline $\mathrm{H}$ & 0.2947930906 & 0.9124400866 & -0.0025470264 \\
\hline $\mathrm{H}$ & 0.7052069094 & 0.0875599064 & 1.0025470464 \\
\hline $\mathrm{H}$ & 0.2052069094 & 0.4124400866 & 0.5025470174 \\
\hline $\mathrm{H}$ & 0.7947930906 & 0.5875599134 & 0.4974529536 \\
\hline $\mathrm{H}$ & 0.3412971857 & 0.8356309038 & 0.0628685598 \\
\hline $\mathrm{H}$ & 0.6587028443 & 0.1643690963 & 0.9371314542 \\
\hline $\mathrm{H}$ & 0.1587028143 & 0.3356309038 & 0.4371314542 \\
\hline $\mathrm{H}$ & 0.8412971557 & 0.6643690962 & 0.5628685458 \\
\hline $\mathrm{H}$ & 0.2602292679 & 0.9038790677 & 0.0725936417 \\
\hline $\mathrm{H}$ & 0.7397707322 & 0.0961209393 & 0.9274063883 \\
\hline $\mathrm{H}$ & 0.2397707321 & 0.4038790677 & 0.4274063583 \\
\hline $\mathrm{H}$ & 0.7602292678 & 0.5961209323 & 0.5725936117 \\
\hline $\mathrm{H}$ & 0.1839424560 & 0.8019189176 & 0.9827887208 \\
\hline $\mathrm{H}$ & 0.8160575590 & 0.1980810675 & 0.0172112922 \\
\hline $\mathrm{H}$ & 0.3160575591 & 0.3019189177 & 0.5172112792 \\
\hline $\mathrm{H}$ & 0.6839424409 & 0.6980810824 & 0.4827887208 \\
\hline $\mathrm{H}$ & 0.2645347817 & 0.7322681589 & 0.9742047418 \\
\hline $\mathrm{H}$ & 0.7354652183 & 0.2677318411 & 0.0257952581 \\
\hline $\mathrm{H}$ & 0.2354652183 & 0.2322681589 & 0.5257952582 \\
\hline
\end{tabular}




\begin{tabular}{|c|c|c|c|}
\hline $\mathrm{H}$ & 0.7645347816 & 0.7677318411 & 0.4742047419 \\
\hline $\mathrm{H}$ & 0.2340874034 & 0.8523166911 & 0.4404012625 \\
\hline $\mathrm{H}$ & 0.7659125966 & 0.1476832788 & 0.5595987375 \\
\hline $\mathrm{H}$ & 0.2659125966 & 0.3523167210 & 0.0595987375 \\
\hline $\mathrm{H}$ & 0.7340874034 & 0.6476833088 & 0.9404012625 \\
\hline $\mathrm{H}$ & 0.2276821240 & 0.7315163631 & 0.4059072388 \\
\hline $\mathrm{H}$ & 0.7723178460 & 0.2684836079 & 0.5940927912 \\
\hline $\mathrm{H}$ & 0.2723178760 & 0.2315163921 & 0.0940927612 \\
\hline $\mathrm{H}$ & 0.7276821539 & 0.7684836369 & 0.9059072090 \\
\hline $\mathrm{H}$ & 0.2236726942 & 0.8378456837 & 0.3524665671 \\
\hline $\mathrm{H}$ & 0.7763273058 & 0.1621543465 & 0.6475334330 \\
\hline $\mathrm{H}$ & 0.2763273058 & 0.3378456534 & 0.1475334329 \\
\hline $\mathrm{H}$ & 0.7236726942 & 0.6621543164 & 0.8524665671 \\
\hline $\mathrm{F}$ & 0.2529903783 & 0.2364235820 & 0.9491451092 \\
\hline $\mathrm{F}$ & 0.7470096217 & 0.7635764330 & 0.0508548719 \\
\hline $\mathrm{F}$ & 0.2470096217 & 0.7364235670 & 0.5508548909 \\
\hline $\mathrm{F}$ & 0.7529903783 & 0.2635764330 & 0.4491451392 \\
\hline $\mathrm{F}$ & 0.2517881148 & 0.0735486801 & 0.9118082899 \\
\hline $\mathrm{F}$ & 0.7482119002 & 0.9264513049 & 0.0881917180 \\
\hline $\mathrm{F}$ & 0.2482119002 & 0.5735486951 & 0.5881917099 \\
\hline $\mathrm{F}$ & 0.7517880998 & 0.4264513049 & 0.4118082901 \\
\hline $\mathrm{F}$ & 0.1413476170 & 0.1504017842 & 0.9254265423 \\
\hline $\mathrm{F}$ & 0.8586523540 & 0.8495982458 & 0.0745734507 \\
\hline $\mathrm{F}$ & 0.3586523830 & 0.6504017542 & 0.5745734579 \\
\hline $\mathrm{F}$ & 0.6413476460 & 0.3495982158 & 0.4254265422 \\
\hline $\mathrm{F}$ & 0.2294293951 & 0.1072249355 & 0.0171793836 \\
\hline $\mathrm{F}$ & 0.7705706349 & 0.8927750645 & 0.9828206003 \\
\hline $\mathrm{F}$ & 0.2705706049 & 0.6072249355 & 0.4828206303 \\
\hline $\mathrm{F}$ & 0.7294293651 & 0.3927750645 & 0.5171793996 \\
\hline $\mathrm{F}$ & 0.2455489098 & 0.6172679739 & 0.8745176508 \\
\hline $\mathrm{F}$ & 0.7544510902 & 0.3827319971 & 0.1254823341 \\
\hline $\mathrm{F}$ & 0.2544510902 & 0.1172680029 & 0.6254823492 \\
\hline $\mathrm{F}$ & 0.7455489098 & 0.8827320261 & 0.3745176508 \\
\hline $\mathrm{F}$ & 0.3415761351 & 0.5273458448 & 0.8414690855 \\
\hline $\mathrm{F}$ & 0.6584238649 & 0.4726541852 & 0.1585309145 \\
\hline $\mathrm{F}$ & 0.1584238649 & 0.0273458149 & 0.6585309145 \\
\hline $\mathrm{F}$ & 0.8415761351 & 0.9726541551 & 0.3414690855 \\
\hline $\mathrm{F}$ & 0.3250197011 & 0.6923817454 & 0.8124286321 \\
\hline $\mathrm{F}$ & 0.6749802990 & 0.3076182246 & 0.1875713829 \\
\hline $\mathrm{F}$ & 0.1749802989 & 0.1923817754 & 0.6875713679 \\
\hline $\mathrm{F}$ & 0.8250197010 & 0.8076182546 & 0.3124286321 \\
\hline $\mathrm{F}$ & 0.3688133542 & 0.6491058573 & 0.9216638558 \\
\hline $\mathrm{F}$ & 0.6311866158 & 0.3508941429 & 0.0783361512 \\
\hline $\mathrm{F}$ & 0.1311866458 & 0.1491058572 & 0.5783361442 \\
\hline $\mathrm{F}$ & 0.8688133842 & 0.8508941427 & 0.4216638558 \\
\hline B & 0.2187615589 & 0.1422038429 & 0.9514075768 \\
\hline B & 0.7812384561 & 0.8577961721 & 0.0485924161 \\
\hline B & 0.2812384561 & 0.6422038279 & 0.5485924232 \\
\hline B & 0.7187615439 & 0.3577961721 & 0.4514075769 \\
\hline B & 0.3199808824 & 0.6210196714 & 0.8629966779 \\
\hline B & 0.6800191176 & 0.3789802985 & 0.1370033371 \\
\hline B & 0.1800191176 & 0.1210197014 & 0.6370033221 \\
\hline B & 0.8199808823 & 0.8789803286 & 0.3629966779 \\
\hline \multicolumn{4}{|l|}{$5-\mathrm{HS}$} \\
\hline \multicolumn{4}{|c|}{ CELL_PARAMETERS (angstrom) } \\
\hline 19.355593277 & 0.000000000 & -0.011377092 & \\
\hline 0.000000000 & 12.920252502 & 0.000000000 & \\
\hline-4.665687532 & 0.000000000 & 20.337183205 & \\
\hline
\end{tabular}




\begin{tabular}{|c|c|c|c|}
\hline \multicolumn{4}{|c|}{ ATOMIC_POSITIONS (crystal) } \\
\hline Fe1 & 0.1028674985 & 0.9773329062 & 0.2264200534 \\
\hline Fe1 & 0.5076788563 & 0.9640107097 & 0.2042952660 \\
\hline Fe1 & 0.8971324945 & 0.0226670828 & 0.7735799316 \\
\hline Fe1 & 0.4923211147 & 0.0359893123 & 0.7957047190 \\
\hline Fe1 & 0.3971324945 & 0.4773329062 & 0.2735799616 \\
\hline Fe1 & 0.9923211437 & 0.4640106807 & 0.2957047190 \\
\hline Fe1 & 0.6028675055 & 0.5226670938 & 0.7264200684 \\
\hline Fe1 & 0.0076788563 & 0.5359892903 & 0.7042952810 \\
\hline $\mathrm{N}$ & 0.0100290528 & 0.0736762620 & 0.2025432242 \\
\hline $\mathrm{N}$ & 0.0805072538 & 0.9232633692 & 0.1272786260 \\
\hline $\mathrm{N}$ & 0.1020111630 & 0.9727188530 & 0.3278594091 \\
\hline $\mathrm{N}$ & 0.0185254060 & 0.8581784298 & 0.2282130420 \\
\hline $\mathrm{N}$ & 0.6218762906 & 0.0121038199 & 0.1985641442 \\
\hline $\mathrm{N}$ & 0.5574472563 & 0.0153033576 & 0.3020636051 \\
\hline $\mathrm{N}$ & 0.4932574331 & 0.0431499388 & 0.1111060872 \\
\hline $\mathrm{N}$ & 0.5566224572 & 0.8188655879 & 0.1831085202 \\
\hline $\mathrm{N}$ & 0.9899709482 & 0.9263236780 & 0.7974567908 \\
\hline $\mathrm{N}$ & 0.9194927752 & 0.0767366088 & 0.8727213890 \\
\hline $\mathrm{N}$ & 0.8979888370 & 0.0272811750 & 0.6721415739 \\
\hline $\mathrm{N}$ & 0.9814745940 & 0.1418215702 & 0.7717869280 \\
\hline $\mathrm{N}$ & 0.3781237394 & 0.9878961801 & 0.8014358858 \\
\hline $\mathrm{N}$ & 0.4425527137 & 0.9846967024 & 0.6979363949 \\
\hline $\mathrm{N}$ & 0.5067425969 & 0.9568500612 & 0.8888939348 \\
\hline $\mathrm{N}$ & 0.4433775428 & 0.1811344271 & 0.8168914798 \\
\hline $\mathrm{N}$ & 0.4899709482 & 0.5736763220 & 0.2974567608 \\
\hline $\mathrm{N}$ & 0.4194927462 & 0.4232633992 & 0.3727213890 \\
\hline $\mathrm{N}$ & 0.3979888370 & 0.4727188240 & 0.1721405909 \\
\hline $\mathrm{N}$ & 0.4814745940 & 0.3581784298 & 0.2717869580 \\
\hline $\mathrm{N}$ & 0.8781237094 & 0.5121038199 & 0.3014358558 \\
\hline $\mathrm{N}$ & 0.9425527437 & 0.5153032976 & 0.1979363799 \\
\hline $\mathrm{N}$ & 0.0067425799 & 0.5431499388 & 0.3888939058 \\
\hline $\mathrm{N}$ & 0.9433775428 & 0.3188655879 & 0.3168914798 \\
\hline $\mathrm{N}$ & 0.5100290518 & 0.4263237080 & 0.7025432092 \\
\hline $\mathrm{N}$ & 0.5805072248 & 0.5767366308 & 0.6272786110 \\
\hline $\mathrm{N}$ & 0.6020111630 & 0.5272811470 & 0.8278584261 \\
\hline $\mathrm{N}$ & 0.5185254060 & 0.6418215702 & 0.7282130720 \\
\hline $\mathrm{N}$ & 0.1218762316 & 0.4878961801 & 0.6985641142 \\
\hline $\mathrm{N}$ & 0.0574472563 & 0.4846966724 & 0.8020636051 \\
\hline $\mathrm{N}$ & 0.9932574031 & 0.4568500322 & 0.6111060652 \\
\hline $\mathrm{N}$ & 0.0566224572 & 0.6811344121 & 0.6831085202 \\
\hline C & 0.0087392143 & 0.1755960615 & 0.1878726254 \\
\hline C & 0.9479190281 & 0.0233912137 & 0.1976020067 \\
\hline C & 0.0175187035 & 0.7980890170 & 0.0521350643 \\
\hline C & 0.9457595419 & 0.2300512574 & 0.1675836705 \\
\hline C & 0.1019893734 & 0.9700780251 & 0.0773272518 \\
\hline C & 0.8813475761 & 0.1782035892 & 0.1620741568 \\
\hline C & 0.0650956003 & 0.8941392409 & 0.3466473574 \\
\hline C & 0.0370964523 & 0.8128441691 & 0.2952638144 \\
\hline C & 0.1327544993 & 0.0441750386 & 0.3722519498 \\
\hline C & 0.0397255912 & 0.8376493067 & 0.1156105448 \\
\hline C & 0.8826105309 & 0.0734106536 & 0.1773577398 \\
\hline C & 0.0397894746 & 0.8465243630 & 0.0004351454 \\
\hline C & 0.0832659371 & 0.9336251844 & 0.0130820731 \\
\hline C & 0.9483050611 & 0.9104011435 & 0.2157159230 \\
\hline C & 0.0230985551 & 0.7841578400 & 0.1750556580 \\
\hline $\mathrm{C}$ & 0.1281643276 & 0.0401785314 & 0.4377626308 \\
\hline
\end{tabular}




\begin{tabular}{|c|c|c|c|}
\hline C & 0.0565998697 & 0.8866413377 & 0.4112588908 \\
\hline C & 0.0891984145 & 0.9598748094 & 0.4573525805 \\
\hline C & 0.2464485604 & 0.9138074252 & 0.2350432325 \\
\hline C & 0.2450863473 & 0.0311552035 & 0.2296839618 \\
\hline C & 0.3680052978 & 0.0299262946 & 0.2235857279 \\
\hline C & 0.3084218481 & 0.8585196371 & 0.2319835252 \\
\hline C & 0.3680142166 & 0.9127565050 & 0.2241276580 \\
\hline C & 0.3063713468 & 0.0854560020 & 0.2267462713 \\
\hline C & 0.5507792778 & 0.0782838814 & 0.0913779709 \\
\hline C & 0.5291502448 & 0.9972770002 & 0.3544228328 \\
\hline C & 0.6228146010 & 0.0466627732 & 0.1310609304 \\
\hline C & 0.6181580378 & 0.0704896626 & 0.3102416869 \\
\hline C & 0.5211402745 & 0.7309537742 & 0.1619984667 \\
\hline C & 0.6272219594 & 0.8222292841 & 0.1865582580 \\
\hline C & 0.6652511886 & 0.9178552397 & 0.2167861527 \\
\hline C & 0.6431794126 & 0.0944128178 & 0.2482107550 \\
\hline C & 0.5542554712 & 0.6440608175 & 0.1432210331 \\
\hline C & 0.5440382819 & 0.1390763417 & 0.0348200058 \\
\hline C & 0.6531575864 & 0.1062264754 & 0.3722534132 \\
\hline C & 0.6634115553 & 0.7392538015 & 0.1665616985 \\
\hline C & 0.4767094365 & 0.1632701045 & 0.9981964638 \\
\hline C & 0.4279024110 & 0.0676722934 & 0.0754838355 \\
\hline C & 0.5614143087 & 0.0297986851 & 0.4176065631 \\
\hline C & 0.6267517021 & 0.6485425527 & 0.1447856540 \\
\hline C & 0.4171441214 & 0.1272359447 & 0.0188479690 \\
\hline C & 0.6247967689 & 0.0851081648 & 0.4266421376 \\
\hline C & 0.9912608067 & 0.8244038795 & 0.8121273896 \\
\hline C & 0.0520809119 & 0.9766087863 & 0.8023979783 \\
\hline C & 0.9824812965 & 0.2019109680 & 0.9478649087 \\
\hline C & 0.0542404581 & 0.7699486836 & 0.8324162995 \\
\hline C & 0.8980106186 & 0.0299219849 & 0.9226727402 \\
\hline C & 0.1186524239 & 0.8217964108 & 0.8379258582 \\
\hline C & 0.9349044077 & 0.1058607591 & 0.6533526426 \\
\hline C & 0.9629035337 & 0.1871558309 & 0.7047361856 \\
\hline C & 0.8672455157 & 0.9558249014 & 0.6277480202 \\
\hline C & 0.9602744268 & 0.1623506633 & 0.8843894402 \\
\hline C & 0.1173894691 & 0.9265893464 & 0.8226422302 \\
\hline C & 0.9602105334 & 0.1534766210 & 0.9995648636 \\
\hline C & 0.9167340709 & 0.0663748126 & 0.9869179549 \\
\hline C & 0.0516949389 & 0.0895988565 & 0.7842841070 \\
\hline C & 0.9769014219 & 0.2158421450 & 0.8249443270 \\
\hline C & 0.8718356724 & 0.9598214686 & 0.5622373692 \\
\hline C & 0.9434001373 & 0.1133586763 & 0.5887411092 \\
\hline C & 0.9108015855 & 0.0401252016 & 0.5426474495 \\
\hline C & 0.7535514396 & 0.0861925448 & 0.7649567975 \\
\hline C & 0.7549136677 & 0.9688447365 & 0.7703160092 \\
\hline C & 0.6319947022 & 0.9700737644 & 0.7764142871 \\
\hline C & 0.6915781519 & 0.1414803929 & 0.7680164898 \\
\hline C & 0.6319857834 & 0.0872435170 & 0.7758723420 \\
\hline C & 0.6936286232 & 0.9145439380 & 0.7732537287 \\
\hline C & 0.4492207522 & 0.9217161186 & 0.9086220291 \\
\hline C & 0.4708497842 & 0.0027229718 & 0.6455771372 \\
\hline C & 0.3771854280 & 0.9533372268 & 0.8689390696 \\
\hline C & 0.3818419332 & 0.9295103974 & 0.6897583131 \\
\hline C & 0.4788597255 & 0.2690462258 & 0.8380015483 \\
\hline C & 0.3727780406 & 0.1777706859 & 0.8134417570 \\
\hline C & 0.3347488414 & 0.0821447603 & 0.7832138623 \\
\hline C & 0.3568206174 & 0.9055872412 & 0.7517892450 \\
\hline
\end{tabular}




\begin{tabular}{|c|c|c|c|}
\hline C & 0.4457445288 & 0.3559391825 & 0.8567789519 \\
\hline C & 0.4559617181 & 0.8609237173 & 0.9651800202 \\
\hline C & 0.3468424426 & 0.8937734646 & 0.6277465868 \\
\hline C & 0.3365884147 & 0.2607462275 & 0.8334383015 \\
\hline C & 0.5232905635 & 0.8367298355 & 0.0018035952 \\
\hline C & 0.5720975590 & 0.9323277066 & 0.9245161645 \\
\hline C & 0.4385856913 & 0.9702013749 & 0.5823934369 \\
\hline C & 0.3732482979 & 0.3514574473 & 0.8552143310 \\
\hline C & 0.5828558486 & 0.8727640553 & 0.9811520610 \\
\hline C & 0.3752032611 & 0.9148918352 & 0.5733578334 \\
\hline C & 0.2535514396 & 0.4138074552 & 0.2649567675 \\
\hline C & 0.2549136377 & 0.5311552635 & 0.2703160382 \\
\hline C & 0.1319946872 & 0.5299262356 & 0.2764142871 \\
\hline C & 0.4912607767 & 0.6755961205 & 0.3121273896 \\
\hline C & 0.5520809719 & 0.5233912137 & 0.3023980083 \\
\hline C & 0.1915781369 & 0.3585196071 & 0.2680164898 \\
\hline C & 0.1319857834 & 0.4127564750 & 0.2758723420 \\
\hline C & 0.1936286382 & 0.5854560620 & 0.2732537287 \\
\hline C & 0.4824812965 & 0.2980890170 & 0.4478649387 \\
\hline C & 0.5542404581 & 0.7300513164 & 0.3324163295 \\
\hline C & 0.3980106186 & 0.4700780251 & 0.4226727402 \\
\hline C & 0.6186524239 & 0.6782036492 & 0.3379258582 \\
\hline C & 0.4349044077 & 0.3941392409 & 0.1533526276 \\
\hline C & 0.4629035627 & 0.3128441691 & 0.2047361706 \\
\hline C & 0.3672455157 & 0.5441750986 & 0.1277480352 \\
\hline C & 0.4602743968 & 0.3376493367 & 0.3843894692 \\
\hline C & 0.6173894691 & 0.5734106536 & 0.3226422602 \\
\hline C & 0.4602105334 & 0.3465243930 & 0.4995648636 \\
\hline C & 0.4167340709 & 0.4336251844 & 0.4869179249 \\
\hline C & 0.5516949389 & 0.4104011435 & 0.2842840770 \\
\hline C & 0.4769014519 & 0.2841578700 & 0.3249443570 \\
\hline C & 0.3718356724 & 0.5401785314 & 0.0622373762 \\
\hline C & 0.4434001373 & 0.3866413377 & 0.0887411172 \\
\hline C & 0.4108015855 & 0.4598748094 & 0.0426474225 \\
\hline C & 0.9492207222 & 0.5782838814 & 0.4086220291 \\
\hline C & 0.9708497552 & 0.4972770302 & 0.1455771522 \\
\hline C & 0.8771854580 & 0.5466627732 & 0.3689390696 \\
\hline C & 0.8818419622 & 0.5704896026 & 0.1897583131 \\
\hline C & 0.9788597255 & 0.2309537592 & 0.3380015183 \\
\hline C & 0.8727780406 & 0.3222293141 & 0.3134417570 \\
\hline C & 0.8347488114 & 0.4178552397 & 0.2832138323 \\
\hline C & 0.8568205874 & 0.5944127588 & 0.2517892450 \\
\hline C & 0.9457445288 & 0.1440608175 & 0.3567789819 \\
\hline C & 0.9559617181 & 0.6390762827 & 0.4651799912 \\
\hline C & 0.8468424136 & 0.6062265354 & 0.1277465868 \\
\hline C & 0.8365884447 & 0.2392537725 & 0.3334383015 \\
\hline C & 0.0232905525 & 0.6632701645 & 0.5018035362 \\
\hline C & 0.0720975820 & 0.5676722934 & 0.4245161645 \\
\hline C & 0.9385856913 & 0.5297986251 & 0.0823934449 \\
\hline C & 0.8732482979 & 0.1485425377 & 0.3552143610 \\
\hline C & 0.0828558786 & 0.6272359447 & 0.4811520310 \\
\hline C & 0.8752032311 & 0.5851081648 & 0.0733578554 \\
\hline C & 0.7464485604 & 0.5861925748 & 0.7350432025 \\
\hline C & 0.7450863323 & 0.4688447665 & 0.7296839908 \\
\hline C & 0.8680052978 & 0.4700737354 & 0.7235857129 \\
\hline C & 0.5087391933 & 0.3244039085 & 0.6878726104 \\
\hline C & 0.4479190581 & 0.4766087863 & 0.6976020217 \\
\hline C & 0.8084218481 & 0.6414803629 & 0.7319835102 \\
\hline
\end{tabular}




\begin{tabular}{|c|c|c|c|}
\hline C & 0.8680142166 & 0.5872434950 & 0.7241276580 \\
\hline C & 0.8063713768 & 0.4145439680 & 0.7267462713 \\
\hline C & 0.5175187035 & 0.7019109830 & 0.5521350913 \\
\hline C & 0.4457595419 & 0.2699486836 & 0.6675837005 \\
\hline C & 0.6019893814 & 0.5299219749 & 0.5773272598 \\
\hline C & 0.3813475761 & 0.3217963808 & 0.6620741418 \\
\hline C & 0.5650955923 & 0.6058607591 & 0.8466473574 \\
\hline C & 0.5370964663 & 0.6871558309 & 0.7952638144 \\
\hline C & 0.6327544843 & 0.4558249014 & 0.8722519798 \\
\hline C & 0.5397255732 & 0.6623506933 & 0.6156105598 \\
\hline C & 0.3826105019 & 0.4265893164 & 0.6773577698 \\
\hline C & 0.5397894666 & 0.6534756370 & 0.5004351364 \\
\hline C & 0.5832659291 & 0.5663748156 & 0.5130820451 \\
\hline C & 0.4483050611 & 0.5895988565 & 0.7157158930 \\
\hline C & 0.5230985781 & 0.7158421600 & 0.6750556730 \\
\hline C & 0.6281643276 & 0.4598214386 & 0.9377626308 \\
\hline C & 0.5565998627 & 0.6133586623 & 0.9112588908 \\
\hline C & 0.5891984145 & 0.5401251906 & 0.9573525505 \\
\hline C & 0.0507792778 & 0.4217160886 & 0.5913779709 \\
\hline C & 0.0291502448 & 0.5027229998 & 0.8544228628 \\
\hline C & 0.1228145420 & 0.4533372268 & 0.6310609304 \\
\hline C & 0.1181580378 & 0.4295103674 & 0.8102416869 \\
\hline C & 0.0211402155 & 0.7690462258 & 0.6619984517 \\
\hline C & 0.1272220194 & 0.6777707159 & 0.6865582430 \\
\hline C & 0.1652511286 & 0.5821447603 & 0.7167861377 \\
\hline C & 0.1431794126 & 0.4055872412 & 0.7482107550 \\
\hline C & 0.0542554122 & 0.8559391825 & 0.6432210481 \\
\hline C & 0.0440382229 & 0.3609237173 & 0.5348199798 \\
\hline C & 0.1531575864 & 0.3937734646 & 0.8722534132 \\
\hline C & 0.1634115553 & 0.7607461985 & 0.6665616985 \\
\hline C & 0.9767094365 & 0.3367298355 & 0.4981964338 \\
\hline C & 0.9279024410 & 0.4323277066 & 0.5754838355 \\
\hline C & 0.0614142497 & 0.4702013449 & 0.9176065631 \\
\hline C & 0.1267517021 & 0.8514574473 & 0.6447856690 \\
\hline C & 0.9171441514 & 0.3727640853 & 0.5188479390 \\
\hline C & 0.1247967089 & 0.4148918352 & 0.9266421666 \\
\hline $\mathrm{H}$ & 0.0601866282 & 0.2134377336 & 0.1938079037 \\
\hline $\mathrm{H}$ & 0.9822080948 & 0.7314673713 & 0.0435250348 \\
\hline $\mathrm{H}$ & 0.9470629822 & 0.3122767800 & 0.1569143778 \\
\hline $\mathrm{H}$ & 0.1353268240 & 0.0385211444 & 0.0897098081 \\
\hline $\mathrm{H}$ & 0.8309464446 & 0.2188652918 & 0.1468643736 \\
\hline $\mathrm{H}$ & 0.0783697460 & 0.7538354903 & 0.2965384674 \\
\hline $\mathrm{H}$ & 0.9915917967 & 0.7732077540 & 0.3078599858 \\
\hline $\mathrm{H}$ & 0.1616438103 & 0.1054497183 & 0.3534251623 \\
\hline $\mathrm{H}$ & 0.8337707949 & 0.0292167611 & 0.1730003553 \\
\hline $\mathrm{H}$ & 0.0227912261 & 0.8172787516 & 0.9504111645 \\
\hline $\mathrm{H}$ & 0.1020467835 & 0.9745890211 & 0.9745746079 \\
\hline $\mathrm{H}$ & 0.9259538729 & 0.9035684651 & 0.2596664435 \\
\hline $\mathrm{H}$ & 0.9109050239 & 0.8702238304 & 0.1758844254 \\
\hline $\mathrm{H}$ & 0.9737929681 & 0.7389596595 & 0.1598216393 \\
\hline $\mathrm{H}$ & 0.0660958289 & 0.7290741566 & 0.1945357266 \\
\hline $\mathrm{H}$ & 0.1539124324 & 0.0982125740 & 0.4729723184 \\
\hline $\mathrm{H}$ & 0.0243048874 & 0.8248027443 & 0.4244602825 \\
\hline $\mathrm{H}$ & 0.0842081881 & 0.9557476140 & 0.5082937360 \\
\hline $\mathrm{H}$ & 0.3084237892 & 0.7740223482 & 0.2345220874 \\
\hline $\mathrm{H}$ & 0.3057658835 & 0.1698794864 & 0.2245329847 \\
\hline $\mathrm{H}$ & 0.4790311535 & 0.9549492332 & 0.3443248475 \\
\hline $\mathrm{H}$ & 0.6423273303 & 0.9820824584 & 0.1054695935 \\
\hline
\end{tabular}




\begin{tabular}{|c|c|c|c|}
\hline $\mathrm{H}$ & 0.6608300194 & 0.1103739403 & 0.1317114510 \\
\hline $\mathrm{H}$ & 0.4645400782 & 0.7322632694 & 0.1609540918 \\
\hline $\mathrm{H}$ & 0.6758335274 & 0.9088499993 & 0.2706248771 \\
\hline $\mathrm{H}$ & 0.7173216401 & 0.9241587907 & 0.2042020865 \\
\hline $\mathrm{H}$ & 0.6184574352 & 0.1671755592 & 0.2268408275 \\
\hline $\mathrm{H}$ & 0.7010124427 & 0.1079330066 & 0.2595986328 \\
\hline $\mathrm{H}$ & 0.5234510456 & 0.5745038156 & 0.1270193749 \\
\hline $\mathrm{H}$ & 0.5914014767 & 0.1685121962 & 0.0208161950 \\
\hline $\mathrm{H}$ & 0.7019134197 & 0.1509684009 & 0.3791858437 \\
\hline $\mathrm{H}$ & 0.7197137593 & 0.7477251297 & 0.1678152087 \\
\hline $\mathrm{H}$ & 0.4703690625 & 0.2117914793 & 0.9545479076 \\
\hline $\mathrm{H}$ & 0.3829870673 & 0.0392479537 & 0.0939224504 \\
\hline $\mathrm{H}$ & 0.5368578284 & 0.0116927427 & 0.4585039016 \\
\hline $\mathrm{H}$ & 0.6538373261 & 0.5818204040 & 0.1298367092 \\
\hline $\mathrm{H}$ & 0.3634185950 & 0.1471047196 & 0.9928965090 \\
\hline $\mathrm{H}$ & 0.6523471910 & 0.1132082678 & 0.4749640807 \\
\hline $\mathrm{H}$ & 0.9398133938 & 0.7865622664 & 0.8061921113 \\
\hline $\mathrm{H}$ & 0.0177919052 & 0.2685326287 & 0.9564749942 \\
\hline $\mathrm{H}$ & 0.0529369588 & 0.6877232200 & 0.8430856072 \\
\hline $\mathrm{H}$ & 0.8646731910 & 0.9614789146 & 0.9102901769 \\
\hline $\mathrm{H}$ & 0.1690535554 & 0.7811347682 & 0.8531356264 \\
\hline $\mathrm{H}$ & 0.9216302460 & 0.2461645097 & 0.7034615326 \\
\hline $\mathrm{H}$ & 0.0084081443 & 0.2267922460 & 0.6921400142 \\
\hline $\mathrm{H}$ & 0.8383561897 & 0.8945502817 & 0.6465748077 \\
\hline $\mathrm{H}$ & 0.1662292051 & 0.9707831789 & 0.8269996597 \\
\hline $\mathrm{H}$ & 0.9772087809 & 0.1827212334 & 0.0495888355 \\
\hline $\mathrm{H}$ & 0.8979531935 & 0.0254109659 & 0.0254253921 \\
\hline $\mathrm{H}$ & 0.0740461271 & 0.0964315199 & 0.7403335265 \\
\hline $\mathrm{H}$ & 0.0890949171 & 0.1297761396 & 0.8241155606 \\
\hline $\mathrm{H}$ & 0.0262070319 & 0.2610403695 & 0.8401783607 \\
\hline $\mathrm{H}$ & 0.9339041861 & 0.2709258144 & 0.8054642734 \\
\hline $\mathrm{H}$ & 0.8460875826 & 0.9017874260 & 0.5270276526 \\
\hline $\mathrm{H}$ & 0.9756950856 & 0.1751972407 & 0.5755397175 \\
\hline $\mathrm{H}$ & 0.9157918349 & 0.0442523820 & 0.4917062940 \\
\hline $\mathrm{H}$ & 0.6915761808 & 0.2259776218 & 0.7654779426 \\
\hline $\mathrm{H}$ & 0.6942341165 & 0.8301205136 & 0.7754670453 \\
\hline $\mathrm{H}$ & 0.5209688765 & 0.0450507588 & 0.6556751235 \\
\hline $\mathrm{H}$ & 0.3576726397 & 0.0179175306 & 0.8945303915 \\
\hline $\mathrm{H}$ & 0.3391700096 & 0.8896261187 & 0.8682885490 \\
\hline $\mathrm{H}$ & 0.5354599518 & 0.2677367596 & 0.8390459382 \\
\hline $\mathrm{H}$ & 0.3241665026 & 0.0911500227 & 0.7293750929 \\
\hline $\mathrm{H}$ & 0.2826783599 & 0.0758411873 & 0.7957979135 \\
\hline $\mathrm{H}$ & 0.3815425938 & 0.8328244408 & 0.7731591725 \\
\hline $\mathrm{H}$ & 0.2989875873 & 0.8920669934 & 0.7404013372 \\
\hline $\mathrm{H}$ & 0.4765489544 & 0.4254962144 & 0.8729806401 \\
\hline $\mathrm{H}$ & 0.4085985233 & 0.8314878038 & 0.9791837780 \\
\hline $\mathrm{H}$ & 0.2980866103 & 0.8490306451 & 0.6208141563 \\
\hline $\mathrm{H}$ & 0.2802872237 & 0.2522748703 & 0.8321848063 \\
\hline $\mathrm{H}$ & 0.5296309375 & 0.7882085207 & 0.0454520924 \\
\hline $\mathrm{H}$ & 0.6170129027 & 0.9607520463 & 0.9060775266 \\
\hline $\mathrm{H}$ & 0.4631421716 & 0.9883072573 & 0.5414960684 \\
\hline $\mathrm{H}$ & 0.3461626739 & 0.4181795660 & 0.8701633058 \\
\hline $\mathrm{H}$ & 0.6365813750 & 0.8528953394 & 0.0071034910 \\
\hline $\mathrm{H}$ & 0.3476528390 & 0.8867917322 & 0.5250359193 \\
\hline $\mathrm{H}$ & 0.4398133638 & 0.7134377336 & 0.3061920813 \\
\hline $\mathrm{H}$ & 0.1915762108 & 0.2740223782 & 0.2654779126 \\
\hline $\mathrm{H}$ & 0.1942341315 & 0.6698794864 & 0.2754670153 \\
\hline $\mathrm{H}$ & 0.5177918452 & 0.2314673563 & 0.4564749652 \\
\hline
\end{tabular}




\begin{tabular}{|c|c|c|c|}
\hline $\mathrm{H}$ & 0.5529370178 & 0.8122767800 & 0.3430856072 \\
\hline $\mathrm{H}$ & 0.3646731910 & 0.5385210854 & 0.4102901769 \\
\hline $\mathrm{H}$ & 0.6690534954 & 0.7188652318 & 0.3531356264 \\
\hline $\mathrm{H}$ & 0.4216302460 & 0.2538354903 & 0.2034615176 \\
\hline $\mathrm{H}$ & 0.5084082033 & 0.2732077540 & 0.1921400282 \\
\hline $\mathrm{H}$ & 0.3383561897 & 0.6054497783 & 0.1465748227 \\
\hline $\mathrm{H}$ & 0.6662291451 & 0.5292168211 & 0.3269996597 \\
\hline $\mathrm{H}$ & 0.4772087809 & 0.3172787516 & 0.5495888355 \\
\hline $\mathrm{H}$ & 0.3979532235 & 0.4745890211 & 0.5254253921 \\
\hline $\mathrm{H}$ & 0.5740461271 & 0.4035684651 & 0.2403335415 \\
\hline $\mathrm{H}$ & 0.5890949761 & 0.3702238604 & 0.3241155606 \\
\hline $\mathrm{H}$ & 0.5262070319 & 0.2389596445 & 0.3401783607 \\
\hline $\mathrm{H}$ & 0.4339041861 & 0.2290741716 & 0.3054642734 \\
\hline $\mathrm{H}$ & 0.3460875826 & 0.5982125740 & 0.0270276706 \\
\hline $\mathrm{H}$ & 0.4756951156 & 0.3248027743 & 0.0755397255 \\
\hline $\mathrm{H}$ & 0.4157918049 & 0.4557476140 & 0.9917062640 \\
\hline $\mathrm{H}$ & 0.0209688535 & 0.4549492332 & 0.1556751375 \\
\hline $\mathrm{H}$ & 0.8576726097 & 0.4820824584 & 0.3945304215 \\
\hline $\mathrm{H}$ & 0.8391699806 & 0.6103738813 & 0.3682885490 \\
\hline $\mathrm{H}$ & 0.0354599328 & 0.2322632404 & 0.3390459082 \\
\hline $\mathrm{H}$ & 0.8241664726 & 0.4088499693 & 0.2293751079 \\
\hline $\mathrm{H}$ & 0.7826783599 & 0.4241588207 & 0.2957979135 \\
\hline $\mathrm{H}$ & 0.8815425648 & 0.6671755592 & 0.2731591725 \\
\hline $\mathrm{H}$ & 0.7989875573 & 0.6079330066 & 0.2404013672 \\
\hline $\mathrm{H}$ & 0.9765489544 & 0.0745037936 & 0.3729806101 \\
\hline $\mathrm{H}$ & 0.9085985233 & 0.6685121962 & 0.4791838080 \\
\hline $\mathrm{H}$ & 0.7980865803 & 0.6509693549 & 0.1208141633 \\
\hline $\mathrm{H}$ & 0.7802862407 & 0.2477251297 & 0.3321848063 \\
\hline $\mathrm{H}$ & 0.0296309305 & 0.7117914793 & 0.5454520924 \\
\hline $\mathrm{H}$ & 0.1170129257 & 0.5392479537 & 0.4060775566 \\
\hline $\mathrm{H}$ & 0.9631421716 & 0.5116927427 & 0.0414960834 \\
\hline $\mathrm{H}$ & 0.8461626739 & 0.0818204190 & 0.3701632758 \\
\hline $\mathrm{H}$ & 0.1365814050 & 0.6471046606 & 0.5071034910 \\
\hline $\mathrm{H}$ & 0.8476528090 & 0.6132082678 & 0.0250359103 \\
\hline $\mathrm{H}$ & 0.5601866062 & 0.2865622364 & 0.6938078887 \\
\hline $\mathrm{H}$ & 0.8084238192 & 0.7259776518 & 0.7345220574 \\
\hline $\mathrm{H}$ & 0.8057658835 & 0.3301205136 & 0.7245329547 \\
\hline $\mathrm{H}$ & 0.4822081248 & 0.7685326287 & 0.5435250058 \\
\hline $\mathrm{H}$ & 0.4470629822 & 0.1877232350 & 0.6569143928 \\
\hline $\mathrm{H}$ & 0.6353268090 & 0.4614788846 & 0.5897098231 \\
\hline $\mathrm{H}$ & 0.3309464746 & 0.2811347682 & 0.6468643736 \\
\hline $\mathrm{H}$ & 0.5783697540 & 0.7461645097 & 0.7965384674 \\
\hline $\mathrm{H}$ & 0.4915918267 & 0.7267922460 & 0.8078599858 \\
\hline $\mathrm{H}$ & 0.6616438103 & 0.3945502517 & 0.8534251923 \\
\hline $\mathrm{H}$ & 0.3337708249 & 0.4707832089 & 0.6730003403 \\
\hline $\mathrm{H}$ & 0.5227912191 & 0.6827212484 & 0.4504111645 \\
\hline $\mathrm{H}$ & 0.6020468065 & 0.5254109789 & 0.4745746369 \\
\hline $\mathrm{H}$ & 0.4259538729 & 0.5964315349 & 0.7596664735 \\
\hline $\mathrm{H}$ & 0.4109050239 & 0.6297761696 & 0.6758844394 \\
\hline $\mathrm{H}$ & 0.4737929681 & 0.7610403405 & 0.6598216393 \\
\hline $\mathrm{H}$ & 0.5660958139 & 0.7709258434 & 0.6945357266 \\
\hline $\mathrm{H}$ & 0.6539124174 & 0.4017874260 & 0.9729723474 \\
\hline $\mathrm{H}$ & 0.5243049144 & 0.6751972557 & 0.9244602825 \\
\hline $\mathrm{H}$ & 0.5842081651 & 0.5442523860 & 0.0082937360 \\
\hline $\mathrm{H}$ & 0.9790311235 & 0.5450507668 & 0.8443248765 \\
\hline $\mathrm{H}$ & 0.1423273903 & 0.5179175416 & 0.6054696085 \\
\hline $\mathrm{H}$ & 0.1608299604 & 0.3896261187 & 0.6317114510 \\
\hline $\mathrm{H}$ & 0.9645400482 & 0.7677367306 & 0.6609540618 \\
\hline
\end{tabular}




\begin{tabular}{|c|c|c|c|}
\hline $\mathrm{H}$ & 0.1758334674 & 0.5911500007 & 0.7706249071 \\
\hline $\mathrm{H}$ & 0.2173215811 & 0.5758412093 & 0.7042020865 \\
\hline $\mathrm{H}$ & 0.1184574352 & 0.3328244108 & 0.7268408275 \\
\hline $\mathrm{H}$ & 0.2010124427 & 0.3920670234 & 0.7595986628 \\
\hline $\mathrm{H}$ & 0.0234510456 & 0.9254961844 & 0.6270193599 \\
\hline $\mathrm{H}$ & 0.0914015357 & 0.3314877748 & 0.5208162220 \\
\hline $\mathrm{H}$ & 0.2019134197 & 0.3490316581 & 0.8791858437 \\
\hline $\mathrm{H}$ & 0.2197137593 & 0.7522748703 & 0.6678151937 \\
\hline $\mathrm{H}$ & 0.9703690625 & 0.2882085207 & 0.4545479376 \\
\hline $\mathrm{H}$ & 0.8829870973 & 0.4607520463 & 0.5939224734 \\
\hline $\mathrm{H}$ & 0.0368578284 & 0.4883072863 & 0.9585039316 \\
\hline $\mathrm{H}$ & 0.1538373861 & 0.9181795960 & 0.6298366942 \\
\hline $\mathrm{H}$ & 0.8634186250 & 0.3528953394 & 0.4928965090 \\
\hline $\mathrm{H}$ & 0.1523471310 & 0.3867917032 & 0.9749640807 \\
\hline 0 & 0.4263433641 & 0.0722977067 & 0.2190510821 \\
\hline 0 & 0.1845622015 & 0.0751611527 & 0.2281317166 \\
\hline 0 & 0.4264620292 & 0.8697095705 & 0.2174909154 \\
\hline 0 & 0.1887883447 & 0.8711283906 & 0.2412091949 \\
\hline 0 & 0.5736566359 & 0.9277023533 & 0.7809479039 \\
\hline 0 & 0.8154377835 & 0.9248388473 & 0.7718682834 \\
\hline 0 & 0.5735379708 & 0.1302904445 & 0.7825090546 \\
\hline 0 & 0.8112116403 & 0.1288715794 & 0.7587908051 \\
\hline 0 & 0.0736566289 & 0.5722976467 & 0.2809479039 \\
\hline 0 & 0.3154378135 & 0.5751611527 & 0.2718682834 \\
\hline 0 & 0.0735379628 & 0.3697095705 & 0.2825090846 \\
\hline 0 & 0.3112116703 & 0.3711284206 & 0.2587908051 \\
\hline 0 & 0.9263433641 & 0.4277023533 & 0.7190520961 \\
\hline 0 & 0.6845622165 & 0.4248388473 & 0.7281317166 \\
\hline 0 & 0.9264620292 & 0.6302904295 & 0.7174909454 \\
\hline 0 & 0.6887883597 & 0.6288716094 & 0.7412091949 \\
\hline $\mathrm{F}$ & 0.2537713357 & 0.2385060506 & 0.9610153438 \\
\hline $\mathrm{F}$ & 0.2408954822 & 0.0855155107 & 0.9011738131 \\
\hline $\mathrm{F}$ & 0.1431529206 & 0.1581348676 & 0.9319966058 \\
\hline $\mathrm{F}$ & 0.2336544656 & 0.0839536753 & 0.0111962598 \\
\hline $\mathrm{F}$ & 0.7462286643 & 0.7614939344 & 0.0389846482 \\
\hline $\mathrm{F}$ & 0.7591045328 & 0.9144845043 & 0.0988261719 \\
\hline $\mathrm{F}$ & 0.8568470794 & 0.8418651324 & 0.0680034172 \\
\hline $\mathrm{F}$ & 0.7663455644 & 0.9160463097 & 0.9888036812 \\
\hline $\mathrm{F}$ & 0.2462286783 & 0.7385060656 & 0.5389846562 \\
\hline $\mathrm{F}$ & 0.2591045328 & 0.5855154957 & 0.5988261869 \\
\hline $\mathrm{F}$ & 0.3568470794 & 0.6581348676 & 0.5680033942 \\
\hline $\mathrm{F}$ & 0.2663455344 & 0.5839536903 & 0.4888036812 \\
\hline $\mathrm{F}$ & 0.7537713357 & 0.2614939644 & 0.4610153438 \\
\hline $\mathrm{F}$ & 0.7408954672 & 0.4144844743 & 0.4011738431 \\
\hline $\mathrm{F}$ & 0.6431529206 & 0.3418651324 & 0.4319965758 \\
\hline $\mathrm{F}$ & 0.7336544356 & 0.4160463097 & 0.5111963188 \\
\hline $\mathrm{F}$ & 0.2729938590 & 0.7195912305 & 0.8409872976 \\
\hline $\mathrm{F}$ & 0.3183309162 & 0.5706511618 & 0.8025511462 \\
\hline $\mathrm{F}$ & 0.3941222026 & 0.7030866460 & 0.8490549971 \\
\hline $\mathrm{F}$ & 0.3388119765 & 0.6018032218 & 0.9148687232 \\
\hline $\mathrm{F}$ & 0.7270061110 & 0.2804077865 & 0.1590127324 \\
\hline $\mathrm{F}$ & 0.6816690838 & 0.4293488082 & 0.1974488388 \\
\hline $\mathrm{F}$ & 0.6058777674 & 0.2969133840 & 0.1509450329 \\
\hline $\mathrm{F}$ & 0.6611880535 & 0.3981968082 & 0.0851312618 \\
\hline $\mathrm{F}$ & 0.2270061410 & 0.2195922135 & 0.6590127024 \\
\hline $\mathrm{F}$ & 0.1816690988 & 0.0706511848 & 0.6974488538 \\
\hline $\mathrm{F}$ & 0.1058777824 & 0.2030866160 & 0.6509450029 \\
\hline $\mathrm{F}$ & 0.1611880385 & 0.1018031998 & 0.5851312768 \\
\hline
\end{tabular}




$\begin{array}{llll}\text { F } & 0.7729938890 & 0.7804087695 & 0.3409872676 \\ \text { F } & 0.8183309162 & 0.9293488382 & 0.3025511762 \\ \text { F } & 0.8941222326 & 0.7969133540 & 0.3490549671 \\ \text { F } & 0.8388119465 & 0.8981967782 & 0.4148687532 \\ \text { B } & 0.2178428430 & 0.1412114488 & 0.9520328718 \\ \text { B } & 0.7821571720 & 0.8587885512 & 0.0479671172 \\ \text { B } & 0.2821571420 & 0.6412114488 & 0.5479671282 \\ \text { B } & 0.7178428280 & 0.3587885512 & 0.4520328718 \\ \text { B } & 0.3305841523 & 0.6483940995 & 0.8520984028 \\ \text { B } & 0.6694158777 & 0.3516059305 & 0.1479015972 \\ \text { B } & 0.1694158477 & 0.1483940695 & 0.6479015972 \\ \text { B } & 0.8305841223 & 0.8516059005 & 0.3520984028\end{array}$

5- is

CELL_PARAMETERS (angstrom)

$\begin{array}{rrr}18.886986685 & 0.000000000 & 0.011250291 \\ 0.000000000 & 12.923090654 & 0.000000000 \\ -4.528156270 & 0.000000000 & 20.074949191\end{array}$

ATOMIC_POSITIONS (crystal)

\begin{tabular}{|c|c|c|c|}
\hline $\mathrm{Fe} 2$ & 0.0944140910 & 0.9636361022 & 0.2258168128 \\
\hline Fe1 & 0.5047513221 & 0.9643875785 & 0.2050097236 \\
\hline $\mathrm{Fe} 2$ & 0.9055859020 & 0.0363638868 & 0.7741831722 \\
\hline Fe1 & 0.4952486489 & 0.0356124435 & 0.7949902614 \\
\hline $\mathrm{Fe} 2$ & 0.4055859020 & 0.4636361022 & 0.2741832022 \\
\hline Fe1 & 0.9952486779 & 0.4643875495 & 0.2949902614 \\
\hline $\mathrm{Fe} 2$ & 0.5944140980 & 0.5363638978 & 0.7258168278 \\
\hline Fe1 & 0.0047513221 & 0.5356124215 & 0.7050097386 \\
\hline $\mathrm{N}$ & 0.0135467810 & 0.0585752129 & 0.2043384551 \\
\hline $\mathrm{N}$ & 0.0803706679 & 0.9268090412 & 0.1317305162 \\
\hline N & 0.0996855100 & 0.9748061688 & 0.3209552031 \\
\hline$N$ & 0.0192561774 & 0.8555618419 & 0.2280629048 \\
\hline$N$ & 0.6227083740 & 0.0119973109 & 0.1988743807 \\
\hline$N$ & 0.5562176509 & 0.0158225665 & 0.3035364838 \\
\hline$N$ & 0.4917260821 & 0.0431959194 & 0.1106976804 \\
\hline$N$ & 0.5557532666 & 0.8193938615 & 0.1835972267 \\
\hline$N$ & 0.9864532200 & 0.9414247271 & 0.7956615599 \\
\hline$N$ & 0.9196293611 & 0.0731909368 & 0.8682694988 \\
\hline $\mathrm{N}$ & 0.9003144900 & 0.0251938592 & 0.6790457799 \\
\hline$N$ & 0.9807438226 & 0.1444381581 & 0.7719370652 \\
\hline $\mathrm{N}$ & 0.3772916560 & 0.9880026891 & 0.8011256493 \\
\hline$N$ & 0.4437823191 & 0.9841774935 & 0.6964635162 \\
\hline $\mathrm{N}$ & 0.5082739479 & 0.9568040806 & 0.8893023416 \\
\hline$N$ & 0.4442467334 & 0.1806061535 & 0.8164027733 \\
\hline $\mathrm{N}$ & 0.4864532200 & 0.5585752729 & 0.2956615299 \\
\hline$N$ & 0.4196293321 & 0.4268090712 & 0.3682694988 \\
\hline$N$ & 0.4003144900 & 0.4748061398 & 0.1790447969 \\
\hline $\mathrm{N}$ & 0.4807438226 & 0.3555618419 & 0.2719370952 \\
\hline $\mathrm{N}$ & 0.8772916260 & 0.5119973109 & 0.3011256193 \\
\hline N & 0.9437823491 & 0.5158225065 & 0.1964635012 \\
\hline N & 0.0082739309 & 0.5431959194 & 0.3893023126 \\
\hline$N$ & 0.9442467334 & 0.3193938615 & 0.3164027733 \\
\hline $\mathrm{N}$ & 0.5135467800 & 0.4414247571 & 0.7043384401 \\
\hline$N$ & 0.5803706389 & 0.5731909588 & 0.6317305012 \\
\hline $\mathrm{N}$ & 0.5996855100 & 0.5251938312 & 0.8209542201 \\
\hline $\mathrm{N}$ & 0.5192561774 & 0.6444381581 & 0.7280629348 \\
\hline$N$ & 0.1227083150 & 0.4880026891 & 0.6988743507 \\
\hline $\mathrm{N}$ & 0.0562176509 & 0.4841774635 & 0.8035364838 \\
\hline $\mathrm{N}$ & 0.9917260521 & 0.4568040516 & 0.6106976584 \\
\hline
\end{tabular}




\begin{tabular}{|c|c|c|c|}
\hline $\mathrm{N}$ & 0.0557532666 & 0.6806061385 & 0.6835972267 \\
\hline C & 0.0187095587 & 0.1606523265 & 0.1916648361 \\
\hline C & 0.9467072574 & 0.0159734775 & 0.1978891396 \\
\hline C & 0.0244225094 & 0.8012459247 & 0.0508305042 \\
\hline C & 0.9576527489 & 0.2224146032 & 0.1716948465 \\
\hline C & 0.1036030338 & 0.9790761535 & 0.0835468226 \\
\hline C & 0.8886285528 & 0.1787912400 & 0.1638528860 \\
\hline C & 0.0666877938 & 0.8950394213 & 0.3456830581 \\
\hline C & 0.0398161886 & 0.8103099670 & 0.2963877634 \\
\hline C & 0.1315894459 & 0.0504343906 & 0.3625513557 \\
\hline C & 0.0424012463 & 0.8375744535 & 0.1160115628 \\
\hline C & 0.8833830438 & 0.0737561273 & 0.1771979719 \\
\hline C & 0.0484995849 & 0.8554268216 & 0.0011234947 \\
\hline C & 0.0895751301 & 0.9450028062 & 0.0178481872 \\
\hline C & 0.9450473302 & 0.9037469646 & 0.2158521063 \\
\hline C & 0.0252884493 & 0.7805380824 & 0.1744983009 \\
\hline C & 0.1316273062 & 0.0496745651 & 0.4301631623 \\
\hline C & 0.0624345087 & 0.8918277909 & 0.4123620384 \\
\hline C & 0.0958678522 & 0.9695985069 & 0.4554002470 \\
\hline C & 0.2368166197 & 0.9098976758 & 0.2355969353 \\
\hline C & 0.2335464776 & 0.0253288263 & 0.2274895778 \\
\hline C & 0.3598303204 & 0.0280758455 & 0.2229036294 \\
\hline C & 0.3010520011 & 0.8556409059 & 0.2350215050 \\
\hline C & 0.3614933306 & 0.9110702247 & 0.2260173284 \\
\hline C & 0.2955808425 & 0.0817234525 & 0.2240627449 \\
\hline C & 0.5509346018 & 0.0778500291 & 0.0908004938 \\
\hline C & 0.5281021238 & 0.9970499247 & 0.3571733462 \\
\hline C & 0.6244052308 & 0.0460504395 & 0.1306815106 \\
\hline C & 0.6186672631 & 0.0704615317 & 0.3114725928 \\
\hline C & 0.5197287237 & 0.7312157017 & 0.1623417737 \\
\hline C & 0.6280128389 & 0.8228062332 & 0.1869577303 \\
\hline C & 0.6669886111 & 0.9180794161 & 0.2176078780 \\
\hline C & 0.6446300525 & 0.0943245768 & 0.2487697549 \\
\hline C & 0.5539062912 & 0.6444961552 & 0.1432046193 \\
\hline C & 0.5450675888 & 0.1389709954 & 0.0338406002 \\
\hline C & 0.6552685118 & 0.1052308545 & 0.3739946470 \\
\hline C & 0.6653073485 & 0.7400577915 & 0.1666604378 \\
\hline C & 0.4765934092 & 0.1646571153 & 0.9969143953 \\
\hline C & 0.4251489916 & 0.0683962211 & 0.0744466695 \\
\hline C & 0.5619847174 & 0.0288078270 & 0.4209934307 \\
\hline C & 0.6280485991 & 0.6492385749 & 0.1445386974 \\
\hline C & 0.4152060282 & 0.1288467594 & 0.0174626989 \\
\hline C & 0.6269077514 & 0.0841006707 & 0.4296148461 \\
\hline C & 0.9812904623 & 0.8393476145 & 0.8083351789 \\
\hline C & 0.0532926826 & 0.9840265225 & 0.8021108454 \\
\hline C & 0.9755774906 & 0.1987540603 & 0.9491694688 \\
\hline C & 0.0423472511 & 0.7775853378 & 0.8283051235 \\
\hline C & 0.8963969582 & 0.0209238565 & 0.9164531694 \\
\hline C & 0.1113714472 & 0.8212087600 & 0.8361471290 \\
\hline C & 0.9333122142 & 0.1049605787 & 0.6543169419 \\
\hline C & 0.9601837974 & 0.1896900330 & 0.7036122366 \\
\hline C & 0.8684105691 & 0.9495655494 & 0.6374486143 \\
\hline C & 0.9575987717 & 0.1624255165 & 0.8839884222 \\
\hline C & 0.1166169562 & 0.9262438727 & 0.8228019981 \\
\hline C & 0.9515004231 & 0.1445741624 & 0.9988765143 \\
\hline C & 0.9104248779 & 0.0549971908 & 0.9821518408 \\
\hline C & 0.0549526698 & 0.0962530354 & 0.7841479237 \\
\hline C & 0.9747115277 & 0.2194619026 & 0.8255016841 \\
\hline
\end{tabular}




\begin{tabular}{|c|c|c|c|}
\hline C & 0.8683726938 & 0.9503254349 & 0.5698368377 \\
\hline C & 0.9375654983 & 0.1081722231 & 0.5876379616 \\
\hline C & 0.9041321478 & 0.0304015041 & 0.5445997830 \\
\hline C & 0.7631833803 & 0.0901022942 & 0.7644030947 \\
\hline C & 0.7664535374 & 0.9746711137 & 0.7725103932 \\
\hline C & 0.6401696796 & 0.9719242135 & 0.7770963856 \\
\hline C & 0.6989479989 & 0.1443591241 & 0.7649785100 \\
\hline C & 0.6385066694 & 0.0889297973 & 0.7739826716 \\
\hline C & 0.7044191275 & 0.9182764875 & 0.7759372551 \\
\hline C & 0.4490654282 & 0.9221499709 & 0.9091995062 \\
\hline C & 0.4718979052 & 0.0029500473 & 0.6428266238 \\
\hline C & 0.3755947982 & 0.9539495605 & 0.8693184894 \\
\hline C & 0.3813327079 & 0.9295385283 & 0.6885274072 \\
\hline C & 0.4802712763 & 0.2687842983 & 0.8376582413 \\
\hline C & 0.3719871611 & 0.1771937368 & 0.8130422847 \\
\hline C & 0.3330114189 & 0.0819205839 & 0.7823921370 \\
\hline C & 0.3553699775 & 0.9056754822 & 0.7512302451 \\
\hline C & 0.4460937088 & 0.3555038448 & 0.8567953657 \\
\hline C & 0.4549324112 & 0.8610290636 & 0.9661594258 \\
\hline C & 0.3447315172 & 0.8947690855 & 0.6260053530 \\
\hline C & 0.3346926215 & 0.2599422375 & 0.8333395622 \\
\hline C & 0.5234065908 & 0.8353428247 & 0.0030856637 \\
\hline C & 0.5748509784 & 0.9316037789 & 0.9255533305 \\
\hline C & 0.4380152826 & 0.9711922330 & 0.5790065693 \\
\hline C & 0.3719514009 & 0.3507614251 & 0.8554612876 \\
\hline C & 0.5847939418 & 0.8711532406 & 0.9825373311 \\
\hline C & 0.3730922786 & 0.9158993293 & 0.5703851249 \\
\hline C & 0.2631833803 & 0.4098977058 & 0.2644030647 \\
\hline C & 0.2664535074 & 0.5253288863 & 0.2725104222 \\
\hline C & 0.1401696646 & 0.5280757865 & 0.2770963856 \\
\hline C & 0.4812904323 & 0.6606523855 & 0.3083351789 \\
\hline C & 0.5532927426 & 0.5159734775 & 0.3021108754 \\
\hline C & 0.1989479839 & 0.3556408759 & 0.2649785100 \\
\hline C & 0.1385066694 & 0.4110701947 & 0.2739826716 \\
\hline C & 0.2044191425 & 0.5817235125 & 0.2759372551 \\
\hline C & 0.4755774906 & 0.3012459247 & 0.4491694988 \\
\hline C & 0.5423472511 & 0.7224146622 & 0.3283051535 \\
\hline C & 0.3963969582 & 0.4790761535 & 0.4164531694 \\
\hline C & 0.6113714472 & 0.6787913000 & 0.3361471290 \\
\hline C & 0.4333122142 & 0.3950394213 & 0.1543169269 \\
\hline C & 0.4601838264 & 0.3103099670 & 0.2036122216 \\
\hline C & 0.3684105691 & 0.5504344506 & 0.1374486293 \\
\hline C & 0.4575987417 & 0.3375744835 & 0.3839884512 \\
\hline C & 0.6166169562 & 0.5737561273 & 0.3228020281 \\
\hline C & 0.4515004231 & 0.3554268516 & 0.4988765143 \\
\hline C & 0.4104248779 & 0.4450028062 & 0.4821518108 \\
\hline C & 0.5549526698 & 0.4037469646 & 0.2841478937 \\
\hline C & 0.4747115577 & 0.2805381124 & 0.3255017141 \\
\hline C & 0.3683726938 & 0.5496745651 & 0.0698368447 \\
\hline C & 0.4375654983 & 0.3918277909 & 0.0876379696 \\
\hline C & 0.4041321478 & 0.4695985069 & 0.0445997560 \\
\hline C & 0.9490653982 & 0.5778500291 & 0.4091995062 \\
\hline C & 0.9718978762 & 0.4970499547 & 0.1428266388 \\
\hline C & 0.8755948282 & 0.5460504395 & 0.3693184894 \\
\hline C & 0.8813327369 & 0.5704614717 & 0.1885274072 \\
\hline C & 0.9802712763 & 0.2312156867 & 0.3376582113 \\
\hline C & 0.8719871611 & 0.3228062632 & 0.3130422847 \\
\hline C & 0.8330113889 & 0.4180794161 & 0.2823921070 \\
\hline
\end{tabular}




\begin{tabular}{|c|c|c|c|}
\hline C & 0.8553699475 & 0.5943245178 & 0.2512302451 \\
\hline C & 0.9460937088 & 0.1444961552 & 0.3567953957 \\
\hline C & 0.9549324112 & 0.6389709364 & 0.4661593968 \\
\hline C & 0.8447314882 & 0.6052309145 & 0.1260053530 \\
\hline C & 0.8346926515 & 0.2400577625 & 0.3333395622 \\
\hline C & 0.0234065798 & 0.6646571753 & 0.5030856047 \\
\hline C & 0.0748510014 & 0.5683962211 & 0.4255533305 \\
\hline C & 0.9380152826 & 0.5288077670 & 0.0790065773 \\
\hline C & 0.8719514009 & 0.1492385599 & 0.3554613176 \\
\hline C & 0.0847939718 & 0.6288467594 & 0.4825373011 \\
\hline C & 0.8730922486 & 0.5841006707 & 0.0703851469 \\
\hline C & 0.7368166197 & 0.5901023242 & 0.7355969053 \\
\hline C & 0.7335464626 & 0.4746711437 & 0.7274896068 \\
\hline C & 0.8598303204 & 0.4719241845 & 0.7229036144 \\
\hline C & 0.5187095377 & 0.3393476435 & 0.6916648211 \\
\hline C & 0.4467072874 & 0.4840265225 & 0.6978891546 \\
\hline C & 0.8010520011 & 0.6443590941 & 0.7350214900 \\
\hline C & 0.8614933306 & 0.5889297753 & 0.7260173284 \\
\hline C & 0.7955808725 & 0.4182765175 & 0.7240627449 \\
\hline C & 0.5244225094 & 0.6987540753 & 0.5508305312 \\
\hline C & 0.4576527489 & 0.2775853378 & 0.6716948765 \\
\hline C & 0.6036030418 & 0.5209238465 & 0.5835468306 \\
\hline C & 0.3886285528 & 0.3212087300 & 0.6638528710 \\
\hline C & 0.5666877858 & 0.6049605787 & 0.8456830581 \\
\hline C & 0.5398162026 & 0.6896900330 & 0.7963877634 \\
\hline C & 0.6315894309 & 0.4495655494 & 0.8625513857 \\
\hline C & 0.5424012283 & 0.6624255465 & 0.6160115778 \\
\hline C & 0.3833830148 & 0.4262438427 & 0.6771980019 \\
\hline C & 0.5484995769 & 0.6445731784 & 0.5011234857 \\
\hline C & 0.5895751221 & 0.5549971938 & 0.5178481592 \\
\hline C & 0.4450473302 & 0.5962530354 & 0.7158520763 \\
\hline C & 0.5252884723 & 0.7194619176 & 0.6744983159 \\
\hline C & 0.6316273062 & 0.4503254049 & 0.9301631623 \\
\hline C & 0.5624345017 & 0.6081722091 & 0.9123620384 \\
\hline C & 0.5958678522 & 0.5304014931 & 0.9554002170 \\
\hline C & 0.0509346018 & 0.4221499409 & 0.5908004938 \\
\hline C & 0.0281021238 & 0.5029500753 & 0.8571733762 \\
\hline C & 0.1244051718 & 0.4539495605 & 0.6306815106 \\
\hline C & 0.1186672631 & 0.4295384983 & 0.8114725928 \\
\hline C & 0.0197286647 & 0.7687842983 & 0.6623417587 \\
\hline C & 0.1280128989 & 0.6771937668 & 0.6869577153 \\
\hline C & 0.1669885511 & 0.5819205839 & 0.7176078630 \\
\hline C & 0.1446300525 & 0.4056754822 & 0.7487697549 \\
\hline C & 0.0539062322 & 0.8555038448 & 0.6432046343 \\
\hline C & 0.0450675298 & 0.3610290636 & 0.5338405742 \\
\hline C & 0.1552685118 & 0.3947690855 & 0.8739946470 \\
\hline C & 0.1653073485 & 0.7599422085 & 0.6666604378 \\
\hline C & 0.9765934092 & 0.3353428247 & 0.4969143653 \\
\hline C & 0.9251490216 & 0.4316037789 & 0.5744466695 \\
\hline C & 0.0619846584 & 0.4711922030 & 0.9209934307 \\
\hline C & 0.1280485991 & 0.8507614251 & 0.6445387124 \\
\hline C & 0.9152060582 & 0.3711532706 & 0.5174626689 \\
\hline C & 0.1269076914 & 0.4158993293 & 0.9296148751 \\
\hline $\mathrm{H}$ & 0.0736220615 & 0.1911962423 & 0.1987586204 \\
\hline $\mathrm{H}$ & 0.9909202464 & 0.7321037245 & 0.0395895557 \\
\hline $\mathrm{H}$ & 0.9644315200 & 0.3044576234 & 0.1630290194 \\
\hline $\mathrm{H}$ & 0.1343190153 & 0.0496996849 & 0.0988340399 \\
\hline $\mathrm{H}$ & 0.8397016028 & 0.2255790669 & 0.1485583736 \\
\hline
\end{tabular}




\begin{tabular}{|c|c|c|c|}
\hline $\mathrm{H}$ & 0.0841029410 & 0.7547984703 & 0.2970388404 \\
\hline $\mathrm{H}$ & 0.9947324825 & 0.7675762013 & 0.3097267600 \\
\hline $\mathrm{H}$ & 0.1573947877 & 0.1116250185 & 0.3394281740 \\
\hline $\mathrm{H}$ & 0.8309559476 & 0.0353602289 & 0.1710166395 \\
\hline $\mathrm{H}$ & 0.0349804900 & 0.8287962983 & 0.9496299546 \\
\hline $\mathrm{H}$ & 0.1099132239 & 0.9900279601 & 0.9810828886 \\
\hline $\mathrm{H}$ & 0.9232941480 & 0.8963981550 & 0.2610605334 \\
\hline $\mathrm{H}$ & 0.9074636062 & 0.8621478744 & 0.1759238456 \\
\hline $\mathrm{H}$ & 0.9756879799 & 0.7337001404 & 0.1593086310 \\
\hline $\mathrm{H}$ & 0.0704025027 & 0.7276993111 & 0.1949237441 \\
\hline $\mathrm{H}$ & 0.1584532915 & 0.1107571380 & 0.4630531585 \\
\hline $\mathrm{H}$ & 0.0330752366 & 0.8288480015 & 0.4297428673 \\
\hline $\mathrm{H}$ & 0.0942988619 & 0.9691987939 & 0.5080121279 \\
\hline $\mathrm{H}$ & 0.3029044163 & 0.7714839840 & 0.2395620289 \\
\hline $\mathrm{H}$ & 0.2940033594 & 0.1659753490 & 0.2206272919 \\
\hline $\mathrm{H}$ & 0.4766265328 & 0.9550551593 & 0.3475547748 \\
\hline $\mathrm{H}$ & 0.6443024906 & 0.9814059294 & 0.1046513878 \\
\hline $\mathrm{H}$ & 0.6634469523 & 0.1097249368 & 0.1314440168 \\
\hline $\mathrm{H}$ & 0.4618652424 & 0.7320762926 & 0.1616548624 \\
\hline $\mathrm{H}$ & 0.6773685734 & 0.9089119258 & 0.2720958862 \\
\hline $\mathrm{H}$ & 0.7204966659 & 0.9246691645 & 0.2050404461 \\
\hline $\mathrm{H}$ & 0.6200983331 & 0.1674250979 & 0.2270539558 \\
\hline $\mathrm{H}$ & 0.7039842399 & 0.1066047215 & 0.2604241549 \\
\hline $\mathrm{H}$ & 0.5226601132 & 0.5746962688 & 0.1269424990 \\
\hline $\mathrm{H}$ & 0.5942472196 & 0.1670915314 & 0.0201214344 \\
\hline $\mathrm{H}$ & 0.7054191583 & 0.1492743790 & 0.3801339149 \\
\hline $\mathrm{H}$ & 0.7229590634 & 0.7491297888 & 0.1683008900 \\
\hline $\mathrm{H}$ & 0.4708631675 & 0.2138973768 & 0.9530967416 \\
\hline $\mathrm{H}$ & 0.3787104071 & 0.0399634089 & 0.0926234639 \\
\hline $\mathrm{H}$ & 0.5377301675 & 0.0100933432 & 0.4629826164 \\
\hline $\mathrm{H}$ & 0.6559941532 & 0.5825361060 & 0.1295156148 \\
\hline $\mathrm{H}$ & 0.3606021702 & 0.1497386541 & 0.9911239905 \\
\hline $\mathrm{H}$ & 0.6554680100 & 0.1121621257 & 0.4782888722 \\
\hline $\mathrm{H}$ & 0.9263779605 & 0.8088037577 & 0.8012413946 \\
\hline $\mathrm{H}$ & 0.0090797536 & 0.2678962755 & 0.9604104733 \\
\hline $\mathrm{H}$ & 0.0355684210 & 0.6955423766 & 0.8369709656 \\
\hline $\mathrm{H}$ & 0.8656809997 & 0.9503003741 & 0.9011659451 \\
\hline $\mathrm{H}$ & 0.1602983972 & 0.7744209931 & 0.8514416264 \\
\hline $\mathrm{H}$ & 0.9158970510 & 0.2452015297 & 0.7029611596 \\
\hline $\mathrm{H}$ & 0.0052674585 & 0.2324237987 & 0.6902732400 \\
\hline $\mathrm{H}$ & 0.8426052123 & 0.8883749815 & 0.6605717960 \\
\hline $\mathrm{H}$ & 0.1690440524 & 0.9646397111 & 0.8289833755 \\
\hline $\mathrm{H}$ & 0.9650195170 & 0.1712036867 & 0.0503700454 \\
\hline $\mathrm{H}$ & 0.8900867531 & 0.0099720269 & 0.0189171114 \\
\hline $\mathrm{H}$ & 0.0767058520 & 0.1036018300 & 0.7389394366 \\
\hline $\mathrm{H}$ & 0.0925363348 & 0.1378520956 & 0.8240761404 \\
\hline $\mathrm{H}$ & 0.0243120201 & 0.2662998886 & 0.8406913690 \\
\hline $\mathrm{H}$ & 0.9295975123 & 0.2723006599 & 0.8050762559 \\
\hline $\mathrm{H}$ & 0.8415467235 & 0.8892428620 & 0.5369468125 \\
\hline $\mathrm{H}$ & 0.9669247364 & 0.1711519835 & 0.5702571327 \\
\hline $\mathrm{H}$ & 0.9057011611 & 0.0308012021 & 0.4919879021 \\
\hline $\mathrm{H}$ & 0.6970955537 & 0.2285159860 & 0.7604380011 \\
\hline $\mathrm{H}$ & 0.7059966406 & 0.8340246510 & 0.7793727381 \\
\hline $\mathrm{H}$ & 0.5233734972 & 0.0449448327 & 0.6524451962 \\
\hline $\mathrm{H}$ & 0.3556974794 & 0.0185940596 & 0.8953485972 \\
\hline $\mathrm{H}$ & 0.3365530767 & 0.8902751222 & 0.8685559832 \\
\hline $\mathrm{H}$ & 0.5381347876 & 0.2679237364 & 0.8383451676 \\
\hline $\mathrm{H}$ & 0.3226314566 & 0.0910880962 & 0.7279040838 \\
\hline
\end{tabular}




\begin{tabular}{|c|c|c|c|}
\hline $\mathrm{H}$ & 0.2795033341 & 0.0753308135 & 0.7949595539 \\
\hline $\mathrm{H}$ & 0.3799016959 & 0.8325749021 & 0.7729460442 \\
\hline $\mathrm{H}$ & 0.2960157901 & 0.8933952785 & 0.7395758151 \\
\hline $\mathrm{H}$ & 0.4773398868 & 0.4253037612 & 0.8730575160 \\
\hline $\mathrm{H}$ & 0.4057527804 & 0.8329084686 & 0.9798785386 \\
\hline $\mathrm{H}$ & 0.2945808717 & 0.8507246670 & 0.6198660851 \\
\hline $\mathrm{H}$ & 0.2770419196 & 0.2508702112 & 0.8316991250 \\
\hline $\mathrm{H}$ & 0.5291368325 & 0.7861026232 & 0.0469032584 \\
\hline $\mathrm{H}$ & 0.6212895629 & 0.9600365911 & 0.9073765131 \\
\hline $\mathrm{H}$ & 0.4622698325 & 0.9899066568 & 0.5370173536 \\
\hline $\mathrm{H}$ & 0.3440058468 & 0.4174638640 & 0.8704844002 \\
\hline $\mathrm{H}$ & 0.6393977998 & 0.8502614049 & 0.0088760095 \\
\hline $\mathrm{H}$ & 0.3445320200 & 0.8878378743 & 0.5217111278 \\
\hline $\mathrm{H}$ & 0.4263779305 & 0.6911962423 & 0.3012413646 \\
\hline $\mathrm{H}$ & 0.1970955837 & 0.2714840140 & 0.2604379711 \\
\hline $\mathrm{H}$ & 0.2059966556 & 0.6659753490 & 0.2793727081 \\
\hline $\mathrm{H}$ & 0.5090796936 & 0.2321037095 & 0.4604104443 \\
\hline $\mathrm{H}$ & 0.5355684800 & 0.8044576234 & 0.3369709656 \\
\hline $\mathrm{H}$ & 0.3656809997 & 0.5496996259 & 0.4011659451 \\
\hline $\mathrm{H}$ & 0.6602983372 & 0.7255790069 & 0.3514416264 \\
\hline $\mathrm{H}$ & 0.4158970510 & 0.2547984703 & 0.2029611446 \\
\hline $\mathrm{H}$ & 0.5052675175 & 0.2675762013 & 0.1902732540 \\
\hline $\mathrm{H}$ & 0.3426052123 & 0.6116250785 & 0.1605718110 \\
\hline $\mathrm{H}$ & 0.6690439924 & 0.5353602889 & 0.3289833755 \\
\hline $\mathrm{H}$ & 0.4650195170 & 0.3287962983 & 0.5503700454 \\
\hline $\mathrm{H}$ & 0.3900867831 & 0.4900279601 & 0.5189171114 \\
\hline $\mathrm{H}$ & 0.5767058520 & 0.3963981550 & 0.2389394516 \\
\hline $\mathrm{H}$ & 0.5925363938 & 0.3621479044 & 0.3240761404 \\
\hline $\mathrm{H}$ & 0.5243120201 & 0.2337001254 & 0.3406913690 \\
\hline $\mathrm{H}$ & 0.4295975123 & 0.2276993261 & 0.3050762559 \\
\hline $\mathrm{H}$ & 0.3415467235 & 0.6107571380 & 0.0369468305 \\
\hline $\mathrm{H}$ & 0.4669247664 & 0.3288480315 & 0.0702571407 \\
\hline $\mathrm{H}$ & 0.4057011311 & 0.4691987939 & 0.9919878721 \\
\hline $\mathrm{H}$ & 0.0233734742 & 0.4550551593 & 0.1524452102 \\
\hline $\mathrm{H}$ & 0.8556974494 & 0.4814059294 & 0.3953486272 \\
\hline $\mathrm{H}$ & 0.8365530477 & 0.6097248778 & 0.3685559832 \\
\hline $\mathrm{H}$ & 0.0381347686 & 0.2320762636 & 0.3383451376 \\
\hline $\mathrm{H}$ & 0.8226314266 & 0.4089118958 & 0.2279040988 \\
\hline $\mathrm{H}$ & 0.7795033341 & 0.4246691945 & 0.2949595539 \\
\hline $\mathrm{H}$ & 0.8799016669 & 0.6674250979 & 0.2729460442 \\
\hline $\mathrm{H}$ & 0.7960157601 & 0.6066047215 & 0.2395758451 \\
\hline $\mathrm{H}$ & 0.9773398868 & 0.0746962468 & 0.3730574860 \\
\hline $\mathrm{H}$ & 0.9057527804 & 0.6670915314 & 0.4798785686 \\
\hline $\mathrm{H}$ & 0.7945808417 & 0.6492753330 & 0.1198660921 \\
\hline $\mathrm{H}$ & 0.7770409366 & 0.2491297888 & 0.3316991250 \\
\hline $\mathrm{H}$ & 0.0291368255 & 0.7138973768 & 0.5469032584 \\
\hline $\mathrm{H}$ & 0.1212895859 & 0.5399634089 & 0.4073765431 \\
\hline $\mathrm{H}$ & 0.9622698325 & 0.5100933432 & 0.0370173686 \\
\hline $\mathrm{H}$ & 0.8440058468 & 0.0825361210 & 0.3704843702 \\
\hline $\mathrm{H}$ & 0.1393978298 & 0.6497385951 & 0.5088760095 \\
\hline $\mathrm{H}$ & 0.8445319900 & 0.6121621257 & 0.0217111188 \\
\hline $\mathrm{H}$ & 0.5736220395 & 0.3088037277 & 0.6987586054 \\
\hline $\mathrm{H}$ & 0.8029044463 & 0.7285160160 & 0.7395619989 \\
\hline $\mathrm{H}$ & 0.7940033594 & 0.3340246510 & 0.7206272619 \\
\hline $\mathrm{H}$ & 0.4909202764 & 0.7678962755 & 0.5395895267 \\
\hline $\mathrm{H}$ & 0.4644315200 & 0.1955423916 & 0.6630290344 \\
\hline $\mathrm{H}$ & 0.6343190003 & 0.4503003441 & 0.5988340549 \\
\hline $\mathrm{H}$ & 0.3397016328 & 0.2744209931 & 0.6485583736 \\
\hline
\end{tabular}




\begin{tabular}{|c|c|c|c|}
\hline $\mathrm{H}$ & 0.5841029490 & 0.7452015297 & 0.7970388404 \\
\hline $\mathrm{H}$ & 0.4947325125 & 0.7324237987 & 0.8097267600 \\
\hline $\mathrm{H}$ & 0.6573947877 & 0.3883749515 & 0.8394282040 \\
\hline $\mathrm{H}$ & 0.3309559776 & 0.4646397411 & 0.6710166245 \\
\hline $\mathrm{H}$ & 0.5349804830 & 0.6712037017 & 0.4496299546 \\
\hline $\mathrm{H}$ & 0.6099132469 & 0.5099720399 & 0.4810829176 \\
\hline $\mathrm{H}$ & 0.4232941480 & 0.6036018450 & 0.7610605634 \\
\hline $\mathrm{H}$ & 0.4074636062 & 0.6378521256 & 0.6759238596 \\
\hline $\mathrm{H}$ & 0.4756879799 & 0.7662998596 & 0.6593086310 \\
\hline $\mathrm{H}$ & 0.5704024877 & 0.7723006889 & 0.6949237441 \\
\hline $\mathrm{H}$ & 0.6584532765 & 0.3892428620 & 0.9630531875 \\
\hline $\mathrm{H}$ & 0.5330752636 & 0.6711519985 & 0.9297428673 \\
\hline $\mathrm{H}$ & 0.5942988389 & 0.5308012061 & 0.0080121279 \\
\hline $\mathrm{H}$ & 0.9766265028 & 0.5449448407 & 0.8475548038 \\
\hline $\mathrm{H}$ & 0.1443025506 & 0.5185940706 & 0.6046514028 \\
\hline $\mathrm{H}$ & 0.1634468933 & 0.3902751222 & 0.6314440168 \\
\hline $\mathrm{H}$ & 0.9618652124 & 0.7679237074 & 0.6616548324 \\
\hline $\mathrm{H}$ & 0.1773685134 & 0.5910880742 & 0.7720959162 \\
\hline $\mathrm{H}$ & 0.2204966069 & 0.5753308355 & 0.7050404461 \\
\hline $\mathrm{H}$ & 0.1200983331 & 0.3325748721 & 0.7270539558 \\
\hline $\mathrm{H}$ & 0.2039842399 & 0.3933953085 & 0.7604241849 \\
\hline $\mathrm{H}$ & 0.0226601132 & 0.9253037312 & 0.6269424840 \\
\hline $\mathrm{H}$ & 0.0942472786 & 0.3329084396 & 0.5201214614 \\
\hline $\mathrm{H}$ & 0.2054191583 & 0.3507256800 & 0.8801339149 \\
\hline $\mathrm{H}$ & 0.2229590634 & 0.7508702112 & 0.6683008750 \\
\hline $\mathrm{H}$ & 0.9708631675 & 0.2861026232 & 0.4530967716 \\
\hline $\mathrm{H}$ & 0.8787104371 & 0.4600365911 & 0.5926234869 \\
\hline $\mathrm{H}$ & 0.0377301675 & 0.4899066858 & 0.9629826464 \\
\hline $\mathrm{H}$ & 0.1559942132 & 0.9174638940 & 0.6295155998 \\
\hline $\mathrm{H}$ & 0.8606022002 & 0.3502614049 & 0.4911239905 \\
\hline $\mathrm{H}$ & 0.1554679500 & 0.3878378453 & 0.9782888722 \\
\hline 0 & 0.4196738505 & 0.0712866087 & 0.2184542066 \\
\hline 0 & 0.1702064452 & 0.0655270472 & 0.2251039207 \\
\hline 0 & 0.4219205429 & 0.8686800655 & 0.2204347626 \\
\hline 0 & 0.1773159460 & 0.8670514515 & 0.2424048164 \\
\hline 0 & 0.5803261495 & 0.9287134513 & 0.7815447794 \\
\hline $\mathrm{O}$ & 0.8297935398 & 0.9344729528 & 0.7748960793 \\
\hline 0 & 0.5780794571 & 0.1313199495 & 0.7795652074 \\
\hline 0 & 0.8226840390 & 0.1329485185 & 0.7575951836 \\
\hline 0 & 0.0803261425 & 0.5712865487 & 0.2815447794 \\
\hline 0 & 0.3297935698 & 0.5655270472 & 0.2748960793 \\
\hline 0 & 0.0780794491 & 0.3686800655 & 0.2795652374 \\
\hline $\mathrm{O}$ & 0.3226840690 & 0.3670514815 & 0.2575951836 \\
\hline 0 & 0.9196738505 & 0.4287134513 & 0.7184552206 \\
\hline 0 & 0.6702064602 & 0.4344729528 & 0.7251039207 \\
\hline 0 & 0.9219205429 & 0.6313199345 & 0.7204347926 \\
\hline 0 & 0.6773159610 & 0.6329485485 & 0.7424048164 \\
\hline $\mathrm{F}$ & 0.2526081138 & 0.2394637292 & 0.9661271063 \\
\hline $\mathrm{F}$ & 0.2378471181 & 0.0922030079 & 0.8998957490 \\
\hline $\mathrm{F}$ & 0.1395994108 & 0.1589847184 & 0.9373783058 \\
\hline $\mathrm{F}$ & 0.2347358059 & 0.0798776672 & 0.0122088502 \\
\hline $\mathrm{F}$ & 0.7473918862 & 0.7605362558 & 0.0338728857 \\
\hline $\mathrm{F}$ & 0.7621528969 & 0.9077970071 & 0.1001042360 \\
\hline $\mathrm{F}$ & 0.8604005892 & 0.8410152816 & 0.0626217172 \\
\hline $\mathrm{F}$ & 0.7652642241 & 0.9201223178 & 0.9877910908 \\
\hline $\mathrm{F}$ & 0.2473919002 & 0.7394637442 & 0.5338728937 \\
\hline $\mathrm{F}$ & 0.2621528969 & 0.5922029929 & 0.6001042510 \\
\hline $\mathrm{F}$ & 0.3604005892 & 0.6589847184 & 0.5626216942 \\
\hline
\end{tabular}




$\begin{array}{llll}\text { F } & 0.2652641941 & 0.5798776822 & 0.4877910908 \\ \text { F } & 0.7526081138 & 0.2605362858 & 0.4661271063 \\ \text { F } & 0.7378471031 & 0.4077969771 & 0.3998957790 \\ \text { F } & 0.6395994108 & 0.3410152816 & 0.4373782758 \\ \text { F } & 0.7347357759 & 0.4201223178 & 0.5122089092 \\ \text { F } & 0.2706683664 & 0.7189287892 & 0.8405384575 \\ \text { F } & 0.3204074623 & 0.5698603410 & 0.8050692296 \\ \text { F } & 0.3952343508 & 0.7053303620 & 0.8511003710 \\ \text { F } & 0.3378685734 & 0.6051529904 & 0.9181421072 \\ \text { F } & 0.7293316036 & 0.2810702278 & 0.1594615725 \\ \text { F } & 0.6795925377 & 0.4301396290 & 0.1949307554 \\ \text { F } & 0.6047656192 & 0.2946696680 & 0.1488996590 \\ \text { F } & 0.6621314566 & 0.3948470396 & 0.0818578778 \\ \text { F } & 0.2293316336 & 0.2189297722 & 0.6594615425 \\ \text { F } & 0.1795925527 & 0.0698603640 & 0.6949307704 \\ \text { F } & 0.1047656342 & 0.2053303320 & 0.6488996290 \\ \text { F } & 0.1621314416 & 0.1051529684 & 0.5818578928 \\ \text { F } & 0.7706683964 & 0.7810712108 & 0.3405384275 \\ \text { F } & 0.8204074623 & 0.9301396590 & 0.3050692596 \\ \text { F } & 0.8952343808 & 0.7946696380 & 0.3511003410 \\ \text { F } & 0.8378685434 & 0.8948470096 & 0.4181421372 \\ \text { B } & 0.2161437589 & 0.1422975060 & 0.9546091300 \\ \text { B } & 0.7838562561 & 0.8577024940 & 0.0453908590 \\ \text { B } & 0.2838562261 & 0.6422975060 & 0.5453908700 \\ \text { B } & 0.7161437439 & 0.3577024940 & 0.4546091300 \\ \text { B } & 0.3305791430 & 0.6494616465 & 0.8540868452 \\ \text { B } & 0.6694208870 & 0.3505383835 & 0.1459131548 \\ \text { B } & 0.1694208570 & 0.1494616165 & 0.6459131548 \\ \text { B } & 0.8305791130 & 0.8505383535 & 0.3540868452 \\ \text { F } & & & \end{array}$

5 -LS

CELL_PARAMETERS (angstrom)

$\begin{array}{lll}18.215268931 & 0.000000000 & -0.000676874\end{array}$

$\begin{array}{lll}0.000000000 & 13.425186656 & 0.000000000\end{array}$

$\begin{array}{lll}-3.622955900 & 0.000000000 & 20.109837866\end{array}$

\begin{tabular}{|c|c|c|c|}
\hline \multicolumn{4}{|c|}{ ATOMIC_POSITIONS (crystal) } \\
\hline $\mathrm{Fe} 2$ & 0.0855758834 & 0.9871136424 & 0.2278826700 \\
\hline $\mathrm{Fe} 2$ & 0.5132060747 & 0.9820389002 & 0.2088658382 \\
\hline Fe2 & 0.9144241166 & 0.0128863316 & 0.7721163170 \\
\hline $\mathrm{Fe} 2$ & 0.4867939253 & 0.0179610868 & 0.7911341768 \\
\hline $\mathrm{Fe} 2$ & 0.4144241166 & 0.4871136724 & 0.2721163170 \\
\hline $\mathrm{Fe} 2$ & 0.9867939253 & 0.4820389002 & 0.2911341768 \\
\hline $\mathrm{Fe} 2$ & 0.5855758834 & 0.5128863576 & 0.7278836830 \\
\hline $\mathrm{Fe} 2$ & 0.0132061337 & 0.5179610998 & 0.7088658232 \\
\hline $\mathrm{N}$ & 0.0002794582 & 0.0736288327 & 0.2041730369 \\
\hline $\mathrm{N}$ & 0.0772589766 & 0.9463315907 & 0.1345429781 \\
\hline $\mathrm{N}$ & 0.0866526619 & 1.0003388095 & 0.3227353368 \\
\hline $\mathrm{N}$ & 0.0111094040 & 0.8787698651 & 0.2326946226 \\
\hline $\mathrm{N}$ & 0.6216893931 & 0.0436943132 & 0.2112892264 \\
\hline $\mathrm{N}$ & 0.5442525655 & 0.0045215320 & 0.3074444151 \\
\hline $\mathrm{N}$ & 0.4958853791 & 0.0299969044 & 0.1145128905 \\
\hline $\mathrm{N}$ & 0.5677480661 & 0.8593896370 & 0.1930281742 \\
\hline $\mathrm{N}$ & 0.9997205568 & 0.9263711673 & 0.7958269781 \\
\hline $\mathrm{N}$ & 0.9227410314 & 0.0536684273 & 0.8654570369 \\
\hline $\mathrm{N}$ & 0.9133473161 & -0.0003387885 & 0.6772646332 \\
\hline $\mathrm{N}$ & 0.9888906090 & 0.1212301349 & 0.7673053924 \\
\hline $\mathrm{N}$ & 0.3783106369 & 0.9563056268 & 0.7887107886 \\
\hline $\mathrm{N}$ & 0.4557474645 & 0.9954794220 & 0.6925555849 \\
\hline
\end{tabular}




\begin{tabular}{|c|c|c|c|}
\hline $\mathrm{N}$ & 0.5041145919 & 0.9700030956 & 0.8854871395 \\
\hline $\mathrm{N}$ & 0.4322519339 & 0.1406103930 & 0.8069718408 \\
\hline $\mathrm{N}$ & 0.4997205268 & 0.5736288327 & 0.2958269491 \\
\hline $\mathrm{N}$ & 0.4227410314 & 0.4463315617 & 0.3654570369 \\
\hline $\mathrm{N}$ & 0.4133473461 & 0.5003387795 & 0.1772646482 \\
\hline $\mathrm{N}$ & 0.4888906090 & 0.3787698651 & 0.2673053624 \\
\hline $\mathrm{N}$ & 0.8783106069 & 0.5436943732 & 0.2887107886 \\
\hline $\mathrm{N}$ & 0.9557474345 & 0.5045205780 & 0.1925555999 \\
\hline $\mathrm{N}$ & 0.0041146109 & 0.5299969044 & 0.3854871095 \\
\hline $\mathrm{N}$ & 0.9322519339 & 0.3593896070 & 0.3069718408 \\
\hline $\mathrm{N}$ & 0.5002794432 & 0.4263711373 & 0.7041730219 \\
\hline $\mathrm{N}$ & 0.5772589686 & 0.5536684093 & 0.6345429631 \\
\hline $\mathrm{N}$ & 0.5866526839 & 0.4996611905 & 0.8227353668 \\
\hline $\mathrm{N}$ & 0.5111093910 & 0.6212301349 & 0.7326946076 \\
\hline $\mathrm{N}$ & 0.1216893931 & 0.4563056268 & 0.7112892114 \\
\hline $\mathrm{N}$ & 0.0442525065 & 0.4954794220 & 0.8074444151 \\
\hline $\mathrm{N}$ & 0.9958854081 & 0.4700030956 & 0.6145128605 \\
\hline $\mathrm{N}$ & 0.0677480661 & 0.6406103630 & 0.6930281592 \\
\hline C & 0.0025279151 & 0.1718488655 & 0.1896100593 \\
\hline C & 0.9329347206 & 0.0278683628 & 0.1967877898 \\
\hline C & 0.0310210210 & 0.8150590671 & 0.0585048040 \\
\hline C & 0.9381132885 & 0.2261195652 & 0.1667569914 \\
\hline C & 0.1021640652 & 0.9946389679 & 0.0847607167 \\
\hline C & 0.8688204210 & 0.1787332669 & 0.1571154121 \\
\hline C & 0.0548169015 & 0.9223695976 & 0.3496665850 \\
\hline C & 0.0287517293 & 0.8389510640 & 0.3019917827 \\
\hline C & 0.1169513636 & 0.0753116888 & 0.3627338274 \\
\hline C & 0.0431562486 & 0.8566327971 & 0.1218878809 \\
\hline C & 0.8666534907 & 0.0778464173 & 0.1722768376 \\
\hline C & 0.0568368211 & 0.8650755872 & 0.0071947202 \\
\hline C & 0.0936904072 & 0.9557862995 & 0.0206976826 \\
\hline C & 0.9335462500 & 0.9209211147 & 0.2178735099 \\
\hline C & 0.0237919681 & 0.8043177066 & 0.1816347992 \\
\hline C & 0.1169461283 & 0.0752647910 & 0.4307948824 \\
\hline C & 0.0512360933 & 0.9190346967 & 0.4171926721 \\
\hline C & 0.0833292538 & 0.9960173087 & 0.4585472646 \\
\hline C & 0.2331480806 & 0.9415291289 & 0.2369908744 \\
\hline C & 0.2282885671 & 0.0524260957 & 0.2277645396 \\
\hline C & 0.3599667688 & 0.0598573320 & 0.2225306051 \\
\hline C & 0.2998650187 & 0.8917651296 & 0.2345244585 \\
\hline C & 0.3614904604 & 0.9471920229 & 0.2236207205 \\
\hline C & 0.2919730160 & 0.1087231416 & 0.2237606840 \\
\hline C & 0.5508013689 & 0.0902342437 & 0.0995359796 \\
\hline C & 0.5111691664 & 0.9672736860 & 0.3561567098 \\
\hline C & 0.6261245345 & 0.0846913314 & 0.1441281371 \\
\hline C & 0.6001816216 & 0.0726426647 & 0.3239319352 \\
\hline C & 0.5336988402 & 0.7732346888 & 0.1703908579 \\
\hline C & 0.6432735120 & 0.8646208040 & 0.2009087254 \\
\hline C & 0.6758874763 & 0.9605885441 & 0.2298527311 \\
\hline C & 0.6298253024 & 0.1176335117 & 0.2659760207 \\
\hline C & 0.5735239997 & 0.6908414609 & 0.1548140085 \\
\hline C & 0.5387914363 & 0.1525932892 & 0.0441682452 \\
\hline C & 0.6262268431 & 0.1003315999 & 0.3896276235 \\
\hline C & 0.6860588547 & 0.7852423524 & 0.1853399388 \\
\hline C & 0.4691508906 & 0.1530490993 & 0.0028249979 \\
\hline C & 0.4288278315 & 0.0301804508 & 0.0735921262 \\
\hline C & 0.5344171646 & 0.9917169794 & 0.4226980294 \\
\hline C & 0.6510071291 & 0.6969170137 & 0.1618004153 \\
\hline
\end{tabular}




\begin{tabular}{|c|c|c|c|}
\hline C & 0.4133127783 & 0.0902125582 & 0.0174643121 \\
\hline C & 0.5932338128 & 0.0586252430 & 0.4399135940 \\
\hline C & 0.9974720789 & 0.8281511345 & 0.8103899557 \\
\hline C & 0.0670652794 & 0.9721316372 & 0.8032131942 \\
\hline C & 0.9689789870 & 0.1849409179 & 0.9414951850 \\
\hline C & 0.0618867115 & 0.7738804948 & 0.8332439926 \\
\hline C & 0.8978359428 & 0.0053610541 & 0.9152392833 \\
\hline C & 0.1311795790 & 0.8212667331 & 0.8428845579 \\
\hline C & 0.9451831065 & 0.0776303874 & 0.6503333850 \\
\hline C & 0.9712482667 & 0.1610489660 & 0.6980082473 \\
\hline C & 0.8830486284 & 0.9246883112 & 0.6372651886 \\
\hline C & 0.9568447394 & 0.1433672029 & 0.8781121411 \\
\hline C & 0.1333465093 & 0.9221536417 & 0.8277231474 \\
\hline C & 0.9431631749 & 0.1349244278 & 0.9928052868 \\
\hline C & 0.9063095998 & 0.0442136745 & 0.9793023184 \\
\hline C & 0.0664537500 & 0.0790789073 & 0.7821264901 \\
\hline C & 0.9762080319 & 0.1956822634 & 0.8183651858 \\
\hline C & 0.8830538567 & 0.9247351490 & 0.5692051176 \\
\hline C & 0.9487639207 & 0.0809652963 & 0.5828073279 \\
\hline C & 0.9166707392 & 0.0039827113 & 0.5414527654 \\
\hline C & 0.7668519344 & 0.0584708751 & 0.7630091556 \\
\hline C & 0.7717114479 & 0.9475739043 & 0.7722354604 \\
\hline C & 0.6400332312 & 0.9401426080 & 0.7774694099 \\
\hline C & 0.7001349513 & 0.1082348774 & 0.7654755565 \\
\hline C & 0.6385095696 & 0.0528079511 & 0.7763792945 \\
\hline C & 0.7080270130 & 0.8912769184 & 0.7762393010 \\
\hline C & 0.4491986311 & 0.9097656973 & 0.9004640274 \\
\hline C & 0.4888308336 & 0.0327263210 & 0.6438432902 \\
\hline C & 0.3738754655 & 0.9153086686 & 0.8558718919 \\
\hline C & 0.3998184084 & 0.9273573943 & 0.6760680348 \\
\hline C & 0.4663011598 & 0.2267652962 & 0.8296081591 \\
\hline C & 0.3567264880 & 0.1353792110 & 0.7990912896 \\
\hline C & 0.3241124937 & 0.0394114709 & 0.7701472839 \\
\hline C & 0.3701746976 & 0.8823665473 & 0.7340240093 \\
\hline C & 0.4264759703 & 0.3091585091 & 0.8451859765 \\
\hline C & 0.4612085637 & 0.8474067108 & 0.9558317288 \\
\hline C & 0.3737731569 & 0.8996673271 & 0.6103723765 \\
\hline C & 0.3139411753 & 0.2147576336 & 0.8146600902 \\
\hline C & 0.5308490804 & 0.8469509607 & 0.9971739981 \\
\hline C & 0.5711721395 & 0.9698195492 & 0.9264078888 \\
\hline C & 0.4655828354 & 0.0082830016 & 0.5773020006 \\
\hline C & 0.3489928409 & 0.3030830163 & 0.8381995697 \\
\hline C & 0.5866872507 & 0.9097875008 & 0.9825356689 \\
\hline C & 0.4067662172 & 0.9413746970 & 0.5600864350 \\
\hline C & 0.2668519344 & 0.4415291289 & 0.2630091256 \\
\hline C & 0.2717114189 & 0.5524260957 & 0.2722354604 \\
\hline C & 0.1400332162 & 0.5598573920 & 0.2774694099 \\
\hline C & 0.4974720789 & 0.6718488655 & 0.3103899557 \\
\hline C & 0.5670652794 & 0.5278683628 & 0.3032122102 \\
\hline C & 0.2001349813 & 0.3917651296 & 0.2654755565 \\
\hline C & 0.1385095396 & 0.4471920529 & 0.2763792645 \\
\hline C & 0.2080269990 & 0.6087230816 & 0.2762393010 \\
\hline C & 0.4689789870 & 0.3150590671 & 0.4414951850 \\
\hline C & 0.5618867115 & 0.7261195052 & 0.3332430086 \\
\hline C & 0.3978359428 & 0.4946389379 & 0.4152392833 \\
\hline C & 0.6311795790 & 0.6787332669 & 0.3428845879 \\
\hline C & 0.4451831065 & 0.4223695976 & 0.1503334000 \\
\hline C & 0.4712482667 & 0.3389510340 & 0.1980082173 \\
\hline
\end{tabular}




\begin{tabular}{|c|c|c|c|}
\hline C & 0.3830486284 & 0.5753116888 & 0.1372661726 \\
\hline C & 0.4568437554 & 0.3566327971 & 0.3781121111 \\
\hline C & 0.6333465093 & 0.5778463583 & 0.3277231474 \\
\hline C & 0.4431631749 & 0.3650755572 & 0.4928052868 \\
\hline C & 0.4063095998 & 0.4557863295 & 0.4793023184 \\
\hline C & 0.5664537500 & 0.4209210857 & 0.2821264901 \\
\hline C & 0.4762080319 & 0.3043177366 & 0.3183652158 \\
\hline C & 0.3830538867 & 0.5752648510 & 0.0692051246 \\
\hline C & 0.4487638917 & 0.4190346967 & 0.0828073419 \\
\hline C & 0.4166707392 & 0.4960172797 & 0.0414527394 \\
\hline C & 0.9491986311 & 0.5902343027 & 0.4004640274 \\
\hline C & 0.9888308336 & 0.4672736860 & 0.1438433052 \\
\hline C & 0.8738754655 & 0.5846913904 & 0.3558718629 \\
\hline C & 0.8998183784 & 0.5726426057 & 0.1760680648 \\
\hline C & 0.9663011598 & 0.2732346888 & 0.3296091421 \\
\hline C & 0.8567264880 & 0.3646207740 & 0.2990912606 \\
\hline C & 0.8241125237 & 0.4605885141 & 0.2701472839 \\
\hline C & 0.8701746976 & 0.6176334527 & 0.2340239943 \\
\hline C & 0.9264760003 & 0.1908414909 & 0.3451860065 \\
\hline C & 0.9612085637 & 0.6525932892 & 0.4558317588 \\
\hline C & 0.8737731569 & 0.6003326729 & 0.1103723685 \\
\hline C & 0.8139411453 & 0.2852423524 & 0.3146600612 \\
\hline C & 0.0308491044 & 0.6530490393 & 0.4971739981 \\
\hline C & 0.0711721615 & 0.5301804508 & 0.4264078588 \\
\hline C & 0.9655828354 & 0.4917170094 & 0.0773019776 \\
\hline C & 0.8489928709 & 0.1969169987 & 0.3381995697 \\
\hline C & 0.0866872287 & 0.5902124992 & 0.4825356989 \\
\hline C & 0.9067661872 & 0.5586253030 & 0.0600864060 \\
\hline C & 0.7331480656 & 0.5584708711 & 0.7369908444 \\
\hline C & 0.7282885521 & 0.4475738743 & 0.7277645396 \\
\hline C & 0.8599667688 & 0.4401426380 & 0.7225305901 \\
\hline C & 0.5025279211 & 0.3281511045 & 0.6896100443 \\
\hline C & 0.4329347206 & 0.4721316082 & 0.6967868058 \\
\hline C & 0.7998650487 & 0.6082348704 & 0.7345244435 \\
\hline C & 0.8614904304 & 0.5528079771 & 0.7236207055 \\
\hline C & 0.7919729870 & 0.3912768884 & 0.7237606990 \\
\hline C & 0.5310210130 & 0.6849409329 & 0.5585048150 \\
\hline C & 0.4381132885 & 0.2738804948 & 0.6667560074 \\
\hline C & 0.6021640572 & 0.5053610321 & 0.5847607167 \\
\hline C & 0.3688204510 & 0.3212667631 & 0.6571154421 \\
\hline C & 0.5548168935 & 0.5776304024 & 0.8496666150 \\
\hline C & 0.5287517333 & 0.6610489360 & 0.8019917527 \\
\hline C & 0.6169513716 & 0.4246883402 & 0.8627348114 \\
\hline C & 0.5431552606 & 0.6433672029 & 0.6218878589 \\
\hline C & 0.3666534607 & 0.4221536417 & 0.6722768526 \\
\hline C & 0.5568368251 & 0.6349244128 & 0.5071947132 \\
\hline C & 0.5936904002 & 0.5442137005 & 0.5206976816 \\
\hline C & 0.4335462500 & 0.5790788853 & 0.7178735099 \\
\hline C & 0.5237919681 & 0.6956822934 & 0.6816348142 \\
\hline C & 0.6169461433 & 0.4247351490 & 0.9307948824 \\
\hline C & 0.5512360793 & 0.5809653033 & 0.9171926721 \\
\hline C & 0.5833292608 & 0.5039826913 & 0.9585472346 \\
\hline C & 0.0508013689 & 0.4097657273 & 0.5995359726 \\
\hline C & 0.0111691664 & 0.5327263140 & 0.8561567098 \\
\hline C & 0.1261244745 & 0.4153086396 & 0.6441281081 \\
\hline C & 0.1001816216 & 0.4273573943 & 0.8239319652 \\
\hline C & 0.0336988402 & 0.7267653112 & 0.6703918409 \\
\hline C & 0.1432735120 & 0.6353791960 & 0.7009087104 \\
\hline
\end{tabular}




\begin{tabular}{|c|c|c|c|}
\hline C & 0.1758874763 & 0.5394114559 & 0.7298527161 \\
\hline C & 0.1298253624 & 0.3823665183 & 0.7659759907 \\
\hline C & 0.0735240597 & 0.8091585391 & 0.6548140235 \\
\hline C & 0.0387914363 & 0.3474067408 & 0.5441682712 \\
\hline C & 0.1262267831 & 0.3996683401 & 0.8896276235 \\
\hline C & 0.1860587947 & 0.7147576476 & 0.6853399098 \\
\hline C & 0.9691509196 & 0.3469509607 & 0.5028260019 \\
\hline C & 0.9288278605 & 0.4698195492 & 0.5735921112 \\
\hline C & 0.0344171046 & 0.5082830206 & 0.9226979994 \\
\hline C & 0.1510071291 & 0.8030829863 & 0.6618004303 \\
\hline C & 0.9133127493 & 0.4097875008 & 0.5174643311 \\
\hline C & 0.0932338128 & 0.4413746970 & 0.9399135650 \\
\hline $\mathrm{H}$ & 0.0580217004 & 0.2051105485 & 0.1973054939 \\
\hline $\mathrm{H}$ & 1.0006781094 & 0.7448892841 & 0.0499309615 \\
\hline $\mathrm{H}$ & 0.9418588662 & 0.3052173229 & 0.1565754348 \\
\hline $\mathrm{H}$ & 0.1300306203 & 0.0657319366 & 0.0973387550 \\
\hline $\mathrm{H}$ & 0.8181408870 & 0.2207995182 & 0.1385127042 \\
\hline $\mathrm{H}$ & 0.0739182843 & 0.7839334759 & 0.3039784581 \\
\hline $\mathrm{H}$ & 0.9807273823 & 0.7997831424 & 0.3162112277 \\
\hline $\mathrm{H}$ & 0.1420138606 & 0.1350838389 & 0.3379604518 \\
\hline $\mathrm{H}$ & 0.8145334133 & 0.0362136793 & 0.1636854189 \\
\hline $\mathrm{H}$ & 0.0479102310 & 0.8342189738 & 0.9570337971 \\
\hline $\mathrm{H}$ & 0.1148658317 & -0.0021109105 & 0.9824529803 \\
\hline $\mathrm{H}$ & 0.9079330241 & 0.9156658937 & 0.2626536632 \\
\hline $\mathrm{H}$ & 0.8988283285 & 0.8768471747 & 0.1787189264 \\
\hline $\mathrm{H}$ & 0.9751941828 & 0.7552148084 & 0.1670992718 \\
\hline $\mathrm{H}$ & 0.0711480334 & 0.7573553087 & 0.2040437285 \\
\hline $\mathrm{H}$ & 0.1428258086 & 0.1358267513 & 0.4621850916 \\
\hline $\mathrm{H}$ & 0.0236771595 & 0.8563612504 & 0.4366508409 \\
\hline $\mathrm{H}$ & 0.0825989044 & 0.9952988824 & 0.5118015105 \\
\hline $\mathrm{H}$ & 0.3032852036 & 0.8107501417 & 0.2383488365 \\
\hline $\mathrm{H}$ & 0.2888089933 & 0.1897480254 & 0.2198457379 \\
\hline $\mathrm{H}$ & 0.4649135108 & 0.9162445071 & 0.3399931431 \\
\hline $\mathrm{H}$ & 0.6617945584 & 0.0346187154 & 0.1205883050 \\
\hline $\mathrm{H}$ & 0.6529456303 & 0.1583513945 & 0.1486730070 \\
\hline $\mathrm{H}$ & 0.4731742130 & 0.7731623075 & 0.1654655215 \\
\hline $\mathrm{H}$ & 0.6869522460 & 0.9530726872 & 0.2843781354 \\
\hline $\mathrm{H}$ & 0.7297244194 & 0.9768273255 & 0.2148959535 \\
\hline $\mathrm{H}$ & 0.5965276050 & 0.1835919491 & 0.2476514523 \\
\hline $\mathrm{H}$ & 0.6880635205 & 0.1424824719 & 0.2809784316 \\
\hline $\mathrm{H}$ & 0.5439590269 & 0.6227767731 & 0.1370024258 \\
\hline $\mathrm{H}$ & 0.5836361073 & 0.2016214292 & 0.0351509020 \\
\hline $\mathrm{H}$ & 0.6717718527 & 0.1538286364 & 0.4017144991 \\
\hline $\mathrm{H}$ & 0.7461905816 & 0.7945263366 & 0.1913844886 \\
\hline $\mathrm{H}$ & 0.4583079456 & 0.2024207824 & 0.9598215171 \\
\hline $\mathrm{H}$ & 0.3865822991 & 0.9812861217 & 0.0879220749 \\
\hline $\mathrm{H}$ & 0.5070559633 & 0.9563925534 & 0.4599082741 \\
\hline $\mathrm{H}$ & 0.6825779442 & 0.6333521298 & 0.1486436346 \\
\hline $\mathrm{H}$ & 0.3580371464 & 0.0883494603 & 0.9867329623 \\
\hline $\mathrm{H}$ & 0.6138147799 & 0.0786873833 & 0.4917043402 \\
\hline $\mathrm{H}$ & 0.9419782706 & 0.7948893915 & 0.8026945361 \\
\hline $\mathrm{H}$ & -0.0006781694 & 0.2551106859 & 0.9500690605 \\
\hline $\mathrm{H}$ & 0.0581410748 & 0.6947826771 & 0.8434245952 \\
\hline $\mathrm{H}$ & 0.8699693947 & 0.9342680634 & 0.9026612520 \\
\hline $\mathrm{H}$ & 0.1818591130 & 0.7792004818 & 0.8614873108 \\
\hline $\mathrm{H}$ & 0.9260816927 & 0.2160665541 & 0.6960215419 \\
\hline $\mathrm{H}$ & 0.0192726177 & 0.2002168276 & 0.6837887723 \\
\hline $\mathrm{H}$ & 0.8579861244 & 0.8649162211 & 0.6620395482 \\
\hline
\end{tabular}




\begin{tabular}{|c|c|c|c|}
\hline $\mathrm{H}$ & 0.1854665867 & 0.9637863207 & 0.8363145811 \\
\hline $\mathrm{H}$ & 0.9520897880 & 0.1657810562 & 0.0429662029 \\
\hline $\mathrm{H}$ & 0.8851341603 & 1.0021109105 & 0.0175470197 \\
\hline $\mathrm{H}$ & 0.0920669159 & 0.0843330773 & 0.7373463368 \\
\hline $\mathrm{H}$ & 0.1011707175 & 0.1231528253 & 0.8212810586 \\
\hline $\mathrm{H}$ & 0.0248058172 & 0.2447851916 & 0.8329007432 \\
\hline $\mathrm{H}$ & 0.9288519666 & 0.2426446913 & 0.7959563005 \\
\hline $\mathrm{H}$ & 0.8571741764 & 0.8641732487 & 0.5378149084 \\
\hline $\mathrm{H}$ & 0.9763228515 & 0.1436387356 & 0.5633491591 \\
\hline $\mathrm{H}$ & 0.9174011176 & 0.0047011436 & 0.4881984595 \\
\hline $\mathrm{H}$ & 0.6967147964 & 0.1892498733 & 0.7616511785 \\
\hline $\mathrm{H}$ & 0.7111909767 & 0.8102520346 & 0.7801542621 \\
\hline $\mathrm{H}$ & 0.5350864892 & 0.0837554709 & 0.6600068869 \\
\hline $\mathrm{H}$ & 0.3382054416 & 0.9653812246 & 0.8794116950 \\
\hline $\mathrm{H}$ & 0.3470543997 & 0.8416486055 & 0.8513270080 \\
\hline $\mathrm{H}$ & 0.5268257870 & 0.2268377075 & 0.8345344785 \\
\hline $\mathrm{H}$ & 0.3130477540 & 0.0469273088 & 0.7156218646 \\
\hline $\mathrm{H}$ & 0.2702755806 & 0.0231726525 & 0.7851040615 \\
\hline $\mathrm{H}$ & 0.4034723650 & 0.8164079909 & 0.7523485477 \\
\hline $\mathrm{H}$ & 0.3119364795 & 0.8575185411 & 0.7190215684 \\
\hline $\mathrm{H}$ & 0.4560409731 & 0.3772231969 & 0.8629975892 \\
\hline $\mathrm{H}$ & 0.4163638927 & 0.7983785708 & 0.9648490760 \\
\hline $\mathrm{H}$ & 0.3282281473 & 0.8461713036 & 0.5982855009 \\
\hline $\mathrm{H}$ & 0.2538094184 & 0.2054736484 & 0.8086154964 \\
\hline $\mathrm{H}$ & 0.5416920844 & 0.7975792176 & 0.0401784829 \\
\hline $\mathrm{H}$ & 0.6134177009 & 0.0187138963 & 0.9120779471 \\
\hline $\mathrm{H}$ & 0.4929440367 & 0.0436074506 & 0.5400917559 \\
\hline $\mathrm{H}$ & 0.3174220558 & 0.3666478702 & 0.8513563954 \\
\hline $\mathrm{H}$ & 0.6419628236 & 0.9116505997 & 0.0132670377 \\
\hline $\mathrm{H}$ & 0.3861852201 & 0.9213126167 & 0.5082956898 \\
\hline $\mathrm{H}$ & 0.4419782996 & 0.7051106085 & 0.3026945061 \\
\hline $\mathrm{H}$ & 0.1967147814 & 0.3107501117 & 0.2616511785 \\
\hline $\mathrm{H}$ & 0.2111910067 & 0.6897479654 & 0.2801542621 \\
\hline $\mathrm{H}$ & 0.4993218906 & 0.2448892991 & 0.4500690305 \\
\hline $\mathrm{H}$ & 0.5581411338 & 0.8052173229 & 0.3434245652 \\
\hline $\mathrm{H}$ & 0.3699693947 & 0.5657319366 & 0.4026612520 \\
\hline $\mathrm{H}$ & 0.6818591130 & 0.7207994582 & 0.3614872808 \\
\hline $\mathrm{H}$ & 0.4260817227 & 0.2839334459 & 0.1960215269 \\
\hline $\mathrm{H}$ & 0.5192726177 & 0.2997831724 & 0.1837887723 \\
\hline $\mathrm{H}$ & 0.3579861544 & 0.6350837789 & 0.1620395482 \\
\hline $\mathrm{H}$ & 0.6854665867 & 0.5362136793 & 0.3363145811 \\
\hline $\mathrm{H}$ & 0.4520897580 & 0.3342189438 & 0.5429662029 \\
\hline $\mathrm{H}$ & 0.3851341603 & 0.4978890895 & 0.5175470197 \\
\hline $\mathrm{H}$ & 0.5920669759 & 0.4156669077 & 0.2373463218 \\
\hline $\mathrm{H}$ & 0.6011716715 & 0.3768471747 & 0.3212810586 \\
\hline $\mathrm{H}$ & 0.5248058172 & 0.2552148084 & 0.3329007132 \\
\hline $\mathrm{H}$ & 0.4288519666 & 0.2573553087 & 0.2959562715 \\
\hline $\mathrm{H}$ & 0.3571741764 & 0.6358267513 & 0.0378149084 \\
\hline $\mathrm{H}$ & 0.4763228515 & 0.3563612504 & 0.0633491661 \\
\hline $\mathrm{H}$ & 0.4174010886 & 0.4952988524 & 0.9881984895 \\
\hline $\mathrm{H}$ & 0.0350865002 & 0.4162445371 & 0.1600068569 \\
\hline $\mathrm{H}$ & 0.8382054416 & 0.5346187754 & 0.3794116950 \\
\hline $\mathrm{H}$ & 0.8470543697 & 0.6583513945 & 0.3513269780 \\
\hline $\mathrm{H}$ & 0.0268257850 & 0.2731623075 & 0.3345344785 \\
\hline $\mathrm{H}$ & 0.8130477540 & 0.4530726872 & 0.2156218646 \\
\hline $\mathrm{H}$ & 0.7702755806 & 0.4768273555 & 0.2851040615 \\
\hline $\mathrm{H}$ & 0.9034723950 & 0.6835920091 & 0.2523485477 \\
\hline $\mathrm{H}$ & 0.8119364795 & 0.6424814589 & 0.2190215834 \\
\hline
\end{tabular}




\begin{tabular}{|c|c|c|c|}
\hline $\mathrm{H}$ & 0.9560409731 & 0.1227767881 & 0.3629975892 \\
\hline $\mathrm{H}$ & 0.9163638927 & 0.7016214292 & 0.4648491060 \\
\hline $\mathrm{H}$ & 0.8282281473 & 0.6538286964 & 0.0982854859 \\
\hline $\mathrm{H}$ & 0.7538094184 & 0.2945263366 & 0.3086154964 \\
\hline $\mathrm{H}$ & 0.0416920584 & 0.7024208424 & 0.5401784829 \\
\hline $\mathrm{H}$ & 0.1134176939 & 0.4812860917 & 0.4120779171 \\
\hline $\mathrm{H}$ & 0.9929440367 & 0.4563925534 & 0.0400917369 \\
\hline $\mathrm{H}$ & 0.8174220558 & 0.1333521298 & 0.3513563654 \\
\hline $\mathrm{H}$ & 0.1419628386 & 0.5883494003 & 0.5132670377 \\
\hline $\mathrm{H}$ & 0.8861852201 & 0.5786874433 & 0.0082956658 \\
\hline $\mathrm{H}$ & 0.5580217294 & 0.2948893915 & 0.6973054639 \\
\hline $\mathrm{H}$ & 0.8032852036 & 0.6892498583 & 0.7383488215 \\
\hline $\mathrm{H}$ & 0.7888090233 & 0.3102520046 & 0.7198457379 \\
\hline $\mathrm{H}$ & 0.5006781094 & 0.7551107159 & 0.5499309395 \\
\hline $\mathrm{H}$ & 0.4418588952 & 0.1947826921 & 0.6565754048 \\
\hline $\mathrm{H}$ & 0.6300306053 & 0.4342680934 & 0.5973387480 \\
\hline $\mathrm{H}$ & 0.3181408870 & 0.2792005118 & 0.6385126892 \\
\hline $\mathrm{H}$ & 0.5739183073 & 0.7160665241 & 0.8039784581 \\
\hline $\mathrm{H}$ & 0.4807274123 & 0.7002168576 & 0.8162112277 \\
\hline $\mathrm{H}$ & 0.6420138756 & 0.3649162211 & 0.8379604518 \\
\hline $\mathrm{H}$ & 0.3145334133 & 0.4637863207 & 0.6636854189 \\
\hline $\mathrm{H}$ & 0.5479102120 & 0.6657810262 & 0.4570337971 \\
\hline $\mathrm{H}$ & 0.6148658397 & 0.5021109105 & 0.4824530093 \\
\hline $\mathrm{H}$ & 0.4079330241 & 0.5843341063 & 0.7626536632 \\
\hline $\mathrm{H}$ & 0.3988293425 & 0.6231528253 & 0.6787189414 \\
\hline $\mathrm{H}$ & 0.4751941828 & 0.7447851916 & 0.6670992568 \\
\hline $\mathrm{H}$ & 0.5711480334 & 0.7426446913 & 0.7040436995 \\
\hline $\mathrm{H}$ & 0.6428258236 & 0.3641732487 & 0.9621850916 \\
\hline $\mathrm{H}$ & 0.5236771485 & 0.6436387496 & 0.9366508409 \\
\hline $\mathrm{H}$ & 0.5825988824 & 0.5047011176 & 0.0118015705 \\
\hline $\mathrm{H}$ & 0.9649135108 & 0.5837554929 & 0.8399931131 \\
\hline $\mathrm{H}$ & 0.1617944994 & 0.4653812246 & 0.6205883050 \\
\hline $\mathrm{H}$ & 0.1529455713 & 0.3416486055 & 0.6486729920 \\
\hline $\mathrm{H}$ & 0.9731742130 & 0.7268376925 & 0.6654655215 \\
\hline $\mathrm{H}$ & 0.1869521870 & 0.5469273128 & 0.7843781354 \\
\hline $\mathrm{H}$ & 0.2297244784 & 0.5231726745 & 0.7148959385 \\
\hline $\mathrm{H}$ & 0.0965276050 & 0.3164079909 & 0.7476514523 \\
\hline $\mathrm{H}$ & 0.1880635205 & 0.3575175581 & 0.7809784316 \\
\hline $\mathrm{H}$ & 0.0439590869 & 0.8772232269 & 0.6370024108 \\
\hline $\mathrm{H}$ & 0.0836361663 & 0.2983785408 & 0.5351509240 \\
\hline $\mathrm{H}$ & 0.1717718527 & 0.3461713336 & 0.9017144991 \\
\hline $\mathrm{H}$ & 0.2461905216 & 0.7054736634 & 0.6913845036 \\
\hline $\mathrm{H}$ & 0.9583079156 & 0.2975791876 & 0.4598215461 \\
\hline $\mathrm{H}$ & 0.8865822991 & 0.5187148913 & 0.5879220529 \\
\hline $\mathrm{H}$ & 0.0070560233 & 0.5436074466 & 0.9599082441 \\
\hline $\mathrm{H}$ & 0.1825780032 & 0.8666478702 & 0.6486436046 \\
\hline $\mathrm{H}$ & 0.8580371764 & 0.4116505997 & 0.4867329623 \\
\hline $\mathrm{H}$ & 0.1138147799 & 0.4213125867 & 0.9917043102 \\
\hline 0 & 0.4217583856 & 0.1044395163 & 0.2194957222 \\
\hline 0 & 0.1619999233 & 0.0889223717 & 0.2251508111 \\
\hline 0 & 0.4226954795 & 0.9050763480 & 0.2144897375 \\
\hline 0 & 0.1724745204 & 0.8982003998 & 0.2459091317 \\
\hline 0 & 0.5782416144 & 0.8955604837 & 0.7805042628 \\
\hline 0 & 0.8380000917 & 0.9110776283 & 0.7748491889 \\
\hline 0 & 0.5773045205 & 0.0949236670 & 0.7855102475 \\
\hline 0 & 0.8275254796 & 0.1017995852 & 0.7540908833 \\
\hline 0 & 0.0782416074 & 0.6044395163 & 0.2805042628 \\
\hline 0 & 0.3380000627 & 0.5889223717 & 0.2748491889 \\
\hline
\end{tabular}




\begin{tabular}{|c|c|c|c|}
\hline 0 & 0.0773045135 & 0.4050763480 & 0.2855102775 \\
\hline $\mathrm{O}$ & 0.3275254796 & 0.3982003998 & 0.2540908533 \\
\hline 0 & 0.9217583856 & 0.3955605137 & 0.7194957372 \\
\hline $\mathrm{O}$ & 0.6619999083 & 0.4110776283 & 0.7251508111 \\
\hline $\mathrm{O}$ & 0.9226954795 & 0.5949236520 & 0.7144897525 \\
\hline 0 & 0.6724745204 & 0.6017996002 & 0.7459091167 \\
\hline $\mathrm{F}$ & 0.2509615652 & 0.2397996109 & 0.9478227972 \\
\hline $\mathrm{F}$ & 0.2477463635 & 0.0750952912 & 0.9117103194 \\
\hline $\mathrm{F}$ & 0.1382746228 & 0.1543278608 & 0.9270247184 \\
\hline $\mathrm{F}$ & 0.2283359864 & 0.1118786545 & 0.0184969719 \\
\hline $\mathrm{F}$ & 0.7490384348 & 0.7602003891 & 0.0521772218 \\
\hline $\mathrm{F}$ & 0.7522536065 & 0.9249047238 & 0.0882897106 \\
\hline $\mathrm{F}$ & 0.8617253622 & 0.8456721252 & 0.0729753046 \\
\hline $\mathrm{F}$ & 0.7716640136 & 0.8881213305 & 0.9815030881 \\
\hline $\mathrm{F}$ & 0.2490384348 & 0.7397996109 & 0.5521772028 \\
\hline $\mathrm{F}$ & 0.2522536215 & 0.5750952762 & 0.5882896806 \\
\hline $\mathrm{F}$ & 0.3617253922 & 0.6543278748 & 0.5729752816 \\
\hline $\mathrm{F}$ & 0.2716640136 & 0.6118786695 & 0.4815030881 \\
\hline $\mathrm{F}$ & 0.7509615652 & 0.2602003891 & 0.4478227672 \\
\hline $\mathrm{F}$ & 0.7477463935 & 0.4249047238 & 0.4117102894 \\
\hline $\mathrm{F}$ & 0.6382746378 & 0.3456721252 & 0.4270246884 \\
\hline $\mathrm{F}$ & 0.7283359864 & 0.3881213305 & 0.5184969119 \\
\hline $\mathrm{F}$ & 0.2434535762 & 0.6242256394 & 0.8761959352 \\
\hline $\mathrm{F}$ & 0.3384308565 & 0.5294063877 & 0.8444468375 \\
\hline $\mathrm{F}$ & 0.3238043859 & 0.6945242198 & 0.8121378466 \\
\hline $\mathrm{F}$ & 0.3672696758 & 0.6549788918 & 0.9230178875 \\
\hline $\mathrm{F}$ & 0.7565464088 & 0.3757743906 & 0.1238040348 \\
\hline $\mathrm{F}$ & 0.6615691735 & 0.4705936123 & 0.1555531475 \\
\hline $\mathrm{F}$ & 0.6761955841 & 0.3054757802 & 0.1878621534 \\
\hline $\mathrm{F}$ & 0.6327303242 & 0.3450211382 & 0.0769821275 \\
\hline $\mathrm{F}$ & 0.2565464088 & 0.1242256174 & 0.6238040648 \\
\hline $\mathrm{F}$ & 0.1615691585 & 0.0294063857 & 0.6555531625 \\
\hline $\mathrm{F}$ & 0.1761955991 & 0.1945242348 & 0.6878621534 \\
\hline $\mathrm{F}$ & 0.1327303102 & 0.1549788618 & 0.5769821125 \\
\hline $\mathrm{F}$ & 0.7434535912 & 0.8757743606 & 0.3761959652 \\
\hline $\mathrm{F}$ & 0.8384308265 & 0.9705936123 & 0.3444468375 \\
\hline $\mathrm{F}$ & 0.8238044159 & 0.8054757802 & 0.3121378466 \\
\hline $\mathrm{F}$ & 0.8672696758 & 0.8450211082 & 0.4230178875 \\
\hline B & 0.2164667659 & 0.1457284985 & 0.9521976182 \\
\hline B & 0.7835332481 & 0.8542715165 & 0.0478024118 \\
\hline B & 0.2835332191 & 0.6457284835 & 0.5478023818 \\
\hline B & 0.7164667519 & 0.3542715165 & 0.4521975882 \\
\hline B & 0.3179166045 & 0.6253173081 & 0.8645106557 \\
\hline B & 0.6820834255 & 0.3746827219 & 0.1354893443 \\
\hline B & 0.1820834105 & 0.1253172861 & 0.6354893443 \\
\hline B & 0.8179165745 & 0.8746826919 & 0.3645106557 \\
\hline \multicolumn{4}{|c|}{$6 \cdot 2 \mathrm{CH}_{2} \mathrm{Cl}_{2} \mathrm{HS}$} \\
\hline \multicolumn{4}{|c|}{ CELL_PARAMETERS (angstrom) } \\
\hline 11.062843513 & 0.000000000 & -0.140545141 & \\
\hline 0.000000000 & 17.392793001 & 0.000000000 & \\
\hline-7.870035648 & 0.000000000 & 13.191453689 & \\
\hline \multicolumn{4}{|c|}{ ATOMIC_POSITIONS (crystal) } \\
\hline $\mathrm{Fe} 1$ & 0.1784645749 & 0.1513161914 & 0.4052382590 \\
\hline Fe1 & 0.8215324251 & 0.6513161914 & 0.0947607410 \\
\hline Fe1 & 0.8215304251 & 0.8486838086 & 0.5947597410 \\
\hline Fe1 & 0.1784615749 & 0.3486838086 & 0.9052372590 \\
\hline 0 & 0.2285305220 & 0.0808481614 & 0.5323827371 \\
\hline
\end{tabular}




\begin{tabular}{|c|c|c|c|}
\hline 0 & 0.7714614780 & 0.5808481614 & 0.9676142629 \\
\hline 0 & 0.7714644780 & 0.9191518386 & 0.4676152629 \\
\hline 0 & 0.2285335220 & 0.4191518386 & 0.0323837371 \\
\hline 0 & 0.9856951383 & 0.0988951305 & 0.3580627605 \\
\hline 0 & 0.0143018617 & 0.5988951305 & 0.1419362395 \\
\hline 0 & 0.0142998617 & 0.9011048695 & 0.6419352395 \\
\hline 0 & 0.9856931383 & 0.4011048695 & 0.8580617605 \\
\hline $\mathrm{N}$ & 0.2764665713 & 0.0642426479 & 0.3592315124 \\
\hline $\mathrm{N}$ & 0.7235304287 & 0.5642426479 & 0.1407674876 \\
\hline $\mathrm{N}$ & 0.7235284287 & 0.9357573521 & 0.6407664876 \\
\hline $\mathrm{N}$ & 0.2764645713 & 0.4357573521 & 0.8592305124 \\
\hline $\mathrm{N}$ & 0.0857012587 & 0.2216471333 & 0.2718379085 \\
\hline $\mathrm{N}$ & 0.9142967413 & 0.7216471333 & 0.2281610915 \\
\hline $\mathrm{N}$ & 0.9142937413 & 0.7783528667 & 0.7281600915 \\
\hline $\mathrm{N}$ & 0.0856982587 & 0.2783528667 & 0.7718369085 \\
\hline $\mathrm{N}$ & 0.3757342429 & 0.2097193933 & 0.4296556687 \\
\hline $\mathrm{N}$ & 0.6242637571 & 0.7097193933 & 0.0703433313 \\
\hline $\mathrm{N}$ & 0.6242607571 & 0.7902806067 & 0.5703423313 \\
\hline $\mathrm{N}$ & 0.3757312429 & 0.2902806066 & 0.9296546687 \\
\hline $\mathrm{N}$ & 0.1863516424 & 0.2567575251 & 0.4849319485 \\
\hline $\mathrm{N}$ & 0.8136463576 & 0.7567575251 & 0.0150670515 \\
\hline $\mathrm{N}$ & 0.8136433576 & 0.7432424749 & 0.5150660515 \\
\hline $\mathrm{N}$ & 0.1863486424 & 0.2432424749 & 0.9849309485 \\
\hline C & 0.1788482364 & 0.2675893059 & 0.2648850760 \\
\hline C & 0.8211497636 & 0.7675893059 & 0.2351139240 \\
\hline C & 0.8211467636 & 0.7324106941 & 0.7351129240 \\
\hline C & 0.1788452364 & 0.2324106941 & 0.7648840760 \\
\hline C & 0.1361428382 & 0.9867876777 & 0.5939832187 \\
\hline C & 0.8638491618 & 0.4867876777 & 0.9060137813 \\
\hline C & 0.8638521618 & 0.0132123223 & 0.4060147813 \\
\hline C & 0.1361458382 & 0.5132123223 & 0.0939842187 \\
\hline $\mathrm{H}$ & 0.2376713045 & 0.9772776609 & 0.6644598717 \\
\hline $\mathrm{H}$ & 0.7623216955 & 0.4772776609 & 0.8355371283 \\
\hline $\mathrm{H}$ & 0.7623236955 & 0.0227223391 & 0.3355381283 \\
\hline H & 0.2376733045 & 0.5227223391 & 0.1644608717 \\
\hline C & 0.9842711944 & 0.0520603869 & 0.4228598084 \\
\hline C & 0.0157268056 & 0.5520603869 & 0.0771391916 \\
\hline C & 0.0157238056 & 0.9479396131 & 0.5771381916 \\
\hline C & 0.9842681944 & 0.4479396131 & 0.9228588084 \\
\hline C & 0.1253343193 & 0.0400346428 & 0.5211779966 \\
\hline C & 0.8746576807 & 0.5400346428 & 0.9788190034 \\
\hline C & 0.8746596807 & 0.9599653572 & 0.4788200034 \\
\hline C & 0.1253373193 & 0.4599653572 & 0.0211789966 \\
\hline C & 0.3171632764 & 0.3716975049 & 0.5657363625 \\
\hline C & 0.6828287236 & 0.8716975049 & 0.9342606375 \\
\hline C & 0.6828317236 & 0.6283024951 & 0.4342616375 \\
\hline C & 0.3171662764 & 0.1283024951 & 0.0657373625 \\
\hline H & 0.4147663096 & 0.4047305024 & 0.5952035296 \\
\hline $\mathrm{H}$ & 0.5852256904 & 0.9047305024 & 0.9047934704 \\
\hline $\mathrm{H}$ & 0.5852286904 & 0.5952694976 & 0.4047944704 \\
\hline $\mathrm{H}$ & 0.4147693096 & 0.0952694976 & 0.0952045296 \\
\hline C & 0.3341232701 & 0.2474133004 & 0.3306115842 \\
\hline C & 0.6658737299 & 0.7474133004 & 0.1693874158 \\
\hline C & 0.6658717299 & 0.7525866996 & 0.6693864158 \\
\hline C & 0.3341212701 & 0.2525866996 & 0.8306105842 \\
\hline $\mathrm{H}$ & 0.4003331198 & 0.2983594516 & 0.3433667022 \\
\hline $\mathrm{H}$ & 0.5996638802 & 0.7983594516 & 0.1566322978 \\
\hline $\mathrm{H}$ & 0.5996618802 & 0.7016405484 & 0.6566312978 \\
\hline
\end{tabular}




\begin{tabular}{|c|c|c|c|}
\hline $\mathrm{H}$ & 0.4003311198 & 0.2016405484 & 0.8433657022 \\
\hline $\mathrm{H}$ & 0.3585163014 & 0.2071736216 & 0.2864595621 \\
\hline $\mathrm{H}$ & 0.6414806986 & 0.7071736216 & 0.2135394379 \\
\hline $\mathrm{H}$ & 0.6414776986 & 0.7928263784 & 0.7135384379 \\
\hline $\mathrm{H}$ & 0.3585143014 & 0.2928263784 & 0.7864585621 \\
\hline C & 0.1327350364 & 0.3281354601 & 0.1951941875 \\
\hline C & 0.8672619636 & 0.8281354601 & 0.3048048125 \\
\hline C & 0.8672599636 & 0.6718645399 & 0.8048038125 \\
\hline C & 0.1327320364 & 0.1718645399 & 0.6951931875 \\
\hline $\mathrm{H}$ & 0.2101347530 & 0.3637970205 & 0.1905159096 \\
\hline $\mathrm{H}$ & 0.7898622470 & 0.8637970205 & 0.3094830904 \\
\hline $\mathrm{H}$ & 0.7898602470 & 0.6362029795 & 0.8094820904 \\
\hline $\mathrm{H}$ & 0.2101317530 & 0.1362029795 & 0.6905149096 \\
\hline C & 0.9454737890 & 0.2345156436 & 0.2108703272 \\
\hline C & 0.0545232110 & 0.7345156436 & 0.2891286728 \\
\hline C & 0.0545212110 & 0.7654843564 & 0.7891276728 \\
\hline C & 0.9454707890 & 0.2654843564 & 0.7108693272 \\
\hline $\mathrm{H}$ & 0.8761711134 & 0.1971137266 & 0.2224929697 \\
\hline $\mathrm{H}$ & 0.1238268866 & 0.6971137266 & 0.2775060303 \\
\hline $\mathrm{H}$ & 0.1238238866 & 0.8028862734 & 0.7775050303 \\
\hline $\mathrm{H}$ & 0.8761681134 & 0.3028862734 & 0.7224919697 \\
\hline C & 0.4811299436 & 0.1482772192 & 0.4527728294 \\
\hline C & 0.5188680564 & 0.6482772192 & 0.0472261706 \\
\hline C & 0.5188650564 & 0.8517227808 & 0.5472251706 \\
\hline C & 0.4811269436 & 0.3517227808 & 0.9527718294 \\
\hline $\mathrm{H}$ & 0.5683247774 & 0.1685533038 & 0.4437179009 \\
\hline $\mathrm{H}$ & 0.4316732226 & 0.6685533038 & 0.0562810991 \\
\hline $\mathrm{H}$ & 0.4316702226 & 0.8314466962 & 0.5562800991 \\
\hline $\mathrm{H}$ & 0.5683217774 & 0.3314466962 & 0.9437169009 \\
\hline $\mathrm{H}$ & 0.5262793179 & 0.1312791235 & 0.5330961871 \\
\hline $\mathrm{H}$ & 0.4737126821 & 0.6312791235 & 0.9669008129 \\
\hline $\mathrm{H}$ & 0.4737146821 & 0.8687208765 & 0.4669018129 \\
\hline $\mathrm{H}$ & 0.5262823179 & 0.3687208765 & 0.0330971871 \\
\hline C & 0.3044806832 & 0.3000282776 & 0.5207133585 \\
\hline C & 0.6955113168 & 0.8000282776 & 0.9792836415 \\
\hline C & 0.6955133168 & 0.6999717224 & 0.4792846415 \\
\hline C & 0.3044836832 & 0.1999717224 & 0.0207143585 \\
\hline C & 0.4121210586 & 0.0783434275 & 0.3868777062 \\
\hline C & 0.5878759414 & 0.5783434275 & 0.1131212938 \\
\hline C & 0.5878739414 & 0.9216565725 & 0.6131202938 \\
\hline C & 0.4121180586 & 0.4216565725 & 0.8868767062 \\
\hline C & 0.8920907455 & 0.2933155681 & 0.1389795968 \\
\hline C & 0.1079062545 & 0.7933155681 & 0.3610194032 \\
\hline C & 0.1079042545 & 0.7066844319 & 0.8610184032 \\
\hline C & 0.8920887455 & 0.2066844319 & 0.6389785968 \\
\hline $\mathrm{H}$ & 0.7780538129 & 0.3007251449 & 0.0898054356 \\
\hline $\mathrm{H}$ & 0.2219431871 & 0.8007251449 & 0.4101935644 \\
\hline $\mathrm{H}$ & 0.2219401871 & 0.6992748551 & 0.9101925644 \\
\hline $\mathrm{H}$ & 0.7780518129 & 0.1992748551 & 0.5898044356 \\
\hline C & 0.0763967913 & 0.2842040612 & 0.4902192766 \\
\hline C & 0.9236012087 & 0.7842040612 & 0.0097797234 \\
\hline C & 0.9235982087 & 0.7157959388 & 0.5097787234 \\
\hline C & 0.0763937913 & 0.2157959388 & 0.9902182766 \\
\hline $\mathrm{H}$ & 0.9815248299 & 0.2483434427 & 0.4569880718 \\
\hline $\mathrm{H}$ & 0.0184731701 & 0.7483434427 & 0.0430109282 \\
\hline $\mathrm{H}$ & 0.0184701701 & 0.7516565573 & 0.5430099282 \\
\hline $\mathrm{H}$ & 0.9815218299 & 0.2516565573 & 0.9569870718 \\
\hline C & 0.2128930237 & -0.0001173453 & 0.3058058439 \\
\hline
\end{tabular}




\begin{tabular}{|c|c|c|c|}
\hline C & 0.7871039763 & 0.4998826547 & 0.1941931561 \\
\hline C & 0.7871009763 & 1.0001173453 & 0.6941921561 \\
\hline C & 0.2128910237 & 0.5001173453 & 0.8058048439 \\
\hline $\mathrm{H}$ & 0.1037066158 & 0.9910803939 & 0.2862568127 \\
\hline $\mathrm{H}$ & 0.8962913842 & 0.4910803939 & 0.2137421873 \\
\hline $\mathrm{H}$ & 0.8962883842 & 0.0089196061 & 0.7137411873 \\
\hline $\mathrm{H}$ & 0.1037036158 & 0.5089196061 & 0.7862558127 \\
\hline C & 0.2811251318 & 0.9471410572 & 0.2777347084 \\
\hline C & 0.7188728682 & 0.4471410572 & 0.2222642916 \\
\hline C & 0.7188698682 & 0.0528589428 & 0.7222632916 \\
\hline C & 0.2811221318 & 0.5528589428 & 0.7777337084 \\
\hline $\mathrm{H}$ & 0.2275664486 & 0.8947614168 & 0.2363324144 \\
\hline $\mathrm{H}$ & 0.7724315514 & 0.3947614168 & 0.2636665856 \\
\hline $\mathrm{H}$ & 0.7724285514 & 0.1052385832 & 0.7636655856 \\
\hline $\mathrm{H}$ & 0.2275634486 & 0.6052385832 & 0.7363314144 \\
\hline C & 0.4251890229 & 0.2651490786 & 0.5150035073 \\
\hline C & 0.5748029771 & 0.7651490786 & 0.9849934927 \\
\hline C & 0.5748049771 & 0.7348509214 & 0.4849944927 \\
\hline C & 0.4251920229 & 0.2348509214 & 0.0150045073 \\
\hline $\mathrm{H}$ & 0.4916956556 & 0.3106403708 & 0.5110654302 \\
\hline $\mathrm{H}$ & 0.5082963444 & 0.8106403708 & 0.9889315698 \\
\hline $\mathrm{H}$ & 0.5082983444 & 0.6893596292 & 0.4889325698 \\
\hline $\mathrm{H}$ & 0.4916986556 & 0.1893596292 & 0.0110664302 \\
\hline $\mathrm{H}$ & 0.4915195339 & 0.2337977645 & 0.5871878236 \\
\hline $\mathrm{H}$ & 0.5084724661 & 0.7337977645 & 0.9128091764 \\
\hline $\mathrm{H}$ & 0.5084754661 & 0.7662022355 & 0.4128101764 \\
\hline $\mathrm{H}$ & 0.4915225339 & 0.2662022355 & 0.0871888236 \\
\hline C & 0.4210275871 & 0.9622274426 & 0.3053512709 \\
\hline C & 0.5789694129 & 0.4622274426 & 0.1946477291 \\
\hline C & 0.5789664129 & 0.0377725574 & 0.6946467291 \\
\hline C & 0.4210255871 & 0.5377725574 & 0.8053502709 \\
\hline $\mathrm{H}$ & 0.4778616536 & 0.9215821436 & 0.2854689049 \\
\hline $\mathrm{H}$ & 0.5221353464 & 0.4215821436 & 0.2145300951 \\
\hline $\mathrm{H}$ & 0.5221323464 & 0.0784178564 & 0.7145290951 \\
\hline $\mathrm{H}$ & 0.4778596536 & 0.5784178564 & 0.7854679049 \\
\hline C & 0.9872861728 & 0.3408592966 & 0.1311698077 \\
\hline C & 0.0127108272 & 0.8408592966 & 0.3688291923 \\
\hline C & 0.0127088272 & 0.6591407034 & 0.8688281923 \\
\hline C & 0.9872841728 & 0.1591407034 & 0.6311688077 \\
\hline $\mathrm{H}$ & 0.9484524053 & 0.3872794333 & 0.0751887063 \\
\hline $\mathrm{H}$ & 0.0515455947 & 0.8872794333 & 0.4248102937 \\
\hline $\mathrm{H}$ & 0.0515425947 & 0.6127205667 & 0.9248092937 \\
\hline $\mathrm{H}$ & 0.9484494053 & 0.1127205667 & 0.5751877063 \\
\hline C & 0.4867100353 & 0.0289479384 & 0.3600650063 \\
\hline C & 0.5132869647 & 0.5289479384 & 0.1399339937 \\
\hline C & 0.5132849647 & 0.9710520616 & 0.6399329937 \\
\hline C & 0.4867070353 & 0.4710520616 & 0.8600640063 \\
\hline $\mathrm{H}$ & 0.5949371696 & 0.0431869779 & 0.3821807749 \\
\hline $\mathrm{H}$ & 0.4050598304 & 0.5431869779 & 0.1178182251 \\
\hline $\mathrm{H}$ & 0.4050578304 & 0.9568130221 & 0.6178172251 \\
\hline $\mathrm{H}$ & 0.5949341696 & 0.4568130221 & 0.8821797749 \\
\hline C & 0.0811379449 & 0.3551775795 & 0.5337861714 \\
\hline C & 0.9188540551 & 0.8551775795 & 0.9662108286 \\
\hline C & 0.9188560551 & 0.6448224205 & 0.4662118286 \\
\hline C & 0.0811409449 & 0.1448224205 & 0.0337871714 \\
\hline $\mathrm{H}$ & 0.9896595031 & 0.3746357675 & 0.5367529822 \\
\hline $\mathrm{H}$ & 0.0103324969 & 0.8746357675 & 0.9632440178 \\
\hline $\mathrm{H}$ & 0.0103344969 & 0.6253642325 & 0.4632450178 \\
\hline
\end{tabular}




\begin{tabular}{|c|c|c|c|}
\hline $\mathrm{H}$ & 0.9896625031 & 0.1253642325 & 0.0367539822 \\
\hline C & 0.2043337815 & 0.3993430294 & 0.5729060442 \\
\hline C & 0.7956582185 & 0.8993430294 & 0.9270909558 \\
\hline C & 0.7956612185 & 0.6006569706 & 0.4270919558 \\
\hline C & 0.2043367815 & 0.1006569706 & 0.0729070442 \\
\hline $\mathrm{H}$ & 0.2125408178 & 0.4551501257 & 0.6080685684 \\
\hline $\mathrm{H}$ & 0.7874511822 & 0.9551501257 & 0.8919284316 \\
\hline $\mathrm{H}$ & 0.7874541822 & 0.5448498743 & 0.3919294316 \\
\hline $\mathrm{H}$ & 0.2125428178 & 0.0448498743 & 0.1080695684 \\
\hline $\mathrm{F}$ & 0.6067770515 & 0.3297781676 & 0.7937876007 \\
\hline $\mathrm{F}$ & 0.3932149485 & 0.8297781676 & 0.7062093993 \\
\hline $\mathrm{F}$ & 0.3932179485 & 0.6702218324 & 0.2062103993 \\
\hline $\mathrm{F}$ & 0.6067800515 & 0.1702218324 & 0.2937886007 \\
\hline $\mathrm{F}$ & 0.6820414359 & 0.2300516204 & 0.9118261887 \\
\hline $\mathrm{F}$ & 0.3179505641 & 0.7300516204 & 0.5881708113 \\
\hline $\mathrm{F}$ & 0.3179525641 & 0.7699483796 & 0.0881718113 \\
\hline $\mathrm{F}$ & 0.6820444359 & 0.2699483796 & 0.4118271887 \\
\hline $\mathrm{F}$ & 0.5189307701 & 0.2062305100 & 0.7399810616 \\
\hline $\mathrm{F}$ & 0.4810612299 & 0.7062305100 & 0.7600159384 \\
\hline $\mathrm{F}$ & 0.4810642299 & 0.7937694900 & 0.2600169384 \\
\hline $\mathrm{F}$ & 0.5189337701 & 0.2937694900 & 0.2399820616 \\
\hline $\mathrm{F}$ & 0.7532172998 & 0.2336038426 & 0.7935830196 \\
\hline $\mathrm{F}$ & 0.2467747002 & 0.7336038426 & 0.7064139804 \\
\hline $\mathrm{F}$ & 0.2467777002 & 0.7663961574 & 0.2064149804 \\
\hline $\mathrm{F}$ & 0.7532202998 & 0.2663961574 & 0.2935840196 \\
\hline B & 0.6408893378 & 0.2503075192 & 0.8099985140 \\
\hline B & 0.3591026622 & 0.7503075192 & 0.6899984860 \\
\hline B & 0.3591056622 & 0.7496924808 & 0.1899994860 \\
\hline B & 0.6408913378 & 0.2496924808 & 0.3099995140 \\
\hline $\mathrm{Cl}$ & 0.7417314003 & 0.4197112712 & 0.5386886538 \\
\hline $\mathrm{Cl}$ & 0.2582605997 & 0.9197112712 & 0.9613083462 \\
\hline $\mathrm{Cl}$ & 0.2582635997 & 0.5802887288 & 0.4613093462 \\
\hline $\mathrm{Cl}$ & 0.7417344003 & 0.0802887288 & 0.0386896538 \\
\hline C & 0.7827430105 & 0.4886692626 & 0.6360411433 \\
\hline C & 0.2172489895 & 0.9886692626 & 0.8639558567 \\
\hline C & 0.2172519895 & 0.5113307374 & 0.3639568567 \\
\hline C & 0.7827450105 & 0.0113307374 & 0.1360421433 \\
\hline $\mathrm{H}$ & 0.8476949577 & 0.4599026073 & 0.7100150630 \\
\hline $\mathrm{H}$ & 0.1522970423 & 0.9599026073 & 0.7899819370 \\
\hline $\mathrm{H}$ & 0.1522990423 & 0.5400973927 & 0.2899829370 \\
\hline $\mathrm{H}$ & 0.8476979577 & 0.0400973927 & 0.2100160630 \\
\hline $\mathrm{H}$ & 0.8374001938 & 0.5370094611 & 0.6256102472 \\
\hline $\mathrm{H}$ & 0.1625918062 & 0.0370094611 & 0.8743867528 \\
\hline $\mathrm{H}$ & 0.1625948062 & 0.4629905389 & 0.3743877528 \\
\hline $\mathrm{H}$ & 0.8374021938 & 0.9629905389 & 0.1256112472 \\
\hline $\mathrm{Cl}$ & 0.6284750978 & 0.5260653826 & 0.6298854765 \\
\hline $\mathrm{Cl}$ & 0.3715169022 & 0.0260653826 & 0.8701115235 \\
\hline $\mathrm{Cl}$ & 0.3715199022 & 0.4739346174 & 0.3701125235 \\
\hline $\mathrm{Cl}$ & 0.6284770978 & 0.9739346174 & 0.1298864765 \\
\hline
\end{tabular}

$\mathbf{6} \cdot \mathbf{2} \mathrm{CH}_{2} \mathrm{Cl}_{2}$--iS

CELL_PARAMETERS (angstrom)
11.049373460
0.000000000
$-0.152254805$
$0.000000000 \quad 16.776924584$
0.000000000
$-7.864622193$
0.000000000
13.475873442

ATOMIC_POSITIONS (angstrom)

$\begin{array}{lr}\text { Fe1 } & -1.3392938625 \\ \text { Fe2 } & 4.3621276722\end{array}$




\begin{tabular}{|c|c|c|c|}
\hline Fe1 & 8.4563562263 & 10.8266432230 & 1.1642676082 \\
\hline $\mathrm{Fe} 2$ & -5.1096875020 & 5.7988342902 & 12.0832143001 \\
\hline 0 & -1.6968249036 & 1.2039327351 & 7.0543282162 \\
\hline 0 & 4.6455552238 & 15.3308137488 & 6.3701541569 \\
\hline 0 & 0.9492650737 & 9.5923946523 & 13.0072271422 \\
\hline 0 & 2.4715071391 & 6.9423509563 & 0.2155277592 \\
\hline 0 & 7.9343218777 & 1.5727610844 & 4.7214586646 \\
\hline 0 & -4.9928967425 & 14.9583505106 & 8.6527457309 \\
\hline 0 & -0.8172595139 & 9.9612231264 & 1.8642232506 \\
\hline 0 & 4.2453369137 & 6.5698882190 & 11.4088096264 \\
\hline $\mathrm{N}$ & 0.0932057555 & 0.9650370010 & 4.7139566812 \\
\hline $\mathrm{N}$ & 3.2321586292 & 15.6286658192 & 8.7002424251 \\
\hline $\mathrm{N}$ & 7.0238566074 & 9.3534990430 & 1.8717252349 \\
\hline $\mathrm{N}$ & -3.9797184590 & 7.2402035276 & 11.3613129322 \\
\hline $\mathrm{N}$ & -1.2828104139 & 3.6771280286 & 3.6892771871 \\
\hline $\mathrm{N}$ & 4.2866263244 & 13.0596646286 & 9.5409679996 \\
\hline $\mathrm{N}$ & 8.3998727778 & 12.0655908201 & 2.8964047289 \\
\hline $\mathrm{N}$ & -5.0341861542 & 4.6712028360 & 10.5205873577 \\
\hline $\mathrm{N}$ & 0.6512284406 & 3.4336091313 & 5.7468829420 \\
\hline $\mathrm{N}$ & 2.5903871706 & 13.3782234362 & 7.5021440593 \\
\hline $\mathrm{N}$ & 6.4658339233 & 11.8220719228 & 0.8387989741 \\
\hline $\mathrm{N}$ & -3.3379469995 & 4.9897616436 & 12.5594112980 \\
\hline $\mathrm{N}$ & -1.8803650771 & 4.2552207967 & 6.4787495944 \\
\hline $\mathrm{N}$ & 5.1043073440 & 12.6270980805 & 7.0515278450 \\
\hline $\mathrm{N}$ & 8.9974274410 & 12.6436835892 & 0.1069323217 \\
\hline $\mathrm{N}$ & -5.8518671729 & 4.2386362879 & 13.0100275123 \\
\hline C & -0.1817367044 & 4.4527104363 & 3.5805701464 \\
\hline C & 3.1855144698 & 12.2753818222 & 9.6513351077 \\
\hline C & 7.2987990683 & 12.8411727288 & 3.0051117697 \\
\hline C & -3.9330742987 & 3.8869195306 & 10.4102202507 \\
\hline C & -3.2268853293 & 16.3917144759 & 7.9507395957 \\
\hline C & 6.1919775754 & 0.1217406348 & 5.4346289709 \\
\hline C & 2.4793254995 & 8.0032521833 & 12.1108157626 \\
\hline C & 0.9250847875 & 8.5102027402 & 1.1510529452 \\
\hline C & 7.4237868141 & 0.7602283488 & 5.5970342388 \\
\hline C & -4.4667077271 & 15.7691654734 & 7.7863677287 \\
\hline C & -0.3067244512 & 9.1486902662 & 0.9886476773 \\
\hline C & 3.7191478973 & 7.3807026808 & 12.2751876296 \\
\hline C & -2.7900429206 & 0.5248856012 & 6.9775631523 \\
\hline C & 5.7485671642 & 16.0028709050 & 6.4236453417 \\
\hline C & 2.0424830907 & 8.9133476432 & 13.0839922050 \\
\hline C & 1.3684951996 & 7.6144086125 & 0.1620365745 \\
\hline C & -1.0534661602 & 6.2473738161 & 7.5267501905 \\
\hline C & 4.5121727441 & 10.7146862959 & 5.7167226368 \\
\hline C & 0.3059063313 & 14.6358356087 & 12.5348051678 \\
\hline C & 2.6048896198 & 2.3262235033 & 0.8689592792 \\
\hline C & 0.9893990703 & 4.1445873613 & 4.4821740186 \\
\hline C & 2.0860224758 & 12.5992748461 & 8.6860891609 \\
\hline C & 6.1276632936 & 12.5330496538 & 2.1035078976 \\
\hline C & -2.8335823056 & 4.2108125536 & 11.3754661964 \\
\hline C & -0.0952925712 & 5.4533940518 & 2.6126010498 \\
\hline C & 3.0888013440 & 11.2865413202 & 10.6285068168 \\
\hline C & 7.2123549350 & 13.8418563443 & 3.9730808652 \\
\hline C & -3.8363611729 & 2.8980790276 & 9.4330485405 \\
\hline C & 8.7233061295 & 3.8792220679 & 2.7082657022 \\
\hline C & -5.7256110488 & 12.8707828745 & 10.5398377038 \\
\hline C & -1.6062437655 & 12.2676846109 & 3.8774162129 \\
\hline C & 4.9780512199 & 4.4823205820 & 9.5217176535 \\
\hline
\end{tabular}




\begin{tabular}{|c|c|c|c|}
\hline C & 1.6262966049 & 2.3464526386 & 5.9964925594 \\
\hline C & 1.6711797053 & 14.5173215907 & 7.2349754580 \\
\hline C & 5.4907657589 & 10.7349151815 & 0.5891893567 \\
\hline C & -2.4187395351 & 6.1288592980 & 12.8265799004 \\
\hline C & -0.8515695514 & 4.9890704265 & 6.9590330860 \\
\hline C & 4.1804058996 & 11.9042701645 & 6.3619079894 \\
\hline C & 0.1040097215 & 13.3775327190 & 13.1025222713 \\
\hline C & 2.9366564633 & 3.5158078719 & 0.2237739267 \\
\hline C & 1.3821364603 & 1.1951113258 & 5.0517763488 \\
\hline C & 1.9531571847 & 15.6042702852 & 8.2342185646 \\
\hline C & 5.7349259036 & 9.5835731174 & 1.5339055662 \\
\hline C & -2.7007170136 & 7.2158074927 & 11.8273367927 \\
\hline C & 8.7492637736 & 4.8458187497 & 1.7106525065 \\
\hline C & -5.7598739694 & 11.9049844686 & 11.5355616352 \\
\hline C & -1.6322014108 & 13.2342805423 & 4.8750294095 \\
\hline C & 5.0123141395 & 3.5165216760 & 8.5259937221 \\
\hline C & -3.1293394354 & 4.7601868746 & 6.5216466143 \\
\hline C & 6.3748714268 & 12.1755611697 & 7.1189567751 \\
\hline C & 10.2464017983 & 13.1486491662 & 0.0640353017 \\
\hline C & -7.1224312567 & 3.7870988772 & 12.9425985822 \\
\hline C & -0.1988524153 & -0.0937083827 & 3.9338013401 \\
\hline C & 3.6000307901 & 16.6381425170 & 9.5171804498 \\
\hline C & 7.3159147783 & 8.2947540423 & 2.6518805760 \\
\hline C & -4.3475906200 & 8.2496802245 & 10.5443749085 \\
\hline C & 0.7764146214 & 15.8172966027 & 3.4487255218 \\
\hline C & 2.7213115064 & 0.8677067222 & 9.9074038047 \\
\hline C & 6.3406477425 & 7.4288338111 & 3.1369563942 \\
\hline C & -3.4688713353 & 9.2561687019 & 10.1541515526 \\
\hline C & 0.5127824451 & 4.3453241888 & 6.9065036917 \\
\hline C & 2.8134494125 & 12.5250071565 & 6.3009636407 \\
\hline C & -1.2603422740 & 12.7337864804 & 13.1550516657 \\
\hline C & 4.3036129513 & 4.1365448639 & 0.2847182754 \\
\hline C & 2.1122917330 & 16.0687189150 & 3.7760921645 \\
\hline C & 1.3978225287 & 0.8189586120 & 9.4637904070 \\
\hline C & 5.0047706298 & 7.6802571235 & 2.8095897516 \\
\hline C & -2.1453823586 & 9.2074211858 & 10.5977649513 \\
\hline C & 9.8844706743 & 5.6435414450 & 1.5842717720 \\
\hline C & -6.8911870021 & 11.1003160086 & 11.6581390454 \\
\hline C & -2.7674083103 & 14.0320037375 & 5.0014101431 \\
\hline C & 6.1436271732 & 2.7118537161 & 8.4034163119 \\
\hline C & 2.4160056483 & 0.3846846688 & 4.5866968971 \\
\hline C & 1.0113488187 & 16.5544283350 & 8.6174036251 \\
\hline C & 4.7010567156 & 8.7731473364 & 1.9989850189 \\
\hline C & -1.7589086486 & 8.1659665424 & 11.4441517322 \\
\hline C & -3.4060668167 & 6.0096634725 & 7.0703805286 \\
\hline C & 6.7746881206 & 10.9999316544 & 6.4884203762 \\
\hline C & 2.6585069878 & 14.3981252642 & 12.9911748287 \\
\hline C & 0.3423742433 & 2.6114688620 & 0.0972615399 \\
\hline C & -2.3490176429 & 6.7610707864 & 7.5874648581 \\
\hline C & 5.8312332023 & 10.2578006878 & 5.7762470377 \\
\hline C & 1.6014578130 & 15.1495325790 & 12.4740905002 \\
\hline C & 1.2858291616 & 1.8693378953 & 0.8094348774 \\
\hline C & 3.4994066449 & 8.0983771650 & 8.4504088100 \\
\hline C & -0.2889509355 & 8.6267448123 & 4.8127747896 \\
\hline C & -4.2469668043 & 16.4868389576 & 11.6111465518 \\
\hline C & 7.4060129698 & 0.2382820198 & 1.7729071309 \\
\hline $\mathrm{H}$ & -2.6129357463 & 16.1784154053 & 8.8269646833 \\
\hline $\mathrm{H}$ & 5.5999985860 & 0.3143209980 & 4.5397326148 \\
\hline
\end{tabular}




\begin{tabular}{|c|c|c|c|}
\hline $\mathrm{H}$ & 1.8653759164 & 7.7899531128 & 11.2345906740 \\
\hline $\mathrm{H}$ & 1.5170637770 & 8.7027833223 & 2.0459493013 \\
\hline $\mathrm{H}$ & -0.2044594312 & 6.8053409980 & 7.9225441658 \\
\hline $\mathrm{H}$ & 3.7454267761 & 10.1743252054 & 5.1603347256 \\
\hline $\mathrm{H}$ & -0.5431003986 & 15.1938027905 & 12.1390111915 \\
\hline $\mathrm{H}$ & 3.3716355868 & 1.7858624130 & 1.4253471894 \\
\hline $\mathrm{H}$ & 1.5547441808 & 5.0638479207 & 4.6964341708 \\
\hline $\mathrm{H}$ & 1.5486564644 & 11.6974730610 & 8.3601455955 \\
\hline $\mathrm{H}$ & 5.5623181821 & 13.4523097132 & 1.8892477453 \\
\hline $\mathrm{H}$ & -2.2962162942 & 3.3090102685 & 11.7014097618 \\
\hline $\mathrm{H}$ & 1.6805868934 & 3.5081763264 & 3.9079476146 \\
\hline $\mathrm{H}$ & 1.3435157415 & 13.2190035609 & 9.2091951431 \\
\hline $\mathrm{H}$ & 5.4364754704 & 11.8966386190 & 2.6777343016 \\
\hline $\mathrm{H}$ & -2.0910755704 & 4.8305412684 & 10.8523602152 \\
\hline $\mathrm{H}$ & 0.8081498739 & 6.0592505471 & 2.5395809543 \\
\hline $\mathrm{H}$ & 2.1817346319 & 10.6866413045 & 10.7042924300 \\
\hline $\mathrm{H}$ & 6.3089124889 & 14.4477123397 & 4.0461009618 \\
\hline $\mathrm{H}$ & -2.9292944617 & 2.2981785120 & 9.3572629274 \\
\hline $\mathrm{H}$ & 7.8464963729 & 3.2579379353 & 2.8828222368 \\
\hline $\mathrm{H}$ & -4.8515223287 & 13.4958817268 & 10.3740575003 \\
\hline $\mathrm{H}$ & -0.7294340100 & 11.6463999773 & 3.7028596793 \\
\hline $\mathrm{H}$ & 4.1039624989 & 5.1074194343 & 9.6874978570 \\
\hline $\mathrm{H}$ & 2.6673072374 & 2.7000183390 & 5.9124649951 \\
\hline $\mathrm{H}$ & 0.6127627644 & 14.2109216890 & 7.2718114343 \\
\hline $\mathrm{H}$ & 4.4497551256 & 11.0884811315 & 0.6732169209 \\
\hline $\mathrm{H}$ & -1.3603225944 & 5.8224598964 & 12.7897439240 \\
\hline $\mathrm{H}$ & 1.4700717864 & 1.9893819944 & 7.0265838367 \\
\hline $\mathrm{H}$ & 1.8975811417 & 14.9000211106 & 6.2284547373 \\
\hline $\mathrm{H}$ & -2.2176312867 & 10.3778442869 & 13.0349715160 \\
\hline $\mathrm{H}$ & 5.2194815507 & 6.5115588180 & 0.3572271743 \\
\hline $\mathrm{H}$ & 7.9001915145 & 4.9538239721 & 1.0390085059 \\
\hline $\mathrm{H}$ & -4.9131756609 & 11.8022154183 & 12.2110994029 \\
\hline $\mathrm{H}$ & -0.7831291516 & 13.3422862647 & 5.5466734101 \\
\hline $\mathrm{H}$ & 4.1656158311 & 3.4137531267 & 7.8504559554 \\
\hline $\mathrm{H}$ & 7.1294804629 & 4.1460078048 & 5.9346909037 \\
\hline $\mathrm{H}$ & -3.9769546895 & 12.7727306433 & 7.8579923924 \\
\hline $\mathrm{H}$ & -0.0124180991 & 12.5344703468 & 0.6509910113 \\
\hline $\mathrm{H}$ & 3.2293948596 & 4.3842683507 & 12.2035629648 \\
\hline $\mathrm{H}$ & -1.2580546364 & 16.5363924495 & 3.7120054496 \\
\hline $\mathrm{H}$ & 4.6402975055 & -0.1555297023 & 9.8358495109 \\
\hline $\mathrm{H}$ & 8.3751170003 & 8.1479301570 & 2.8736764665 \\
\hline $\mathrm{H}$ & -5.3878573354 & 8.2329327150 & 10.2257058474 \\
\hline $\mathrm{H}$ & 0.5040994987 & 14.9487283697 & 2.8489437688 \\
\hline $\mathrm{H}$ & 3.0687143810 & 1.6843920408 & 10.5410341915 \\
\hline $\mathrm{H}$ & 6.6129628642 & 6.5602660771 & 3.7367381472 \\
\hline $\mathrm{H}$ & -3.8162742109 & 10.0728545838 & 9.5205211658 \\
\hline $\mathrm{H}$ & 1.3054873279 & 5.1129483308 & 6.9175915752 \\
\hline $\mathrm{H}$ & 2.0248005560 & 11.7635681043 & 6.1841315821 \\
\hline $\mathrm{H}$ & -2.0530471568 & 13.5014106234 & 13.1439637821 \\
\hline $\mathrm{H}$ & 5.0922618078 & 3.3751058127 & 0.4015503329 \\
\hline $\mathrm{H}$ & 0.6250710168 & 3.7516769124 & 7.8274317263 \\
\hline $\mathrm{H}$ & 2.7859007570 & 13.1709283215 & 5.4096852661 \\
\hline $\mathrm{H}$ & -1.3726308458 & 12.1401389555 & 12.2341236320 \\
\hline $\mathrm{H}$ & 4.3311616069 & 4.7824660290 & 1.1759966500 \\
\hline $\mathrm{H}$ & 2.8969609125 & 15.3998738455 & 3.4211402489 \\
\hline $\mathrm{H}$ & 0.6836503255 & 1.5910494588 & 9.7552231288 \\
\hline $\mathrm{H}$ & 4.2201014503 & 7.0114115529 & 3.1645416672 \\
\hline $\mathrm{H}$ & -1.4312101554 & 9.9795117513 & 10.3063322296 \\
\hline
\end{tabular}




\begin{tabular}{|c|c|c|c|}
\hline $\mathrm{H}$ & 9.9379221306 & 6.4033559714 & 0.8041736190 \\
\hline $\mathrm{H}$ & -6.9370121680 & 10.3357627186 & 12.4326045863 \\
\hline $\mathrm{H}$ & -2.8208597678 & 14.7918187640 & 5.7815082971 \\
\hline $\mathrm{H}$ & 6.1894523381 & 1.9473009260 & 7.6289507710 \\
\hline $\mathrm{H}$ & 3.4418752302 & 0.6198808066 & 4.8602801962 \\
\hline $\mathrm{H}$ & -0.0095232460 & 16.4942977246 & 8.2393896086 \\
\hline $\mathrm{H}$ & 3.6751868032 & 9.0083432863 & 1.7254017234 \\
\hline $\mathrm{H}$ & -0.7380369134 & 8.1058354330 & 11.8221657532 \\
\hline $\mathrm{H}$ & 6.6145454950 & 6.3702484438 & 6.9415410750 \\
\hline $\mathrm{H}$ & -3.2313231224 & 10.6930909762 & 6.7110092129 \\
\hline $\mathrm{H}$ & -7.3621053249 & 14.7587107363 & 13.1200142823 \\
\hline $\mathrm{H}$ & 10.3483854852 & 2.3046286837 & -0.1253272978 \\
\hline $\mathrm{H}$ & -2.5260762099 & 7.7420482102 & 8.0302383395 \\
\hline $\mathrm{H}$ & 6.1124237359 & 9.3318863549 & 5.2712516526 \\
\hline $\mathrm{H}$ & 1.7785163801 & 16.1305105028 & 12.0313170178 \\
\hline $\mathrm{H}$ & 1.0046386270 & 0.9434240624 & 1.3144302635 \\
\hline $\mathrm{H}$ & 3.6427679906 & 7.5785193302 & 9.4053556690 \\
\hline $\mathrm{H}$ & -0.4503718414 & 9.1295362910 & 3.8504111504 \\
\hline $\mathrm{H}$ & -4.3903276548 & 15.9669816227 & 10.6561996861 \\
\hline $\mathrm{H}$ & 7.5674343691 & 0.7410739984 & 2.7352707634 \\
\hline $\mathrm{H}$ & 4.1677978442 & 8.9581563994 & 8.3321943768 \\
\hline $\mathrm{H}$ & -0.9456848401 & 7.7618223781 & 4.9561523214 \\
\hline $\mathrm{H}$ & -4.9153580036 & 0.5696941078 & 11.7293609860 \\
\hline $\mathrm{H}$ & 8.0627468735 & 16.1502846706 & 1.6295295992 \\
\hline $\mathrm{F}$ & 0.1606785251 & 5.6947604789 & 10.6408882729 \\
\hline $\mathrm{F}$ & 2.8315246507 & 11.0632634456 & 2.8686841081 \\
\hline $\mathrm{F}$ & -0.9082383550 & 14.0832232704 & 9.4206670844 \\
\hline $\mathrm{F}$ & 4.2855377122 & 2.6748016530 & 3.7169978069 \\
\hline $\mathrm{F}$ & 0.5650721594 & 3.9026401431 & 12.0647549157 \\
\hline $\mathrm{F}$ & 2.7441393564 & 12.6077667475 & 1.1283355460 \\
\hline $\mathrm{F}$ & -1.3126316598 & 12.2911024356 & 7.9968004371 \\
\hline $\mathrm{F}$ & 4.3729233948 & 4.2193044549 & 5.4573462649 \\
\hline $\mathrm{F}$ & -0.2801907477 & 3.5243310371 & 9.9288789573 \\
\hline $\mathrm{F}$ & 3.4127439861 & 13.2772020851 & 3.2532566833 \\
\hline $\mathrm{F}$ & -0.4673690822 & 11.9127928288 & 10.1326764001 \\
\hline $\mathrm{F}$ & 3.7043182603 & 4.8887392925 & 3.3324254328 \\
\hline $\mathrm{F}$ & 1.9150449981 & 4.2243209667 & 10.1917579013 \\
\hline $\mathrm{F}$ & 1.1939670450 & 12.7174501457 & 2.8585033512 \\
\hline $\mathrm{F}$ & -2.6626051565 & 12.6127832592 & 9.8697974605 \\
\hline $\mathrm{F}$ & 5.9230949893 & 4.3289878532 & 3.7271785695 \\
\hline B & 0.5982428784 & 4.3446437465 & 10.7101968310 \\
\hline B & 2.5381551573 & 12.4088953537 & 2.5244816788 \\
\hline B & -1.3458023788 & 12.7331060381 & 9.3513585218 \\
\hline B & 4.5789075351 & 4.0204330612 & 4.0612002317 \\
\hline $\mathrm{Cl}$ & 3.8568601288 & 6.9400381728 & 7.1451594696 \\
\hline $\mathrm{Cl}$ & -0.6345563865 & 9.7951612789 & 6.1129107262 \\
\hline $\mathrm{Cl}$ & -4.6044196291 & 15.3285004653 & 12.9163958841 \\
\hline $\mathrm{Cl}$ & 7.7516190789 & 1.4066989864 & 0.4727711853 \\
\hline $\mathrm{Cl}$ & 1.8314806749 & 8.7125726506 & 8.4052417205 \\
\hline $\mathrm{Cl}$ & 1.3898735825 & 8.0356918434 & 4.8500068965 \\
\hline $\mathrm{Cl}$ & -2.5790405047 & 0.3241103580 & 11.6563136367 \\
\hline $\mathrm{Cl}$ & 5.7271887804 & 16.4241541359 & 1.7356750196 \\
\hline \multicolumn{4}{|c|}{$6 \cdot 2 \mathrm{CH}_{2} \mathrm{Cl}_{2--L S}$} \\
\hline \multicolumn{4}{|c|}{ CELL_PARAMETERS (angstrom) } \\
\hline & $26 \quad 0.000000000$ & -0.224378863 & \\
\hline & $00 \quad 16.694072122$ & 0.000000000 & \\
\hline & $5 \quad 0.000000000$ & 13.596333387 & \\
\hline
\end{tabular}


ATOMIC_POSITIONS (crystal)

\begin{tabular}{|c|c|c|c|}
\hline $\mathrm{Fe} 2$ & 0.1789711039 & 0.1565174368 & 0.3977957351 \\
\hline Fe2 & 0.8210258961 & 0.6565174368 & 0.1022032649 \\
\hline $\mathrm{Fe} 2$ & 0.8210238961 & 0.8434825632 & 0.6022022649 \\
\hline Fe2 & 0.1789681039 & 0.3434825632 & 0.8977947351 \\
\hline 0 & 0.2375854504 & 0.0747473885 & 0.5304969407 \\
\hline 0 & 0.7624065496 & 0.5747473885 & 0.9695000593 \\
\hline 0 & 0.7624095496 & 0.9252526115 & 0.4695010593 \\
\hline 0 & 0.2375884504 & 0.4252526115 & 0.0304979407 \\
\hline 0 & 0.9856605494 & 0.1023584698 & 0.3614059269 \\
\hline 0 & 0.0143364506 & 0.6023584698 & 0.1385930731 \\
\hline 0 & 0.0143344506 & 0.8976415302 & 0.6385920731 \\
\hline 0 & 0.9856585494 & 0.3976415302 & 0.8614049269 \\
\hline $\mathrm{N}$ & 0.2502169743 & 0.0674639310 & 0.3473006797 \\
\hline $\mathrm{N}$ & 0.7497800257 & 0.5674639310 & 0.1526983203 \\
\hline $\mathrm{N}$ & 0.7497780257 & 0.9325360690 & 0.6526973203 \\
\hline $\mathrm{N}$ & 0.2502149743 & 0.4325360690 & 0.8472996797 \\
\hline $\mathrm{N}$ & 0.0989344778 & 0.2238901037 & 0.2789715231 \\
\hline $\mathrm{N}$ & 0.9010635222 & 0.7238901037 & 0.2210274769 \\
\hline $\mathrm{N}$ & 0.9010605222 & 0.7761098963 & 0.7210264769 \\
\hline $\mathrm{N}$ & 0.0989314778 & 0.2761098963 & 0.7789705231 \\
\hline $\mathrm{N}$ & 0.3699334828 & 0.2086334961 & 0.4311752170 \\
\hline $\mathrm{N}$ & 0.6300645172 & 0.7086334961 & 0.0688237830 \\
\hline $\mathrm{N}$ & 0.6300615172 & 0.7913665039 & 0.5688227830 \\
\hline $\mathrm{N}$ & 0.3699304828 & 0.2913665039 & 0.9311742170 \\
\hline $\mathrm{N}$ & 0.1676040850 & 0.2535079029 & 0.4697076208 \\
\hline $\mathrm{N}$ & 0.8323939150 & 0.7535079029 & 0.0302913792 \\
\hline $\mathrm{N}$ & 0.8323909150 & 0.7464920971 & 0.5302903792 \\
\hline $\mathrm{N}$ & 0.1676010850 & 0.2464920971 & 0.9697066208 \\
\hline C & 0.1910514368 & 0.2702161424 & 0.2685118670 \\
\hline C & 0.8089465632 & 0.7702161424 & 0.2314871330 \\
\hline C & 0.8089435632 & 0.7297838576 & 0.7314861330 \\
\hline C & 0.1910484368 & 0.2297838576 & 0.7685108670 \\
\hline C & 0.1362271972 & 0.9820320353 & 0.5886902280 \\
\hline C & 0.8637648028 & 0.4820320353 & 0.9113067720 \\
\hline C & 0.8637678028 & 0.0179679647 & 0.4113077720 \\
\hline C & 0.1362301972 & 0.5179679647 & 0.0886912280 \\
\hline $\mathrm{H}$ & 0.2379300732 & 0.9676115175 & 0.6551304011 \\
\hline $\mathrm{H}$ & 0.7620629268 & 0.4676115175 & 0.8448665989 \\
\hline $\mathrm{H}$ & 0.7620649268 & 0.0323884825 & 0.3448675989 \\
\hline $\mathrm{H}$ & 0.2379320732 & 0.5323884825 & 0.1551314011 \\
\hline C & 0.9867235293 & 0.0542097526 & 0.4255288176 \\
\hline C & 0.0132744707 & 0.5542097526 & 0.0744701824 \\
\hline C & 0.0132714707 & 0.9457902474 & 0.5744691824 \\
\hline C & 0.9867205293 & 0.4457902474 & 0.9255278176 \\
\hline C & 0.1294469526 & 0.0372209377 & 0.5190518229 \\
\hline C & 0.8705450474 & 0.5372209377 & 0.9809451771 \\
\hline C & 0.8705470474 & 0.9627790623 & 0.4809461771 \\
\hline C & 0.1294499526 & 0.4627790623 & 0.0190528229 \\
\hline C & 0.2966315176 & 0.3691603421 & 0.5651228214 \\
\hline C & 0.7033604824 & 0.8691603421 & 0.9348741786 \\
\hline C & 0.7033634824 & 0.6308396579 & 0.4348751786 \\
\hline C & 0.2966345176 & 0.1308396579 & 0.0651238214 \\
\hline $\mathrm{H}$ & 0.3960984247 & 0.4022343356 & 0.6035156478 \\
\hline $\mathrm{H}$ & 0.6038935753 & 0.9022343356 & 0.8964813522 \\
\hline $\mathrm{H}$ & 0.6038965753 & 0.5977656644 & 0.3964823522 \\
\hline $\mathrm{H}$ & 0.3961014247 & 0.0977656644 & 0.1035166478 \\
\hline C & 0.3450184231 & 0.2533504984 & 0.3406852346 \\
\hline
\end{tabular}




\begin{tabular}{|c|c|c|c|}
\hline C & 0.6549785769 & 0.7533504984 & 0.1593137654 \\
\hline C & 0.6549765769 & 0.7466495016 & 0.6593127654 \\
\hline C & 0.3450164231 & 0.2466495016 & 0.8406842346 \\
\hline $\mathrm{H}$ & 0.4084440154 & 0.3085136178 & 0.3622000693 \\
\hline $\mathrm{H}$ & 0.5915529846 & 0.8085136178 & 0.1377989307 \\
\hline $\mathrm{H}$ & 0.5915509846 & 0.6914863822 & 0.6377979307 \\
\hline $\mathrm{H}$ & 0.4084420154 & 0.1914863822 & 0.8621990693 \\
\hline $\mathrm{H}$ & 0.3838409024 & 0.2160939682 & 0.3023794337 \\
\hline $\mathrm{H}$ & 0.6161560976 & 0.7160939682 & 0.1976195663 \\
\hline $\mathrm{H}$ & 0.6161530976 & 0.7839060318 & 0.6976185663 \\
\hline $\mathrm{H}$ & 0.3838389024 & 0.2839060318 & 0.8023784337 \\
\hline C & 0.1438775010 & 0.3268080552 & 0.1926836326 \\
\hline C & 0.8561194990 & 0.8268080552 & 0.3073153674 \\
\hline C & 0.8561174990 & 0.6731919448 & 0.8073143674 \\
\hline C & 0.1438745010 & 0.1731919448 & 0.6926826326 \\
\hline $\mathrm{H}$ & 0.2206837753 & 0.3623939394 & 0.1856944953 \\
\hline $\mathrm{H}$ & 0.7793132247 & 0.8623939394 & 0.3143045047 \\
\hline $\mathrm{H}$ & 0.7793112247 & 0.6376060606 & 0.8143035047 \\
\hline $\mathrm{H}$ & 0.2206807753 & 0.1376060606 & 0.6856934953 \\
\hline C & 0.9581629206 & 0.2333671522 & 0.2151309502 \\
\hline C & 0.0418340794 & 0.7333671522 & 0.2848680498 \\
\hline C & 0.0418320794 & 0.7666328478 & 0.7848670498 \\
\hline C & 0.9581599206 & 0.2666328478 & 0.7151299502 \\
\hline $\mathrm{H}$ & 0.8896587310 & 0.1967482231 & 0.2296089096 \\
\hline $\mathrm{H}$ & 0.1103392690 & 0.6967482231 & 0.2703900904 \\
\hline $\mathrm{H}$ & 0.1103362690 & 0.8032517769 & 0.7703890904 \\
\hline $\mathrm{H}$ & 0.8896557310 & 0.3032517769 & 0.7296079096 \\
\hline C & 0.4691187367 & 0.1408152002 & 0.4519873532 \\
\hline C & 0.5308792633 & 0.6408152002 & 0.0480116468 \\
\hline C & 0.5308762633 & 0.8591847998 & 0.5480106468 \\
\hline C & 0.4691157367 & 0.3591847998 & 0.9519863532 \\
\hline $\mathrm{H}$ & 0.5611796749 & 0.1595814632 & 0.4477743710 \\
\hline $\mathrm{H}$ & 0.4388183251 & 0.6595814632 & 0.0522246290 \\
\hline $\mathrm{H}$ & 0.4388153251 & 0.8404185368 & 0.5522236290 \\
\hline $\mathrm{H}$ & 0.5611766749 & 0.3404185368 & 0.9477733710 \\
\hline $\mathrm{H}$ & 0.5068407941 & 0.1194613200 & 0.5279565466 \\
\hline $\mathrm{H}$ & 0.4931512059 & 0.6194613200 & 0.9720404534 \\
\hline $\mathrm{H}$ & 0.4931532059 & 0.8805386800 & 0.4720414534 \\
\hline $\mathrm{H}$ & 0.5068437941 & 0.3805386800 & 0.0279575466 \\
\hline C & 0.2889851917 & 0.2975145439 & 0.5171788774 \\
\hline C & 0.7110068083 & 0.7975145439 & 0.9828181226 \\
\hline C & 0.7110088083 & 0.7024854561 & 0.4828191226 \\
\hline C & 0.2889881917 & 0.2024854561 & 0.0171798774 \\
\hline C & 0.3906627547 & 0.0732536812 & 0.3802373216 \\
\hline C & 0.6093342453 & 0.5732536812 & 0.1197616784 \\
\hline C & 0.6093322453 & 0.9267463188 & 0.6197606784 \\
\hline C & 0.3906597547 & 0.4267463188 & 0.8802363216 \\
\hline C & 0.9046710634 & 0.2882243020 & 0.1375976534 \\
\hline C & 0.0953259366 & 0.7882243020 & 0.3624013466 \\
\hline C & 0.0953239366 & 0.7117756980 & 0.8624003466 \\
\hline C & 0.9046690634 & 0.2117756980 & 0.6375966534 \\
\hline $\mathrm{H}$ & 0.7907914230 & 0.2926854306 & 0.0865130261 \\
\hline $\mathrm{H}$ & 0.2092055770 & 0.7926854306 & 0.4134859739 \\
\hline $\mathrm{H}$ & 0.2092025770 & 0.7073145694 & 0.9134849739 \\
\hline $\mathrm{H}$ & 0.7907894230 & 0.2073145694 & 0.5865120261 \\
\hline C & 0.0517027020 & 0.2805643259 & 0.4681526274 \\
\hline C & 0.9482952980 & 0.7805643259 & 0.0318463726 \\
\hline C & 0.9482922980 & 0.7194356741 & 0.5318453726 \\
\hline
\end{tabular}




\begin{tabular}{|c|c|c|c|}
\hline C & 0.0516997020 & 0.2194356741 & 0.9681516274 \\
\hline $\mathrm{H}$ & 0.9563599210 & 0.2441912688 & 0.4270918671 \\
\hline $\mathrm{H}$ & 0.0436380790 & 0.7441912688 & 0.0729071329 \\
\hline $\mathrm{H}$ & 0.0436350790 & 0.7558087312 & 0.5729061329 \\
\hline $\mathrm{H}$ & 0.9563569210 & 0.2558087312 & 0.9270908671 \\
\hline C & 0.1765181797 & 0.0046879519 & 0.2891853432 \\
\hline C & 0.8234788203 & 0.5046879519 & 0.2108136568 \\
\hline C & 0.8234758203 & 0.9953120481 & 0.7108126568 \\
\hline C & 0.1765161797 & 0.4953120481 & 0.7891843432 \\
\hline $\mathrm{H}$ & 0.0654335383 & 1.0025310356 & 0.2667361314 \\
\hline $\mathrm{H}$ & 0.9345644617 & 0.5025310356 & 0.2332628686 \\
\hline $\mathrm{H}$ & 0.9345614617 & -0.0025310356 & 0.7332618686 \\
\hline $\mathrm{H}$ & 0.0654305383 & 0.4974689644 & 0.7667351314 \\
\hline C & 0.2379372193 & 0.9459991965 & 0.2610222118 \\
\hline C & 0.7620607807 & 0.4459991965 & 0.2389767882 \\
\hline C & 0.7620577807 & 0.0540008035 & 0.7389757882 \\
\hline C & 0.2379342193 & 0.5540008035 & 0.7610212118 \\
\hline $\mathrm{H}$ & 0.1758006606 & 0.8950120268 & 0.2161708203 \\
\hline $\mathrm{H}$ & 0.8241973394 & 0.3950120268 & 0.2838281797 \\
\hline $\mathrm{H}$ & 0.8241943394 & 0.1049879732 & 0.7838271797 \\
\hline $\mathrm{H}$ & 0.1757976606 & 0.6049879732 & 0.7161698203 \\
\hline C & 0.4153073089 & 0.2616991860 & 0.5190509646 \\
\hline C & 0.5846846911 & 0.7616991860 & 0.9809460354 \\
\hline C & 0.5846866911 & 0.7383008140 & 0.4809470354 \\
\hline C & 0.4153103089 & 0.2383008140 & 0.0190519646 \\
\hline $\mathrm{H}$ & 0.4888607566 & 0.3086343718 & 0.5243091665 \\
\hline $\mathrm{H}$ & 0.5111312434 & 0.8086343718 & 0.9756878335 \\
\hline $\mathrm{H}$ & 0.5111332434 & 0.6913656282 & 0.4756888335 \\
\hline $\mathrm{H}$ & 0.4888637566 & 0.1913656282 & 0.0243101665 \\
\hline $\mathrm{H}$ & 0.4720252458 & 0.2247415883 & 0.5867385129 \\
\hline $\mathrm{H}$ & 0.5279667542 & 0.7247415883 & 0.9132584871 \\
\hline $\mathrm{H}$ & 0.5279697542 & 0.7752584117 & 0.4132594871 \\
\hline $\mathrm{H}$ & 0.4720282458 & 0.2752584117 & 0.0867395129 \\
\hline C & 0.3809777143 & 0.9531177940 & 0.2925479327 \\
\hline C & 0.6190192857 & 0.4531177940 & 0.2074510673 \\
\hline C & 0.6190162857 & 0.0468822060 & 0.7074500673 \\
\hline C & 0.3809757143 & 0.5468822060 & 0.7925469327 \\
\hline $\mathrm{H}$ & 0.4324039055 & 0.9080222814 & 0.2719880737 \\
\hline $\mathrm{H}$ & 0.5675930945 & 0.4080222814 & 0.2280109263 \\
\hline $\mathrm{H}$ & 0.5675900945 & 0.0919777186 & 0.7280099263 \\
\hline $\mathrm{H}$ & 0.4324019055 & 0.5919777186 & 0.7719870737 \\
\hline C & 0.9986598344 & 0.3356234823 & 0.1261410829 \\
\hline C & 0.0013371656 & 0.8356234823 & 0.3738579171 \\
\hline C & 0.0013351656 & 0.6643765177 & 0.8738569171 \\
\hline C & 0.9986578344 & 0.1643765177 & 0.6261400829 \\
\hline $\mathrm{H}$ & 0.9584821474 & 0.3788601359 & 0.0655361287 \\
\hline $\mathrm{H}$ & 0.0415158526 & 0.8788601359 & 0.4344628713 \\
\hline $\mathrm{H}$ & 0.0415128526 & 0.6211398641 & 0.9344618713 \\
\hline $\mathrm{H}$ & 0.9584791474 & 0.1211398641 & 0.5655351287 \\
\hline C & 0.4577974660 & 0.0178417120 & 0.3527137166 \\
\hline C & 0.5421995340 & 0.5178417120 & 0.1472852834 \\
\hline C & 0.5421975340 & 0.9821582880 & 0.6472842834 \\
\hline C & 0.4577944660 & 0.4821582880 & 0.8527127166 \\
\hline $\mathrm{H}$ & 0.5698081197 & 0.0245674068 & 0.3794718019 \\
\hline $\mathrm{H}$ & 0.4301888803 & 0.5245674068 & 0.1205271981 \\
\hline $\mathrm{H}$ & 0.4301868803 & 0.9754325932 & 0.6205261981 \\
\hline $\mathrm{H}$ & 0.5698051197 & 0.4754325932 & 0.8794708019 \\
\hline C & 0.0525747127 & 0.3514907002 & 0.5149593620 \\
\hline
\end{tabular}




\begin{tabular}{|c|c|c|c|}
\hline C & 0.9474172873 & 0.8514907002 & 0.9850376380 \\
\hline C & 0.9474192873 & 0.6485092998 & 0.4850386380 \\
\hline C & 0.0525777127 & 0.1485092998 & 0.0149603620 \\
\hline $\mathrm{H}$ & 0.9565054475 & 0.3700549564 & 0.5124701079 \\
\hline $\mathrm{H}$ & 0.0434865525 & 0.8700549564 & 0.9875268921 \\
\hline $\mathrm{H}$ & 0.0434885525 & 0.6299450436 & 0.4875278921 \\
\hline $\mathrm{H}$ & 0.9565084475 & 0.1299450436 & 0.0124711079 \\
\hline C & 0.1769982700 & 0.3963980540 & 0.5643294023 \\
\hline C & 0.8229937300 & 0.8963980540 & 0.9356675977 \\
\hline C & 0.8229967300 & 0.6036019460 & 0.4356685977 \\
\hline C & 0.1770012700 & 0.1036019460 & 0.0643304023 \\
\hline $\mathrm{H}$ & 0.1814823893 & 0.4522460268 & 0.6018469230 \\
\hline $\mathrm{H}$ & 0.8185096107 & 0.9522460268 & 0.8981500770 \\
\hline $\mathrm{H}$ & 0.8185126107 & 0.5477539732 & 0.3981510770 \\
\hline $\mathrm{H}$ & 0.1814843893 & 0.0477539732 & 0.1018479230 \\
\hline $\mathrm{F}$ & 0.5887811385 & 0.3417106037 & 0.7902396181 \\
\hline $\mathrm{F}$ & 0.4112108615 & 0.8417106037 & 0.7097573819 \\
\hline $\mathrm{F}$ & 0.4112138615 & 0.6582893963 & 0.2097583819 \\
\hline $\mathrm{F}$ & 0.5887841385 & 0.1582893963 & 0.2902406181 \\
\hline $\mathrm{F}$ & 0.6937726846 & 0.2374743864 & 0.9037477816 \\
\hline $\mathrm{F}$ & 0.3062193154 & 0.7374743864 & 0.5962492184 \\
\hline $\mathrm{F}$ & 0.3062213154 & 0.7625256136 & 0.0962502184 \\
\hline $\mathrm{F}$ & 0.6937756846 & 0.2625256136 & 0.4037487816 \\
\hline $\mathrm{F}$ & 0.5087613588 & 0.2113976890 & 0.7419846041 \\
\hline $\mathrm{F}$ & 0.4912306412 & 0.7113976890 & 0.7580123959 \\
\hline $\mathrm{F}$ & 0.4912336412 & 0.7886023110 & 0.2580133959 \\
\hline $\mathrm{F}$ & 0.5087643588 & 0.2886023110 & 0.2419856041 \\
\hline $\mathrm{F}$ & 0.7287611961 & 0.2481564281 & 0.7707189387 \\
\hline $\mathrm{F}$ & 0.2712308039 & 0.7481564281 & 0.7292780613 \\
\hline $\mathrm{F}$ & 0.2712338039 & 0.7518435719 & 0.2292790613 \\
\hline $\mathrm{F}$ & 0.7287641961 & 0.2518435719 & 0.2707199387 \\
\hline B & 0.6310803693 & 0.2601691817 & 0.8020095106 \\
\hline B & 0.3689116307 & 0.7601691817 & 0.6979874894 \\
\hline B & 0.3689146307 & 0.7398308183 & 0.1979884894 \\
\hline B & 0.6310823693 & 0.2398308183 & 0.3020105106 \\
\hline $\mathrm{Cl}$ & 0.7342437145 & 0.4144555288 & 0.5431578370 \\
\hline $\mathrm{Cl}$ & 0.2657482855 & 0.9144555288 & 0.9568391630 \\
\hline $\mathrm{Cl}$ & 0.2657512855 & 0.5855444712 & 0.4568401630 \\
\hline $\mathrm{Cl}$ & 0.7342467145 & 0.0855444712 & 0.0431588370 \\
\hline C & 0.7697125686 & 0.4823423255 & 0.6408298589 \\
\hline C & 0.2302794314 & 0.9823423255 & 0.8591671411 \\
\hline C & 0.2302824314 & 0.5176576745 & 0.3591681411 \\
\hline C & 0.7697145686 & 0.0176576745 & 0.1408308589 \\
\hline $\mathrm{H}$ & 0.8340021224 & 0.4508089019 & 0.7118999871 \\
\hline $\mathrm{H}$ & 0.1659898776 & 0.9508089019 & 0.7880970129 \\
\hline $\mathrm{H}$ & 0.1659918776 & 0.5491910981 & 0.2880980129 \\
\hline $\mathrm{H}$ & 0.8340051224 & 0.0491910981 & 0.2119009871 \\
\hline $\mathrm{H}$ & 0.8236604033 & 0.5346448934 & 0.6339365469 \\
\hline $\mathrm{H}$ & 0.1763315967 & 0.0346448934 & 0.8660604531 \\
\hline $\mathrm{H}$ & 0.1763345967 & 0.4653551066 & 0.3660614531 \\
\hline $\mathrm{H}$ & 0.8236624033 & 0.9653551066 & 0.1339375469 \\
\hline $\mathrm{Cl}$ & 0.6134118535 & 0.5172164406 & 0.6342408900 \\
\hline $\mathrm{Cl}$ & 0.3865801465 & 0.0172164406 & 0.8657561100 \\
\hline $\mathrm{Cl}$ & 0.3865831465 & 0.4827835594 & 0.3657571100 \\
\hline $\mathrm{Cl}$ & 0.6134138535 & 0.9827835594 & 0.1342418900 \\
\hline \multicolumn{4}{|c|}{$6-\mathrm{HS}$} \\
\hline \multicolumn{4}{|c|}{ CELL_PARAMETERS (angstrom) } \\
\hline & $2 \quad 0.005428407$ & 0.313446291 & \\
\hline
\end{tabular}




$\begin{array}{lll}-0.001399682 & 18.102240649 & -0.002608771 \\ -7.677339554 & -0.010278591 & 11.883427898\end{array}$

ATOMIC_POSITIONS (crystal)

\begin{tabular}{|c|c|c|c|}
\hline Fe1 & 0.1555375426 & 0.1560552883 & 0.3985676786 \\
\hline Fe1 & 0.8012009427 & 0.8405447730 & 0.5633878923 \\
\hline Fe1 & 0.8008779736 & 0.6557473108 & 0.0639874386 \\
\hline Fe1 & 0.1557717848 & 0.3409788331 & 0.8979037141 \\
\hline $\mathrm{N}$ & 0.2693724659 & 0.0807295208 & 0.3541255168 \\
\hline $\mathrm{N}$ & 0.0589742498 & 0.2284452713 & 0.2577398637 \\
\hline $\mathrm{N}$ & 0.3457782530 & 0.2211043659 & 0.4300252378 \\
\hline $\mathrm{N}$ & 0.1464638458 & 0.2542114923 & 0.4839819370 \\
\hline $\mathrm{N}$ & 0.6873332116 & 0.9158817385 & 0.6078048652 \\
\hline $\mathrm{N}$ & 0.8977161959 & 0.7681644040 & 0.7042125171 \\
\hline $\mathrm{N}$ & 0.6109271791 & 0.7755117337 & 0.5318894497 \\
\hline $\mathrm{N}$ & 0.8102711946 & 0.7424291957 & 0.4779557205 \\
\hline $\mathrm{N}$ & 0.6875167179 & 0.5802685053 & 0.1085817954 \\
\hline $\mathrm{N}$ & 0.8974103281 & 0.7284338900 & 0.2045992578 \\
\hline $\mathrm{N}$ & 0.6104135938 & 0.7205816953 & 0.0325974029 \\
\hline $\mathrm{N}$ & 0.8092291330 & 0.7537585424 & 0.9782516415 \\
\hline $\mathrm{N}$ & 0.2690988586 & 0.4164417323 & 0.8532979091 \\
\hline $\mathrm{N}$ & 0.0592639132 & 0.2682643995 & 0.7573214229 \\
\hline $\mathrm{N}$ & 0.3462518445 & 0.2761496543 & 0.9293312408 \\
\hline $\mathrm{N}$ & 0.1474259379 & 0.2429583742 & 0.9836642104 \\
\hline C & 0.1495586898 & 0.2784078721 & 0.2579952253 \\
\hline C & 0.2421387935 & 0.3752403140 & 0.5473356950 \\
\hline C & 0.3026660879 & 0.2576838541 & 0.3228286356 \\
\hline C & 0.1027921288 & 0.3436022683 & 0.1975838551 \\
\hline C & 0.9200157620 & 0.2414723090 & 0.1962846916 \\
\hline C & 0.4601643677 & 0.1676897909 & 0.4586343966 \\
\hline C & 0.2521937199 & 0.3034189372 & 0.5163182449 \\
\hline C & 0.4041735147 & 0.0983513091 & 0.3897533878 \\
\hline C & 0.8658607147 & 0.3050554049 & 0.1326486487 \\
\hline C & 0.0279953359 & 0.2755031792 & 0.4799294069 \\
\hline C & 0.2161753631 & 0.0170391947 & 0.2982696431 \\
\hline C & 0.2946529037 & 0.9683053820 & 0.2756290032 \\
\hline C & 0.3817529223 & 0.2748227111 & 0.5198799158 \\
\hline C & 0.4337170879 & 0.9868733036 & 0.3110988224 \\
\hline C & 0.9585339592 & 0.3571163138 & 0.1339065280 \\
\hline C & 0.4885180893 & 0.0529976702 & 0.3680758211 \\
\hline C & 0.0097775795 & 0.3464276308 & 0.5085974195 \\
\hline C & 0.1191492095 & 0.3970489462 & 0.5430672242 \\
\hline C & 0.1123319128 & 0.9830012414 & 0.5818426320 \\
\hline C & 0.8444004711 & 0.0136221595 & 0.3801434752 \\
\hline C & 0.9635301906 & 0.0540836770 & 0.4042934457 \\
\hline C & 0.9932001925 & 0.9425376405 & 0.5576928194 \\
\hline C & 0.1017409466 & 0.0395158766 & 0.5095945253 \\
\hline C & 0.8549912569 & 0.9571038928 & 0.4523891675 \\
\hline C & 0.8071166533 & 0.7181988304 & 0.7039270841 \\
\hline C & 0.7145656677 & 0.6214193360 & 0.4144938733 \\
\hline C & 0.6540085347 & 0.7389196047 & 0.6390714546 \\
\hline C & 0.8538667203 & 0.6529984660 & 0.7643236975 \\
\hline C & 0.0366719393 & 0.7551347210 & 0.7656837493 \\
\hline C & 0.4965404515 & 0.8289269008 & 0.5032756255 \\
\hline C & 0.7045287765 & 0.6932243729 & 0.4455757001 \\
\hline C & 0.5525257016 & 0.8982649771 & 0.5721590654 \\
\hline C & 0.0908098842 & 0.6915454867 & 0.8293068967 \\
\hline C & 0.9287342967 & 0.7211506487 & 0.4819890075 \\
\hline
\end{tabular}




\begin{tabular}{|c|c|c|c|}
\hline C & 0.7405234488 & 0.9795761507 & 0.6636473064 \\
\hline C & 0.6620333450 & 0.0283185876 & 0.6862622703 \\
\hline C & 0.5749672792 & 0.7218040434 & 0.4420257755 \\
\hline C & 0.5229644578 & 0.0097538143 & 0.6507806632 \\
\hline C & 0.9981219655 & 0.6394819600 & 0.8280184865 \\
\hline C & 0.4681675981 & 0.9436263736 & 0.5938121826 \\
\hline C & 0.9469335162 & 0.6502424660 & 0.4532581991 \\
\hline C & 0.8375492018 & 0.5996239680 & 0.4187433460 \\
\hline C & 0.8066909974 & 0.7784667111 & 0.2040893969 \\
\hline C & 0.7123192803 & 0.8743993257 & 0.9137964891 \\
\hline C & 0.6536937197 & 0.7574818010 & 0.1396947598 \\
\hline C & 0.8532560058 & 0.8439907855 & 0.2637812651 \\
\hline C & 0.0363084172 & 0.7417184512 & 0.2656357167 \\
\hline C & 0.4963435372 & 0.6670812558 & 0.0044454464 \\
\hline C & 0.7030413110 & 0.8027190407 & 0.9455451850 \\
\hline C & 0.5527589518 & 0.5978054977 & 0.0732623090 \\
\hline C & 0.0902789970 & 0.8056511566 & 0.3285564766 \\
\hline C & 0.9274470400 & 0.7751769133 & 0.9820466102 \\
\hline C & 0.7409400827 & 0.5165752491 & 0.1642351690 \\
\hline C & 0.6627470882 & 0.4677770179 & 0.1869906764 \\
\hline C & 0.5738493368 & 0.7740175796 & 0.9424059192 \\
\hline C & 0.5237433616 & 0.4862732489 & 0.1518625886 \\
\hline C & 0.9974562676 & 0.8577779255 & 0.3270045594 \\
\hline C & 0.4687008394 & 0.5523889494 & 0.0950737614 \\
\hline C & 0.9449226819 & 0.8459851625 & 0.9526945394 \\
\hline C & 0.8350321275 & 0.8963383995 & 0.9177731824 \\
\hline C & 0.8444451430 & 0.4831443548 & 0.8799364873 \\
\hline C & 0.1121587976 & 0.5135184672 & 0.0820059467 \\
\hline C & 0.9929761004 & 0.5539084122 & 0.0579948649 \\
\hline C & 0.9636313767 & 0.4427623826 & 0.9039420017 \\
\hline C & 0.8548571320 & 0.5394479362 & 0.9525285957 \\
\hline C & 0.1017514083 & 0.4572270875 & 0.0094040553 \\
\hline C & 0.1500065775 & 0.2182416223 & 0.7578437851 \\
\hline C & 0.2443791718 & 0.1223257898 & 0.0481346891 \\
\hline C & 0.3029957593 & 0.2392384749 & 0.8222457434 \\
\hline C & 0.1034725745 & 0.1527140409 & 0.6981571134 \\
\hline C & 0.9203710048 & 0.2549633885 & 0.6962834711 \\
\hline C & 0.4603079412 & 0.3296549292 & 0.9574680245 \\
\hline C & 0.2536370740 & 0.1940099933 & 0.0163880241 \\
\hline C & 0.4038639984 & 0.3989168585 & 0.8886231320 \\
\hline C & 0.8664310728 & 0.1910254740 & 0.6333710269 \\
\hline C & 0.0292042666 & 0.2215221784 & 0.9798513975 \\
\hline C & 0.2156426597 & 0.4801185544 & 0.7976033359 \\
\hline C & 0.2938127599 & 0.5289135935 & 0.7748198650 \\
\hline C & 0.3828271996 & 0.2227272158 & 1.0195405682 \\
\hline C & 0.4328268158 & 0.5104321805 & 0.8099608774 \\
\hline C & 0.9592782791 & 0.1389111252 & 0.6349316745 \\
\hline C & 0.4878981209 & 0.4443299471 & 0.8667833681 \\
\hline C & 0.0117487621 & 0.1507091226 & 0.0092018805 \\
\hline C & 0.1216636856 & 0.1003690204 & 0.0441399789 \\
\hline $\mathrm{H}$ & 0.3295484860 & 0.4135342629 & 0.5738309266 \\
\hline $\mathrm{H}$ & 0.3696570682 & 0.3058414084 & 0.3352157894 \\
\hline $\mathrm{H}$ & 0.3217567842 & 0.2182876482 & 0.2726301926 \\
\hline $\mathrm{H}$ & 0.1786768710 & 0.3824478059 & 0.1994480182 \\
\hline $\mathrm{H}$ & 0.8524009765 & 0.1999094358 & 0.2008724546 \\
\hline $\mathrm{H}$ & 0.5432657486 & 0.1920411306 & 0.4481993128 \\
\hline $\mathrm{H}$ & 0.5062588042 & 0.1527867517 & 0.5463087278 \\
\hline $\mathrm{H}$ & 0.7528574751 & 0.3130475554 & 0.0841329888 \\
\hline
\end{tabular}




\begin{tabular}{|c|c|c|c|}
\hline $\mathrm{H}$ & 0.9444037635 & 0.2345062705 & 0.4504354235 \\
\hline $\mathrm{H}$ & 0.1065511002 & 1.0059052879 & 0.2714250499 \\
\hline $\mathrm{H}$ & 0.2487469765 & 0.9167134757 & 0.2314168779 \\
\hline $\mathrm{H}$ & 0.4456847906 & 0.3207681101 & 0.5181330213 \\
\hline $\mathrm{H}$ & 0.4454431320 & 0.2459111685 & 0.5991016029 \\
\hline $\mathrm{H}$ & 0.4985286645 & 0.9494994588 & 0.2956093880 \\
\hline $\mathrm{H}$ & 0.9194592181 & 0.4076517777 & 0.0853186117 \\
\hline $\mathrm{H}$ & 0.5957940595 & 0.0698561031 & 0.3955737881 \\
\hline $\mathrm{H}$ & 0.9105823225 & 0.3604248043 & 0.5021214845 \\
\hline $\mathrm{H}$ & 0.1091955471 & 0.4532870317 & 0.5659625045 \\
\hline $\mathrm{H}$ & 0.2121478173 & 0.9721680548 & 0.6578641968 \\
\hline $\mathrm{H}$ & 0.7445857878 & 0.0244508855 & 0.3041190384 \\
\hline $\mathrm{H}$ & 0.6271438351 & 0.5831281185 & 0.3879592061 \\
\hline $\mathrm{H}$ & 0.5870187152 & 0.6907562203 & 0.6266573673 \\
\hline $\mathrm{H}$ & 0.6348861234 & 0.7783027688 & 0.6892665884 \\
\hline $\mathrm{H}$ & 0.7779722505 & 0.6141477488 & 0.7624324896 \\
\hline $\mathrm{H}$ & 0.1042999757 & 0.7966997544 & 0.7611188709 \\
\hline $\mathrm{H}$ & 0.4134303536 & 0.8045739668 & 0.5136979558 \\
\hline $\mathrm{H}$ & 0.4504589089 & 0.8438327476 & 0.4156036472 \\
\hline $\mathrm{H}$ & 0.2038111056 & 0.6835487567 & 0.8778357203 \\
\hline $\mathrm{H}$ & 0.0123368148 & 0.7621433802 & 0.5115200322 \\
\hline $\mathrm{H}$ & 0.8501529584 & -0.0092939994 & 0.6905029700 \\
\hline $\mathrm{H}$ & 0.7079342683 & 0.0799141124 & 0.7304653046 \\
\hline $\mathrm{H}$ & 0.5110487714 & 0.6758485009 & 0.4437639693 \\
\hline $\mathrm{H}$ & 0.5112666729 & 0.7507188560 & 0.3628081234 \\
\hline $\mathrm{H}$ & 0.4581444784 & 0.0471320692 & 0.6662546056 \\
\hline $\mathrm{H}$ & 0.0371830439 & 0.5889408364 & 0.8765931190 \\
\hline $\mathrm{H}$ & 0.3608865784 & 0.9267713164 & 0.5663030592 \\
\hline $\mathrm{H}$ & 0.0461222037 & 0.6362542844 & 0.4597173549 \\
\hline $\mathrm{H}$ & 0.8474864441 & 0.5433983051 & 0.3957965432 \\
\hline $\mathrm{H}$ & 0.6245125385 & 0.9124774455 & 0.8869463622 \\
\hline $\mathrm{H}$ & 0.5864763082 & 0.8055670601 & 0.1272305401 \\
\hline $\mathrm{H}$ & 0.6351287991 & 0.7181928548 & 0.1902237646 \\
\hline $\mathrm{H}$ & 0.7772777211 & 0.8829013206 & 0.2616976869 \\
\hline $\mathrm{H}$ & 0.1040195291 & 0.7000695625 & 0.2612756614 \\
\hline $\mathrm{H}$ & 0.4134628536 & 0.6913886767 & 0.0152384089 \\
\hline $\mathrm{H}$ & 0.4498179906 & 0.6520706255 & 0.9167377050 \\
\hline $\mathrm{H}$ & 0.2032396269 & 0.8138848049 & 0.3766846844 \\
\hline $\mathrm{H}$ & 0.0114492688 & 0.7343962105 & 0.0119116503 \\
\hline $\mathrm{H}$ & 0.8504972013 & 0.5054902071 & 0.1907970546 \\
\hline $\mathrm{H}$ & 0.7087918795 & 0.4161715080 & 0.2309968695 \\
\hline $\mathrm{H}$ & 0.5096561809 & 0.8199102838 & 0.9440019137 \\
\hline $\mathrm{H}$ & 0.5102198839 & 0.7448137110 & 0.8633805119 \\
\hline $\mathrm{H}$ & 0.4591708145 & 0.4488462985 & 0.1674595129 \\
\hline $\mathrm{H}$ & 0.0363594946 & 0.9085938321 & 0.3749924594 \\
\hline $\mathrm{H}$ & 0.3614316970 & 0.5691725988 & 0.0677645547 \\
\hline $\mathrm{H}$ & 1.0439546008 & 0.8601326384 & 0.9590182766 \\
\hline $\mathrm{H}$ & 0.8443938100 & 0.9524789575 & 0.8943304839 \\
\hline $\mathrm{H}$ & 0.7446941462 & 0.4724004588 & 0.8037914190 \\
\hline $\mathrm{H}$ & 0.2119113036 & 0.5242644133 & 0.1581492280 \\
\hline $\mathrm{H}$ & 0.3322034252 & 0.0842580672 & 0.0749955482 \\
\hline $\mathrm{H}$ & 0.3702296710 & 0.1911576198 & 0.8347280114 \\
\hline $\mathrm{H}$ & 0.3215600123 & 0.2785247864 & 0.7717134104 \\
\hline $\mathrm{H}$ & 0.1794708843 & 0.1138154470 & 0.7002457254 \\
\hline $\mathrm{H}$ & 0.8526406361 & 0.2966031233 & 0.7006368220 \\
\hline $\mathrm{H}$ & 0.5431922049 & 0.3053488935 & 0.9466774614 \\
\hline $\mathrm{H}$ & 0.5068343805 & 0.3446773875 & 0.0451709622 \\
\hline $\mathrm{H}$ & 0.7534737324 & 0.1827797635 & 0.5852398464 \\
\hline
\end{tabular}




\begin{tabular}{|c|c|c|c|}
\hline $\mathrm{H}$ & 0.9451820790 & 0.2622918819 & 0.9499716051 \\
\hline $\mathrm{H}$ & 0.1060797472 & 0.4911927496 & 0.7710327871 \\
\hline $\mathrm{H}$ & 0.2477454898 & 0.5805081006 & 0.7307880461 \\
\hline $\mathrm{H}$ & 0.4470593533 & 0.1768428209 & 1.0179787341 \\
\hline $\mathrm{H}$ & 0.4464203866 & 0.2519493730 & 0.0985541805 \\
\hline $\mathrm{H}$ & 0.4973819943 & 0.5478595566 & 0.7943456187 \\
\hline $\mathrm{H}$ & 0.9203970708 & 0.0880919483 & 0.5869484505 \\
\hline $\mathrm{H}$ & 0.5951734513 & 0.4275546240 & 0.8940998369 \\
\hline $\mathrm{H}$ & 0.9127116320 & 0.1365469734 & 0.0028605104 \\
\hline $\mathrm{H}$ & 0.1123177986 & 0.0442247701 & 0.0675800041 \\
\hline 0 & 0.2033345113 & 0.0820521284 & 0.5274445218 \\
\hline 0 & 0.7534047729 & 0.9145641253 & 0.4345297180 \\
\hline $\mathrm{O}$ & 0.9659134288 & 0.1048986640 & 0.3411420446 \\
\hline 0 & 0.9908126790 & 0.8917230041 & 0.6208387352 \\
\hline $\mathrm{O}$ & 0.7531540002 & 0.5818529741 & 0.9348746371 \\
\hline 0 & 0.2034724893 & 0.4148360877 & 0.0270414773 \\
\hline 0 & 0.9904860424 & 0.6045737475 & 0.1213962669 \\
\hline 0 & 0.9661295459 & 0.3921035823 & 0.8405306465 \\
\hline $\mathrm{F}$ & 0.5778914533 & 0.2886256996 & 0.7947930762 \\
\hline $\mathrm{F}$ & 0.6686517468 & 0.1930813538 & 0.9232097739 \\
\hline $\mathrm{F}$ & 0.4964779663 & 0.1685913938 & 0.7401391598 \\
\hline $\mathrm{F}$ & 0.7212358801 & 0.1998571112 & 0.7836438161 \\
\hline $\mathrm{F}$ & 0.3786885772 & 0.7080115372 & 0.1670791442 \\
\hline $\mathrm{F}$ & 0.2880065530 & 0.8035729783 & 0.0386892338 \\
\hline $\mathrm{F}$ & 0.4601380691 & 0.8280340976 & 0.2217800335 \\
\hline $\mathrm{F}$ & 0.2353482994 & 0.7967883456 & 0.1782118222 \\
\hline $\mathrm{F}$ & 0.3806633170 & 0.7890948943 & 0.6676937937 \\
\hline $\mathrm{F}$ & 0.2874512540 & 0.6939883241 & 0.5384557019 \\
\hline $\mathrm{F}$ & 0.4606170466 & 0.6687312229 & 0.7209024224 \\
\hline $\mathrm{F}$ & 0.2369111236 & 0.7008307130 & 0.6791702666 \\
\hline $\mathrm{F}$ & 0.5759771965 & 0.2075990198 & 0.2941930347 \\
\hline $\mathrm{F}$ & 0.6692822934 & 0.3026754488 & 0.4234779287 \\
\hline $\mathrm{F}$ & 0.4960771048 & 0.3279804644 & 0.2410596502 \\
\hline $\mathrm{F}$ & 0.7197429702 & 0.2958558832 & 0.2827170631 \\
\hline B & 0.6170686841 & 0.2126094181 & 0.8112412579 \\
\hline B & 0.3395403694 & 0.7840305930 & 0.1506451257 \\
\hline B & 0.3404189934 & 0.7132490859 & 0.6507774331 \\
\hline B & 0.6162624071 & 0.2834399960 & 0.3111405204 \\
\hline \multicolumn{4}{|c|}{6 -is } \\
\hline \multicolumn{4}{|c|}{ CELL PARAMETERS (angstrom) } \\
\hline 10.956183467 & 0.000000000 & -0.374528150 & \\
\hline 0.000000000 & 16.865344540 & 0.000000000 & \\
\hline-8.430030853 & 0.000000000 & 13.433752791 & \\
\hline \multicolumn{4}{|c|}{ ATOMIC_POSITIONS (crystal) } \\
\hline Fe1 & 0.1507538140 & 0.1431938966 & 0.3972838682 \\
\hline $\mathrm{Fe} 2$ & 0.8370633487 & 0.8464788038 & 0.6076936982 \\
\hline Fe1 & 0.8492462010 & 0.6431938966 & 0.1027161388 \\
\hline $\mathrm{Fe} 2$ & 0.1629366513 & 0.3464788338 & 0.8923063018 \\
\hline $\mathrm{N}$ & 0.2415729973 & 0.0574624775 & 0.3452890464 \\
\hline $\mathrm{N}$ & 0.0547841424 & 0.2159920877 & 0.2653014609 \\
\hline $\mathrm{N}$ & 0.3552634849 & 0.2034092959 & 0.4282832329 \\
\hline $\mathrm{N}$ & 0.1614862552 & 0.2493604569 & 0.4780694979 \\
\hline $\mathrm{N}$ & 0.7839621042 & 0.9319219866 & 0.6648172235 \\
\hline $\mathrm{N}$ & 0.9187209330 & 0.7780724806 & 0.7246278114 \\
\hline $\mathrm{N}$ & 0.6420396909 & 0.8019442707 & 0.5660679247 \\
\hline $\mathrm{N}$ & 0.8463232457 & 0.7557486164 & 0.5349905246 \\
\hline $\mathrm{N}$ & 0.7584270327 & 0.5574624775 & 0.1547109536 \\
\hline
\end{tabular}




\begin{tabular}{|c|c|c|c|}
\hline $\mathrm{N}$ & 0.9452158426 & 0.7159921027 & 0.2346985541 \\
\hline $\mathrm{N}$ & 0.6447365151 & 0.7034092809 & 0.0717167741 \\
\hline $\mathrm{N}$ & 0.8385137148 & 0.7493604569 & 0.0219305101 \\
\hline $\mathrm{N}$ & 0.2160378958 & 0.4319219866 & 0.8351827765 \\
\hline $\mathrm{N}$ & 0.0812790670 & 0.2780725106 & 0.7753721886 \\
\hline $\mathrm{N}$ & 0.3579603091 & 0.3019442707 & 0.9339320753 \\
\hline $\mathrm{N}$ & 0.1536766943 & 0.2557486454 & 0.9650094754 \\
\hline C & 0.1501888712 & 0.2640909573 & 0.2616419424 \\
\hline C & 0.2996420393 & 0.3664366716 & 0.5641308529 \\
\hline C & 0.3126513006 & 0.2471566776 & 0.3340848221 \\
\hline C & 0.1009588744 & 0.3236912762 & 0.1884788486 \\
\hline C & 0.9087506966 & 0.2253279039 & 0.1969159845 \\
\hline C & 0.4601607638 & 0.1390109174 & 0.4484387821 \\
\hline C & 0.2853462053 & 0.2931715178 & 0.5186044027 \\
\hline C & 0.3822667227 & 0.0706674484 & 0.3758276453 \\
\hline C & 0.8525219800 & 0.2823371606 & 0.1207857812 \\
\hline C & 0.0481482092 & 0.2774075949 & 0.4801614225 \\
\hline C & 0.1685143829 & 0.9948706898 & 0.2846889741 \\
\hline C & 0.2328625495 & 0.9427134525 & 0.2524368692 \\
\hline C & 0.4095610066 & 0.2559340631 & 0.5172657973 \\
\hline C & 0.3779524899 & 0.9566450229 & 0.2830711937 \\
\hline C & 0.9500907006 & 0.3324675278 & 0.1165255378 \\
\hline C & 0.4530175344 & 0.0218412893 & 0.3450465871 \\
\hline C & 0.0544398809 & 0.3500147676 & 0.5241883487 \\
\hline C & 0.1828747033 & 0.3950750133 & 0.5673134632 \\
\hline C & 0.1404280959 & 0.9783398262 & 0.5947722886 \\
\hline C & 0.8465577041 & 0.0025466425 & 0.4110806916 \\
\hline C & 0.9676710827 & 0.0420816344 & 0.4235349860 \\
\hline C & 0.0184339937 & 0.9396084277 & 0.5820917673 \\
\hline C & 0.1197368194 & 0.0306372085 & 0.5190393623 \\
\hline C & 0.8676850958 & 0.9518655868 & 0.4884272196 \\
\hline C & 0.8202302762 & 0.7316014583 & 0.7276670821 \\
\hline C & 0.7040508475 & 0.6484534747 & 0.4226918706 \\
\hline C & 0.6620057286 & 0.7530422980 & 0.6520548997 \\
\hline C & 0.8649979764 & 0.6715642847 & 0.7996176704 \\
\hline C & 0.0644250130 & 0.7662258708 & 0.7933512045 \\
\hline C & 0.5468616314 & 0.8717708406 & 0.5478891604 \\
\hline C & 0.7165787432 & 0.7162555757 & 0.4771340319 \\
\hline C & 0.6376194714 & 0.9342408720 & 0.6263841792 \\
\hline C & 0.1159546944 & 0.7077160754 & 0.8673200044 \\
\hline C & 0.9662684522 & 0.7284436212 & 0.5404467619 \\
\hline C & 0.8727266851 & 0.9886950833 & 0.7312051632 \\
\hline C & 0.8190713697 & 0.0497878396 & 0.7608604860 \\
\hline C & 0.5888698008 & 0.7547941926 & 0.4720640695 \\
\hline C & 0.6692253423 & 0.0516818124 & 0.7226349320 \\
\hline C & 1.0153213461 & 0.6594340177 & 0.8705115415 \\
\hline C & 0.5774770236 & 0.9924936862 & 0.6548753813 \\
\hline C & 0.9608904921 & 0.6613607975 & 0.4872633897 \\
\hline C & 0.8278066655 & 0.6207641650 & 0.4273593735 \\
\hline C & 0.8498111138 & 0.7640909873 & 0.2383580426 \\
\hline C & 0.7003579907 & 0.8664366716 & 0.9358691471 \\
\hline C & 0.6873486994 & 0.7471566626 & 0.1659151779 \\
\hline C & 0.8990411406 & 0.8236912472 & 0.3115211514 \\
\hline C & 0.0912493634 & 0.7253279039 & 0.3030840155 \\
\hline C & 0.5398392362 & 0.6390109174 & 0.0515612259 \\
\hline C & 0.7146537947 & 0.7931715178 & 0.9813955973 \\
\hline C & 0.6177333073 & 0.5706674484 & 0.1241723627 \\
\hline C & 0.1474780200 & 0.7823371906 & 0.3792142038 \\
\hline
\end{tabular}




\begin{tabular}{|c|c|c|c|}
\hline C & 0.9518518168 & 0.7774076249 & 0.0198385835 \\
\hline C & 0.8314856461 & 0.4948707198 & 0.2153110259 \\
\hline C & 0.7671364665 & 0.4427134525 & 0.2475631458 \\
\hline C & 0.5904389934 & 0.7559340920 & 0.9827342027 \\
\hline C & 0.6220475101 & 0.4566450529 & 0.2169288213 \\
\hline C & 0.0499092994 & 0.8324674988 & 0.3834744472 \\
\hline C & 0.5469824356 & 0.5218413023 & 0.1549534129 \\
\hline C & 0.9455601191 & 0.8500147976 & 0.9758116513 \\
\hline C & 0.8171253107 & 0.8950749843 & 0.9326865367 \\
\hline C & 0.8595718891 & 0.4783398552 & 0.9052277114 \\
\hline C & 0.1534422959 & 0.5025466135 & 0.0889193084 \\
\hline C & 0.0323289173 & 0.5420816494 & 0.0764650140 \\
\hline C & 0.9815660143 & 0.4396084277 & 0.9179091867 \\
\hline C & 0.8802631576 & 0.5306372085 & 0.9809606377 \\
\hline C & 0.1323148452 & 0.4518655868 & 0.0115727674 \\
\hline C & 0.1797697838 & 0.2316014583 & 0.7723329179 \\
\hline C & 0.2959490925 & 0.1484534597 & 0.0773081294 \\
\hline C & 0.3379942714 & 0.2530422980 & 0.8479451003 \\
\hline C & 0.1350020236 & 0.1715642697 & 0.7003823296 \\
\hline C & 0.9355749790 & 0.2662259008 & 0.7066487955 \\
\hline C & 0.4531383686 & 0.3717708106 & 0.9521108396 \\
\hline C & 0.2834211968 & 0.2162555757 & 0.0228659811 \\
\hline C & 0.3623805286 & 0.4342408720 & 0.8736158208 \\
\hline C & 0.8840452906 & 0.2077160894 & 0.6326799956 \\
\hline C & 0.0337315478 & 0.2284435762 & 0.9595532381 \\
\hline C & 0.1272733149 & 0.4886950833 & 0.7687948368 \\
\hline C & 0.1809286303 & 0.5497878616 & 0.7391395140 \\
\hline C & 0.4111301992 & 0.2547941926 & 0.0279359265 \\
\hline C & 0.3307745987 & 0.5516818234 & 0.7773650680 \\
\hline C & -0.0153213461 & 0.1594340627 & 0.6294884585 \\
\hline C & 0.4225230364 & 0.4924936562 & 0.8451246187 \\
\hline C & 0.0391095679 & 0.1613607975 & 0.0127366033 \\
\hline C & 0.1721933345 & 0.1207641500 & 0.0726406295 \\
\hline $\mathrm{H}$ & 0.4022905524 & 0.3996108746 & 0.5983332164 \\
\hline $\mathrm{H}$ & 0.3767816146 & 0.3022891613 & 0.3531023442 \\
\hline $\mathrm{H}$ & 0.3455465338 & 0.2111490582 & 0.2920568326 \\
\hline $\mathrm{H}$ & 0.1803905974 & 0.3614740030 & 0.1868577106 \\
\hline $\mathrm{H}$ & 0.8379705825 & 0.1862175621 & 0.2063882883 \\
\hline $\mathrm{H}$ & 0.5510294142 & 0.1599102978 & 0.4432554159 \\
\hline $\mathrm{H}$ & 0.5073059885 & 0.1175480903 & 0.5264568111 \\
\hline $\mathrm{H}$ & 0.7343574468 & 0.2865744109 & 0.0652283594 \\
\hline $\mathrm{H}$ & 0.9493912310 & 0.2406945481 & 0.4442443610 \\
\hline $\mathrm{H}$ & 0.0554562906 & 0.9871445134 & 0.2630105352 \\
\hline $\mathrm{H}$ & 0.1723320792 & 0.8914365968 & 0.2056338663 \\
\hline $\mathrm{H}$ & 0.4846671307 & 0.3018533499 & 0.5215784080 \\
\hline $\mathrm{H}$ & 0.4735952434 & 0.2198324360 & 0.5867677212 \\
\hline $\mathrm{H}$ & 0.4322095777 & 0.9162900699 & 0.2601840104 \\
\hline $\mathrm{H}$ & 0.9090750793 & 0.3774676112 & 0.0571296032 \\
\hline $\mathrm{H}$ & 0.5657520306 & 0.0351655539 & 0.3702702941 \\
\hline $\mathrm{H}$ & 0.9596650236 & 0.3696229190 & 0.5242032917 \\
\hline $\mathrm{H}$ & 0.1924255327 & 0.4519587137 & 0.6033028796 \\
\hline $\mathrm{H}$ & 0.2501581057 & 0.9674941353 & 0.6627571880 \\
\hline $\mathrm{H}$ & 0.7375107741 & 0.0119156159 & 0.3418613572 \\
\hline $\mathrm{H}$ & 0.5973801341 & 0.6194633012 & 0.3747931797 \\
\hline $\mathrm{H}$ & 0.5901688868 & 0.7005484778 & 0.6231418395 \\
\hline $\mathrm{H}$ & 0.6247067414 & 0.7885092193 & 0.6917164080 \\
\hline $\mathrm{H}$ & 0.7826854928 & 0.6361563930 & 0.8007954602 \\
\hline $\mathrm{H}$ & 0.1378879145 & 0.8039903780 & 0.7852492783 \\
\hline
\end{tabular}




\begin{tabular}{|c|c|c|c|}
\hline $\mathrm{H}$ & 0.4509934075 & 0.8557577342 & 0.5497563494 \\
\hline $\mathrm{H}$ & 0.5079517114 & 0.8956532693 & 0.4722834171 \\
\hline $\mathrm{H}$ & 0.2339044093 & 0.7008420130 & 0.9220337498 \\
\hline $\mathrm{H}$ & 0.0680963647 & 0.7613536515 & 0.5895383339 \\
\hline $\mathrm{H}$ & 0.9885995167 & 0.9840008212 & 0.7586391566 \\
\hline $\mathrm{H}$ & 0.8936913953 & 0.0955009807 & 0.8128712192 \\
\hline $\mathrm{H}$ & 0.5046933788 & 0.7110383525 & 0.4580317211 \\
\hline $\mathrm{H}$ & 0.5372887806 & 0.7957554512 & 0.4069085590 \\
\hline $\mathrm{H}$ & 0.6237905202 & 0.0987289721 & 0.7443824535 \\
\hline $\mathrm{H}$ & 0.0536802818 & 0.6130265516 & 0.9278854458 \\
\hline $\mathrm{H}$ & 0.4596572726 & 0.9925956849 & 0.6229138606 \\
\hline $\mathrm{H}$ & 0.0611233888 & 0.6427864908 & 0.4937955047 \\
\hline $\mathrm{H}$ & 0.8197363298 & 0.5681459739 & 0.3844396481 \\
\hline $\mathrm{H}$ & 0.5977094776 & 0.8996109046 & 0.9016667836 \\
\hline $\mathrm{H}$ & 0.6232183854 & 0.8022891613 & 0.1468976408 \\
\hline $\mathrm{H}$ & 0.6544534362 & 0.7111490432 & 0.2079431674 \\
\hline $\mathrm{H}$ & 0.8196094026 & 0.8614740030 & 0.3131422894 \\
\hline $\mathrm{H}$ & 0.1620294175 & 0.6862175471 & 0.2936117267 \\
\hline $\mathrm{H}$ & 0.4489705858 & 0.6599102978 & 0.0567445981 \\
\hline $\mathrm{H}$ & 0.4926940115 & 0.6175481053 & 0.9735431889 \\
\hline H & 0.2656424932 & 0.7865744109 & 0.4347716406 \\
\hline $\mathrm{H}$ & 0.0506087690 & 0.7406945331 & 0.0557556280 \\
\hline $\mathrm{H}$ & 0.9445437014 & 0.4871445134 & 0.2369894798 \\
\hline H & 0.8276679348 & 0.3914365968 & 0.2943661337 \\
\hline $\mathrm{H}$ & 0.5153328692 & 0.8018533499 & 0.9784215920 \\
\hline $\mathrm{H}$ & 0.5264047866 & 0.7198324510 & 0.9132322788 \\
\hline $\mathrm{H}$ & 0.5677904223 & 0.4162900699 & 0.2398159756 \\
\hline $\mathrm{H}$ & 0.0909249797 & 0.8774676412 & 0.4428703888 \\
\hline $\mathrm{H}$ & 0.4342479994 & 0.5351655799 & 0.1297297209 \\
\hline $\mathrm{H}$ & 0.0403350364 & 0.8696229190 & 0.9757967083 \\
\hline $\mathrm{H}$ & 0.8075744673 & 0.9519587137 & 0.8966971204 \\
\hline $\mathrm{H}$ & 0.7498419233 & 0.4674941353 & 0.8372428120 \\
\hline $\mathrm{H}$ & 0.2624892849 & 0.5119156069 & 0.1581386428 \\
\hline H & 0.4026198059 & 0.1194633082 & 0.1252068134 \\
\hline $\mathrm{H}$ & 0.4098311132 & 0.2005484778 & 0.8768581605 \\
\hline $\mathrm{H}$ & 0.3752932586 & 0.2885092193 & 0.8082835920 \\
\hline $\mathrm{H}$ & 0.2173145072 & 0.1361553950 & 0.6992045398 \\
\hline $\mathrm{H}$ & 0.8621130765 & 0.3039903480 & 0.7147506617 \\
\hline $\mathrm{H}$ & 0.5490065925 & 0.3557577642 & 0.9502436506 \\
\hline H & 0.4920482886 & 0.3956532393 & 0.0277165969 \\
\hline $\mathrm{H}$ & 0.7660955907 & 0.2008420279 & 0.5779662502 \\
\hline $\mathrm{H}$ & 0.9319036243 & 0.2613536215 & 0.9104616661 \\
\hline H & 0.0114004833 & 0.4840008212 & 0.7413608433 \\
\hline $\mathrm{H}$ & 0.1063086047 & 0.5955009957 & 0.6871287208 \\
\hline $\mathrm{H}$ & 0.4953065612 & 0.2110383826 & 0.0419682899 \\
\hline $\mathrm{H}$ & 0.4627112194 & 0.2957554212 & 0.0930914480 \\
\hline $\mathrm{H}$ & 0.3762094798 & 0.5987289721 & 0.7556175465 \\
\hline H & 0.9463197182 & 0.1130265216 & 0.5721145542 \\
\hline $\mathrm{H}$ & 0.5403426674 & 0.4925956849 & 0.8770861394 \\
\hline $\mathrm{H}$ & 0.9388766192 & 0.1427864608 & 0.0062045053 \\
\hline $\mathrm{H}$ & 0.1802636702 & 0.0681459849 & 0.1155603449 \\
\hline 0 & 0.2233993866 & 0.0710546870 & 0.5242157504 \\
\hline 0 & 0.7638047845 & 0.9131282432 & 0.4855049934 \\
\hline 0 & 0.9586898750 & 0.0896403919 & 0.3562767101 \\
\hline 0 & 0.0244979096 & 0.8915977644 & 0.6480418744 \\
\hline 0 & 0.7766006284 & 0.5710547020 & 0.9757842496 \\
\hline 0 & 0.2361952745 & 0.4131282432 & 0.0144950006 \\
\hline 0 & 0.0413101250 & 0.5896413969 & 0.1437232749 \\
\hline
\end{tabular}




\begin{tabular}{|c|c|c|c|}
\hline $\mathrm{O}$ & 0.9755021084 & 0.3915977644 & 0.8519581256 \\
\hline$F$ & 0.5738387129 & 0.3300982468 & 0.8074817329 \\
\hline $\mathrm{F}$ & 0.6897334477 & 0.2152205067 & 0.8991993017 \\
\hline $\mathrm{F}$ & 0.4785947108 & 0.2058685394 & 0.7355440922 \\
\hline $\mathrm{F}$ & 0.6966310889 & 0.2487896389 & 0.7593071741 \\
\hline $\mathrm{F}$ & 0.4181694346 & 0.6710320021 & 0.1946059479 \\
\hline $\mathrm{F}$ & 0.3093598804 & 0.7794559614 & 0.0866536079 \\
\hline $\mathrm{F}$ & 0.5119613888 & 0.7979114616 & 0.2523501413 \\
\hline $\mathrm{F}$ & 0.2867340129 & 0.7626801661 & 0.2232866767 \\
\hline $\mathrm{F}$ & 0.4261612871 & 0.8300982468 & 0.6925182671 \\
\hline $\mathrm{F}$ & 0.3102665524 & 0.7152205067 & 0.6008006983 \\
\hline $\mathrm{F}$ & 0.5214052592 & 0.7058685544 & 0.7644559078 \\
\hline $\mathrm{F}$ & 0.3033689411 & 0.7487896099 & 0.7406928259 \\
\hline $\mathrm{F}$ & 0.5818305354 & 0.1710320021 & 0.3053940521 \\
\hline $\mathrm{F}$ & 0.6906400896 & 0.2794559614 & 0.4133463771 \\
\hline $\mathrm{F}$ & 0.4880386112 & 0.2979114906 & 0.2476508417 \\
\hline $\mathrm{F}$ & 0.7132659571 & 0.2626801361 & 0.2767133233 \\
\hline B & 0.6102911846 & 0.2503476902 & 0.8006719347 \\
\hline B & 0.3805584902 & 0.7526017764 & 0.1887538155 \\
\hline B & 0.3897088154 & 0.7503477192 & 0.6993280653 \\
\hline B & 0.6194415098 & 0.2526017614 & 0.3112461695 \\
\hline \multicolumn{4}{|l|}{ 6-LS } \\
\hline \multicolumn{4}{|c|}{ CELL_PARAMETERS (angstrom) } \\
\hline 11.033504933 & 0.000000000 & -0.366556482 & \\
\hline 0.000000000 & 16.858754857 & 0.000000000 & \\
\hline-8.608474119 & 0.000000000 & 13.467948228 & \\
\hline \multicolumn{4}{|c|}{ ATOMIC_POSITIONS (angstrom) } \\
\hline Fe2 $\quad-1$ & -1.5351125052 & 2.6066296099 & 5.2220641736 \\
\hline $\mathrm{Fe} 2$ & 3.9601427338 & 14.2521247446 & 7.8793279849 \\
\hline $\mathrm{Fe} 2$ & 8.2643805790 & 11.0360065357 & 1.1453536489 \\
\hline $\mathrm{Fe} 2$ & -5.8393489796 & 5.8227478189 & 11.9560378755 \\
\hline $\mathrm{N}$ & -0.3807049539 & 1.1347032747 & 4.4770581927 \\
\hline $\mathrm{N}$ & -1.4537595234 & 3.7514291655 & 3.6519000368 \\
\hline $\mathrm{N}$ & 0.3181820433 & 3.4481165086 & 5.6673930349 \\
\hline $\mathrm{N}$ & -2.2297175000 & 4.1854460507 & 6.2492790112 \\
\hline $\mathrm{N}$ & 2.8057360965 & 15.7240513308 & 8.6243335423 \\
\hline $\mathrm{N}$ & 3.8787901727 & 13.1073256911 & 9.4494917156 \\
\hline $\mathrm{N}$ & 2.1068490992 & 13.4106380970 & 7.4339987001 \\
\hline $\mathrm{N}$ & 4.6547484784 & 12.6733093078 & 6.8521127303 \\
\hline $\mathrm{N}$ & 7.1099730278 & 9.5640809535 & 1.8903596297 \\
\hline $\mathrm{N}$ & 8.1830273607 & 12.1808065943 & 2.7155174006 \\
\hline $\mathrm{N}$ & 6.4110861584 & 11.8774941884 & 0.7000245858 \\
\hline $\mathrm{N}$ & 8.9589854579 & 12.6148239815 & 0.1181387415 \\
\hline $\mathrm{N}$ & -4.6849423423 & 7.2946739031 & 11.2110323180 \\
\hline $\mathrm{N}$ & -5.7579970758 & 4.6779482633 & 10.3858741675 \\
\hline $\mathrm{N}$ & -3.9860553450 & 4.9812611712 & 12.4013671602 \\
\hline $\mathrm{N}$ & -6.5339547242 & 4.2439311260 & 12.9832531309 \\
\hline C & -0.3564358490 & 4.5440731976 & 3.5248272175 \\
\hline C & -1.5766128102 & 6.0384935664 & 7.6322324139 \\
\hline C & 0.7663609947 & 4.2507384392 & 4.4800838186 \\
\hline C & -0.2648907284 & 5.5080258216 & 2.5235847505 \\
\hline C & 8.5510452299 & 3.8984802681 & 2.4161545702 \\
\hline C & 1.2379908259 & 2.2919820088 & 5.8814099232 \\
\hline C & -1.2686757449 & 4.8852403005 & 6.9146783251 \\
\hline C & 0.9136820700 & 1.1886529377 & 4.9026184755 \\
\hline C & 8.5933511073 & 4.8305909210 & 1.3895366308 \\
\hline C & -3.5041533951 & 4.6310694002 & 6.2730614659 \\
\hline
\end{tabular}




\begin{tabular}{|c|c|c|c|}
\hline C & -0.7589520525 & 0.1183874440 & 3.6702805413 \\
\hline C & 0.1244746866 & 15.9799522278 & 3.2646323719 \\
\hline C & 0.1127319568 & 4.2754827016 & 6.8932324471 \\
\hline C & 1.4553806306 & 16.0446031674 & 3.6842141598 \\
\hline C & 9.7131957828 & 5.6515590548 & 1.2569638360 \\
\hline C & 1.8528054846 & 0.2410217850 & 4.5078819067 \\
\hline C & -3.8766159947 & 5.7743027305 & 6.9745845886 \\
\hline C & -2.8986030766 & 6.4867455944 & 7.6688421028 \\
\hline C & -3.4903315047 & 16.6015818953 & 7.9091166811 \\
\hline C & 5.9153624831 & 0.2571732436 & 5.1922750604 \\
\hline C & 7.1579893620 & 0.8882886679 & 5.3273013246 \\
\hline C & -4.7329583836 & 15.9704658127 & 7.7740904160 \\
\hline C & -3.0216094564 & 0.6319146354 & 6.9303380801 \\
\hline C & 5.4466405265 & 16.2268574927 & 6.1710532644 \\
\hline C & 2.7814667348 & 12.3146982400 & 9.5765649191 \\
\hline C & 4.0016436236 & 10.8202617922 & 5.4691593331 \\
\hline C & 1.6586698187 & 12.6080164174 & 8.6213079274 \\
\hline C & 2.6899212127 & 11.3507290350 & 10.5778070074 \\
\hline C & -6.1260144156 & 12.9602745884 & 10.6852371768 \\
\hline C & 1.1870403166 & 14.5667725967 & 7.2199818119 \\
\hline C & 3.6937066307 & 11.9735150580 & 6.1867138125 \\
\hline C & 1.5113488158 & 15.6701015419 & 8.1987736620 \\
\hline C & -6.1683206321 & 12.0281634336 & 11.7118555204 \\
\hline C & 5.9291839528 & 12.2276859593 & 6.8283306826 \\
\hline C & 3.1839827017 & 16.7403672402 & 9.4311112112 \\
\hline C & 2.3005557986 & 0.8788021893 & 9.8367593860 \\
\hline C & 2.3122991142 & 12.5832721549 & 6.2081588984 \\
\hline C & 0.9696499271 & 0.8141517517 & 9.4171779886 \\
\hline C & -7.2881651987 & 11.2071952997 & 11.8444281151 \\
\hline C & 0.5722259146 & 16.6177163979 & 8.5935094278 \\
\hline C & 6.3016468090 & 11.0844526280 & 6.1268071584 \\
\hline C & 5.3236336343 & 10.3720092622 & 5.4325500446 \\
\hline C & 7.0857033935 & 12.9734511284 & 2.8425904252 \\
\hline C & -0.3025934356 & 14.4678704932 & 12.2031334473 \\
\hline C & 5.9629070067 & 12.6801329509 & 1.8873336134 \\
\hline C & 6.9941582729 & 13.9374032503 & 3.8438328931 \\
\hline C & -1.8217773564 & 12.3278576959 & 3.9512630625 \\
\hline C & 5.4912774722 & 10.7213596886 & 0.4860075471 \\
\hline C & -0.6105308301 & 13.3146172273 & 12.9206875471 \\
\hline C & 5.8155861326 & 9.6180307424 & 1.4647991462 \\
\hline C & -1.8640838909 & 13.2599678467 & 4.9778810237 \\
\hline C & 10.2334212372 & 13.0604473310 & 0.0943562170 \\
\hline C & 7.4882198898 & 8.5477650452 & 2.6971368962 \\
\hline C & 6.6047935160 & 7.5505753020 & 3.1027852499 \\
\hline C & -1.9919382026 & 12.7048601294 & 12.9421334132 \\
\hline C & 5.2738872429 & 7.6152257396 & 2.6832034728 \\
\hline C & -2.9839286953 & 14.0809369856 & 5.1104540192 \\
\hline C & 4.8764621887 & 8.6703988045 & 1.8595355361 \\
\hline C & 1.9974097488 & 14.2036806613 & 12.8607812727 \\
\hline C & 1.0193968308 & 14.9161230232 & 12.1665237575 \\
\hline C & 1.6111254229 & 8.1722039645 & 11.9262491748 \\
\hline C & 0.8139061126 & 8.6865503891 & 1.1751424500 \\
\hline C & -0.4287208952 & 9.3176664727 & 1.0401163857 \\
\hline C & 2.8537523029 & 7.5410888859 & 12.0612754388 \\
\hline C & 1.1424032106 & 9.0612918747 & 12.9050277802 \\
\hline C & 1.2826274749 & 7.7974629819 & 0.1963641675 \\
\hline C & -4.6606729806 & 3.8853042312 & 10.2588009412 \\
\hline C & 2.7276241860 & 2.3908841124 & 0.8982584000 \\
\hline
\end{tabular}




\begin{tabular}{|c|c|c|c|}
\hline C & -3.5378760645 & 4.1786389886 & 11.2140579329 \\
\hline C & -4.5691274585 & 2.9213513552 & 9.2575588539 \\
\hline C & 4.2468081697 & 4.5308971607 & 9.1501286845 \\
\hline C & -3.0662465624 & 6.1373956710 & 12.6153840484 \\
\hline C & 3.0355611465 & 3.5441371273 & 0.1807039708 \\
\hline C & -3.3905550616 & 7.2407246161 & 11.6365921993 \\
\hline C & 4.2891137905 & 3.5987867588 & 8.1235111456 \\
\hline C & -7.8083895404 & 3.7983080286 & 13.0070351568 \\
\hline C & -5.0631889476 & 8.3109898124 & 10.4042546491 \\
\hline C & -4.1797620445 & 9.3081800565 & 9.9986064752 \\
\hline C & 4.4169688232 & 4.1538949782 & 0.1592586339 \\
\hline C & -2.8488566853 & 9.2435291169 & 10.4181886756 \\
\hline C & 5.4089588515 & 2.7778181219 & 7.9909377496 \\
\hline C & -2.4514321604 & 8.1883555491 & 11.2418564334 \\
\hline C & 0.4276209441 & 2.6550744462 & 0.2406106620 \\
\hline C & 1.4056335171 & 1.9426318334 & 0.9348677093 \\
\hline $\mathrm{H}$ & -0.7918144579 & 6.5552263327 & 8.1852543415 \\
\hline $\mathrm{H}$ & 1.2788307602 & 5.1689553410 & 4.8023283273 \\
\hline $\mathrm{H}$ & 1.5248091922 & 3.6658292597 & 3.9400125970 \\
\hline $\mathrm{H}$ & 0.6361995270 & 6.1173145568 & 2.4425174877 \\
\hline $\mathrm{H}$ & 7.6860860320 & 3.2597609380 & 2.5962225089 \\
\hline $\mathrm{H}$ & 2.2950094360 & 2.5862814266 & 5.7846373460 \\
\hline $\mathrm{H}$ & 1.0639231330 & 1.9142197693 & 6.9002120009 \\
\hline $\mathrm{H}$ & 7.7585896817 & 4.8982829149 & 0.6966075177 \\
\hline $\mathrm{H}$ & 6.8075475434 & 4.0511735464 & 5.3320361285 \\
\hline $\mathrm{H}$ & -1.8091394682 & 0.1252714079 & 3.3735017374 \\
\hline $\mathrm{H}$ & -0.2245142096 & 15.1594518968 & 2.6374949139 \\
\hline $\mathrm{H}$ & 0.8946652676 & 5.0457868800 & 6.9969146764 \\
\hline $\mathrm{H}$ & 0.1871817796 & 3.6136337459 & 7.7690053145 \\
\hline $\mathrm{H}$ & 2.1742196336 & 15.2786801443 & 3.3900084460 \\
\hline $\mathrm{H}$ & 9.7644609976 & 6.3879983598 & 0.4543109118 \\
\hline $\mathrm{H}$ & 2.8794040439 & 0.3083143332 & 4.8667282207 \\
\hline $\mathrm{H}$ & 6.1092255940 & 6.0762217483 & 6.5981663383 \\
\hline $\mathrm{H}$ & -3.1571830318 & 7.3825040621 & 8.2356798571 \\
\hline $\mathrm{H}$ & -2.8820506312 & 16.3850654272 & 8.7886200429 \\
\hline $\mathrm{H}$ & 5.3070810249 & 0.4736897439 & 4.3127721100 \\
\hline $\mathrm{H}$ & 3.2168452724 & 10.3035285239 & 4.9161374045 \\
\hline $\mathrm{H}$ & 1.1461997976 & 11.6897995156 & 8.2990638202 \\
\hline $\mathrm{H}$ & 0.9002219503 & 13.1929258479 & 9.1613791391 \\
\hline $\mathrm{H}$ & 1.7888317442 & 10.7414397978 & 10.6588740476 \\
\hline $\mathrm{H}$ & -5.2610554179 & 13.5989944216 & 10.5051690482 \\
\hline $\mathrm{H}$ & 0.1300216351 & 14.2724734299 & 7.3167539995 \\
\hline $\mathrm{H}$ & 1.3611074247 & 14.9445350872 & 6.2011801476 \\
\hline $\mathrm{H}$ & -5.3335586107 & 11.9604719416 & 12.4047838278 \\
\hline $\mathrm{H}$ & -4.3825165559 & 12.8075818121 & 7.7693552198 \\
\hline $\mathrm{H}$ & 4.2341701990 & 16.7334830091 & 9.7278900123 \\
\hline $\mathrm{H}$ & 2.6495450230 & 1.6993027087 & 10.4638968330 \\
\hline $\mathrm{H}$ & 1.5303655458 & 11.8129679766 & 6.1044770706 \\
\hline $\mathrm{H}$ & 2.2378487771 & 13.2451208596 & 5.3323868330 \\
\hline $\mathrm{H}$ & 0.2508112532 & 1.5800748373 & 9.7113836915 \\
\hline $\mathrm{H}$ & -7.3394298868 & 10.4707559948 & 12.6470804324 \\
\hline $\mathrm{H}$ & -0.4543732295 & 16.5504409316 & 8.2346635253 \\
\hline $\mathrm{H}$ & -3.6841946165 & 10.7825336102 & 6.5032254023 \\
\hline $\mathrm{H}$ & 5.5822134244 & 9.4762507945 & 4.8657122958 \\
\hline $\mathrm{H}$ & -1.0873917878 & 14.9846037615 & 11.6501115188 \\
\hline $\mathrm{H}$ & 5.4504369856 & 13.5983327697 & 1.5650895051 \\
\hline $\mathrm{H}$ & 5.2044584811 & 12.0952064375 & 2.4274048459 \\
\hline $\mathrm{H}$ & 6.0930681462 & 14.5466924876 & 3.9248999551 \\
\hline
\end{tabular}




\begin{tabular}{|c|c|c|c|}
\hline $\mathrm{H}$ & -0.9568180296 & 11.6891378638 & 3.7711949230 \\
\hline $\mathrm{H}$ & 4.4342588306 & 11.0156588554 & 0.5827801754 \\
\hline $\mathrm{H}$ & -2.9431297069 & 10.3435971981 & 12.9351538713 \\
\hline $\mathrm{H}$ & -1.0293224654 & 13.3276603437 & 5.6708101368 \\
\hline $\mathrm{H}$ & -0.0782802641 & 12.4805514772 & 1.0353814257 \\
\hline $\mathrm{H}$ & 8.5384072591 & 8.5546492762 & 2.9939158980 \\
\hline $\mathrm{H}$ & 6.9537820832 & 6.7300749700 & 3.7299227187 \\
\hline $\mathrm{H}$ & -2.7738715134 & 13.4751643087 & 12.8384511849 \\
\hline $\mathrm{H}$ & -2.0663880254 & 12.0430114257 & 12.0663605458 \\
\hline $\mathrm{H}$ & 4.5550484412 & 6.8493027165 & 2.9774093764 \\
\hline $\mathrm{H}$ & -3.0351931240 & 14.8173752855 & 5.9131067209 \\
\hline $\mathrm{H}$ & 3.8498634367 & 8.7376913538 & 1.5006895223 \\
\hline $\mathrm{H}$ & -7.9884311825 & 14.5055986751 & 13.2371995002 \\
\hline $\mathrm{H}$ & 1.2779766219 & 15.8118814899 & 11.5996860086 \\
\hline $\mathrm{H}$ & 1.0028442213 & 7.9556874965 & 11.0467458229 \\
\hline $\mathrm{H}$ & 1.4221860626 & 8.9030668581 & 2.0546457452 \\
\hline $\mathrm{H}$ & 3.5124226661 & 1.8741509701 & 1.4512801278 \\
\hline $\mathrm{H}$ & -3.0254053851 & 3.2604223388 & 11.5363020183 \\
\hline $\mathrm{H}$ & -2.7794281961 & 4.7635484191 & 10.6739867222 \\
\hline $\mathrm{H}$ & -3.6680379891 & 2.3120626210 & 9.1764918137 \\
\hline $\mathrm{H}$ & 3.3818495012 & 5.1696164908 & 9.3301968012 \\
\hline $\mathrm{H}$ & -2.0092285381 & 5.8430960012 & 12.5186118836 \\
\hline $\mathrm{H}$ & 5.3681611060 & 6.5151576584 & 0.1662374633 \\
\hline $\mathrm{H}$ & 3.4543528782 & 3.5310945128 & 7.4305812305 \\
\hline $\mathrm{H}$ & 2.5033102275 & 4.3782038814 & 12.0660106433 \\
\hline $\mathrm{H}$ & -6.1133764448 & 8.3041060834 & 10.1074758489 \\
\hline $\mathrm{H}$ & -4.5287512688 & 10.1286803885 & 9.3714690273 \\
\hline $\mathrm{H}$ & 5.1989023592 & 3.3835907998 & 0.2629405119 \\
\hline $\mathrm{H}$ & 4.4914183104 & 4.8157434308 & 1.0350310213 \\
\hline $\mathrm{H}$ & -2.1300174991 & 10.0094521400 & 10.1239821698 \\
\hline $\mathrm{H}$ & 5.4602236817 & 2.0413791940 & 7.1882854276 \\
\hline $\mathrm{H}$ & -1.4248323581 & 8.1210630008 & 11.6007023132 \\
\hline $\mathrm{H}$ & 10.4134625661 & 2.3531556796 & -0.1358076376 \\
\hline $\mathrm{H}$ & 1.1470543843 & 1.0468733032 & 1.5017054373 \\
\hline 0 & -1.9009372336 & 1.2445410266 & 7.0256964276 \\
\hline 0 & 4.3259684687 & 15.6142140820 & 6.0756949124 \\
\hline 0 & 7.6219023943 & 1.6885245953 & 4.4371580841 \\
\hline 0 & -5.1968721150 & 15.1702306383 & 8.6642340727 \\
\hline 0 & 0.0217398754 & 9.6739182034 & 12.8096557805 \\
\hline 0 & 2.4033001585 & 7.1848361512 & 0.2917225478 \\
\hline 0 & -0.8926346495 & 10.1179016471 & 1.9302597493 \\
\hline 0 & 3.3176655918 & 6.7408527075 & 11.1711317970 \\
\hline $\mathrm{F}$ & -0.5030546959 & 5.5468436063 & 10.6772604177 \\
\hline $\mathrm{F}$ & -0.0806941057 & 3.6361070845 & 11.9334996322 \\
\hline $\mathrm{F}$ & -0.9680057290 & 3.4416576396 & 9.7947479084 \\
\hline $\mathrm{F}$ & 1.2326814382 & 4.1154323553 & 10.0661381627 \\
\hline $\mathrm{F}$ & 2.9280852526 & 11.3119112502 & 2.4241317308 \\
\hline $\mathrm{F}$ & 2.5057362916 & 13.2226482750 & 1.1678914423 \\
\hline $\mathrm{F}$ & 3.3930365423 & 13.4170969660 & 3.3066438386 \\
\hline$F$ & 1.1923491749 & 12.7433225013 & 3.0352533945 \\
\hline $\mathrm{F}$ & -1.3761515500 & 13.9762210342 & 9.1581054436 \\
\hline $\mathrm{F}$ & -1.7985009604 & 12.0654840103 & 7.9018658567 \\
\hline $\mathrm{F}$ & -0.9112005169 & 11.8710353194 & 10.0406179530 \\
\hline $\mathrm{F}$ & -3.1118880131 & 12.5448097830 & 9.7692277095 \\
\hline $\mathrm{F}$ & 3.8011826210 & 2.8825335714 & 3.9432859019 \\
\hline$F$ & 4.2235426978 & 4.7932703443 & 5.1995259194 \\
\hline $\mathrm{F}$ & 3.3362313312 & 4.9877195372 & 3.0607737940 \\
\hline $\mathrm{F}$ & 5.5369182417 & 4.3139450735 & 3.3321644498 \\
\hline
\end{tabular}




\begin{tabular}{|c|c|c|c|}
\hline B & -0.0726163186 & 4.1897998402 & 10.6236296914 \\
\hline B & 2.4976466750 & 12.6689550163 & 2.4777622663 \\
\hline B & -1.8065902563 & 12.6191772680 & 9.2117361799 \\
\hline B & 4.2316207416 & 4.2395778395 & 3.8896555780 \\
\hline \multirow{2}{*}{\multicolumn{4}{|c|}{$\begin{array}{l}\text { 3-reg HS } \\
\text { ATOMIC_POSITIONS (angstrom) }\end{array}$}} \\
\hline & & & \\
\hline Fe1 & 6.5487994166 & 4.2705223379 & 4.2850579582 \\
\hline 0 & 7.3825514936 & 6.1956685822 & 4.4342296286 \\
\hline 0 & 6.9080745562 & 4.3532857888 & 6.2699087823 \\
\hline $\mathrm{N}$ & 4.5469666507 & 5.1119668847 & 4.0534361924 \\
\hline $\mathrm{N}$ & 6.2107991020 & 4.2320515915 & 2.0331508534 \\
\hline $\mathrm{N}$ & 5.7229101230 & 2.3152884608 & 4.0660500443 \\
\hline $\mathrm{N}$ & 8.4363798365 & 3.4970137077 & 3.5003677224 \\
\hline C & 3.6267356784 & 5.2001805277 & 5.0344808307 \\
\hline H & 3.9711063005 & 4.9294637135 & 6.0358212341 \\
\hline C & 2.3147750573 & 5.6070797559 & 4.7959733221 \\
\hline $\mathrm{H}$ & 1.6038298360 & 5.6645764452 & 5.6207125018 \\
\hline C & 1.9382199771 & 5.9244049043 & 3.4887753902 \\
\hline $\mathrm{H}$ & 0.9166814884 & 6.2353552331 & 3.2638805526 \\
\hline C & 2.8892601937 & 5.8309439572 & 2.4687318104 \\
\hline $\mathrm{H}$ & 2.6295215352 & 6.0685197710 & 1.4354098105 \\
\hline C & 4.1871279657 & 5.4301411567 & 2.7881133539 \\
\hline C & 5.3028633328 & 5.3748908613 & 1.7672235988 \\
\hline $\mathrm{H}$ & 4.8947366754 & 5.3516257900 & 0.7406197807 \\
\hline $\mathrm{H}$ & 5.9008254773 & 6.2957009869 & 1.8685563849 \\
\hline C & 5.6008490027 & 2.9232798714 & 1.6689163791 \\
\hline $\mathrm{H}$ & 6.2176531510 & 2.4367457424 & 0.8946568862 \\
\hline $\mathrm{H}$ & 4.6177393839 & 3.1043732780 & 1.2029893037 \\
\hline C & 4.8805700438 & 0.6656545793 & 2.5440373407 \\
\hline $\mathrm{H}$ & 4.6304546394 & 0.3838792197 & 1.5189054050 \\
\hline C & 4.6843454492 & -0.2287838917 & 3.5960774294 \\
\hline $\mathrm{H}$ & 4.2771983208 & -1.2230872912 & 3.4054915213 \\
\hline C & 5.0177716992 & 0.1692020993 & 4.8947107536 \\
\hline H & 4.8810022624 & -0.4976732800 & 5.7462938049 \\
\hline C & 5.5343409009 & 1.4461169109 & 5.0862986136 \\
\hline $\mathrm{H}$ & 5.8162349897 & 1.8138853942 & 6.0760046015 \\
\hline C & 5.4019336930 & 1.9356569262 & 2.8117203318 \\
\hline C & 7.5572919123 & 4.4213728949 & 1.4410299683 \\
\hline $\mathrm{H}$ & 7.5667458949 & 4.2101209497 & 0.3564273034 \\
\hline $\mathrm{H}$ & 7.8251564591 & 5.4823583527 & 1.5767426139 \\
\hline C & 8.5955012124 & 3.5873841897 & 2.1597977411 \\
\hline C & 9.6784202901 & 3.0004633732 & 1.5040136610 \\
\hline $\mathrm{H}$ & 9.7707920605 & 3.0835242655 & 0.4194677699 \\
\hline C & 10.6361755129 & 2.3151256645 & 2.2567805713 \\
\hline $\mathrm{H}$ & 11.4923764220 & 1.8484928089 & 1.7668983924 \\
\hline C & 10.4759393479 & 2.2326859772 & 3.6420008502 \\
\hline $\mathrm{H}$ & 11.1986763564 & 1.7062000959 & 4.2660440087 \\
\hline C & 9.3595415660 & 2.8321585492 & 4.2233720736 \\
\hline $\mathrm{H}$ & 9.1790397797 & 2.7887775799 & 5.3004612349 \\
\hline C & 7.7346334573 & 6.5030096689 & 5.6250250810 \\
\hline C & 7.4668672500 & 5.4498530329 & 6.6890308906 \\
\hline C & 8.3372815487 & 7.7285984476 & 5.9763509652 \\
\hline H & 8.5269954065 & 8.4787593176 & 5.2074084837 \\
\hline 0 & 8.7681211015 & 7.2242675966 & 9.5557254814 \\
\hline 0 & 9.2451963385 & 9.0655417396 & 7.7192111168 \\
\hline C & 8.4175348419 & 6.9161465524 & 8.3645243379 \\
\hline C & 8.6861192784 & 7.9689062055 & 7.3003476115 \\
\hline C & 7.8158033931 & 5.6902148786 & 8.0131430534 \\
\hline
\end{tabular}




\begin{tabular}{|c|c|c|c|}
\hline $\mathrm{H}$ & 7.6261769410 & 4.9400310714 & 8.7821096080 \\
\hline Fe1 & 9.6039290707 & 9.1488576687 & 9.7035135402 \\
\hline $\mathrm{N}$ & 11.6041602023 & 8.3021529035 & 9.9364157473 \\
\hline $\mathrm{N}$ & 9.9387202208 & 9.1839266318 & 11.9554367130 \\
\hline $\mathrm{N}$ & 10.4362946405 & 11.1007851501 & 9.9248130845 \\
\hline $\mathrm{N}$ & 7.7174408169 & 9.9267792339 & 10.4865013620 \\
\hline C & 12.5254986064 & 8.2110948930 & 8.9566689383 \\
\hline $\mathrm{H}$ & 12.1837137844 & 8.4838190083 & 7.9549803233 \\
\hline C & 13.8354338530 & 7.7986285711 & 9.1967343509 \\
\hline $\mathrm{H}$ & 14.5472239754 & 7.7383127435 & 8.3729003066 \\
\hline C & 14.2089588469 & 7.4792595352 & 10.5043138244 \\
\hline $\mathrm{H}$ & 15.2288923564 & 7.1639675607 & 10.7304545784 \\
\hline C & 13.2568881747 & 7.5759908698 & 11.5230750165 \\
\hline $\mathrm{H}$ & 13.5142577983 & 7.3368300654 & 12.5566420550 \\
\hline C & 11.9609393916 & 7.9816431388 & 11.2020237948 \\
\hline C & 10.8437586698 & 8.0387612356 & 12.2212562675 \\
\hline $\mathrm{H}$ & 11.2504429763 & 8.0599884609 & 13.2484767755 \\
\hline $\mathrm{H}$ & 10.2438844305 & 7.1194101439 & 12.1179645771 \\
\hline C & 10.5511286578 & 10.4909081786 & 12.3219377094 \\
\hline $\mathrm{H}$ & 9.9324036854 & 10.9793571657 & 13.0934368380 \\
\hline $\mathrm{H}$ & 11.5317403068 & 10.3071038414 & 12.7920724812 \\
\hline C & 11.2848986625 & 12.7450267582 & 11.4491800074 \\
\hline $\mathrm{H}$ & 11.5351317896 & 13.0246768801 & 12.4748698755 \\
\hline C & 11.4869201076 & 13.6392705654 & 10.3980915117 \\
\hline $\mathrm{H}$ & 11.8985937539 & 14.6314475347 & 10.5900231356 \\
\hline C & 11.1534812590 & 13.2438498260 & 9.0986689323 \\
\hline $\mathrm{H}$ & 11.2949196739 & 13.9105806726 & 8.2477400260 \\
\hline C & 10.6308832550 & 11.9696452802 & 8.9054171809 \\
\hline $\mathrm{H}$ & 10.3488099769 & 11.6039364001 & 7.9149842177 \\
\hline C & 10.7574815112 & 11.4778916889 & 11.1798720495 \\
\hline C & 8.5910374991 & 8.9969725502 & 12.5457377231 \\
\hline $\mathrm{H}$ & 8.5808078406 & 9.2070607083 & 13.6305578877 \\
\hline $\mathrm{H}$ & 8.3209271575 & 7.9367445956 & 12.4085251500 \\
\hline C & 7.5556879221 & 9.8342636097 & 11.8266200966 \\
\hline C & 6.4728124094 & 10.4223250517 & 12.4815071970 \\
\hline $\mathrm{H}$ & 6.3782209045 & 10.3375128965 & 13.5657278242 \\
\hline C & 5.5179701069 & 11.1112199352 & 11.7282835949 \\
\hline $\mathrm{H}$ & 4.6618766335 & 11.5788159763 & 12.2174457836 \\
\hline C & 5.6809744053 & 11.1960173099 & 10.3435243721 \\
\hline $\mathrm{H}$ & 4.9606226528 & 11.7253537740 & 9.7191434206 \\
\hline C & 6.7971234748 & 10.5951890995 & 9.7630983782 \\
\hline $\mathrm{H}$ & 6.9797279352 & 10.6401896595 & 8.6864314102 \\
\hline \multicolumn{4}{|c|}{ 3-reg -iS } \\
\hline \multicolumn{4}{|c|}{ ATOMIC_POSITIONS (angstrom) } \\
\hline Fe1 & 6.6294321210 & 4.3462393102 & 4.4486894090 \\
\hline 0 & 7.4557403452 & 6.2643217895 & 4.5960118696 \\
\hline 0 & 6.9821809740 & 4.4270479076 & 6.4389277255 \\
\hline $\mathrm{N}$ & 4.6237787157 & 5.1925541250 & 4.2288884388 \\
\hline $\mathrm{N}$ & 6.3136625340 & 4.3614993117 & 2.2023443839 \\
\hline $\mathrm{N}$ & 5.7913568994 & 2.4025216990 & 4.1870729934 \\
\hline $\mathrm{N}$ & 8.5280089139 & 3.5869506658 & 3.6658680830 \\
\hline C & 3.6926418112 & 5.2540784965 & 5.2014342641 \\
\hline $\mathrm{H}$ & 4.0293316868 & 4.9700333402 & 6.2017038970 \\
\hline C & 2.3788977040 & 5.6517121828 & 4.9565019104 \\
\hline $\mathrm{H}$ & 1.6586792901 & 5.6867562148 & 5.7744344361 \\
\hline C & 2.0131350869 & 5.9900986385 & 3.6516008688 \\
\hline $\mathrm{H}$ & 0.9909695627 & 6.2952354500 & 3.4215106499 \\
\hline C & 2.9759287553 & 5.9257497945 & 2.6403460557 \\
\hline
\end{tabular}




\begin{tabular}{|c|c|c|c|}
\hline $\mathrm{H}$ & 2.7250129227 & 6.1815662859 & 1.6091969089 \\
\hline C & 4.2739969108 & 5.5304049762 & 2.9657480432 \\
\hline C & 5.4004862860 & 5.5048575838 & 1.9557009120 \\
\hline $\mathrm{H}$ & 5.0034361383 & 5.5038899794 & 0.9245869585 \\
\hline $\mathrm{H}$ & 5.9924926443 & 6.4257381814 & 2.0865486491 \\
\hline C & 5.7208692678 & 3.0564688284 & 1.7988894083 \\
\hline $\mathrm{H}$ & 6.3776573057 & 2.5762434629 & 1.0542948248 \\
\hline $\mathrm{H}$ & 4.7625357522 & 3.2387469093 & 1.2842291315 \\
\hline C & 4.9176688001 & 0.8083842982 & 2.6245473890 \\
\hline $\mathrm{H}$ & 4.6681419228 & 0.5548619935 & 1.5919400530 \\
\hline C & 4.6901870464 & -0.1027501422 & 3.6558589636 \\
\hline $\mathrm{H}$ & 4.2585828527 & -1.0817135458 & 3.4410954735 \\
\hline C & 5.0235035024 & 0.2586773969 & 4.9650685760 \\
\hline $\mathrm{H}$ & 4.8627732415 & -0.4221577050 & 5.8012465098 \\
\hline C & 5.5708273320 & 1.5177524368 & 5.1873783900 \\
\hline $\mathrm{H}$ & 5.8520535813 & 1.8570614958 & 6.1873972851 \\
\hline C & 5.4711609856 & 2.0578445953 & 2.9222672416 \\
\hline C & 7.6653961763 & 4.5768614207 & 1.6297702351 \\
\hline $\mathrm{H}$ & 7.6861639845 & 4.4029145925 & 0.5388033118 \\
\hline $\mathrm{H}$ & 7.9268638158 & 5.6333396621 & 1.8061873881 \\
\hline C & 8.6987115016 & 3.7214194934 & 2.3302598721 \\
\hline C & 9.7870623947 & 3.1554827011 & 1.6650697695 \\
\hline $\mathrm{H}$ & 9.8890748846 & 3.2741463418 & 0.5847143093 \\
\hline C & 10.7372957772 & 2.4439642972 & 2.4029930990 \\
\hline $\mathrm{H}$ & 11.5970215179 & 1.9922506179 & 1.9053111287 \\
\hline C & 10.5648961601 & 2.3157022375 & 3.7831775689 \\
\hline $\mathrm{H}$ & 11.2816097595 & 1.7678904175 & 4.3957121998 \\
\hline C & 9.4443076894 & 2.8978199028 & 4.3743637563 \\
\hline $\mathrm{H}$ & 9.2557896895 & 2.8204952899 & 5.4481766542 \\
\hline C & 7.8030545878 & 6.5780347127 & 5.7917499014 \\
\hline C & 7.5332158351 & 5.5262932410 & 6.8551982605 \\
\hline C & 8.3981079694 & 7.8006196526 & 6.1474196593 \\
\hline $\mathrm{H}$ & 8.5933476066 & 8.5557400271 & 5.3848237131 \\
\hline 0 & 8.8132239583 & 7.3179416939 & 9.7271005424 \\
\hline 0 & 9.2839055018 & 9.1216070565 & 7.9273387899 \\
\hline C & 8.4627681098 & 6.9922658547 & 8.5284422361 \\
\hline C & 8.7320954432 & 8.0330905152 & 7.4818217669 \\
\hline C & 7.8722010705 & 5.7653200950 & 8.1872045438 \\
\hline $\mathrm{H}$ & 7.6799453424 & 5.0127109336 & 8.9529612181 \\
\hline $\mathrm{Fe} 2$ & 9.5817833486 & 9.1263508021 & 9.8525112725 \\
\hline $\mathrm{N}$ & 11.3868869174 & 8.3799542727 & 9.7805009983 \\
\hline $\mathrm{N}$ & 9.8456932057 & 9.0555288174 & 11.8342017886 \\
\hline $\mathrm{N}$ & 10.3322374342 & 10.9005981709 & 9.9521266381 \\
\hline $\mathrm{N}$ & 7.8217737361 & 9.8416577021 & 10.3129152234 \\
\hline C & 12.2308477538 & 8.3727370813 & 8.7265414640 \\
\hline $\mathrm{H}$ & 11.8212513885 & 8.7655749730 & 7.7946529941 \\
\hline C & 13.5367233793 & 7.8951273822 & 8.8266361571 \\
\hline $\mathrm{H}$ & 14.1842399132 & 7.9083293599 & 7.9495012833 \\
\hline C & 13.9929473949 & 7.4183649761 & 10.0570913703 \\
\hline $\mathrm{H}$ & 15.0137337204 & 7.0497301845 & 10.1681973695 \\
\hline C & 13.1212752376 & 7.4273662198 & 11.1513117178 \\
\hline $\mathrm{H}$ & 13.4430168700 & 7.0661655013 & 12.1295318984 \\
\hline C & 11.8247416762 & 7.9044409196 & 10.9790042282 \\
\hline C & 10.7686548352 & 7.9009880027 & 12.0548741650 \\
\hline $\mathrm{H}$ & 11.2080936019 & 7.9054007075 & 13.0667761838 \\
\hline $\mathrm{H}$ & 10.1678471319 & 6.9848331243 & 11.9434608252 \\
\hline C & 10.4358026318 & 10.3524296703 & 12.3185410903 \\
\hline $\mathrm{H}$ & 9.7513555966 & 10.8109533219 & 13.0499248671 \\
\hline
\end{tabular}




\begin{tabular}{|c|c|c|c|}
\hline $\mathrm{H}$ & 11.3759139834 & 10.1495419891 & 12.8557462627 \\
\hline C & 11.2563313980 & 12.5812220984 & 11.4019076611 \\
\hline $\mathrm{H}$ & 11.5345974406 & 12.8902812230 & 12.4112402953 \\
\hline C & 11.4573849153 & 13.4301182072 & 10.3121564940 \\
\hline $\mathrm{H}$ & 11.8978412718 & 14.4177742039 & 10.4557095790 \\
\hline C & 11.0851331369 & 12.9913936052 & 9.0378310125 \\
\hline $\mathrm{H}$ & 11.2242374804 & 13.6211643826 & 8.1588231136 \\
\hline C & 10.5277755240 & 11.7251200426 & 8.8945203251 \\
\hline $\mathrm{H}$ & 10.2176111948 & 11.3237047649 & 7.9287647701 \\
\hline C & 10.6914981524 & 11.3232367444 & 11.1899863216 \\
\hline C & 8.4781056431 & 8.8228365403 & 12.3905576448 \\
\hline $\mathrm{H}$ & 8.4299938014 & 9.0169267276 & 13.4755357670 \\
\hline $\mathrm{H}$ & 8.2378746228 & 7.7621585463 & 12.2178237278 \\
\hline C & 7.4894024881 & 9.6617253553 & 11.6211446414 \\
\hline C & 6.3140032312 & 10.1792802337 & 12.1585193950 \\
\hline H & 6.0852938349 & 10.0275498881 & 13.2147043302 \\
\hline C & 5.4406763986 & 10.8878989232 & 11.3263388615 \\
\hline $\mathrm{H}$ & 4.5137046152 & 11.3026460701 & 11.7250378685 \\
\hline C & 5.7796237109 & 11.0611368377 & 9.9830484647 \\
\hline $\mathrm{H}$ & 5.1292559975 & 11.6098172917 & 9.3013171689 \\
\hline C & 6.9793957461 & 10.5294492439 & 9.5126268504 \\
\hline $\mathrm{H}$ & 7.3015139707 & 10.6393901775 & 8.4760112822 \\
\hline \multirow{2}{*}{\multicolumn{4}{|c|}{$\begin{array}{l}\text { 3-reg -LS } \\
\text { ATOMIC_POSITIONS (angstrom) }\end{array}$}} \\
\hline & & & \\
\hline $\mathrm{Fe} 2$ & 6.6325356659 & 4.3879415346 & 4.2884034280 \\
\hline 0 & 7.4028310379 & 6.1957845236 & 4.4221681978 \\
\hline 0 & 6.9199463418 & 4.3864863388 & 6.2142097806 \\
\hline $\mathrm{N}$ & 4.8273589384 & 5.1358663508 & 4.3526363524 \\
\hline $\mathrm{N}$ & 6.3825095844 & 4.4710636601 & 2.3058616278 \\
\hline$N$ & 5.8810361375 & 2.6148348972 & 4.1731205339 \\
\hline $\mathrm{N}$ & 8.3958610194 & 3.6757921376 & 3.8366256037 \\
\hline C & 3.9742882262 & 5.1352723093 & 5.3993369367 \\
\hline $\mathrm{H}$ & 4.3755931075 & 4.7362463740 & 6.3321760334 \\
\hline C & 2.6686451025 & 5.6118200749 & 5.2913972847 \\
\hline H & 2.0132618558 & 5.5906977497 & 6.1625374533 \\
\hline C & 2.2224824511 & 6.0970340288 & 4.0605611502 \\
\hline $\mathrm{H}$ & 1.2021555654 & 6.4650165010 & 3.9431726119 \\
\hline C & 3.1035907713 & 6.0967500976 & 2.9739355437 \\
\hline $\mathrm{H}$ & 2.7900834424 & 6.4644711197 & 1.9954810426 \\
\hline C & 4.3989394969 & 5.6192172669 & 3.1538892400 \\
\hline C & 5.4631510636 & 5.6289200324 & 2.0863654170 \\
\hline $\mathrm{H}$ & 5.0316120258 & 5.6323703209 & 1.0711119447 \\
\hline H & 6.0647177861 & 6.5430025437 & 2.2096171485 \\
\hline C & 5.7933600502 & 3.1783345079 & 1.8094423813 \\
\hline $\mathrm{H}$ & 6.4803028723 & 2.7244811579 & 1.0775230099 \\
\hline H & 4.8557683433 & 3.3864829397 & 1.2698635131 \\
\hline C & 4.9683743261 & 0.9430024269 & 2.7059077350 \\
\hline $\mathrm{H}$ & 4.6980699452 & 0.6401787178 & 1.6925396817 \\
\hline C & 4.7590984485 & 0.0872178477 & 3.7886877886 \\
\hline $\mathrm{H}$ & 4.3199791973 & -0.8996040606 & 3.6356142742 \\
\hline C & 5.1214020356 & 0.5180552386 & 5.0685087390 \\
\hline $\mathrm{H}$ & 4.9754999561 & -0.1170189722 & 5.9425864407 \\
\hline C & 5.6775637529 & 1.7834115567 & 5.2238328581 \\
\hline $\mathrm{H}$ & 5.9804971243 & 2.1780855443 & 6.1945786182 \\
\hline C & 5.5310533017 & 2.1998992255 & 2.9299363440 \\
\hline C & 7.7538820822 & 4.7062270034 & 1.7599566192 \\
\hline $\mathrm{H}$ & 7.8092117166 & 4.5164392235 & 0.6746083188 \\
\hline $\mathrm{H}$ & 7.9925354415 & 5.7662151924 & 1.9387609612 \\
\hline
\end{tabular}




\begin{tabular}{|c|c|c|c|}
\hline C & 8.7386161623 & 3.8657824941 & 2.5325905252 \\
\hline C & 9.9217402352 & 3.3582989423 & 2.0023364980 \\
\hline $\mathrm{H}$ & 10.1588715608 & 3.5182961880 & 0.9492588451 \\
\hline C & 10.7919913652 & 2.6498592057 & 2.8377316813 \\
\hline $\mathrm{H}$ & 11.7253462267 & 2.2438752574 & 2.4448477874 \\
\hline C & 10.4418922970 & 2.4653995813 & 4.1766080480 \\
\hline $\mathrm{H}$ & 11.0892445523 & 1.9160204639 & 4.8606252553 \\
\hline C & 9.2348075519 & 2.9868041177 & 4.6395442411 \\
\hline $\mathrm{H}$ & 8.9043501616 & 2.8673371779 & 5.6724314311 \\
\hline C & 7.7535718611 & 6.5148446268 & 5.6256818132 \\
\hline C & 7.4749266978 & 5.4680969970 & 6.6654873121 \\
\hline C & 8.3485149250 & 7.7315165080 & 5.9804538714 \\
\hline $\mathrm{H}$ & 8.5516514034 & 8.4928033170 & 5.2262142033 \\
\hline $\mathrm{O}$ & 8.7502829941 & 7.2199082729 & 9.5663561839 \\
\hline 0 & 9.2316756490 & 9.0300603300 & 7.7748848219 \\
\hline C & 8.3989751917 & 6.9012896715 & 8.3628539370 \\
\hline C & 8.6771605463 & 7.9483155748 & 7.3232148774 \\
\hline C & 7.8038566363 & 5.6847106673 & 8.0080738434 \\
\hline $\mathrm{H}$ & 7.6008644069 & 4.9231006719 & 8.7620314229 \\
\hline $\mathrm{Fe} 2$ & 9.5204259466 & 9.0276074653 & 9.7011323494 \\
\hline $\mathrm{N}$ & 11.3257822689 & 8.2807757804 & 9.6350915420 \\
\hline $\mathrm{N}$ & 9.7715098426 & 8.9434114446 & 11.6832356848 \\
\hline $\mathrm{N}$ & 10.2718892923 & 10.8010974106 & 9.8171417303 \\
\hline $\mathrm{N}$ & 7.7573232046 & 9.7401132835 & 10.1544966375 \\
\hline C & 12.1785002210 & 8.2825884575 & 8.5880899695 \\
\hline $\mathrm{H}$ & 11.7770325066 & 8.6829661961 & 7.6558862345 \\
\hline C & 13.4839908630 & 7.8053194681 & 8.6947659160 \\
\hline $\mathrm{H}$ & 14.1390659572 & 7.8273626233 & 7.8234177423 \\
\hline C & 13.9304983470 & 7.3180928617 & 9.9247329350 \\
\hline $\mathrm{H}$ & 14.9506092613 & 6.9491782688 & 10.0410722917 \\
\hline C & 13.0499573792 & 7.3176713839 & 11.0118084794 \\
\hline $\mathrm{H}$ & 13.3638605151 & 6.9486959779 & 11.9896654965 \\
\hline C & 11.7547370718 & 7.7960562634 & 10.8331060229 \\
\hline C & 10.6912660906 & 7.7856621124 & 11.9013715650 \\
\hline $\mathrm{H}$ & 11.1236763428 & 7.7819472888 & 12.9162424703 \\
\hline $\mathrm{H}$ & 10.0898128512 & 6.8714865504 & 11.7782954958 \\
\hline C & 10.3605336038 & 10.2359383713 & 12.1803543094 \\
\hline $\mathrm{H}$ & 9.6726299765 & 10.6900236261 & 12.9112518259 \\
\hline $\mathrm{H}$ & 11.2972974748 & 10.0276919825 & 12.7213384993 \\
\hline C & 11.1902113245 & 12.4696786316 & 11.2846915968 \\
\hline $\mathrm{H}$ & 11.4627084130 & 12.7709480360 & 12.2979810468 \\
\hline C & 11.4002036323 & 13.3260012973 & 10.2024562113 \\
\hline $\mathrm{H}$ & 11.8420206108 & 14.3115409058 & 10.3558104534 \\
\hline C & 11.0349939031 & 12.8971382875 & 8.9227146663 \\
\hline $\mathrm{H}$ & 11.1813256077 & 13.5327410595 & 8.0490757469 \\
\hline C & 10.4757415654 & 11.6331828946 & 8.7670192609 \\
\hline $\mathrm{H}$ & 10.1709692235 & 11.2400782583 & 7.7961814480 \\
\hline C & 10.6242558773 & 11.2143535922 & 11.0602397231 \\
\hline C & 8.4004119123 & 8.7078471615 & 12.2297830810 \\
\hline $\mathrm{H}$ & 8.3459005146 & 8.8964291910 & 13.3153772333 \\
\hline $\mathrm{H}$ & 8.1613779036 & 7.6481513706 & 12.0498130936 \\
\hline C & 7.4157221464 & 9.5498430561 & 11.4588072619 \\
\hline C & 6.2341876777 & 10.0592246445 & 11.9907907488 \\
\hline $\mathrm{H}$ & 5.9980713827 & 9.8992059082 & 13.0440969429 \\
\hline C & 5.3642768912 & 10.7699505673 & 11.1569448736 \\
\hline $\mathrm{H}$ & 4.4322154601 & 11.1775198520 & 11.5512212096 \\
\hline C & 5.7132585159 & 10.9547968582 & 9.8177812085 \\
\hline $\mathrm{H}$ & 5.0662764086 & 11.5061904333 & 9.1350274116 \\
\hline
\end{tabular}




\begin{tabular}{|c|c|c|c|}
\hline C & 6.9188127519 & 10.4313888083 & 9.3531210433 \\
\hline $\mathrm{H}$ & 7.2483846223 & 10.5512778935 & 8.3199703280 \\
\hline \multicolumn{4}{|c|}{ 5-dis -HS } \\
\hline \multicolumn{4}{|c|}{ ATOMIC_POSITIONS (angstrom) } \\
\hline Fe1 & 16.1734806903 & 6.1363016043 & 14.5134348442 \\
\hline $\mathrm{Fe} 1$ & 8.2196899595 & 6.2986762258 & 15.0580256182 \\
\hline 0 & 14.5224383907 & 7.5380672598 & 14.6221335217 \\
\hline 0 & 14.5363554778 & 4.9221494549 & 14.7080971650 \\
\hline 0 & 9.7872333278 & 7.5746187075 & 14.9263762832 \\
\hline 0 & 9.8203844352 & 4.9510528329 & 15.2027360110 \\
\hline $\mathrm{N}$ & 16.4826207769 & 7.0870468875 & 12.5671847020 \\
\hline $\mathrm{N}$ & 6.4381478490 & 4.8838905407 & 15.1667133015 \\
\hline $\mathrm{N}$ & 18.4345523922 & 6.8216360533 & 14.5020285019 \\
\hline $\mathrm{N}$ & 7.8108789474 & 6.1153849320 & 17.1846660916 \\
\hline $\mathrm{N}$ & 7.9338517422 & 5.6860186455 & 12.9701827625 \\
\hline $\mathrm{N}$ & 6.6250703351 & 7.6769134660 & 14.6497522619 \\
\hline $\mathrm{N}$ & 16.6200140736 & 6.8474477542 & 16.5058931044 \\
\hline $\mathrm{N}$ & 17.3427031226 & 4.2978980733 & 14.2962535635 \\
\hline C & 10.9662037911 & 5.4988275190 & 15.0515307146 \\
\hline C & 13.3740336986 & 6.9981239512 & 14.7420403422 \\
\hline C & 18.6798116362 & 4.3716628932 & 14.5053520915 \\
\hline C & 17.6469738582 & 7.7186391926 & 16.6444442291 \\
\hline C & 16.8193733543 & 3.1302168239 & 13.8640981770 \\
\hline $\mathrm{H}$ & 15.7359758647 & 3.1241913478 & 13.7324932781 \\
\hline C & 10.9601522193 & 7.0157255837 & 14.9130767350 \\
\hline C & 12.1799687806 & 4.7854932438 & 14.9938442859 \\
\hline $\mathrm{H}$ & 12.1737848233 & 3.6972136894 & 15.0683843610 \\
\hline C & 12.1564930767 & 7.7123871724 & 14.7842131795 \\
\hline $\mathrm{H}$ & 12.1625263198 & 8.8006365105 & 14.7077356854 \\
\hline C & 19.1855565099 & 5.6815505871 & 15.0671924523 \\
\hline $\mathrm{H}$ & 19.0200362203 & 5.6746195494 & 16.1567979922 \\
\hline $\mathrm{H}$ & 20.2748337002 & 5.7832227776 & 14.9064962425 \\
\hline C & 5.4006005162 & 7.1088221652 & 14.5470191459 \\
\hline C & 17.7035427683 & 7.5740869013 & 12.2507313280 \\
\hline C & 5.2225056910 & 5.7431597120 & 15.1834753204 \\
\hline $\mathrm{H}$ & 4.9549037451 & 5.9141957252 & 16.2395292097 \\
\hline $\mathrm{H}$ & 4.3682238925 & 5.2177800358 & 14.7217501695 \\
\hline C & 6.8051708073 & 8.9339380517 & 14.1896660822 \\
\hline $\mathrm{H}$ & 7.8174856365 & 9.3325983186 & 14.2917980782 \\
\hline C & 13.3730138446 & 5.4796910580 & 14.8171753841 \\
\hline C & 17.6015300224 & 2.0029650302 & 13.6153885581 \\
\hline $\mathrm{H}$ & 17.1313558461 & 1.0828729828 & 13.2672631320 \\
\hline C & 18.4381407302 & 8.0034062530 & 15.3853593303 \\
\hline $\mathrm{H}$ & 17.9555048451 & 8.8299075102 & 14.8341325330 \\
\hline $\mathrm{H}$ & 19.4630569987 & 8.3356915233 & 15.6393896574 \\
\hline C & 18.8547899782 & 7.1263600108 & 13.1229755116 \\
\hline $\mathrm{H}$ & 19.2547561648 & 6.1964006001 & 12.6839117776 \\
\hline $\mathrm{H}$ & 19.6761922470 & 7.8657692950 & 13.0942576712 \\
\hline C & 7.0077558268 & 5.0904784941 & 17.5551784106 \\
\hline C & 6.6026054134 & 4.1453505316 & 16.4432050513 \\
\hline $\mathrm{H}$ & 7.4158073962 & 3.4154629129 & 16.2961978994 \\
\hline $\mathrm{H}$ & 5.6942176921 & 3.5810045505 & 16.7223007742 \\
\hline C & 17.9104935666 & 8.3829344703 & 11.1301491838 \\
\hline $\mathrm{H}$ & 18.9065945719 & 8.7770333425 & 10.9202263360 \\
\hline C & 6.5671750135 & 4.4067931227 & 11.4636490728 \\
\hline $\mathrm{H}$ & 5.8029315987 & 3.6401389884 & 11.3226559602 \\
\hline C & 5.7754762111 & 9.6738394403 & 13.6117895738 \\
\hline $\mathrm{H}$ & 5.9649217987 & 10.6881078228 & 13.2594652442 \\
\hline
\end{tabular}




\begin{tabular}{|c|c|c|c|}
\hline C & 8.4742693978 & 6.3244730541 & 11.9128645603 \\
\hline $\mathrm{H}$ & 9.2200141019 & 7.0877821083 & 12.1494455306 \\
\hline C & 6.6165471732 & 4.9031070247 & 18.8814322785 \\
\hline $\mathrm{H}$ & 5.9571072519 & 4.0737869536 & 19.1441370044 \\
\hline C & 8.1073853016 & 6.0413778993 & 10.5983178570 \\
\hline $\mathrm{H}$ & 8.5729803734 & 6.5815279454 & 9.7734704502 \\
\hline C & 16.0980263561 & 7.1152549967 & 18.8370963530 \\
\hline $\mathrm{H}$ & 15.4652162502 & 6.8433801648 & 19.6822450748 \\
\hline C & 17.1556613742 & 8.0174151115 & 18.9814581106 \\
\hline H & 17.3701582318 & 8.4732219453 & 19.9494540626 \\
\hline C & 16.8342677130 & 8.6680469867 & 10.2894989947 \\
\hline $\mathrm{H}$ & 16.9731355570 & 9.2951646978 & 9.4071800596 \\
\hline C & 7.1328592928 & 5.0667199320 & 10.3698631237 \\
\hline $\mathrm{H}$ & 6.8138862581 & 4.8255629099 & 9.3545279106 \\
\hline C & 7.0802350117 & 5.7898097153 & 19.8570774755 \\
\hline H & 6.7865289480 & 5.6644741023 & 20.9005513903 \\
\hline C & 7.9159894139 & 6.8415320214 & 19.4737596076 \\
\hline $\mathrm{H}$ & 8.2956447272 & 7.5586214621 & 20.2020977908 \\
\hline C & 4.5153166680 & 9.0841769831 & 13.4884206814 \\
\hline $\mathrm{H}$ & 3.6888969767 & 9.6280825658 & 13.0280497034 \\
\hline C & 6.5083397041 & 4.0008904132 & 13.9797862534 \\
\hline H & 5.5405773271 & 3.5065795392 & 13.7788193113 \\
\hline $\mathrm{H}$ & 7.2407619849 & 3.2081722123 & 14.2055959235 \\
\hline C & 17.9434906384 & 8.3197996558 & 17.8688113013 \\
\hline $\mathrm{H}$ & 18.7847512153 & 9.0109328963 & 17.9470334114 \\
\hline C & 15.8622970147 & 6.5558860358 & 17.5838747545 \\
\hline $\mathrm{H}$ & 15.0482880322 & 5.8470245579 & 17.4154098564 \\
\hline C & 19.5261763043 & 3.2887048633 & 14.2667501916 \\
\hline H & 20.5996672952 & 3.3899374752 & 14.4369348046 \\
\hline C & 4.3277535377 & 7.7824194036 & 13.9611954542 \\
\hline $\mathrm{H}$ & 3.3552298788 & 7.2929811053 & 13.8806427186 \\
\hline C & 6.9970045545 & 4.7362726920 & 12.7502691409 \\
\hline C & 18.9803286925 & 2.0836230648 & 13.8155620826 \\
\hline $\mathrm{H}$ & 19.6236366987 & 1.2235580206 & 13.6222995343 \\
\hline C & 15.4432384584 & 7.3737918448 & 11.7535443282 \\
\hline $\mathrm{H}$ & 14.4750194777 & 6.9759037216 & 12.0602337025 \\
\hline C & 15.5778098834 & 8.1422475090 & 10.6009600738 \\
\hline $\mathrm{H}$ & 14.7089578148 & 8.3376852517 & 9.9722164218 \\
\hline C & 8.2545966231 & 6.9708408283 & 18.1279518908 \\
\hline $\mathrm{H}$ & 8.8979442889 & 7.7792912009 & 17.7719619306 \\
\hline \multirow{2}{*}{\multicolumn{4}{|c|}{$\begin{array}{l}\text { 5-dis -iS } \\
\text { ATOMIC_POSITIONS (angstrom) }\end{array}$}} \\
\hline & & & \\
\hline Fe1 & 8.6029583106 & -0.5376634419 & 4.1152514120 \\
\hline $\mathrm{Fe} 2$ & 0.7464982507 & -0.4956226416 & 4.5070417988 \\
\hline 0 & 6.9462921918 & 0.8381881142 & 4.2027222498 \\
\hline 0 & 2.1949191431 & 0.8028102895 & 4.3919730262 \\
\hline 0 & 6.9660370400 & -1.7744600768 & 4.3151721775 \\
\hline 0 & 2.2369929196 & -1.7692253439 & 4.6873870411 \\
\hline $\mathrm{N}$ & 10.8671237398 & 0.1439505573 & 4.1157751369 \\
\hline $\mathrm{N}$ & -0.6949930478 & 0.7641958919 & 4.2718083432 \\
\hline $\mathrm{N}$ & 9.0386052665 & 0.1753734588 & 6.1111007871 \\
\hline $\mathrm{N}$ & 0.6787885013 & -0.8847406225 & 2.5906335348 \\
\hline $\mathrm{N}$ & 8.9236491926 & 0.4281915616 & 2.1729547103 \\
\hline $\mathrm{N}$ & 0.5488580758 & -0.3965238496 & 6.4487611090 \\
\hline $\mathrm{N}$ & -0.6808194064 & -1.8910982352 & 4.6389895302 \\
\hline$N$ & 9.7691970267 & -2.3769850565 & 3.8915074830 \\
\hline C & 3.3980076008 & -1.2209404050 & 4.5616608792 \\
\hline C & 3.3809843665 & 0.2752560312 & 4.4095843116 \\
\hline
\end{tabular}




\begin{tabular}{|c|c|c|c|}
\hline C & 5.7932960726 & 0.2942270238 & 4.3009898983 \\
\hline C & -0.5416633763 & 2.0944953713 & 4.0637488200 \\
\hline $\mathrm{H}$ & 0.4888380009 & 2.4519834483 & 4.0801396548 \\
\hline C & 10.1469515952 & 0.9157150209 & 1.8668668659 \\
\hline C & -1.9452481828 & 0.2378596047 & 4.2657170814 \\
\hline C & 8.2707588261 & -0.1132965734 & 7.1825230683 \\
\hline $\mathrm{H}$ & 7.4474266878 & -0.8086496134 & 7.0039237884 \\
\hline C & 11.2933634297 & 0.4584581173 & 2.7404190190 \\
\hline $\mathrm{H}$ & 11.6935371188 & -0.4686619397 & 2.2956090682 \\
\hline $\mathrm{H}$ & 12.1163259960 & 1.1964098909 & 2.7210510550 \\
\hline C & 10.0762743512 & 1.0315907357 & 6.2616444404 \\
\hline C & 4.6073769098 & -1.9315130035 & 4.5505200928 \\
\hline $\mathrm{H}$ & 4.6113402646 & -3.0187915355 & 4.6378475027 \\
\hline C & 5.8004778172 & -1.2229605131 & 4.3928367482 \\
\hline C & 4.5721325648 & 0.9940365042 & 4.3058044752 \\
\hline $\mathrm{H}$ & 4.5627401723 & 2.0815379505 & 4.2205601535 \\
\hline C & 9.2448449328 & -3.5411843214 & 3.4509974649 \\
\hline $\mathrm{H}$ & 8.1615006193 & -3.5456082270 & 3.3199541594 \\
\hline C & 11.1063778467 & -2.3066225109 & 4.1019633907 \\
\hline C & 11.6141860679 & -1.0021691689 & 4.6737704276 \\
\hline $\mathrm{H}$ & 11.4475757700 & -1.0162896782 & 5.7632212199 \\
\hline $\mathrm{H}$ & 12.7039065195 & -0.9021485344 & 4.5149626588 \\
\hline C & 10.8763230335 & 1.3185150464 & 5.0087392473 \\
\hline $\mathrm{H}$ & 10.4042980183 & 2.1540069328 & 4.4620912772 \\
\hline $\mathrm{H}$ & 11.9030020940 & 1.6390785308 & 5.2705940614 \\
\hline C & -0.2294704657 & -2.4256920672 & 0.9797218899 \\
\hline $\mathrm{H}$ & -0.7862742434 & -3.3423690481 & 0.7783598460 \\
\hline C & -1.6254421667 & 2.9375076649 & 3.8410532851 \\
\hline $\mathrm{H}$ & -1.4552959606 & 4.0020702718 & 3.6784866159 \\
\hline C & 1.1499116946 & -0.1233243086 & 1.5799324594 \\
\hline $\mathrm{H}$ & 1.6816914589 & 0.7804787595 & 1.8814346408 \\
\hline C & -2.9149140242 & 2.3973268962 & 3.8260348585 \\
\hline $\mathrm{H}$ & -3.7836283518 & 3.0327156563 & 3.6481988177 \\
\hline C & -0.1427680271 & -1.4439530710 & 6.9775071930 \\
\hline C & 10.0256332394 & -4.6675383489 & 3.1943818813 \\
\hline $\mathrm{H}$ & 9.5542126144 & -5.5845337239 & 2.8398589009 \\
\hline C & -0.4691284246 & -2.5237231251 & 5.9770831300 \\
\hline $\mathrm{H}$ & 0.4057501914 & -3.1859049025 & 5.8845153548 \\
\hline $\mathrm{H}$ & -1.3330674675 & -3.1332301612 & 6.2921507818 \\
\hline C & 0.9430679220 & 0.6048332830 & 7.2642759281 \\
\hline $\mathrm{H}$ & 1.4938264884 & 1.4137569229 & 6.7817508779 \\
\hline C & 10.3611230314 & 1.7347033346 & 0.7549607051 \\
\hline $\mathrm{H}$ & 11.3588274127 & 2.1294617266 & 0.5540813208 \\
\hline C & 0.0012305237 & -2.0278141536 & 2.2939962268 \\
\hline C & -3.0735259083 & 1.0272279133 & 4.0419636322 \\
\hline $\mathrm{H}$ & -4.0650499300 & 0.5705453156 & 4.0375684161 \\
\hline C & 10.3748899680 & 1.6185202302 & 7.4924929842 \\
\hline $\mathrm{H}$ & 11.2252444446 & 2.2971952285 & 7.5806537365 \\
\hline C & 11.9513348560 & -3.3889958411 & 3.8559484639 \\
\hline $\mathrm{H}$ & 13.0248292153 & -3.2903899255 & 4.0275165166 \\
\hline C & 0.2641166556 & -1.6378555472 & -0.0654089417 \\
\hline $\mathrm{H}$ & 0.0959758686 & -1.9298164762 & -1.1030636884 \\
\hline C & 9.2897675608 & 2.0292814447 & -0.0886465045 \\
\hline $\mathrm{H}$ & 9.4341055217 & 2.6646170741 & -0.9641942639 \\
\hline C & 7.8890219069 & 0.7242484474 & 1.3565907383 \\
\hline $\mathrm{H}$ & 6.9182092850 & 0.3272443550 & 1.6557098987 \\
\hline C & 8.5074792839 & 0.4327866438 & 8.4414830920 \\
\hline $\mathrm{H}$ & 7.8658889717 & 0.1639233236 & 9.2809284239 \\
\hline
\end{tabular}




\begin{tabular}{|c|c|c|c|}
\hline C & 0.9664556247 & -0.4710601770 & 0.2425294481 \\
\hline $\mathrm{H}$ & 1.3633234405 & 0.1739446496 & -0.5418522815 \\
\hline C & 11.4043766090 & -4.5900482487 & 3.3956159539 \\
\hline $\mathrm{H}$ & 12.0466913070 & -5.4494601532 & 3.1963431192 \\
\hline C & -2.0361024079 & -1.2455242225 & 4.5350048456 \\
\hline $\mathrm{H}$ & -2.5830231159 & -1.4092536073 & 5.4772754204 \\
\hline $\mathrm{H}$ & -2.6224684533 & -1.7387669574 & 3.7435858932 \\
\hline C & 8.0310078578 & 1.5025319330 & 0.2113878190 \\
\hline $\mathrm{H}$ & 7.1656714612 & 1.7052467380 & -0.4199098677 \\
\hline C & -0.4110761324 & -2.8255143837 & 3.5048034799 \\
\hline $\mathrm{H}$ & -1.2706029894 & -3.4839390548 & 3.2933896309 \\
\hline $\mathrm{H}$ & 0.4402323417 & -3.4562383867 & 3.8051817623 \\
\hline C & 9.5773292285 & 1.3181232479 & 8.5986853610 \\
\hline $\mathrm{H}$ & 9.7932985026 & 1.7629980776 & 9.5714458833 \\
\hline C & 0.6633353340 & 0.6012748833 & 8.6300760277 \\
\hline H & 1.0002735843 & 1.4314866140 & 9.2514049359 \\
\hline C & -0.4674822904 & -1.5027877986 & 8.3299690212 \\
\hline $\mathrm{H}$ & -1.0344294925 & -2.3509602802 & 8.7173653594 \\
\hline C & -0.0552190975 & -0.4653957537 & 9.1734041041 \\
\hline $\mathrm{H}$ & -0.2980617786 & -0.4895827011 & 10.2367636962 \\
\hline 5-dis & & & \\
\hline \multicolumn{4}{|c|}{ ATOMIC_POSITIONS (angstrom) } \\
\hline $\mathrm{Fe} 2$ & 16.0885556728 & 6.0408079334 & 14.6007488407 \\
\hline $\mathrm{Fe} 2$ & 8.1772956053 & 6.4115679266 & 14.8656835664 \\
\hline 0 & 14.4229138929 & 7.5230741992 & 14.6987471123 \\
\hline 0 & 14.3194147065 & 4.7955977726 & 14.7347282326 \\
\hline 0 & 9.6898584573 & 7.6355385148 & 14.7752851549 \\
\hline 0 & 9.5993006092 & 5.0518292999 & 14.9754426954 \\
\hline $\mathrm{N}$ & 16.1066880381 & 6.2573946858 & 12.5986522949 \\
\hline $\mathrm{N}$ & 6.6830692752 & 5.0875833440 & 14.9759550659 \\
\hline $\mathrm{N}$ & 17.6931014389 & 7.3740356043 & 14.5014188068 \\
\hline $\mathrm{N}$ & 8.0249792686 & 6.4454266752 & 16.8132067629 \\
\hline $\mathrm{N}$ & 8.0545649386 & 6.1018400573 & 12.9390419467 \\
\hline $\mathrm{N}$ & 6.8004705945 & 7.7524113023 & 14.7128043957 \\
\hline $\mathrm{N}$ & 16.3880658681 & 6.2431088412 & 16.5801676749 \\
\hline $\mathrm{N}$ & 17.4737922853 & 4.6704313843 & 14.4517829703 \\
\hline C & 10.7892930409 & 5.5492513351 & 14.8911785237 \\
\hline C & 13.2659596362 & 6.9689061055 & 14.7388276369 \\
\hline C & 18.7580828661 & 5.1008396469 & 14.3326202822 \\
\hline C & 17.1934860181 & 7.2943504217 & 16.9109771486 \\
\hline C & 17.2190779877 & 3.3388883335 & 14.3875579009 \\
\hline $\mathrm{H}$ & 16.1678465633 & 3.0622245992 & 14.4884583190 \\
\hline C & 10.8456549615 & 7.0478576768 & 14.7939526768 \\
\hline C & 11.9642713734 & 4.7937107436 & 14.8788313532 \\
\hline $\mathrm{H}$ & 11.9225846961 & 3.7048538752 & 14.9304911138 \\
\hline C & 12.0709202166 & 7.7109825818 & 14.7360685354 \\
\hline $\mathrm{H}$ & 12.1108653874 & 8.7999041826 & 14.6845331578 \\
\hline C & 18.9783489943 & 6.5931991079 & 14.4620773480 \\
\hline $\mathrm{H}$ & 19.5346359435 & 6.7883355662 & 15.3929624023 \\
\hline $\mathrm{H}$ & 19.6103714999 & 6.9566206307 & 13.6361428383 \\
\hline C & 5.5240639237 & 7.2927182474 & 14.7209676035 \\
\hline C & 16.9009949610 & 7.2766212854 & 12.1614722093 \\
\hline C & 5.3615289932 & 5.8052945723 & 14.9285571226 \\
\hline $\mathrm{H}$ & 4.8241805692 & 5.6277020057 & 15.8738340878 \\
\hline $\mathrm{H}$ & 4.7362588646 & 5.3781112977 & 14.1285204343 \\
\hline C & 7.0185049896 & 9.0807218008 & 14.5550132768 \\
\hline $\mathrm{H}$ & 8.0665873184 & 9.3831829303 & 14.5567480471 \\
\hline C & 13.2053047780 & 5.4423164298 & 14.7842347860 \\
\hline
\end{tabular}




\begin{tabular}{|c|c|c|c|}
\hline C & 18.2279910390 & 2.4004055688 & 14.2002247504 \\
\hline $\mathrm{H}$ & 17.9758593023 & 1.3407642174 & 14.1551546709 \\
\hline C & 17.5734582581 & 8.1900599591 & 15.7516294741 \\
\hline $\mathrm{H}$ & 16.7514656122 & 8.9053580885 & 15.5892323063 \\
\hline $\mathrm{H}$ & 18.4932719051 & 8.7618375338 & 15.9622039952 \\
\hline C & 17.4588267341 & 8.1642712889 & 13.2526290820 \\
\hline $\mathrm{H}$ & 18.3702721430 & 8.6904874495 & 12.9219574130 \\
\hline $\mathrm{H}$ & 16.6952832458 & 8.9214748266 & 13.4925982398 \\
\hline C & 7.2919866700 & 5.4140280613 & 17.3170212369 \\
\hline C & 6.8914604839 & 4.3923760468 & 16.2831709952 \\
\hline $\mathrm{H}$ & 7.7316902119 & 3.6944037679 & 16.1442585563 \\
\hline $\mathrm{H}$ & 6.0057638451 & 3.8126137075 & 16.5940004722 \\
\hline C & 17.1126325978 & 7.5101823971 & 10.8045995062 \\
\hline $\mathrm{H}$ & 17.7626244823 & 8.3280324866 & 10.4889525403 \\
\hline C & 7.0354768627 & 4.6752474539 & 11.2895298992 \\
\hline $\mathrm{H}$ & 6.4262304921 & 3.7981643159 & 11.0649445399 \\
\hline C & 5.9756676675 & 9.9877595137 & 14.3982959929 \\
\hline $\mathrm{H}$ & 6.1980089792 & 11.0478748989 & 14.2742405813 \\
\hline C & 8.5485045602 & 6.8753506648 & 11.9485717057 \\
\hline $\mathrm{H}$ & 9.1348589966 & 7.7364264874 & 12.2729545880 \\
\hline C & 6.9923849492 & 5.3186664634 & 18.6731484623 \\
\hline $\mathrm{H}$ & 6.3909793647 & 4.4854653489 & 19.0405415301 \\
\hline C & 8.3229212041 & 6.5898511998 & 10.6028981637 \\
\hline $\mathrm{H}$ & 8.7414460321 & 7.2420997134 & 9.8359532815 \\
\hline C & 16.2750480647 & 5.6275263137 & 18.9010637524 \\
\hline $\mathrm{H}$ & 15.8890794568 & 4.9458783061 & 19.6594041222 \\
\hline C & 17.1094067176 & 6.6936140597 & 19.2406538260 \\
\hline $\mathrm{H}$ & 17.3980390825 & 6.8665334664 & 20.2785514731 \\
\hline C & 16.4793147434 & 6.6911990521 & 9.8646862557 \\
\hline $\mathrm{H}$ & 16.6318288544 & 6.8577010193 & 8.7972298646 \\
\hline C & 7.5524134284 & 5.4754620676 & 10.2651939246 \\
\hline $\mathrm{H}$ & 7.3500986240 & 5.2333981871 & 9.2207974200 \\
\hline C & 7.4741572620 & 6.3002569397 & 19.5461204668 \\
\hline $\mathrm{H}$ & 7.2517648109 & 6.2470144444 & 20.6128741175 \\
\hline C & 8.2353744826 & 7.3496873614 & 19.0279545326 \\
\hline $\mathrm{H}$ & 8.6275343071 & 8.1366611092 & 19.6726968361 \\
\hline C & 4.6592294946 & 9.5168247992 & 14.3994930250 \\
\hline $\mathrm{H}$ & 3.8210658373 & 10.2036633650 & 14.2739533014 \\
\hline C & 6.8809463794 & 4.1864495144 & 13.8007909431 \\
\hline $\mathrm{H}$ & 5.9841505854 & 3.5819666603 & 13.5830410766 \\
\hline $\mathrm{H}$ & 7.7042553988 & 3.5018500206 & 14.0581220968 \\
\hline C & 17.5771222577 & 7.5370401209 & 18.2274276436 \\
\hline $\mathrm{H}$ & 18.2317823289 & 8.3797313711 & 18.4560978292 \\
\hline C & 15.9407758857 & 5.4305349822 & 17.5619414611 \\
\hline $\mathrm{H}$ & 15.2983682836 & 4.6111137122 & 17.2342820802 \\
\hline C & 19.8127679495 & 4.2094650642 & 14.1342355786 \\
\hline $\mathrm{H}$ & 20.8317405333 & 4.5882712950 & 14.0346917896 \\
\hline C & 4.4339214521 & 8.1488082428 & 14.5636313016 \\
\hline $\mathrm{H}$ & 3.4196263615 & 7.7452426037 & 14.5702899780 \\
\hline C & 7.3112981803 & 5.0087424417 & 12.6129356878 \\
\hline C & 19.5481905538 & 2.8406839853 & 14.0669384953 \\
\hline $\mathrm{H}$ & 20.3599226925 & 2.1286051802 & 13.9115668426 \\
\hline C & 15.4993817772 & 5.4673216174 & 11.6867337404 \\
\hline $\mathrm{H}$ & 14.8774089689 & 4.6704093403 & 12.0986313164 \\
\hline C & 15.6562145955 & 5.6574203315 & 10.3147599038 \\
\hline $\mathrm{H}$ & 15.1449021388 & 4.9948732701 & 9.6160059056 \\
\hline C & 8.4866799117 & 7.3927414663 & 17.6573578969 \\
\hline $\mathrm{H}$ & 9.0681227254 & 8.1913289120 & 17.1940602072 \\
\hline
\end{tabular}

